\title{
Texturentwicklung in Zwei-Phasen Strömungen
}

\author{
Dissertation \\ zur Erlangung des Doktorgrades \\ der Mathematischen-Naturwissenschaftlichen Fakultäten \\ der Georg-August-Universität zu Göttingen
}

vorgelegt von

Ulf Garbe

aus Höxter

Göttingen 2005 
Referentin/Referent: Prof. Dr.W. F. Kuhs

Korreferentin/Korreferent: Prof. Dr. R.A. Schwarzer

Tag der mündlichen Prüfung: 17.03.2005 


\section{Inhaltsverzeichnis}

$\begin{array}{ll}\text { Einleitung } & 7\end{array}$

1 Beschreibung von Strömungen $\quad 9$

1.1 Fluide und Strömung . . . . . . . . . . . . . . . . . 9

1.2 Laminare und Turbulente Strömung . . . . . . . . . . . . . 9

1.2.1 Laminare Couette-Strömung . . . . . . . . . . . . . 9

1.2.2 Laminare Rohrströmung . . . . . . . . . . . . . . . . . . 11

1.2.3 Mechanische Ähnlichkeit von Strömungen . . . . . . . . . 12

1.2.4 Turbulent strömende Flüssigkeiten . . . . . . . . . . . . . 13

1.3 Ein Teilchen in der Strömung . . . . . . . . . . . . . . . . . . . 14

1.3.1 Eine Kugel in der Strömung . . . . . . . . . . . . . . 15

1.3.2 Gleichgewichtsorientierung eines einzelnen Teilchens . . . 15

1.3.3 Gleichgewichtsposition von festen Teilchen in einer Strömung . . . . . . . . . . . . . . . . 19

1.4 Das Vielteilchenproblem . . . . . . . . . . . . . . . . 20

1.4.1 Der Ort und die Geschwindigkeit von Partikeln . . . . . . 20

1.4.2 Orientierungsverteilung von Partikeln . . . . . . . . . 22

1.4.3 Bestimmung der Partikelorientierung mittels Diffraktion . 25

2 Texturanalyse $\quad 29$

2.1 Die Texturbestimmung . . . . . . . . . . . . . . . . . . . . . 29

2.2 Die Polfigurmessung . . . . . . . . . . . . . . . . . . . . . . 29

2.2.1 Die Beamline BW5 . . . . . . . . . . . . . . . 32

2.2.1.1 Vorversuche . . . . . . . . . . . . 34

2.2.2 Polfigurmessung am BW5 . . . . . . . . . . . . . . 35

2.3 Polfigursoftware . . . . . . . . . . . . . . . . . 38

2.3.1 Berechnung der integrale Intensität . . . . . . . . . 38

2.3.2 Korrektur des beugenden Volumens . . . . . . . . . . 40

2.3.3 Korrektur der Primärstrahlintensität . . . . . . . . . . . 40

2.3.4 Die Absorptionskorrektur . . . . . . . . . . . . . . 41

2.3.5 Interpolation des Polfigurrasters . . . . . . . . . . 45

2.4 Die Textur . . . . . . . . . . . . . . . . . . 46

2.5 Die Orientierungsverteilungsfunktion . . . . . . . . . . . 47

2.5.1 Orientierungsverteilungen . . . . . . . . . . . 47

2.5 .2 Polfiguren . . . . . . . . . . . . . . . . 48

2.5.3 Berechnung der ODF mittels Reihenentwicklung . . . . . 49 
2.5.4 Konvergenz der Reihenentwicklung . . . . . . . . . . 51

2.5.5 Fehler der ODF-Berechnung . . . . . . . . . . . 52

3 Die kristallinen Partikel $\quad 55$

3.1 Das Muskovitpulver . . . . . . . . . . . . . . . . 55

3.1.1 Lichtmikroskopische Bestimmung der Partikel . . . . . . . 56

3.2 Die industriellen Aluminiumoxidpulver . . . . . . . . . . . . 58

3.2.1 Elektronenmikroskopische Bestimmung der Aluminiumoxidpartikel . . . . . . . . . . . . . . . 59

3.2.1.1 Charakterisierung der Proben A, B und C . . 59

3.2.1.2 Charakterisierung der Proben HVAFG, CTC20 und Gilox . . . . . . . . . . . . . 61

4 Konstruktion der Küvetten $\quad 63$

4.1 Die Rotationsküvette . . . . . . . . . . . . . . . . . . 63

4.2 Die Durchflussküvette . . . . . . . . . . . . . . . . . 65

5 Orientierung der Partikel $\quad 69$

5.1 Muskovitpartikel in Glyzerin . . . . . . . . . . . . . . 69

5.1.1 Muskovit-/Glyzeringemisch in der Rotationsküvette . . . 71

5.1.2 Muskovit-/Glyzeringemische in der Durchflussküvette . . 77

5.2 Aluminiumoxidpartikel in Glyzerin . . . . . . . . . . . . . 80

5.2.1 Ermittlung der Probensymmetrie . . . . . . . . . . . 81

5.2.2 Ermittlung des Reihenentwicklungsgrades . . . . . . . . . 85

5.2.3 Vergleich der rückgerechneten Polfiguren mit den Gemessenen . . . . . . . . . . . . . 88

5.2.4 Die Proben A,B und C in der Rotationsküvette . . . . . 95

5.2.4.1 Partikelform . . . . . . . . . . . 95

5.2.4.2 Strömungsgeschwindigkeit . . . . . . . . . . . 99

5.2.4.3 Feststoffkonzentration . . . . . . . . . . . 101

5.2.4.4 Zusammenfassung . . . . . . . . . . . . . 102

5.2.5 Die Proben HVAFG, CTC20 und GILOX in der Rotati-

onsküvette . . . . . . . . . . . . . . 103

5.2.5.1 Partikelform . . . . . . . . . . . . . . 104

5.2.5.2 Strömungsgeschwindigkeit . . . . . . . . . . . 108

5.2.5.3 Feststoffkonzentration . . . . . . . . . . . . . 109

5.2.5.4 Zusammenfassung . . . . . . . . . . . . . . 112

5.2.6 Die Proben HVAFG, CTC20 und Gilox in der Durchflussküvette . . . . . . . . . . . . . . . . 113

5.2.6.1 Partikelform . . . . . . . . . . . . . . . 114

5.2.6.2 Strömungsgeschwindigkeit . . . . . . . . . . . . . . . . . . . . . . . . .

5.2.6.3 Feststoffkonzentration . . . . . . . . . . . . . . 119

5.2.6.4 Zusammenfassung . . . . . . . . . . . . . 121

6 Diskussion 
$\begin{array}{ll}\text { Abbildungsverzeichnis } & 146\end{array}$

$\begin{array}{lr}\text { A Diffraktionsdaten } & 147\end{array}$

A.1 Pulverdaten für Muskovit . . . . . . . . . . . . . . . . . 147

A.2 Pulverdaten für Aluminiumoxid . . . . . . . . . . . . . . . . 148

$\begin{array}{lr}\mathrm{B} \text { Rotationsküvette }(\mathrm{A}, \mathrm{B}, \mathrm{C}) & 149\end{array}$

B.1 Probe A . . . . . . . . . . . . . . . . . 150

B.2 Probe B . . . . . . . . . . . . . . . 156

B.3 Probe C . . . . . . . . . . . . . . . . . . . 168

C Rotationsküvette (HVAFG, CTC20, Gilox) 175

C.1 Probe HVAFG 26Vol\% . . . . . . . . . . . . . . . 176

C.2 Probe HVAFG 13Vol\% . . . . . . . . . . . . . . . . . 182

C.3 Probe HVAFG 9Vol\% . . . . . . . . . . . . . . . 186

C.4 Probe CTC20 26Vol\% . . . . . . . . . . . . . . . . . . 192

C.5 Probe CTC20 13Vol\% . . . . . . . . . . . . . . . . 198

C.6 Probe CTC20 9Vol\% . . . . . . . . . . . . . . . . . . . 204

C.7 Probe Gilox 13Vol\% . . . . . . . . . . . . . . . . . . 210

D Durchflussküvette (HVAFG, CTC20, GILOX) 217

D.1 Probe HVAFG 10Vol\% . . . . . . . . . . . . . . . 218

D.2 HVAFG 12,5 Vol\% $\ldots \ldots \ldots \ldots \ldots \ldots$

D.3 Probe CTC20 7,5Vol\% . . . . . . . . . . . . . . 238

D.4 Probe CTC20 10Vol\% . . . . . . . . . . . . . . . . 248

D.5 Probe CTC20 12,5Vol\% . . . . . . . . . . . . . 258

D.6 Probe Gilox $7,5 \mathrm{Vol} \% \ldots \ldots \ldots \ldots$

D.7 Probe Gilox 10Vol\% . . . . . . . . . . . . . . . 278

D.8 Probe Gilox $12,5 \mathrm{Vol} \% \ldots \ldots$. . . . . . . . . . . . . . 288 
INHALTSVERZEICHNIS 


\section{Einleitung}

Strömungen begegnen uns alltäglich in der Natur. In fast allen Lebensbereichen, sowohl bei komplizierten technischen Anwendungen als auch bei Dingen des täglichen Lebens, lässt sich das unterschiedliche Strömungsverhalten von Flüssigkeiten und Gasen beobachten. Eine besondere Form der Strömung ist die Zwei-Phasen-Strömung. Sie entsteht, wenn ein Gemisch aus Feststoff und Flüssigkeit/Gas in Bewegung gerät. Hierbei ist ein besonderes Augenmerk auf die Bewegung der festen Phase gerichtet, wie sie z.B. bei granularen Schüttungen zu beobachten ist. Für solche Schüttungen wurden anhand von Reishaufen $[1,2,3]$ Modelle entwickelt um Lawinenabgänge vorzusagen.

Eine weitere Art der Zwei-Phasen-Strömung tritt auf, wenn eine Flüssigkeit zum Transport von Feststoff verwendet wird. Dies geschieht bei der Grünformung von Keramiken, sowie auch bei Erzschlämmen in Minen. Auch bei einigen geologischen Fragestellungen, wie z.B. Sedimentation [4], spielt das Verhalten der Zwei-Phasen-Gemische eine wichtige Rolle.

Jede dieser Strömungen zeichnet sich durch eine Vielzahl charakteristischer Eigenschaften (Strömungsgeschwindigkeiten, Feststoffkonzentration, Partikelform, Partikelgröße) aus. Jede dieser Eigenschaften lässt sich teilweise leicht mit gravimetrischen und mikroskopischen Methoden bestimmen. Aus dem Zusammenspiel dieser Eigenschaften entsteht in Gemischen aus einer flüssigen Phase und einer kristallinen Phase eine charakteristische Orientierung der Partikel, die Textur [5]. Da die physikalischen Eigenschaften von polykristallinen Materialien durch die Textur beeinflusst werden, ist die Texturanalyse von großem industriellem Interesse.

Eine strömende viskose Flüssigkeit bildet ein Strömungsprofil aus, in dem die kristallinen Teilchen Scherkräfte durch die Flüssigkeit erfahren. Dies führt zu unterschiedlichen Partikelgeschwindigkeiten und zur Partikelrotation. Durch die Formanisotropie der Partikel nehmen diese Vorzugsorientierungen ein bei denen möglichst geringen Scherkräfte auf die Partikel einwirken. Für einfache rotationsellipsoide Partikel in einer Strömung wurden von Jeffery [6] und Anczurowski et al. [7] theoretische Modelle entwickelt. Komplexere Teilchenformen und Vielteilchen-Systeme wurden durch theoretische Arbeiten von Batchelor [8, 9] und Herczyński et al. [10] beschrieben.

Um das Verhalten der festen Teilchen in der Strömung zu untersuchen, wurde in vielen Fällen mittels Lichtstreuung [11] die Position, die Geschwindigkeit oder die Orientierung der Teilchen bestimmt. Diese Methode stößt jedoch bei hohen Feststoffkonzentrationen aufgrund der Lichtundurchlässigkeit der Gemische an ihre Grenzen. Auch an komplexen Teilchenformen kann über die Licht- 
streuung die Orientierung nur schwer bestimmt werden.

Eine Weiterentwicklung der Messmethoden ermöglicht die Untersuchung strömender Zwei-Phasen-Gemische mittels NMR (nuclear magnetic resonance) $[12,13]$ und SANS (small angle neutron scattering) [14]. Durch NMR-Experimente wurde die Position und Geschwindigkeit der Partikel bei unterschiedlichen Strömungsverhältnissen bestimmt. Mit Hilfe die Kleinwinkelstreuung konnten erste Aussagen über die Orientierung der Partikel in konzentrierten Suspensionen gemacht werden. Eine vollständige Beschreibung der Orientierung ist aber auch mit dieser Methode nicht möglich.

Röntgenographische Texturbestimmungen an Zwei-Phasen-Gemischen wurden von Böcker [15] durchgeführt. Es wurden im Rahmen der Arbeit die Einflüsse von Kornform und Viskosität der flüssigen Phase auf die Textur keramischer Produkte bestimmt. Die Methode war allerdings nur geeignet um Grünlinge nach vollendet Formgebung zu untersuchen.

Erst die modernen Hochenergie Synchrotron Messplätze, wie z.B. BW5 und Petra 2 am HASYLAB at DESY [16, 17] oder ID15A/B an der ESRF [18], geben die Möglichkeit mittels Diffraktion die Orientierung kristalliner Partikel in einer strömenden Flüssigkeit zu ermitteln. Aufgrund der hohen Strahlenergie (60-120 keV) ist es möglich die strömenden Gemische direkt in der Probenumgebung (Küvette) messen. Über dies hinaus lassen sich durch den Einsatz von Flächendetektoren [19, 20, 21] die bisherigen Methoden der röntgenographischen Texturmessung deutlich verbessern. Im Bereich der klassischen Texturmessung verkürzt sich die Messzeit deutlich, da die Proben zur Polfigurmessung nur noch um eine Achse gedreht werden muss, welches die Texturbestimmung an strömenden Flüssigkeiten erst möglich macht. Bei einer Drehung um zwei Achsen würden die Strömungsküvetten zeitweise auf dem Kopf stehen und auslaufen.

Diffraktion an Kaolinitpartikeln in Wasser mittels Synchrotronstrahlung wurde von Barè et al. für Rohrströmungen bei unterschiedlichen Orientierungen durchgeführt. Die Untersuchungen waren jedoch auf einige wenige Orientierungen beschränkt. In dieser Arbeit soll zum ersten Mal eine Polfigurmessung mit anschließender 3-dimensionaler Texturanalyse an strömenden Zwei-PhasenGemischen mit Hochenergetischer Synchrotronstrahlung durchgeführt werden. Die kristalline Phase bildeten Muskovit als Modellsubstanz und $\mathrm{Al}_{2} \mathrm{O}_{3}$ als werkstoffkundlich relevantes Beispiel.

Die vollständige Orientierungsbestimmung während des Strömungsvorgangs soll erstmals einen detaillierten Einblick in die Abhängigkeiten der Texturentwicklung von kristallinen Partikeln von unterschiedlichen Parametern, wie Strömungsgeschwindigkeit, Partikelform, Feststoffkonzentration und Strömungsprofil geben.

Hierzu wurde der Messplatz BW5 des HASYLAB zur Polfigurmessung [19] weiterentwickelt und der Einsatz eines MAR345 Flächendetektors erarbeitet. Außerdem wurden zwei unterschiedliche Küvetten konstruiert, welche eine Scherströmung und eine Rohrströmung erzeugen. Mit diesen Mitteln wurde die Texturbestimmung von der Einzelmessung über die Polfigur bis zur 3-dimensionalen Texturanalyse vollendet. 


\section{Kapitel 1}

\section{Beschreibung von Strömungen}

\subsection{Fluide und Strömung}

Als Fluide versteht man, im Gegensatz zu Festkörpern, Substanzen, die in Ruhelage Scherkräften nicht widerstehen können [22]. Dies gilt für Flüssigkeiten und Gase. Sind Fluide in Bewegung (Strömung), kann die Strömungsgeschwindigkeit jedes einzelnen Fluidteilchens am Ort $r$ durch einen Geschwindigkeitsvektor $v$ beschrieben werden. Die gesamte Strömung wird durch die Menge aller Vektoren, das Vektorfeld $v(r)$ beschrieben. Hängt dieses Vektorfeld nicht von der Zeit ab, heißt die Strömung stationär [23].

Die untersuchten Gemische bestehen aus einer Flüssigkeit und einem kristallinen Pulver. In diesen strömenden Gemischen ist die Dichte der einzelnen Phasen überall gleich, da der Druck sich während der gesamten Zeit nicht stark genug ändert, um dieses Gemisch zu komprimieren. Solche Strömungen mit konstanter Dichte heißen inkompressibel.

\subsection{Laminare und Turbulente Strömung}

Alle realen Flüssigkeiten besitzen eine gewissen "Zähigkeit", die sich als innere Reibung bei der Formänderung äußert.

Eine Strömung, deren Verhalten durch die innere Reibung bestimmt wird, heißt laminare Strömung. Betrachtet man ein strömendes Fluid als ein System aus vielen Schichten, so gleiten die einzelnen Schichten übereinander ohne sich zu vermischen. Verwirbeln die einzelnen Schichten miteinander, spricht man von turbulenten Strömungen [22].

\subsubsection{Laminare Couette-Strömung}

Die Orientierung von Partikel in einer laminaren Couette-Strömung stellt einen Teil dieser Arbeit dar.

Befindet sich eine Flüssigkeit mit der Schichtdicke $h$ zwischen einer festen Wand und einer beweglichen Platte, kann eine Scherströmung durch die bewegliche Platte erzeugt werden (Abb. 1.1). Ist der Abstand zwischen der ruhenden und der beweglichen Platte kleiner als die laminare Grenzschichtdicke (Kap. 
1.2.4) stellt sich ein lineares Geschwindigkeitsprofil ein. Diese Strömung wird gewöhnlich als Couette-Strömung bezeichnet. Die Strömungsgeschwindigkeit $v_{x}$ in Richtung $x$ an der Position $z$ wird bestimmt durch,

$$
v_{x}=v_{x, 0} *(z / h) .
$$

Die unmittelbar an der bewegten Platte befindliche Schicht hat dieselbe Geschwindigkeit $\left(v_{x, 0}\right)$ wie die Platte selber [24].

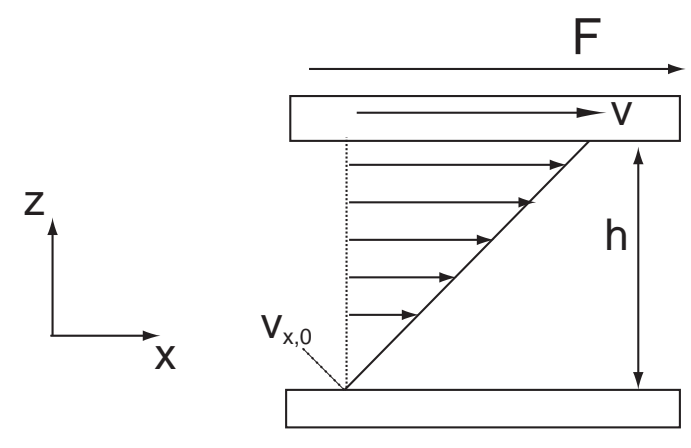

Abbildung 1.1: Scherströmung zwischen einer festen Wand und einer bewegten Platte

Um die Platte mit einer Fläche $A$ mit konstanter Geschwindigkeit $v$ parallel zur Wand zu verschieben, braucht man eine Kraft $F$.

$$
\mathbf{F}=\eta A \frac{v}{h}
$$

Die Viskosität $\eta$, ist eine physikalische Eigenschaft der Fluide. Diese verursacht Reibungskräfte, die auf das Fluid einwirken und dafür sorgen, dass bewegte Fluide auch ohne Einwirkung äußerer Kräfte zur Ruhe kommen [23].

Durch die Adhäsionskraft kann der Verlauf des Geschwindigkeitsprofils vom linearen Verhalten abweichen. Ist die Scherkraft zwischen der beweglichen Platte und der Flüssigkeit deutlich stärker als die Adhäsionskraft, kann die Flüssigkeit selber als Scheibe beschrieben werden. Diese bewegt sich dann mit halber Geschwindigkeit der beweglichen Platte in die gleich Richtung (Abb. 1.2) [25].

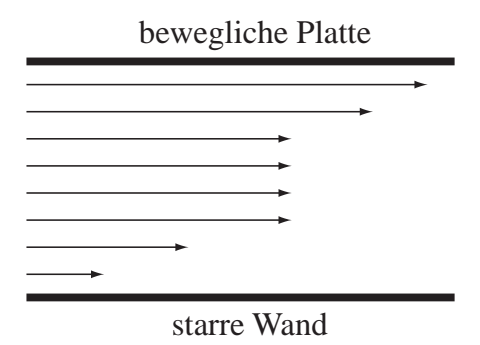

Abbildung 1.2: Geschwindigkeitsverteilung einer Scherströmung für eine Flüssigkeit mit schwachen Adhäsionskräften [25] 


\subsubsection{Laminare Rohrströmung}

Eine weitere Art von Strömungen, welche in der vorliegenden Arbeit ebenfalls auftritt, ist die laminare Rohrströmung. Wenn eine Flüssigkeit durch ein Druckgefälle angetrieben wird und in einem geraden Rohr mit Kreisquerschnitt strömt, bildet sich ein Geschwindigkeitsprofil wie in Abbildung 1.3 aus [24].

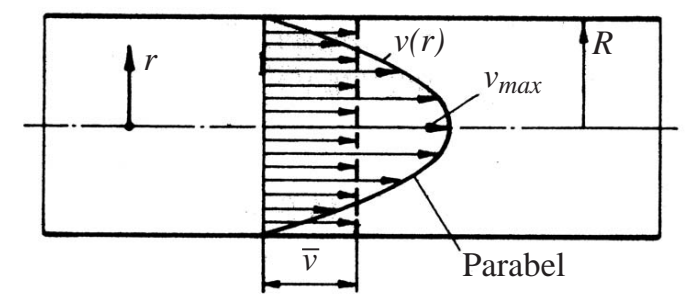

Abbildung 1.3: Geschwindigkeitsverlauf $v=v(r)$ bei laminarer Rohrströmung [24]

An der Wand haftet die Flüssigkeit und besitzt somit die Geschwindigkeit $v=0$. In der Mitte der beiden Wände ist die Strömungsgeschwindigkeit entsprechend am höchsten $\left(v_{\max }\right)$. Mit Hilfe der kinematischen Viskosität, dem Verhältnis von Viskosität $\eta$ und Dichte $\varrho$,

$$
\nu=\frac{\eta}{\varrho}
$$

lässt sich mit der spezifische Verlustenergie $Y_{v}$ und der Benetzungslänge $L$ die Geschwindigkeit $v(r)$ am Punkt $r$ nach dem Gesetz von Stokes berechnen. Sie ist definiert für Rohre mit einem Radius $R$.

$$
v(r)=\frac{Y_{v}}{4 \nu L}\left(R^{2}-r^{2}\right)
$$

Das in Gleichung 1.4 beschriebene Geschwindigkeitsprofil gilt nur für vollständig ausgebildete Rohrströmungen. Diese wird bei einem Rohr mit konstantem Querschnitt jedoch erst nach einer gewissen Anlaufstrecke erreicht (Abb. 1.4). Näherungsweise kann die Strömungsgeschwindigkeit beim Rohreinlauf $(\bar{v})$ über den gesamten Eintrittsquerschnitt als konstant angenommen werden.

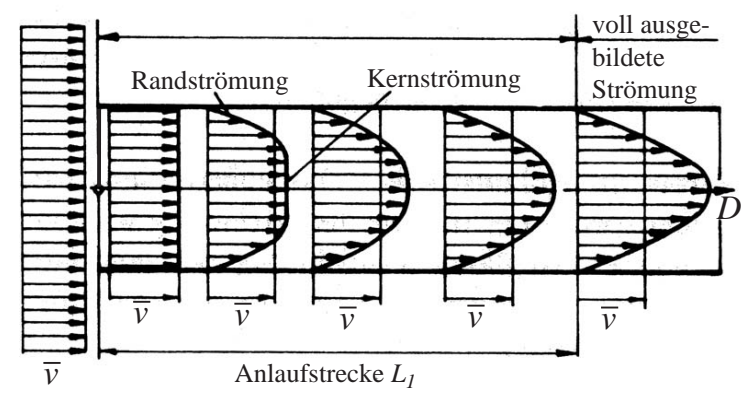

Abbildung 1.4: Anlaufströmung [24] 
Die Berechnung der laminaren Anlaufstrecke mit der Länge $L_{1}$ folgt in Abhängigkeit vom Durchmesser $D$ und der Reynoldszahl $R_{e}$ (Kap. 1.2.3):

$$
L_{1} / D=0,06 * R_{e}
$$

\subsubsection{Mechanische Ähnlichkeit von Strömungen}

Ein Kriterium für die mechanische Ähnlichkeit von Strömungen ist die Reynoldssche Zahl $R_{e}$. Strömungen die sich in ihren Abmessungen, Geschwindigkeiten oder Art der Flüssigkeiten unterscheiden, sind bei gleicher $R_{e}$-Zahl mechanisch ähnlich. Dies gibt die Möglichkeit schwer zu fassende Problemstellungen, wie das Tankerunglück in Abbildung 1.5 a), als Modell nachzuvollziehen und zu untersuchen (Abb. 1.5 b)) [26, 22].

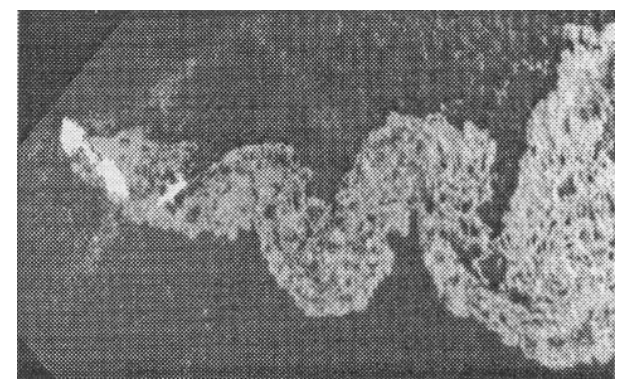

(a) Luftaufnahme eines Tankerunglücks

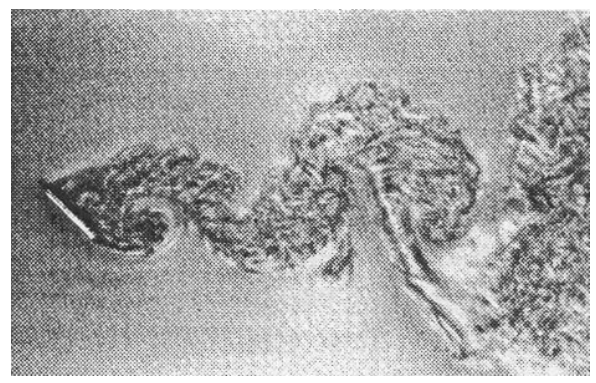

(b) Modell des Tankers in Glyzerin

Abbildung 1.5: Mechanische Ähnlichkeit von Strömungen [27]

Man spricht von mechanisch ähnlichen Strömungen, wenn in geometrisch ähnlichen Körpern die Bewegung der inkompressiblen Flüssigkeit ähnlich verläuft. Zwei verschiedene Rohrströmungen sind charakterisiert durch ihre Rohrdurchmesser $D_{1}$ und $D_{2}$ und ihre Strömungsgeschwindigkeiten $v_{1}$ bzw. $v_{2}$. Gleiches gilt für die Dichten $\left(\rho_{1}\right.$ und $\left.\rho_{2}\right)$, sowie die Viskositäten $\eta_{1}$ und $\eta_{2}$. Mechanische Ähnlichkeit gilt, wenn

$$
\frac{\rho_{1} v_{1} D_{1}}{\eta_{1}}=\frac{\rho_{2} v_{2} D_{2}}{\eta_{2}}
$$

Durch Einsetzen der kinematischen Viskosität $\nu$ (Gl. 1.3) ergibt sich die Reynoldszahl

$$
R_{e}=\frac{\rho v D}{\eta}=\frac{v D}{\nu}
$$

Für den Fall einer Strömung, welche durch eine rotierende Scheibe mit dem Radius $R$ und der Winkelgeschwindigkeit $\omega$ erzeugt wird (Kap. 4.1), gilt:

$$
R_{e}=\frac{R^{2} \omega}{\nu}
$$

Die Reynoldszahl beschreibt auch den Übergang von laminarer zur turbulenten Strömung. 
- Die laminare Strömung erreicht einen stationären Zustand. Es können zwei unterschiedliche Zustände entstehen. Entweder schmiegen sich die Stromlinien eng um Hindernisse $\left(R_{e}<1\right)$, oder es bilden sich stabile Rückströmungsgebiete $\left(R_{e}>4\right)$ aus, die zeitlich unverändert bleiben.

- Für Reynoldszahlen $\left(R_{e}>40\right)$ trennen sich die Rückströmgebiete vom Hindernis ab und bilden sich hinter dem Hindernis neu. Die Strömung wird periodisch.

- Für noch größere Reynoldszahlen werden die zeitliche Abfolge, sowie die Größe der Wirbel unregelmäßiger. Die Strömung wird quasi-periodisch.

- Bei sehr großen Reynoldszahlen bildet sich eine völlig irreguläre, chaotische Strömung aus. Die Strömung ist turbulent [22].

\subsubsection{Turbulent strömende Flüssigkeiten}

Turbulente Strömungen haben im Gegensatz zu laminaren Strömungen andere charakteristische Eigenschaften.

- Turbulente Strömungen sind instationär und im Allgemeinen dreidimensional.

- Sie sind unregelmäßig, chaotisch und unvorhersagbar.

- Der Strömungswiderstand ist nicht mehr wie im laminaren Fall, proportional zur Geschwindigkeit, sondern proportional zum Quadrat der Geschwindigkeit.

- Die Diffusionsraten sind in turbulenten Strömungen stark erhöht.

- Es treten Wirbel unterschiedlicher Größe auf.

- Durch die viskose Reibung wird ständig Energie verbraucht. Um eine turbulente Strömung aufrecht zu erhalten muss Energie zugeführt werden [22].

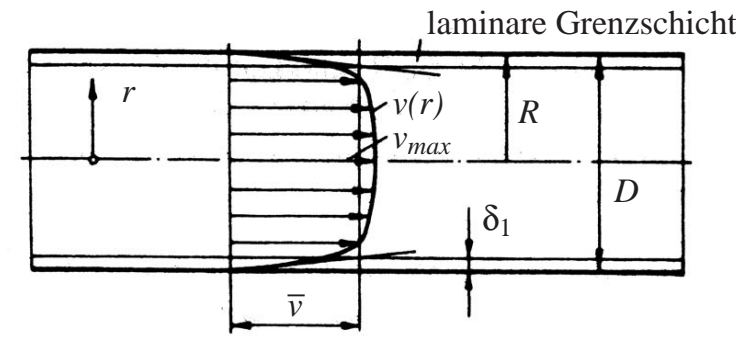

Abbildung 1.6: Geschwindigkeitsverteilung der turbulenten Strömung in einem Rohr mit dem Durchmesser D [24]

Bei Strömungen längs einer Wand wird die Turbulenzbewegung durch die Wand behindert. Die direkt an der Wand befindliche Flüssigkeitsschicht haftet, 
wie bei der laminaren Strömung, fest. Die nachfolgenden, sehr dünnen, Schichten zeigen noch laminares Verhalten. Es entsteht eine laminare Grenzschicht $\delta_{1}$. Dies beeinflusst die Geschwindigkeitsverteilung in einer Rohrströmung (Abb. 1.6). Der Gradient der Strömungsgeschwindigkeit ist deutlich kleiner als beim laminaren Verhalten (Abb. 1.3). Die mittlere Geschwindigkeit beträgt bei der turbulenten Strömung etwa $83 \%$ der Maximalgeschwindigkeit $\left(v_{\max }\right)$. Im laminaren Fall sind es nur 50\%.

Der Geschwindigkeitsverlauf lässt sich nach einem Potenzgesetz berechnen, welches im Wesentlichen empirisch ermittelt wurde.

$$
v(r)=\left(1-\frac{r}{R}\right)^{n} * v_{\max }
$$

Die Geschwindigkeit $v(r)$ an Punkt $r$ ist abhängig vom Rohrdurchmesser $R$ und der Rohrrauhigkeit. Mit zunehmender Rauhigkeit der Rohre nimmt der Exponent $n \mathrm{zu}$ [24].

\subsection{Ein Teilchen in der Strömung}

Nach der Beschreibung von strömenden einphasigen Flüssigkeiten werden nun die Modelle erweitert. Als erster Schritt eignet sich Darstellung des Verhaltens von einem Teilchen in einer strömenden Flüssigkeit um sich so dem Verhalten eines fest/flüssig Gemisches langsam anzunähern.

Ein festes Teilchen in einer strömenden Flüssigkeit erfährt unterschiedliche Kräfte. Für ein Volumenelement $d V=d x d y d z$ in einer laminar strömenden Flüssigkeit ergeben sich Reibungskräfte an den Flächen parallel zur Strömungsrichtung $y$. Gibt es einen Geschwindigkeitsgradienten senkrecht zur Strömungsrichtung (Abb. 1.7) treten an beiden Seiten des Volumenelements entgegengesetzte Reibungskräfte $F_{1}$ und $F_{2}$ auf.

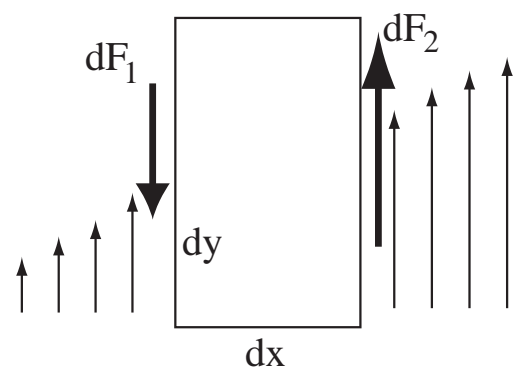

Abbildung 1.7: Volumenelement in einer inhomogenen Strömung [23]

Für die linke Seite gilt:

$$
d F_{1}=-\left.\eta \frac{\partial v}{\partial x}\right|_{l i n k s} d y d z
$$

Für die rechte Seite gilt:

$$
d F_{2}=\left.\eta \frac{\partial v}{\partial x}\right|_{\text {rechts }} d y d z
$$




\subsubsection{Eine Kugel in der Strömung}

Ist das Volumenelement eine Kugel mit dem Radius $r$ und wird diese von einer Flüssigkeit mit der Geschwindigkeit $v$ umströmt, so entsteht ein Geschwindigkeitsprofil mit der Geschwindigkeit $v=0$ direkt an der Kugel (Abb. 1.8).

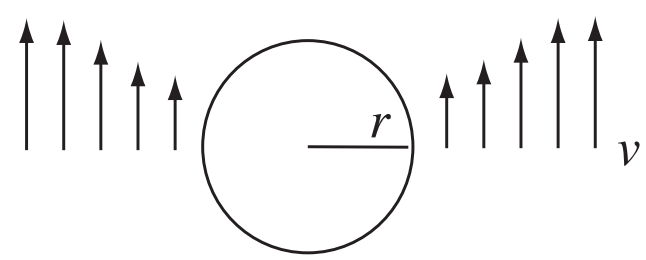

Abbildung 1.8: Geschwindigkeitsprofil um eine Kugel, die von einer viskosen Flüssigkeit umströmt wird [23]

Die Kraft $F$, die eine Kugel erfährt, die von einer Flüssigkeit umströmt wird, ist gegeben durch

$$
F=-6 \pi \eta v r .
$$

Dieser Zusammenhang wird auch zur Bestimmung der Viskosität nach der Kugelfallmethode benutzt [23].

\subsubsection{Gleichgewichtsorientierung eines einzelnen Teilchens}

Ein festes Teilchen in einer strömenden Flüssigkeit wird vom Geschwindigkeitsgradienten beeinflusst und verändert diesen gleichzeitig. Da reale Teilchen selten kugelförmig sind, ist es eigentlich notwendig die Bewegung eines unregelmäßigen Spheroids zu beschreiben. Zur mathematischen Behandlung des Problems $[28,6,29]$ ist ein Rotationsellipsoid als erste Näherung gut geeignet, da zunächst die Orientierung der Hauptachsen beschrieben werden soll. In Abbildung 1.9 ist eine zufällige Orientierung eines Ellipsoids dargestellt. Je nach Achsenverhältnis $r_{e}=a / b$ lassen sich so Stäbchen $\left(r_{e}>1\right)$ oder Plättchen $\left(r_{e}<1\right)$ beschreiben.

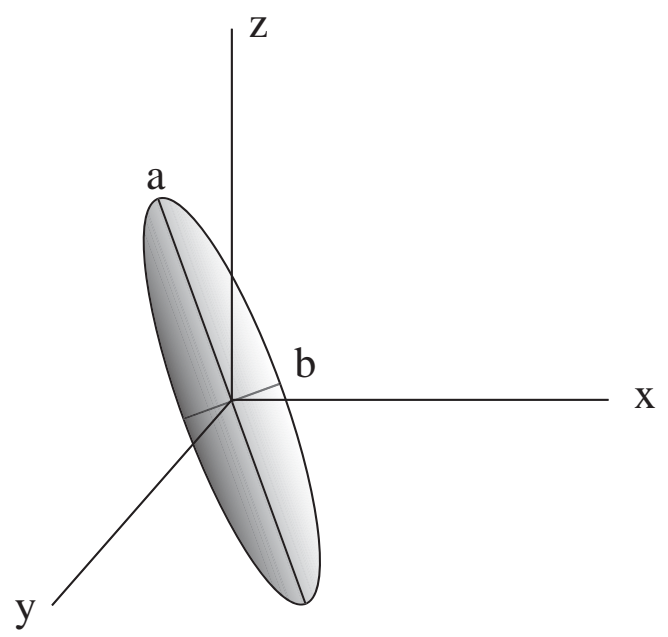

Abbildung 1.9: Rotationsellipsoid im kartesischen Koordinatensystem 
Die Orientierung des Partikels lässt sich nur durch die Lage der Hauptachse beschreiben. Aufgrund der Rotation sind unterschiedliche Orientierungen der zweiten Achse nicht unterscheidbar. Liegt der Ursprung des Koordinatensystems im Zentrum des Partikels bewegen sich die Enden des Ellipsoids auf einer Kugeloberfläche. Eine Formulierung in Polarkoordinaten ist also zweckmäßig. Für den Fall der Couette-Strömung mit der Scherrate $G$ gilt für die Orientierung der Hauptachse $a$ des Ellipsoids mit den Hauptachsen ( $a, b$ siehe Abb. 1.9) in Polarkoordinaten $\phi$ und $\theta$, nach Jeffery's [6]:

$$
\begin{aligned}
& \omega(\phi)=\frac{d \phi}{d t} \\
& \omega(\theta)=\frac{d \theta}{d t}
\end{aligned}
$$

Für die Orientierung der Normalen der Basisebene in Polarkoordinaten liefert Jeffery [6]:

$$
\begin{aligned}
\tan \phi & =\frac{a}{b} \tan \left(\frac{G a b t}{a^{2}+b^{2}}\right) \\
\tan ^{2} \theta & =\frac{a^{2} b^{2}}{k^{2}\left(a^{2} \cos ^{2} \phi+\sin ^{2} \phi\right)}
\end{aligned}
$$

Wobei $k$ die Integrationskonstante ist. Die Betrachtung des Grenzfalls für ein unendlich dünnes Plättchen $(a=0)$ zeigt, das die Plättchenebene jede Position parallel zur Strömungsrichtung einnehmen kann. Der andere Grenzfall für unendlich dünne Stäbchen $(b=0)$ führt zu einer Orientierung der Stabachse parallel zur Strömungsrichtung. Das Stäbchen erfährt so keinen Einfluss des Strömungsgradienten.

Eine Projektion der Rotation auf eine der drei Hauptebenen (ZY,ZX und $\mathrm{XY}$ ) liefert für die ZY-Ebene [7]:

$$
\begin{aligned}
\tan \phi_{x} & =C \cos \left(\frac{2 \pi t}{T}\right) \\
\text { und } & \\
\cos \theta_{x} & =\frac{C r_{e} \sin \phi_{z}}{\sqrt{r_{e}^{2} \cos ^{2} \phi_{z}+\sin ^{2} \phi_{z}+C^{2} r_{e}^{2}}}
\end{aligned}
$$

Für die ZX-Ebene:

$$
\begin{aligned}
\tan \phi_{y} & =C r_{e} \sin \left(\frac{2 \pi t}{T}\right) \\
\text { und } & \\
\cos \theta_{y} & =\frac{C r_{e} \cos \phi_{z}}{\sqrt{r_{e}^{2} \cos ^{2} \phi_{z}+\sin ^{2} \phi_{z}+C^{2} r_{e}^{2}}}
\end{aligned}
$$


Für die XY-Ebene:

$$
\begin{aligned}
\tan \phi_{z} & =r_{e} \tan \left(\frac{2 \pi t}{T}\right) \\
\text { und } & \\
\tan \theta_{z} & =\frac{C r_{e}}{\sqrt{r_{e}^{2} \cos ^{2} \phi_{z}+\sin ^{2} \phi_{z}}}
\end{aligned}
$$

$T$ gibt den Zeitraum für eine Rotation um die a-Achse an. Die Konstante $C$ setzt sich aus der Achsenlänge $a$ und der Integrationskonstante $k$ aus Gleichung 1.14 zusammen. Sie beschreibt die Umlaufbahn, die durch die Enden eines rotierenden Teilchens aufspannt wird (siehe Abb.1.10).

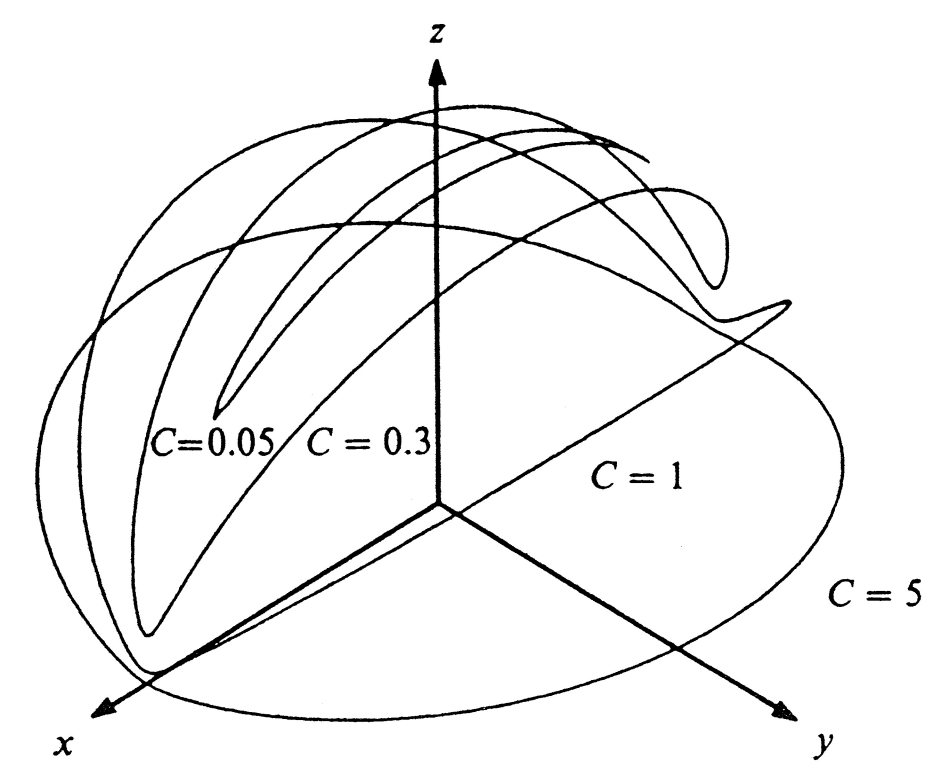

Abbildung 1.10: Darstellung der Flugbahnen für ein Stäbchen in einer Scherströmung $\left(r_{e}>1\right)$ für unterschiedliche Werte der Orbitkonstante $C$ [30]

Jede der in Abbildung 1.10 dargestellten Flugbahnen für ein Stäbchen (auch ' 'Jeffery's orbits'" genannt, lässt sich durch eine so genannte Orbitkonstante beschreiben. Diese Orbitkonstante errechnet sich nach:

$$
C=\frac{\tan \theta\left(r_{e}^{2} \cos ^{2} \phi+\sin ^{2} \phi\right)^{1 / 2}}{r_{e}^{2}} .
$$

In Abbildung 1.11 (i-iii) ist die Rotation der Hauptachse eines Ellipsoids in sphärischen Polarkoordinaten dargestellt. Die Abbildungen (iv - ix) zeigen die Projektion des Ellipsoidorbits auf eine der drei Hauptebenen.

Die XY-Projektion, berechnet nach Gleichung 1.17, in Abbildung 1.11 zeigt, dass die Polarachse des Partikels eine vollständige Umdrehung durch $\phi_{z}=2 \pi$ beschreibt. Die Hauptachse der projizierten planaren Ellipse liegt in X-Richtung für Stäbchen und in Y-Richtung für Plättchen (Abb. 1.11 (iv) u. (vii)). 


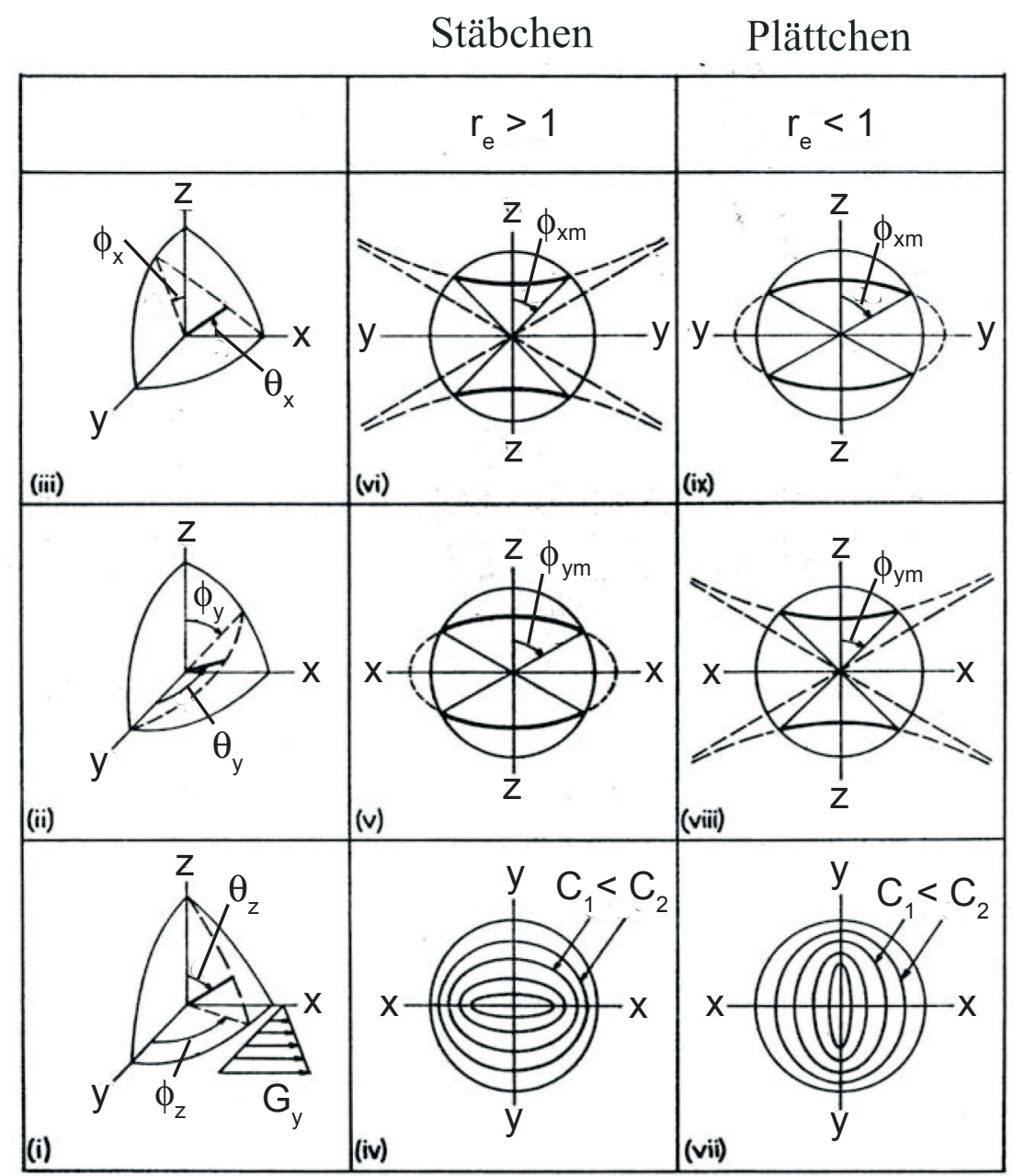

Abbildung 1.11: Stereographische Projektion der Bewegung eines Partikels in einer Scherströmung mit x als Strömungsrichtung und y als Gradientenrichtung für die drei Hauptebenen [7]

In der ZY- (siehe Gl. 1.15) und der ZX-Ebene (siehe Gl. 1.16) vollführt die Polarachse eine Taumelbewegung, welche entweder durch ein Segment einer Ellipse oder einer Hyperbel beschrieben werden kann (Abb. 1.11 (v, vi) u. (viii, ix)). Für $r_{e}<1$ ist die minimale Winkelgeschwindigkeit $\omega\left(\phi_{z}\right)=0$ bei $\phi_{x}=$ $\pm \phi_{x m}$ (ix). Dies entspricht einer stabilen Orientierung. Für $r_{e}>1$ ist diese stabile Orientierung bei $\phi_{y}= \pm \phi_{y m}$ erreicht [7]. Der Index $m$ bezeichnet die Maximale Auslenkung der Achse.

Zusätzlich zur Bewegung der Polarachse erfährt das Teilchen in der CouetteStrömung mit der Scherrate $G$ auch eine Rotation um die Polarachse, der Spin $\omega_{s}$. Die so erhaltene Rotationsenergie kann das Teilchen in der Strömung stabilisieren. Der Spin ergibt sich nach

$$
\omega_{s}=\frac{d \Omega}{d t}=\frac{G}{2} \cos \theta_{z},
$$


mit $\Omega$ als Winkel des axialen Spins. Eine stabile Position ergibt sich somit aus der Kombination aus einer günstigen Lage zum Strömungsgradienten und der maximalen Rotationsenergie.

\subsubsection{Gleichgewichtsposition von festen Teilchen in einer Strö- mung}

Ist das Teilchen nicht ortsfest stellt sich eine Gleichgewichtsposition in einer horizontalen Scherströmung ein. Für ein kreisförmiges Teilchen mit dem Durchmesser $d$ in einer zweidimensionalen Scherströmung wurde durch Simulationsrechnungen von Feng et al. [31] eine Gleichgewichtsposition für unterschiedliche Reynoldszahlen ermittelt. Die Simulationsrechnung wurde mit einem Dichteverhältnis (fest/flüssig) von 1,005 durchgeführt.

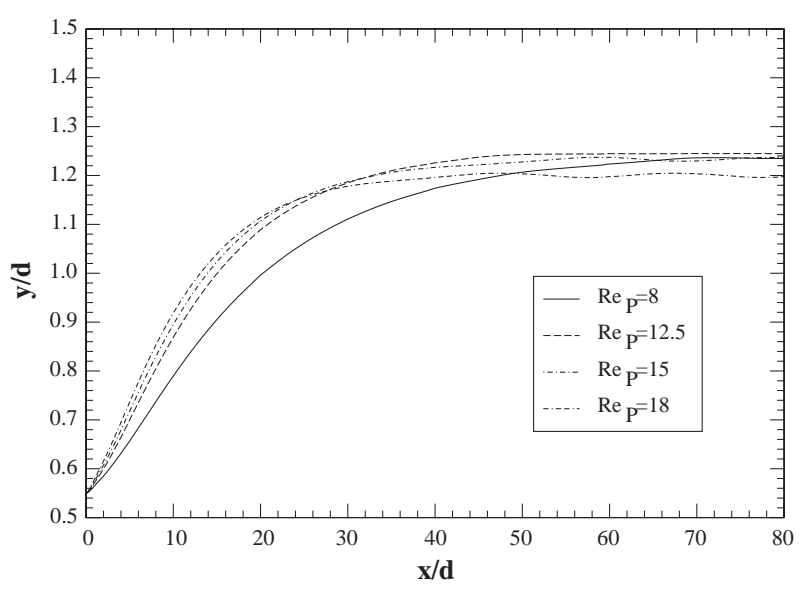

Abbildung 1.12: Simulierte Flugbahn eines Partikels als Funktion der Reynolds$z a h l[31]$

In Abbildung 1.12 ist $x$ die Strömungsrichtung und $y$ die Richtung des Geschwindigkeitsgradienten. Nach einer Strecke von $x / d=40$ stellt sich die Gleichgewichtsposition in einer Höhe von ca. $y / d=1.2$ ein.

Dieses Verhalten lässt sich auch durch Simulationsrechnungen von vielen Partikeln in einer Rohrströmung beschreiben. Die Eigenschaft der Strömung, ein ruhendes Teilchen von der Wand zu lösen, ist auch für viele Teilchen in einer Rohrströmung von $\mathrm{Hu}$ [32] berechnet worden. Die simulierte Strömung zeigt, dass sich an den Wänden ein Schmierfilm ausbildet und die Partikel nicht in Kontakt mit der Wand kommen (Abbildung 1.13).

Auch für den Fall einer turbulenten Strömung wurden Experimente im Hinblick auf Schwankungen in der Partikelkonzentration von Hardalupas et al. [33] und Alipchenkov [34] durchgeführt. Der Schmierfilm tritt trotz einiger Unterschiede auch hier auf. Turbulente Strömungen wurden jedoch in dieser Arbeit nicht erzeugt. 


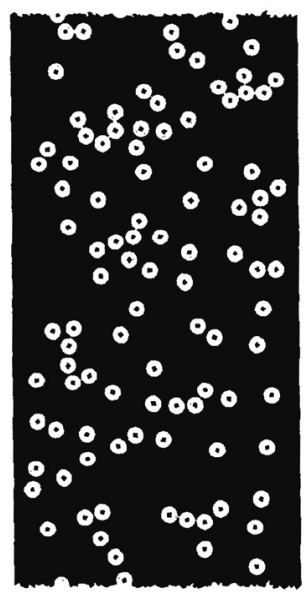

Abbildung 1.13: Simulation der Fließbewegung von 400 Teilchen in einer periodischen Zellen [32]

\subsection{Das Vielteilchenproblem}

Befindet sich ein Teilchen mit vielen anderen in einer strömenden Flüssigkeit wird die Orientierung nicht nur durch die Strömung beeinflusst, sondern auch durch Teilchen-Teilchen Interaktionen [35, 36, 37]. Um dies zu beschreiben, benötigt man Informationen über die Teilchenform, die Teilchenkonzentration, die Geschwindigkeit der Strömung und der Teilchen, sowie den Aufenthaltsort der Teilchen. Dies führt zu einem sehr komplexen Zusammenhang zwischen den beschriebenen Parametern und der daraus resultierenden Orientierungsverteilung. Zur Vereinfachung des Problems ist es nötig die Bewegung der Teilchen unabhängig von ihrer Orientierung zu betrachten. Anschließend können die Beobachtungen zur Bewegung und die Orientierung einzelner Teilchen zu einem Gesamtbild zusammengeführt werden.

\subsubsection{Der Ort und die Geschwindigkeit von Partikeln}

Für vielteilchen fest-flüssig Systeme wurden numerische Simulationen von Tran et al. [38] durchgeführt. Die Beschreibung der Wechselwirkungen zwischen den Teilchen wurde hierbei auf Zwei- und Drei-Teilchen-Systeme beschränkt. Die Berechnungen nach der "boundary element method (BEM)" ergaben für einen sinkenden Abstand auf unter 5 Teilchendurchmesser zwischen zwei Teilchen eine steigende Sinkgeschwindigkeit. Bei noch geringerem Abstand wird auch die Rotationsgeschwindigkeit der Teilchen stark beeinflusst.

Die experimentelle Bestimmung der Teilchenbewegung in konzentrierten Fest-Flüssig-Gemischen wurde mittels NMR (nuclear magnetic resonance) sowohl für die Couette-Strömung [12] als auch für die Poisseuille-Strömung [13] (laminare Rohrströmung) durchgeführt.

Die Couette-Strömung wird durch zwei ineinander gestellte Zylinder erzeugt. Zwischen den beiden Zylindern befindet sich das Gemisch aus fester und flüssiger Phase. Der innere Zylinder rotiert und es entsteht eine Scherströmung. 


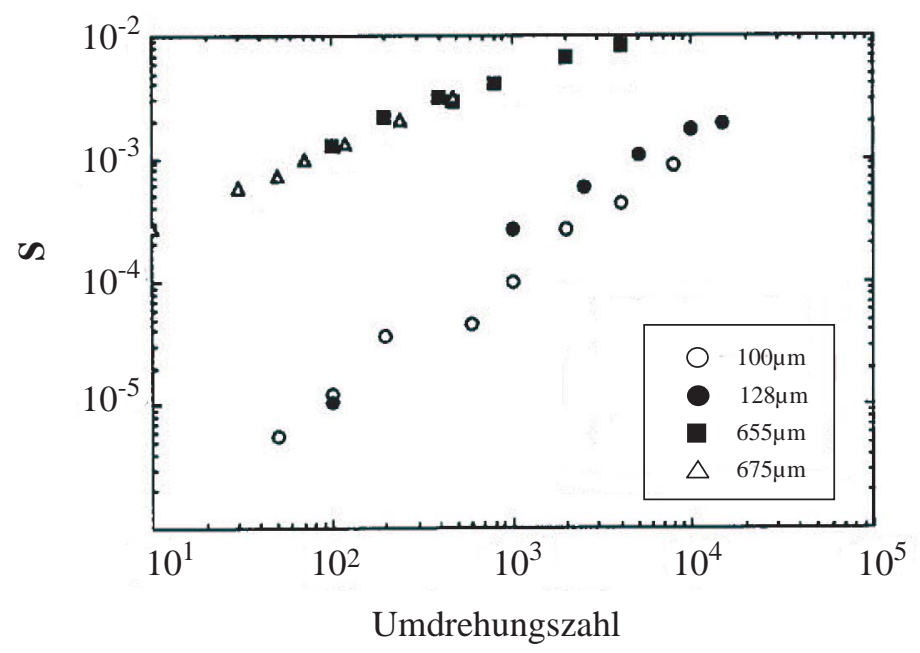

Abbildung 1.14: Grad der Partikelbewegung für unterschiedliche Partikelgrößen bei $50 \mathrm{Vol} \%$ Feststoffkonzentration [12]

Der Grad der Partikelbewegung $S$ ist in Abhängigkeit von der Umdrehungszahl des inneren Zylinders in Abbildung 1.14 dargestellt. In einer Couette-Strömung ist der Grad der Bewegung für kleine Partikel deutlich kleiner als für große Partikel [12].

Der Grad für die Partikelbewegung $S$ ist definiert als:

$$
S(N)=\left[\sum_{i=1}^{m}\left(\phi_{r_{i}, N}-\langle\phi\rangle\right)^{2} r_{i}\right] / \sum_{i=1}^{m} r_{i},
$$

wobei $\phi_{r_{i}, N}$ der lokale Volumenanteil der festen Phase im $i$-ten konzentrischen Ring nach $N$ Drehungen ist. $\langle\phi\rangle$ ist die mittlere Konzentration der festen Phase und $r_{i}$ der Abstand vom Drehzentrum.

Wie in Kapitel 1.2.2 in Gleichung 1.4 beschrieben nimmt der Geschwindigkeitsgradient für Poisseuille-Strömungen eine Parabelform an. Der Einfluss auf den Gradienten durch Zusatz von festen Partikeln ist von S.W. Sinton et al. [13] bestimmt worden. In Abbildung 1.15 ist der Geschwindigkeitsgradient für unterschiedliche Feststoffkonzentrationen dargestellt. Es ist jeweils die Geschwindigkeit für den gesamten Rohrdurchmesser aufgetragen, wobei $Y=0$ der Rohrmitte entspricht.

In Abbildung 1.15(a) konnte eine Parabel (durchgezogene Linie) an die Messwerte (Quadrate) angepasst werden. Bei höheren Feststoffkonzentrationen (Abb. 1.15(b,c)) ist die Anpassung einer einzelnen Parabel nicht mehr möglich. Es wurde jeweils eine Parabel (gestrichelte Linie) für die maximale Geschwindigkeit und eine Parabel (durchgezogene Linie) für die minimale Geschwindigkeit angepasst. Durch die Zugabe von festen Teilchen gelten ab einer bestimmten Konzentration für die Randbereiche einer Strömung und die maximale Geschwindigkeit andere Geschwindigkeitsgesetze [13]. 
(a)

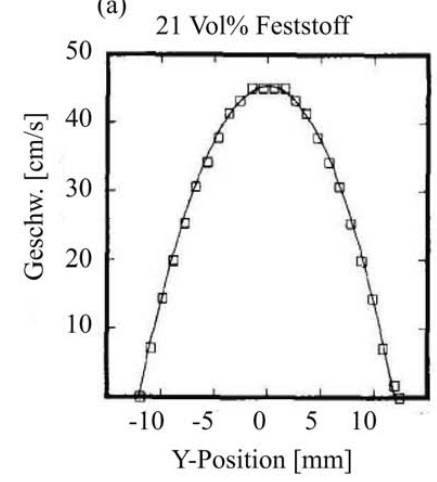

(b)

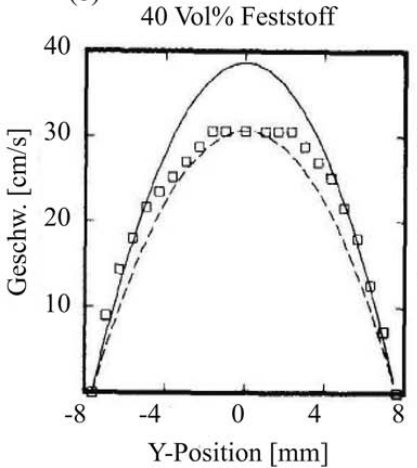

(c)

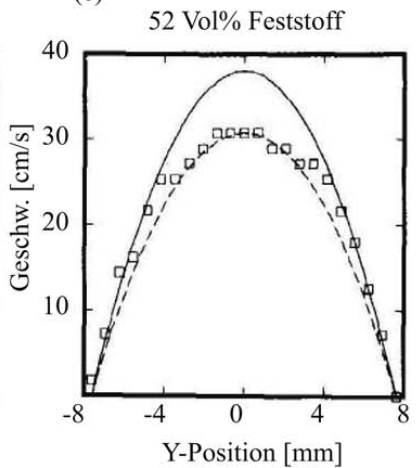

Abbildung 1.15: Geschwindigkeitsverteilung der Rohrströmung für verschiedene Feststoffkonzentrationen [13]

\subsubsection{Orientierungsverteilung von Partikeln}

Durch die Partikelbewegung kommt es zu Kollisionen zwischen Partikeln, welche die Orientierung ändern können. Jeffery [6] nahm an, dass die starre Rotation der Partikel beeinflusst wird, wenn ein anderes Teilchen in der Nähe ist. Die Orientierung vom Teilchen wurde in Kapitel 1.3.2 durch die Polarkoordinaten $\phi_{i}$ und $\theta_{i}$ beschrieben. Diese Orientierung in Abhängigkeit von $\phi_{i}$ ist gegeben durch die Kontinuitätsgleichung

$$
\frac{\partial p\left(\phi_{i}, t\right)}{\partial t}=\frac{\partial}{\partial \phi_{i}}\left[p\left(\phi_{i}, t\right) \omega\left(\phi_{i}\right)\right],
$$

mit $p\left(\phi_{i}, t\right) d \phi_{i}$ als dem Anteil von Partikeln welcher einer Orientierung im Intervall $d \phi_{i}$ zur Zeit $t$ einnimmt. Die Bewegung der Hauptachse des Partikels ist durch die Winkelgeschwindigkeit $\omega\left(\phi_{i}\right)$ gegeben. Befindet sich das System im Gleichgewicht, ist die Verteilung zeitunabhängig und lässt sich integrieren:

$$
p\left(\phi_{i}\right)=\frac{k_{i}}{\omega\left(\phi_{i}\right)} .
$$

Hier ist $k_{i}$ die Integrationskonstante. Die entsprechende integrale Verteilungsfunktion ist gegeben durch

$$
P\left(\phi_{i}\right)=\int_{0}^{\phi_{i}} p\left(\phi_{i}\right) d \phi_{i} .
$$

$P\left(\phi_{i}\right)$ ist der Anteil mit der Orientierung zwischen 0 und $\phi_{i}$. Die Konstante $k_{i}$ verschwindet durch Normierung von $P\left(\phi_{i}\right)$. Die experimentelle Bestimmung der Verteilungsfunktion für eine Couette-Strömung wurde von Anczurowski et al. [39] für stark verdünnte Systeme (0,004 - 0,25 Vol\%) ermittelt. Hierzu wurde in einer Küvette die Orientierung der Teilchen mittels Fotografie zu unterschiedlichen Zeiten ermittelt. 


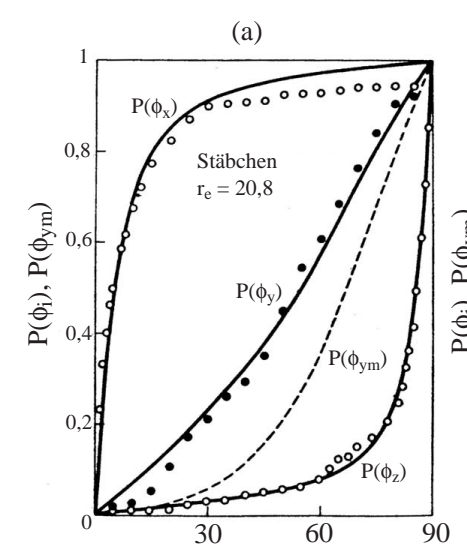

$\left(\phi_{\mathrm{i}}\right),\left(\phi_{\mathrm{ym}}\right)\left[^{\circ}\right]$

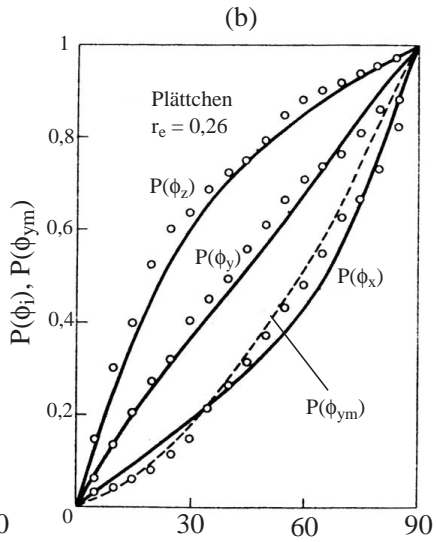

$\left(\phi_{\mathrm{i}}\right),\left(\phi_{\mathrm{ym}}\right)\left[^{\circ}\right]$

Abbildung 1.16: Integrale Orientierungsverteilung $P\left(\phi_{i}\right)$ als Funktion der Winkel $\phi_{i}$ (siehe Abb. 1.11) für a) Stäbchen und b) Plättchen in stark verdünnten Systemen [39]

Die integrale Orientierungsverteilung für Stäbchen (a) und Plättchen (b) ist in Abbildung 1.16 aufgetragen. Die Punkte stellen experimentelle Daten dar, welche für die Verteilung von $\phi_{z}$ direkt ermittelt wurden. $\phi_{x}$ und $\phi_{y}$ stammen aus der Projektion der XY-Ebene.

Die Kurven in Abbildung 1.16 beruhen auf Berechnungen nach Jeffery's Gleichungen (Kap. 1.3.2). Für Stäbchen ist die Vorzugsorientierung für $\phi_{z}=90^{\circ}$ und für $\phi_{x}=0^{\circ}$. Plättchen verhalten sich bezogen auf die zwei Winkel gegensätzlich, jedoch nicht so stark ausgeprägt. Hier ist die Vorzugsorientierung $\phi_{z}=0^{\circ}$ und $\phi_{x}=90^{\circ} . \phi_{y}$ zeigt für beide Teilchenformen eine isotrope Verteilung.

(a)

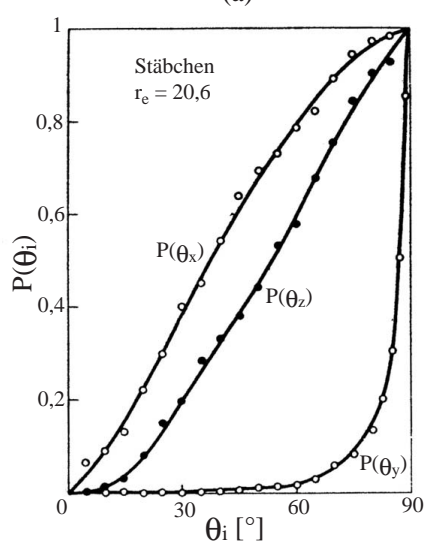

(b)

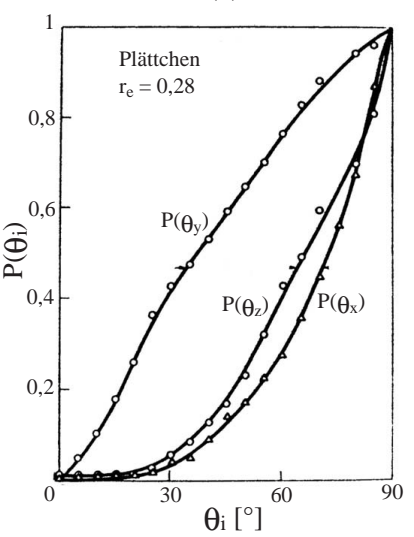

Abbildung 1.17: Integrale Orientierungsverteilung $P \theta_{i}$ als Funktion der Winkel $\theta_{i}$ (siehe Abb. 1.11) für a) Stäbchen und b) Plättchen in stark verdünnten Systemen [39]

Abbildung 1.17 zeigt für die gleichen Stäbchen und Plättchen die integrale 
Orientierungsverteilung mit der Polarkoordinate $\theta_{i}$. Auch hier stellen wieder die Punkte die experimentellen Daten dar. Die Kurven sind ebenfalls aus Jeffery's Gleichungen berechnet.

Wenn man von sehr kleinen Werten für $\theta_{z}$ absieht, sind $\theta_{x}$ und $\theta_{z}$ für Stäbchen nahezu isotrop verteilt. Ungefähr $92 \%$ aller Stäbchen haben eine Orientierung zwischen $75^{\circ}-90^{\circ}$ für $\theta_{y}$. Plättchen hingegen zeigen bei kleinen Winkeln für $\theta_{x}$ und $\theta_{z}$ nur geringe Werte. Ein Großteil der Partikel bewegen sich zwischen $30^{\circ}-90^{\circ}$. Die Verteilung von $\theta_{y}$ ist beinahe isotrop.

Eine andere Art der Darstellung ergibt sich, wenn man die Enden der Polarachse des Rotationsellipsoids auf eine der drei Hauptebenen projiziert. Die experimentellen Daten den Abbildungen 1.16 und 1.17 sind auf diese Weise in den Abbildungen 1.18 und 1.19 zu sehen.

Die stark ausgeprägte Vorzugsorientierung der Stäbchen (Abb. 1.18) ist auch hier gut zu erkennen. Die Stäbchen sind parallel zur Strömungsrichtung orientiert $\left(\phi_{y}\right.$ ist nahe $90^{\circ}$ Abb.1.18 (a)). In der YZ-Ebene ist eine Verteilung der Stäbchenenden entlang der Z-Achse zu sehen $\left(\phi_{z}=0^{\circ}\right)$. Der Winkel $\theta_{y}=90^{\circ}$ entspricht dem Kreisumfang in der XZ-Ebene, auf dem sich die Teilchenenden abbilden. Die Stäbchen orientieren sich den theoretischen Überlegungen von Jeffery entsprechend so in der Strömung, dass um das Stäbchen herum ein möglichst kleiner Strömungsgradient herrscht [6].
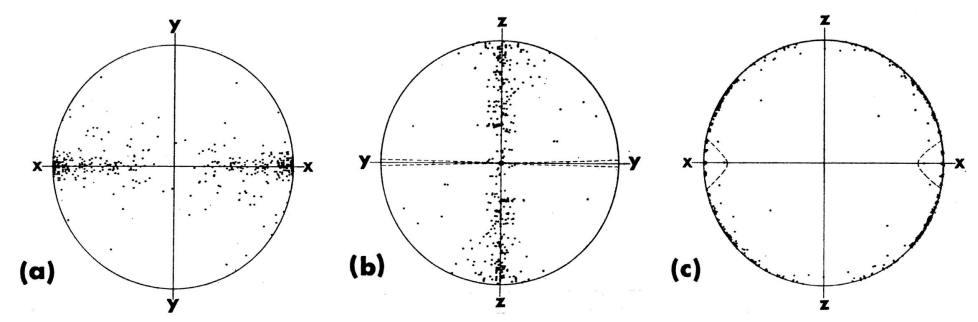

Abbildung 1.18: Projektion der Enden der Polarachsen von Stäbchen mit dem Radienverhältnis $r_{e}=20.8$ nach Anczurowski et al. [39]

Die in Abbildung 1.19 dargestellte Projektion der Enden der Polarachsen von den Plättchen zeigt, dass die ermittelten Orientierungen stärker um die von Jeffery vorhergesagte ideale Position schwanken. Für die XY-Ebene und die YZ- Ebene in Abbildung 1.19 (a) und (c) ist zu beobachten, dass sich die kurze Polarachse der Plättchen um die Y-Achse und im Zentrum befindet.
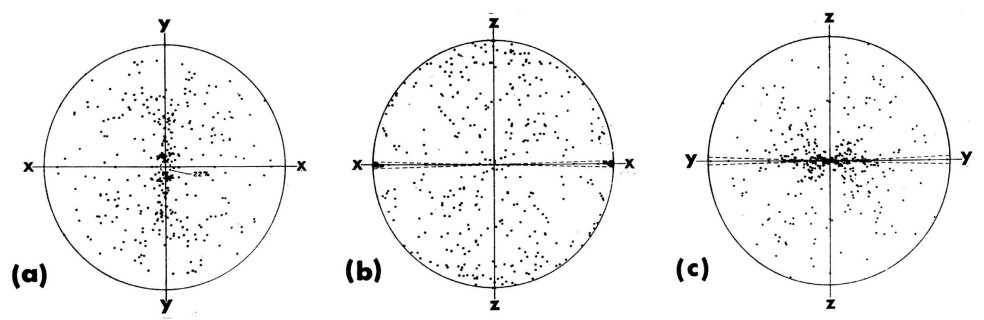

Abbildung 1.19: Projektion der Enden der Polarachsen von Plättchen mit dem Radienverhältnis $r_{e}=0,26$ nach Anczurowski et al. [39] 


\subsubsection{Bestimmung der Partikelorientierung mittels Diffraktion}

Die von Anczurowski et al. [39] durchgeführten optischen Orientierungsbestimmungen an plättchenförmigen und stäbchenförmigen Partikeln haben die Vorzugsorientierungen wie von Jeffery [6] berechnet aufgezeigt. Es handelt sich hierbei lediglich um Messungen von Einzelorientierungen. Für die Bestimmung einer Orientierungsverteilung ist eine bessere Statistik notwendig. Diese lässt sich mit Diffraktionsmethoden sehr gut erreichen, da hier die Orientierung vieler Partikel gleichzeitig bestimmt werden kann.

Die Messung der Partikelorientierung mittels Diffraktion ist nur an kristallinen Partikeln möglich. An den Netzebenen mit dem Abstand $d_{h k l}$ der Kristallite in dem Gemisch beugen die Röntgenstrahlen mit der Wellenlänge $\lambda$ um einen Winkel $\theta$. Die reflektierten Wellen überlagern sich und löschen sich teilweise aus. Bei einem bestimmten Winkel ist der Gangunterschied zwischen den an benachbarten Netzebenen reflektierten Wellen ein Vielfaches $n$ von $\lambda$. Es kommt zur konstruktiven Interferenz. Nach der Bragg'schen Gleichung ist diese Reflexionsbedingung:

$$
2 d_{h k l} \sin \theta=n \lambda .
$$

Erste Orientierungsbestimmungen mittels Diffraktion wurden an plättchenförmigen Pulvern nach vollendeter Strömung in einem stabilen Zustand (z.B. in Gelform) durchgeführt [40]. Diese Methode hat jedoch den Nachteil, dass beim Erzeugen eines stabilen Zustands der Strömungsvorgang für das Zwei-PhasenGemisch beendet wird und sich die Orientierungen der Partikel nochmals ändern kann. Ob sich diese lediglich durch starre Rotation der Partikel im Strömungsgradienten der Flüssigkeit eingestellt hat, oder diese beim Überführen in die Gelform noch schärfer oder schwächer geworden ist kann so nicht beurteilt werden. Mit dieser Methode wird auch bei Brandon et al. [41] eine Vorzugsorientierung für die Basisebene der plättchenförmigen Partikel parallel zur Strömungsrichtung gefunden (Kap. 1.4.2).

Direkte Messungen zur Orientierungsbestimmung an strömenden fest/flüssig Gemischen wurden mittels Neutronenbeugung von Clarke et al. [42] durchgeführt. Die eingesetzte Scherküvette wurde während der Messung mit einem 1-dimensionalem Detektor jedoch nur um eine Achse gedreht, so dass lediglich die Intensität eines Beugungsreflexes in Abhängigkeit eines Winkels zwischen Neutronenstrahl und Strömungsgradient gemessen wurde. Clarke et al. [42] machte Annahme, dass sich die Partikel um die Normale der Basisebene frei drehen.

Erste Orientierungsmessungen mit Hochenergetischer Synchrotronstrahlung an Gemischen aus Wasser und Kaolinit wurden von Barè et al. [43] durchgeführt. Es wurden, wie in Abbildung 1.20 dargestellt, drei unterschiedliche Orientierungen eines strömenden Gemisches zum Primärstrahl detektiert. Die Beugungsbilder für diese Messungen sind mit einem Flächendetektor aufgenommen worden, so dass schon mehr Orientierungen als bei einem 1-dimensionalem Detektor bestimmt werden konnten. Diese zusätzliche Information findet aber keine Berücksichtigung.

Die mit der Methode von Barè et al. erzielten Ergebnisse sind in Abbildung 1.21 dargestellt. Die plättchenförmigen Partikel (ii) richten sich parallel zur 

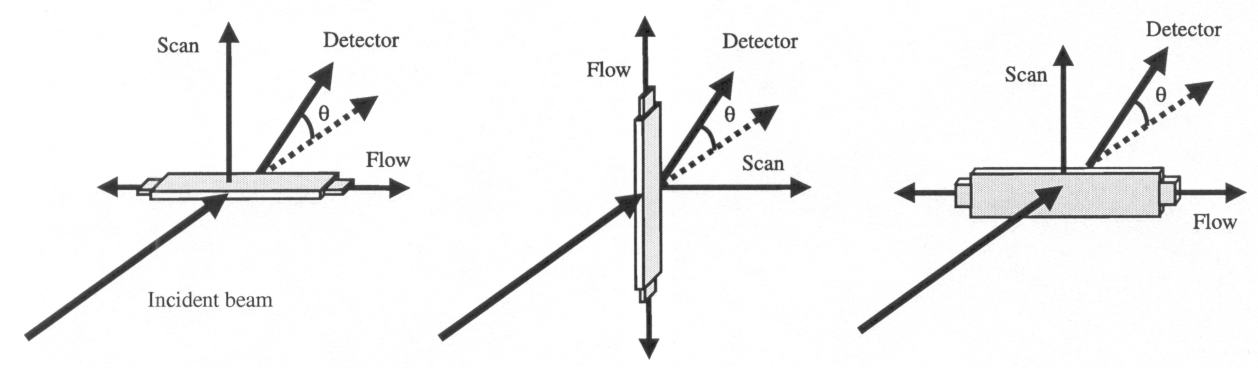

Abbildung 1.20: Unterschiedliche Probenorientierung bei Orientierungsmessungen an strömenden Kaolinit/Wasser Gemischen von Barè et al. [43]

Strömungsrichtung aufgrund des Strömungsgradienten aus. Dies stimmt mit den Ergebnissen in Kapitel 5.2 überein.

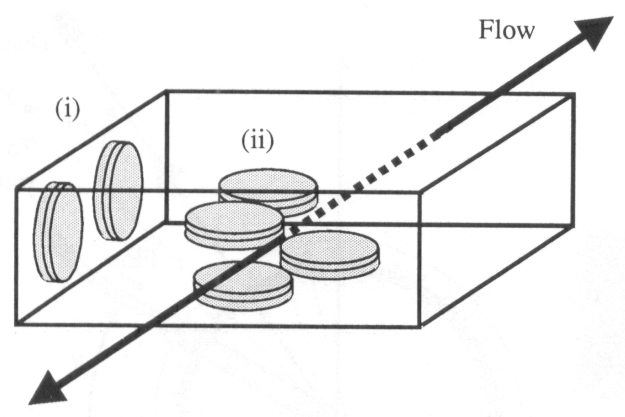

Abbildung 1.21: Schematische Darstellung der Orientierung von Kaolinitpartikeln in einer Strömung nach Barè et al. [43]

Für die Orientierung der Partikel (i) in Abbildung 1.21 ist nach Barè et al. die Küvettenwand der Ausschlag gebende Faktor. Da jedoch die Partikel, wie von Feng et al. [31] beschrieben, in einer Strömung eine Gleichgewichtsposition einnehmen, welche nicht direkt an der Wand ist (es bildet sich ein Schmierfilm aus), können auch andere Einflüsse diese Orientierung erzeugen. Da in der Durchflussküvette zwei Strömungsgradienten vorliegen, jeweils zwischen den Wänden im Strömungskanal, kann in den Randbereichen der Einfluss des zweiten Strömungsgradienten stärker werden und so für diese Orientierung verantwortlich sein.

Eine weitere Möglichkeit die Orientierung von Partikeln in einer strömenden Flüssigkeit zu bestimmen ist die Kleinwinkelstreuung [14]. Diese Methode beschreibt aber auch nur einen kleinen Teil des gesamten Orientierungsraums und gibt zusätzlich Informationen über Ordnungsphenomäne der Partikel untereinander. Die Ergebnisse zu plättchenförmigen Partikel (Abb. 1.22 b)) von Brown et al. [44] bestätigen, dass sich die Partikel parallel zur Strömungsrich- 
tung ausrichten.

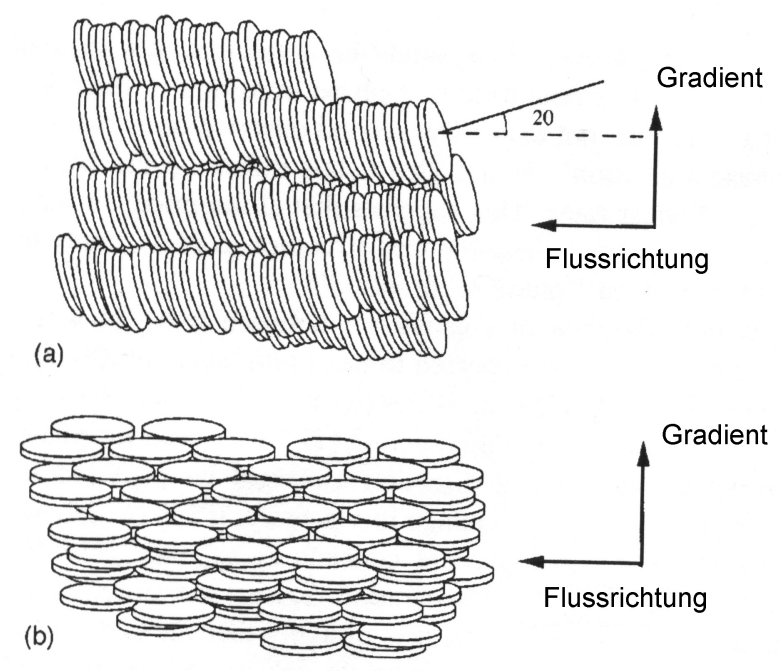

Abbildung 1.22: a) Orientierung bei geringer Scherrate b) Orientierung bei hoher Scherrate nach Brown et al. [44]

Es wird hier allerdings noch eine weitere Orientierung der Plättchen (Abb. 1.22 a)) beschrieben, welche sich bei geringen Scherraten nahezu senkrecht zur Strömungsrichtung einregeln. Solche Unterschiede wurden bei allen in dieser Arbeit gemessenen Polfiguren nicht beobachtet. Da hier jedoch eine andere Küvettenform (siehe Kap. 4.1 Abb. 4.1) verwendet wurde lassen sich die Ergebnisse auch nicht in jedem Detail miteinander vergleichen. Die Untersuchung des Einflusses der Strömungsgeschwindigkeit auf die Textur teilt sich in zwei Aspekte auf. Eine Änderung der Strömungsgeschwindigkeit kann eine Änderung der Vorzugsorientierung bewirken und/oder eine Änderung der Texturschärfe. Die Abhängigkeit der Orientierungsdichte von der Strömungsgeschwindigkeit oder Scherrate wurde von Brown et al. [44, 45, 46] an wässrigen Dispersionen von Kaolinit und Nickel(II)Hydroxid untersucht. In Abbildung 1.23 ist die Intensität für den Basisebenenreflex in Abhängigkeit von Winkel $\alpha$ zwischen Strömungsgradient und Flächennormale der Basisebene dargestellt.

Die Messungen haben ergeben, dass die hier dargestellten Orientierungsdichten im Maximum mit steigender Strömungsgeschwindigkeit zunehmen. 


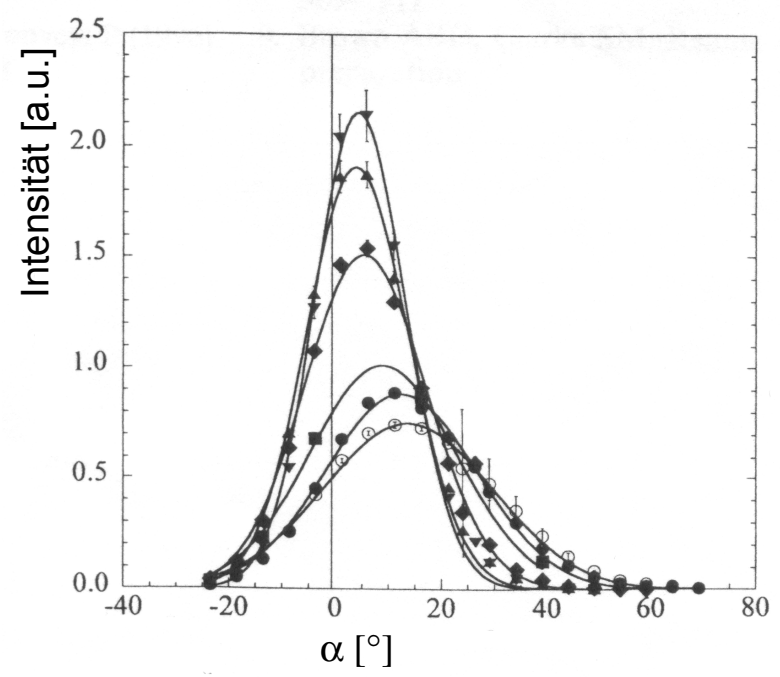

Abbildung 1.23: Intensität für den Basisebenereflex für unterschiedliche Scherraten $0.22 s^{-1}(\bigcirc), 1.0 s^{-1}(\bullet), 1.1 s^{-1}(\mathbf{\square}), 3.6 s^{-1}(\bullet), 8.4 s^{-1}(\mathbf{\Delta}), 23 s^{-1}(\mathbf{\nabla})$ nach Brown et al. [44] 


\section{Kapitel 2}

\section{Texturanalyse}

\subsection{Die Texturbestimmung}

Die zur Texturbestimmung am häufigsten verwendete Methode ist die Polfigurmessung, welche einen Zugang zur ODF ermöglicht. Diese Polfiguren können durch Röntgen- [47], Neutronen- [48] und Elektronenbeugung [49] bestimmt werden. Die Unterschiede dieser Methoden zeigen sich im Wesentlichen in der Eindringtiefe ins Material, der Ortsauflösung und der Orientierungsauflösung. Die klassische Methode der Polfigurmessung durch Röntgenstrahlung (Röntgenröhre) ist für die Bestimmung von Strömungstexturen nur mit hohem konstruktivem Aufwand geeignet [50]. Aufgrund der geringeren Eindringtiefe der Röntgenstrahlung, im Vergleich mit Hochenergie Synchrotronstrahlung, müssten die Küvette mit speziellen Fenstern (Kapton, Beryllium) ausgestattet werden. Die Elektronenbeugung eignet sich gar nicht. Die Eindringtiefe ist viel zu gering um Strömungstexturen in den in Kapitel 4 beschriebenen Küvetten zu bestimmen. Die Neutronenbeugung erreicht zwar die nötige Eindringtiefe, ist jedoch aufgrund des geringen Neutronenflusses nicht geeignet. Die Messzeit übersteigt die Belastbarkeit der Strömungsküvette. Die neu entwickelte Methode der Texturmessung mittels Hochenergie Synchrotronstrahlung [19] bietet dank der hohen Eindringtiefe und des hohen Photonenflusses gute Voraussetzungen zur Bestimmung von Strömungstexturen.

\subsection{Die Polfigurmessung}

Zur Polfigurmessung wird die entsprechende Küvette so auf einem $\omega$-Tisch montiert, dass der monochromatische Röntgenstrahl das strömende fest/flüssig Gemisch an einer Position trifft, an der stationäre Strömungsverhältnisse zu erwarten sind (siehe Kap. 4).

Die klassische Texturmessung erfolgt in Texturgoniometern, die mit einer Eulerwiege ausgestattet sind (Abb. 2.1). Hier wird ein Detektor auf einen $2 \theta$ Winkel eines Bragg-Reflexes eingestellt. Durch Variation des Drehwinkels $\varphi$ und des Kippwinkels $\chi$ können alle Kristallite der Probe in Reflexionsstellung gebracht werden. Die gemessenen Intensitäten entsprechen dem Volumenanteil der Netzebenen, welche sich in Reflexionsstellung befinden. 


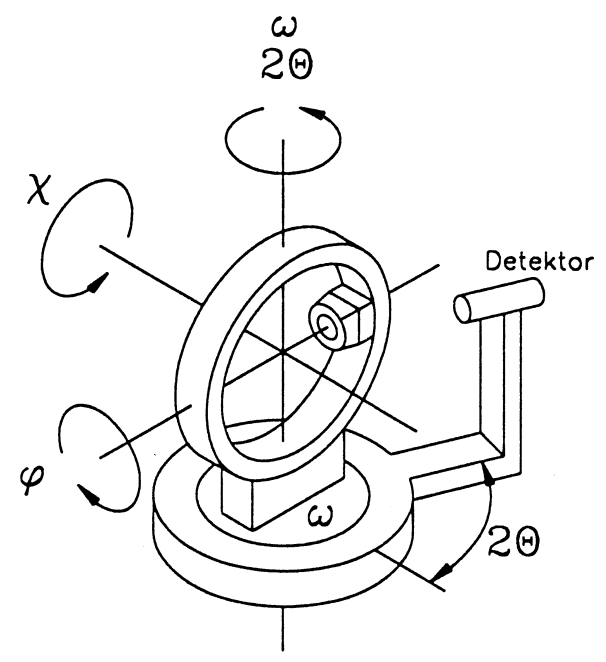

Abbildung 2.1: Texturgoniometer mit Eulerwiege [51]

Die Textur kann nach dem Rückstrahlverfahren (siehe Abb. 2.2 Reflexion) nach Schulz [52] gemessen werden oder nach dem Durchstrahlverfahren (siehe Abb. 2.2 Transmission) nach Decker, Asp und Harker [53].

a)

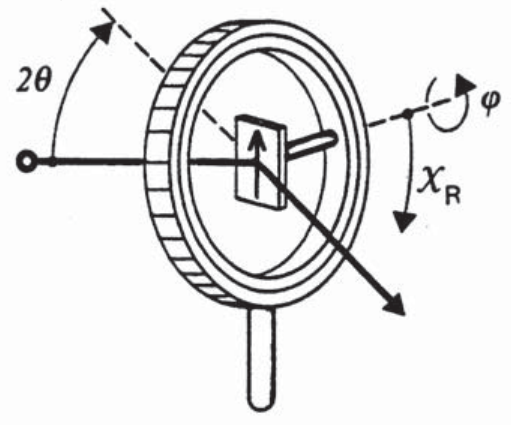

Reflexion

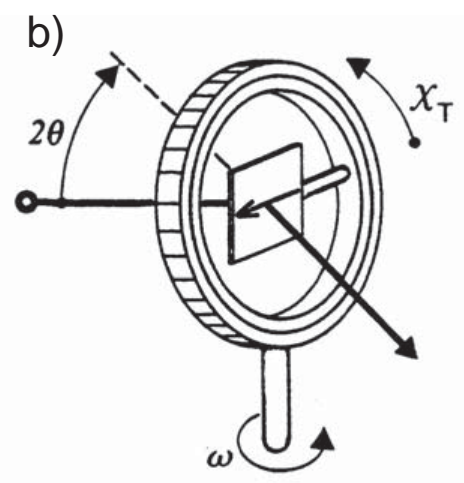

Transmission

Abbildung 2.2: Texturmessung nach a) dem Rückstrahlverfahren und b) dem Durchstrahlverfahren [54]

Bei dem Rückstrahlverfahren wird die Probe mit der Flächennormale parallel zur Drehachse $\varphi$ auf dem Probenhalter montiert. Die Probe wird nun um den Winkel $\varphi$ von $0-360^{\circ}$ in vorher festgelegten Schritten $\Delta \varphi$ gedreht und die entsprechende Intensität wird detektiert. Nach vollendeter Drehung wird die Probe um einen bestimmten Winkel $\chi_{R}$ gekippt und die Drehung um $\varphi$ wird erneut durchgeführt. Aufgrund der Defokussierung bei der Messgeometrie wird die Messung in den meisten Fällen ab einem Winkel von $\chi=70^{\circ}$ abgebrochen.

Mit dem Durchstrahlverfahren wird ein anderer Orientierungsbereich bei der Polfigurmessung abgedeckt (siehe Abb. 2.3). Hier wird die Probe mit der 
Flächennormalen parallel zur Drehachse $\chi_{T}$ montiert. Während der Messung wird die Probe in $\chi_{T}$ von $0-360^{\circ}$ und in $\omega$ von $0-70^{\circ}$ gedreht.

Der Zusammenhang zwischen den Eulerwiegenwinkel $\{\omega, \chi, \varphi\}$ und den Polfigurwinkeln $\{\alpha, \beta\}$ ist in Tabelle 2.2 dargestellt.

\begin{tabular}{c|c|c} 
& $\begin{array}{c}\text { Rückstrahl- } \\
\text { verfahren }\end{array}$ & $\begin{array}{c}\text { Durchstrahl- } \\
\text { verfahren }\end{array}$ \\
\hline$\alpha$ & $\chi_{R}$ & $90-\theta_{(h k l)}-\omega$ \\
\hline$\beta$ & $\varphi$ & $\chi_{T}$
\end{tabular}

Durch Kombination von beiden Methoden lassen sich vollständige Polfiguren bestimmen. In Abbildung 2.3 sind die gemessenen Bereiche in den Polfiguren der beiden Methoden dargestellt.

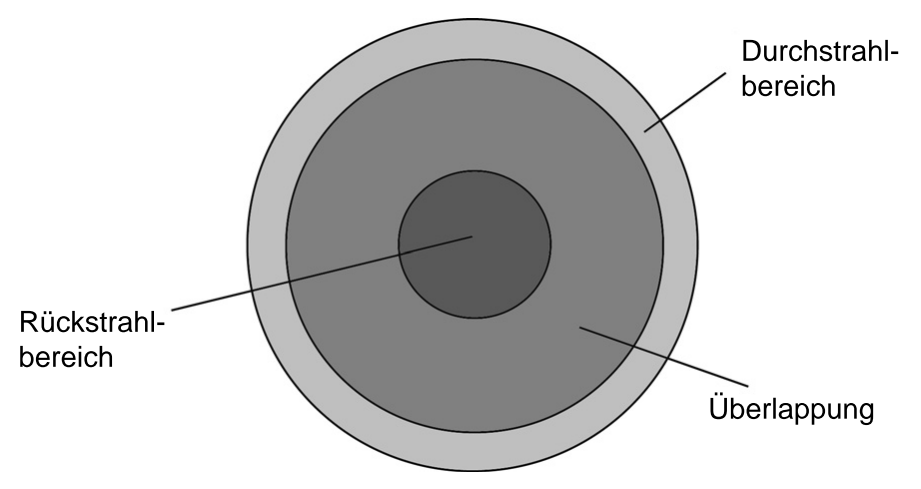

Abbildung 2.3: Messbare Bereiche in der Polfigur nach a) dem Rückstrahlverfahren und b) dem Durchstrahlverfahren

Polfiguren sind als kontinuierliche Poldichteverteilungen definiert. Für experimentell ermittelte Daten steht jedoch nur eine begrenzte Anzahl an Messpunkten zur Verfügung. Die einzelnen Schrittweiten bei den gemessenen Polfiguren können so gewählt werden, dass die Messpunkte entweder jeweils die gleichen Winkelbereiche in der Polfigur beschreiben (Abb. 2.4 a)) oder aber jeweils die gleiche Fläche in der Polfigur (Abb. 2.4 b)).

a)

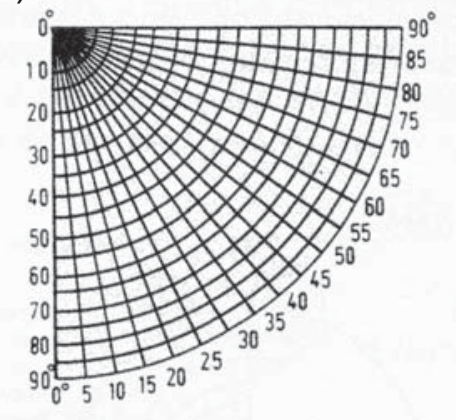

b)

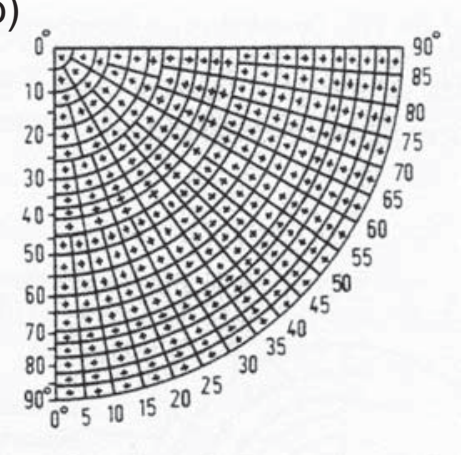

Abbildung 2.4: Polfigurmessraster a) Winkeltreu und b) Flächentreu 
Die Messpunkte bei der Polfigurmessung mit Hochenergie Synchrotronstrahlung und Flächendetektor befinden sich jedoch auf keinem dieser bei Polfigurraster und müssen erst umgerechnet werden (siehe Kap. 2.3.5, da die Software zur 3-Dimensionalen Texturanalyse auf dem Winkeltreuen Raster aufbaut.

\subsubsection{Die Beamline BW5}

Das Pulverdiffraktometer der hochenergie Beamline BW5 [16] lässt sich sehr gut zur Polfigurbestimmung nutzen (Abb. 2.5). Die Beamline befindet sich am 4.5GeV Synchrotronring DORIS des HASYLAB in Hamburg.

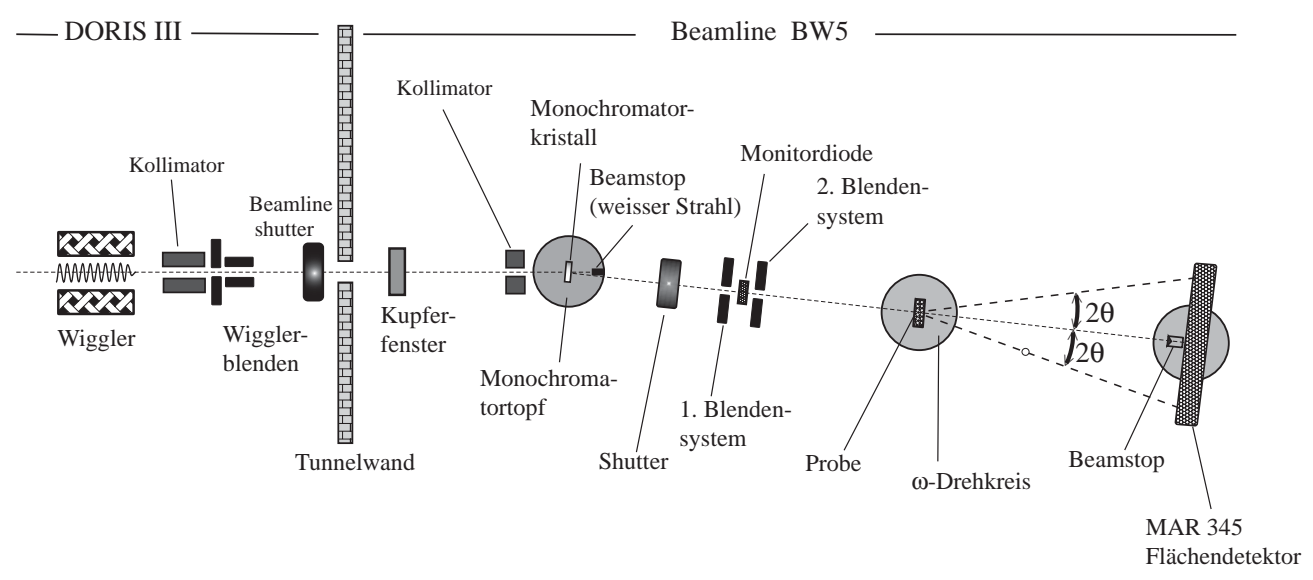

Abbildung 2.5: Schematische Darstellung des Texturmessplatzes BW5 am HASYLAB [19]

Die durch den Wiggler erzeugte Strahlung liefert ein breites Spektrum, wie in Abbildung 2.6 gezeigt. Der durch Kollimator und Wigglerblenden begrenzte Strahl passiert den Beamlineshutter und trifft auf ein Kupferfenster. Hier wird der niederenergetische Teil der Strahlung absorbiert, um die Hitzelast auf dem Monochromatorkristall zu verringern.

Nach dem Kupferfenster endet das Hochvakuum und der eigentliche Messplatz BW5 beginnt. Der Strahl wird erneut kollimiert und trifft auf den Monochromatorkristall im Helium gespülten Monochromatortank. Durch die Stellung des Monochromatorkristalls lässt sich die Wellenlänge des monochromatischen Strahls für die Messung wählen. Hier stehen drei Monochromatorkristalle zur Auswahl (Tab. 2.1), welche sich in ihrer Mosaizität unterscheiden und somit einen unterschiedlichen Wellenlängenbereich $\Delta \lambda$ und unterschiedliche Intensität auf der Probe liefern.

Der Teil des Spektrums, welcher den Monochromatorkristall durchdringt, wird im Monochromatortank durch einen Wasser gekühlten Kupferbeamstop aufgefangen. Der abgebeugte monochromatische Strahl verlässt den Monochromatortank durch ein Captonfenster.

Daran anschließend folgt eine optische Bank, welche mit unterschiedlichen Komponenten bestückt werden kann. Für die Polfigurmessung wird ein Shutter benötigt um die Belichtungszeiten des Flächendetektors zu steuern. Anschlie- 


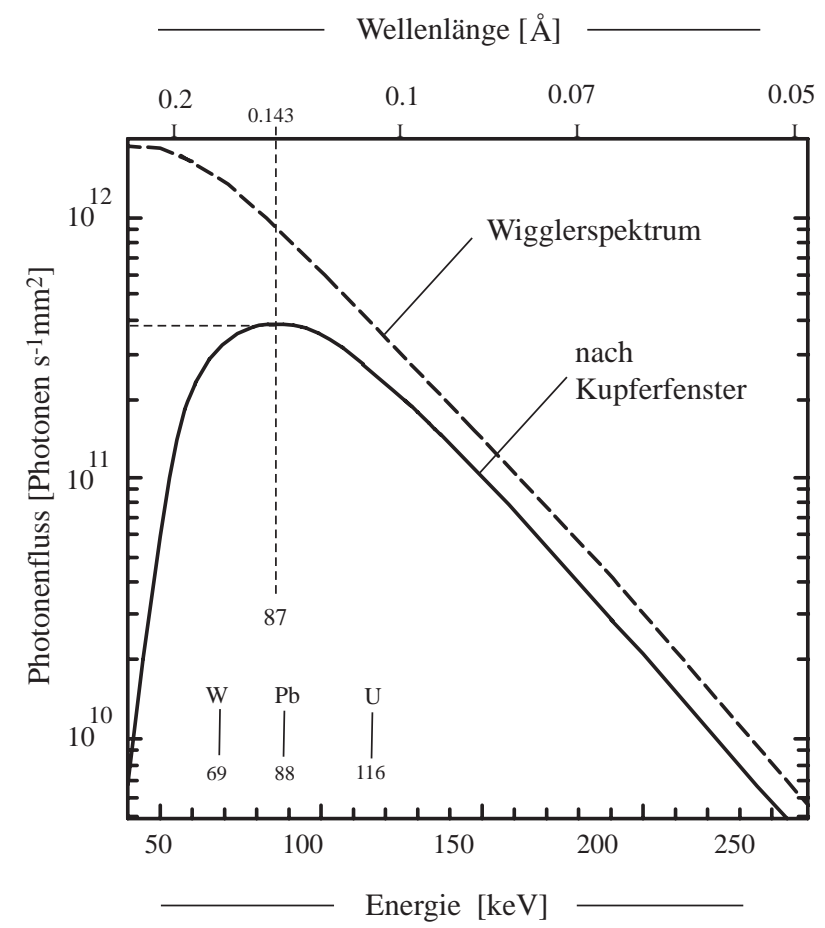

Abbildung 2.6: Spektrum des Wiggler des BW5 in Abhängigkeit von Photonenfluss (mit u. ohne Kupferfenster) [19]

\begin{tabular}{c|cccc} 
& perfekt & imperfekt & gradient & gradient \\
& $\mathrm{Si}$ & $\mathrm{Si}$ & $\mathrm{Si} / \mathrm{Ge}$ & $\mathrm{Si} / \mathrm{Ge}$ \\
\hline Mosaizität $(\operatorname{arcsec})$ & 0.5 & 5.6 & 13.5 & 80 \\
Spektrale Breite $\Delta \lambda / \lambda\left(* 10^{-3}\right)$ & 0.14 & 1.6 & 3.8 & 22.5 \\
Intensität $\left(\right.$ Photonen $\left.s^{-1} \mathrm{~mm}^{2}\right)$ & $2 * 10^{7}$ & $3 * 10^{9}$ & $2 * 10^{11}$ & $5 * 10^{12}$
\end{tabular}

Tabelle 2.1: Mosaizität, spektrale Breite und Intensität in Abhängigkeit vom Monochromatorkristall $(\lambda=0.124)[19]$

ßend wird der Strahlquerschnitt durch eine mit einem Schrittmotor gesteuerte Blende definiert. Darauf folgt eine Diode zur Bestimmung der Strahlintensität, welche mit fallendem Ringstrom abnimmt. Mit einer weiteren Blende, welche ein wenig weiter als die Erste geöffnet ist, werden Beugungsreflexe der ersten Blende und der Diode entfernt.

Der so definierte Strahl trifft auf die Probe. Zur Polfigurmessung ist die Probe auf einem $\omega$-Drehtisch befestigt. Die Beugungsreflexe werden mit einem MAR345 Flächendetektor detektiert und zur weiteren Auswertung auf einem Steuerrechner gespeichert. Der Teil des monochromatischen Strahls, der die Probe durchdringt wird mit einem weiteren Beamstop aus Blei vor dem Detektor abgefangen. 


\subsubsection{Vorversuche}

Um die möglichen Feststoffkonzentrationen für die Experimente zu ermitteln wurden Referenzmessungen in einem aufgebohrten Plexiglasquader durchgeführt (Abb. 2.7). In die Bohrung wurde das Gemisch eingefüllt und anschließend geschüttelt. Die Bohrung wurde mit $2 \mathrm{~mm}$ Durchmesser so gewählt, dass dies dem beugenden Volumen des Gemisches in den Küvetten entspricht.

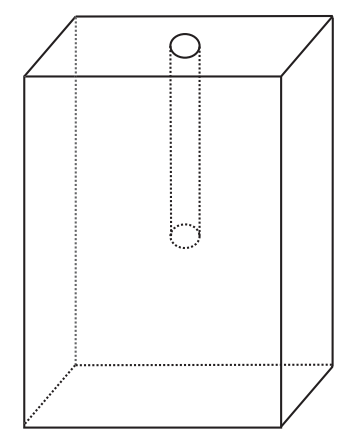

Abbildung 2.7: Plexiglasquader

Hierbei galt es die Feststoffkonzentration zu ermitteln, mit der eine Auswertung der gemessenen Daten noch möglich war. Da mit sinkender Feststoffkonzentration die Belichtungszeit erhöht werden muss, war auch die Dichtigkeit der Küvette ein beschränkender Faktor. In Abbildung 2.8 sind Beugungsbilder von Muskovit/Glyzerin Gemischen mit unterschiedlicher Feststoffkonzentration dargestellt. Beide Aufnahmen wurden 40sec belichtet.
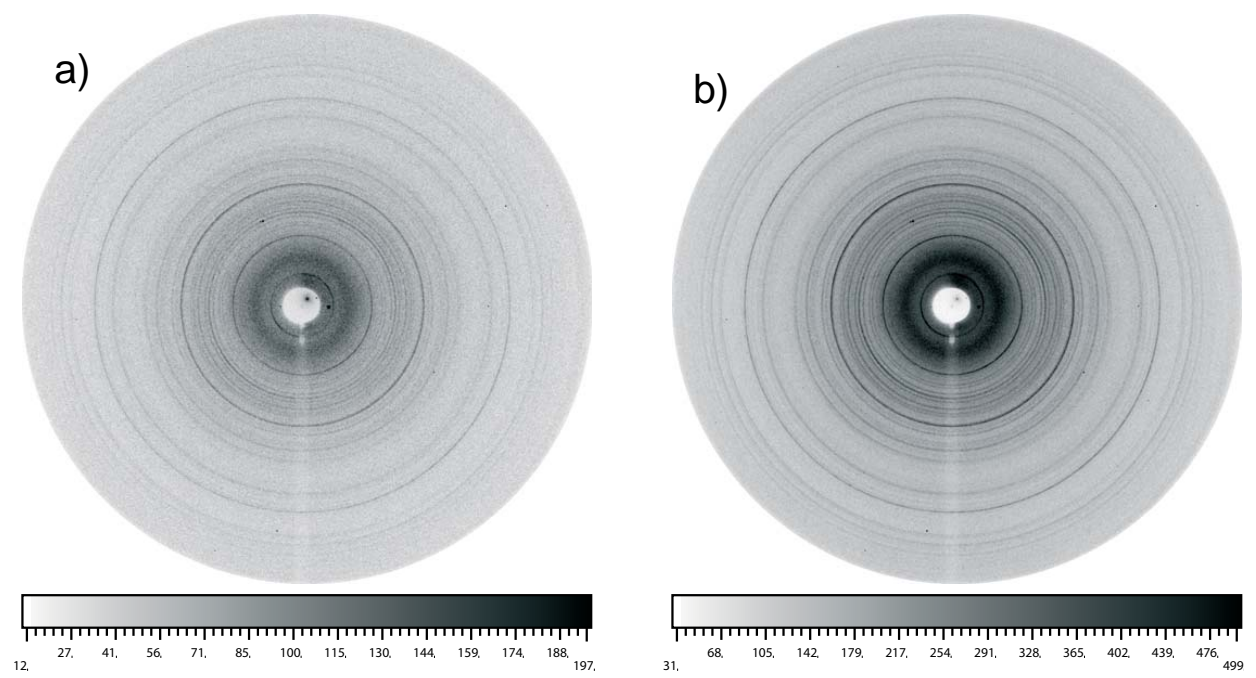

Abbildung 2.8: Beugungsbilder von Muskovit/Glyzerin Gemischen a) 10Vol\% b) $20 \mathrm{Vol} \%$ Feststoffkonzentration

In Abbildung 2.8 a) sind die Beugungsringe nur noch sehr schwach zu erkennen und heben sich kaum vom Untergrund ab. Eine Feststoffkonzentration von $10 \mathrm{Vol} \%$ ist für Muskovit nicht mehr zur Polfigurauswertung geeignet. Bei 
einer Feststoffkonzentration von 20Vol\% (Abb. 2.8 b)) sind die Beugungsringe schon sehr deutlich zu sehen.

Ein weiterer Punkt ist die Zähigkeit des Gemisches. Ist die Feststoffkonzentration zu hoch, ist die Motorkraft nicht mehr ausreichend um die Rotationsküvette zu bewegen. Gleiches gilt auch für die Druckkraft in der Durchflussküvette. Hier sind die möglichen Konzentrationen noch geringer. Andererseits läuft die Durchflussküvette über einen längeren Zeitraum stabil und es können so längere Belichtungszeiten realisiert werden. Die ermittelten Parameter werden in Kapitel 5 zu den einzelnen Proben detailliert dargestellt.

\subsubsection{Polfigurmessung am BW5}

Die Polfigurmessung an der Beamline BW5 unterscheidet sich von der in Kapitel 2.2 beschriebenen klassischen Methode. Durch den Einsatz des MAR345 Flächendetektors ist es für eine Messung mit der Durchstrahlmethode nicht nötig die Probe in einer Eulerwiege um die Achsen $\varphi$ und $\chi$ zu drehen. Die Probe wird auf einem $\omega$-Drehtisch gedreht.

Wird eine polykristalline Probe bestrahlt, bilden sich auf dem Flächendetektor Beugungsringe mit entsprechendem 2 $\theta$-Winkel ab (Abb. 2.9).

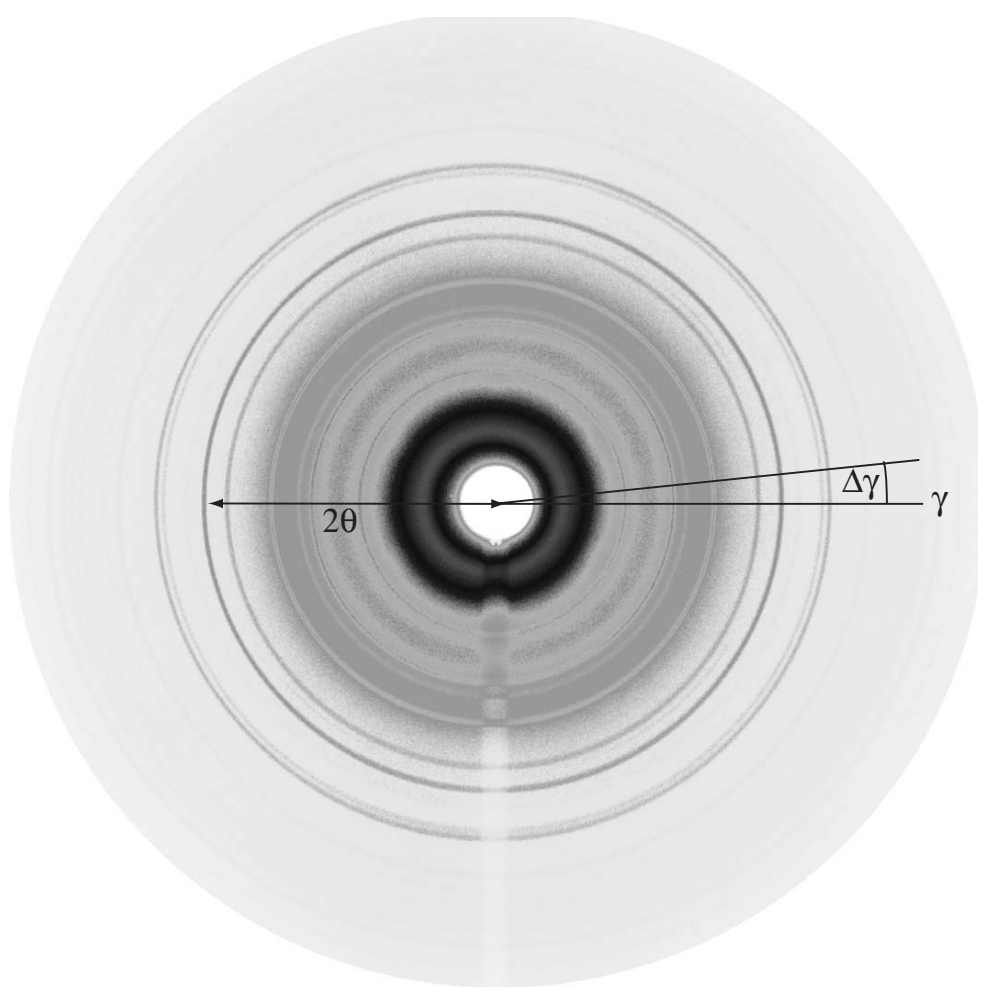

Abbildung 2.9: Aufteilung eines Beugungsbildes zur Polfigurauswertung

Jeder Punkt auf einem Beugungsring entspricht einer bestimmten Orientierung der in Reflexion befindlichen Netzebene. Diese Punkte lassen sich mit Kenntnis der Orientierung der Probe in der Eulerwiege $\{\omega, \chi, \varphi\}$ und der Winkel 
$2 \theta$ und $\gamma$ (Abb. 2.9) in der Flächendetektoraufnahme in die Polfigurpositionen $\{\alpha, \beta\}$ umrechnen [55].

Der abgebeugte Strahl $\vec{R}$ trifft unter den Winkeln $\theta$ und $\gamma$ auf den Detektor. Der Beugungsvektor $\vec{S}$ in Abbildung 2.10a lässt sich in Komponenten im Diffraktometerkoordinatensystem $K_{D}$ durch die Winkel $(\theta, \gamma)$ darstellen.

$$
\vec{S}=\left(\begin{array}{l}
s_{1} \\
s_{2} \\
s_{3}
\end{array}\right)=\left(\begin{array}{c}
-\sin \theta \\
\cos \theta \cdot \cos \gamma \\
\cos \theta \cdot \sin \gamma
\end{array}\right)
$$

Der oben definierte Beugungsvektor $\vec{S}$ lässt sich auch im Polfigurkoordinatensystem $K_{D}$ (Abb.2.10b) darstellen durch $\{\alpha, \beta\}$.

$$
\vec{S}=\left(\begin{array}{l}
y_{1} \\
y_{2} \\
y_{3}
\end{array}\right)=\left(\begin{array}{c}
\sin \alpha \cdot \cos \beta \\
\cos \alpha \\
\sin \alpha \cdot \sin \beta
\end{array}\right)
$$

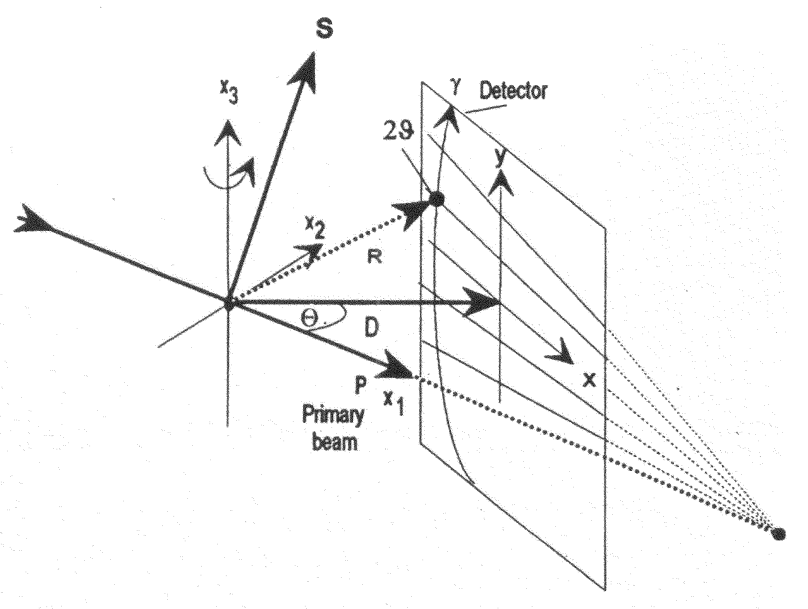

(a) $\vec{s}$ in Diffraktometerkoordinaten

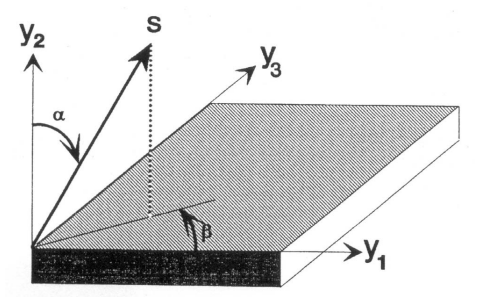

(b) $\vec{s}$ in Probenkoordinaten

Abbildung 2.10: Schematische Darstellung der Beugungsvektors [55]

Die beiden Koordinatensysteme sind über die Probenorientierung $G$ miteinander verknüpft.

$$
y_{i}=G_{i k} \cdot s_{k}
$$

Diese Orientierung $G$ ist gegeben durch die Eulerwiegenwinkel $\{\omega, \chi, \varphi\}$ und wird durch die Orientierungsmatrix $G_{i k}$ beschrieben.

$$
G_{i k}=\left(\begin{array}{ccc}
\cos \omega \cos \varphi & \sin \omega \cos \varphi & \sin \varphi \sin \chi \\
-\sin \omega \sin \varphi \cos \chi & +\cos \omega \sin \varphi \cos \chi & \\
-\cos \omega \cos \varphi & -\sin \omega \cos \varphi & \cos \varphi \sin \chi \\
-\sin \omega \cos \varphi \cos \chi & +\cos \omega \cos \varphi \cos \chi & \\
\sin \omega \sin \chi & -\cos \omega \sin \chi & \cos \chi
\end{array}\right)
$$


Die Messgeometrie am BW5 mittels Durchstrahltechnik vereinfacht die Orientierungsmatrix, da hier die Orientierung nur noch vom der Winkel $\omega$ abhängig ist.

$$
G_{i k}=\left(\begin{array}{ccc}
\cos \omega & \sin \omega & 0 \\
-\sin \omega & \cos \omega & 0 \\
0 & 0 & 0
\end{array}\right) .
$$

In Abbildung 2.11 sind die Polfigurpositionen $\{\alpha, \beta\}$ in Abhängigkeit von Drehwinkel der Küvette $\omega$ und dem Winkel $\gamma$ im Beugungsbild (Abb. 2.9) aufgetragen.

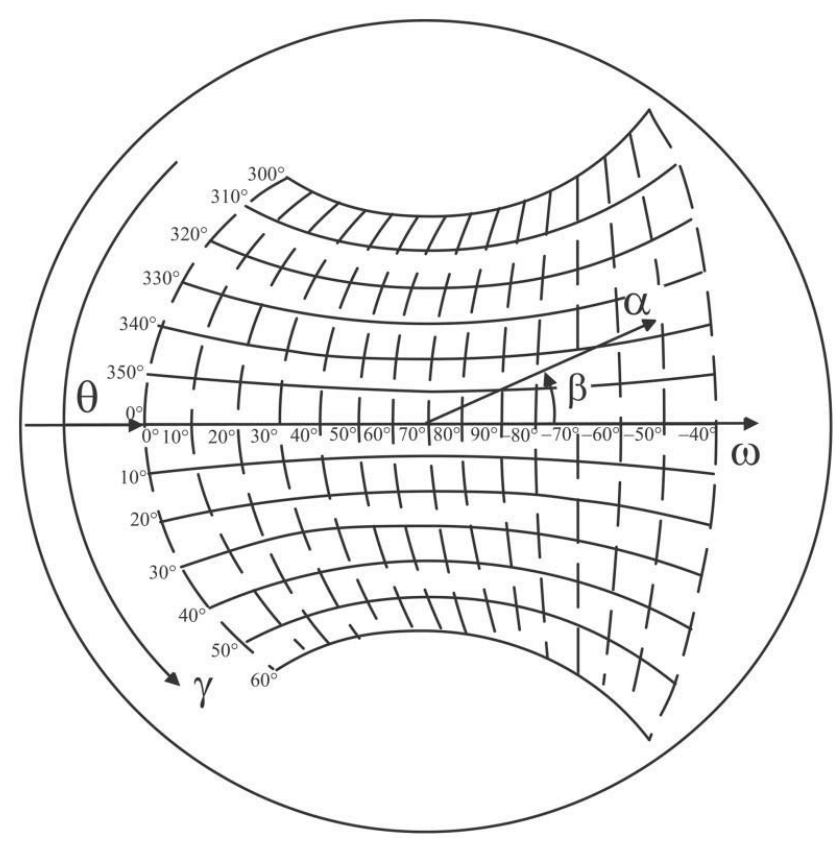

Abbildung 2.11: Schematische Darstellung der Polfigurpositionen $\{\alpha, \beta\}$ in Abhängigkeit von $\omega$ und $\gamma$ [21]

Da sich mit steigendem Drehwinkel $\omega$ Teile der Küvette in den Strahlengang drehen ist der mögliche Aufnahmebereich begrenzt. Für die Rotationsküvette ist dieser bei $56^{\circ}$ erreicht. Der maximale Durchstrahlwinkel für die Durchflussküvette ist Bauart bedingt höher und beträgt $70^{\circ}$. So bleibt ein linsenförmiger nicht gemessener Bereich übrig.

Je nach Feststoffkonzentration, Feststoffart und verwendeter Küvette wurde mit unterschiedlichen Parametern gemessen, welche im Einzelnen in den Kapiteln 5.1 und 5.2 aufgeführt sind. Die Methode ist jedoch für alle Proben gleich. In der Startposition $\omega_{0}$ wird die Probe für eine bestimmte Zeit belichtet und anschließend wird der Flächendetektor ausgelesen. Die Probe wird auf dem $\omega$ Tisch (siehe Abb. 2.1 und 2.5) um einen bestimmten Winkel $\Delta \omega$ gedreht und wieder belichtet. Dieser Vorgang wird bis zum maximalen Durchstrahlwinkel wiederholt.

Aus den so aufgenommenen Beugungsbildern werden anschließend mittels einer eigens entwickelten Software die Polfiguren berechnet. 


\subsection{Polfigursoftware}

Aus den gemessenen Daten sollen Polfiguren berechnet werden, welche selber schon Rückschlüsse auf die Partikelorientierung zulassen. Außerdem werden die Polfigurdaten zur Berechnung der Orientierungsverteilungsfunktion benötigt, welche Vergleiche unter den Proben aufgrund der Normierung (Kap.2.5.1) zulassen. Die Auswertung der einzelnen Flächendetektordaten erfolgt einer speziell entwickelten Software, die folgende Aspekte berücksichtigen muss:

- Berechnung der integralen Intensität für die einzelnen Reflexe

- Korrektur des veränderten beugenden Volumens

- Korrektur des abnehmenden Primärstrahlintensität

- Absorptionskorrektur

- Berechnung der Polfigurkoordinaten

- Interpolation der gemessenen Polfigurdaten auf ein äquidistantes Raster

\subsubsection{Berechnung der integrale Intensität}

Zur Berechnung der integralen Intensität der Beugungsreflexe bedarf es einiger Vorarbeiten. Die während der Messung verwendete Wellenlänge und der Abstand zwischen Probe und Detektor müssen bekannt sein. Für die Beugungsbilder einer Versuchsreihe wird zunächst die Beugungsmitte ermittelt. Hierzu eignet sich die Software FIT2D von A.P. Hammersley [56].

Die zur Berechnung notwendigen Daten wie, Beugungsmitte, Dateinamen (MAR-Bilder), Wellenlänge usw. werden über Eingabefelder (Abb. 2.12) dem Programm zugänglich gemacht. In einem ersten Schritt wird ein Beugungsbild geladen. Vorzugsweise wird das Beugungsbild bei $\omega=0^{\circ}$ benutzt, da die Peakpositionen bei einer Drehung der Probe sich leicht verschieben können, wenn Strahlachse und Drehachse des $\omega$-Kreises nicht exakt übereinander liegen.

Ausgehend von diesem ersten Beugungsbild wird ab einer Entfernung von 50 Pixeln von der Beugungsmitte von der Polfigursoftware ein Beugungsdiagramm erstellt. Vom Programm wird zunächst die Intensität des Pixels für $\gamma=0^{\circ}$ (siehe Abb. 2.9) in dieser Entfernung ausgelesen. Anschließend wird in gleicher Entfernung von der Mitte in Schritten von $\Delta \gamma=1^{\circ}$ die Intensität ausgelesen und summiert bis die $360^{\circ}$-Drehung komplett ist. Die Entfernung von der Beugungsmitte wird in Schritten von 0,5 Pixeln erhöht und es wird wieder für die $360^{\circ}$-Drehung alle Intensität summiert. Dieser Vorgang wird bis 20 Pixel vor dem Ende des Beugungsbilds wiederholt und es entsteht ein Beugungsdiagramm (Abb. 2.12 oberes Fenster).

Aus diesem ersten Beugungsdiagramm werden die Startwerte zur Berechnung der integralen Intensität für die einzelnen Peaks ermittelt. Da jeder Peak mit der Strahlungsintensität der amorphen Substanzen (Glyzerin und Plexiglas) gemeinsam auftritt, müssen diese von einander getrennt werden. 


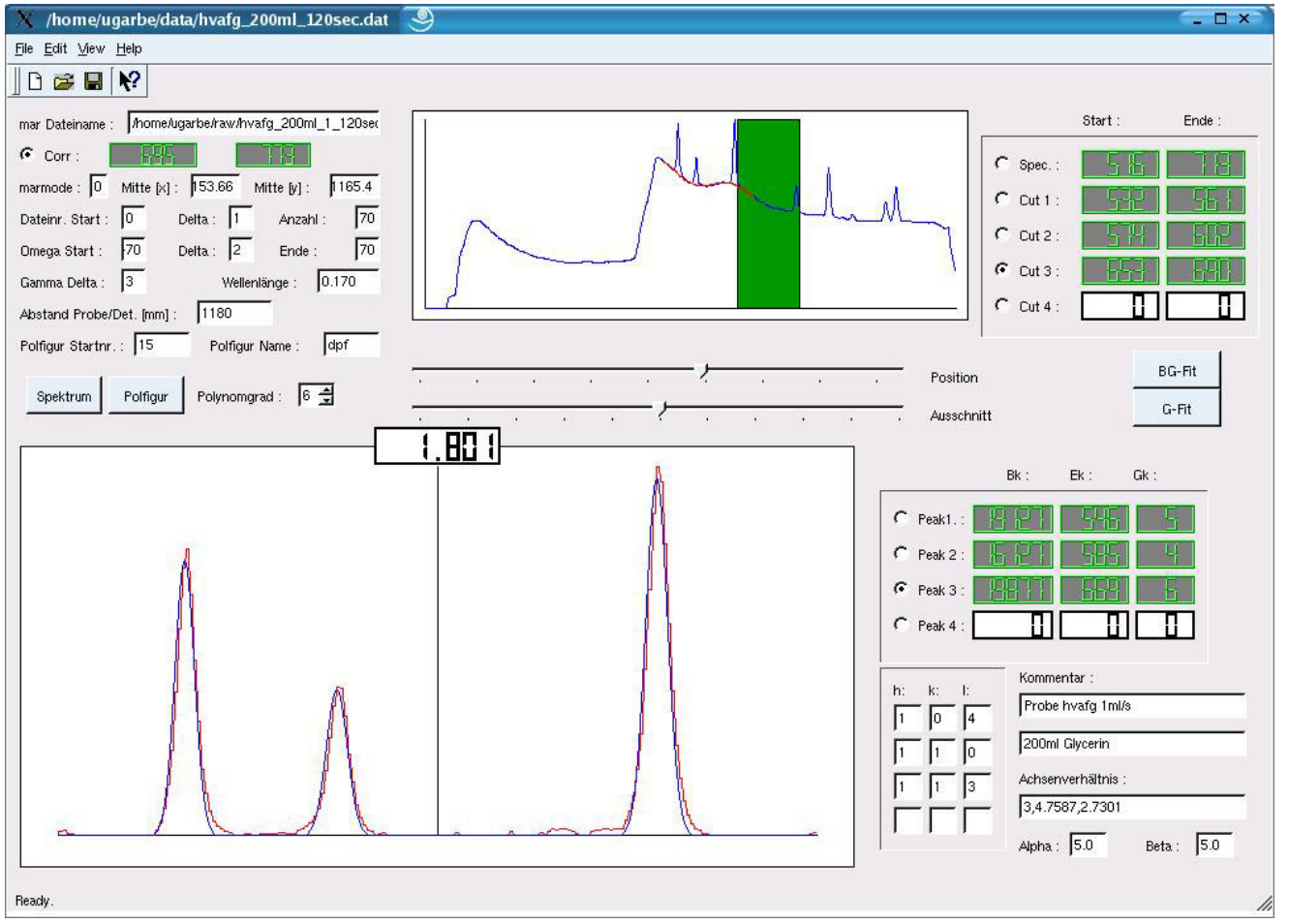

Abbildung 2.12: Die Polfigursoftware

Der amorphe Untergrund kann durch ein Polynom beschrieben werden. Als erstes wird der Bereich markiert, welcher durch ein Polynom beschrieben werden soll. Um die Markierungspunkte möglichst genau setzen zu können ist der grau unterlegte Bereich, dessen Position und Ausdehnung variiert werden kann, im unteren Fenster vergrößert dargestellt. Nachdem der Teil markiert wurde, werden nun die Bereiche in denen Beugungsreflexe auftreten ebenfalls markiert. Diese Peaks werden bei der Polynomanpassung nicht berücksichtigt. Die Anpassung erfolgt mit einem frei wählbaren Polynomgrad nach den Routinen der "Numerical Recipies" [57]. Die berechneten Intensitäten werden vom Beugungsdiagramm subtrahiert und im unteren Programmfenster (Abb. 2.12) neu dargestellt.

Die isolierten Peaks werden durch eine Gaußfunktion beschrieben. Die im unteren Fenster dargestellten Peaks werden erneut markiert. Es wird die Peakposition, Peakintensität und die Halbwertbreite per Mausklick bestimmt und dem Programm übergeben. Mit diesen Daten wird über eine weitere Routine der "Numerical Recipies" [58] eine Gaußfunktion an die jeweiligen Peaks angepasst. Die so berechnete Peakposition, Peakintensität und Halbwertsbreite werden als Startwerte für die Verarbeitung aller Flächendetektorbilder gespeichert.

Um eine Polfigur aus den Flächendetektorbildern zu berechnen muss eine ganze Serie von Bildern (bis zu 70 Bilder pro Polfigur) verarbeitet werden. Durch die Polfigursoftware wird jede Aufnahme in Segmente von $\Delta \gamma=3^{\circ}$ aufgeteilt (Abb. 2.9). Kleinere Segmente führen zu einer dichteren Belegung mit Messpunkten in der Polfigur. Der Wert von $3^{\circ}$ für $\Delta \gamma$ ist so gewählt, dass die 
Belegung der Polfigur die zu erwartende Poldichteverteilung genügend genau beschreibt. Sehr scharfe Texturen benötigen deutlich mehr Messpunkte und somit kleinere Segmente.

Auch aus den Segmenten wird nach der oben beschrieben Methode ein Beugungsdiagramm erstellt. Jedoch stehen jetzt für jedes Diagramm nur $3^{\circ}$ in $\gamma$ zur Verfügung und nicht die vollen $360^{\circ}$. Für jedes Segment wird der Untergrund durch ein Polynom angepasst und subtrahiert. Die integrale Intensität und der $2 \theta$-Wert der einzelnen Peaks wird mit den berechneten Startwerten durch eine Gaußfunktion ermittelt und die Polfigurkoordinaten werden berechnet. Dieser Vorgang wird Bild für Bild wiederholt. Für jede neue Peakanpassung werden jeweils die Peakposition, Peakintensität und Halbwertbreite der letzten Berechnung als Startwert benutzt. Sollte an einigen Positionen kein Peak vorhanden sein und die Peakanpassung somit einen Fehler ausgeben, werden die Startwerte aus der ersten Bestimmung wieder neu geladen und die Berechnung läuft weiter. Die Anzahl der Fehler während einer Anpassung wird protokolliert.

\subsubsection{Korrektur des beugenden Volumens}

Die in Kapitel 2.3.1 berechnete Intensität der einzelnen Polfigurpunkte wird nicht nur durch die Textur beeinflusst. Während der Messung ändert sich das bestrahlte Volumen der Probe in Abhängigkeit vom Drehwinkel $\omega$. Im Fall einer planparallelen Platte, welche für den bestrahlten Bereich der eingesetzten Küvetten gilt, ändert sich das bestrahlte Volumen nach:

$$
V=V_{0} / \cos \omega
$$

So lässt sich für jedes aufgenommene Beugungsbild die Intensität auf das beugende Volumen korrigieren.

\subsubsection{Korrektur der Primärstrahlintensität}

Während einer Messung nimmt die Intensität des Primärstrahls ständig ab. Der Ringstrom des DORIS Speicherrings nimmt unter normalen Betriebsbedingungen um ca. $50 \%$ innerhalb von 8 Stunden ab, weil die Zahl der umlaufenden Positronen sich durch Kollisionen mit Schmutzteilchen oder der Wand verringert. Ist der minimale Ringstrom erreicht wird der Synchrotronbetrieb unterbrochen und der Ring wird mit neuen Positronpaketen gefüllt. Da die Polfigurmessung 1-3 Stunden dauert, muss jede Aufnahme gegen die Primärstrahlintensität normiert werden. Für die ersten Messungen wurde hierzu eine Diode im Strahlengang platziert. Die gemessene Intensität $I_{\text {Diode }, 0 \ldots n}$ wurde in einer Datei für jede Aufnahme gespeichert. Nach Beendigung der Messung wurde aus dem Verhältnis

$$
K_{n}=\frac{I_{\text {Diode }, n}}{I_{\text {Diode }, 0}}
$$

ein Korrekturfaktor $K_{n}$ ermittelt, welcher die Intensität für ein Beugungsbild korrigiert. Diese Methode hat sich aber als zu ungenau erwiesen und wurde 
durch eine andere ersetzt. Da die Intensität des amorphen Untergrunds summiert über ein komplettes Beugungsbild nur von der Intensität des Primärstrahls und des beugenden Volumens abhängt und nicht von der Textur, lässt sich diese für eine Korrektur des beugenden Volumens und der Primärstrahlintensität verwenden.

Es wird ein Bereich des Beugungsdiagramms benutzt, in dem keine Beugungsreflexe auftreten. Die hier ermittelte Intensität ist ausschließlich durch amorphe Substanzen gegeben. Die integrale Intensität $I_{a m o r p h, 0 \ldots n}$ für einen so gewählten Bereich wird gespeichert und es wird ein Korrekturfaktor $K_{n}$ ermittelt, welcher sowohl von dem beugenden Volumen als auch von der Primärstrahlintensität abhängig ist.

$$
K_{n}=\frac{I_{a m o r p h, n}}{I_{a m o r p h, 0}}
$$

\subsubsection{Die Absorptionskorrektur}

Beim Durchgang eines Röntgenstrahls durch ein Medium wird die Intensität durch Absorption geschwächt. Die Absorption lässt sich für monochromatische Strahlung durch ein Exponentialgesetz beschreiben.

$$
I=I_{0} \exp (-\mu d)
$$

Hierbei bedeutet $I_{0}$ die eingestrahlte Intensität, $I$ die jeweilige Intensität nach dem Durchdringen einer Schicht mit der Dicke $d$ und dem Absorptionskoeffizienten $\mu$.

Die Absorptionskorrektur bei der Polfigurmessung ist deutlich komplizierter. Hier ist die Länge des gesamten Strahlwegs des Primärstrahls und des abgebeugten Strahls für den jeweiligen Korrekturfaktor verantwortlich. Der Strahlweg für einen bestimmten Punkt auf dem Flächendetektor ist in Abbildung 2.13 dargestellt. Die Weglänge ist von den Parametern $\omega, 2 \theta$ und $\gamma$ abhängig.

Die Intensität für die in Abbildung 2.13 dargestellte Durchstrahlgeometrie berechnet sich nach [59]:

$$
\begin{aligned}
I_{\omega, \gamma}=K A \int_{0}^{t} e^{B x} \frac{d x}{\cos \omega} & =K \frac{A\left(e^{B t}-1\right)}{B \cos \omega} \\
\text { mit } & \\
A & =e^{-\mu t C} \\
B & =-\mu\left(\frac{1}{\cos \omega}-C\right) \\
C & =\frac{1}{\cos \omega \cos 2 \theta-\sin \omega \sin 2 \theta \cos \gamma}
\end{aligned}
$$

$\mu=$ linearer Absorptionskoeffizient

$\omega=$ Winkel zwischen Primärstrahl und Probennormalen.

$\gamma=$ Azimuthwinkel entlang des Beugungsrings (Abb. 2.9 Kap. 2.3.1). 
$\mathrm{t}=$ Probendicke.

$\mathrm{x}=$ Position an der Beugung stattfindet.

$\mathrm{K}=$ Konstante, welche die Intensität des Primärstrahls und den Strukturfaktor beinhaltet.

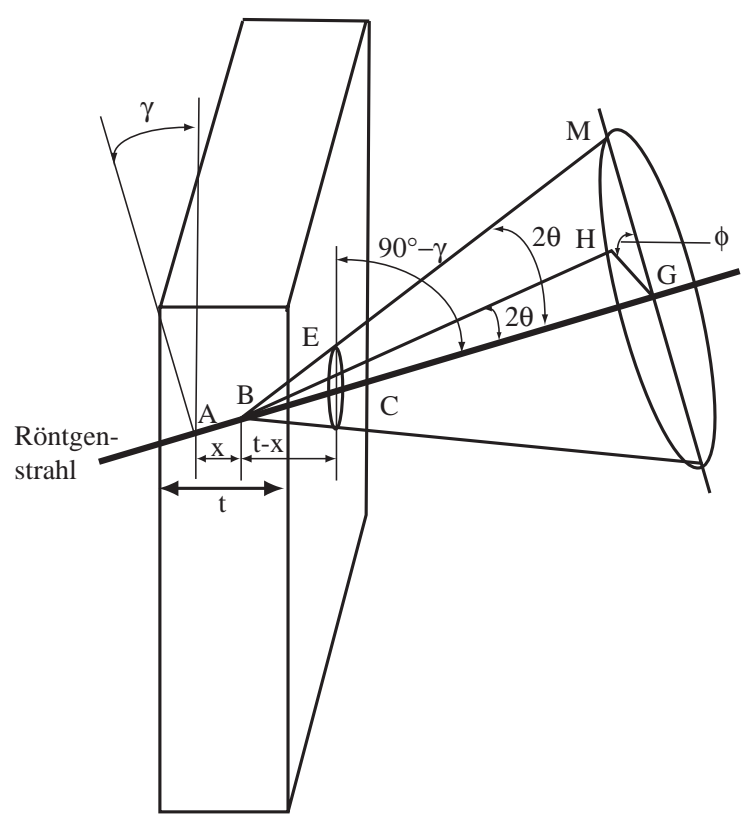

Abbildung 2.13: Schematische Darstellung des Strahlwegs [59]

Die Berechnung der absorptionskorrigierten Intensität gilt nur für ein durchstrahltes Medium mit homogenem Absorptionskoeffizienten. Der Strahlweg in den eingesetzten Küvetten passiert jedoch drei unterschiedliche Medien, welche nicht homogen verteilt sind. Zuerst wird eine Plexiglasschicht durchstrahlt. Darauf folgt eine Schicht aus Glyzerin und $\mathrm{Al}_{2} \mathrm{O}_{3}$ und noch mal eine Plexiglasschicht. Die linearen Absorptionskoeffizienten für die drei durchstrahlten Substanzen wurden mit dem Programm ' 'absorb' " [60] erstellt und sind in Tabelle 2.2 aufgelistet.

\begin{tabular}{c|c|c} 
Substanz & $\mu\left[\mathrm{mm}^{-1}\right]$ bei $80 \mathrm{keV}$ & $\mu\left[\mathrm{mm}^{-1}\right]$ bei $100 \mathrm{keV}$ \\
\hline Plexiglas & 0,022 & 0,021 \\
Glyzerin & 0,020 & 0,019 \\
$\mathrm{Al}_{2} \mathrm{O}_{3}$ & 0,051 & 0,045 \\
Küvette & 0,023 & 0,022
\end{tabular}

Tabelle 2.2: Lineare Absorptionskoeffizienten bei $80 \mathrm{keV}$ und $100 \mathrm{keV}$ Strahlenergie [60]

Der Absorptionskoeffizient für die Küvette entspricht einer Mittelung der einzelnen Koeffizienten unter Berücksichtigung der jeweiligen Volumenanteile der durchstrahlten Substanzen. 
In Abbildung 2.14 ist der Korrekturfaktor zur Berechnung der gebeugten Intensität mit einem linearen Absorptionskoeffizient $\mu=23$ und der Schichtdicke $t=20 \mathrm{~mm}$ aufgetragen. Der maximalen Beugungswinkeln $2 \theta=6,5^{\circ}$ der untersuchten Reflexe wird hier zur Berechnung des Korrekturfaktors verwendet, da so der größtmögliche Längenunterschied für die Strahlwege erreicht wird.

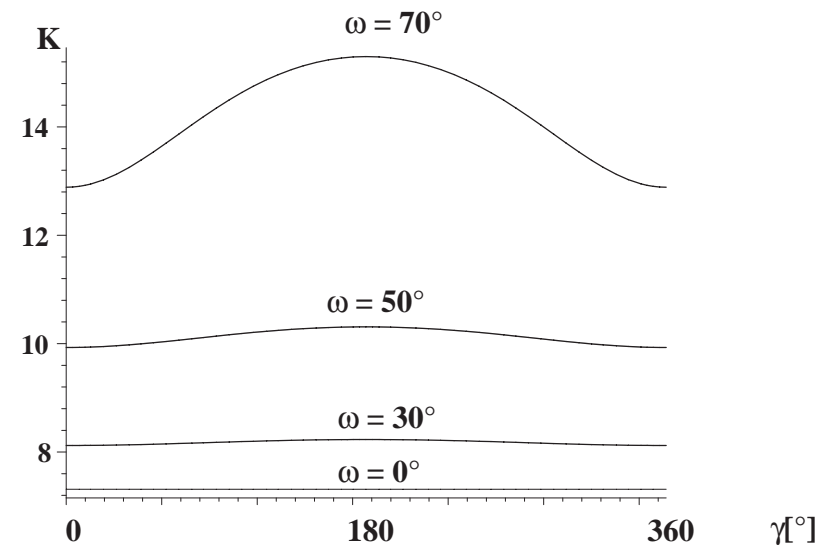

Abbildung 2.14: Korrekturfaktor der Absorption für unterschiedliche Absorptionskoeffizienten

Die aufgetragenen Korrekturfaktoren zeigen deutlich, dass auch bei den hohen Strahlenergien am BW5 die Absorption einen Einfluss auf die Intensität der gebeugten Strahlung hat. Der Korrekturfaktor schwankt von minimal ca. 7,5 bei $\omega=0^{\circ}$ bis maximal ca. 15 bei $\omega=70^{\circ}$. Auch bei konstantem $\omega=70^{\circ}$ innerhalb eines Beugungsrings $\left(\gamma=0-360^{\circ}\right)$ ist die Absorption nicht zu vernachlässigen. Hier ändert sich der Korrekturfaktor von ca. 13-15.

Eine Überprüfung des mittleren Koeffizienten mit realen Daten ist also notwendig. Hierzu wird die Intensität der amorphen Substanzen für einen bestimmten $2 \theta$-Wert ausgelesen und korrigiert. Da für den amorphen Anteil ein regelloses Verhalten angenommen werden kann, sollte die Intensität lediglich von den geometrischen Verhältnissen und nicht von der Textur abhängen. Nach einer gelungenen Korrektur muss für jede Position in $\omega$ und $\gamma$ die gleiche Intensität bestimmt werden. In Abbildung 2.15 sind die korrigierten Intensitäten für verschiedene Messungen einer nicht korrigierten Messung in Abhängigkeit von $\omega$ und $\gamma$ gegenübergestellt. Nach der Korrektur ändert sich die Intensität mit unterschiedlichen Detektorpositionen in $\gamma$ kaum noch. Die Korrekturen führen zu deutlich geringeren Intensitätsschwankungen innerhalb eines Beugungsrings. Mit steigendem Kippwinkel $\omega$ führt die Korrektur der Absorption jedoch zu geringeren Intensitäten.

Eine zufrieden stellende Korrektur ist mit dieser Methode nicht möglich. Die geometrischen Verhältnisse eines Systems mit mehreren Schichten lassen sich durch einen mittleren linearen Absorptionskoeffizienten nicht beschreiben. Da die Absorption jedoch auch bei diesen hohen Energien einen großen Einfluss auf die Intensität der gebeugten Strahlung hat muss ein anderer Weg zur Korrektur gefunden werden.

Hierzu eignet sich der Teil der gebeugten Strahlung, welcher von den amor- 

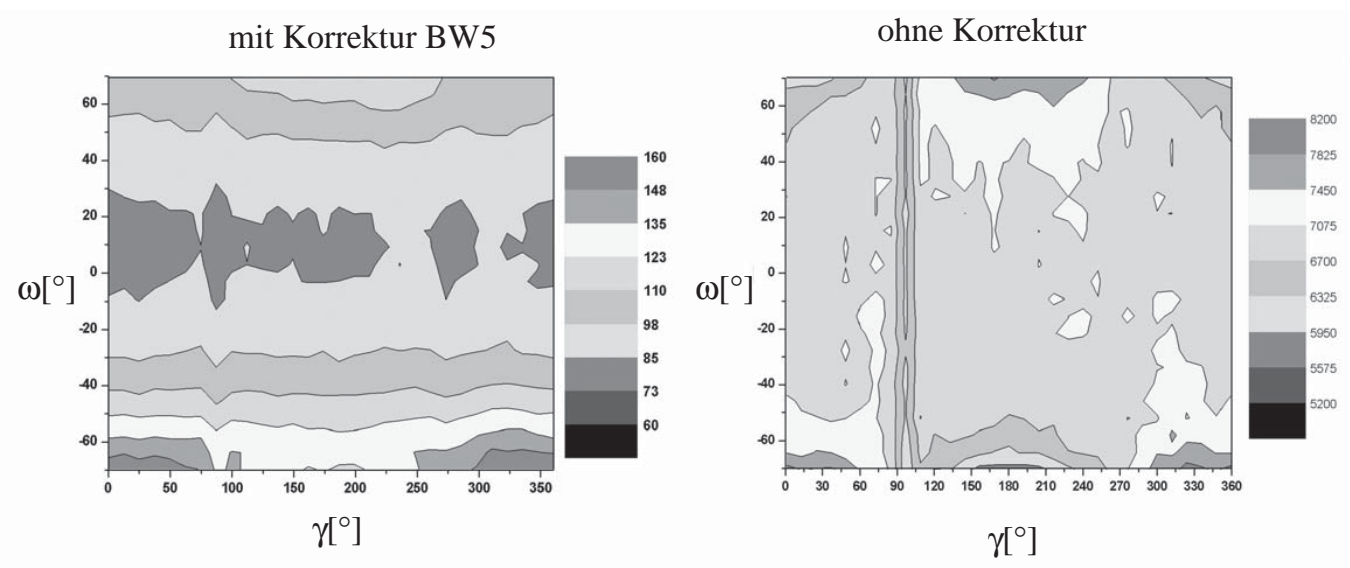

Abbildung 2.15: Korrigierte amorphe Intensitäten

phen Substanzen verursacht wird. Der an einer amorphen Substanz gebeugte Primärstrahl wird im weiteren Verlauf genauso absorbiert, wie ein gebeugter Strahl an einer kristallinen Substanz. Die Beugung findet für die amorphen Medien zwar teilweise an anderen Orten statt, wie im kristallinen Fall. Eine Korrektur mit der Intensität der amorphen Medien hat jedoch den Vorteil, dass sie unter gleichen Bedingungen entsteht, wie die Bragg Peaks. Dies ist auch nur eine Näherung für die tatsächliche Absorptionskorrektur, welche jedoch für diesen Fall die bestmögliche darstellt. Die errechneten Korrekturfaktoren beinhalten sowohl die Absorptionskorrektur, als auch die Volumenkorrektur und die Korrektur des Ringstroms.

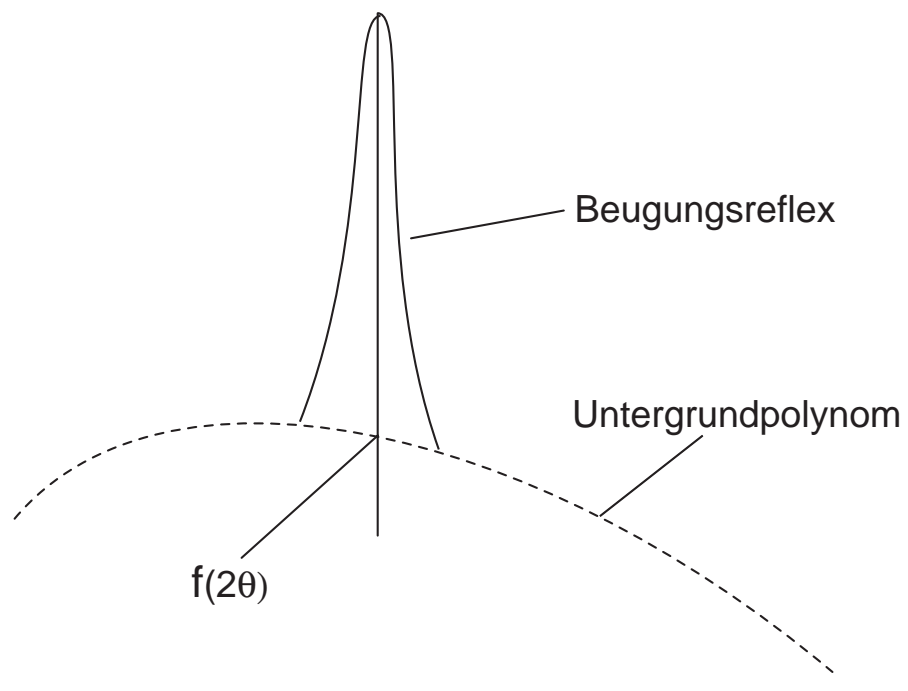

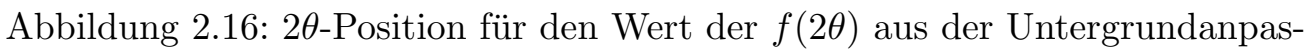
sung

Die Beschreibung des Untergrunds durch ein Polynom liefert auch für die $2 \theta$-Position einen Wert $f(2 \theta)$, welcher direkt unter einem Bragg Peak sitzt (siehe Abb. 2.16). Dieser Wert wird im Programm berechnet und zur Korrektur 
weiterverarbeitet. Die Korrektur erfolgt nach dem gleichen Prinzip, wie bei der Diode oder der mathematischen Korrektur.

$$
K_{n}=\frac{f_{2 \theta, n}}{f_{2 \theta, 0}}
$$

Alle Polfiguren im weiteren Verlauf sind nach dieser Methode korrigiert worden.

\subsubsection{Interpolation des Polfigurrasters}

Die weitere Verarbeitung der so berechneten Polfigurdaten erfordert eine nochmalige Umrechnung. Die ODF-Programme, welche zur weiteren Bearbeitung der Daten nötig sind, benötigen die Daten auf einem äquidistanten Polfigurraster $(\Delta \alpha=$ const. und $\Delta \beta=$ const.). In Abbildung 2.17 sind die Positionen der beiden Raster dargestellt. Die gefüllten Punkte befinden sich auf dem Raster der gemessenen Polfigurdaten und die leeren Punkte zeigen das äquidistante Polfigurraster.

Zur Umrechnung wird für jeden Punkt des äquidistanten Polfigurrasters der Abstand zu den gemessenen Polfigurpunkten berechnet. Für jeden dieser äquidistanten Rasterpunkte wird aus den drei nächstgelegenen gemessenen Punkten die Intensität ermittelt. Die drei Intensitäten der gemessenen Polfigurpunkte werden über den Abstand zum Rasterpunkt gewichtet und es wird der Mittelwert gebildet. Die so berechneten Polfiguren werden im Weiteren direkt dargestellt oder zur ODF-Rechnung verwendet.

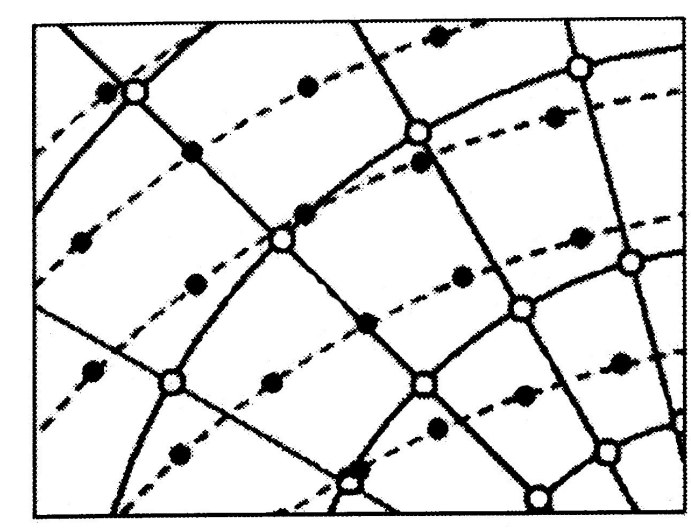

Abbildung 2.17: Polfigurausschnitt des Messrasters und des äquidistanten Rasters [61] 


\subsection{Die Textur}

Die kristallographische Orientierung der Kristallite in einem polykristallinen Material wird als Textur bezeichnet. Um die Orientierung der Kristallite beschreiben zu können, legt man in der zu untersuchenden Probe ein rechtwinkliges Koordinatensystem fest $K_{A}$. Im Falle von strömenden zwei-phasen Gemischen fällt eine Achse mit der Flussrichtung FR zusammen. Die zweite Koordinatenachse ist senkrecht zur ersten Richtung und befindet sich in der Ebene der Strömungsküvette. Die dritte Achse ergibt sich aus der Lage der ersten beiden Koordinatenachsen (Abb. 2.18).

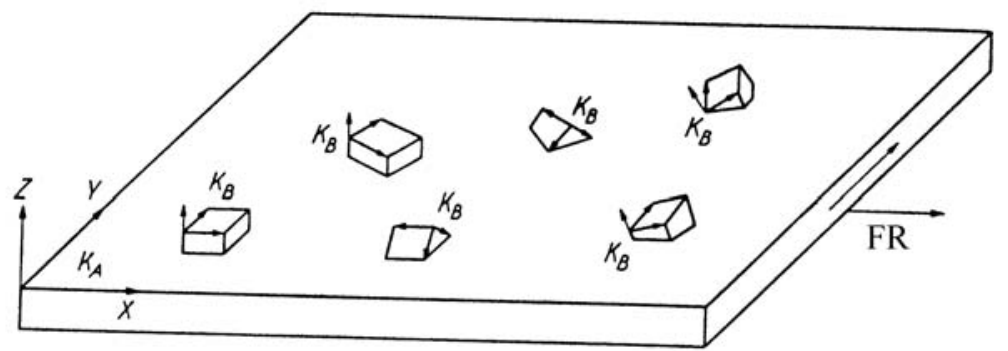

Abbildung 2.18: Probenfestes Koordinatensystem $K_{A}$ und kristallfestes Koordinatensystem $K_{B}$ [62]

Für die Kristallite in der strömenden Flüssigkeit wird ein weiteres Koordinatensystem $K_{B}$ festgelegt, welches sich an den kristallographischen Achsen orientiert. Da die zu untersuchenden $\mathrm{Al}_{2} \mathrm{O}_{3}$-Partikel hexagonale Kristalle sind, muss das Koordinatensystem entsprechend angepasst werden:

$$
\begin{aligned}
& \text { - } \mathrm{x} \Rightarrow(100) \\
& \text { - } \mathrm{y} \Rightarrow(110) \\
& \text { - } \mathrm{z} \Rightarrow(001)
\end{aligned}
$$

Die Orientierung der Netzebenen in einem Kristallit innerhalb der Strömung wird durch die Angabe der Drehung $g$ beschrieben, die das probenfeste Koordinatensystem $K_{A}$ in das kristallfeste Koordinatensystem $K_{B}$ überführt.

$$
K_{B}=g * K_{A}
$$

Um die Drehung $g$ zu beschreiben gibt es verschiedene Möglichkeiten. Im Bereich der Texturanalyse ist die Eulersche Darstellung der Drehung weit verbreitet. Zunächst wird das Koordinatensystem um die Z-Achse um den Winkel $\varphi_{1}$ gedreht. Anschließend dreht man um die neue X'-Achse um $\Phi$ und letztlich noch um die neue Z'-Achse um $\varphi_{2}$ (Abb. 2.19)[62].

Um eine Orientierung darstellen zu können werden die drei Drehwinkel als Koordinatenachsen eines kartesischen Koordinatensystems dargestellt. Sie spannen den so genannten Eulerraum auf, in dem sich eine einzelne Orientierung als Punkt darstellt (Abb.2.20a ). Trägt man die Gesamtheit aller gemessenen 


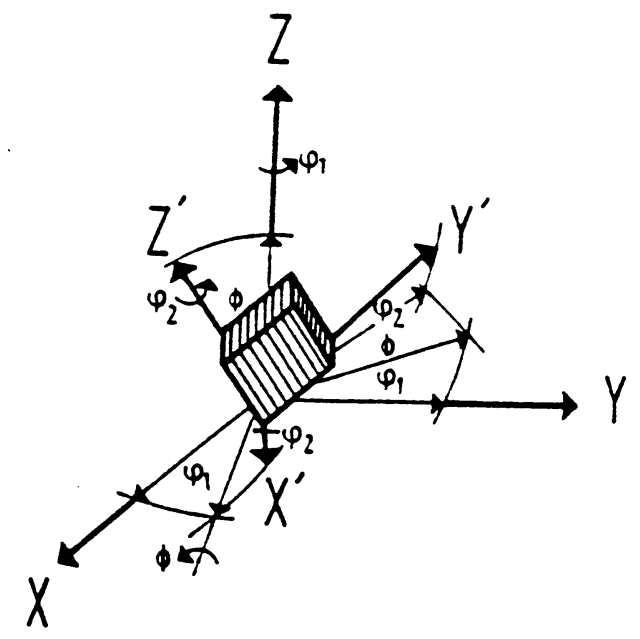

Abbildung 2.19: Definition der Eulerschen Winkel $\left\{\varphi_{1}, \phi, \varphi_{2}\right\}[62]$

Orientierungen von polykristallinen Substanzen auf erhält man eine Wolke von Punkten (Abb.2.20b). Die Orientierungsverteilungsfunktion, welche die gesamte Textur beschreibt ist in Abbildung .2.20c dargestellt [51].
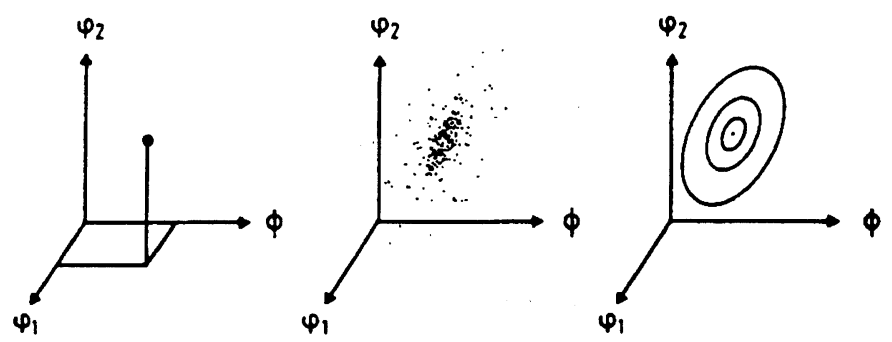

Abbildung 2.20: Eulerraum: a) einzelne Orientierung b) Orientierung aller Kristallite in der Probe c) Orientierungsverteilung [62]

\subsection{Die Orientierungsverteilungsfunktion}

\subsubsection{Orientierungsverteilungen}

Die in Kapitel 2.4 beschriebene Drehung um die Eulerwinkel $\left\{\varphi_{1}, \phi, \varphi_{2}\right\}$ beschreibt eine einzelne Orientierung $g$. Um die Orientierungen der in der Strömung befindlichen Kristallite beschreiben zu können benötigt man für jeden Ort mit den Koordinaten $x, y, z$ innerhalb der Probe die zugehörige Orientierung g.

Die röntgenographische Polfigurmessung (Kap. 2.2) erfasst ein bestimmtes Probenvolumen. Die mit dieser Methode gemessene Intensität lässt sich keinem Ort $(x, y, z)$ innerhalb des bestrahlten Volumens zuordnen. Es ist lediglich ein Volumenanteil von Netzebenen, welcher nach der Braggschen Gleichung die Reflexionsbedingungen erfüllt [63].

Eine Darstellung der Textur aus röntgenographisch gemessenen Daten berück- 
sichtigt nur die Orientierungen der Volumenelemente in der Probe. Bezeichnet man mit $d V$ die Gesamtheit aller Volumenanteile der Probe, die eine Orientierung $g$ innerhalb des Orientierungselements $d g$ besitzen, und mit $V$ das gesamte Probenvolumen, so ist durch

$$
f(g)=f\left\{\varphi_{1}, \phi, \varphi_{2}\right\}=\frac{d V(g) / V}{d g}
$$

eine Orientierungsverteilungsfunktion $f(g)$ des Volumens definiert. Dabei wird die Orientierungsdichte $f$ in Vielfachen der regellosen Verteilung angegeben und entsprechend normiert.

$$
f_{\text {regellos }}=\oint f(g) d g=1
$$

Eine solche Normierung lässt auch den Texturvergleich von unterschiedlich gemessenen Proben zu. Die erhaltene Orientierungsdichte ist nicht von den Messbedingungen ( z.B. Primärstrahlintensität, Wellenlänge, Belichtungszeit) abhängig, sondern nur von der Orientierung der Kristallite.

\subsubsection{Polfiguren}

Da die Orientierung der Kristallite nicht direkt röntgenographisch bestimmt werden kann, ist die Berechnung der Orientierungsverteilungsfunktion (Orientation Distribution Function $=$ ODF) mittels Reihenentwicklung aus Polfigurdaten notwendig.

Eine bestimmte Probenrichtung $y$ kann durch ihre kartesischen Koordinaten oder Polarkoordinaten im Probenkoordinatensystem $K_{A}$ festgelegt werden.

$$
y=\left[y_{1}, y_{2}, y_{3}\right]=\{\alpha, \beta\}
$$

Entsprechend ist eine Kristallrichtung $h$ im Kristallkoordinatensystem $K_{B}$ definiert.

$$
h=\left[h_{1}, h_{2}, h_{3}\right]=\{\theta, \gamma\}
$$

Betrachtet man diejenigen Kristallite, die eine bestimmte Kristallrichtung $h$ parallel zu einer bestimmten Probenrichtung $y$ besitzen, so ist die Achsenverteilungsfunktion $A(h, y)$ dieser Kristallite durch das Integral über die Orientierungsverteilungsfunktion $\mathrm{f}(\mathrm{g})$ gegeben.

$$
A(h, y)=\frac{1}{2 \pi} \int_{h \| y} f(g) d \psi
$$

Hält man eine bestimmte Kristallrichtung $h$ fest und variiert die Probenrichtung $y$, erhält man die Poldichteverteilung oder Polfigur, welche man auch als Ergebnis der hier durchgeführten röntgenographischen Untersuchungen erhält (siehe Kap. 2.2) Es gilt:

$$
A(h, y)=P_{h}(y)=P_{h}(\alpha, \beta)
$$


Polfiguren werden durch Beugung an der zur Kristallrichtung $h$ senkrechten Netzebenenschar (hkl) bestimmt. Diese Polfiguren sind mit der Orientierungsverteilungsfunktion (ODF) folgendermaßen verknüpft.

$$
P_{(h k l)}(\alpha, \beta)=\frac{1}{2 \pi} \int_{(h k l) \perp(\alpha, \beta)} f(g) d \psi
$$

Eine Drehung der Kristallite um die Richtung $h$ kann bei der röntgenographischen Messung nicht registriert werden. Die Intensität im Punkt $y=\{\alpha, \beta\}$ der Polfigur ist somit das Integral über alle Orientierungen, die durch Drehung um diese Richtung hervorgehen. Polfiguren sind 2-dimensionale Projektionen der dreidimensionalen ODF [62, 51].

\subsubsection{Berechnung der ODF mittels Reihenentwicklung}

Das Verfahren der Reihenentwicklung erstellt aus den 2-dimensionalen Polfiguren die 3-dimensionale Abbildung des Orientierungsraums. Es ist mit der Computertomographie in der Medizin vergleichbar. Die Texturfunktion $f(g)$ wird in einer Reihe nach symmetrisch verallgemeinerten Kugelfunktionen entwickelt.

$$
f(g)=\sum_{l=0}^{\infty} \sum_{m=-l}^{+l} \sum_{n=-l}^{+l} C_{l}^{m n} T_{l}^{m n}(g)
$$

Die ODF berücksichtigt zwei Arten von Symmetrien:

- die Kristallsymmetrie (z. B. kubisch (AlMn), hexagonal $\left(\mathrm{Al}_{2} \mathrm{O}_{3}\right)$ )

- die statistische Probensymmetrie (z. B. orthorhombisch (Bleche), rotationssymmetrisch (Drähte))

Symmetrisch äquivalente Punkte besitzen in der Regel die gleichen Werte der Orientierungsverteilungsfunktion.

$$
f\left(g^{c} \cdot g \cdot g^{s}\right)=f(g)
$$

$g^{c}$ bezeichnet die Symmetriedrehung der Kristallsymmetrie und $g^{s}$ die Symmetriedrehung der Probensymmetrie. Wenn $f(g)$ beide Symmetriebedingungen erfüllt, werden je nach vorhandener Symmetrie einige Koeffizienten $C_{l}^{m n}$ Null oder untereinander gleich sein. Die Berücksichtigung der Kristall- und Probensymmetrie führt zu einer anderen Reihenentwicklung, welche für jedes Reihenglied beide Arten von Symmetriebedingungen erfüllt.

$$
f(g)=\sum_{l=0}^{\infty} \sum_{\mu=1}^{M(l)} \sum_{\nu=1}^{N(l)} C_{l}^{\mu \nu} \dot{T}_{l}^{\mu \nu}(g)
$$

Darin sind die $\dot{T}_{l}^{\mu \nu}$ die symmetrisch verallgemeinerten Kugelfunktionen und $C_{l}^{\mu \nu}$ die Koeffizienten der Reihenentwicklung. Jede Funktion $\ddot{T}_{l}^{\mu \nu}(g)$ genügt 
der Kristallsymmetrie (linksseitiger Punkt) und der Probensymmetrie (rechtsseitiger Punkt). Die verschiedenen Symmetriegruppen werden durch die unterschiedliche Anzahl von Punkten charakterisiert. $M(l)$ gibt die Zahl der linear unabhängigen Funktionen des Grades ( $l$ ) für die Probensymmetrie an und $N(l)$ die linear unabhängigen Funktionen für die Kristallsymmetrie. Setzt man die Reihenentwicklung in Gl. 2.26 ein, so erhält man

$$
A(h, y)=\sum_{l=0}^{L_{\max }} \sum_{\mu=1}^{M(l)} \sum_{\nu=1}^{N(l)} \frac{4 \pi}{2 l+1} C_{l}^{\mu \nu} \cdot \dot{k}_{l}^{\star \mu}(h) \cdot \dot{k}_{l}^{\nu}(y)
$$

Dabei sind $\dot{k}_{l}^{\star \mu}$ und $\dot{k}_{l}^{\nu}$ symmetrische Kugelflächenfunktionen der Kristallrichtung $h$ und der Probenrichtung $y$. Hält man die Kristallrichtung $h$ konstant, ergibt sich für die Polfigur:

$$
P_{h k l}(\alpha, \beta)=\sum_{l=0}^{L_{\max }} \sum_{\nu=1}^{N(l)} F_{l}^{\nu} \cdot \dot{k}_{l}^{\nu}(\alpha, \beta)
$$

Die Funktion $P_{h k l}(\alpha, \beta)$ gibt an, für welchen Volumenanteil der Probe die Netzebenennormale (hkl) in die verschiedenen Probenrichtungen fällt. Sie entspricht der Polfigur. Die in Gleichung 2.28 auftretenden Reihenentwicklungskoeffizienten $F_{l}^{\nu}$ sind mit den $C_{l}^{\mu \nu}$-Koeffizienten folgendermaßen verknüpft:

$$
F_{l}^{\nu}(h k l)=\frac{4 \pi}{2 l+1} \sum_{\mu=1}^{M(l)} C_{l}^{\mu \nu} \dot{k}_{l}^{\star \nu}(y)
$$

Für jedes Indexpaar $l, \nu$ stellt Gleichung 2.29 ein Gleichungssystem mit $M(l)$ Unbekannten $C_{l}^{\mu \nu}$ dar. Dieses Gleichungssystem kann nach $C_{l}^{\mu \nu}$ aufgelöst werden, wenn mindestens ebenso viele $F_{l}^{\nu}(h k l)$ für verschiedene (hkl) gegeben sind. Die $F_{l}^{\nu}(h k l)$ erhält man durch Umkehrung von Gleichung 2.28 in der Form

$$
F_{l}^{\nu}(h k l)=\int_{\alpha=0}^{\pi} \int_{\beta=0}^{2 \pi} P_{h k l}(\alpha, \beta) \cdot \dot{k}_{l}^{\star \nu}(\alpha, \beta) \cdot \sin \alpha d \alpha d \beta
$$

aus den gemessenen Polfiguren. Das Integral ist über die vollständige Polfigur zu erstrecken. Da die Polfiguren röntgenographisch nicht vollständig gemessen werden können, muss man den fehlenden Teil durch Näherungen ermitteln. Dabei setzt man voraus, dass jede Kristallorientierung g innerhalb der gemessenen Bereiche der Polfiguren "Bildpunkte" besitzt, so dass die fehlenden Bereiche einer Polfigur durch vorhandene einer anderen ersetzt werden können. Als Startwert für die Berechnung genügt irgendeine Näherungsannahme. Die rückgerechneten Polfiguren können jetzt negative Werte besitzen. Diese sind physikalisch sinnlos und werden durch Null ersetzt. Dann wird das Verfahren wiederholt, bis die Polfigurwerte $\geq 0$ sind. Dieses Verfahren nennt sich Herstellen der "Polfigurenpositivität" [51].

Um die Orientierungsverteilungsfunktion zu berechnen, ist eine gewisse Anzahl $I_{P}$ von Polfiguren nötig. Je nach Reihenentwicklungsgrad $l$ erhält man 
ein Gleichungssystem von $I_{P}$ linearen Gleichungen mit $M(l)$ unbekannten CKoeffizienten $\left(C_{l}^{\mu \nu}\right)$ (siehe Gl. 2.29). Um dieses Gleichungssytem eindeutig zu lösen muss die Zahl der Gleichungen (Polfiguren) mindestens gleich der Zahl der Unbekannten sein.

$$
M(l) \leqq I_{P}
$$

In Abbildung 2.21 ist der Zusammenhang zwischen dem Reihenentwicklungsgrad, der Kristallsymmetrie und der Anzahl der benötigten Polfiguren dargestellt.

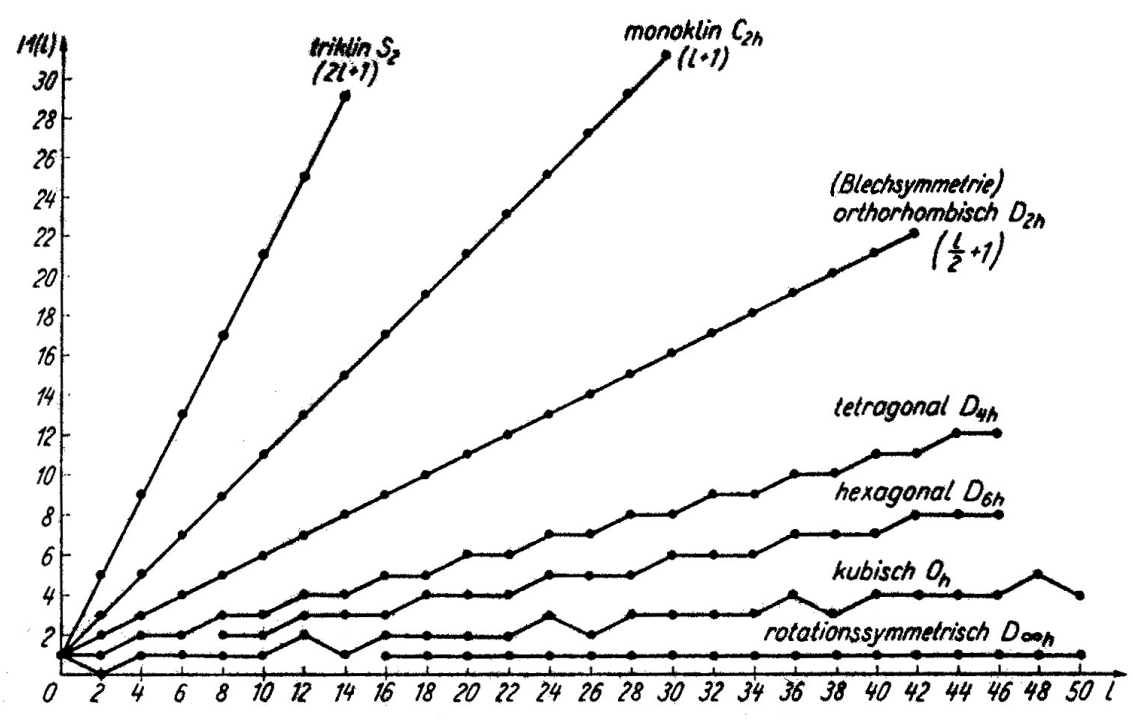

Abbildung 2.21: Anzahl der linear unabhängigen Polfiguren der verschiedenen Symmetrien in Abhängigkeit vom Reihenentwicklungsgrad $l$

\subsubsection{Konvergenz der Reihenentwicklung}

Erstreckt man die Reihenentwicklung bis zu sehr hohen, aber endlichen Werten von $l$, so wird die unstetige Funktion $f(g)$ durch eine stetige Funktion angenähert, die überall dort Maxima besitzt, wo $f(g)$ von Null verschieden ist (Abb. 2.22). Da so jedoch auch statistische Zufälligkeiten beschrieben werden, muss die Reihenentwicklung vorher abgebrochen werden. Mit kleiner werdendem Reihenentwicklungsgrad $L$ erreicht die Halbwertsbreite der einzelnen Maxima die Größenordnung ihres gegenseitigen Abstandes, so dass diese nicht mehr aufgelöst werden können. Andererseits muss $L$ so groß gewählt werden, das die wesentlichen Merkmale der Orientierungsverteilung durch die Funktion $f(g)$ wiedergegeben werden.

Um scharfe Texturen in einer Reihe zu entwickeln benötigt man einen höheren Reihenentwicklungsgrad als bei flacheren Texturen. Je nach Qualität der gemessenen Daten und nach Schärfe der Textur beginnt der mittlere Absolutwert der Koeffizienten $C_{l}^{\mu \nu}$ mit steigendem Reihenentwicklungsgrad zu divergieren. 


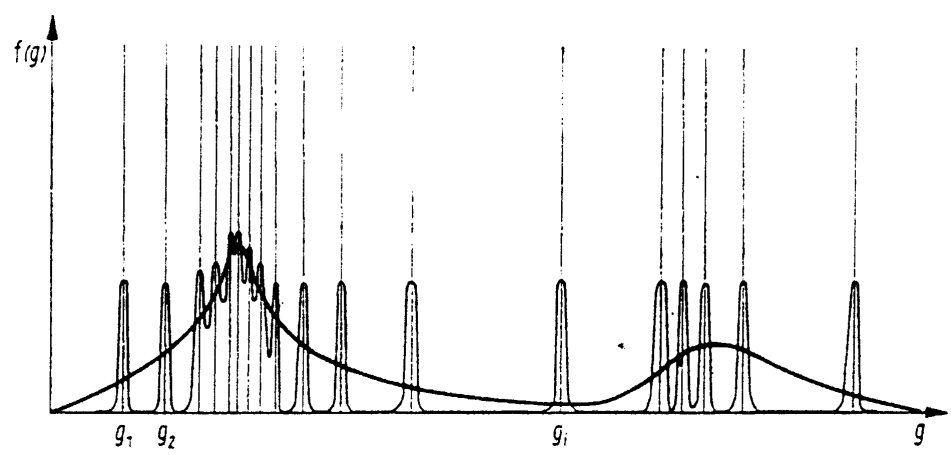

Abbildung 2.22: Annäherung der unstetigen Funktion $f(g)$ durch ihre Dichteverteilung [62]

Die Reihenentwicklung muss vor diesem Punkt abgebrochen werden.

$$
C_{l}=\left|\overline{C_{l}^{\mu \nu}}\right|=\frac{1}{M(l) \cdot N(l)} \sum_{\mu=1}^{M(l)} \sum_{\nu=1}^{N(l)}\left|C_{l}^{\mu \nu}\right|
$$

\subsubsection{Fehler der ODF-Berechnung}

Die Genauigkeit der ODF-Rechnung kann anhand von Differenzpolfiguren abgeschätzt werden.

$$
\Delta P_{(h k l)}(\alpha, \beta)=P_{(h k l)}(\alpha, \beta)_{e x p}-P_{(h k l)}(\alpha, \beta)_{c a l c}
$$

Im idealen Fall sollten die Differenzpolfiguren überall den Wert Null besitzen. Es ist jedoch sinnvoll ein Genauigkeitsmaß zu haben, welches mit dem Konvergenzmaß $C_{l}$ vergleichbar ist. Das erhält man, wenn man die Differenzpolfigur in einer Reihe entwickelt.

$$
\Delta P_{(h k l)}(\alpha, \beta)=\sum_{l=0}^{L_{\max }} \sum_{\nu=1}^{N(l)} \epsilon_{l}^{\nu}(h k l) \cdot \dot{k}_{l}^{\nu}(\alpha, \beta)
$$

mit den Koeffizienten

$$
\epsilon_{l}^{\nu}(h k l)=F_{l}^{\nu}(h k l)_{e x p}-F_{l}^{\nu}(h k l)_{c a l c}
$$

Der wahrscheinliche Fehler der Koeffizienten $C_{l}^{\mu \nu}$ errechnet sich nach der Leastsquares Methode.

$$
\Delta C_{l}^{\mu \nu}=\sqrt{\beta^{\mu \mu}} \cdot \sqrt{\frac{\sum_{i}\left[\epsilon_{l}^{\nu}(h k l)_{i}\right]^{2}}{I_{P}-M(l)}}
$$

Hier ist $I_{P}$ die Zahl der verwendeten Polfiguren. Die Größen $\Delta C_{l}^{\mu \nu}$ können als Näherung für den Fehler der Koeffizienten $C_{l}^{\mu \nu}$ angesehen werden. $\beta^{\mu \mu}$ ist ein 
Koeffizient, der ein Gütekriterium für die Lösung der linearen Gleichungssysteme in Gleichung 2.29 darstellt. Ein gemitteltes Fehlermaß ergibt sich dann durch

$$
\Delta C_{l}=\left|\overline{\Delta C_{l}^{\mu \nu}}\right|=\frac{1}{M(l) \cdot N(l)} \sum_{\mu=1}^{M(l)} \sum_{\nu=1}^{N(l)}\left|\Delta C_{l}^{\mu \nu}\right|
$$

Die $\Delta C_{l}$-Werte können mit den $\overline{C_{l}}$-Werten verglichen werden, wobei die Konvergenz der $\overline{C_{l}}$ im Verhältnis zum Fehler gesehen werden muss [62, 51]. In Abbildung 2.23 ist jeweils ein gutes (Abb. 2.23 a) und ein schlechtes Beispiel (Abb. 2.23 b) für den Verlauf der $\overline{C_{l}}$ und $\Delta C_{l}$ bei einer ODF-Rechnung dargestellt.

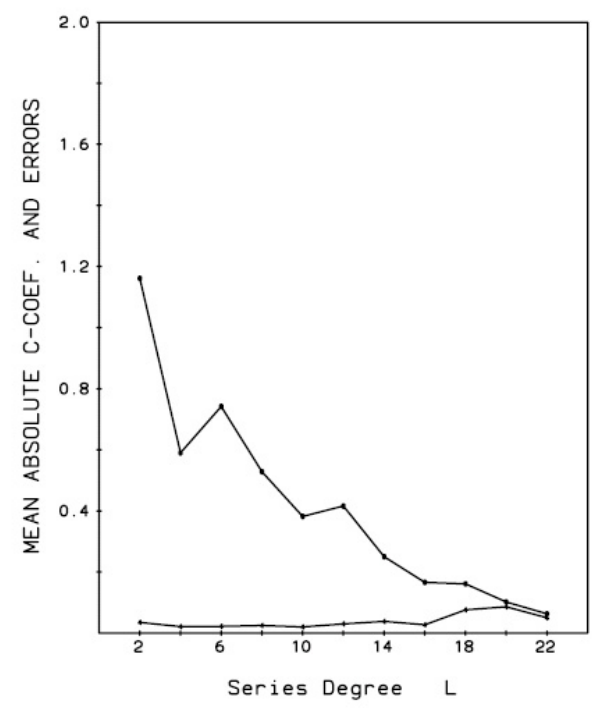

(a) korrekter Verlauf von $\overline{C_{l}}$ und $\Delta C_{l}$

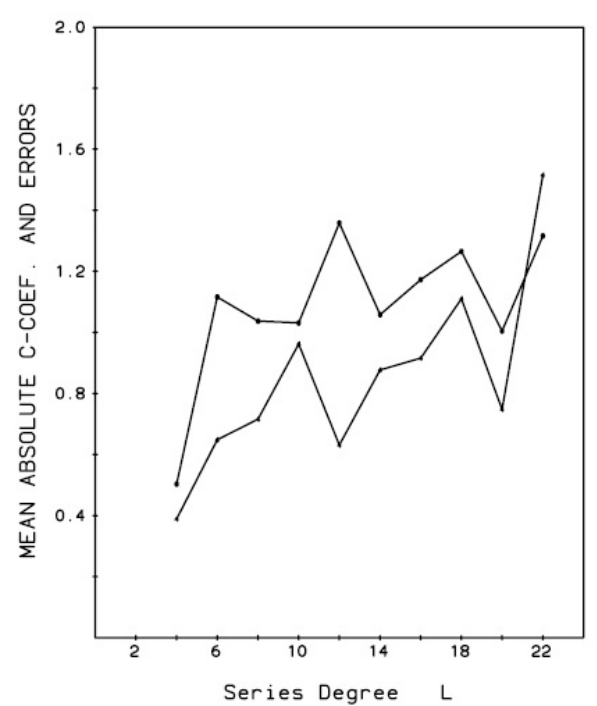

(b) fehlerhafter Verlauf von $\overline{C_{l}}$ und $\Delta C_{l}$

Abbildung 2.23: Darstellung des mittleren Betrags der Koeffizienten $\overline{C_{l}}$ und des Fehlers $\Delta C_{l}[55]$ 


\section{Kapitel 3}

\section{Die kristallinen Partikel}

Die für die vorliegende Arbeit notwendigen kristallinen Pulver wurden im Falle des Muskovits natürliche Mineralien gemahlen und durch Sieben in verschiedene Korngrößenfraktionen getrennt. Die unterschiedlichen $\mathrm{Al}_{2} \mathrm{O}_{3}$-Pulver stammen einerseits aus einer früheren Arbeit [15] und wurden andererseits von der Firma Alcoa zur Verfügung gestellt.

\subsection{Das Muskovitpulver}

Das natürliche Mineral Muskovit $\left(\mathrm{KAl}_{2}\left[(\mathrm{OH}, \mathrm{F})_{2} \mid \mathrm{AlSi}_{3} \mathrm{O}_{10}\right]\right)$ gehört zu der Gruppe der Glimmerminerale. Diese zeichnen sich dadurch aus, dass zwischen die Schichtpakete große Kationen $\left(\mathrm{K}^{+}, \mathrm{Na}^{+}, \mathrm{Ca}^{2+}, \mathrm{Ba}^{2+}\right)$ eingebaut sind. Im Falle des Muskovits sind Kaliumionen zwischen den Silikatschichten eingebaut (Abb. 3.1). Die kristallographischen Pulverdaten sind im Anhang A.1 zusammengestellt.

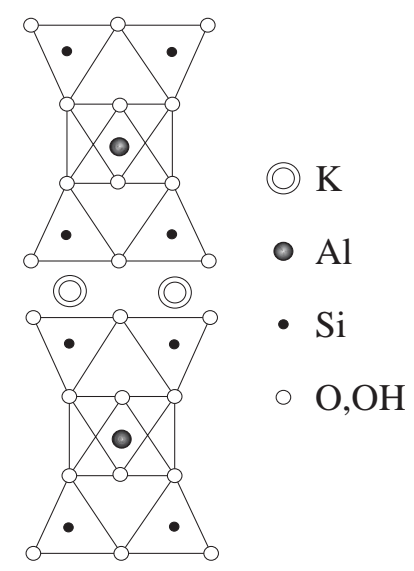

Abbildung 3.1: Kristallstruktur von Muskovit [63]

Der Muskovit bildet tafelig plattige Kristalle mit hexagonalem Umriss aus. Die Aggregate sind blättrig dicht (Abb. 3.2). Aufgrund der Schichtstruktur von Muskovit ist die Spaltbarkeit sehr vollkommen nach $\{001\}$ ausgeprägt [64]. 


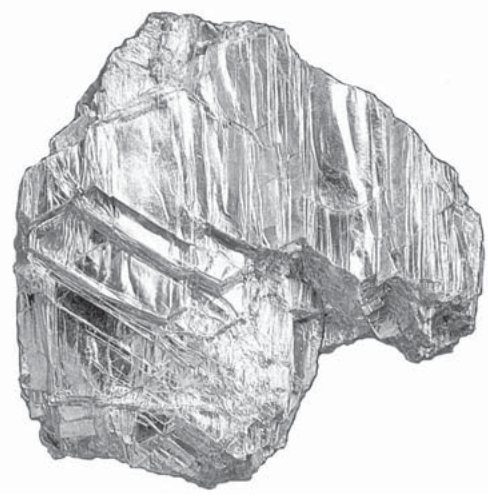

Abbildung 3.2: Natürliches Muskovitmineral [64]

Sehr dünne Schichten des Muskovits lassen sich von Hand vom Mineral lösen. Diese sind lichtdurchlässig und sehr biegsam. Sie haben folienähnlichen Charakter.

Die Muskovitmineralien wurden mit einer Schere in ca. daumengroße Stücke zerkleinert, dann 6 Stunden in einer Achat-Kugelmühle gemahlen und anschlieBend durch Sieben in die Fraktionen $<63 \mu m$ und 63-125 $\mu m$ getrennt. Der Siebvorgang dauerte zwischen 3-6 Stunden, da sich die sehr stark plättchenförmig ausgeprägten Partikel flach auf das Sieb legen und somit diese sehr schnell zusetzen. In Abständen von 30-40 Minuten musste die Muskovitplättchen vom Sieb mit einem Pinsel entfernt werden. Die Siebrückstände wurden erneut zum Mahlgut zurückgegeben.

\subsubsection{Lichtmikroskopische Bestimmung der Partikel}

Die relativ großen Körner des Muskovits lassen eine lichtmikroskopische Bestimmung zu. Die Untersuchung wurde am Mikroskop Axioskop der Firma Zeiss durchgeführt. Dieses Mikroskop ist mit einer Kamera MC 80 der Firma Zeiss ausgestattet. Die Aufnahmen wurden im Durchlichtverfahren erstellt. Das Muskovitpulver wurde hierzu auf einen Objektträger gestreut und mit Tesafilm fixiert.

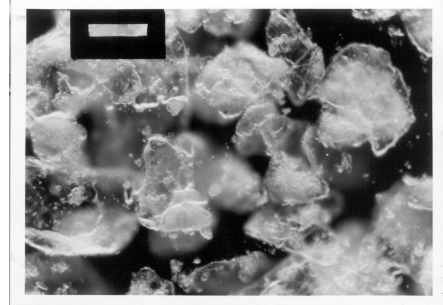

(a) Fraktion $<63 \mu m$ 25fach vergrößert

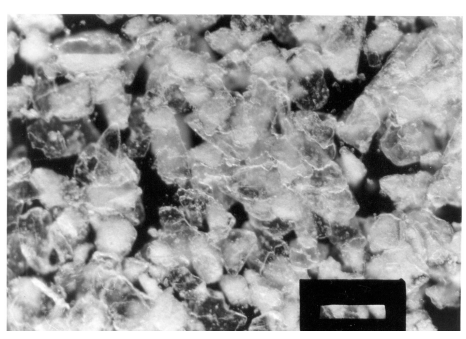

(b) Fraktion $63-123 \mu m$ 10fach vergrößert

Abbildung 3.3: Durchlichtmikroskopische Aufnahme des Muskovitpulvers 
In Abbildung 3.3 ist der stark ausgeprägte plättchenförmige Charakter der Muskovitpartikel zu sehen. Die Plättchen richten sich durch die Fixierung mit ihrer Basisebene parallel zum Objektträger aus. Somit lässt sich die Ausdehnung der Basisebene sehr gut bestimmen, die Plättchendicke jedoch nicht.

Die Größe der Basisebene wurde anhand von vier Aufnahmen für jede Fraktion mittels Linienschnittverfahren ermittelt. In Abbildung 3.4 ist die Korngrößenverteilung für die Fraktion $<63 \mu m$ dargestellt.

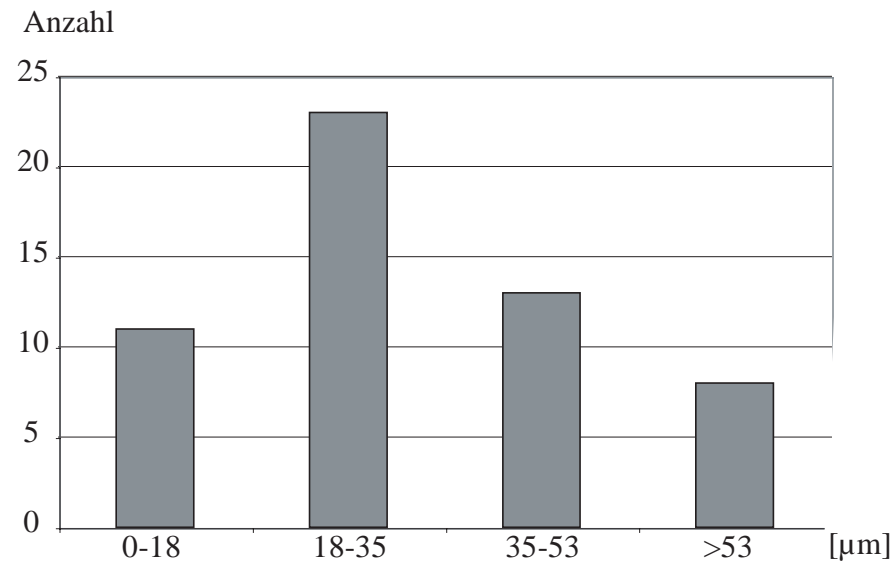

Abbildung 3.4: Korngrößenverteilung der Muskovitpulverfraktion $<63 \mu m$

Unter der Annahme, dass die Oberfläche der Basisebene kreisförmig ist, ergibt sich für den mittleren Partikelradius ein Wert von ca. $30 \mu \mathrm{m}$. Die durchschnittliche Plättchendicke lässt sich nur abschätzen. Eine Ausdehnung der Plättchen in die Breite ist zwar zu erkennen, liegt jedoch im Bereich des Auflösungsvermögens des Mikroskops. Ein Wert von ca. $2 \mu m$ knapp oberhalb des Auflösungsvermögens ergibt somit ein Radienverhältnis (Kap. 1.3.2) von $r_{e}=$ 0.06 .

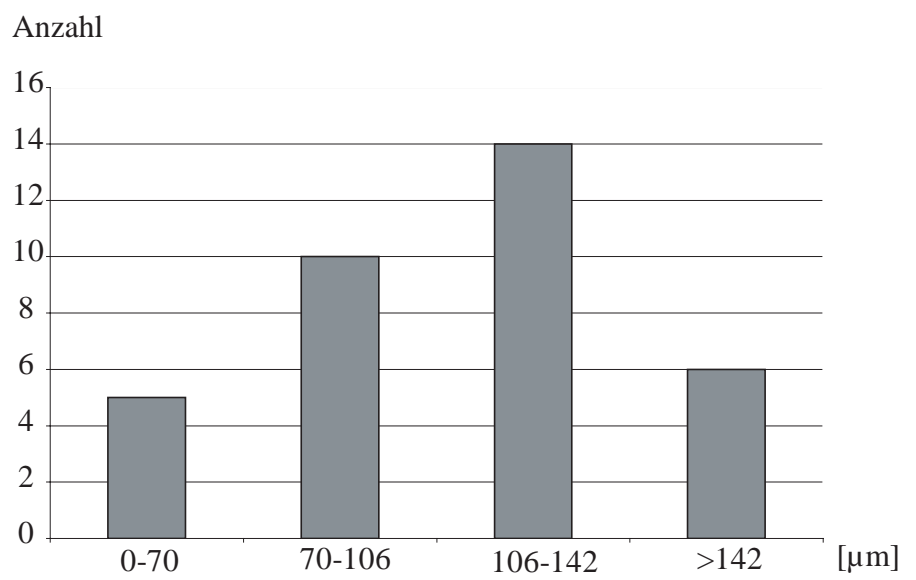

Abbildung 3.5: Korngrößenverteilung der Muskovitpulverfraktion $63-125 \mu \mathrm{m}$

Die Korngröße der Fraktion 63-125 $\mu m$ wurde nach dem gleichen Verfah- 
ren ermittelt. Anhand der in Abbildung 3.5 dargestellten Korngrößenverteilung ergibt sich ein mittlerer Radius der Basisebene von $120 \mu \mathrm{m}$. Die Partikel der Fraktion 63-125 $\mu \mathrm{m}$ unterscheiden sich nicht messbar in ihrer Teilchendicke. Sie ist auch hier wieder im Bereich des Auflösungsvermögens des Mikroskops. Daraus ergibt sich ein deutlich kleineres Radienverhältnis mit $r_{e}=0.017$.

\subsection{Die industriellen Aluminiumoxidpulver}

$\mathrm{Al}_{2} \mathrm{O}_{3}$-Pulver ist als Grundstoff für Keramiken von besonderer werkstoffkundlicher Bedeutung. Die Kristallstruktur von $\mathrm{Al}_{2} \mathrm{O}_{3}$ ist trigonal (Abb. 3.6). Im Kristallgitter des Korunds $\left(\mathrm{Al}_{2} \mathrm{O}_{3}\right)$ bilden die Sauerstoffatome eine hexagonaldichteste Kugelpackung, also eine Schichtenfolge ABAB.... Von den vorhandenen oktaedrischen Lücken werden $2 / 3$ von $A l^{+3}$-Ionen besetzt. Die kristallographischen Pulverdaten sind im Anhang A.2 zusammengestellt.

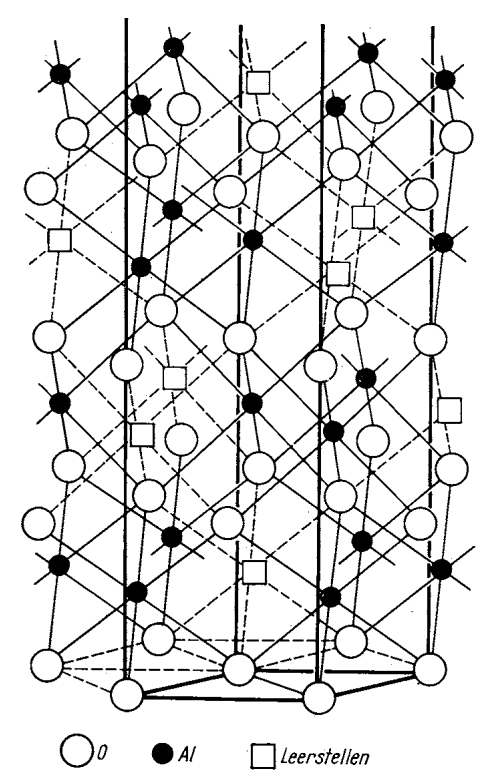

Abbildung 3.6: Kristallstruktur von $\mathrm{Al}_{2} \mathrm{O}_{3}$ [63]

Der hier verwendete technische Korund wird aus Bauxit hergestellt. Dazu wird $\mathrm{Al}_{2} \mathrm{O}_{3}$ im elektrischen Ofen geschmolzen und nach Bedarf zerkleinert. Die einzelnen Kristallite können in ihrer Form von plättchenförmig über kubisch bis hin zu globularen Körnern variieren. Dies ist in erster Linie abhängig von Herstellungsprozess. $\mathrm{Al}_{2} \mathrm{O}_{3}$-Kristalle sind nach $\{001\}$ und $\{101\}$ spaltbar. Natürlich gewachsene Kristalle zeichnen sich durch unebene raue Flächen aus und weisen einen tonnenförmigen Habitus auf (Abb. 3.7).

Aufgrund der Spaltbarkeit nach $\{001\}$ können von dieser Form Plättchen abgespalten werden, bei denen die Basisebene der (001)-Ebene entspricht. Findet nur ein Bruch in der Mitte statt, entstehen kubische Kristallite. Eine Kombination der beiden möglichen Spaltebenen kann zu einer globularen Kristallitform (Abb. 3.7) führen. 


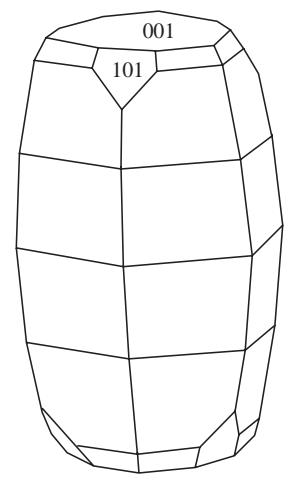

(a) Tonnenförmiger Habitus

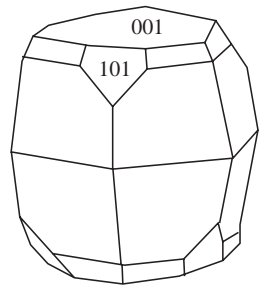

(b) Kubischer Habitus

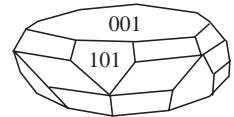

(c) plättchenförmiger Habitus

Abbildung 3.7: Kristallhabitus von Korund [65]

Die hier untersuchten Proben [15] stammen von der Firma Hoechst Ceramtec und werden im weiteren Verlauf mit A, B und C bezeichnet. Weitere $\mathrm{Al}_{2} \mathrm{O}_{3}$-Proben, welche von der Firma Alcoa zur Verfügung gestellt wurden, werden unter deren industriellen Bezeichnungen HVAFG, GILOX 63 und CTC 20 geführt. Diese Proben wurden von der Firma Alcoa als Musterbeispiele für plättchenförmige, globulare und gemischte Kornformen ausgewählt.

\subsubsection{Elektronenmikroskopische Bestimmung der Aluminium- oxidpartikel}

Die $\mathrm{Al}_{2} \mathrm{O}_{3}$-Partikel sind mit einer mittleren Korngröße von $2-5 \mu \mathrm{m}$ zur lichtmikroskopischen Untersuchung zu klein. Um die Partikelform zu charakterisieren ist ein Rasterelektronenmikroskop (REM) sehr gut geeignet. Die Partikel der Proben A, B und C wurden mit dem Elektronenmikroskop Zeiss LEO 1530 Gemini des Geowissenschaftlichen Zentrums Göttingen in der Abteilung Geologie untersucht. Hierzu wurden von allen verwendeten $\mathrm{Al}_{2} \mathrm{O}_{3}$-Partikeln mehrere Aufnahmen angefertigt, um das charakteristisches Radienverhältnis $r_{e}$ (Kap. 1.3.2) zu bestimmen.

\subsubsection{Charakterisierung der Proben A, B und C}

Zur Bestimmung des Radienverhältnisses $r_{e}$ wurden in den REM-Aufnahmen einige Partikel vermessen und der Durchmesser, sowie die Dicke ermittelt. In Abbildung 3.8 ist skizziert, wie die Auswertung durchgeführt wurde. Die Oberfläche der Partikel wurde durch eine Ellipse angenähert. Die mittlere Achsenlänge ergibt dann den Durchmesser. Die Partikeldicke wurde an Partikeln, welche für die Auswertung günstig lagen gemessen. Aus dem Mittelwert der gesamten Messungen ergibt sich das charakteristische Radienverhältnis von $r_{e}=0,3$ für die Probe A.

Die Partikel der Probe A zeichnen sich durch eine Mischung von plättchenförmigen und globularen Partikeln aus. Die Kanten der plättchenförmigen 


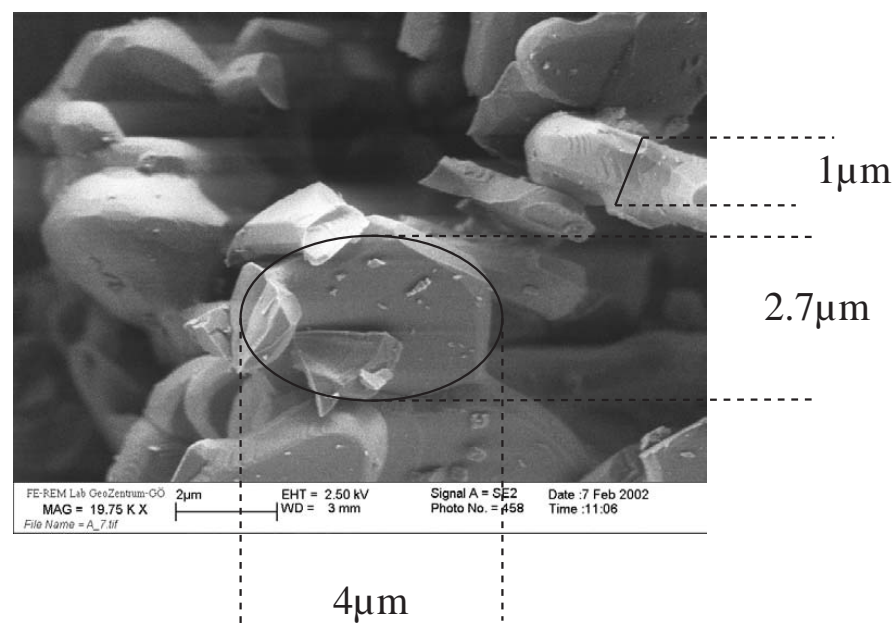

Abbildung 3.8: REM-Aufnahmen für die Probe A

Teilchen sind teilweise abgerundet und teilweise sind scharfe Bruchkanten vorhanden. Der globulare Anteil besitzt nur wenige scharfe Kanten. Die Partikelgröße variiert zwischen 1,5 und $6 \mu m$.

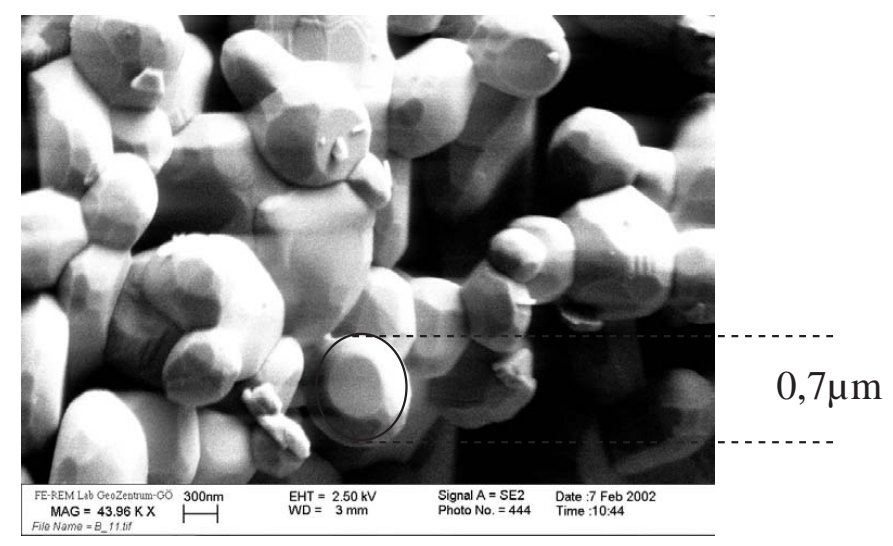

Abbildung 3.9: REM-Aufnahmen für die Probe B

Die Abbildung 3.9 zeigt die Partikel der Probe B. Die globularen Kristallite bilden sehr leicht Agglomerate. Die Kanten sind glatt ohne scharfe Brüche. Mit einer Korngröße von 0,3 bis 1,2 $\mu \mathrm{m}$ sind die Partikel deutlich kleiner als die der Probe A. Das Radienverhältnis $r_{e}$ beträgt für diese Probe 1.

Im Vergleich zu den Proben A und B variiert die Partikelgröße der Probe $\mathrm{C}$ mit 0,5 bis $5 \mu \mathrm{m}$ am stärksten. Es ist auffällig, dass hier die großen Partikel einen plättchenförmige Habitus mit teilweise scharfen Bruchkanten besitzen (Abb. 3.10). Die kleinen Kristallite sind hingegen meistens globular geformt ohne scharfe Kanten. Das Radienverhältnis $r_{e}$ für die plättchenförmigen Partikel beträgt 6 und für die globularen ca. 1 . 


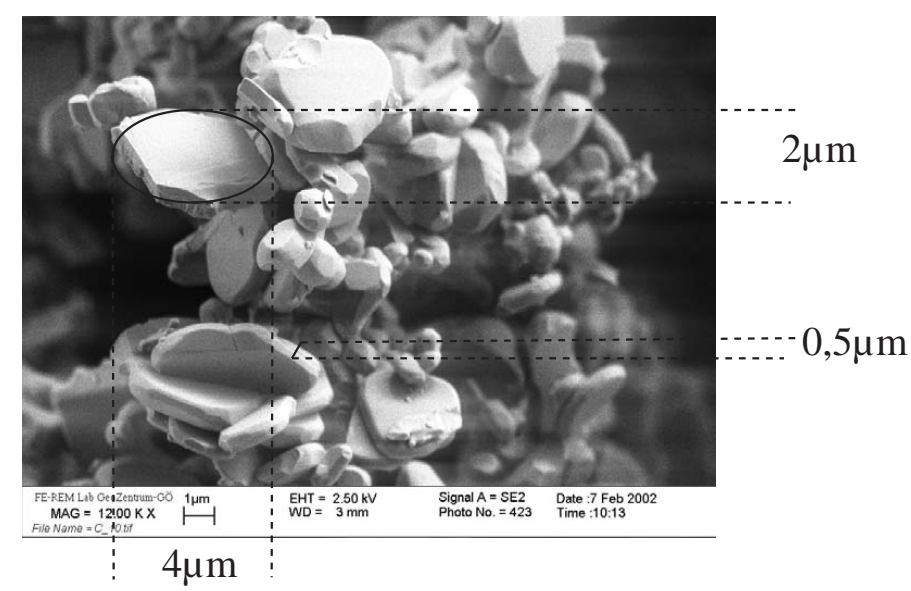

Abbildung 3.10: REM-Aufnahmen für die Probe C

\subsubsection{Charakterisierung der Proben HVAFG, CTC20 und Gilox}

Darüber hinaus wurden für diese Arbeit die industriellen $\mathrm{Al}_{2} \mathrm{O}_{3}$-Pulver der Firma Alcoa untersucht. Die Proben wurden mit dem Elektronenmikroskop JSM 5400 der Firma Jeol analysiert. Diese Materialien finden als Schleifmittel Verwendung und unterscheiden sich durch ihren Kalzinationsgrad. Die Partikel mit dem geringsten Kalzinationsgrad haben die Bezeichnung HVAFG. CTC20 besitzt einen hohen Kalzinationsgrad und Gilox den höchsten. Mit steigendem Kalzinationsgrad nimmt auch die Korngröße zu.

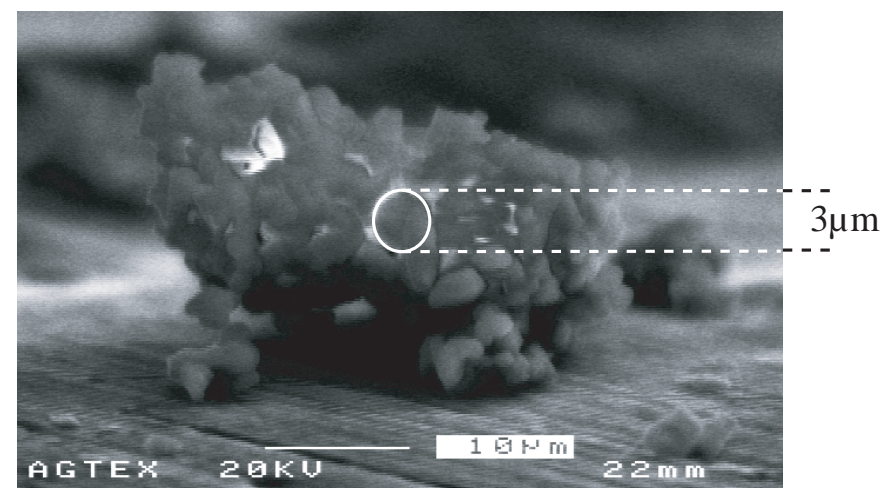

Abbildung 3.11: REM-Aufnahme der Probe HVAFG

Die in Abbildung 3.11 dargestellten Partikel der Probe HVAFG sind von einem globularen Habitus. Sie neigen zur Bildung von Agglomeraten welche $50 \mu \mathrm{m}$ und größer sind. Die einzelnen Partikel sind zwischen 2 und $5 \mu \mathrm{m}$ groß. Es sind keine scharfen Kanten oder Bruchkanten zu erkennen.

Die Probe CTC20 zeigt eine Mischung aus plättchenförmigen und globularen Körnern (Abb. 3.12). Dies ist auch bei der oben gezeigten Probe C (Abb. 3.10) zu beobachten. Die großen Partikel sind auch hier wieder plättchenförmig, mit einer Korngröße von 30 bis $50 \mu m$ und einem Radienverhältnis von $r_{e}=0.25$. Die Plättchendicke wurde hier mit $5 \mu m$ abgeschätzt. Die kleinen globularen 


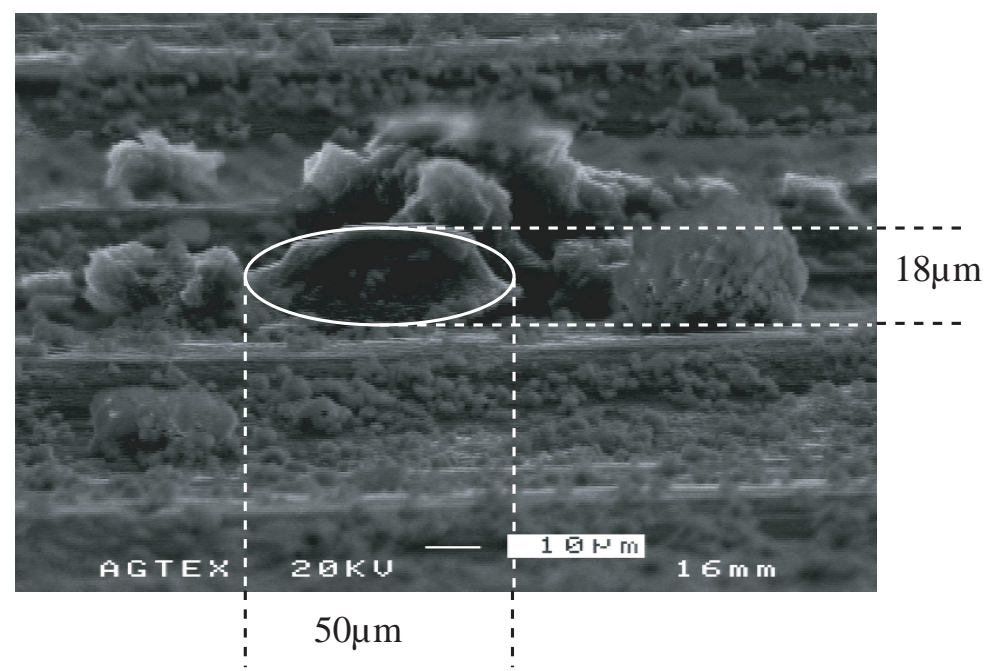

Abbildung 3.12: REM-Aufnahme der Probe CTC20

Körner sind nur ca 3 bis $6 \mu m$ groß, mit einem $r_{e}=1$. Agglomerate der Teilchen können auch hier wieder bis zu 50 $\mu$ m groß werden.

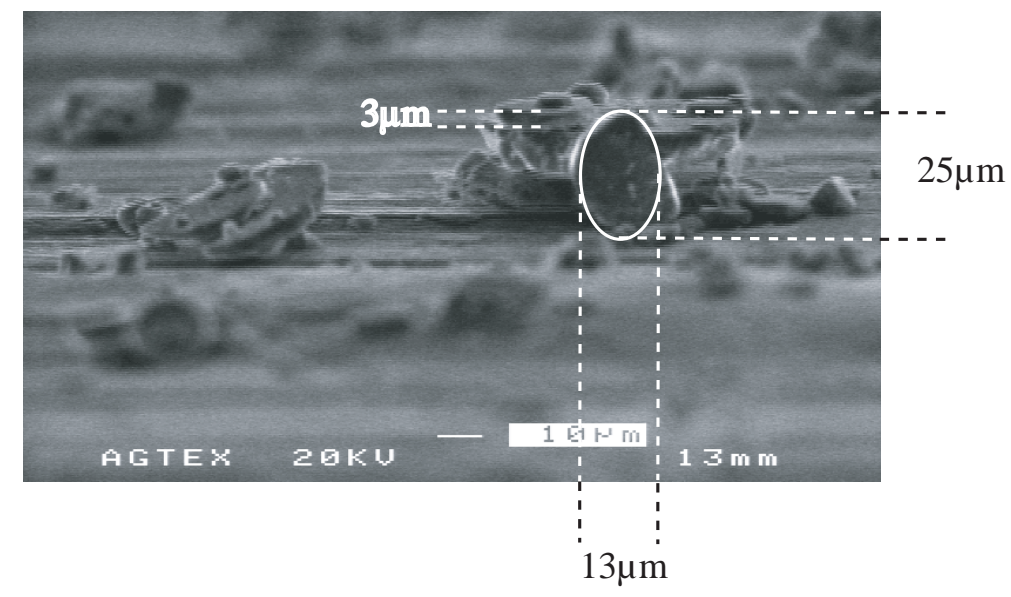

Abbildung 3.13: REM-Aufnahme der Probe Gilox

Den plättchenförmigsten Charakter haben die Partikel der Probe Gilox (Abb. 3.13). Die Plättchen haben ein Radienverhältnis von $r_{e}=0.15$ und sind zwischen 15 bis $35 \mu \mathrm{m}$ groß. Die Bruchkanten der Partikel sind sehr scharf. Es sind nur sehr wenige globulare Teilchen vorhanden. Die Bildung von Agglomeraten konnte nicht beobachtet werden. 


\section{Kapitel 4}

\section{Konstruktion der Küvetten}

\subsection{Die Rotationsküvette}

Zur Erzeugung einer stationären Scherströmung wurde eine Rotationsküvette konstruiert. Die Küvette soll über einen Zeitraum von mehreren Stunden in der Lage sein stationäre Strömungsverhältnisse zu gewährleisten. Bisherige Untersuchungen der Partikelorientierung in Scherströmungen wurden an zwei unterschiedlichen Küvetten durchgeführt. Eine mögliche Küvette besteht aus zwei Zylindern, wobei ein Zylinder sich im Inneren des anderen Zylinders befindet [44]. Der innere Zylinder ist feststehend und der Äußere rotiert (Abb. 4.1a). Zwischen den beiden Zylindern entwickelt sich wie in Kapitel 1.2.1 ein Geschwindigkeitsprofil innerhalb des Zwei-Phasen-Gemisches.

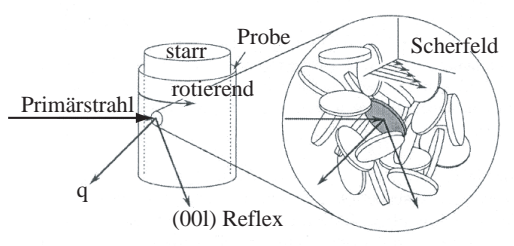

(a) Partikel in Reflexion

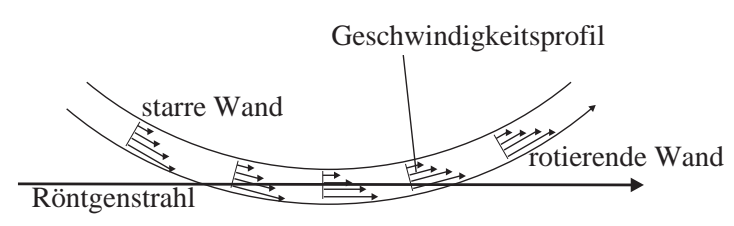

(b) Strahllage zum Geschwindigkeitsprofil

Abbildung 4.1: Scherküvette mit rotierenden Zylindern [44]

Für die Orientierungsbestimmung mittels Hochenergie Synchrotronstrahlung ist diese Konstruktion jedoch nicht geeignet, da nicht in Reflexionsgeometrie (siehe Kap. 2.2) gemessen werden kann. Der Röntgenstrahl würde einen relativ großen Bereich erfassen. Über diesen Bereich ändert der Gradient des Scherfelds aufgrund der Krümmung des Zylinders seine Orientierung zum Primärstrahl (Abb. 4.1b). Da während der Messung nur die Orientierung nicht aber der Ort bestimmt werden kann, lässt sich die Orientierung keinem Strömungsfeld zuordnen. Ein Zusammenhang zwischen Strömungsfeld und Orientierung der Teilchen kann so nicht hergestellt werden. Eine weitere Möglichkeit eine stationäre Scherströmung zu erzeugen bieten zwei kreisförmige Scheiben, von denen eine starr ist und die andere rotiert [45]. Zwischen den beiden Scheiben bildet sich eine Scherströmung aus. Zweckmäßigerweise ist die starre Scheibe 
der Boden eines Topfs. In diesem Topf rotiert die andere Scheibe und schließt mit der Wandung des Topfs ab. Diese Küvette hat den Vorteil, dass sich die Orientierung des Strömungsfelds zu Primärstrahl im detektierten Volumen nicht ändert (Abb. 4.2).

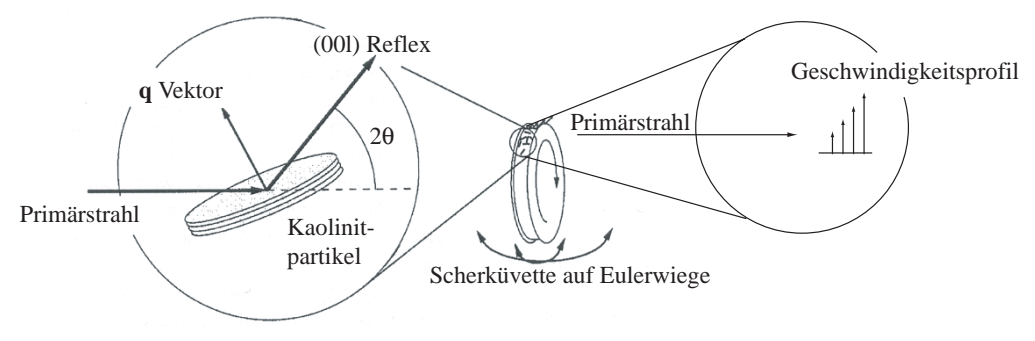

Abbildung 4.2: Schematische Darstellung einer Rotationsküvette im Strahlengang [45]

Als Küvettenmaterial wurde Plexiglas ausgewählt (Abb. 4.3). Dieses hat den Vorteil, dass der Absorptionskoeffizient sehr gering ist. Ein möglichst geringer Absorptionskoeffizient erhöht die Intensität an der Probe und verringert somit die notwendige Messzeit. Außerdem treten beim amorphen Plexiglas keine Beugungsringe auf, welche sich mit den Beugungsringen der festen Phase überlagern können.

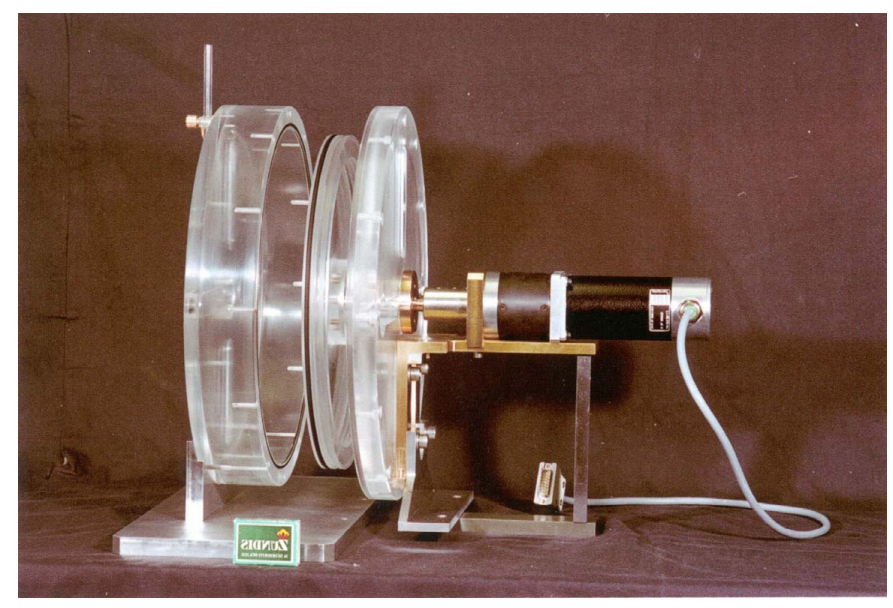

Abbildung 4.3: Die Rotationsküvette

Der amorphe Zustand des Plexiglases führt jedoch zu einer Erhöhung des Untergrunds, welcher die Intensität der Beugungsreflexe der kristallinen Phase jedoch nicht beeinflusst. Die flüssige Phase des zu untersuchenden Gemischs erzeugt ebenfalls einen amorphen Untergrund, welcher sich zum Untergrund des Plexiglases addiert. Bei der späteren Auswertung wird die Intensität des Untergrunds subtrahiert (Kap. 2.3). Um möglichst wenig Plexiglas während der Messung durchdringen zu müssen, wurden im Durchstrahlbereich Vertiefungen eingefräst. Diese sind so platziert, dass sich über einen möglichst breiten Bereich 
des Durchstrahlwinkels (siehe Kap. 2.2.2) kein zusätzliches Küvettenmaterial im Strahlengang befindet (Abb. 4.4).

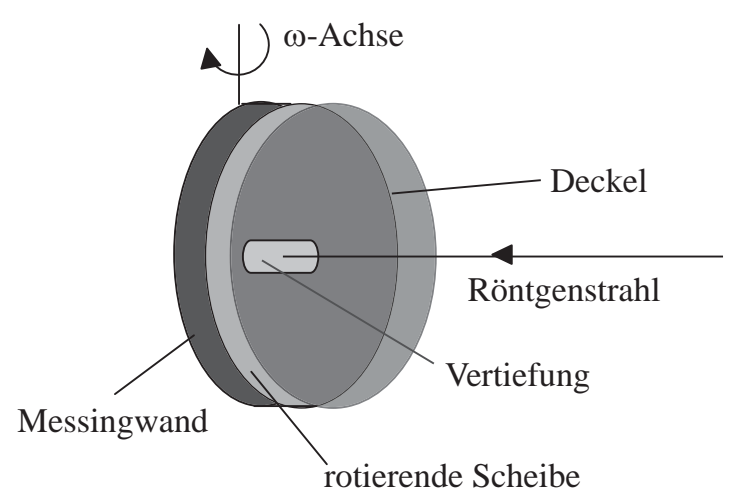

Abbildung 4.4: Die Rotationsküvette in schematischer Darstellung

Die rotierende Scheibe wird mittels Schrittmotor angetrieben und mit einem Dichtring aus Viton versehen. Dieses Material soll den schleifenden Eigenschaften des Zwei-Phasen-Gemisches (z.B. $\mathrm{Al}_{2} \mathrm{O}_{3}$-Pulver in Glyzerin) standhalten. Erste Tests zeigten jedoch, dass im Plexiglas der Küvettenwand sehr schnell Schleifspuren zu beobachten waren, welche zu Undichtigkeiten führten. Diese ist durch den Einsatz eines Messingrings als Wandmaterial behoben worden. Zusätzlich wurde eine neue Dichtung aus Teflon gefertigt, welche aus einer Dichtlippe in Verbindung mit einem Dichtring (Viton) besteht (Abb. 4.5). In dieser Konfiguration lässt sich die Rotationsküvette über einen Zeitraum von 4 Stunden ohne größere Flüssigkeitsverluste betreiben.

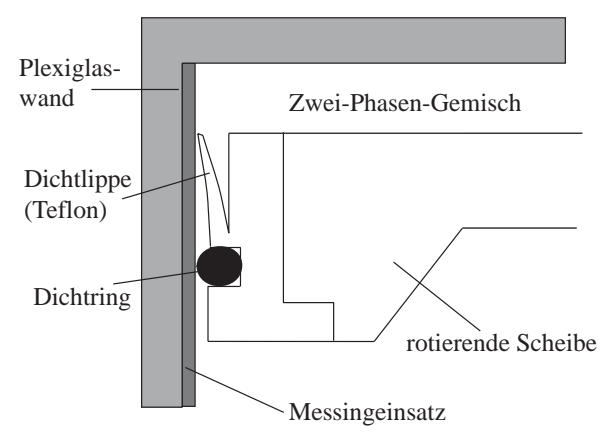

Abbildung 4.5: Spezielle Dichtung der Rotationsküvette

\subsection{Die Durchflussküvette}

Der Einfluss des Strömungsfelds auf die Textur lässt sich durch den Einsatz einer Fließküvette bestimmen. Die hierbei erzeugte Rohrströmung (Kap. 1.2.2) unterscheidet sich im Strömungsprofil von der Scherströmung (Kap. 1.2.1) in einer Rotationsküvette. Frühere Messungen an Kaolinitpartikeln [43] in Was- 
ser wurden mittels Spritzenpumpe, welche an eine Küvette angeschlossen ist durchgeführt (Abb. 4.6).

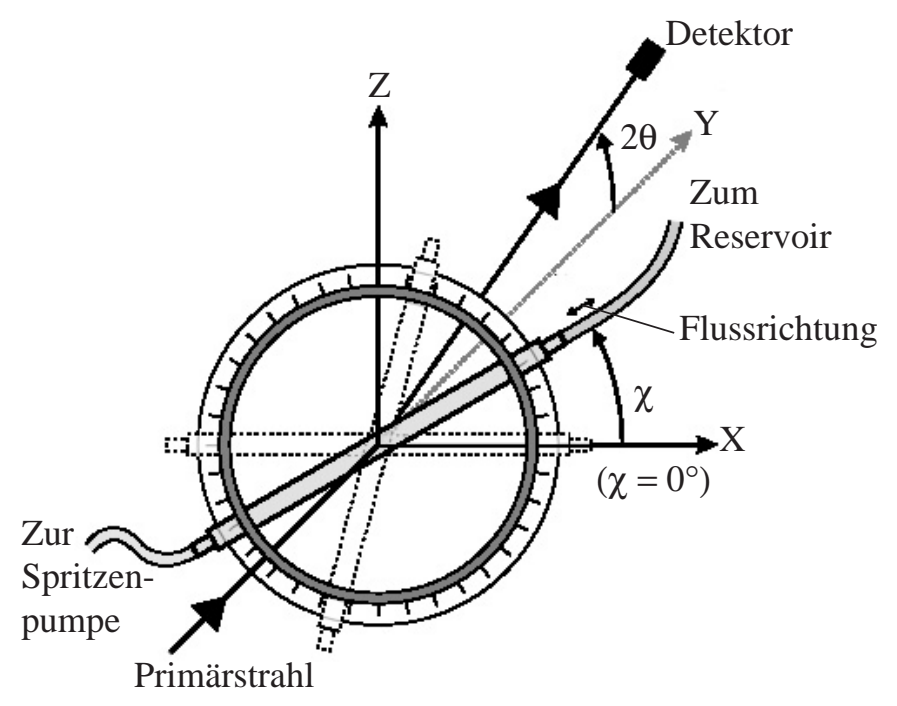

Abbildung 4.6: Schematische Darstellung der Orientierungsbestimmung von Zwei-Phasen-Gemischen mittels Spritzenpumpe [43]

Aufgrund der beobachteten stark schleifenden Eigenschaften der $\mathrm{Al}_{2} \mathrm{O}_{3}$ Partikel bei längerem Einsatz ist ein Spritzensystem nicht geeignet. Der bewegliche Kolben der Spritze führt bei längerer Benutzung zu Undichtigkeiten. Um diese $\mathrm{Al}_{2} \mathrm{O}_{3}$-Partikelgemische zu untersuchen wurde eine Durchflussküvette ohne bewegliche Teile konstruiert (Abb. 4.7).

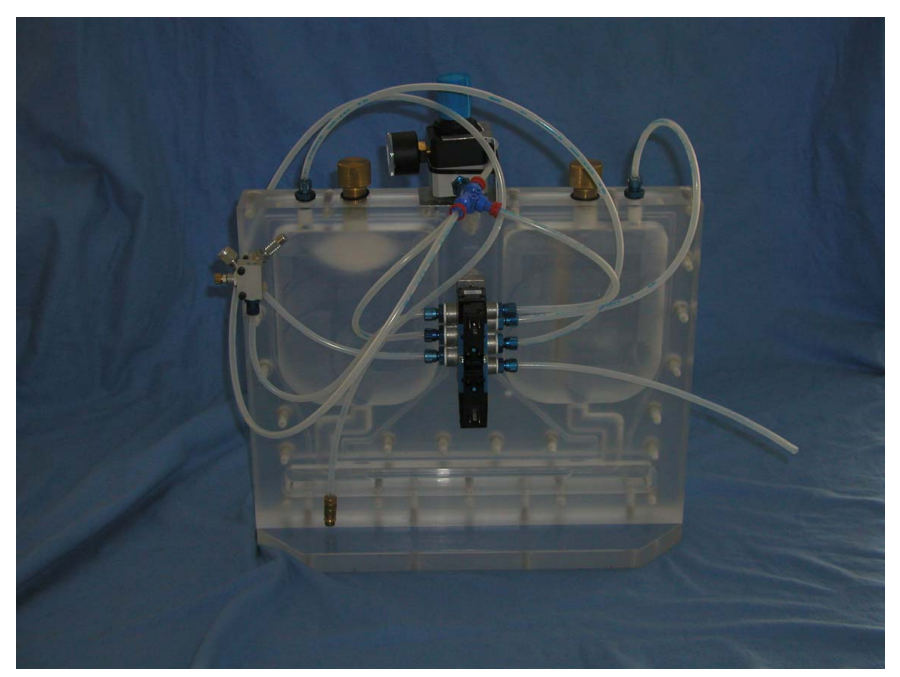

Abbildung 4.7: Die Durchflussküvette

Die Reservoire, sowie der Strömungskanal sind komplett in ein Plexiglasstück gefräst worden. Um die gesamten Kanäle ist eine Nut gefräst, in die eine 
Dichtung eingebracht wird. Durch eine von außen angeschraubte Plexiglasplatte ist die Küvette abgedichtet und die beiden Reservoire sind nur über den Strömungskanal miteinander verbunden. Der Fluss wird mittels Druckluft erzeugt. Wie bei der Rotationsküvette wurde auch hier Plexiglas als Küvettenmaterial verwendet. Die Küvette besteht aus zwei Reservoiren die über einen definierten $2 \mathrm{~mm}$ breiten und $20 \mathrm{~mm}$ hohen Strömungskanal miteinander verbunden sind. Durch ein 3-5-Wege Schaltventil der Firma Festo wird der Fluss des Gemisches gesteuert (Abb. 4.8). Das Ventil verfügt über drei Schaltzustände:

Position 1: die Durchgänge 1/6 und $4 / 7$ sowie der Drucklufteingang 5 sind geschlossen

Position 2: Verbindung zwischen 5 und 2, sowie 4 und 7 sind geöffnet

Position 3: Verbindung zwischen 5 und 3, sowie 1 und 6 sind geöffnet

Die Position 1 beschreibt den Ruhezustand der Küvette. In Position 2 wird das linke Reservoir mit Druckluft gefüllt und der Auslass des rechten Reservoirs geöffnet. Das Gemisch fließt durch den Kanal in das rechte Reservoir. Die Fließgeschwindigkeit wird über das Feinventil am Ausgang des rechten Reservoirs geregelt. In Position 3 sind jeweils die anderen Durchgänge geöffnet und das Gemisch wird zurückgepumpt. Da immer nur in Position 2 gemessen wird, befindet sich am Ausgang des linken Reservoirs kein Feinventil. Es wird mit maximaler Geschwindigkeit gepumpt.

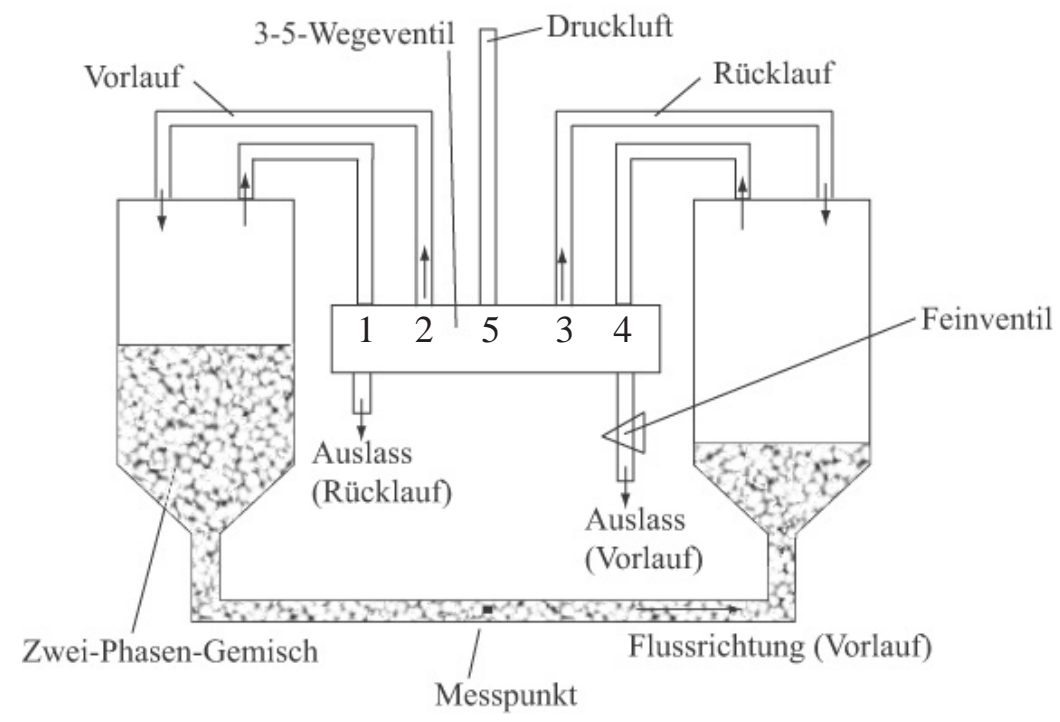

Abbildung 4.8: Schematische Darstellung der Durchflussküvette

Durch eine am linken Reservoir angebrachte Skala kann mit einer Stoppuhr die jeweils eingestellte Fließgeschwindigkeit ermittelt werden. Die Konstruktion erlaubt Fließgeschwindigkeiten von $0.2 \mathrm{ml} / \mathrm{s}$ bis maximal $5 \mathrm{ml} / \mathrm{s}$. Der Betriebsdruck variiert je nach Fließgeschwindigkeit von 0,7-2bar. 


\section{Kapitel 5}

\section{Orientierung der Partikel}

\subsection{Muskovitpartikel in Glyzerin}

Bisherige Untersuchungen zur Orientierung von kristallinen Partikeln in strömenden Flüssigkeiten fanden meistens an Tonmineralien wie z.B. Kaolinit [45, 43] oder an Nickelhydroxid [66] statt. Aufgrund der stark ausgeprägten Plättchenform dieser kristallinen Partikel ist die Orientierung in der Strömung, wie in Kapitel 1.3.2 beschrieben, besonders stark ausgeprägt und somit auch die kristallographische Textur. In dieser Arbeit dient Muskovit, welches ein Glimmermineral ist, aufgrund des in Kapitel 3.1 und 3.1.1 beschriebenen plättchenförmigen Charakters als Modellsubstanz. Die zu erwartende Strömungstextur sollte im Fall des Muskovits besonders stark sein.

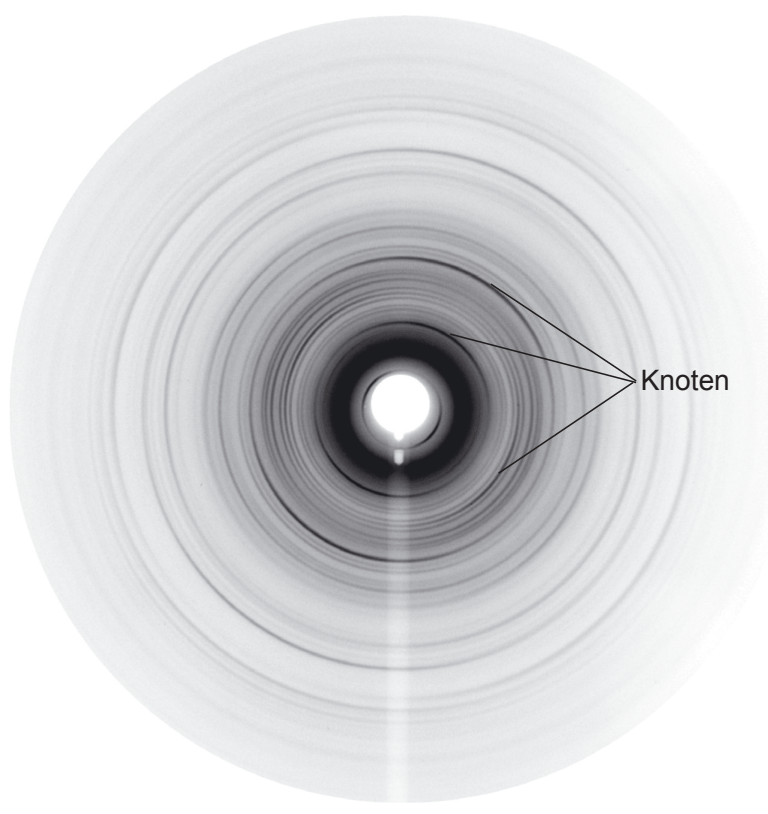

Abbildung 5.1: Beugungsbild von Muskovit (Korngröße $<63 \mu m$ ) in Glyzerin

Das Beugungsbild in Abbildung 5.1 zeigt aufgrund der monoklinen Kristallstruktur von Muskovit eine Vielzahl von Beugungsreflexen des Muskovits. Da 
viele der Reflexe nur sehr schwach sind und das Gemisch auch noch texturiert ist, wie an den Knoten in Abbildung $5.1 \mathrm{zu}$ sehen, sind in dem aus Abbildung 5.1 berechneten Beugungsdiagramm in Abbildung 5.2 nur zwei Reflexe (( 111$)$ und (116)) zu erkennen. Die anderen heben sich nur sehr schwach von Untergrund ab und überlagern sich. Die Aufnahme wurde mit der Rotationsküvette in der Position $\omega=0^{\circ}$ aufgenommen.

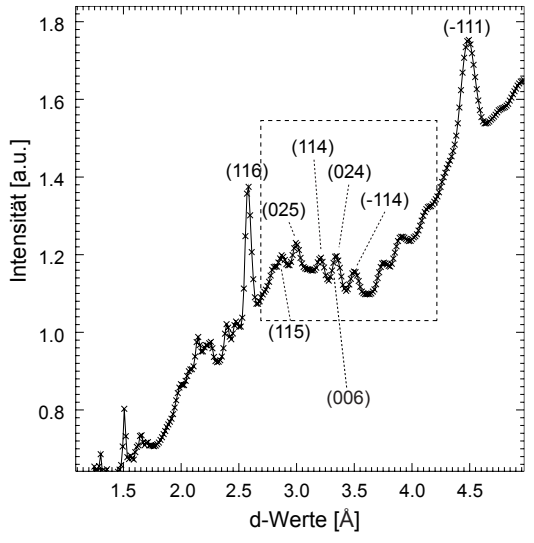

(a) Integriertes Beugungsdiagramm aus dem Beugungsbild von Muskovit (Abb. 5.1) in Glyzerin

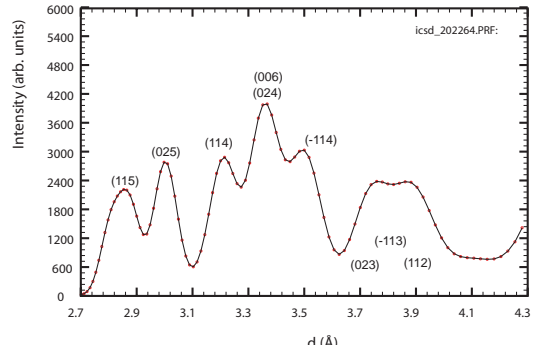

(b) Simuliertes Beugungsdiagramm für den in Abbildung (a) gekennzeichneten Bereich

Abbildung 5.2: Beugungsdiagramme für Muskovit

Um eine Polfigur aus den gemessenen Daten zu berechnen ist es notwendig die integrale Intensität eines einzelnen Peaks zu bestimmen (Kap. 2.3). In Abbildung 5.2 ist mittels FIT2D [56] die integrale Intensität des gesamten Beugungsbild als Beugungsdiagramm berechnet worden. So lassen sich die einzelnen Peaks, welche sich über den gesamten Bereich des Beugungsdiagramms überlagern und aufgrund der Textur teilweise nur in wenigen Aufnahmen zu sehen sind besser identifizieren. Die meisten Reflexe lassen sich jedoch aufgrund der Überlagerungen, wie auch in Abbildung 5.2b zu sehen, nicht auswerten.

Der (006)-Reflex des Muskovits ist sehr aussagekräftig, da er die Orientierung der Basisebene der Partikel wiederspiegelt. Im Beugungsdiagramm in Abbildung 5.2 ist der (006)-Reflex, welcher sich mit dem (024)-Reflex überlagert kaum zu sehen. Da sich die plättchenförmigen Partikel, wie in Kapitel 1.4.2 beschrieben, parallel zur Strömungsrichtung ausrichten, ist für die (001)Reflexe die maximale Intensität für einen Durchstrahlwinkel von $\omega=90^{\circ} \mathrm{zu}$ messen (minimale Intensität bei $\omega=0^{\circ}$ ). Da mit der in Kapitel 2.2.2 beschriebenen Messgeometrie bei der Rotationsküvette nur ein Durchstrahlwinkel von $\omega=50^{\circ}$ erreicht werden kann, ist aufgrund der oben beschriebenen Orientierung der Partikel kaum Intensität für den (001)-Reflexe zu erwarten.

Für alle weiteren Muskovitpolfiguren wurde jeweils der (111)-Reflex ausgewertet. Die $\{\overline{1} 11\}$-Ebene ist um ca. $82^{\circ}$ zur Basisebene der Muskovitpartikel geneigt. Der Peak ist bei $\omega=0^{\circ}$ deutlich zu sehen und eignet sich gut für die Polfigurbestimmung, da keine weiteren Reflexe die Auswertung beeinträchtigen. Die maximale Intensität ist bei der oben angenommenen Orientierung der 
Teilchen bei $\omega=0-15^{\circ}$ zu erwarten.

\subsubsection{Muskovit-/Glyzeringemisch in der Rotationsküvette}

Die Polfigurmessungen mit der Rotationsküvette (siehe Kap. 4.1) wurden mit den beiden Korngrößenfraktionen $<63 \mu m$ und $63-125 \mu m$ durchgeführt. In Tabelle 5.1 sind die einzelnen Messungen mit den entsprechenden Parametern aufgeführt.

\begin{tabular}{|c|c|c|c|c|}
\hline $\begin{array}{l}\text { Korngrößen- } \\
\text { fraktion }\end{array}$ & $\begin{array}{l}\text { Rotations- } \\
\text { geschwindigkeit }\end{array}$ & $\begin{array}{c}\text { Drehwinkel } \\
\omega\left[^{\circ}\right]\end{array}$ & $\begin{array}{l}\text { Belichtungs- } \\
\text { zeit [sec] }\end{array}$ & $\begin{array}{c}\text { Feststoff- } \\
\text { konz. [Vol\%] }\end{array}$ \\
\hline$<63 \mu m$ & $\begin{array}{c}0 \mathrm{U} / \min \\
\text { eine Drehung } \\
1 \mathrm{U} / \mathrm{min} \\
4 \mathrm{U} / \mathrm{min}\end{array}$ & $0-50$ & 120 & 20 \\
\hline $63-125 \mu \mathrm{m}$ & $\begin{array}{c}0 \mathrm{U} / \min \\
0.1 \mathrm{U} / \mathrm{min} \\
0.3 \mathrm{U} / \mathrm{min} \\
1 \mathrm{U} / \mathrm{min}\end{array}$ & $0-50$ & 120 & 38 \\
\hline
\end{tabular}

Tabelle 5.1: Messparameter für Muskovit/Glyzerin in der Rotationsküvette

Zu Beginn einer Messreihe wurde zunächst der direkt nach dem Befüllen der Küvette eine Polfigurmessung gestartet, ohne dabei die Scheibe der Küvette rotieren zu lassen (entspricht $0 \mathrm{U} / \mathrm{min}$ Tab. 5.1). Unterscheiden sich die bei rotierender Scheibe gemessen Polfiguren von denen bei $0 \mathrm{U} / \mathrm{min}$ ist sichergestellt, dass sich die Ergebnisse auf die anschließend erzeugte Scherströmung beziehen und nicht schon beim Einfüllen erzeugt wurden. Der Strahlquerschnitt für alle Messungen beträgt $2 * 2 \mathrm{~mm}$ und nach jeder Aufnahme wurde die Küvette, wie in Kapitel 2.2.2 beschrieben, um $2^{\circ}$ in $\omega$ gedreht.

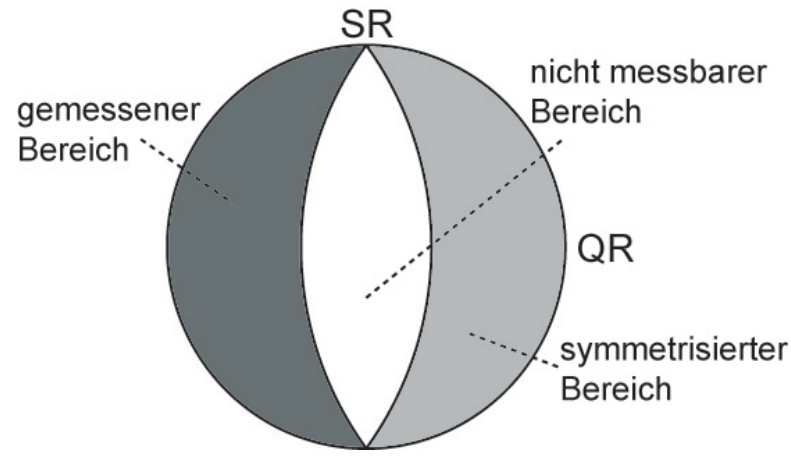

Abbildung 5.3: Gemessener (dunkelgrau) und nicht gemessener Bereich (hellgrau) in der Polfigur

Aufgrund der Bauweise der Küvette ist die Drehung um $\omega$ nur in eine Richtung $\left(0-50^{\circ}\right)$ möglich. In der anderen Richtung (bis $\left.-50^{\circ}\right)$ würde sich aufgrund der Messgeometrie der Antriebsmotor der Küvettenscheiben in den Strahlengang drehen. Es wird so nur eine Hälfte der Polfigur gemessen, in Abbildung 
5.3 dunkelgrau dargestellt. Die andere Hälfte (hellgrau) der im weiteren Verlauf dargestellten Polfiguren ist durch Symmetrisierung der Polfigur entstanden. Dies ist bei einer orthorhombischen Probensymmetrie erlaubt.

Da die Probensymmetrie jedoch nicht bekannt ist, sollte eigentlich von $\left(-\omega<0^{\circ}<\omega\right)$ gemessen werden. Die Probensymmetrie lässt aus den Polfiguren direkt bestimmen, da aber der gemessene Bereich nur eine Hälfte der Polfigur abdeckt ist dies hier nicht geeignet. Eine weitere Möglichkeit zur Ermittlung der Probensymmetrie bietet die Betrachtung der C-Koeffizienten aus der Berechnung der Orientierungsverteilungsfunktion. Dies ist für diese Messungen jedoch nicht möglich (siehe Kap. 5.1.2). 5.4).

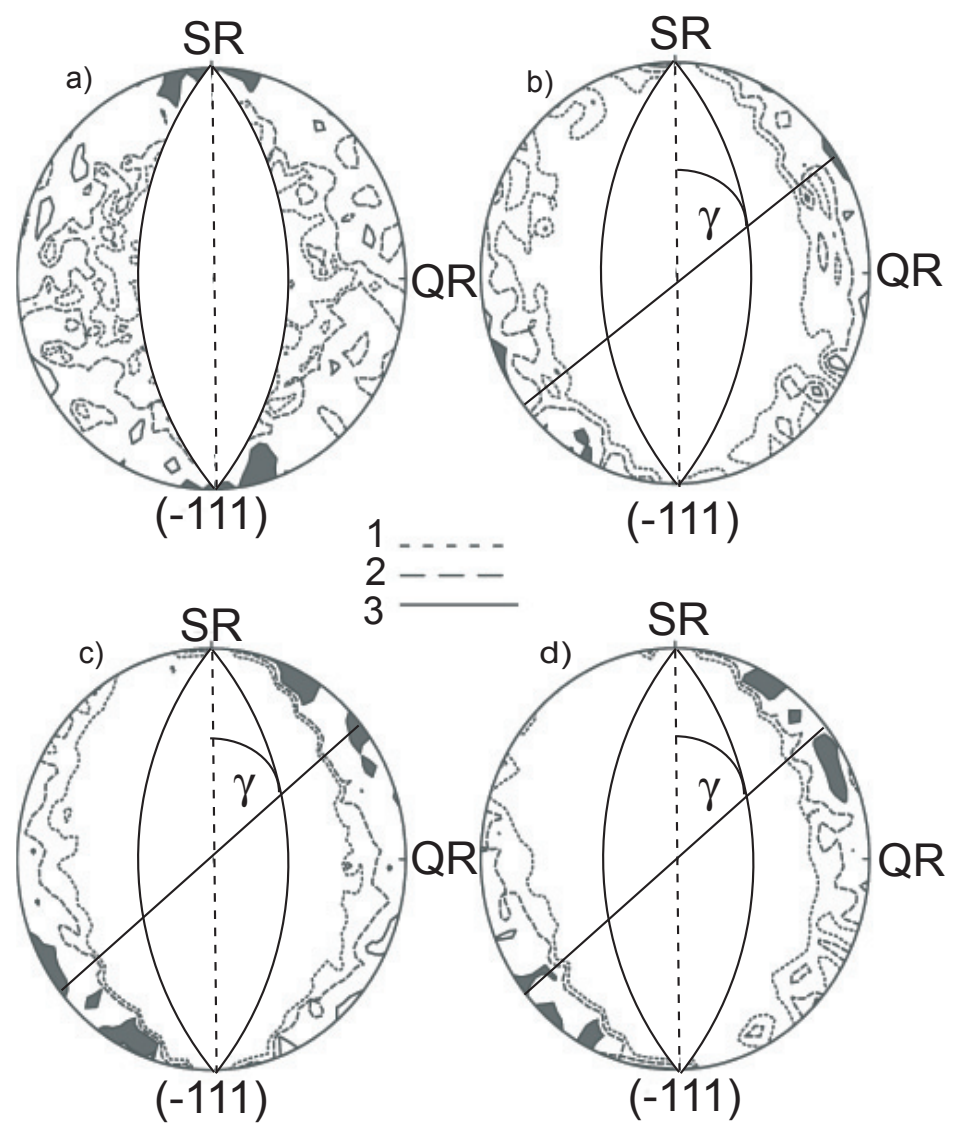

Abbildung 5.4: Muskovitpolfiguren $(<63 \mu \mathrm{m})$ gemessen bei a) $0 \mathrm{U} / \mathrm{min}$ b) eine Drehung c) $1 \mathrm{U} / \mathrm{min}$ d) $4 \mathrm{U} / \mathrm{min}$

Die Abbildung 5.4 zeigt die mit der Rotationsküvette gemessenen (111)Polfiguren von Muskovit in Glyzerin der Korngrößenfraktion $<63 \mu m$. Die Polfigur 5.4 a), welche direkt nach dem Befüllen gemessen wurde, weist über den gesamten gemessenen Bereich Intensität auf. Nach dem Befüllen sind die Partikel noch nicht stark ausgerichtet. Lediglich im oberen und unteren Teil der Polfigur bildet sich ein Maximum aus. Hier ist eine orthorhombische Proben- 
symmetrie möglich. Da die Partikel während der gesamten Aufnahmezeit sedimentieren, können sie sich so einregeln, dass sich die Basisebene senkrecht zur Rotationsrichtung (SR) und parallel zur Querrichtung (QR) orientiert. Da der (111)-Reflex ebenfalls nahezu senkrecht zur Basisebene steht, ergibt sich die maximale Intensität in Richtung (SR) (dunkelgraue Bereich in Abb. Nach der Ruheaufnahme wurde für eine weitere Polfiguraufnahme (Abb. 5.4 b)) die Küvettenscheibe ein Umdrehung gedreht und dann gestoppt. Anschließend wurde die Messung gestartet. Da die Sedimentation aufgrund der hohen Viskosität des Glyzerins gegenüber Wasser verlangsamt wird, wurde auch eine Polfigurmessung nach nur einer Umdrehung der Scheibe gestartet. Es ist anzunehmen, dass sich die erzeugte Orientierung während der Messung kaum ändert. Im Vergleich mit der Polfigur bei $0 \mathrm{U} / \mathrm{min}$ ist deutlich zu sehen, dass sich die Muskovitteilchen durch die Scherströmung einregeln. Die maximale Intensität befindet nicht mehr in Richtung SR, sondern um ca. $45^{\circ}$ verschoben. Die Probensymmetrie ändert sich und ist mit einsetzender Strömung maximal monoklin. Insgesamt konzentriert sich die gemessene Intensität mehr auf die Randbereiche der Polfigur.

Die weiteren Polfiguren (Abb. 5.4 c,d)) wurden bei konstanter Rotationsgeschwindigkeit der Scheibe aufgenommen. Die maximale Intensität ist für den (111)-Reflex am Rand der Polfigur zu beobachten. Für die Basisebene der Muskovitplättchen bedeutet dies, dass sie sich parallel zur Strömungsrichtung (SR) und senkrecht zum Strömungsgradienten ausrichten. Da die Intensitätsverteilung am Rand der Polfigur nicht gleichmäßig ist, sondern ein Maximum (grau) vorhanden ist, gibt es eine weitere Vorzugsorientierung.

Die in der Theorie in Kapitel 1.3.2 beschriebenen rotationsellipsoiden Plättchen richten sich mit ihrer Basisebene parallel zur Strömungsrichtung aus und rotieren frei um die Normale der Basisebene. Diese freie Rotation würde für den (111)-Reflex zu einer gleichmäßigen Intensitätsverteilung am Rand der Polfigur führen. Da dies nicht der Fall ist, gibt es für die Orientierung der Basisebene um ihre Normale eine Vorzugsrichtung und die Annahme rotationsellipsoider Plättchen ist nicht zulässig. Es gibt sowohl in Strahlrichtung zwischen der Wand und der rotierenden Scheibe der Küvette einen Strömungsgradienten (Abb. 1.1, als auch vom Drehzentrum der Küvette zum Rand (Abb. 4.4) einen weiteren Gradienten. Im bestrahlten Bereich der Küvette ändert sich die Strömungsgeschwindigkeit zwischen der Wand und der Scheibe von 0 bis zur absoluten Geschwindigkeit der Scheibe. Die Änderung der absoluten Geschwindigkeit in Querrichtung, vom Drehzentrum zum Rand, ist über den bestrahlten Bereich deutlich schwächer, da sich der Radius nur um $2 \mathrm{~mm}$ ändert, und hat somit einen deutlich geringeren Einfluss auf die Orientierung der Basisebene. Er kann jedoch dazu führen, dass bereits nach der Basisebene orientierte Plättchen aufgrund unterschiedlicher Strömungsgeschwindigkeiten aufeinander treffen und sich zu Agglomeraten zusammenschließen. Innerhalb dieser Agglomerate können die Partikel eine weitere Vorzugsorientierung einnehmen, indem sich die $\{110\}$ Ebenen aneinander legen. Diese ist wie in den Polfiguren in Abbildung 5.4 b,c,d) zu sehen schwächer ausgeprägt als die Orientierung der Basisebene.

Für plättchenförmige hexagonale Partikel ist dies bei Brown et al. [66] in Abbildung $5.5 \mathrm{zu}$ sehen. Die Partikel ordnen sich in der Ebene nicht aufgrund 


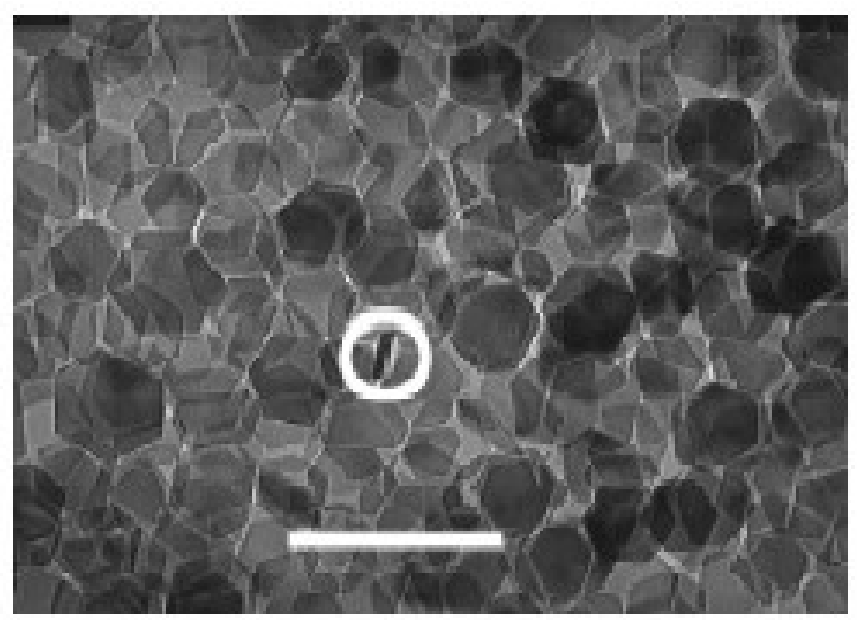

Abbildung 5.5: Agglomerat hexagonaler Nickel(II)Hydroxid Partikel nach Brown et al. [66]

der Strömungsverhältnisse, sondern nehmen einen geordneten Zustand ein, welche einer dichten Packung entspricht.

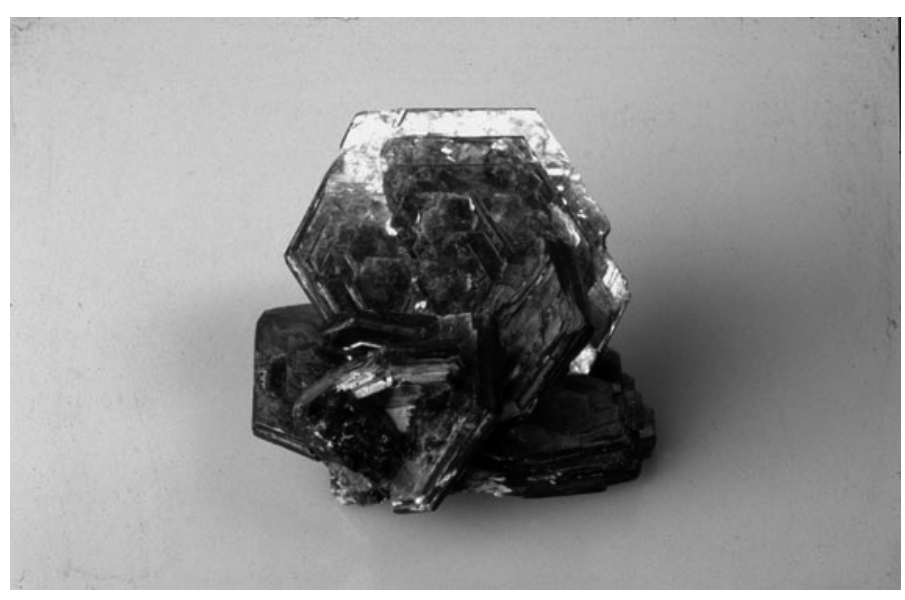

Abbildung 5.6: Pseudohexagonales Muskovitpartikel [67]

Die monoklinen Muskovitpartikel können auch in einer pseudohexagonalen Kristallitform, wie in Abbildung 5.6 dargestellt, auftreten. Diese Form ist jedoch bei den hier verwendeten gemahlenen Muskovitpulvern (siehe Kap. 3.1.1) nicht ist vorhanden. Es sind jedoch einzelne gerade Kanten zu erkennen (Abb. 3.3). Diese Kanten können im Agglomerat ebenfalls zu einer Ausrichtung der Partikel führen, welche jedoch nicht so stark ausgeprägt ist, wie bei den Nickel(II)Hydroxid Teilchen [66] (Abb. 5.5).

Die einzelnen Winkel zwischen den Netzebenen im Muskovitkristall sind in Tabelle 5.2 zusammengestellt. Mit diesen Winkeln lässt sich die Orientierung des gesamten Partikels in der Strömung abschätzen.

Ein pseudohexagonales Muskovitteilchen kann sich entsprechend der vor- 


\begin{tabular}{c|c|c|c|c|c|c}
$\begin{array}{c}\text { Netz- } \\
\text { ebenen }\end{array}$ & $\begin{array}{c}(001) \\
(010)\end{array}$ & $\begin{array}{c}(001) \\
(110)\end{array}$ & $(\overline{1} 11)$ & $\begin{array}{c}(\overline{1} 11) \\
(010)\end{array}$ & $\begin{array}{c}(111) \\
(110)\end{array}$ & $\begin{array}{c}(110) \\
(010)\end{array}$ \\
\hline Winkel & $85^{\circ}$ & $85^{\circ}$ & $82^{\circ}$ & $60^{\circ}$ & $119^{\circ}$ & 60
\end{tabular}

Tabelle 5.2: Winkel zwischen den Netzebenen von Muskovit

handenen Kristallitkanten ausrichten. In Abbildung 5.7 ist die Skizze eines pseudohexagonalen Teilchens in eine Polfigur eingezeichnet. Als Anhaltspunkt dient die maximale Intensität des (111). Von dieser Orientierung ausgehend ist die Orientierung der anderen Ebenen durch die Winkelverhältnisse rekonstruiert worden.

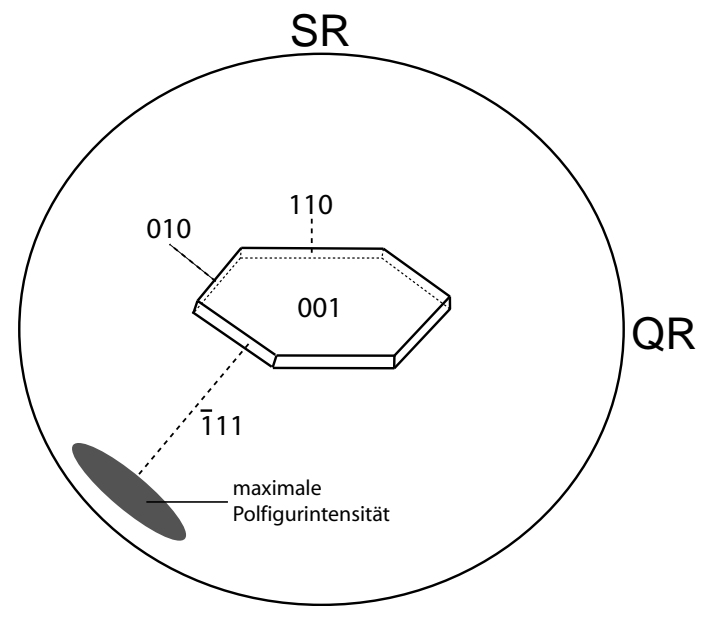

Abbildung 5.7: Schematische Darstellung der Orientierung eines Muskovitteilchens in einer Polfigur für die Rotationsküvette

Die Position der grauen Fläche entspricht ungefähr der des Maximums in den gemessenen (111)-Polfiguren in Abbildung 5.4 c-d). Entspricht eine Bruchkante der 110-Ebene der gemahlenen Muskovitpartikel kann diese durch Einregeln mit anderen Partikeln für die Ausbildung des Maximums am Rand der (111)-Polfigur verantwortlich sein.

Der Winkel $\gamma$, welche die Position des Maximums in Abbildung 5.4 beschreibt, ändert sich ab einer Rotationsgeschwindigkeit von $1 \mathrm{U} / \mathrm{min}$ nicht mehr. Die Vorzugsorientierung um die Normale der Basisebene scheint bei $1 \mathrm{U} / \mathrm{min}$ schon im Gleichgewicht zu sein. Darum wurde für eine weitere Messreihe der Geschwindigkeitsbereich zwischen 0-1 U/min untersucht. Hierzu wurde die Korngrößenfraktion von 63-125 $\mu \mathrm{m}$ verwendet, da das Radienverhältnis (Abb. 1.9) mit $r_{e}=0,017$ auf der Grundlage der in Kapitel 1.3.2 beschriebenen Theorie zu einem noch größeren Effekt führen sollte. Die Partikel sind auch größer und erfahren so über die gesamte Partikeloberfläche unterschiedliche Strömungsgeschwindigkeiten. Dies sollte noch zusätzlich zu einem größeren Effekt führen. Die Messungen wurden mit den Parametern in Tabelle 5.1 durchgeführt.

Wie bei der ersten Messreihe mit der Muskovitfraktion (Korngröße $<63 \mu m$ ) stellt sich nach dem Befüllen der Küvette die maximale Intensität in Rotationsrichtung (SR) am Rand der Polfigur (Abb. 5.8 a)) ein. Die Probensymmetrie 

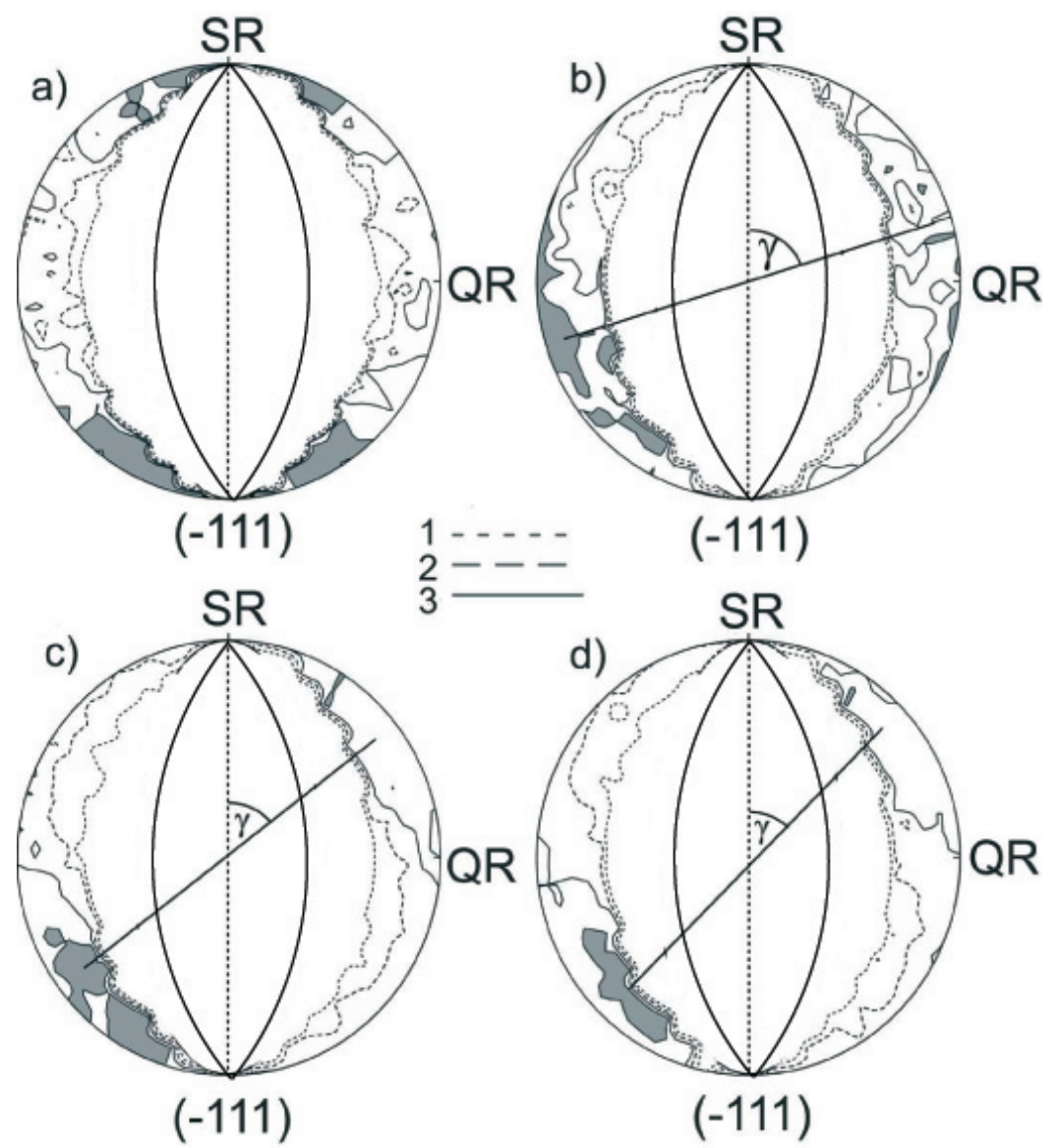

Abbildung 5.8: Muskovitpolfiguren (Korngröße 63-125 $\mu \mathrm{m}$ ) gemessen bei a) $0 \mathrm{U} / \min$ b) $0.1 \mathrm{U} / \min$ c) $0.3 \mathrm{U} / \min$ d) $1 \mathrm{U} / \min$

ändert sich auch hier wieder von maximal orthorhombisch im Ruhezustand zu maximal monoklin bei einsetzender Strömung. Die Textur ist jedoch etwas schärfer als bei der ersten Messung. Der innere Bereich der Polfigur weist nur wenig Intensität auf. Die Partikel dieser Messreihe sinken aufgrund ihrer Größe schneller ab und können sich somit leichter orientieren.

Die Intensität in den Muskovitpolfiguren (Korngröße $63-125 \mu m$ ) in den Abbildungen 5.8 b,c,d) nimmt am Rand der Polfigur deutlich zu. Dies ist auch schon in der ersten Messreihe in Abbildung 5.4 zu sehen. Die Polfigurmessung im Geschwindigkeitsbereich zwischen 0-1 U/min zeigt, das sich die Position des Maximums (grau) mit der Drehgeschwindigkeit ändert. Die Position ist durch den Winkel $\gamma$ ist in den Abbildungen 5.8 b,c,d) gekennzeichnet. Des Weiteren ist in Abbildung $5.8 \mathrm{~d}$ ) zu beobachten, dass sich das Maximum nicht mehr am Rand der Polfigur befindet, sondern um $5-10^{\circ}$ zur Mitte hin gekippt ist.

Die Änderung des Orientierungswinkels $\gamma$ mit der Drehgeschwindigkeit ist in Abbildung 5.9 aufgetragen. Zur Verdeutlichung wurden die einzelnen Punkte miteinander verbunden. Der Winkel $\gamma$ ändert sich zwischen $0,3 \mathrm{U} / \mathrm{min}$ und $1 \mathrm{U} / \mathrm{min}$ kaum noch und aus der vorherigen Messung ist zu sehen, dass auch zwischen $1 \mathrm{U} / \mathrm{min}$ und $4 \mathrm{U} / \mathrm{min}$ keine signifikanten Änderungen der Position 


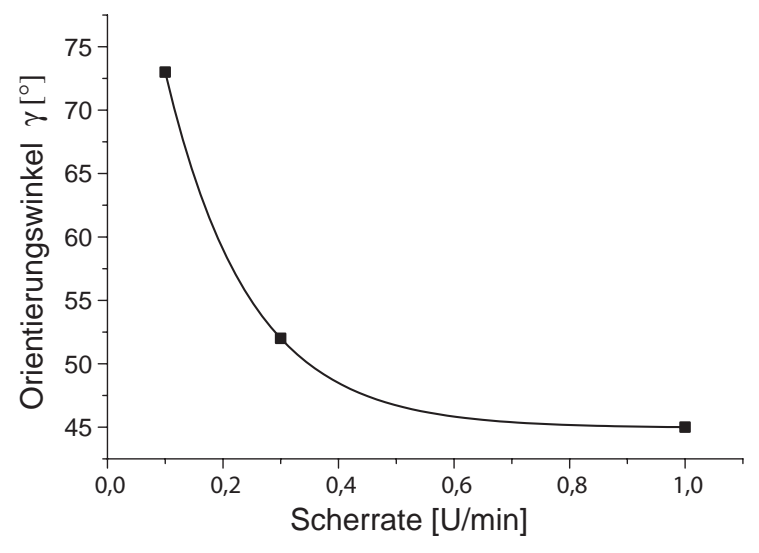

Abbildung 5.9: Darstellung des Orientierungswinkels $\gamma$ in Abhängigkeit von der Rotationsgeschwindigkeit ('"guide to the eye'")

der Vorzugorientierung auftreten. Mit steigender Rotationsgeschwindigkeit nehmen die Partikel eine Gleichgewichtsposition ein. Ob die Partikel bei wesentlich höheren Strömungsgeschwindigkeiten diese Vorzugsorientierung beibehalten ist konstruktionsbedingt mit dieser Küvette nicht zu klären.

Die Messung mit den Partikeln der Korngrößenfraktion 63-125 $\mu$ m zeigt ein deutlich sensitiveres Verhalten auf die Änderung der Strömungsgeschwindigkeit als die Fraktion $<63 \mu m$. Dies Verhalten lässt sich in Übereinstimmung mit den Theorien von Anczurowski et al. [7, 39, 68] in Kapitel 1.3 und 1.4 durch die unterschiedlichen Radienverhältnisse $r_{e}$ erklären. Außerdem tragen auch die absoluten Teilchengrößen zu einem unterschiedlichen Verhalten bei.

\subsubsection{Muskovit-/Glyzeringemische in der Durchflussküvette}

Um den Einfluss von unterschiedlichen Strömungsgradienten auf die Textur zu bestimmen wurden mit der Muskovitfraktion (Korngröße $<63 \mu m$ ) Polfiguraufnahmen mit der Durchflussküvette (siehe Kap. 4.2) gemessen. Außerdem wurde auch hier der Einfluss der Strömungsgeschwindigkeit untersucht. Die entsprechenden Messparameter sind in Tabelle 5.3 zusammengestellt. Alle Messungen wurden mit einem Strahlquerschnitt von $2 * 2 \mathrm{~mm}$ und einer Schrittweite $\Delta \omega=2^{\circ}$ durchgeführt.

\begin{tabular}{c|c|c|c|c}
$\begin{array}{c}\text { Korngrößen- } \\
\text { fraktion }\end{array}$ & $\begin{array}{c}\text { Durchfluss } \\
{[\mathrm{ml} / \mathrm{s}]}\end{array}$ & $\begin{array}{c}\text { Drehwinkel } \\
\omega\left[^{\circ}\right]\end{array}$ & $\begin{array}{c}\text { Belichtungs- } \\
\text { zeit [sec] }\end{array}$ & $\begin{array}{c}\text { Konzentration } \\
\text { Vol\% }\end{array}$ \\
\hline$<63 \mu m$ & 0.2 & $-70-70$ & 40 & 20 \\
& 0.5 & -70 & &
\end{tabular}

Tabelle 5.3: Messparameter von Muskovit/Glyzerin in der Durchflussküvette

Eine Messung, die den Zustand direkt nach dem Befüllen widerspiegelt, wie bei der Rotationsküvette, ist mit der Durchflussküvette aufgrund der Funkti- 
onsweise nicht möglich. Ein weiterer Unterschied zur Rotationsküvette besteht darin, dass es mit der Durchflussküvette möglich ist, aus der Position $\omega=0^{\circ}$, bei der der Strahl senkrecht auf die Probe trifft, in beide Richtungen zu drehen. Für die im weiteren Verlauf dargestellten Polfiguren wurde sowohl der dunkelgraue als auch der hellgraue Bereich (Abb. 5.3) gemessen.

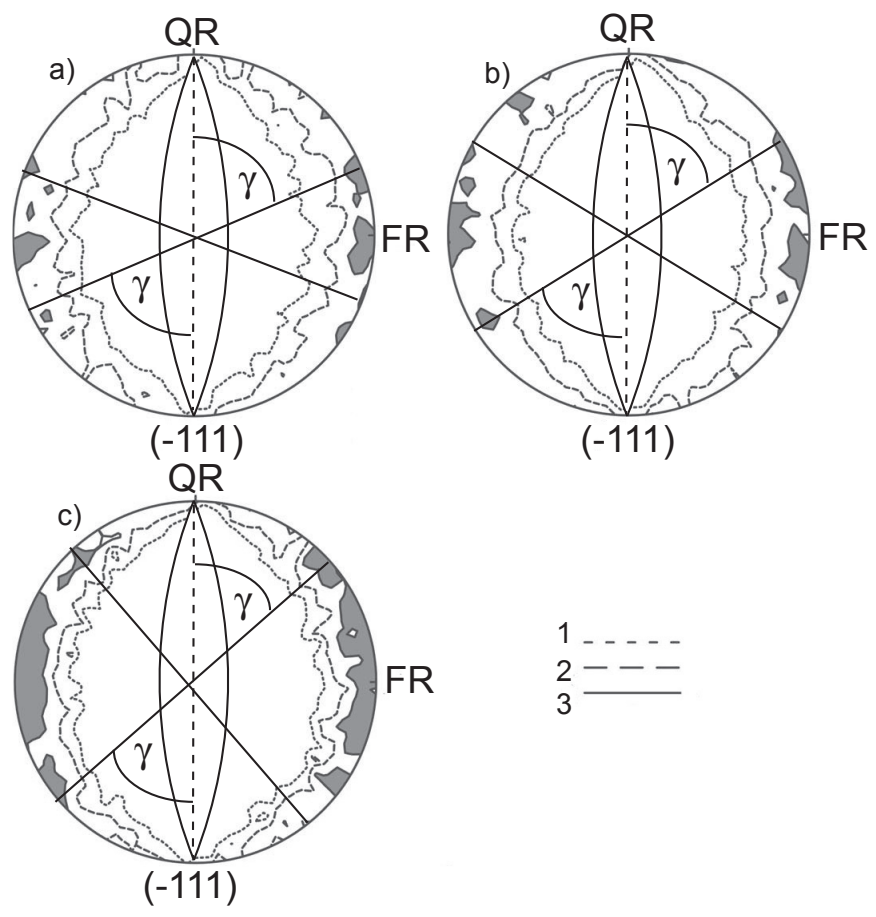

Abbildung 5.10: Muskovitpolfiguren (Korngröße $<63 \mu \mathrm{m}$ ) gemessen bei unterschiedlichen Fliessgeschwindigkeiten a) $0,2 \mathrm{ml} / \mathrm{s}$ b) $0,5 \mathrm{ml} / \mathrm{s}$ c) $1 \mathrm{ml}$

Auch im Strömungsprofil der Durchflussküvette nehmen die Muskovitpartikel eine Vorzugsorientierung ein. Diese ist denen der Rotationsküvette sehr ähnlich. Jedoch liegt in der Durchflussküvette eine orthorhombische Probensymmetrie vor. Die Polfiguren in Abbildung 5.10 a-c) zeigen wie die Polfiguren der Rotationsküvette, dass die maximale Intensität am Rand der Polfigur zu beobachten ist.

Die maximale Intensität scheint sich mit steigender Fließgeschwindigkeit von einem lokalen Maximum ausgehend über den Randbereich breiter zu verteilen. Es ist jedoch im Bereich $\pm 40^{\circ}$ in Flussrichtung (FR) in allen Polfiguren in Abbildung 5.10 ein lokales Maximum mit unterschiedlicher Ausprägung zu beobachten. Das in Kapitel 5.1.1 beschriebene Verhalten der Partikel, dass sie aufgrund unterschiedlicher Geschwindigkeit Agglomerate bilden und so eine weitere Vorzugsorientierung ausbilden, kann auch hier wieder zu Vorzugsorientierungen um die Normale der Basisebene führen. Da aber bei der Durchflussküvette das Strömungsfeld höher symmetrisch ist (siehe Kap. 1.2.1 und 1.2.2) als bei der 
Rotationsküvette treten sowohl über und unter der horizontalen Ebene Maxima auf. Diese können sehr breit sein und sich so zu einem großen Maximum überlagern.
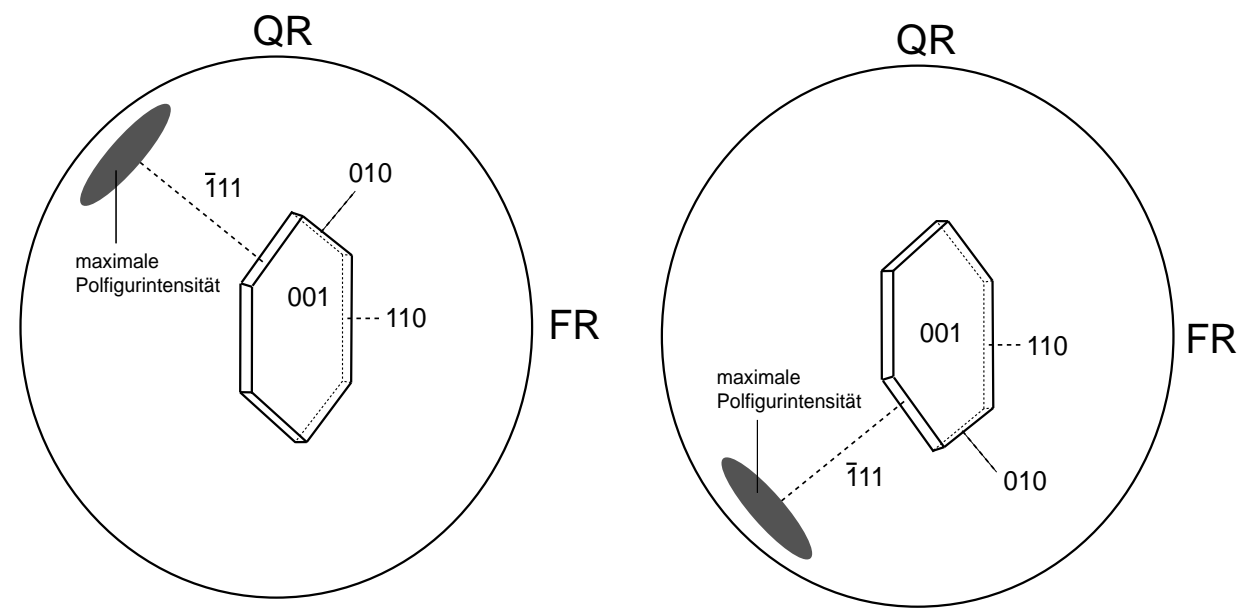

Abbildung 5.11: Schematische Darstellung der Orientierung eines Muskovitteilchen in einer Polfigur im Strömungsfeld der Durchflussküvette

In Abbildung 5.11 sind zwei mögliche Orientierungen von pseudohexagonalen Muskovitkristalliten dargestellt. Zwei weitere Orientierungen, welche die Maxima in Flussrichtung erzeugen sind nicht dargestellt. Sie ergeben sich durch eine vertikale Spiegelung. Trotz unterschiedlicher Position der Maxima in den Polfiguren der Rotations- und Durchflussküvette können die Vorzugsorientierungen durch den gleichen Vorgang entstanden sein. Lediglich das unterschiedliche Strömungsprofil, wie in Abbildung 1.1 und 1.3 gezeigt, kann für diese Unterschiede verantwortlich sein.

Da sich die Intensitäten der einzelnen Messungen jedoch nur bedingt miteinander vergleichen lassen, ist ein Vergleich der Texturschärfe in Abhängigkeit mit der Strömungsgeschwindigkeit oder des Strömungsfeldes nicht möglich. Dies wäre nur möglich, wenn rückgerechnete Polfiguren aus der Orientierungsverteilungsfunktion (ODF) zugänglich sind. Diese sind wie in Kapitel 2.5.1 beschrieben auf ein regelloses Verhalten normiert. Für das monokline Kristallsystem des Muskovits ist eine solche ODF-Rechnung mit den gemessenen Daten jedoch nicht möglich. Aufgrund der vielen überlagerten Reflexe des Muskovits sind einzelne Polfiguren nur sehr schwer zu bestimmen. Hinzu kommt noch der Einfluss der amorphen Substanzen, welche eine zusätzliche Störung bedeuten.

Zur Berechnung der ODF wird eine bestimmte Anzahl an gemessenen Polfiguren benötigt (siehe Kap. 2.5.3). Für niedrige Reihenentwicklungsgrade, wie z.B. $L_{\max }=6$, werden bei monokliner Kristallsymmetrie des Muskovits mindestens 7 Polfiguren benötigt. Diese Anzahl an Polfiguren ist durch den hier verwendeten Aufbau nicht zugänglich. Die Messung müsste mit einer deutlich höheren Auflösung in $2 \vartheta$ durchgeführt werden. Außerdem ist aufgrund des schwachen Streuvermögens des Muskovits eine deutlich längere Messzeit erforderlich, welche mit den verwendeten Küvetten nicht realisierbar ist. 


\subsection{Aluminiumoxidpartikel in Glyzerin}

Nach den ersten Messungen mit Muskovitpartikeln in Glyzerin und den damit erzielten Ergebnissen, ist eine andere Substanz nötig, welche sich für die Berechnung der Orientierungsverteilungsfunktion (ODF) eignet. Aufgrund der Normierung der ODF-Daten gegen den regellosen Fall, lassen dann auch einzelne Messungen hinsichtlich der Texturschärfe miteinander vergleichen (siehe Kap. 2.5.1). Da dies wie in Kapitel 5.1.2 beschrieben mit Muskovit nicht möglich ist, werden im weiteren Verlauf $\mathrm{Al}_{2} \mathrm{O}_{3}$-Partikel in Glyzerin untersucht. Die Texturentwicklung während der Grünformung von $\mathrm{Al}_{2} \mathrm{O}_{3}$-Substraten ist von großem industriellen Interesse und wurde bereits von A. Böcker [15] beschrieben. Es wurden dabei jedoch nur die Zustände vor und nach der Formgebung durch Gießen oder ausstreichen betrachtet. In dieser Arbeit wird der Teil der Texturentwicklung untersucht, welcher direkt während der Strömung stattfindet und damals noch nicht experimentell zugänglich war.

Die $\mathrm{Al}_{2} \mathrm{O}_{3}$-Partikel eignen sich aufgrund der trigonalen Kristallsymmetrie deutlich besser zur Berechnung der Orientierungsverteilungsfunktion. Es werden weniger Polfiguren als bei Muskovit benötigt (siehe Abb. 2.21). Beim $\mathrm{Al}_{2} \mathrm{O}_{3}$ sind weniger Beugungsreflexe vorhanden als beim Muskovit (Abb. 5.1) und somit auch weniger Überlagerungen von den Reflexen, wie im Beugungsbild in Abbildung 5.12 zu sehen.

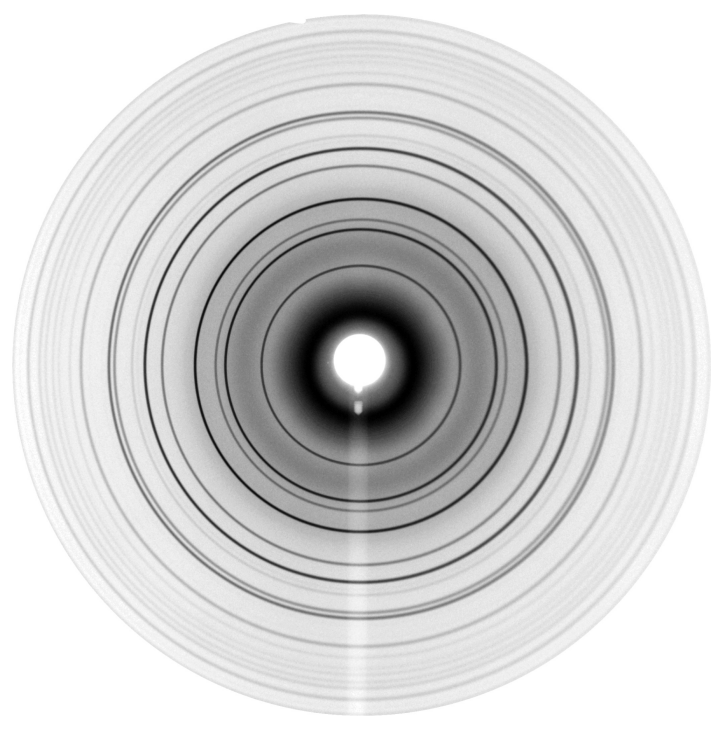

Abbildung 5.12: Beugungsbild von $\mathrm{Al}_{2} \mathrm{O}_{3}$-Partikeln in Glyzerin in der Rotationsküvette

Bei $\mathrm{Al}_{2} \mathrm{O}_{3}$ lassen sich mit der vorhandenen Messmethode (siehe Kapitel 2.1) sieben linear unabhängige Polfiguren messen und auswerten, welches Reihenentwicklungsgrade bis $L_{\max }=40$ für vollständig gemessene Polfiguren zulassen würde. Die einzelnen Beugungsreflexe sind in Abbildung 5.13a unter realen Messbedingungen mit amorphem Untergrund und in Abbildung 5.13b ohne Untergrund dargestellt. Es überschneiden sich lediglich der (300)-Reflex und der (214)-Reflex, welche sich jedoch mathematisch mit der eigens entwickelten 
Software (Kap. 2.3) trennen lassen.

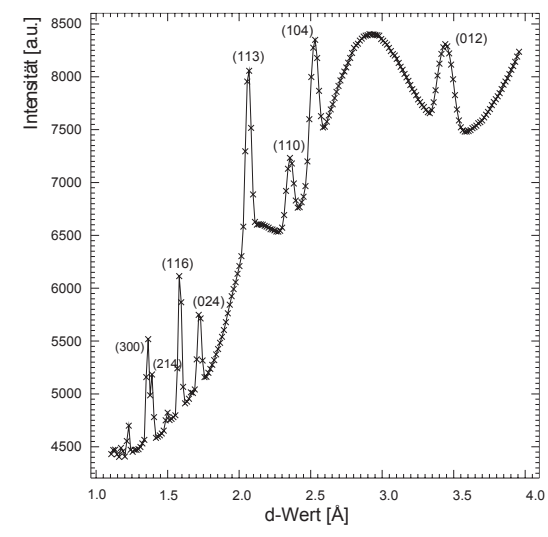

(a) Integriertes Beugungsdiagramm aus dem Beugungsbild (Abb. 5.12) $\mathrm{Al}_{2} \mathrm{O}_{3}$-Partikel in Glyzerin

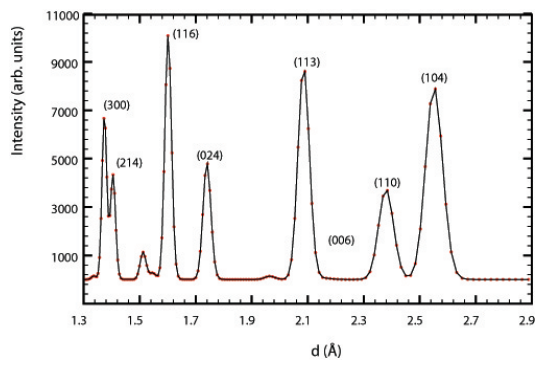

(b) Simuliertes Beugungsdiagramm für Abbildung a)

Abbildung 5.13: Beugungsdiagramme für $\mathrm{Al}_{2} \mathrm{O}_{3}$

Bevor die einzelnen Messungen miteinander verglichen werden können, müssen für alle zu berechnenden Orientierungsverteilungsfunktionen die gleichen Parameter (Probensymmetrie, Reihenentwicklungsgrad) verwendet werden. Die für alle Proben geeigneten Parameter werden im Folgenden ermittelt. Hierzu werden einige charakteristische Messungen ausgewählt an denen exemplarisch die ODF aus den gemessenen Polfiguren gerechnet wird. Da sich die Probensymmetrie nicht sprunghaft von einer Messung zur nächsten ändern sollte, wird jeweils pro Substanz und Küvette eine Messung untersucht.

Durch Variieren des Reihenentwicklungsgrades (Kap. 5.2.2) bei zuvor bestimmter Probensymmetrie und Gegenüberstellung der Ergebnisse wird der Reihenentwicklungsgrad ermittelt, mit welchem die vorhandene Textur am besten beschrieben wird (siehe Kap. 2.5.4). Als letzter Schritt werden noch zur Überprüfung der Ergebnisse die rückgerechneten Polfiguren aus der ODF mit den gemessenen verglichen (Kap. 5.2.3). Sind diese Polfiguren identisch, sind die notwendigen Parameter ermittelt und es werden alle weiteren Orientierungsverteilungsfunktionen mit den ermittelten Parametern gerechnet. Es kann eine systematische Betrachtung der Ergebnisse beginnen.

\subsubsection{Ermittlung der Probensymmetrie}

Zur Bestimmung der Probensymmetrie wird zunächst die ODF mit einer triklinen Symmetrie berechnet. Mit trikliner Probensymmetrie erhält man aus der ODF-Rechnung alle C-Koeffizienten, welche zur Abschätzung der Symmetrie notwendig sind. Wie in Kapitel 2.5.3 beschrieben enthalten die C-Koeffizienten aus der Reihenentwicklung sämtliche Texturinformationen, wie z.B. auch die Probensymmetrie. Zur Bestimmung der Probensymmetrie sind besonders die niedrig indizierten C-Koeffizienten bei geringem Reihenentwicklungsgrad geeignet. Je nach vorhandener Probensymmetrie wird ein Teil der C-Koeffizienten aufgrund von Symmetrieoperationen $\left|C_{l}^{m n}\right| \approx 0$ [69]. 
Bei monokliner Probensymmetrie sind nur die C-Koeffizienten des Realteils von Null verschieden. Die C-Koeffizienten des Imaginärteils sind $\left|C_{l}^{m n}\right| \approx 0$. Da es sich bei der Probensymmetrie um eine statistische Symmetrie handelt, bei der Kristallite bestimmte Orientierungen mit gleicher Wahrscheinlichkeit einnehmen, ist der imaginäre Teil der C-Koeffizienten nicht exakt gleich Null. Ein Vergleich zwischen realem und imaginärem Teil der C-Koeffizienten liefert aber die Information über mindestens monokline Probensymmetrie.

\begin{tabular}{c|c|c} 
Proben & real Teil & imaginär Teil \\
\hline A (rot $\left.{ }^{1}\right)$ & 4.161 & 0.645 \\
B (rot) & 4.643 & 0.371 \\
C (rot) & 3.451 & 0.321 \\
HVAFG (rot) & 3.182 & 0.265 \\
CTC20 (rot) & 6.230 & 0.666 \\
Gilox (rot) & 4.684 & 0.930 \\
HVAFG (durch ${ }^{2}$ ) & 4.309 & 0.176 \\
CTC20 (durch) & 4.621 & 0.334 \\
Gilox (durch) & 6.518 & 0.339
\end{tabular}

Tabelle 5.4: Betrag des realen und imaginären Teils der $\left|C_{l}^{m n}\right|$ bis $l=6$ für unterschiedliche Proben

In Tabelle 5.4 sind die Beträge aller C-Koeffizienten $\left|C_{l}^{m n}\right|$ bis $l=6$ der unterschiedlichen Substanzen für real und imaginär Teil aufsummiert und gegenübergestellt. Zur Berechnung der ODF konnten mit der vorhandenen Software maximal 5 gemessene Polfiguren herangezogen werden. In diesem Fall sind es die (104)-,(110)-,(113)-,(116)- und (300)-Polfigur. Die Anzahl der Polfiguren ist für die Abschätzung der Probensymmetrie jedoch völlig ausreichend. Die unterschiedlichen verwendeten Küvetten sind in der Tabelle 5.4 mit "(rot)" für die Rotationsküvette und mit "(durch)" für die Durchflussküvette gekennzeichnet.

Der Vergleich zwischen real und imaginär Teil zeigt deutlich, dass mindestens monokline Probensymmetrie für alle gemessenen Proben vorliegt. Der imaginäre Teil ist für die meisten Proben sehr klein. Auch ein Betrag von 0.93 bei der Rechnung "Gilox (rot)" ist nicht sehr hoch, da dieser Betrag sich aus insgesamt 14 C-Koeffizienten zusammensetzt. Die einzelnen Beträge der C-Koeffizienten sind überwiegend kleiner 0.1 oder sogar Null.

Da alle Proben mindestens monokline Probensymmetrie zeigen (siehe Tab. 5.4), ist eine weitere Überprüfung nach höherer Probensymmetrie notwendig. Die nächst höhere orthorhombische Probensymmetrie lässt sich an den C-Koeffizienten des realen Teils der Reihenentwicklung erkennen. Die Koeffizienten $\left|C_{l}^{m n}\right|$ mit $n=$ gerade sind bei orthorhombischer Symmetrie deutlich größer als die Koeffizienten mit $n=$ ungerade. In Abbildung 5.14 ist der Verlauf der C-Koeffizienten über den Reihenentwicklungsgrad $n$ für $l=6$ und $m=1$ dargestellt. Die zugrunde liegende Messung für die Proben A,B und C fand in der Rotationsküvette bei $0.25 \mathrm{U} / \mathrm{min}$ statt. Diesen Messungen wurden exemplarisch

\footnotetext{
${ }^{1}$ rot $=$ Rotationsküvette

${ }^{2}$ durch $=$ Durchflussküvette
} 
zur Abschätzung der Probensymmetrie ausgesucht.

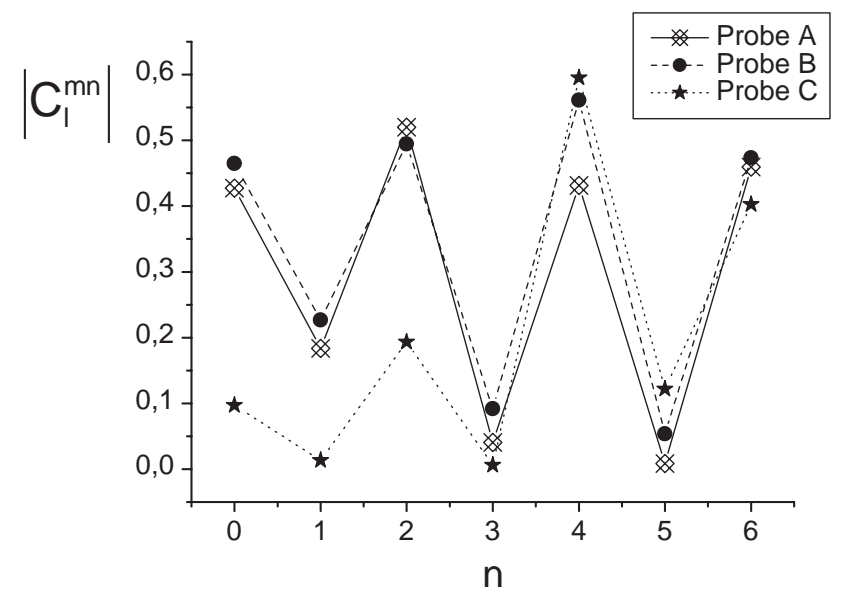

Abbildung 5.14: $\left|C_{l}^{m n}\right|$ für $l=6$ und $m=1$ für die Proben A,B,C in der Rotationsküvette

Anhand der dargestellten C-Koeffizienten in Abbildung 5.14 lässt sich die orthorhombische Probensymmetrie [69] für die Substanzen A,B und C erkennen. Immer von Wechsel $n=$ gerade zu $n=$ ungerade sinkt der Betrag für den CKoeffizienten. Die C-Koeffizienten mit $n=$ ungerade sind deutlich kleiner als die mit $n=$ gerade .

Die orthorhombische Probensymmetrie wird auch für C-Koeffizienten mit $l=4$ oder $l=2$ deutlich. Es sind dann nur fünf bzw. drei C-Koeffizienten vorhanden an denen die Probensymmetrie zu erkennen ist. Auf die Darstellung dieser C-Koeffizienten wurde jedoch verzichtet, weil kein zusätzlicher Informationsgehalt zu erwarten ist.

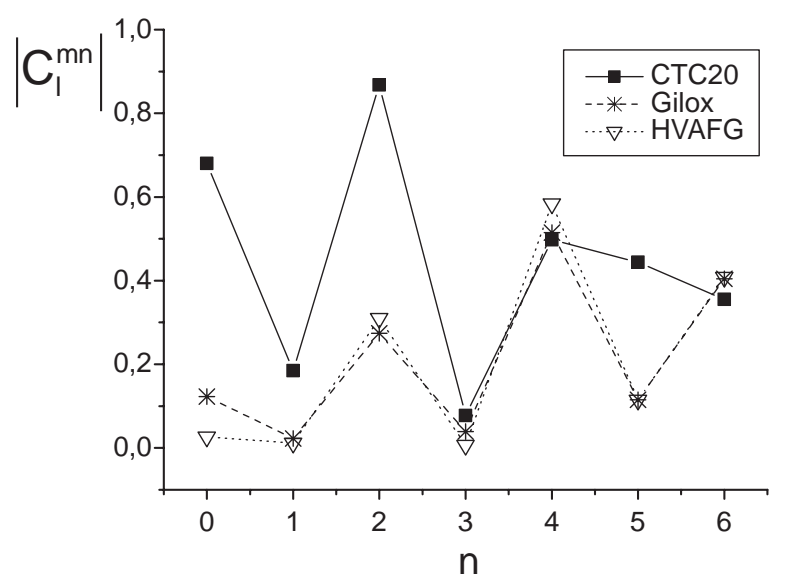

Abbildung 5.15: C-Koeffizienten $l=6$ für die Proben CTC20, Gilox und HVAFG in der Rotationsküvette 
Da es durchaus möglich ist, dass sich die einzelnen Substanzen in der Strömung im Hinblick auf die Probensymmetrie unterschiedlich verhalten, ist auch eine Überprüfung der Proben HVAFG, CTC20 und Gilox notwendig. In Abbildung 5.15 sind auch hier wieder $\left|C_{l}^{m n}\right|$ über den Reihenentwicklungsgrad $n$ für $l=6$ und $m=1$ dargestellt.

Auch hier ist wieder eine orthorhombische Probensymmetrie zu beobachten. Lediglich für $n=5$ bei der Probe CTC20 ist der Wert für den C-Koeffizienten sehr hoch. Allerdings ist er gegenüber $n=4$ leicht geringer. Da es sich bei der Probensymmetrie um eine statistische Symmetrie handelt, können solche geringen Abweichungen vom orthorhombischen Verhalten aufgrund der geringen Kornstatistik auftreten. Die Probensymmetrie der Messungen mit der Probe CTC20 können dennoch als orthorhombisch angesehen werden.

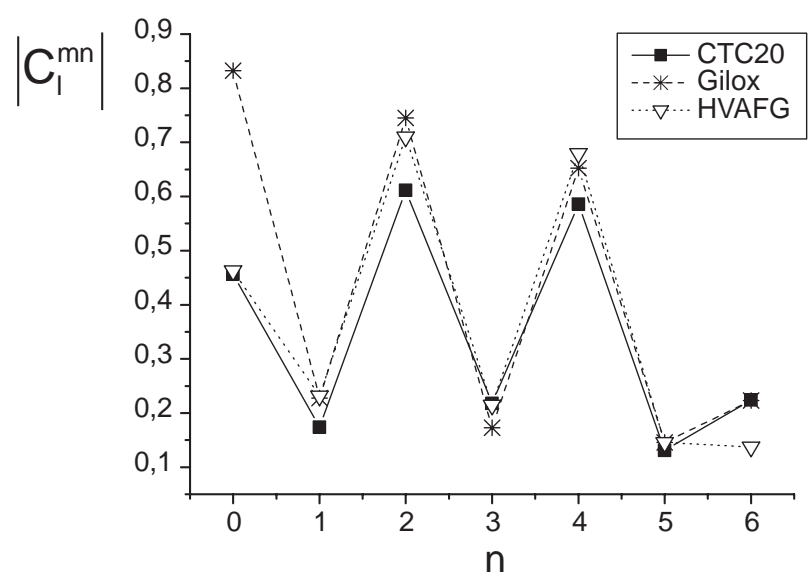

Abbildung 5.16: C-Koeffizienten $l=6$ für die Proben CTC20, Gilox und HVAFG in der Durchflussküvette

Ein weiterer Einfluss auf die Probensymmetrie kann ein unterschiedliches Strömungsfeld sein. Da sich die Strömungsfelder der beiden Küvetten, wie in Kapitel 1.2.1 und 1.2.2 beschrieben, unterscheiden, galt es auch noch die Probensymmetrie der Substanzen HVAFG, CTC20 und Gilox in der Durchflussküvette zu bestimmen. Die C-Koeffizienten in Abbildung 5.16 wurden mit den gleichen Parametern wie bei der Rotationsküvette ermittelt. Es stehen jedoch bei der Durchflussküvette gemessene Polfiguren in einem Bereich $(-70 \leq$ $\omega \leq 70)^{\circ}$ zur Verfügung. Da diese Ergebnisse auf deutlich mehr gemessenen Daten beruhen sind sie noch aussagekräftiger als die der Rotationsküvette.

Im Vergleich mit den ermittelten C-Koeffizienten der Rotationsküvette ist der Verlauf der Koeffizienten für alle Proben ähnlich. Mit der Durchflussküvette ließen sich deutlich längere Messzeiten, als mit der Rotationsküvette realisieren. So kommt hier noch zusätzlich die bessere Kornstatistik zum tragen und die Proben verhalten sich ohne Abweichung orthorhombisch. Die Unterschiede zwischen den Beträgen der C-Koeffizienten für $n=$ gerade und $n=$ ungerade (Abb. 5.16) sind hier noch deutlicher. Dies lässt auf eine sehr hohe orthorhombische Probensymmetrie schließen. 


\subsubsection{Ermittlung des Reihenentwicklungsgrades}

Um die Textur durch die Reihenentwicklungsmethode zu beschreiben muss der Reihenentwicklungsgrad so gewählt werden, dass die Funktion eine gute Näherung der gemessenen einzelnen Orientierungen darstellt. Die Reihenentwicklung wird abgebrochen wenn die Funktion die Dichteverteilung der Einzelorientierungen beschreibt (siehe Kap. 2.5.4). Ein Kriterium für den richtigen Reihenentwicklungsgrad ist die Konvergenz der C-Koeffizienten. Ab einem bestimmten Reihenentwicklungsgrad, welcher von der vorhandenen Schärfe der Textur abhängig ist, divergieren die C-Koeffizienten. Ab diesem Punkt ist der zulässige Reihenentwicklungsgrad $L_{\max }$ überschritten. Je "schärfer" eine Textur ausgeprägt ist, umso größer muss der Reihenentwicklungsgrad gewählt werden um diese Textur zu beschreiben.

Da es sich bei den hier gemessenen Orientierungen um sehr schwache Texturen handelt, ist ein niedriger Reihenentwicklungsgrad, wie er auch schon bei den Betrachtungen zur Probensymmetrie (Kap. 5.2.1) verwendet wurde, ausreichend. Die Überprüfung wurde exemplarisch an drei kommerziellen Proben (HVAFG, GILOX, CTC20) in der Durchflussküvette bei $1 \mathrm{ml} / \mathrm{s}$ Fluss durchgeführt. Die Berechnung der ODF ist hier aufgrund einer anderen Software mit mehr Polfiguren möglich als bei trikliner Probensymmetrie. Bis zu einem Reihenentwicklungsgrad von $L_{\max }=16$ werden zwar nur 4 Polfiguren benötigt (siehe Abb. 2.21), die gemessenen Polfiguren besitzen aber alle einen nicht messbaren Bereich. Durch weitere Polfiguren wird dies ausgeglichen und es stehen mehr Daten zur Berechnung zur Verfügung. Aus den gemessenen Daten wurden 6 unabhängige Beugungsreflexe zur Polfigurauswertung verwendet. Es handelt sich um die (104)-,(110)-,(113)-,(024)-,(116)- und (300)-Polfigur.

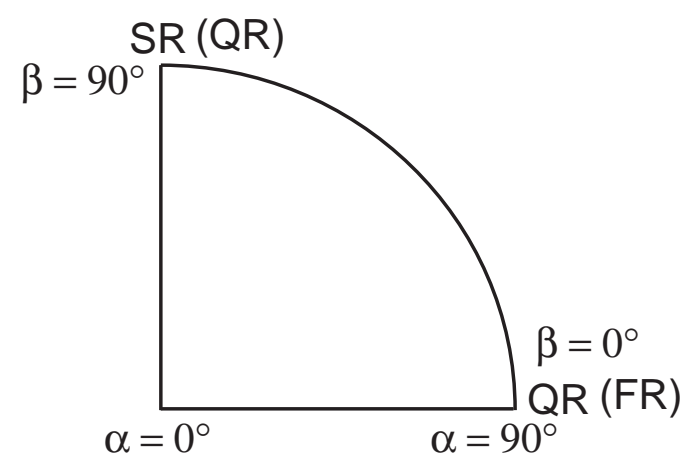

Abbildung 5.17: Darstellung der kleinsten asymmetrischen Einheit einer rückgerechneten Polfigur mit orthorhombischer Probensymmetrie

Die im weiteren Verlauf gezeigten rückgerechneten Polfiguren werden nur noch aufgrund der orthorhombischen Probensymmetrie als Quadrant einer Polfigur dargestellt. Für die Rotationsküvette ist die Scherrichtung mit SR und die dazugehörigen Querrichtung mit QR gekennzeichnet. Die Bezeichnungen für die Durchflussküvette sind in Klammern zu sehen (FR = Flussrichtung). Die Polfigurwinkel $\alpha$ und $\beta$ sind in Abbildung 5.17 exemplarisch dargestellt und werden im weiteren Verlauf nicht mehr aufgeführt. 
Der Verlauf der C-Koeffizienten in Abbildung 5.18a) zeigt, dass sie bis zu einem Reihenentwicklungsgrad von $L_{\max }=12$ konvergieren. Auch der Fehler in den Polfiguren (siehe Kap. 2.5.5) sinkt mit steigendem $L_{\max }$.

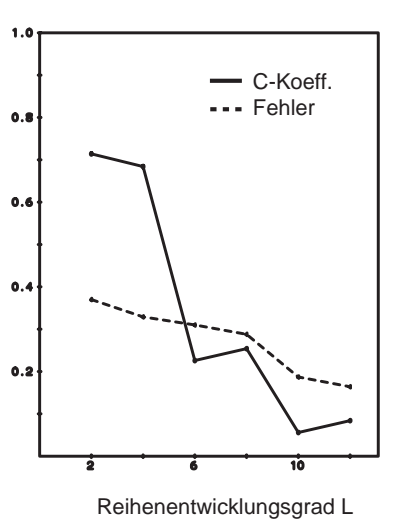

(a) $\bar{C}$-Koeffizienten und Fehler

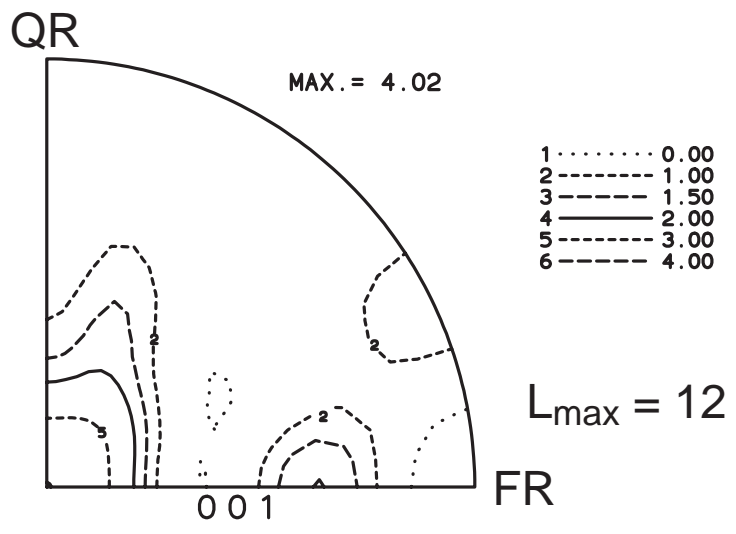

(b) Rückgerechnete (001)-Polfigur

Abbildung 5.18: Ergebnisse der ODF-Rechnung für die Probe Gilox (10Vol\%) in der Rotationsküvette bei $1 \mathrm{ml} / \mathrm{s}$ Fluss

Nach den Konvergenzkriterien würde dies für einen Reihenentwicklungsgrad von $L_{\max }=12$ sprechen. Ein weiteres Kriterium für die Güte der Reihenentwicklung befindet sich in den einzelnen Intensitätsdaten der rückgerechneten Polfiguren. Die in Abbildung 5.18b) dargestellte Polfigur weist im Wesentlichen zwei Vorzugsorientierungen auf. Eine befindet sich im Zentrum der Polfigur bei $\alpha=0^{\circ}$ und $\beta=0^{\circ}$. Die andere Vorzugsorientierung ist bei $\alpha=70^{\circ}$ und $\beta=$ $0^{\circ}$.

Um die Ergebnisse der rückgerechneten Polfiguren bei unterschiedlichem Reihenentwicklungsgrad besser miteinander vergleichen zu können, ist ein Polfigurschnitt bei $\beta=0^{\circ}$ am besten geeignet. Dieser erfasst beide auftretenden Vorzugsorientierungen.

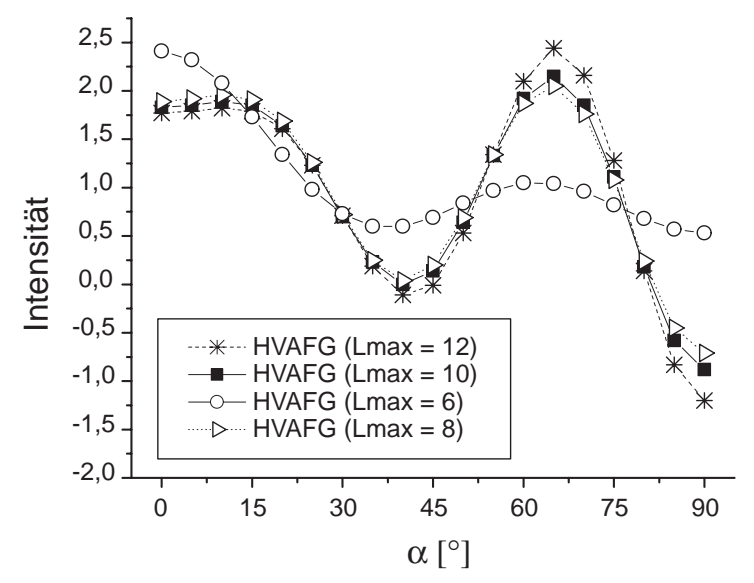

Abbildung 5.19: Polfigurschnitt bei $\beta=0^{\circ}$ für verschiedene $L_{\max }$ von HVAFG in der Durchflussküvette 
Der Intensitätsverlauf in Abhängigkeit von Polfigurwinkel $\alpha$ in Abbildung 5.19 zeigt für die Probe HVAFG eine deutliche Änderung ab einem Reihenentwicklungsgrad von $L_{\max }=6$. Die Funktion bildet ab diesem Reihenentwicklungsgrad das zweite Maximum bei $\alpha=70^{\circ}$ und $\beta=0^{\circ}$ aus. Außerdem treten negative Intensitäten auf, welche physikalisch nicht sinnvoll sind.

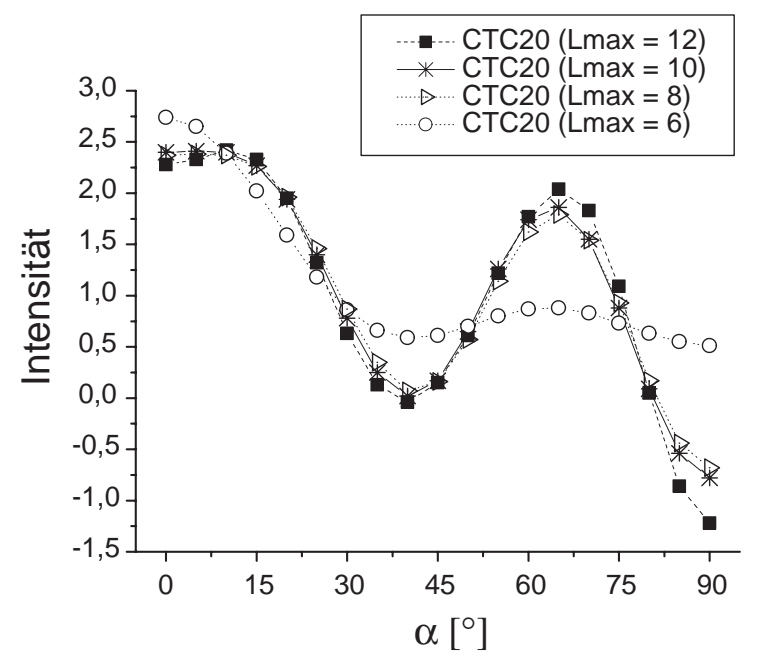

Abbildung 5.20: Polfigurschnitt bei $\beta=0^{\circ}$ für verschiedene $L_{\max }$ von CTC20 in der Durchflussküvette

Auch die Probe CTC20 (Abb. 5.20) weist bei einem Reihenentwicklungsgrad $L_{\max }>6$ ein Maximum bei $\alpha=70^{\circ}$ und $\beta=0^{\circ}$ auf. Dieses Verhalten ist ein guter Hinweis darauf, dass es sich bei dem zweiten Maximum $\left(\alpha=70^{\circ}\right)$ nicht um eine real vorhandene Vorzugsorientierung handelt, sondern ein aufgrund von Abbruchfehlern bei der Reihenentwicklung entstandenes Maximum ist. Bis zu einem $L_{\max }=6$ treten keine negativen Intensitäten auf.

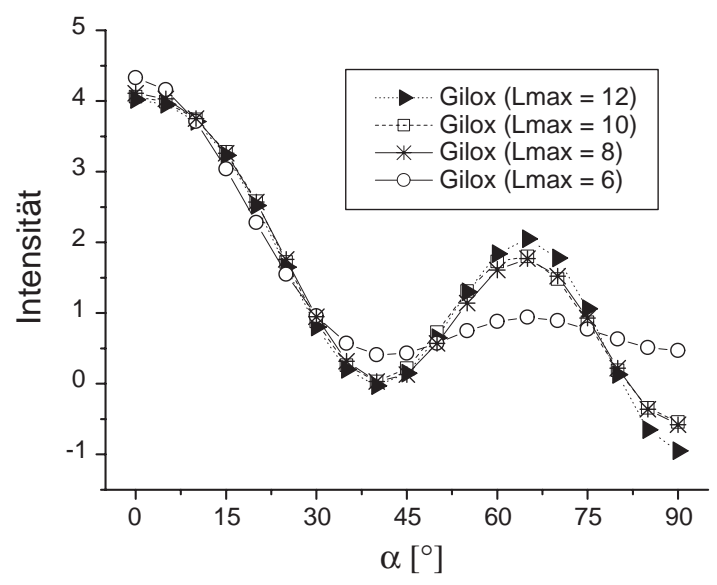

Abbildung 5.21: Polfigurschnitt bei $\beta=0^{\circ}$ für verschiedene $L_{\max }$ von Gilox in der Durchflussküvette

Das Verhalten der oben beschriebenen Proben (HVAFG u. CTC20) ist auch 
bei der Probe Gilox im Polfigurschnitt in Abbildung $5.21 \mathrm{zu}$ beobachten. Ab einem Reihenentwicklungsgrad von $L_{\max }>6$ tritt wieder ein weiteres Maximum auf, sowie negative Intensitäten.

Für alle weiteren Auswertungen wird die Reihenentwicklung bei $L_{\max }=6$ abgebrochen. Das zweite Maximum tritt bei allen drei Proben nur zusammen mit negativen Intensitäten auf. Es ist darum sehr wahrscheinlich, dass es sich hierbei um Abbruchfehler aus der Reihenentwicklung handelt und nicht um eine tatsächlich vorhandene Orientierung.

\subsubsection{Vergleich der rückgerechneten Polfiguren mit den Gemes- senen}

Eine abschließende Überprüfung der Ergebnisse aus Kapitel 5.2.1 und 5.2.2 zur Berechnung der ODF ist der Vergleich der rückgerechneten Polfiguren mit den gemessenen Polfiguren. Beschreiben die rückgerechneten Polfiguren die gemessene Textur, sollten jeweils die Polfiguren für die gleiche Netzebene übereinstimmen.

Da in einigen gemessenen Polfiguren kaum Vorzugsorientierungen, und somit Intensitätsunterschiede, zu erkennen sind, eignen sich diese nicht für einen Vergleich. Die (116)- und die (110)-Polfiguren zeigen je nach Ausprägung der Textur deutliche Maxima. Diese sollten in beiden Polfiguren zu finden sein. AuBerdem sollten durch die Berechnung der ODF keine weiteren Vorzugsorientierungen hinzugefügt werden. In den folgenden Abbildungen sind die Positionen der Vorzugsorientierungen durch Graustufen gekennzeichnet. Die Intensitäten in den gezeigten Polfiguren sind nicht normiert.

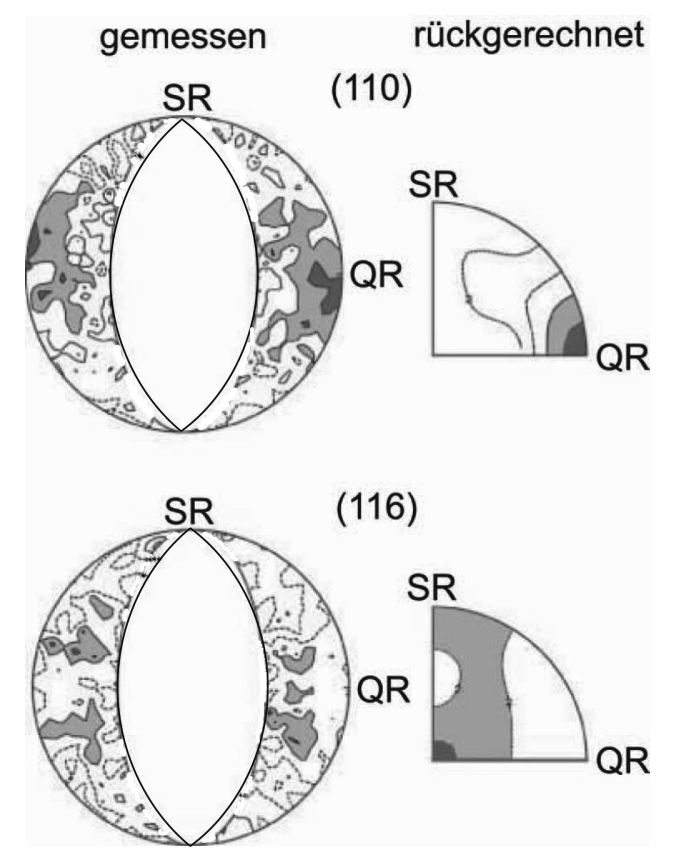

Abbildung 5.22: Gemessene und rückgerechnete Polfiguren der Probe B (36Vol\%) (Rotationsküvette $0,25 \mathrm{U} / \mathrm{min}$ 
In Abbildung 5.22 ist für die Proben $\mathrm{B}$ exemplarisch die (116)- und die (110)-Polfiguren als rückgerechnete und als gemessene Polfigur gegenübergestellt. Alle weiteren Polfiguren befinden sich im Anhang (B). Die gemessene (110)-Polfigur in Abbildung 5.22 zeigt eine deutliche Übereinstimmung mit der rückgerechneten Polfigur. Die maximale Intensität ist hauptsächlich am Rand der Polfigur zu finden. Das Maximum senkrecht zur Scherrichtung (SR) am Rand der Polfigur ist in beiden Polfiguren zu sehen. Die Position des Maximums ist in der gemessenen Polfigur jedoch leicht von der Querrichtung verschoben. Da die berechnete Textur jedoch mit ca. 2-fach regellos sehr schwach ist, liegen diese Abweichungen im Rahmen der Messgenauigkeit.

Die (116)-Polfiguren (Abb. 5.22) weisen noch schwächere Intensitätsunterschiede auf, als bei der (110)-Polfigur. Außerdem befindet sich bei der rückgerechneten Polfigur die maximale Intensität im Zentrum. Dieser Bereich ist aufgrund des eingeschränkten Durchstrahlwinkels (siehe Kap. 4) nicht messbar. Ein leichter Anstieg der Intensität in Richtung des Polfigurzentrums ist jedoch beim Vergleich von gemessener und rückgerechneter (116)-Polfigur zu erkennen.

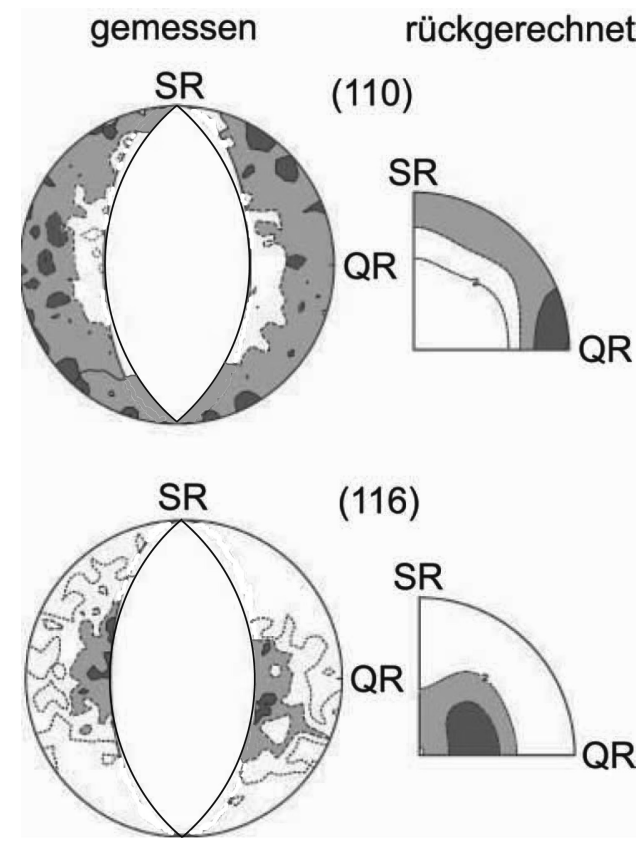

Abbildung 5.23: Gemessene und rückgerechnete Polfiguren von Gilox (13Vol\%) der Rotationsküvette bei $1 \mathrm{U} / \mathrm{min}$

Auch für die kommerziellen Proben sind die Polfiguren der Probe Gilox in der Rotationsküvette exemplarisch in Abbildung 5.23 dargestellt (weitere Ergebnisse befinden sich im Anhang C). Aufgrund einer Umbaumassnahme an der Rotationsküvette ist der Durchstrahlwinkel von $50^{\circ}$ auf $56^{\circ}$ erweitert worden. So ergibt sich ein größerer Bereich der mit den rückgerechneten Polfiguren verglichen werden kann. Dies ist besonders für die (116)-Polfigur interessant.

Die gemessenen Texturen sind für diese Substanzen etwas stärker ausge- 
prägt. Die Gegenüberstellung von gemessenen und rückgerechneten (110)-Polfiguren zeigt im Gegensatz zur Probe B in Abbildung 5.22 einen Unterschied. Die rückgerechnete Polfigur weist auch hier wieder ein Maximum am Rand der Polfigur senkrecht zur Scherrichtung auf. Dies befindet sich jedoch in der gemessenen (110)-Polfigur gleichmäßig über den gesamten Rand verteilt wieder.

Andererseits sind die Übereinstimmungen in der (116)-Polfigur deutlich besser. Der größere gemessene Bereich zeigt deutlich, dass das Maximum in der rückgerechneten Polfigur auch in der gemessenen Polfigur wieder zu finden ist. Dies ließ sich bei der Probe B (Abb. 5.22) nur erahnen.

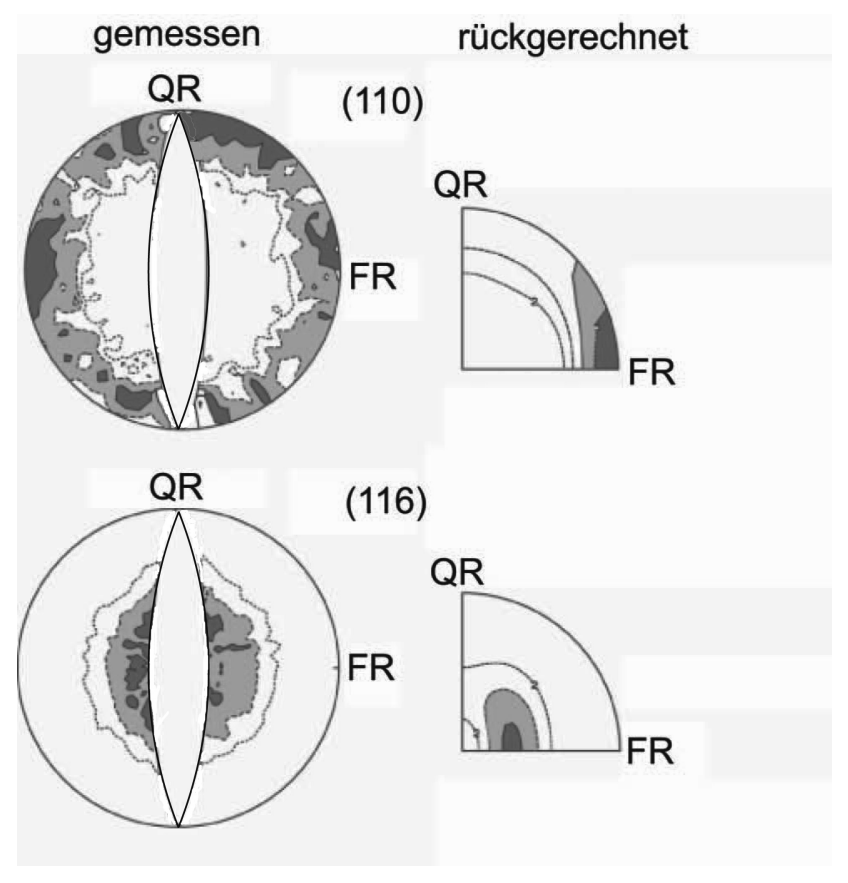

Abbildung 5.24: Gemessene und rückgerechnete Polfiguren von Gilox (10Vol\%) der Durchflussküvette bei $1 \mathrm{ml} / \mathrm{s}$

Im Falle der Durchflussküvette ist der gemessene Bereich mit $\omega=70^{\circ}$ am größten. Außerdem ist aufgrund der längeren Messzeiten eine bessere Zählstatistik vorhanden. In Abbildung 5.24 sind auch hier wieder exemplarisch die Polfiguren der Probe Gilox in der Durchflussküvette dargestellt. Alle weiteren Polfiguren sind im Anhang D zu finden.

In der gemessenen und der normierten (110)-Polfigur in Abbildung 5.24 sind in Scherrichtung und senkrecht dazu Maxima vorhanden. Von diesen Maxima ist in der rückgerechneten Polfigur nur das Maximum senkrecht zu SR vorhanden. In beiden (110)-Polfiguren ist die insgesamt hohe Intensität in den Randbereichen zu sehen.

Die (116)-Polfiguren zeigen, wie schon in Abbildung 5.23 gute Übereinstimmung. Dies gilt vor allem für den Bereich, der mit der Rotationsküvette noch nicht messbar ist. Die Auswirkungen der längeren Messzeiten und des größeren Durchstrahlwinkels sind bei diesen Vergleichen deutlich zu beobachten.

Für die (116)-Polfiguren gilt, dass die gemessenen Intensitäten bei allen Pro- 
ben mit den Rückgerechneten gut übereinstimmen. Alle rückgerechneten (110)Polfiguren weisen hohe Intensitäten mit einem lokalen Maximum in Querrichtung in den Randbereichen auf, welche bei der Probe B auch in der gemessenen Polfigur auftreten. Unterschiede zwischen den gemessenen und rückgerechneten Polfiguren bei lokalen Maxima lassen sich auf den nicht messbaren linsenförmigen Bereich in der Mitte aller Polfiguren zurückführen. Dies wird durch eine $\mathrm{Al}_{2} \mathrm{O}_{3}$-Probe bekannter Textur bestätigt, welche unter anderen Bedingungen gemessen wurde.

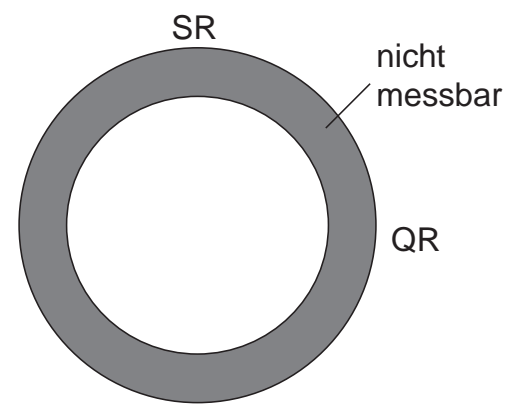

Abbildung 5.25: Schematische Darstellung des nicht messbaren Bereichs nach dem Durchstrahlverfahren

Die Probe H1 mit ausgeprägter Fasertextur der Basisebene wurde mit konventioneller Röntgenstrahlung (Röntgenröhre) nach dem Reflexionsverfahren nach Schulz [52] gemessen. Bei diesem Verfahren befindet sich der nicht messbare Bereich am Rand der Polfigur, wie in Abbildung 5.25 dargestellt.

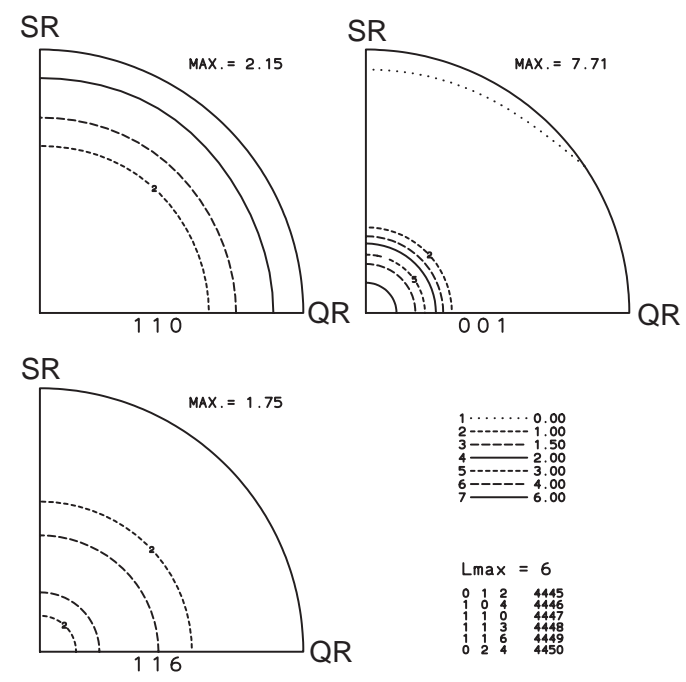

Abbildung 5.26: Rückgerechnete Polfiguren der Proben H1 aus konventionell röntgenographisch ermittelten Daten nach dem Reflexionsverfahren

Anhand der rückgerechneten Polfiguren in Abbildung 5.26 lässt sich so der Einfluss des nicht messbaren Bereichs erkennen. Allerdings ist die konventionell gemessene Textur aufgrund der geringeren Eindringtiefe der Röntgenstrahlung 
nur lokal an der Oberfläche bestimmt worden. Die rückgerechneten Polfiguren aus gemessenen Daten nach dem Reflexionsverfahren zeigen eine Fasertextur ohne eine Vorzugsorientierung in der (110)-Polfigur.

Zum Vergleich wurde die Probe H1 unter den gleichen Bedingungen, wie bei den Zwei-Phasen-Gemischen in der Rotationsküvette gemessen und die Orientierungsverteilungsfunktion gerechnet. Mit der Hochenergetischen Synchrotronstrahlung wird jedoch im Durchstrahlverfahren die globale Textur gemessen. Es ist in den rückgerechneten Polfiguren in Abbildung 5.27 eine Vorzugsorientierung senkrecht zu SR zu beobachten. Beobachtete Unterschiede zwischen den beiden Messungen können auch in den rückgerechneten Polfiguren aufgrund der unterschiedlichen Messmethode entstehen.
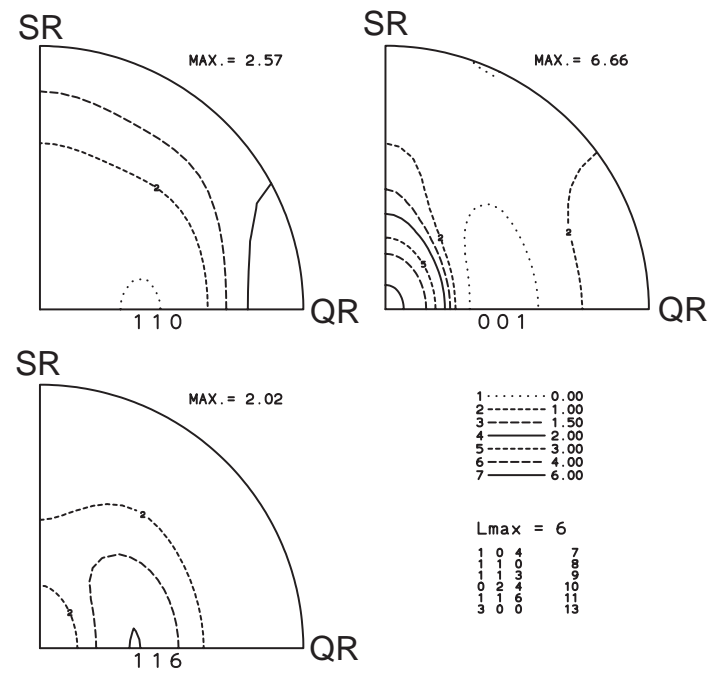

Abbildung 5.27: Rückgerechnete Polfiguren der Proben H1 aus 6 gemessen Polfiguren in der Rotationsküvette

Zur Berechnung der ODF wurden die unter den vorliegenden Bedingungen messbaren Polfiguren (104), (110), (113), (024), (116) und (300) verwendet. In der Polfiguren (104),(113) und (116) lässt sich ein großer Teil des Bereichs mit maximaler Intensität nicht messen. Aufgrund der Fasertextur nach der Basisebene (001) ist in diesen drei Polfiguren rotationssymmetrisch um das Polfigurzentrum die maximale Intensität zu beobachten. Dieser Teil wird durch den nicht messbaren linsenförmigen Bereich zerschnitten und führt so in der Berechnung der ODF zu einer Vorzugsorientierung.

Da zur Berechnung der ODF mit einem Reihenentwicklungsgrad von $L_{\max }=$ 6 nur 3 vollständige Polfiguren notwendig sind, kann diese auch mit den 4 verfügbaren unvollständigen Polfiguren (110),(024),(214) und (300) aus der Röntgentexturmessung berechnet werden. Diese Variation führt zu einer deutlich stärker ausgeprägten Fasertextur mit maximalen Intensitäten, welche den Ergebnissen mit dem Reflexionsverfahren schon sehr ähnlich sind.

In der (110)-Polfigur in Abbildung 5.28 ist die Vorzugsorientierung deutlich schwächer ausgeprägt als in Abbildung 5.27. Da durch diese Kombination von Polfiguren einige Orientierungsbereiche nicht abgedeckt, kommt es im Randbe- 

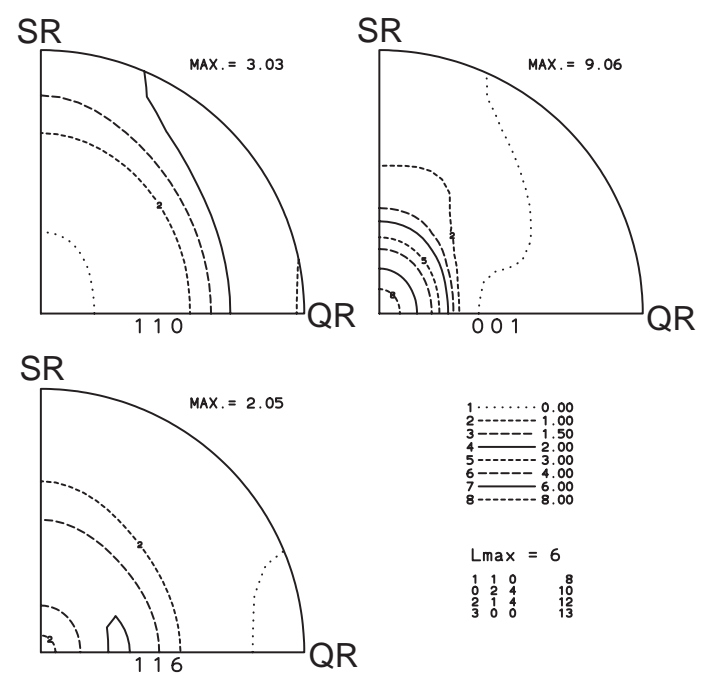

$\begin{array}{rrrr}1 & 1 & 0 \\ 0 & 2 & 4 \\ 2 & 1 & 4 \\ 3 & 0 & 4 & 10 \\ 3 & 13\end{array}$

Abbildung 5.28: Rückgerechnete Polfiguren der Proben H1 aus 4 gemessen Polfiguren in der Rotationsküvette

reich der rückgerechneten (001)-Polfigur zu hohen negativen Intensitäten. Ein Vergleich der negativen Intensitäten in den (001)-Polfiguren in Abbildung 5.29 zeigt, dass mit den 4 Polfiguren zwar eine stärker ausgeprägte Fasertextur berechnet wird, der Fehler aber deutlich höher ist. Somit ist diese Kombination von Polfiguren zur Berechnung der ODF nicht geeignet. Der geringere Fehler bei der Berechnung der ODF mit 6 Polfiguren macht die einzelnen Ergebnisse besser miteinander vergleichbar und führt nur zu sehr geringen negativen Intensitäten.

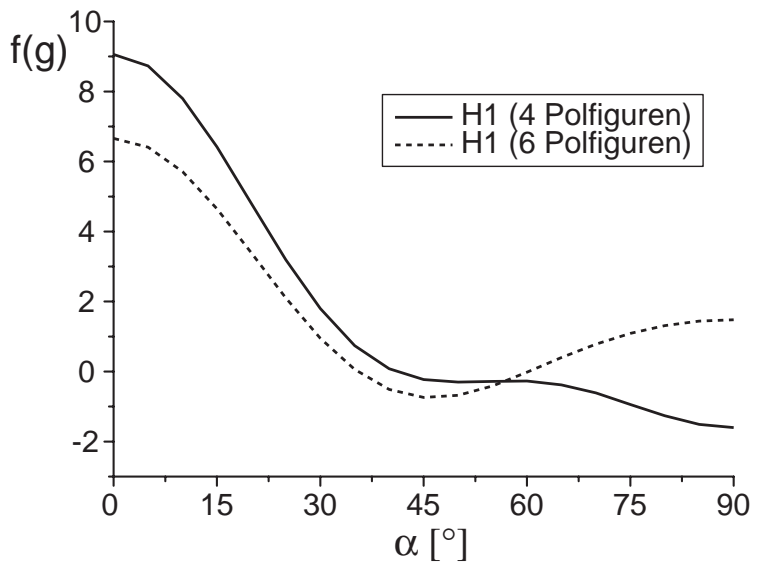

Abbildung 5.29: Horizontaler (001)-Polfigurschnitt in Querrichtung der Probe H1 in der Rotationsküvette

Da es sich gezeigt hat, dass die Vorzugsorientierung in der rückgerechneten (110)-Polfigur durch die ODF-Rechnung entsteht und nicht in den gemessen Polfiguren zu finden ist, wird diese im weiteren nicht berücksichtigt. Die Vorzugsorientierung der Basisebene lässt sich jedoch mit dieser Methode sehr gut 
beschreiben.

Sowohl die schwachen Texturen, als auch die Messbedingungen, würden bei höheren Reihenentwicklungsgraden dazu führen, dass noch weitere Orientierungen beschrieben werden, welche sich nur aufgrund der ODF-Rechnung ergeben. Dies ist ebenfalls ein Grund für den geringen Reihenentwicklungsgrad.

Die bessere Zählstatistik bei den Experimenten mit der Durchflussküvette, sowie der höhere Durchstrahlwinkel zeigen sich besonders anhand der (116)Polfiguren. Diese stimmen sehr gut mit den rückgerechneten Ergebnissen überein. Mit den, in diesem Kapitel, sowie in den Kapiteln 5.2.1 und 5.2.2 erhaltenen Ergebnissen lassen sich die Parameter für die ODF-Rechnung wie folgt zusammenfassen:

- die Probensymmetrie kann orthorhombisch berechnet werden

- der beste Reihenentwicklungsgrad ist $L_{\max }=6$

- zur ODF-Rechnung werden die Polfiguren (104), (110), (113), (024), (116) und (300) herangezogen 


\subsubsection{Die Proben A,B und C in der Rotationsküvette}

Für erste Messungen standen verschiedene $\mathrm{Al}_{2} \mathrm{O}_{3}$-Pulver in geringen Mengen aus einer früheren Arbeit von Angelika Böcker [15] zur Verfügung. Diese wurden in ersten Experimenten in der Rotationsküvette untersucht. Nach einer Messung ließen sich die Pulver nicht ohne sehr großen Aufwand wiedergewinnen, so dass nur einige Messungen möglich waren. Die Experimente mit diesen Substanzen sollen es erstmals ermöglichen die Orientierungsverteilungsfunktion zu berechnen. Mit diesen neu gewonnenen Daten kann der Einfluss der Strömungsparameter auf die Texturschärfe ermittelt werden, welche anschließend mit anderen Substanzen systematisch untersucht werden. Die untersuchten Parameter sind:

- Einfluss der Partikelform

- Einfluss der Strömungsgeschwindigkeit

- Einfluss der Feststoffkonzentration

In Tabelle 5.5 sind die Parameter für die Rotationsküvette der jeweiligen Polfigurmessungen nach Proben aufgeführt.

\begin{tabular}{c|c|c|c|c} 
Probe & $\begin{array}{c}\text { Konzentration } \\
{[\text { Vol\% }]}\end{array}$ & $\begin{array}{c}\text { Drehwinkel } \omega \\
{\left[{ }^{\circ}\right]}\end{array}$ & $\begin{array}{c}\text { Strahlquerschnitt } \\
{\left[\mathrm{mm}^{2}\right]}\end{array}$ & $\begin{array}{c}\text { Belichtungszeit } \\
{[\mathrm{sec}]}\end{array}$ \\
\hline A & 36 & $0-50$ & $2 * 2$ & 20 \\
B1 & 36 & $0-50$ & $2 * 2$ & 20 \\
B2 & 24 & $0-50$ & $2 * 2$ & 40 \\
C & 28 & $0-50$ & $2 * 2$ & 30
\end{tabular}

Tabelle 5.5: Messparameter für die Rotationsküvette

Die längeren Messzeiten von den Messungen B2 und C sind durch eine Umbaumassnahme an der Rotationsküvette möglich geworden. Der Einsatz der neu entwickelten Dichtung (siehe Kap. 4.1) führt zu stabileren Bedingungen während der Messung.

\subsubsection{Partikelform}

Die untersuchten Partikel zeigen eine mehr oder weniger ausgeprägte Plättchenform (siehe Kap. 3.2.1). Da die Basisebene der Plättchen der kristallographischen (001)-Ebene entspricht, lässt sich die Abhängigkeit der Textur von der Partikelform an der (001)-Polfigur am deutlichsten beobachten. Aus den gemessenen Polfiguren wurden die (001)-Polfiguren mit den in Kapitel 5.2.1 und 5.2.2 ermittelten Parametern berechnet.

In Abbildung 5.30 sind für die Proben A,B und C die (001)-Polfiguren dargestellt. Alle drei Proben sind bei gleichen Strömungsgeschwindigkeiten $(1 \mathrm{U} / \mathrm{min})$ gemessen worden.

Anhand der (001)-Polfiguren ist die Ausrichtung der Basisebene der Plättchen in der Strömung zu beobachten. Der Zusammenhang zwischen Basisebene und (001)-Ebene ist in Kapitel 3.2 beschrieben. In allen Polfiguren ist die 

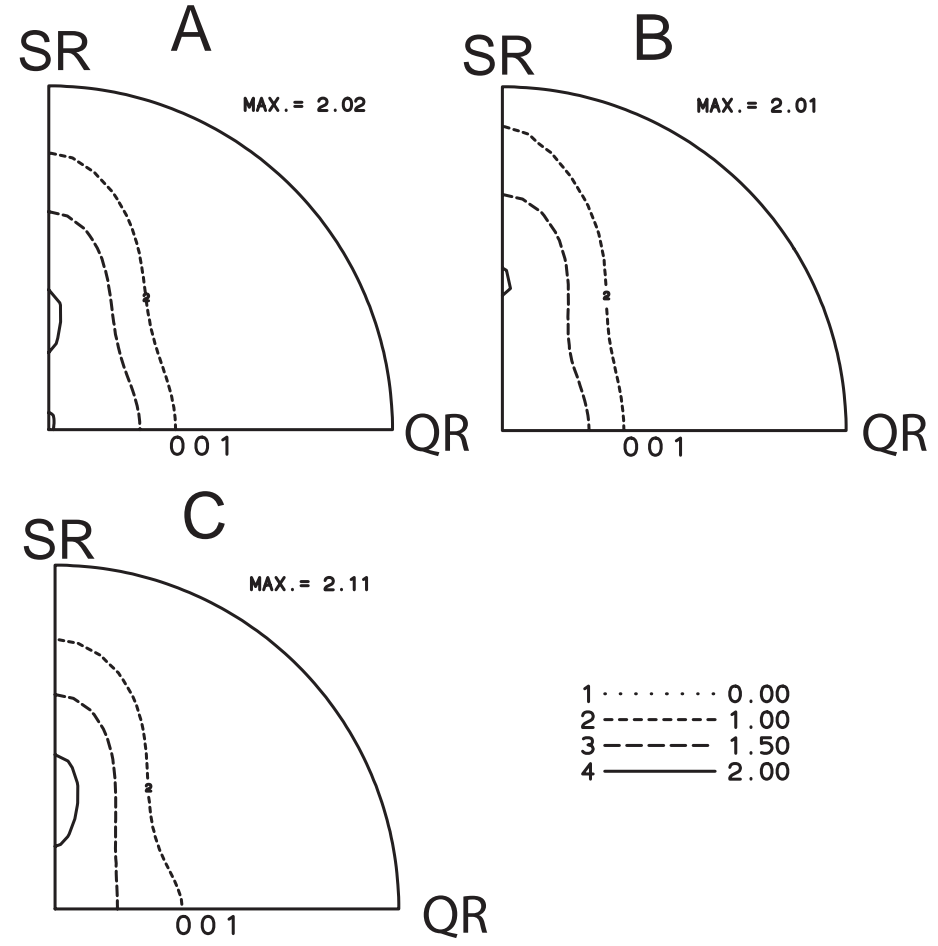

Abbildung 5.30: (001)-Polfiguren der Proben A,B,C bei $1 \mathrm{U} / \mathrm{min}$

Vorzugsorientierung in der Mitte der Polfigur zu sehen. Dies entspricht einer Ausrichtung der Basisebene parallel zur Scherrichtung (die Scherrichtung (SR) ist auch die Strömungsrichtung des Gemisches). In der Strömung schwanken die Plättchen, wie von Anczurowski et al. [7] beschrieben um die stabile Lage parallel zur Strömungsrichtung. Die stabile Lage ist durch den Gradienten der Strömungsgeschwindigkeit bestimmt. Ein Teilchen richtet sich immer so in der Strömung aus, dass es einen möglichst geringen Strömungsgradienten erfährt [6].

An den Höhenlinien in den (001)-Polfiguren ist zu beobachten, dass die Auslenkung der Basisebene in Strömungsrichtung deutlich stärker ist, als senkrecht zur Strömungsrichtung. Dies stimmt mit den Berechnungen von Jeffery [6] und den Experimenten Anczurowski et al. [7, 39] nicht überein. Diese Auslenkung wird durch die nicht gemessenen Bereiche der verwendeten Polfiguren erzeugt. Das in Kapitel 5.2.3 beschriebene Maximum in Querrichtung in der rückgerechneten (110)-Polfigur führt in der (001)-Polfigur zu dieser höheren Intensität in Scherrichtung.

Die unterschiedlichen Kornformen der $\mathrm{Al}_{2} \mathrm{O}_{3}$-Pulver A,B und $\mathrm{C}$ (siehe Kap. 3.2.1.1) führen nur zu sehr geringen Unterschieden in den (001)-Polfiguren. Sowohl die Auslenkung der Basisebene aus der Ideallage, als auch die maximale Intensität in der Polfigurmitte sind für alle Polfiguren nahezu gleich. Als geringer Unterschied bildet sich in Scherrichtung ein Maximum aus, welches mit steigendem plättchenförmigen Charakter stärker wird (siehe maximale Intensität in Abbildung 5.30). 


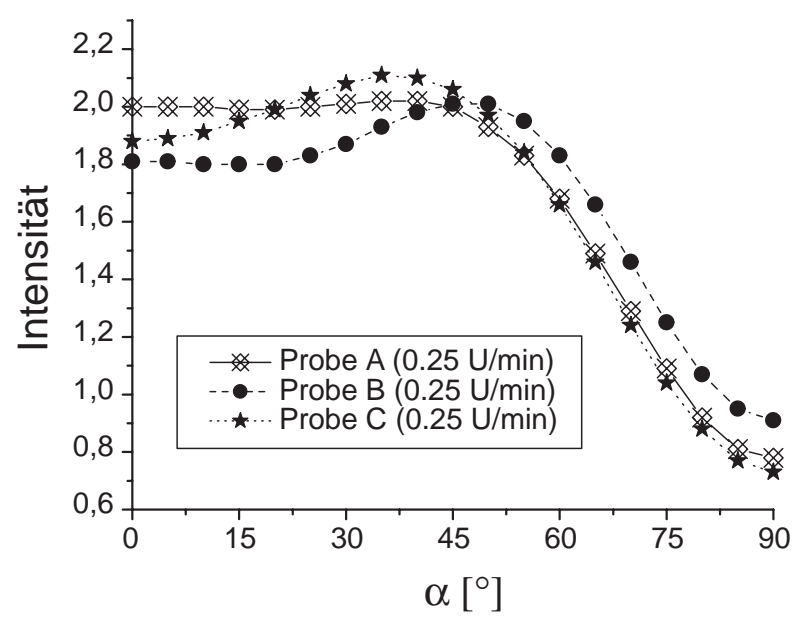

Abbildung 5.31: (001)-Polfigurschnitt in Scherrichtung der Proben A,B,C bei $0,25 \mathrm{U} / \mathrm{min}$

Die Darstellung eines Schnitts durch die Polfigur von Zentrum ausgehend in Scherrichtung (von $\{0,90\}$ bis $\{90,90\}$ ) lässt diese Abhängigkeit deutlicher werden. In Abbildung 5.31 ist dieser Schnitt für die Proben A, B und C dargestellt. Die Polfiguren der entsprechenden Polfigurschnitte der Proben A, B und C befinden sich im Anhang B.

Hier ist noch mal deutlich zu sehen, dass für alle drei Substanzen die Auslenkung von der Ideallage im Zentrum der Polfigur gleich ist. Die Basisebene verkippt bis zu $50^{\circ}$ in Scherrichtung. Zwischen der maximalen Verkippung und der Ideallage gibt es nur geringe Intensitätsschwankungen. Da aber die drei Substanzen unterschiedliche Kornformen aufweisen, sollte sich ein Unterschied beobachten lassen. Dies ist ein weiteres Indiz dafür, dass die breite Ausdehnung des Maximums in Scherrichtung auf die nicht messbaren Bereiche in den Polfiguren zurückzuführen ist. Insgesamt sind in diesem Polfigurschnitt nur geringe Unterschiede zwischen den einzelnen Kornformen zu beobachten.

Eine weitere interessante Möglichkeit die einzelnen Substanzen miteinander zu vergleichen bietet auch ein Polfigurschnitt senkrecht zur Scherrichtung (SR). Die Ergebnisse sind in Abbildung 5.32 zu sehen. Die geringen Unterschiede der einzelnen Proben werden in diese Richtung deutlicher. Die Probe C, welche aus plättchenförmigen und globularen Körnern besteht zeigt hier ein anderes Verhalten, als die Proben A und B. Von der maximalen Intensität bei $0^{\circ}$ ausgehend fällt sie bei der Probe $\mathrm{C}$ bis $90^{\circ}$ gleichmäßig ab. Diese Orientierungsverteilung lässt sich durch die Mischung von plättchenförmigen und globularen Körnern erklären. Beide Kornformen beeinflussen sich gegenseitig so, dass alle Orientierungen vorkommen. Da die Plättchen aber größer sind, haben sie auch ein größeres Beugungsvolumen und bilden so ein Maximum aus.

Die beiden anderen Proben bilden ein schärferes Maximum bei $0^{\circ}$ aus, welches ab $20^{\circ}$ stark abnimmt. Die globularen Körner der Probe B, welche dazu neigen, sich zu größeren Agglomeraten zusammenzuschließen, können dadurch eine ähnliche Strömungstextur ausbilden, wie die plättchenförmige Probe A. 


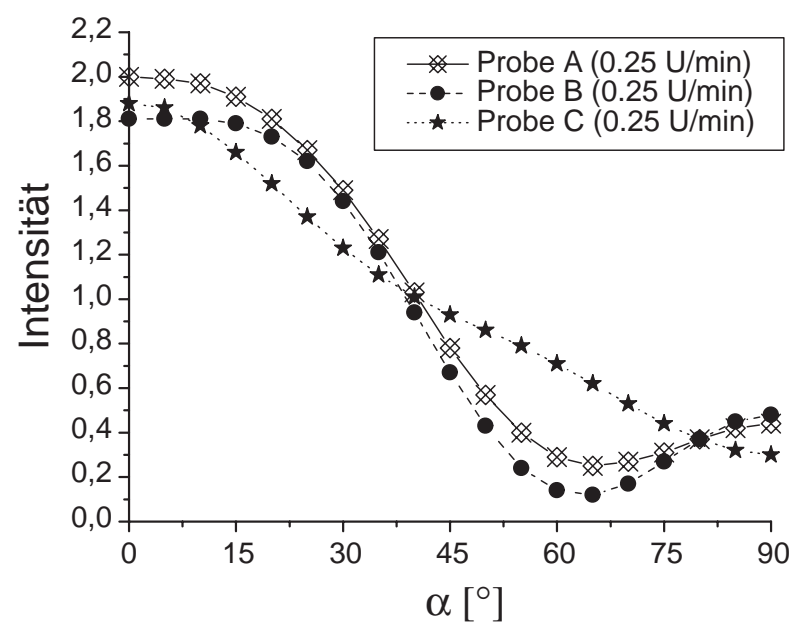

Abbildung 5.32: (001)-Polfigurschnitt senkrecht zur Scherrichtung der Proben $\mathrm{A}, \mathrm{B}, \mathrm{C}$ bei $0,25 \mathrm{U} / \mathrm{min}$

Das in Kapitel 3.2.1.1 beschriebene Radienverhältnis $\left(A\left(r_{e}\right)=3\right.$ und $\left.C\left(r_{e}\right)=6\right)$ hat für diese Konzentration an Feststoff nur einen sehr geringen Einfluss auf die Textur. Auch die Probe B mit einem Radienverhältnis von $B\left(r_{e}\right)=1$ weist hier kaum Unterschiede in der Textur auf, obwohl hier das Maximum am schwächsten ist.
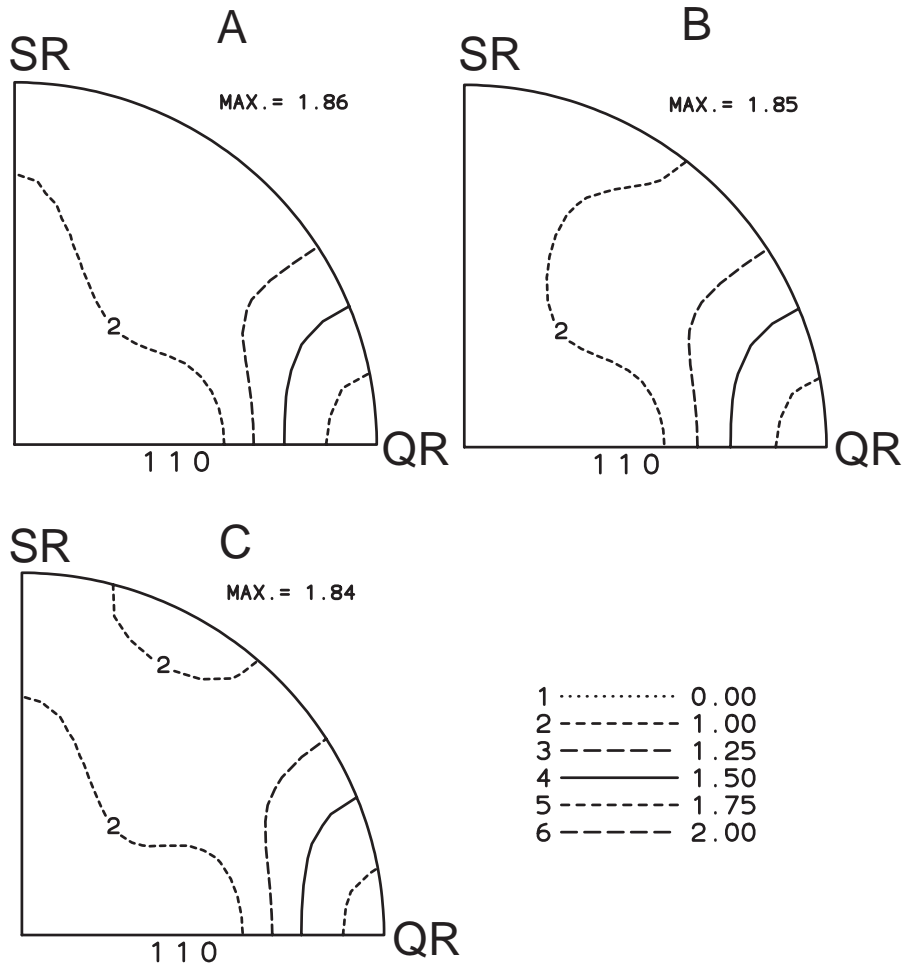

Abbildung 5.33: (110)-Polfiguren der Proben A,B,C bei 1U/min 
Neben der Vorzugsorientierung der Basisebene tritt hier, wie schon in Kapitel 5.2.3, eine weitere auf. Diese wird in der (110)-Polfigur in Abbildung 5.33 am deutlichsten. Die (110)-Polfiguren weisen neben den Polfiguren der Basisebene (001) die höchsten Intensitäten auf. Bei allen drei Proben ist das Maximum in der (110)-Polfigur senkrecht zur Scherrichtung SR. Trotz großer Unterschiede in den Kornformen sind in diesen Polfiguren kaum Unterschiede zu beobachten. Lediglich der Verlauf der Höhenlinien weist geringe Unterschiede auf, welche sich jedoch nicht durch die Kornform erklären lassen. Die Maximale Intensität ist bei allen drei Proben identisch.

\subsubsection{Strömungsgeschwindigkeit}

Der Einfluss der Strömungsgeschwindigkeit auf die Textur ist eine weitere interessante Fragestellung. In Abbildung 5.34 ist in einem Polfigurschnitt in Scherrichtung die Intensität für verschiedene Strömungsgeschwindigkeiten der Probe A dargestellt. Die maximale Intensität ist für alle drei Strömungsgeschwindigkeiten gleich. Sie ist jedoch nicht bei $0^{\circ} \mathrm{zu}$ beobachten, welche einer Ausrichtung der Plättchen parallel zur Strömung entspricht, sonder bei ca. $45^{\circ}$ in Scherrichtung.

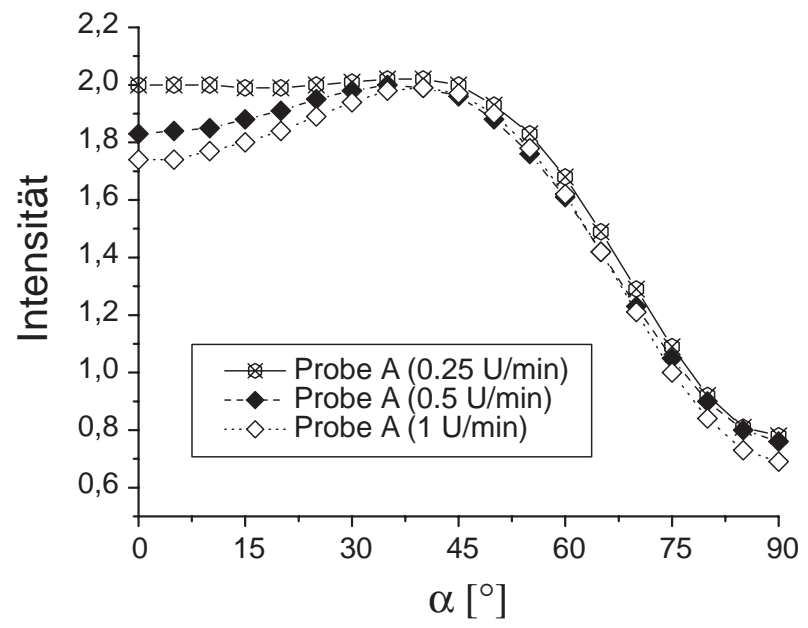

Abbildung 5.34: (001)-Polfigurschnitt in Scherrichtung der Proben A für unterschiedliche Strömungsgeschwindigkeiten

Die Orientierungsdichte bei $0^{\circ}$ weist jedoch unterschiedliche Intensitäten in Abhängigkeit von der Strömungsgeschwindigkeit auf. Die Intensitäten sind für alle Messungen der Proben A, B und C in Tabelle 5.6 zusammengestellt.

Die beiden mit * dargestellten Intensitäten beruhen auf Messungen bei denen die Rotationsküvette aufgrund von mechanischen Problemen nicht gleichmäßig rotiert. Eine Abhängigkeit der Orientierungsdichte für die (001)-Ebene bei $\alpha=0^{\circ}$ und $\beta=0^{\circ}$ mit der Strömungsgeschwindigkeit lässt sich nicht eindeutig erkennen. Ausgenommen der bei mit * gekennzeichneten Werte, ist für die Proben A und B ein Trend zu erkennen. Die Orientierungsdichte nimmt mit steigender Strömungsgeschwindigkeit ab. Für die Probe $\mathrm{C}$ gilt dies nicht. Da 


\begin{tabular}{c||c|c|c|c}
$\mathrm{U} / \min$ & $\mathrm{A}$ & $\mathrm{B}_{1}$ & $\mathrm{~B}_{2}$ & $\mathrm{C}$ \\
\hline 0,25 & 2 & 1,81 & 1,91 & 1,88 \\
0,5 & 1,83 & $1,4^{*}$ & 1,88 & $0,98^{*}$ \\
1 & 1,74 & 1,77 & 1,66 & 1,96
\end{tabular}

Tabelle 5.6: Intensität der Proben A, B und C in der (001)-Polfigur bei $\alpha=0^{\circ}$ und $\beta=0^{\circ}$

die Unterschiede nur sehr gering sind und außerdem nur wenige Messungen zur Verfügung stehen können es nur zufällige Abhängigkeiten sein.

Da für alle Substanzen die maximale Intensität in Scherrichtung bei $\alpha=45^{\circ}$ zu beobachten ist, sind auch diese in Tabelle 5.7 zusammengestellt. ${ }^{1}$ Alle maxi-

\begin{tabular}{c||c|c|c|c}
$\mathrm{U} / \mathrm{min}$ & $\mathrm{A}$ & $\mathrm{B}_{1}$ & $\mathrm{~B}_{2}$ & $\mathrm{C}$ \\
\hline 0,25 & 2,02 & 2,01 & 1,96 & 2,06 \\
0,5 & 1,99 & $2,12^{*}$ & 2,05 & $1,8^{*}$ \\
1 & 1,99 & 1,06 & 2,03 & 1,98
\end{tabular}

Tabelle 5.7: Intensität der Proben A, B und C in der (001)-Polfigur bei $\alpha=45^{\circ}$ und $\beta=0^{\circ}$

malen Intensitäten zeigen hier nahezu den gleichen Wert. Dies gilt auch für die beiden mit * gekennzeichneten Werte. Eine Abhängigkeit mit der Strömungsgeschwindigkeit ist mit den Messungen nicht zu bestimmen. Da aber auch schon bei der Betrachtung der Partikelform nur sehr geringe Unterschiede aufgetreten sind, kann eine Abhängigkeit von der Strömungsgeschwindigkeit unterhalb der Messungenauigkeit liegen.

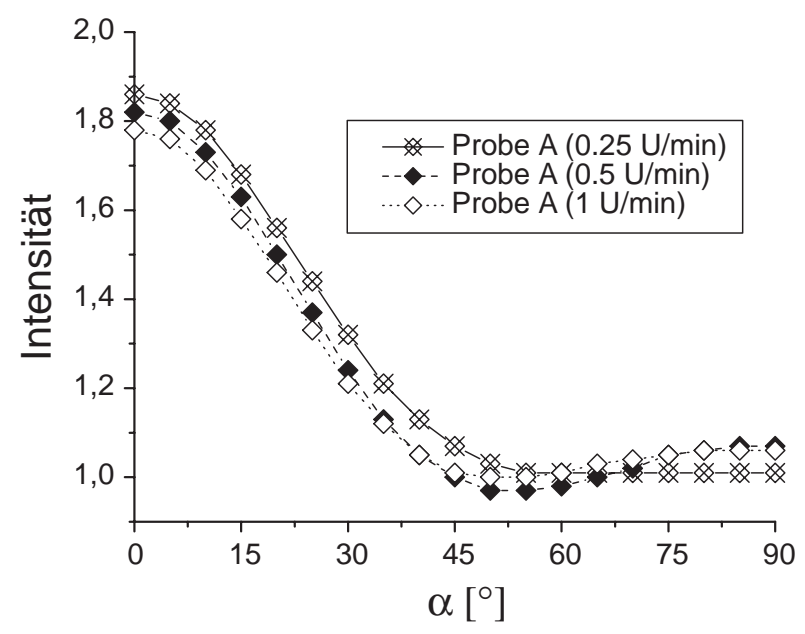

Abbildung 5.35: (110)-Polfigurschnitt bei $\alpha=90^{\circ}$ der Proben A für unterschiedliche Strömungsgeschwindigkeiten

Da die Strömungsabhängigkeit bei den Muskovitmessungen in der Position der Vorzugsorientierung senkrecht zur Basisebene zu beobachten ist, ist auch

\footnotetext{
${ }^{1 *}=$ Störung der Küvettenrotation währen der Messung
} 
für die $\mathrm{Al}_{2} \mathrm{O}_{3}$-Proben eine solche Betrachtung notwendig. Die Vorzugsorientierung in der rückgerechneten (110)-Polfigur ist zwar durch den nicht messbaren Bereich in den originalen Polfiguren entstanden, Unterschiede in der Intensität können jedoch nur durch unterschiedliches Verhalten der Partikel und durch die ODF-Rechnung erzeugt werden. In Abbildung 5.35 ist für die Probe A die Polfigurintensität am Rand der rückgerechneten (110)-Polfigur aufgetragen. Die Probe A mit der ausgeprägtesten Plättchenform zeigt auch hier keine signifikante Abhängigkeit mit der Strömungsgeschwindigkeit. Weder die maximale Intensität bei $\beta=0^{\circ}$ noch die Position des Maximums ändert sich. Unter diesen Strömungsbedingungen treten keine signifikanten Änderungen der Textur mit der Strömungsgeschwindigkeit auf.

\subsubsection{Feststoffkonzentration}

Durch eine Verringerung der Feststoffkonzentration verringert sich die Anzahl der Teilchen-Teilchen Stöße. Dies hat zur Folge, dass die resultierende Textur stärker durch die Orientierung des einzelnen Teilchens in der Strömung bestimmt wird. Um dies zu untersuchen wurde für die Probe B die Feststoffkonzentration von 36 auf 24 Vol\% reduziert. Da in den vorangegangenen Polfigurschnitten senkrecht zur Scherrichtung größere Unterschiede zu beobachten sind, werden auch hier die beiden Konzentrationen anhand des gleichen Schnittes verglichen.

In Abbildung 5.36 ist der Polfigurschnitt der (001)-Polfigur senkrecht zu SR für die Probe B zu sehen. Mit sinkender Feststoffkonzentration nimmt auch die maximale Intensität ab. Die Unterschiede sind jedoch nur sehr gering. Die höher konzentrierte Probe bildet ein Maximum bis ca. $20^{\circ}$ aus. Anschließend fällt die Intensität stark ab.

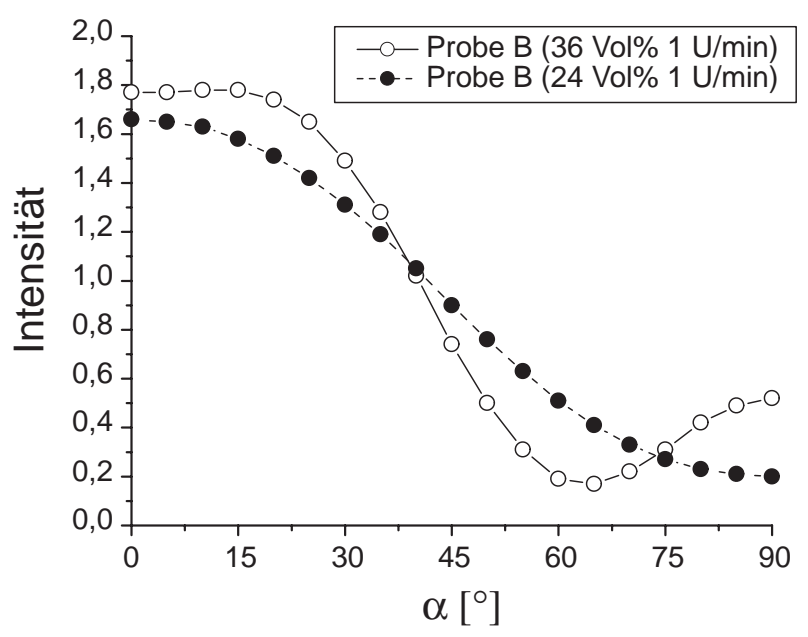

Abbildung 5.36: (001)-Polfigurschnitt senkrecht zu SR der Proben B unterschiedlichen Feststoffkonzentrationen

Bei der geringer konzentrierten Probe nimmt der Verlauf der Intensität über den gesamten Bereich gleichmäßig in Richtung $\alpha=90^{\circ} \mathrm{ab}$. Da für die Bestimmung der Konzentrationsabhängigkeit nur zwei Messreihen der Probe B 
zur Verfügung stehen, lässt sich nur feststellen, dass sich die Textur mit unterschiedlicher Konzentration ändert. Um Abhängigkeiten zu bestimmen sind jedoch weitere Messungen nötig, welche in Kapitel 5.2.5 und 5.2.6 durchgeführt wurden.

\subsubsection{Zusammenfassung}

Erste Messungen mit $\mathrm{Al}_{2} \mathrm{O}_{3}$-Proben zeigen, dass auch diese eine Vorzugsorientierung ausbilden. Die Ausrichtung der Plättchen ist parallel zur Strömungsrichtung und entspricht der Theorie von Jeffery [6] und Anczurowski et al. [7, 39]. Obwohl der plättchenförmige Charakter viel geringer ausgeprägt ist als bei dem Muskovitpulver, entstehen messbare Texturen. Der Einfluss der Kornform und der Feststoffkonzentration ist zu erkennen, lässt sich jedoch mit der geringen Anzahl von Messungen nicht näher beschreiben. Im Gegensatz zu den Messungen mit Muskovitpartikeln ist eine zweite Vorzugsorientierung um die Basisebene nicht erkennbar. Eine Abhängigkeit mit der Strömungsgeschwindigkeit lässt sich nicht beobachten. Die Ergebnisse sind dank der hexagonalen Kristallsymmetrie und der Möglichkeit normierte Polfiguren aus der ODF rückzurechnen besser untereinander vergleichbar. Die Texturschärfe unterschiedlicher Messungen kann für dieses System direkt gegenüber gestellt werden. Dies geht über den reinen Vergleich von Orientierungen beim Muskovit hinaus. Weitere Untersuchung an kommerziellen Proben der Firma Alcoa werden im nächsten Kapitel anhand von weitreichenderen Messreihen die bisherigen Ergebnisse überprüfen. 


\subsubsection{Die Proben HVAFG, CTC20 und GILOX in der Rotati- onsküvette}

Aufgrund der bisherigen Ergebnisse mit den ersten $\mathrm{Al}_{2} \mathrm{O}_{3}$-Proben, welche zu sehr schwachen Texturen führten, wurde im weiteren die Feststoffkonzentration herabgesetzt. Der Einfluss der Teilchen-Teilchen Stöße nimmt bei geringeren Feststoffkonzentrationen ab. Dadurch haben die Teilchen im Mittel mehr Zeit sich im Strömungsgradienten einzuregeln, ohne dass dies durch Stöße gestört wird. Dies entspricht eher der Situation eines Teilchens in der Strömung wie von Jeffery beschrieben (siehe Kap. 1.3.2).

Aufgrund einer weiteren Umbaumassnahme an der Rotationsküvette (siehe Kap. 4.1 Abb. 4.4) ist nun ein maximaler Durchstrahlwinkel von $58^{\circ}$ möglich. Dies hat den Vorteil, dass für die Berechnung der Orientierungsverteilungsfunktion mehr gemessene Daten zur Verfügung stehen.

Ziel dieser Versuchsreihe ist es, die bisherigen Ergebnisse zu bestätigen und die noch unsicheren Trends zu vertiefen. Im Einzelnen geht es hierbei um:

- Überprüfung der Partikelformabhängigkeit der Textur

- Überprüfung der Texturabhängigkeit von der Strömungsgeschwindigkeit

- Erweiterung der Messreihe zu unterschiedlichen Feststoffkonzentrationen

Zu diesem Zweck wurden Messreihen mit unterschiedlichen Substanzen bei Rotationsgeschwindigkeiten von $0,5 \mathrm{U} / \mathrm{min}, 1 \mathrm{U} / \mathrm{min}$ und $2 \mathrm{U} / \mathrm{min}$ durchgeführt. Die Proben HVAFG und CTC20 wurden mit unterschiedlichen Konzentrationen gemessen. Die einzelnen Messparameter sind in Tabelle 5.8 zusammengefasst.

\begin{tabular}{c|c|c|c|c} 
Probe & $\begin{array}{c}\text { Konzentration } \\
{[\text { Vol\% }]}\end{array}$ & $\begin{array}{c}\text { Drehwinkel } \omega \\
{\left[{ }^{\circ}\right]}\end{array}$ & $\begin{array}{c}\text { Strahlquerschnitt } \\
{\left[\mathrm{mm}^{2}\right]}\end{array}$ & $\begin{array}{c}\text { Belichtungszeit } \\
{[\mathrm{sec}]}\end{array}$ \\
\hline \multirow{3}{*}{ HVAFG } & 26 & $0-58$ & $1 * 1$ & 20 \\
& 13 & $0-58$ & $1^{*} 1$ & 20 \\
\hline Gilox & 9 & $0-58$ & $1^{*} 1$ & 20 \\
\hline \multirow{3}{*}{ CTC20 } & 13 & $0-58$ & $1^{*} 1$ & 20 \\
& 26 & $0-58$ & $2 * 2$ & 30 \\
& 13 & $0-58$ & $2 * 2$ & 40
\end{tabular}

Tabelle 5.8: Messparameter der Alcoa Proben für die Rotationsküvette

Die unterschiedlichen Strahlquerschnitte und Messzeiten sind durch eine weitere Variation im Messaufbau möglich. Die Messungen der Probe CTC20 wurden mit einer weiteren Blende durchgeführt. Diese Braggblende, bestehend aus einer Aluminium/Blei Sandwichplatte, befindet sich zwischen der Probe und dem Detektor (Abb. 5.37). Sie ist kreisrund zugeschnitten und absorbiert so, den Teil der Beugungsringe, welcher für die Polfigurauswertung nicht nötig ist. In diesem Fall handelt es sich um den inneren Bereich bis zum (104)-Reflex, in dem auch die Intensität des amorphen Teils (Plexiglas, Glyzerin) besonders 
hoch ist. Mit der Blende lässt sich der dynamische Bereich des Detektors besser ausnutzen, da die hohen Intensitäten im Zentrum nicht auf den Detektor treffen und ihn zum überlaufen bringen. Die schwächeren Reflexe im äußeren Bereich lassen sich aufgrund einer höheren Intensität, ermöglicht durch längere Messzeit und größeren Strahlquerschnitt, besser auswerten.

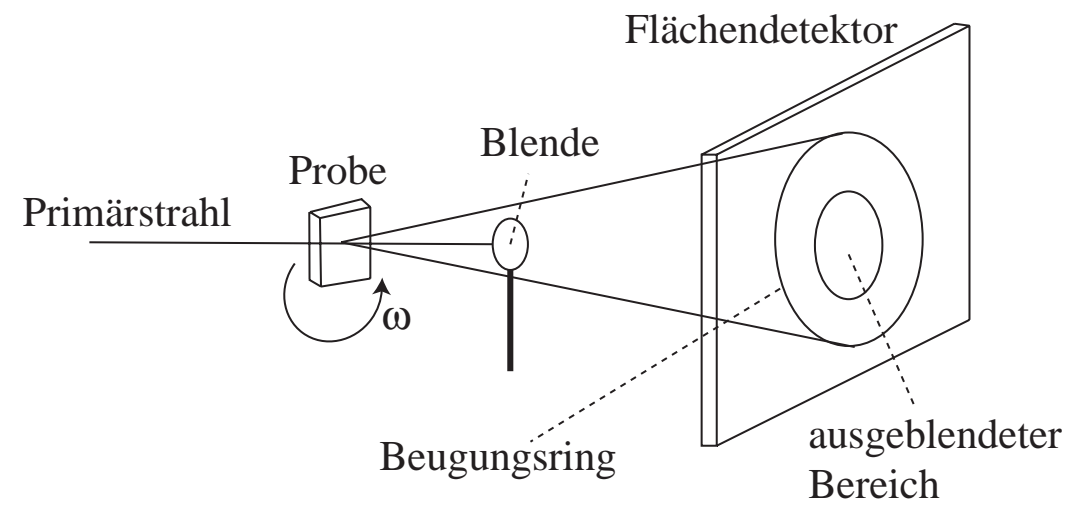

Abbildung 5.37: schematische Darstellung der Braggblende im Strahlengang

Mit dieser verbesserten Messmethode lässt sich auch im Vergleich mit bisherigen Messungen abschätzen, ob die Messbedingungen der Proben A, B und $\mathrm{C}$ genügen um die ODF aus den Messdaten mittels Reihenentwicklung zu bestimmen. Sollten sich die Ergebnisse grundlegend unterscheiden, sind die Kornstatistik der ersten Messungen und/oder der maximale Durchstrahlwinkel nicht ausreichend für eine ODF-Rechnung.

\subsubsection{Partikelform}

Ähnlich den bisher untersuchten Proben A, B und C, zeigen auch die kommerziellen Substanzen HVAFG, CTC20 und Gilox Unterschiede im plättchenförmigen Charakter (siehe Kap. 3.2.1.2). Unter den zuvor beschriebenen Messbedingungen sollen die Unterschiede der Partikelform deutlicher werden als in Kapitel 5.2 .4 beobachtet.

Zum Vergleich der Vorzugsorientierung, bedingt durch die Partikelform, sind in Abbildung 5.38 die aus der ODF rückgerechneten (001)-Polfiguren dargestellt, da auch bei diesen $\mathrm{Al}_{2} \mathrm{O}_{3}$-Proben die Basisebene der Plättchen der (001)Ebene im Kristall entspricht. Alle drei Proben sind bei gleicher Strömungsgeschwindigkeit und Konzentration gemessen worden.

Die maximalen Intensitäten der (001)-Polfiguren sind bei den $\mathrm{Al}_{2} \mathrm{O}_{3}$-Pulvern HVAFG und CTC20 nahezu gleich. Obwohl sich die Radienverhältnisse, mit $r_{e}=1$ für HVAFG und $r_{e}=4$ für CTC20, deutlich unterscheiden. Der Einfluss des globularen Anteils bei CTC20 führt hier zu einem vergleichbaren Verhalten der unterschiedlichen Substanzen. Der Verlauf der Höhenlinien der Proben HVAFG und CTC20, sind jedoch voneinander verschieden. Die Probe CTC20 zeigt um das Maximum eine breitere kreisförmige Ausdehnung. Für HVAFG ist diese Ausdehnung nur in Richtung SR zu beobachten. 


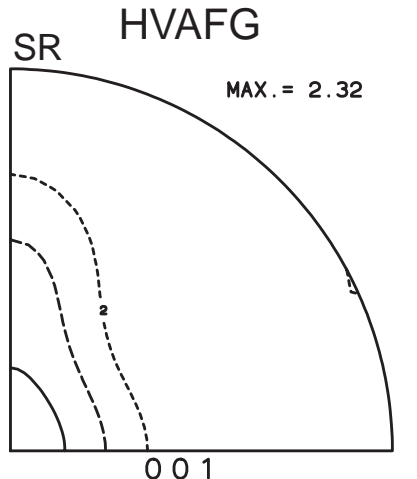

SR CTC20

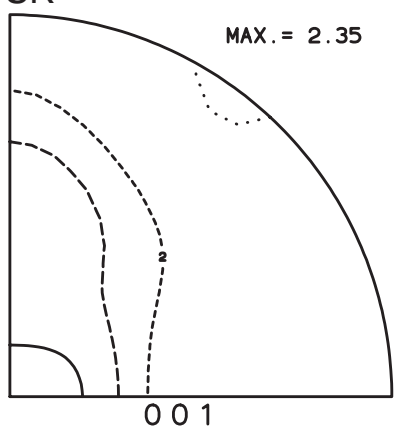

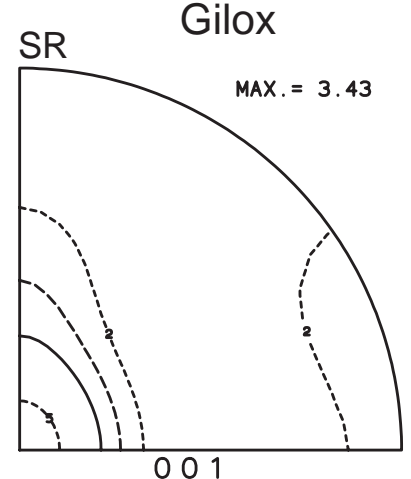

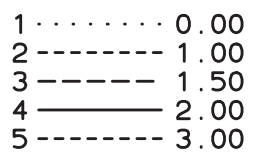

Abbildung 5.38: (001)-Polfiguren der Proben HVAFG, CTC20 und Gilox bei $1 \mathrm{U} / \mathrm{min}$

Das Produkt Gilox mit der ausgeprägtesten Plättchenform $\left(r_{e}=7\right)$ zeigt eine deutlich schärfere Textur in der (001)-Polfigur im Vergleich zu den Proben HVAFG und CTC20. Anhand der Höhenlinien ist auch hier eine Ausdehnung des Maximums in Scherrichtung aufgrund des nicht messbaren Bereichs in den originalen Polfiguren zu sehen. Die gegenüber den Proben A, B und C deutlich schärferen Texturen sind hier auf die insgesamt geringere Feststoffkonzentration zurückzuführen.

Eine genauere Betrachtung der Ergebnisse erlauben die Polfigurschnitte. Alle Polfigurschnitte verlaufen im weiteren von $\alpha=0^{\circ}$ bis $\alpha=90^{\circ}$. Der vertikale Schnitt in Richtung SR befindet sich bei $\beta=90^{\circ}$, der Horizontale in Richtung QR bei $\beta=0^{\circ}$. Dieser ist für die oben beschriebenen Polfiguren in Abbildung 5.39 zu sehen. Die bei den Polfiguren gezeigten Unterschiede finden sich auch in den Schnitten wieder.

Lediglich Gilox zeigt aufgrund des stark ausgeprägten plättchenförmigen Charakters eine deutliche Vorzugsorientierung der Basisebene parallel zur Strömungsrichtung. Die Unterschiede zwischen HVAFG und CTC20 finden sich in der Ausdehnung des Maximums in $\alpha$. Außerdem lässt sich durch den Schnitt außerdem feststellen, dass die Intensität bei CTC20 fast bis auf 0 bei $\alpha=65^{\circ}$ zurückgeht. Außerdem wird der aus der (001)-Polfigur gewonnene Eindruck, dass das Maximum für CTC20 sich in beide Richtungen ausdehnt, hier nicht bestätigt. Alle drei Proben haben bei ca. $40^{\circ}$ in $\alpha$ einen Wert von 1 erreicht. $\mathrm{Zu}$ 


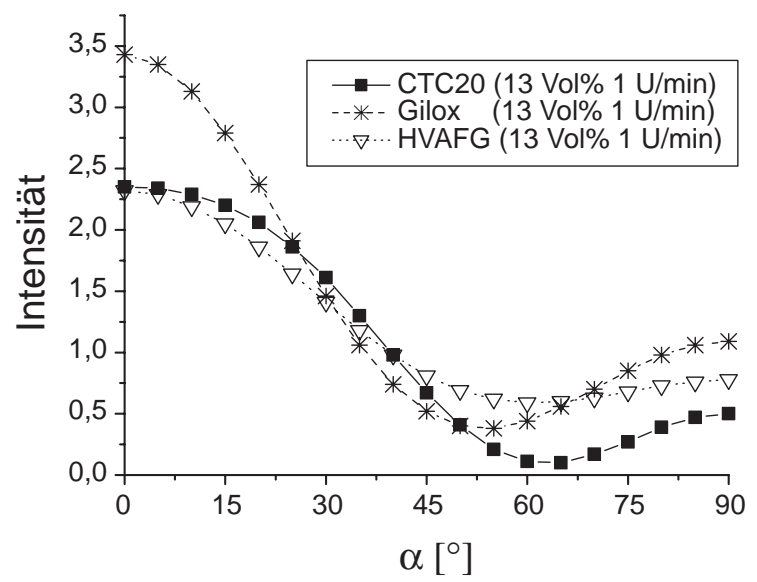

Abbildung 5.39: (001)-Polfigurschnitte senkrecht zu SR der Proben HVAFG, CTC20 und Gilox bei 1U/min

noch größeren Winkeln $\alpha$ erreichen alle Substanzen ein Minimum. Anschließend weist HVAFG bei $\alpha=90^{\circ}$ den höchsten Wert von 1 auf.

Auch der in Abbildung 5.40 gezeigte Polfigurschnitt der Proben HVAFG, CTC20 und Gilox in Scherrichtung führt zu weiteren Erkenntnissen. Das bei den Proben A, B und $\mathrm{C}$ zu beobachtende Maximum bei $\alpha=50^{\circ}$ und die große Ausdehnung in Scherrichtung ist hier nur für CTC20 auch zu sehen. Jedoch ist dies nicht so stark ausgeprägt wie bei A, B, C. Die beiden anderen Substanzen haben an dieser Position kein Maximum.

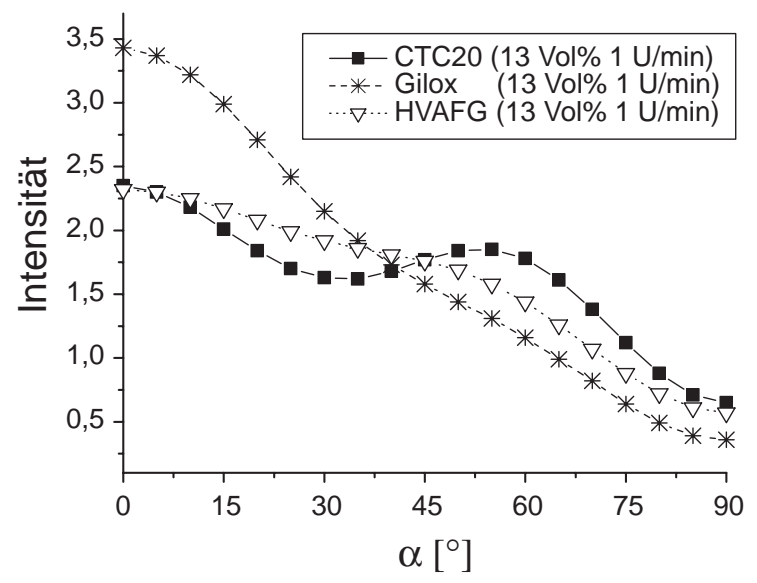

Abbildung 5.40: (001)-Polfigurschnitte in Richtung SR der Proben HVAFG, CTC20 und Gilox bei $1 \mathrm{U} / \mathrm{min}$

Jedoch ist die Ausdehnung der hohen Intensität in Scherrichtung auch hier sehr groß. Ein Wert von 1-fach regellos wird erst nach $\alpha=70^{\circ}$ erreicht. Für HVAFG und Gilox befindet sich das Maximum bei $\alpha=0^{\circ}$. Von dort sinkt die Intensität kontinuierlich bis $\alpha=90^{\circ}$ ab. Die im Vergleich mit A, B und 
C geringere Feststoffkonzentration mit 13Vol \% führt zumindest für Gilox zu einer deutlich schärferen Textur.

Die bei den Proben A, B und C beobachtete weitere Vorzugsorientierung ist auch bei den Proben HVAFG, CTC20 und Gilox zu beobachten. In den in Abbildung 5.41 gezeigten (110)-Polfiguren tritt das Maximum wie bei den anderen Proben auch senkrecht zur Scherrichtung SR auf.
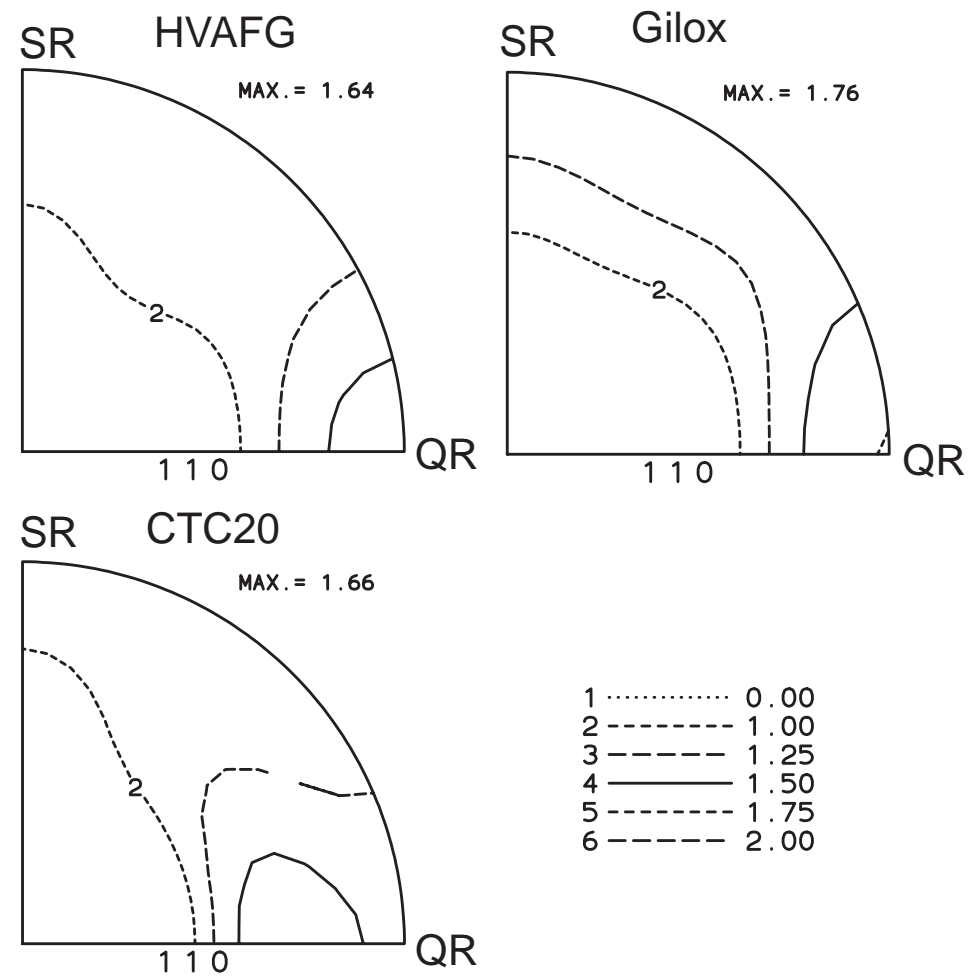

Abbildung 5.41: (110)-Polfiguren der Proben HVAFG, CTC20 und Gilox bei $1 \mathrm{U} / \mathrm{min}$

Die Position des Maximums in der (110)-Polfigur der Probe CTC20 unterscheidet sich von den beiden anderen. Das Maximum befindet sich hier zwar auch senkrecht zur Strömungsgeschwindigkeit, ist jedoch in $\alpha$ bei ca. $70^{\circ} \mathrm{zu}$ sehen. Auch in dem Schnitt der (001)-Polfigur (Abb. 5.40) ist schon ein lokales Maximum bei $\alpha=50^{\circ}$ zu sehen, welches bei den beiden anderen Proben nicht auftritt. Diese Orientierung lässt sich durch einen plättchenförmigen Charakter nicht erklären und ist trotz der nicht messbaren Bereiche in den Polfiguren entstanden. Richtet sich ein Plättchen in der Strömung entsprechend den Theorien von Jeffery [6] und Anczurowski et al. [7] mit der Basisebene aus, sollte die maximale Intensität des (110)-Reflexes am Rand der Polfigur auftreten.

Da jedoch die Probe CTC20 als einzige einen großen Anteil von globularen Partikeln aufweist, ist es möglich, dass die globularen Partikel die Ausrichtung der plättchenförmigen Partikel stören und so eine weitere Vorzugsorientierung erzeugen. 


\subsubsection{Strömungsgeschwindigkeit}

Die hohen Feststoffkonzentrationen der Proben A, B und C haben keine Abhängigkeit von der Strömungsgeschwindigkeit erkennen lassen. Da die $\mathrm{Al}_{2} \mathrm{O}_{3}$-Pulver von Alcoa bei geringeren Konzentrationen gemessen wurden ist auch hier eine Überprüfung der Abhängigkeit von der Strömungsgeschwindigkeit notwendig. Die Abbildung 5.42 zeigt exemplarisch die Polfigurschnitte der Probe CTC20 bei unterschiedlichen Strömungsgeschwindigkeiten.

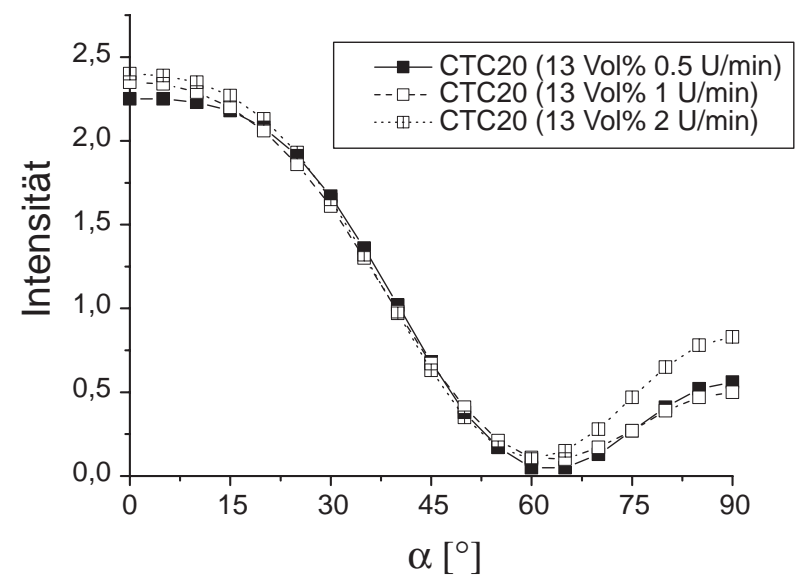

Abbildung 5.42: (001)-Polfigurschnitte senkrecht zu SR der Proben CTC20 mit $13 \mathrm{Vol} \%$

Die maximale Intensität nimmt bei dieser Messreihe mit steigender Strömungsgeschwindigkeit zu. Dieser Effekt ist jedoch nur sehr schwach. Der weitere Verlauf der Intensität mit $\alpha$ weist kaum Unterschiede auf. Erst bei $\alpha=90^{\circ}$ sind wieder Unterschiede in der Intensität vorhanden. Diese hängen aber nicht systematisch mit der Strömungsgeschwindigkeit zusammen. Es sind auch keine neuen Vorzugsorientierungen vorhanden.

Für einen Vergleich bei den anderen Messungen ist es also ausreichend die Intensität bei $\alpha=0^{\circ}$ und $\beta=0^{\circ}$ gegenüberzustellen. In Tabelle 5.9 sind für alle durchgeführten Messungen die Intensitäten in Abhängigkeit von der Strömungsgeschwindigkeit aufgeführt.

\begin{tabular}{c||c|c|c||c|c|c||c}
\multirow{2}{*}{\multicolumn{1}{c||}{ U/min }} & \multicolumn{3}{c||}{ HVAFG } & \multicolumn{3}{c||}{ CTC20 } & Gilox \\
\cline { 2 - 8 } & $26 \mathrm{Vol} \%$ & $13 \mathrm{Vol} \%$ & $9 \mathrm{Vol} \%$ & $26 \mathrm{Vol} \%$ & $13 \mathrm{Vol} \%$ & $9 \mathrm{Vol} \%$ & $13 \mathrm{Vol} \%$ \\
\hline 0,25 & 2,12 & 2,41 & 2,61 & $*$ & 2,25 & 2,14 & 3,43 \\
0,5 & 2,12 & 2,32 & 2,66 & 2,17 & 2,35 & $*$ & 3,43 \\
1 & 2.15 & $*$ & 2,64 & 2,25 & 2,4 & 2,63 & 3,4
\end{tabular}

Tabelle 5.9: Intensität der Proben HVAFG, CTC20 und Gilox in der (001)Polfigur bei $\alpha=0^{\circ}$ und $\beta=0^{\circ}$

Die mit * gekennzeichneten Intensitäten resultieren aus Messungen, bei denen es aufgrund einer undichten Küvette oder Schwierigkeiten mit dem Antrieb der Scheibe zu verfälschten Ergebnissen kam. 
Der schwache Trend aus der Abbildung 5.42 setzt sich bei den anderen Messungen nicht fort. Die Änderungen in der Intensität sind nur sehr gering und über die gemessenen Geschwindigkeiten zufällig verteilt.

Die bei Muskovit beobachtete Positionsänderung des Maximums in der (111)-Polfigur in Abhängigkeit von der Strömungsgeschwindigkeit konnte bei den $\mathrm{Al}_{2} \mathrm{O}_{3}$-Proben $\mathrm{A}, \mathrm{B}$ und $\mathrm{C}$ in der (110)-Polfigur nicht beobachtet werden. Für die Probe CTC20 wurde dieser Effekt anhand von Polfigurschnitten (Abb. 5.43 bei konstantem Polfigurwinkel $\alpha$ untersucht.

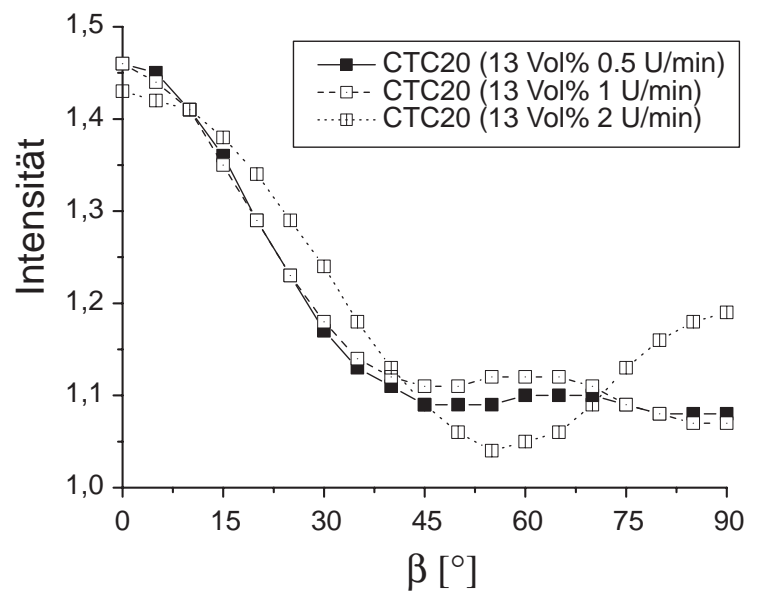

Abbildung 5.43: (110)-Polfigurschnitte mit $\alpha=90^{\circ}$ der Proben CTC20 bei unterschiedlichen Strömungsgeschwindigkeiten

Auch in der Darstellung des Maximums in Abbildung 5.43 ist keine Positionsänderung des Maximums mit der Strömungsgeschwindigkeit zu beobachten. Auch treten keinerlei andere systematische Zusammenhänge der Textur mit der Strömungsgeschwindigkeit auf. Um den in Kapitel 5.1 beschriebene Effekt zu bestimmen ist der plättchenförmige Charakter der $\mathrm{Al}_{2} \mathrm{O}_{3}$-Partikel in dieser Scherströmung nicht ausreichend.

\subsubsection{Feststoffkonzentration}

Die in Kapitel 5.2.4 beschriebene Änderung der maximalen Intensität in der (001)-Polfigur bei $\alpha=0^{\circ}$ und $\beta=0^{\circ}$ mit unterschiedlicher Feststoffkonzentration in der rückgerechneten (001)-Polfigur konnte dort nur anhand einer Messung beobachtet werden. Mit den neuen $\mathrm{Al}_{2} \mathrm{O}_{3}$-Pulvern von Alcoa, welche in großer Menge vorhanden sind, war nun eine systematischere Messung möglich. Da bei der in Kapitel untersuchten Probe B 5.2.4 die maximale Intensität mit sinkender Feststoffkonzentration zunahm, wurde die Konzentration für die Proben HVAFG und CTC20 weiter herabgesetzt um einen möglichst großen Effekt zu erzielen.

Aufgrund der in Tabelle 5.9 gezeigten Ausfälle bei einigen Messungen, reduzierte sich die Zahl der zu Verfügung stehenden Ergebnisse auf drei Messreihen. Für drei weitere Messreihen stehen nur zwei Messwerte zur Verfügung. Die Auswertung der Messergebnisse erfolgt unter zwei Gesichtspunkten. Zum 
einen ist der Verlauf der Intensität mit veränderter Feststoffkonzentration interessant. Zum anderen ist auch eine Gegenüberstellung der Intensitäten bei $\alpha=$ $0^{\circ}$ und $\beta=0^{\circ}$, welche der Orientierung der Basisebene der Partikel parallel zur Strömung entspricht von Bedeutung.

Der Verlauf der Intensität für unterschiedliche Konzentrationen der Probe CTC20 ist in einem (001)-Polfigurschnitt in Abbildung 5.44 dargestellt. Mit sinkender Feststoffkonzentration nimmt auch hier die Intensität für die Vorzugsorientierung bei $\alpha=0^{\circ}$ und $\beta=0^{\circ}$ zu. Die bei der Probe B (Kap. 5.2.4) beobachteten Ergebnisse, dass sich mit geringerer Feststoffkonzentration die Basisebene stärker parallel zur Strömungsrichtung einregelt, werden durch diese Messreihe bestätigt.

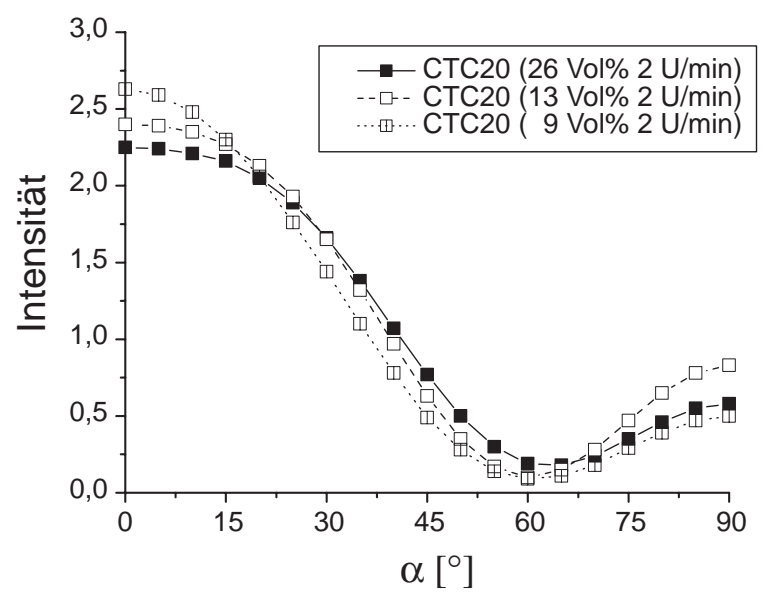

Abbildung 5.44: 001)-Polfigurschnitt senkrecht zu SR von CTC20 bei unterschiedlichen Feststoffkonzentrationen

Der Schnitt zeigt außerdem, dass sich der Verlauf der Intensität kaum ändert. Es treten bei den unterschiedlichen Konzentrationen keine neuen Vorzugsorientierungen auf.

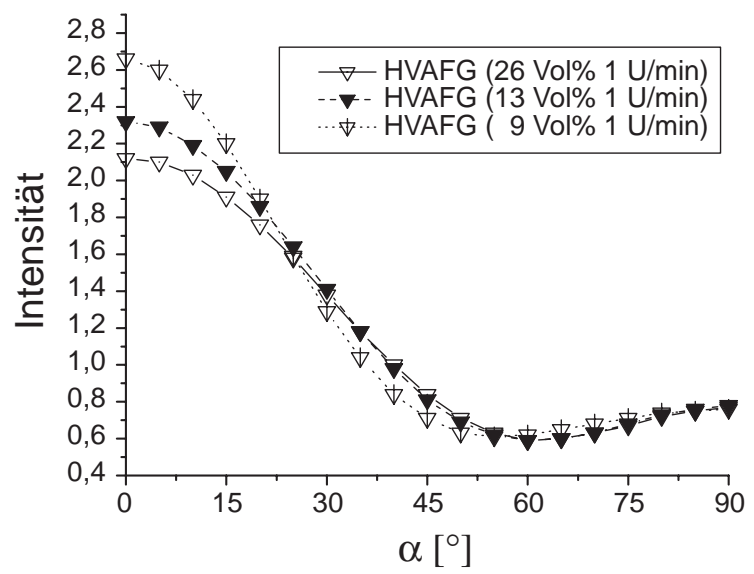

Abbildung 5.45: 001)-Polfigurschnitt senkrecht zu SR von HVAFG bei unterschiedlichen Feststoffkonzentrationen 
Auch für das $\mathrm{Al}_{2} \mathrm{O}_{3}$-Pulver HVAFG ist in Abbildung 5.45 der Verlauf der Intensität für verschiedene Konzentrationen im horizontalen (001)-Polfigurschnitt dargestellt. Die Unterschiede bei $\alpha=0^{\circ}$ und $\beta=0^{\circ}$ sind hier etwas deutlicher zu sehen als bei CTC20.

Es treten aber auch hier keine neuen Vorzugsorientierungen bei unterschiedlichen Konzentrationen auf. Der Effekt, dass die Texturschärfe mit sinkender Konzentration zunimmt ist in allen Messreihen nur schwach ausgeprägt. Der maximale Fehler in den rückgerechneten Polfiguren (siehe Kap. 2.5.5) beträgt bei allen Rechnungen ca. 0,3fach regellos (siehe Anhang C). Somit ist der Fehler aus der ODF-Rechnung auf ähnlichem Niveau wie der beobachtete Effekt. Da sich aber für alle Messungen und Rechnungen ein Zusammenhang zwischen Texturschärfe und Konzentration darstellt ist eine zufällige Abhängigkeit unwahrscheinlich.

In Abbildung 5.46 sind die Intensitäten bei $\alpha=0^{\circ}$ und $\beta=0^{\circ}$ der drei stehenden Messreihen in Abhängigkeit von der Feststoffkonzentration aufgetragen.

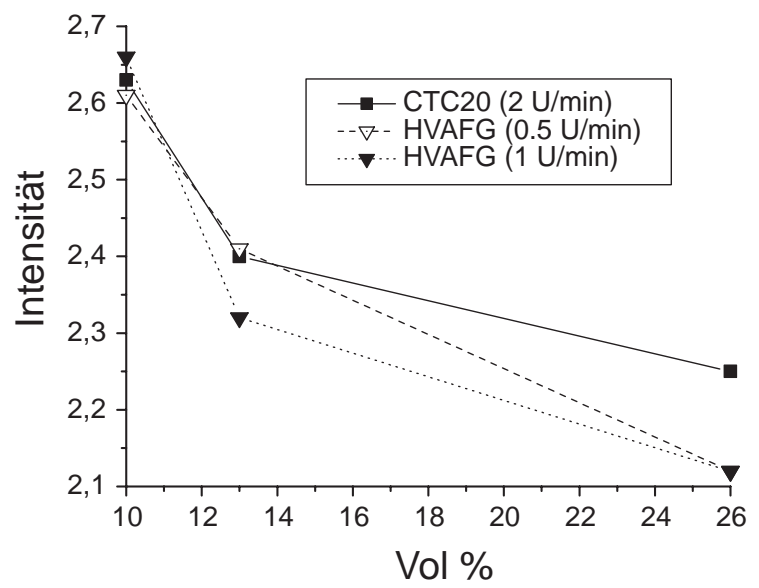

Abbildung 5.46: Intensität der Vorzugsorientierung bei $\{0,0\}$ von HVAFG und CTC20 für unterschiedliche Feststoffkonzentrationen

Die einzelnen Messpunkte sind nur zur Verdeutlichung miteinander verbunden. Eine nichtlineare Konzentrationsabhängigkeit ist denkbar, lässt sich aber aufgrund der wenigen Datenpunkte nicht mit Sicherheit vorhersagen. Die geringere Feststoffkonzentration führt zu weniger Teilchen-Teilchen-Stößen und somit zu weniger Störungen bereits eingeregelter Teilchen. Außerdem kann sich durch eine veränderte Feststoffkonzentration, wie in Kapitel 1.4.1 beschrieben, der Strömungsgradient ändern [13]. Mit steigender Feststoffkonzentration wird der Strömungsgradient schwächer und somit auch die daraus resultierende Textur. 


\subsubsection{Zusammenfassung}

Die Ergebnisse der Proben A, B und C in Kapitel 5.2.4 werden durch die Messungen mit den Proben HVAFG, CTC20 und Gilox bestätigt. Die Abhängigkeit der Textur von der Partikelform ist in allen Messungen zu beobachten. Die Veränderung der Messparameter zu geringeren Konzentrationen hat sich als positiv herausgestellt, da die ermittelten Intensitäten der Vorzugsorientierungen deutlich höher sind.

Eine Abhängigkeit der Textur von der Strömungsgeschwindigkeit lässt sich auch bei geringeren Konzentrationen nicht beobachten. Die Konzentrationsabhängigkeit ist jedoch aufgrund der zusätzlichen Messungen deutlich geworden. 


\subsubsection{Die Proben HVAFG, CTC20 und Gilox in der Durch- flussküvette}

Die Durchflussküvette besitzt aufgrund der Bauart zwei wesentliche Vorteile.

- keine beweglichen Teile in Kontakt mit der Suspension

- keine Motorteile drehen sich in den Strahlengang

Dies ermöglicht lange Messzeiten bei sehr stabilen Bedingungen und mit einem erweiterten Messbereich in $\omega$ von $-70^{\circ}$ bis $70^{\circ}$ (siehe Kap. 4.2). Die Orientierungsverteilungsfunktion und die rückgerechnete (001)- und (110)-Polfiguren lassen sich mit mehr gemessenen Daten berechnen.

Außerdem bietet die Durchflussküvette ein anderes Strömungsprofil gegenüber der Rotationsküvette (Kap. 1.2). Dieser Einfluss kann im Vergleich der Ergebnisse mit der Rotationsküvette herausgearbeitet werden, da in beiden Systemen die gleichen $\mathrm{Al}_{2} \mathrm{O}_{3}$-Pulver und Glyzerin verwendet werden. Lediglich die gewählten Feststoffkonzentrationen sind geringer, da für die Durchflussküvette die Fließfähigkeit der Suspension gewährleistet werden muss.

Unter diesen veränderten Bedingungen sollen folgende Effekte untersucht werden.

- Überprüfung der Partikelformabhängigkeit der Textur im veränderten Strömungsfeld

- Überprüfung der Texturabhängigkeit von der Strömungsgeschwindigkeit

- Verhalten bei unterschiedlichen Feststoffkonzentrationen

- Vergleich der Strömungsfelder von Rotations- und Durchflussküvette

Es wurde für jede Suspension eine Messreihe mit unterschiedlichen Strömungsgeschwindigkeiten $(0,2,0,5,1,2 \mathrm{u} .5 \mathrm{ml} / \mathrm{s})$ durchgeführt. Die einzelnen Parameter der Messreihen sind in Tabelle 5.10 zusammengefasst.

\begin{tabular}{c|c|c|c|c} 
Probe & $\begin{array}{c}\text { Konzentration } \\
{[\text { Vol\%] }}\end{array}$ & $\begin{array}{c}\text { Drehwinkel } \\
\omega\left[{ }^{\circ}\right]\end{array}$ & $\begin{array}{c}\text { Strahlquer- } \\
\text { schnitt }[\mathrm{mm}]\end{array}$ & $\begin{array}{c}\text { Belichtungs- } \\
\text { zeit }[\mathrm{sec}]\end{array}$ \\
\hline \multirow{3}{*}{ HVAFG } & 12,5 & $-70-70$ & $1^{*} 1$ & 60 \\
\hline \multirow{3}{*}{ Gilox } & 10 & $-70-70$ & $1^{*} 1$ & 120 \\
\hline \multirow{3}{*}{ CTC20 } & 12,5 & $-70-70$ & $1^{*} 1$ & 40 \\
& 10 & $-70-70$ & $2^{*} 2$ & 60 \\
& 7,5 & $-70-70$ & $1^{*} 1$ & 60 \\
\hline & 12,5 & $-70-70$ & $1^{*} 1$ & 40 \\
& 10 & $-70-70$ & $2^{*} 2$ & 60 \\
& 7,5 & $-70-70$ & $1 * 1$ & 60
\end{tabular}

Tabelle 5.10: Messparameter der Alcoa Proben für die Durchflussküvette

Die Strahlquerschnitte und Messzeiten wurden jeweils so gewählt, dass der dynamische Bereich des Detektors optimal ausgenutzt wurde. Die maximale 
Intensität pro Pixel beträgt 100000 counts [70]. Da einige Messungen an der Petra II Beamline (identisch zu BW5 Kap. 2.2.1) mit höherem Photonenfluss durchgeführt wurden, war ein Strahlquerschnitt von $1^{*} 1 \mathrm{~mm}$ ausreichend. Das in Kapitel 5.2.5 beschriebene Blendensystem wurde auch hier wieder eingesetzt.

\subsubsection{Partikelform}

Wie schon bei den Messungen mit der Rotationsküvette ist auch hier der Einfluss der Partikelform auf die Textur am deutlichsten an der (001)-Polfigur in Abbildung $5.47 \mathrm{zu}$ sehen. Die Partikel richten sich auch hier im Gradienten der Strömung mit der Basisebene parallel zur Strömungsrichtung aus.
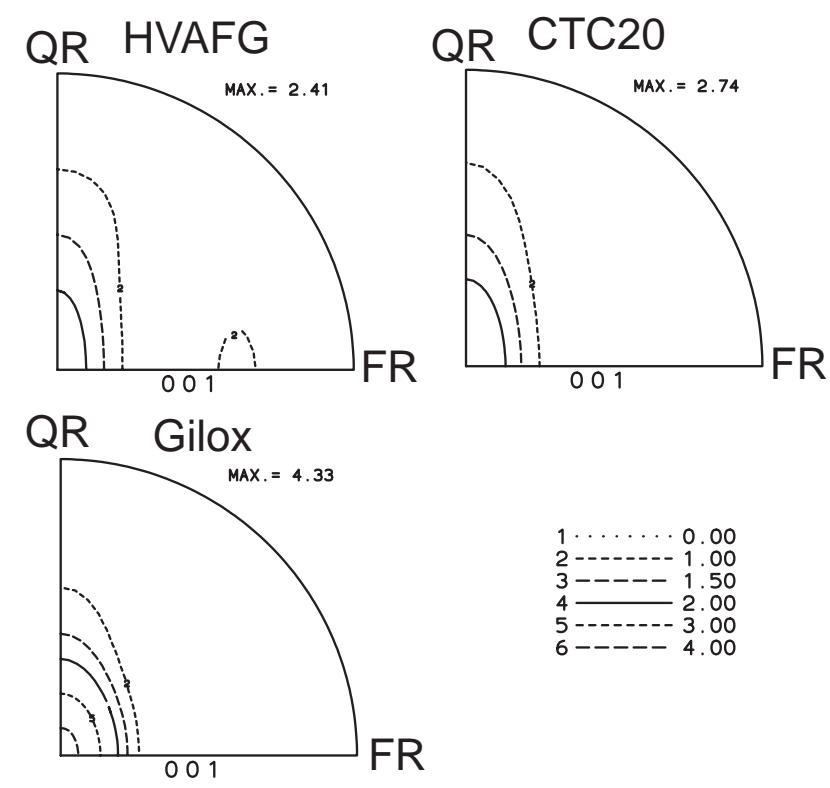

Abbildung 5.47: (001)-Polfiguren der Proben HVAFG, CTC20 und Gilox bei $1 \mathrm{ml} / \mathrm{s}$

Die mit der Durchflussküvette gemessenen Texturen sind schärfer als bei der Rotationsküvette. So wird auch der Unterschied zwischen den globularen Partikeln der Probe HVAFG und den leicht plättchenförmigen Partikel der Probe CTC20 deutlicher. Gilox bildet auch hier wieder die schärfsten Texturen aus. Durch den veränderten Geschwindigkeitsgradienten (Kap. 1.2.2) in der Strömung treten aber sonst keine weiteren Unterschiede, wie z.B. neue Komponenten, zwischen den Texturen der Rotations- und der Durchflussküvette auf.

Die Verläufe der Höhenlinien sind denen der Rotationsküvette sehr ähnlich. Das Maximum bei $\alpha=0^{\circ}$ und $\beta=0^{\circ}$ fällt in Flussrichtung FR schnell ab. Senkrecht zur Flussrichtung ist es deutlich breiter ausgedehnt. Die Strömungsrichtung in den beiden Küvetten ist zwar unterschiedlich, die Richtung des Geschwindigkeitsgradienten ist jedoch gleich geblieben. Für alle Messungen verläuft der größte Geschwindigkeitsgradient in Strahlrichtung. So sind die 
(001)-Polfiguren direkt miteinander vergleichbar, obwohl sich die Strömungsrichtung ändert.

Da auch hier die wesentlichen Änderungen in der Textur in Flussrichtung und senkrecht dazu zu beobachten sind, ist die Darstellung eines Polfigurschnitts in die entsprechende Richtung geeignet, um Unterschiede zwischen den einzelnen Substanzen besser sichtbar zu machen.

In Abbildung 5.48 ist der horizontale Schnitt durch die (001)-Polfigur dargestellt. Der Schnitt gibt über die Unterschiede in der maximalen Intensität hinaus noch weitere Informationen. Bei ca. $\alpha=30^{\circ}$ erreicht die Intensitäten für alle Substanzen der Wert 1, welches einem regellosen Verhalten entspricht.

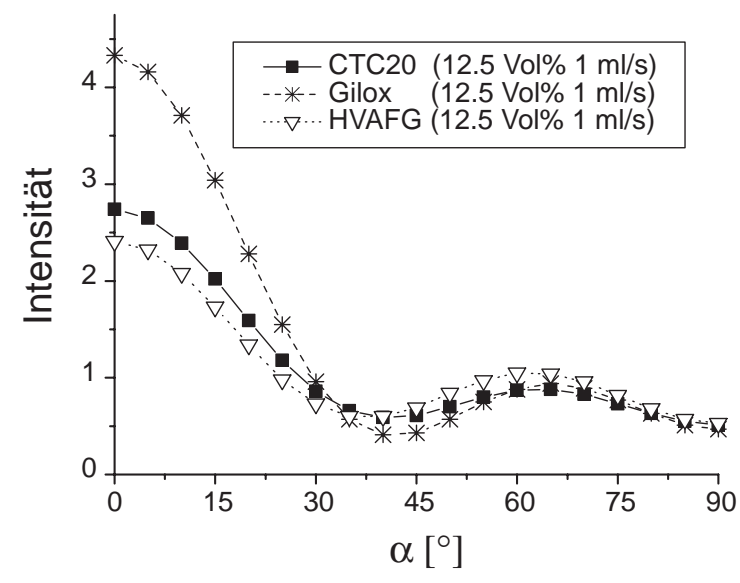

Abbildung 5.48: (001)-Polfigurschnitte in Richtung FR der Proben HVAFG, CTC20 und Gilox bei $1 \mathrm{ml} / \mathrm{s}$

Die Auslenkung aus der Ideallage, in Flussrichtung, ist somit unabhängig von der Partikelform. Sie hat nur einen Einfluss auf die maximale Intensität.

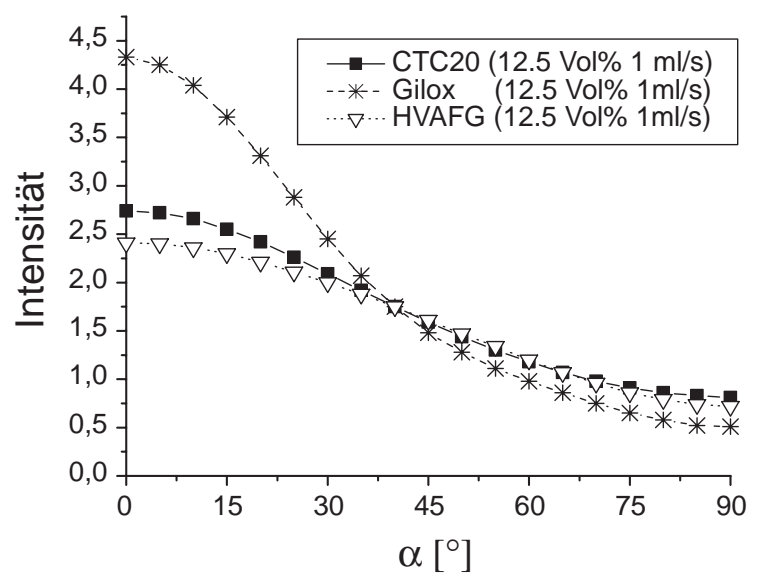

Abbildung 5.49: (001)-Polfigurschnitte senkrecht zu FR der Proben HVAFG, CTC20 und Gilox bei $1 \mathrm{ml} / \mathrm{s}$ 
Ein weiterer Aspekt ist die Auslenkung senkrecht zur Flussrichtung. Entspricht der Scherrichtung in der Rotationsküvette. Zum Vergleich der Proben ist ein Polfigurschnitt senkrecht zu FR notwendig. In dem in Abbildung 5.49 dargestellten Schnitt zeigt sich ein ähnliches Verhalten, wie bei dem horizontalen Schnitt. Eine Abhängigkeit der Auslenkung von der Partikelform ist nicht vorhanden.

Ab einem Winkel $\alpha=40^{\circ}$ verhalten sich alle drei Proben ähnlich. Jedoch ist hier erst ein Wert von 1,5 erreicht. Regelloses Verhalten ist erst am Rand der Polfigur zu beobachten. Diese stärkere Auslenkung der Basisebene aus der Ideallage in Richtung des Geschwindigkeitsgradienten ist auch von Anczurowski et al. [7] so vorher gesagt.
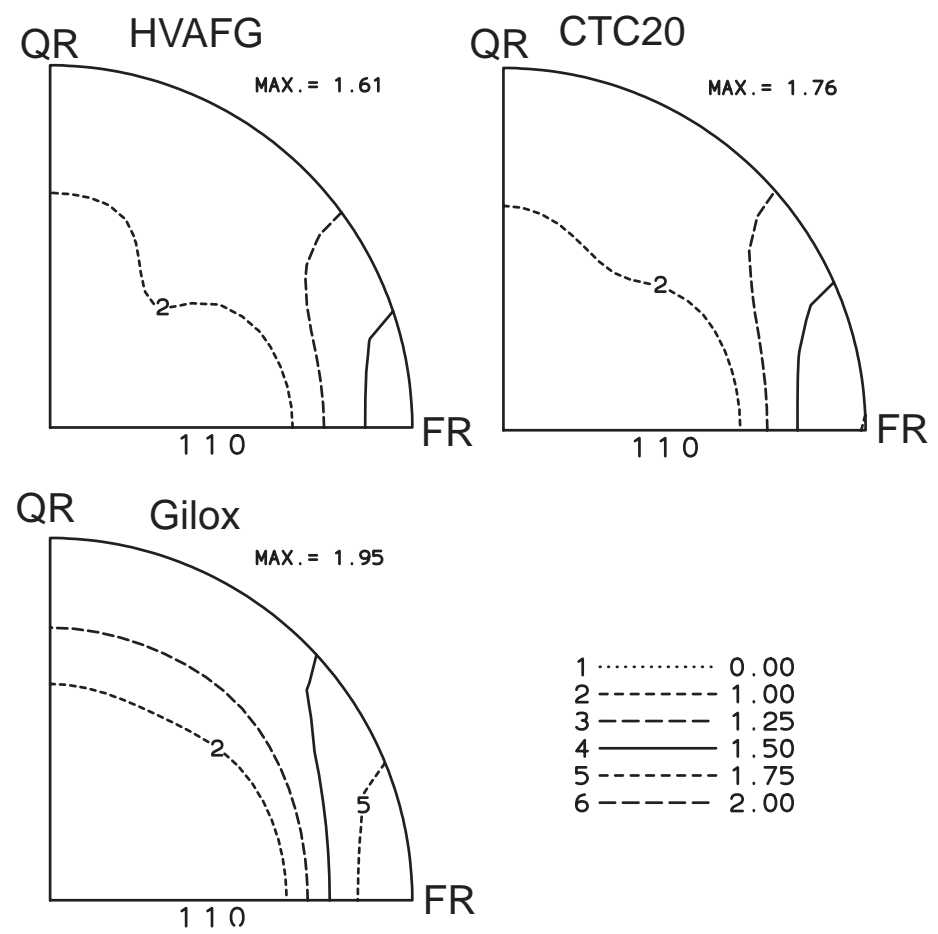

Abbildung 5.50: (001)-Polfiguren der Proben HVAFG, CTC20 und Gilox bei $1 \mathrm{ml} / \mathrm{s}$

Ein weiterer Aspekt bei der Betrachtung der Abhängigkeit der Textur von der Partikelform, ist neben der Orientierung der Basisebene, die Orientierung um die Normale der Basisebene. Wie schon in den Kapiteln 5.2.4 und 5.2.5 ist die Textur in den rückgerechneten (110)-Polfiguren nicht rotationssymmetrisch. Auch die (110)-Polfiguren aus der Durchflussküvette, der Proben HVAFG, CTC20 und Gilox in Abbildung 5.50 zeigen, wie bei den Messungen mit der Rotationsküvette ein Maximum in Flussrichtung. Trotz unterschiedlicher Flussrichtungen befindet sich das Maximum der (110)-Polfigur an der gleichen Position wie bei der Rotationsküvette. Dies ist ein weiteres Indiz für ein Maximum, welches aufgrund des nicht messbaren Bereichs entsteht.

Die Probe Gilox, mit dem größten plättchenförmigen Charakter, zeigt im 
Gegensatz zu den anderen Proben eine gleichmäßigere Intensitätsverteilung am Rand der Polfigur. Es tritt zwar auch ein Maximum in Flussrichtung auf, jedoch ist die Intensität in den anderen Randbereichen der Polfigur deutlich größer und weist einen hohen rotationssymmetrischen Anteil auf. Der deutlich kleinere nicht messbare Bereich mit der Durchflussküvette durchschneidet den rotationssymmetrischen Teil in der (104), (113) und (116)-Polfigur nicht mehr so stark und beeinflusst somit die ODF-Rechnung weniger.

Da bei den Proben HVAFG und CTC20 dies nicht zu beobachten ist, besteht die Möglichkeit, dass es bei Proben mit einer weniger stark ausgeprägten Plättchenform tatsächlich eine Vorzugsorientierung in der Basisebene gibt. Die gemessenen (110)-Polfiguren im Anhang D geben hierauf einen Hinweis, da auch hier schon ein Maximum in Strömungsrichtung zu beobachten ist.

\subsubsection{Strömungsgeschwindigkeit}

Alle bisherigen Messungen haben keine Abhängigkeiten der Textur von der Strömungsgeschwindigkeit gezeigt. Die in der Strömung der Durchflussküvette entstandenen Texturen sind jedoch deutlich schärfer als die aus der Rotationsküvette. Dies kann besonders bei der Probe Gilox, mit der schärfsten Textur, dazu führen, dass Unterschiede bei der Strömungsgeschwindigkeit in den rückgerechneten Polfiguren zu erkennen sind. Da die Texturen wie in Kapitel 5.2.2 nur mit einem geringen Reihenentwicklungsgrad gerechnet werden können, lassen sich die geringen Abweichungen nicht darstellen.

In Abbildung 5.51 sind im Polfigurschnitt senkrecht zur Fließrichtung für unterschiedliche Strömungsgeschwindigkeiten bei gleicher Feststoffkonzentration (10Vol\%) gegenübergestellt. Der Verlauf der Intensität ist für alle Geschwindigkeiten fast über den gesamten Bereich gleich, obwohl gerade senkrecht zu FR die Auslenkung der Basisebene am größten ist und somit ein unterschiedliches Verhalten am wahrscheinlichsten ist. Lediglich im Maximum bei $\alpha=0^{\circ}$ und $\beta=0^{\circ}$ treten Abweichungen auf.

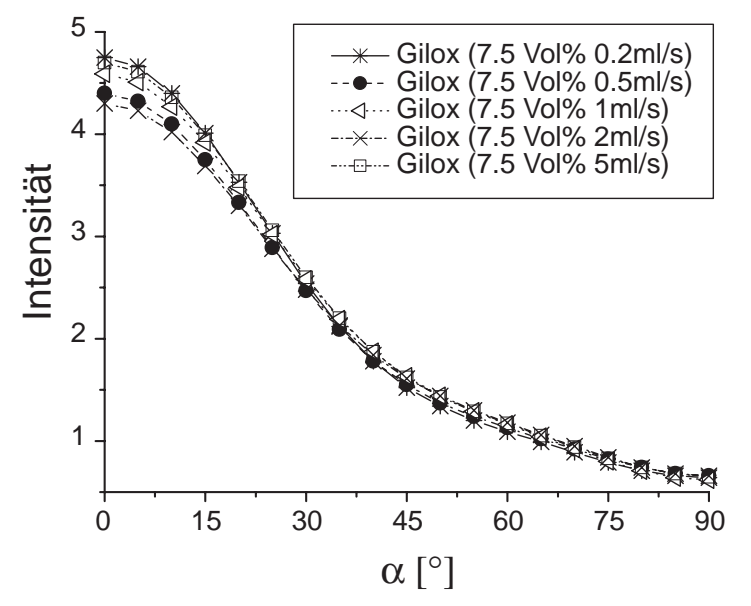

Abbildung 5.51: (001)-Polfigurschnitte senkrecht zu FR der Proben Gilox bei unterschiedlichen Strömungsgeschwindigkeiten 
Die unterschiedlichen maximalen Intensitäten weisen allerdings keinerlei Systematik auf. Es ist weder eine Zunahme der Intensität der Basisebenenorientierung noch eine Abnahme mit der Strömungsgeschwindigkeit zu beobachten. Da jedoch die maximale Intensität um ca. $\pm 0,3$-fach regellos variiert, ist ein Einfluss der Strömungsgeschwindigkeit nicht auszuschließen.

Eine Zusammenstellung der maximalen Intensitäten bei $\alpha=0^{\circ}$ und $\beta=0^{\circ}$ in Tabelle 5.11 bestätigt dies auch für die anderen Substanzen. Die maximale Abweichung zwischen den Intensitäten der einzelnen Strömungsgeschwindigkeiten einer Probe beträgt ca. 10\%. Die minimale Abweichung ist mit ca. $1 \%$ unterhalb der Genauigkeit der ODF-Rechnung.

\begin{tabular}{lc||c|c|c|c|c} 
& Vol $\%$ & $0,2 \mathrm{ml} / \mathrm{s}$ & $0,5 \mathrm{ml} / \mathrm{s}$ & $1 \mathrm{ml} / \mathrm{s}$ & $2 \mathrm{ml} / \mathrm{s}$ & $5 \mathrm{ml} / \mathrm{s}$ \\
\hline \multirow{2}{*}{ HVAFG } & 12,5 & 2,4 & 2,43 & 2,41 & 2,47 & 2,46 \\
& 10 & 2,55 & 2,56 & 2,59 & 2,55 & 2,65 \\
\hline \multirow{2}{*}{ CTC20 } & 12,5 & 2,5 & 2,54 & 2,78 & 2,66 & 2,68 \\
& 10 & 2,59 & 2,69 & 2,74 & 2,81 & 2,84 \\
& 7,5 & 2,78 & 2,99 & 3,01 & $*$ & 2,87 \\
\hline \multirow{2}{*}{ Gilox } & 12,5 & 4,19 & $*$ & 4,24 & 4,19 & 4,24 \\
& 10 & 4,5 & 4,59 & 4,33 & 4,5 & 4,19 \\
& 7,5 & 4,75 & 4,4 & 4,59 & $*$ & 4,7
\end{tabular}

Tabelle 5.11: Intensität der Proben HVAFG, CTC20 und Gilox in der (001)Polfigur bei $\alpha=0^{\circ}$ und $\beta=0^{\circ}$

Die Variation der Intensitäten ist bei der plättchenförmigen Substanz Gilox am größten. Ein Einfluss der Strömungsgeschwindigkeit auf die Textur, kann auch hier vorhanden sein. Die $\mathrm{Al}_{2} \mathrm{O}_{3}$-Pulver sind jedoch aufgrund des Radienverhältnisses und der Kristallsymmetrie jedoch nicht sensitiv genug um diesen Effekt sichtbar zu machen.

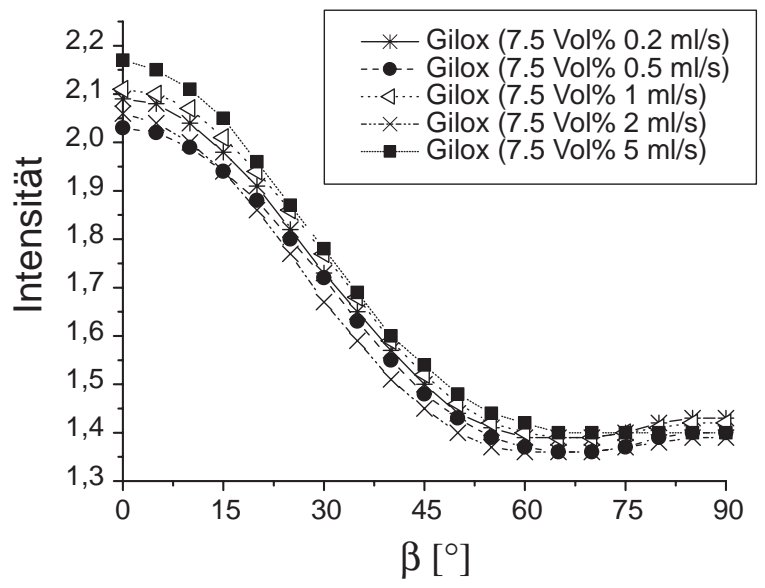

Abbildung 5.52: (110)-Polfigurschnitte mit $\alpha=90^{\circ}$ der Proben Gilox bei unterschiedlichen Strömungsgeschwindigkeiten

Eine weitere Abhängigkeit der Textur von der Strömungsgeschwindigkeit, 
wie bei den Muskovitmessungen (z.B. Kap. 5.1 Abb. 5.10 o. 5.8), ist die maximale Orientierungsdichte in einer Polfigur senkrecht zu Basisebene. Aufgrund der günstigeren Messbedingungen ist die (110)-Polfigur hier besser geeignet um die freie Rotation der Partikel um die Normale der Basisebene in der Strömung zu untersuchen. Der Einfluss des nicht messbaren Bereichs in den originalen Polfiguren ist deutlich geringer. Wie schon in den Kapiteln 5.2.4 und 5.2.5 dient auch hier zur Bestimmung der Position des Maximum am Rand der (110)Polfigur ein Polfigurschnitt bei $\alpha=90^{\circ}$ von $\beta=0^{\circ}$ bis $\beta=90^{\circ}$.

Der Polfigurschnitt in Abbildung 5.52 zeigt keine Änderung der Position des Maximums mit der Strömungsgeschwindigkeit. Auch die insgesamt schärfere Textur der Probe Gilox ist nicht ausreichend um einen systematischen Zusammenhang zwischen Textur und Strömungsgeschwindigkeit darzustellen.

\subsubsection{Feststoffkonzentration}

Mit Hilfe der größeren Anzahl von Messungen mit der Durchflussküvette bei unterschiedlicher Feststoffkonzentration soll die in Kapitel 5.2.5 beobachtete Abhängigkeit der Textur von der Konzentration bestätigt werden. Die schärferen Texturen im Strömungsfeld der Durchflussküvette sind besser geeignet um Unterschiede besser darzustellen.

Eine Gegenüberstellung von Messungen einer Substanz bei gleicher Strömungsgeschwindigkeit im Schnitt durch die rückgerechnete (001)-Polfigur gibt die Unterschiede im Profil des Maximums. In Abbildung 5.53 ist der horizontale Polfigurschnitt der Probe Gilox bei unterschiedlichen Feststoffkonzentrationen dargestellt. Die Probe Gilox, welche die stärksten Texturen ausbildet, lässt erkennen, dass mit steigender Feststoffkonzentration die Texturschärfe abnimmt.

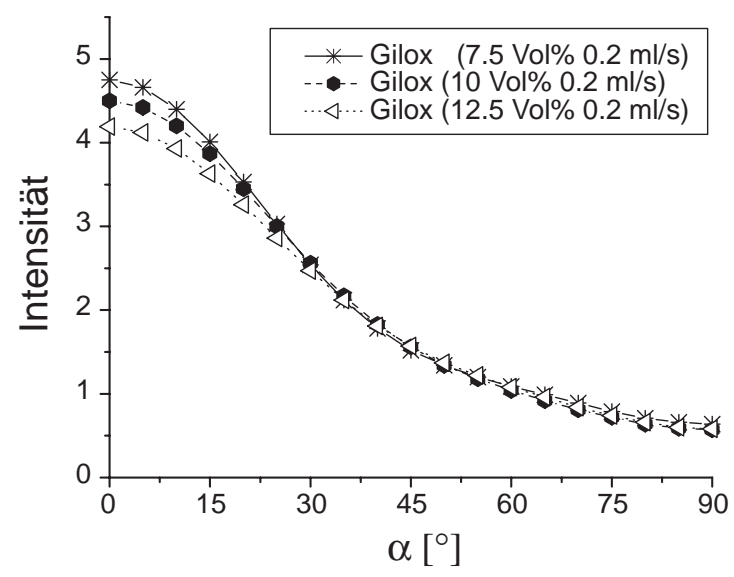

Abbildung 5.53: (001)-Polfigurschnitte senkrecht zu FR der Proben Gilox bei unterschiedlichen Strömungsgeschwindigkeiten

Das Auftreten von anderen oder zusätzlichen Orientierungen, welches mit den sensitiveren Messungen mit der Durchflussküvette möglich wäre, lässt sich nicht beobachten. Der Verlauf der Intensität ist für alle Konzentrationen gleich. 
Es treten lediglich Unterschiede in der maximalen Konzentration bei $\alpha=0^{\circ}$ und $\beta=0^{\circ}$ auf.

Da sich mit unterschiedlicher Feststoffkonzentration nur die maximale Intensität ändert, sind in Abbildung 5.54 die Intensitäten bei $\alpha=0^{\circ}$ und $\beta=0^{\circ}$ in Abhängigkeit von der Konzentration aufgetragen. Die Bezeichnung Gilox $(\overline{m l / s})$ steht für die gemittelte Intensität über alle Strömungsgeschwindigkeiten.

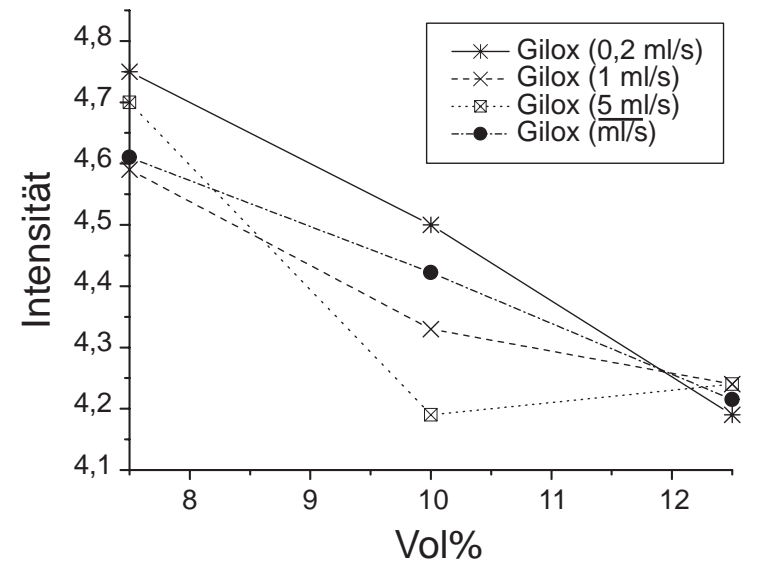

Abbildung 5.54: Intensität der Vorzugsorientierung bei $\{0,0\}$ von Gilox für unterschiedliche Feststoffkonzentrationen

Es sind nur die Strömungsgeschwindigkeiten aufgetragen, welche für alle drei Konzentrationen korrekt gemessen wurden. Die einzelnen Messpunkte sind nur zur Verdeutlichung des Verlaufs der Intensität miteinander verbunden. Die Messungen bestätigen die Ergebnisse aus Kapitel 5.2.4 und 5.2.5. Sie zeigen deutlich die Abhängigkeit der Texturschärfe von der Feststoffkonzentration, auch wenn bei der Messung für Gilox $5 \mathrm{ml} / \mathrm{s}$ ein Ausreißer bei 10Vol\% auftritt. Die gemittelte Intensität zeigt jedoch einen nahezu linearen Verlauf in Abhängigkeit von der Konzentration.

Die gleiche Messreihe mit drei unterschiedlichen Konzentrationen bei fünf verschiedenen Strömungsgeschwindigkeiten wurde auch für die Substanz CTC20 durchgeführt. Hier sind die absoluten Intensitäten aufgrund des weniger ausgeprägten plättchenförmigen Charakters bei $\alpha=0^{\circ}$ und $\beta=0^{\circ}$ nicht so hoch wie bei Gilox.

Die in Abbildung 5.55 aufgetragenen maximalen Intensitäten zeigen jedoch deutlich, dass auch hier die Texturschärfe mit steigender Feststoffkonzentration abnimmt. Der Fehler aus der ODF-Rechnung (siehe Kap. 2.5.5) ist im Vergleich zur Rotationsküvette mit ca. 0,2fach regellos (siehe Anhang D) aufgrund der besseren Messbedingungen geringer. Gleiches gilt aber auch für den beobachteten Effekt. Betrachtet man die gemittelte Intensität (CTC20 $(\overline{\mathrm{ml} / \mathrm{s}})$, zeigt sich ein fast linearer Verlauf in Abhängigkeit von der Konzentration.

Die Messung von CTC20 bei $2 \mathrm{ml} / \mathrm{s}$ wurde nicht aufgetragen, da nur zwei 


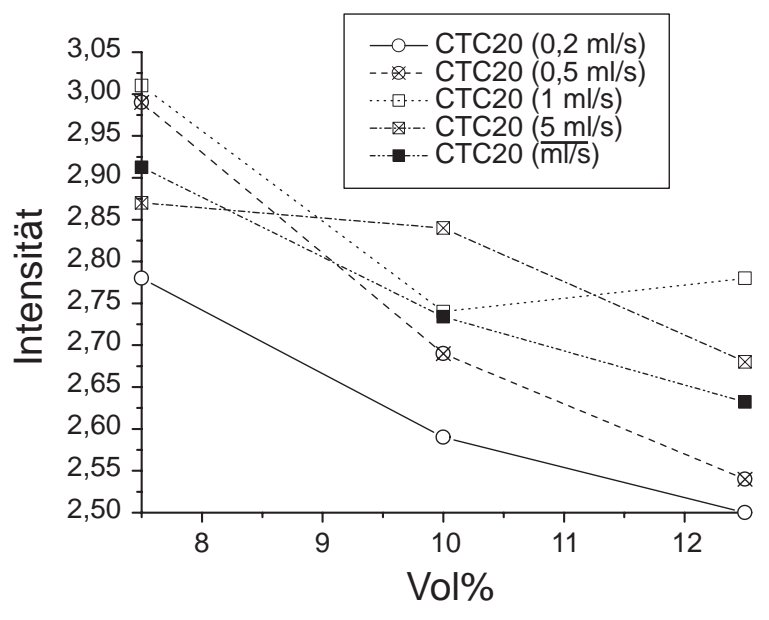

Abbildung 5.55: Intensität der Vorzugsorientierung bei $\{0,0\}$ von CTC20 für unterschiedliche Feststoffkonzentrationen

Messwerte zur Verfügung stehen. Der nicht stetig abnehmende Verlauf der Intensität von CTC20 bei $1 \mathrm{ml} / \mathrm{s}$ ist als Ausreißer zu bewerten. Die höchste und geringste Konzentration der $1 \mathrm{ml} / \mathrm{s}$-Messung spiegeln aber den Trend wieder.

Für beide Substanzen (CTC20 und Gilox) nimmt die mittlere Intensität über den gemessenen Bereich um ca. $10 \%$ ab. Die Abweichungen liegen zwar innerhalb der Messungenauigkeit, treten aber bei allen Proben auf, so dass der Zusammenhang zwischen Feststoffkonzentration und schärfe der Vorzugsorientierung der Partikel parallel zur Strömungsrichtung eindeutig ist.

\subsubsection{Zusammenfassung}

Die Vielzahl der Messungen mit der Durchflussküvette hat die Ergebnisse, welche mit der Rotationsküvette erzielt wurden bestätigt. Unterschiedliche Strömungsgeschwindigkeiten zeigen auch hier keinen systematischen Einfluss auf die Textur. Die Schwankungen der maximalen Intensität bei $\alpha=0^{\circ}$ und $\beta=0^{\circ}$ zwischen den einzelnen Geschwindigkeiten sind mit der Durchflussküvette jedoch deutlich stärker geworden. Auch eine weitere Untersuchung der Ebene senkrecht zur Basisebene hat keinen Einfluss der Strömungsgeschwindigkeit auf die Textur ergeben. Die insgesamt stärkeren Texturen machen auch die Unterschiede in der Partikelform deutlicher. Dieses gilt besonders für die eher geringen Unterschiede zwischen HVAFG und CTC20. Mit den Messungen der Durchflussküvette wurde bestätigt, dass mit steigender Feststoffkonzentration die Orientierungsdichte der Basisebene parallel zur Strömungsrichtung abnimmt. Außerdem zeigt sich, dass der geringere nicht messbare Bereich dazu führt, das ein rotationssymmetrisches Verhalten bei der Probe Gilox zu beobachten ist, welches mit der Rotationsküvette nicht möglich ist. 


\section{Kapitel 6}

\section{Diskussion}

Die Ergebnisse aus Kapitel 5 zeigen, dass die Methode der Polfigurmessung mit Hochenergetischer Synchrotronstrahlung sehr gut geeignet ist, um die Orientierung von kristallinen Partikeln in Flüssigkeitsströmungen zu bestimmen. Die hohe Intensität der Beamline BW5 am HASYLAB reduziert die Messzeit auf wenige Stunden. Andere Beugungsmethoden Mittels Röntgenröhre oder Neutronenstrahlung sind nicht geeignet, da aufgrund des geringen Flusses die Messzeit mehrere Wochen betragen würde.

Die beiden konstruierten Küvetten (siehe Kap. 4) haben über einen ausreichend großen Zeitraum eine konstante Strömung erzeugt, wobei sich die Durchflussküvette aufgrund fehlender beweglicher Teile als vorteilhaft erwiesen hat. Es kam auch nach mehr als 10 Stunden Betriebszeit zu keinerlei Undichtigkeiten, im Gegensatz zur Rotationsküvette. Auch Plexiglas als verwendetes Küvettenmaterial ist sehr gut geeignet, da es nur den amorphen Anteil des Beugungsdiagramms erhöht und keinerlei weitere texturierte Beugungsreflexe auftreten, welche die Messung stören. Mit beiden verwendeten Küvetten ist es möglich die Abhängigkeit der Textur von der Partikelform, der Strömungsgeschwindigkeit, der Feststoffkonzentration und dem Strömungsprofil zu untersuchen.

Texturentwicklung mit unterschiedlicher Partikelform Bei der Untersuchung des Einflusses unterschiedlicher Partikelformen auf die Textur stand die mehr oder weniger stark ausgeprägte Plättchenform (siehe Kap. 3) der einzelnen Partikel im Vordergrund. Jedoch galt es nicht einzelne Partikel in der Strömung zu charakterisieren, wie von Jeffery in Kapitel 1.3.2 beschrieben, sondern das Verhalten von Partikelgemengen. Orientierungen einzelner Partikel mit exakt definierter Form in einer strömenden Flüssigkeit lassen sich mit diesen Polfigurmessungen nicht beschreiben, da bei dieser Methode immer sehr viele Partikel gleichzeitig detektiert werden. Die Messung vieler Partikel gleichzeitig liefert eine Orientierungsverteilung, welche charakteristisch für das gesamte Gemisch ist.

Die Polfigurmessung mit Hochenergetischer Synchrotronstrahlung zeigt, wie sich die Partikel des Gemisches im Strömungsgradienten der Flüssigkeit durch starre Rotation einregeln. Somit ist sie auch deutlich besser geeignet, als die Methode von Brandon et al. [41] (siehe Kap. 1.4.3), da die Orientierung direkt in 
der Strömung bestimmt wird. Zwar wird auch bei dort eine Vorzugsorientierung für die Basisebene der plättchenförmigen Partikel parallel zur Strömungsrichtung gefunden, es wurde jedoch keine 3-dimensionale Texturanalyse gerechnet. So lassen sich die Aussagen über die Texturschärfe nicht miteinander vergleichen.

Die in Kapitel 1.4.3 beschriebene Orientierungsbestimmung mittels Neutronenbeugung von Clarke et al. [42] liefert im Vergleich mit der in dieser Arbeit durchgeführten Polfigurmessung auch nur einen kleinen Teil der Ergebnisse. Die von Clarke et al. [42] gemachte Annahme, dass sich die Partikel um die Normale der Basisebene frei drehen, konnte durch die Muskovitpolfiguren (Kap. 5.1) nicht bestätigt werden. Aufgrund des stark eingeschränkten Orientierungsbereichs bei der Messgeometrie von Clarke et al. [42] ist es leicht möglich eine Veränderung der Position des Maximum, wie bei den Muskovitmessungen in Kapitel 5.1 beobachtet, als eine Veränderung in der Texturschärfe zu deuten. Die Aussage, dass sich die Partikel mit ihrer Basisebene parallel zur Strömungsrichtung ausrichten stimmt aber mit den Ergebnissen dieser Arbeit überein.

Auch bei den ersten Messungen mit Hochenergetischer Synchrotronstrahlung an Gemischen aus Wasser und Kaolinit von Barè et al. [43] wurden keine Polfiguren gemessen. Die Beugungsbilder wurden für diese Messungen mit einem Flächendetektor für aufgenommen, so dass schon mehr Orientierungen als bei einem 1-dimensionalem Detektor bestimmt werden konnten. Diese zusätzliche Information wurde aber nicht berücksichtigt.

Die mit der Methode von Barè et al. erzielten Ergebnisse (siehe Kap.1.4.3 Abb. 1.21) zeigen, das sich die plättchenförmigen Partikel (ii) parallel zur Strömungsrichtung aufgrund des Strömungsgradienten ausrichten. Dies stimmt mit den Ergebnissen in Kapitel 5.2 überein. Da von Barè et al. [43] hierfür jedoch nur Beugungsbilder von drei unterschiedlichen Probenorientierungen (Abb. 1.20) zur Verfügung standen, ist eine Aussage über das Vorhandensein weiterer Orientierungen nicht möglich. Auch können so keine Aussagen über die freie Rotation der Plättchen in der Strömung gemacht werden. Hierzu ist die Polfigurmessung unerlässlich.

Für die Orientierung der Partikel (i) in Abbildung 1.21 ist nach Barè et al. die Küvettenwand der Ausschlag gebende Faktor. Da jedoch die Partikel, wie von Feng et al. [31] beschrieben, in einer Strömung eine Gleichgewichtsposition einnehmen, welche nicht direkt an der Wand ist (es bildet sich ein Schmierfilm aus), können auch andere Einflüsse diese Orientierung erzeugen. Da in der Durchflussküvette zwei Strömungsgradienten vorliegen, jeweils zwischen den Wänden im Strömungskanal, kann in den Randbereichen der Einfluss des zweiten Strömungsgradienten stärker werden und so für diese Orientierung verantwortlich sein.

Es wird hier allerdings noch eine weitere Orientierung der Plättchen (Abb. 1.22 a)) beschrieben, welche sich bei geringen Scherraten nahezu senkrecht zur Strömungsrichtung einregeln. Solche Unterschiede wurden bei allen in dieser Arbeit gemessenen Polfiguren nicht beobachtet. Da hier jedoch eine andere Küvettenform (siehe Kap. 4.1 Abb. 4.1) verwendet wurde lassen sich die Ergebnisse auch nicht in jedem Detail miteinander vergleichen.

Um jedoch die Orientierung der Basisebene vollständig zu beschreiben, und 
nicht nur einzelne Orientierungen, ist es notwendig mit den gemessenen Polfiguren durch eine 3-dimensionale Texturanalyse die vollständige Basisebenenpolfigur (001) zu berechnen. In allen bisherigen Arbeiten ist dies für strömende Zwei-Phasen-Gemische noch nicht geschehen.

In diesem Zusammenhang sind gerade die $\mathrm{Al}_{2} \mathrm{O}_{3}$-Pulver aufgrund der trigonalen Kristallstruktur besonders gut geeignet (siehe Kap. 5.2). Mit diesen Proben konnte erstmalig eine 3-dimensionale Texturanalyse für strömende ZweiPhasen-Gemische berechnet werden. Aus der 3-dimensionalen Texturanalyse lassen sich auch die (001)-Polfiguren, welche für $\mathrm{Al}_{2} \mathrm{O}_{3}$ nicht messbar sind, zurückrechnen. Der (001)-Reflex ist bei $\mathrm{Al}_{2} \mathrm{O}_{3}$ ein sehr schwacher Reflex, welcher außerdem bei einer Orientierung der $\{001\}$-Ebene parallel zur Strömungsrichtung für Probenwinkel $\omega=90^{\circ}$ die maximale Intensität hat. Diese Orientierung befindet sich im Zentrum der Polfigur und ist mit dieser Messgeometrie nicht zu erfassen.

Anhand der rückgerechneten Polfiguren wurde für unterschiedliche Partikelformen gezeigt, dass eine ausgeprägte Plättchenform zur schärferen Texturen bei gleichen Strömungsbedingungen führt. Dies ließ sich sowohl für die Rotationsküvette als auch für die Durchflussküvette nachweisen (siehe Kap. 5.2.4, 5.2.5 und 5.2.6).

Neben der Ausrichtung der Basisebene parallel zur Strömungsrichtung tritt in anderen Polfiguren eine weitere Vorzugsorientierung auf. Die Muskovitpartikel zeigen für den (111)-Reflex, welcher sich fast senkrecht zum Basisebenenreflex befindet, ein deutliches Maximum (Abb. 5.4, 5.8 und 5.10). Dieses Maximum weist auf eine in der Basisebene befindliche Vorzugsorientierung hin, so dass nicht von einer freien Rotation der Plättchen um ihre Normale der Basisebene ausgegangen werden kann. Auch in den rückgerechneten (110)-Polfiguren der $\mathrm{Al}_{2} \mathrm{O}_{3}$-Pulver ist solch eine Vorzugsorientierung zu beobachten. Die Position dieser Vorzugsorientierung ist jedoch für beide Küvetten gleich, obwohl sich die Strömungsrichtung ändert. Dieser Effekt könnte deshalb auch durch die Reihenentwicklung entstanden sein.

Die zur Reihenentwicklung ausgewählten Polfiguren (siehe Kap. 5.2.3) beinhalten die (110)-, (113)- und (116)-Polfiguren, welche einen ähnlichen Orientierungsbereich beschreiben. Die Zugrunde liegenden drei Beugungsreflexe unterscheiden sich lediglich in der Neigung zur c-Achse. Somit ist der Einfluss auf einen bestimmten Orientierungsbereich sehr hoch. Dies könnte zu einem Maximum in der rückgerechneten (110)-Polfigur führen, welches durch die Reihenentwicklung erzeugt wird. Da aber eine 3-dimensionale Texturanalyse mit anderen Polfiguren (Kap. 5.2.3) zu stark negativen Intensitäten führt, welche physikalisch nicht sinnvoll sind, kann auf die ausgewählten Polfiguren nicht verzichtet werden. Die Überprüfung anhand der Referenzprobe H1 hat ergeben, dass die zur Berechnung ausgewählten Polfiguren (104), (110), (113), (024), (116) und (003) zu den besten Ergebnissen führen (siehe Kap. 5.2.3). Aber auch mit der $\mathrm{H} 1$ Probe lässt sich nicht abschließend klären, ob sich bei den $\mathrm{Al}_{2} \mathrm{O}_{3}$-Proben eine Fasertextur mit der Faserachse parallel zur c-Achse ausgebildet haben könnte.

Die unterschiedlichen maximalen Intensitäten in den rückgerechneten (001)Polfiguren der einzelnen Proben sind aber dennoch miteinander vergleichbar. Da der nicht messbare Bereich bei gleichem Durchstrahlwinkel gleich ist, ist auch 
der Einfluss auf die berechnete Intensität immer gleich. Die beobachteten Unterschiede in der maximalen Intensität für eine Ausrichtung der Plättchen mit ihrer Basisebene parallel zur Strömungsrichtung $\left(\left\{0^{\circ}, 0^{\circ}\right\}\right.$ in der rückgerechneten (001)-Polfigur) können daher nicht durch die Reihenentwicklung erzeugt worden sein. Ein Vergleich der Texturschärfe anhand dieser rückgerechneten Polfiguren ist somit zulässig. Nur ein Vergleich der Texturschärfe zwischen den beiden Küvetten ist problematisch, da bauartbedingt unterschiedliche nicht messbare Bereiche zu Grunde liegen.

Die experimentelle Überprüfung neuer Simulationsergebnisse von Tsuji et al. [71], welche die Interaktionen von zwei gleichen Partikeln beschreiben, sowie die Berechnung von Prokunin [72] für die Bewegung von Partikeln an einer Wand, ist mit der hier verwendeten Orientierungsbestimmung nicht möglich. Auch der Einfluss von rauen Oberflächen auf das Strömungsverhalten der Flüssigkeit wie bei Pakdel und Kim [73] beschrieben, lässt sich nicht mit den in dieser Arbeit erzielten Ergebnissen belegen. Die Vorgänge an isolierten Teilchen in der Strömung tragen aber zur Texturbildung bei. Die in Kapitel 1.3 und 1.4 beschriebenen Zusammenhängen zwischen Teilchenform und Strömung von Jeffrey [6] und Anczurowski et al. [7, 39, 68] finden sich jedoch in den hier gemessenen Ergebnissen wieder. In allen durchgeführten Experimenten ließ sich nachweisen, dass sich die plättchenförmigen Partikel mit der Basisebene parallel zur Strömungsrichtung ausrichten.

Texturentwicklung bei unterschiedlicher Strömungsgeschwindigkeit Die Untersuchung des Einflusses der Strömungsgeschwindigkeit auf die Textur teilt sich in zwei Aspekte auf. Eine Änderung der Strömungsgeschwindigkeit kann eine Änderung der Vorzugsorientierung bewirken und/oder eine Änderung der Texturschärfe. Die Vorzugsorientierungen lassen sich teilweise direkt an den gemessenen Polfiguren beobachten. Zur Bestimmung der Texturschärfe ist die Berechnung der Orientierungsverteilungsfunktion unerlässlich.

Die in Kapitel 5.1 gezeigten Polfiguren für die Muskovitpartikel sind zur Bestimmung der Partikelorientierung direkt aus den gemessenen Daten besonders gut geeignet, da aufgrund der extremen Plättchenform die Vorzugsorientierungen sehr stark sind. Eine Änderung der Vorzugsorientierung mit der Strömungsgeschwindigkeit konnte jedoch nur für die Fraktion $63-125 \mu \mathrm{m}$ beobachtet werden. Da die Partikelgröße einen Einfluss auf den Grad der Partikelbewegung [12] in einer Strömung hat, ist ein sensitiveres Verhalten der größeren Muskovitpartikel, hinsichtlich der Abhängigkeit der Textur von der Strömungsgeschwindigkeit möglich. Außerdem erfahren größere Partikel im gleichen Strömungsfeld einen größeren Gradienten der Strömungsgeschwindigkeit, welches ebenfalls das unterschiedliche Verhalten der beiden Korngrößenfraktionen erklären kann.

Die von Brown et al. [44] mittels Diffraktion beobachtete Veränderung in der Orientierungsdichte (Kap. 1.4.3 Abb. 1.23 lässt sich aber auch auf eine Änderung der Orientierung des Maximums zurückführen, wie sie bei den Muskovitmessungen in Kapitel 5.1 beobachtet wurde. Verändert sich die Position des Maximums ändert sich die Intensität in dem von Brown et al. [44] gemessenen Bereich. Um sicherzustellen, dass tatsächlich eine Veränderung in der Ori- 
entierungsdichte vorliegt ist es notwendig den gesamten Orientierungsraum zu betrachten. Eine sehr gute Möglichkeit bietet die 3-dimensionale Texturanalyse, da die Ergebnisse normiert sind (siehe Kap. 2.5.3) und somit auch unterschiedliche Messungen miteinander verglichen werden können. Zur Berechnung stehen für die Muskovitgemische unter den vorhandenen Messbedingungen jedoch nicht genügend Polfiguren zur Verfügung (siehe Kap. 5.1). Diese Berechnungen waren nur mit den $\mathrm{Al}_{2} \mathrm{O}_{3}$-Proben durchführbar, welche jedoch insgesamt aufgrund der schwach ausgeprägten Plättchenform zu schwachen Texturen führen und somit nicht direkt mit den Ergebnissen der Muskovitmessungen vergleichbar sind. Effekte, die bei den Muskovitmessungen zu beobachten waren, sind bei den schwachen Texturen der $\mathrm{Al}_{2} \mathrm{O}_{3}$-Pulver nicht mehr messbar.

Alle gemessenen $\mathrm{Al}_{2} \mathrm{O}_{3}$-Proben, sowohl in der Rotationsküvette als auch in der Durchflussküvette, zeigen geringe Schwankungen in der maximalen Intensität der (001)-Polfigur. Es ist jedoch keine systematische Ab- oder Zunahme des Maximums mit steigender Strömungsgeschwindigkeit zu erkennen. Somit lässt sich für die $\mathrm{Al}_{2} \mathrm{O}_{3}$-Proben zeigen, dass die Strömungsgeschwindigkeit keinen messbaren Einfluss auf die Orientierungsdichte für die Basisebene parallel zur Strömungsrichtung hat. Sollten solche Abhängigkeiten dennoch vorhanden sein, sind sie unterhalb des Auflösungsvermögens der 3-dimensionalen Texturanalyse.

Veränderungen in der Position des Maximums, sowohl für die (001)-Polfigur als auch die (110)-Polfigur, sind bei allen $\mathrm{Al}_{2} \mathrm{O}_{3}$-Messungen nicht zu beobachten. Auch eine Änderung der Texturschärfe in Abhängigkeit von der Strömungsgeschwindigkeit ist bei den $\mathrm{Al}_{2} \mathrm{O}_{3}$-Partikeln nicht zu beobachten. Die deutlich schwächer ausgeprägte Plättchenform der Partikel führt aber auch insgesamt zu schwachen Texturen, bei denen Abhängigkeiten, so fern vorhanden, unterhalb des Auflösungsvermögens der Polfigurmessung und der Reihenentwicklung liegen könnten.

Texturentwicklung bei unterschiedlicher Feststoffkonzentration Eine weitere untersuchte Einflussgröße auf die Strömungstextur ist die Feststoffkonzentration. Erste Orientierungsmessungen wurden zwar mit unterschiedlichen Feststoffkonzentrationen durchgeführt [43, 42], eine Gegenüberstellung der ermittelten Orientierungsparameter führt jedoch nicht zu einem eindeutigen Ergebnis. Die von Clarke et al. [42] bestimmten Ordnungsparameter wurden, wie zuvor beschrieben, ohne Polfiguren zu messen berechnet. Für die Messung wurde die verwendete Küvette nur um eine Achse gedreht. Auf der Grundlage eines Modells von Straley [74], welches einen Ordnungsparameter für zweiachsige Partikel beschreibt, wurde von einer Rotationssymmetrie ausgehend, dieser Parameter aus den Beugungsdaten ermittelt. Um den Einfluss der Feststoffkonzentration auf die Partikelorientierung zu beschreiben sind die von Clarke et al. [42] und Barè et al. [43] durchgeführten Untersuchungen nicht ausreichend, da die Ergebnisse aus den Muskovitmessungen in Kapitel 5.1 gezeigt haben, dass die Rotationssymmetrie nicht unbedingt gegeben ist.

Für die untersuchten Feststoffkonzentrationen ließ sich jedoch durch die Texturbestimmung an $\mathrm{Al}_{2} \mathrm{O}_{3}$-Partikeln und anschließender 3-dimensionaler Textur- 
analyse erstmals nachweisen, dass mit steigender Feststoffkonzentration die Orientierungsdichte der Basisebene parallel zur Strömungsrichtung schwächer wird. Diese Abhängigkeiten sind, wie in Kapitel 5.2.4, 5.2.5 und 5.2.6 beschrieben, zwar nur schwach, so dass es sich auch um eine zufällige Schwankung innerhalb der Messgenauigkeit handeln könnte. Da dieser Effekt aber für alle $\mathrm{Al}_{2} \mathrm{O}_{3}$ Messungen sowohl in der Rotationsküvette als auch in der Durchflussküvette, beobachtet wurde, ist diese Abhängigkeit tatsächlich vorhanden. Die bereits beschriebene Problematik der unvollständig gemessenen Polfiguren, kann einen solchen Effekt nicht künstlich erzeugen. 


\section{Kapitel 7}

\section{Zusammenfassung und Ausblick}

Erstmalig wurden in dieser Arbeit an strömenden Zwei-Phasen-Gemischen Polfiguren gemessen und die 3-dimensionale Texturanalyse durchgeführt. Sowohl die Polfigurmessung als auch die 3-dimensionale Texturanalyse gehen weit über bisherige Untersuchungen in diesem Gebiet hinaus. Der Texturmessplatz an der Hochenergie Beamline BW5 des HASYLAB/DESY ermöglicht durch den Einsatz eines Flächendetektors Polfigurmessungen, bei denen die Probe, im Unterschied zur konventionellen Messmethode, nur um eine Achse gedreht werden muss und somit zu kürzeren Messzeiten führt. Außerdem konnten die zur Erzeugung der Strömung benötigten Küvetten Dank dieser Messmethode mit einem deutlich geringeren Aufwand konstruiert werden.

Mit dieser Methode konnte neben der Orientierung der Basisebene von plättchenförmigen Partikeln parallel zur Strömungsrichtung eine weitere Vorzugsorientierung bei den Muskovitpartikeln bestimmt werden. Diese Orientierung zeigt, dass die Rotationssymmetrie um die Normale der Basisebene nicht, wie bisher angenommen, immer für plättchenförmige Partikel gegeben ist, sondern dass sich nach dem Einregeln der Basisebene diese noch um ihre Normale orientieren. Darüber hinaus konnte für sehr große Muskovitplättchen nachgewiesen werden, dass die Position dieser Vorzugsorientierung von der Strömungsgeschwindigkeit abhängig ist.

Die 3-dimensionale Texturanalyse ermöglicht es unterschiedliche Messungen hinsichtlich der Texturschärfe miteinander zu vergleichen. So konnte für $\mathrm{Al}_{2} \mathrm{O}_{3}$ der Einfluss von der Partikelform, der Strömungsgeschwindigkeit und der Feststoffkonzentration untersucht werden. Partikel mit stark ausgeprägter Plättchenform zeigten bei allen Untersuchungen stärkere Orientierungsdichten der Basisebene parallel zur Strömungsrichtung. Auch eine geringere Feststoffkonzentration führt zu höheren Orientierungsdichten der Basisebene. Eine Abhängigkeit der Textur von der Strömungsgeschwindigkeit konnte für $\mathrm{Al}_{2} \mathrm{O}_{3}$ Pulver nicht nachgewiesen werden. Sollte sie vorhanden sein, ist sie sehr schwach.

Zur theoretischen Beschreibung des Verhaltens von kristallinen Partikeln in einer strömenden Flüssigkeit ist Muskovit besser geeignet. Aufgrund der monoklinen Kristallsymmetrie gibt es weniger symmetrisch äquivalente Ori- 
entierungen in einer Polfigur. Neuere Messplätze, wie sie z.B. bei Petra III am HASYLAB in Hamburg in Zukunft zur Verfügung stehen werden, liefern die Möglichkeit die Polfiguren mit einer höheren Auflösung in $2 \theta$ zu messen. So werden auch für monokline Materialien genügend Polfiguren auswertbar sein um die Orientierungsverteilungsfunktion zu berechnen.

Ein weiterer Aspekt ist der nicht messbare Bereich in den Polfiguren, welche die Texturberechnung der $\mathrm{Al}_{2} \mathrm{O}_{3}$-Proben beeinflusst. Dieser Einfluss lässt sich durch Umbaumassnahmen an den beiden Küvetten noch weiter verringern. Ab einem Durchstrahlwinkel von $\omega=80^{\circ}$ sind aber die Grenzen der Messmethode erreicht, da die Küvetten aufgrund der Druckfestigkeit eine gewisse Materialstärke nicht unterschreiten dürfen und sonst Teile des Küvettenrands im Strahlengang sind. Die Größe des nicht messbaren Bereichs würde sich aber halbieren, welches zu verbesserten Ergebnissen für die Texturbestimmung führen sollte. 


\section{Literaturverzeichnis}

[1] S. Luding, Langkorn- oder Kurzkornreis? Phys. Bl. 52 (1996) 203. 7

[2] V. Frette, K. Christensen, A. Malthe-Sørensen, J. Feder, T. Jøssang, P. Meakin, Avalanche Dynamics in a Pile of Rice Nature 379 (1996) 49. 7

[3] H.M. Jäger, S.R. Nagel, Physics of Granular State, Science 255 (1992) 1523. 7

[4] G.K. Batchelor, Sedimentation in a Dilute Polydisperse System of Interacting Spheres. Part1. General Theory, J. Fluid Mech. 119 (1982) 379. 7

[5] W. Skrotzki, Mechanisms of Texture Development in Rocks, Textures of Geological Materials, DGM Informationsgesellschaft, (1994) 167. 7

[6] G.B. Jeffery, The Motion of Ellipsoidal Particles in a Viscous Fluid, Proc. Roy. Soc. (London) A102 (1923) 161. 7, 15, 16, 22, 24, 25, 96, 102, 107, 126

[7] E. Anczurowski, S.G. Mason, The Kinetics of Flowing Dispersions, II. Equilibrium Orientations of Rods and Discs (Theoretical), J. Colloid Interface Sci. 23 (1967) 522. 7, 16, 18, 77, 96, 102, 107, 116, 126, 137

[8] G.K. Batchelor, Transport Properties of Two-Phase Materials with Random Structure, Ann. Rev. Fluid Mech. 6 (1974) 227. 7

[9] G.K. Batchelor, Brownian Diffussion of Particles with Hydrodynamic Interaction, J. Fluid Mech. 74 (1976) 1. 7

[10] R. Herczyński, I. Pieńkowska, Toward a Statistical Theory of Suspension, Ann. Rev. Fluid Mech. 12 (1980) 237. 7

[11] R.G. Avery, J.D.F. Ramsay, Colloidal Properties of Synthetic Hectorite Clay Dispersions, II. Light and Small Angle Neutron Scattering, J. Colloid Interface Sci. 109 (1986) 448. 7

[12] J.R. Abbott, N. Tetlow, A.L. Graham, S.A. Altobelli, Eiichi Fukushima, L.A. Mondy, T.S. Stephens, Experimental Observations of Particle Migration in Concentrated Suspensions: Couette Flow, J. Rheol. 35 (5) (1991) 773. 8, 20, 21, 126, 137 
[13] S.W. Sinton, A.W. Chow, NMR Flow Imaging of Fluids and Solid Suspensions in Poiseuille Flow, J. Rheol. 35 (5) (1991) 735. 8, 20, 21, 22, 111,137

[14] S. Ashdown, I. Marković, R.H. Ottewill, P. Lindner, R.C. Oberthür, A.R. Rennie, Small-Angle Neutron-Scattering Studies on Ordered Polymer Colloid Dispersions, Langmuir 6 (1990) 303. 8, 26

[15] A. Böcker, Einfluss von Material und Verarbeitung auf die Texturbildung in $\mathrm{Al}_{2} \mathrm{O}_{3}$, Dissertation TU-Clausthal 1994. 8, 55, 59, 80, 95

[16] R. Bouchard, D. Hupfeld, T. Lippmann, J. Neuefeind, H. Neumann, H.F. Poulsen, U. Rütt, T. Schmidt, J.R. Schneider, J.Süssenbach, M. von Zimmermann, A Triple-Crystal Diffratometer for High-Energy Synchrotron Radiation at the HASYLAB High-Field Wiggler Beamline BW5, J. Synchrotron Rad. 5 (1998) 90. 8, 32

[17] H.F. Poulsen, S. Garbe, T. Lorentzen, D. Juul-Jensen, W.F. Poulsen, N.H. Anderson, T. Frello, R. Feidenhansl, H. Graafsma, Applications of HighEnergy Synchrotron Radiation for Structural Studies of Polycrystalline Materials, J. Synchrotron Rad. 4 (1997) 147. 8

[18] P. Suortti and T. Tschentscher High energy scattering beamlines at European Synchrotron Radiation Facility Rev. Sci. Instrum. 66 (1995) 1798 . 8

[19] L. Wcislak, H. Klein, H.J. Bunge, U. Garbe, T. Tschentscher, J.R. Schneider, Texture Analysis with High-Energy Synchrotron Radiation, J. Appl. Cryst. 35 (2002) 82. 8, 29, 32, 33, 138

[20] H.J. Bunge, H. Klein, L. Wcislak, U. Garbe, W. Weiß, J.R. Schneider, High-Resolution Imaging of Texture and Microstructure by the Moving Detector Method, Texture Microstruct. 35 (2003) 253. 8

[21] H.J. Bunge, L. Wcislak, H. Klein, U. Garbe, J.R. Schneider, Texture and Microstructure Imaging in Six Dimensions with High-Energy Synchrotron Radiation, J. Appl. Cryst. 36 (2003) 1240. 8, 37, 138

[22] M. Griebel, T. Dornseifer, T. Neunhoeffer, Numerische Simulation in der Strömungslehre, Vieweg 1995. 9, 12, 13

[23] C. Gerthsen, H.O. Kneser, H. Vogel, Physik, Springer-Verlag Berlin 1989. $9,10,14,15,137$

[24] H. Sigloch, Technische Fluidmechanik, Springer-Verlag Berlin 2003. 10, $11,13,14,137$

[25] A. Magerl, H. Zabel, B. Frick, P. Lindner, Flow Dynamics of Sheared Liquids Explored by Inelastic Neutron Scattering, Appl. Phys. Lett. 74 No. 23 (1999) 3475. 10, 137 
[26] L. Prandtl, K. Oswatitsch, K. Wieghardt, Führer durch die Strömungslehre, Vieweg 1993. 12

[27] B. Cantwell, Organized Motion in Turbulent Flow, Ann. Rev. Fluid Mech. 13 (1981) 457. 12, 137

[28] S.F. Grace, Free Motion of a Sphere in a Rotating Liquid parallel to the Axis of Rotation, Proc. Roy. Soc. (London) A102 (1923) 89. 15

[29] G.I. Taylor, The Motion of a Sphere in a Rotating Liquid, Proc. Roy. Soc. (London) A102 (1923) 180. 15

[30] M. Manga, Orientation Distribution of Microlites in Obsidian, J. Volcanol. Geotherm. Res. 86 (1998) 107. 17, 137

[31] Z.-G. Feng, E.E. Michaelides, Equilibrium Position for a Particle in a Horizontal Shear Flow, J. Multiphase Flow 29 (2003) 943. 19, 26, 124, 137

[32] H.H. Hu, Direct Simulation of Flows of Solid-Liquid Mixtures, J. Multiphase Flow 22 (1996) 335. 19, 20, 137

[33] Y. Hardalupas, S. Horender, Fluctuations of Particle Concentration in a Turbulent Two-Phase Shear Layer, J. Multiphase Flow 29 (2003) 1645. 19

[34] V.M. Alipchenkov, L.I. Zaichik, Particle Clustering in Isotropic Turbulent Flow, Fluid Dynamics 38 (2003) 417. 19

[35] I.L. Claeys, J.F. Brady, Suspensions of Prolate Spheroids in Stokes Flow. Part 1. Dynamic of a Finite Number of Particles in an Unbounded Fluid, J. Fluid Mech. 251 (1993) 411. 20

[36] I.L. Claeys, J.F. Brady, Suspensions of Prolate Spheroids in Stokes Flow. Part 2. Statistically Homogeneous Dispersions, J. Fluid Mech. 251 (1993) 443. 20

[37] I.L. Claeys, J.F. Brady, Suspensions of Prolate Spheroids in Stokes Flow. Part 3. Hydrodynamic Transport Properties of Crystalline Dispersions, J. Fluid Mech. 251 (1993) 479. 20

[38] T. Tran-Cong, N. Phan-Thien, Stokes Problems of Multipartikel Systems: A Numerical Method for Arbitary Flows, Phys. Fluids A 1 (1989) 453. 20

[39] E. Anczurowski, S.G. Mason, The Kinetics of Flowing Dispersions, III. Equilibrium Orientations of Rods and Discs (Experimental), J. Colloid Interface Sci. 23 (1967) 533. 22, 23, 24, 25, 77, 96, 102, 126, 137, 138

[40] M. Sintubin, H.-R. Wenk, D.S. Phillips, Texture Development in Platy Materials: Comparsion of Bi2223 Aggregates with Phyllosilicate Fabrics, Mat. Sci. Eng. A202 (1995) 157. 25 
[41] D. Brandon, D. Chen, H.Chan, Control of Texture in Monolithic Alumina, Mat. Sci. Eng. A195 (1995) 189. 25, 123

[42] S.M. Clarke, A.R. Rennie, P. Convert, A Diffraction Technique to Investigate the Orientational Alignment of Anisotropic Particles: Studies of Clay Under Flow, Europhys. Lett. 35 (1996) 233. 25, 124, 127

[43] S. Barè, J.K. Cockcroft, S.L. Colston, A. Jupe and A.R. Rennie, X-ray study of the orientational order of a concentrated dispersion of kaolinte under flow, J. Appl. Cryst. 34 (2001) 573. 25, 26, 65, 66, 69, 124, 127, 138,139

[44] A.B.D. Brown, A.R. Rennie, Monodisperse colloidal plates under shear, Phys. Rev. E 62 (2000) 851. 26, 27, 28, 63, 126, 138, 139

[45] A.B.D. Brown, S.M. Clarke, P. Convert, A.R. Rennie, Oreintational order in concentrated dispersions of plate-like particles under shear, J. Rheol. 44 (2000) 221. 27, 63, 64, 69, 139

[46] A.B.D. Brown, S.M. Clarke, A.R. Rennie, Shear Induced Alignment of Kaolinite: Studies Using a Diffraction Technique, Progr. Colloid Polym. Sci. 110 (1998) 80. 27

[47] G. Wassermann, J. Grewen, Texturen metallischer Werkstoffe, Berlin: Springer Verlag 1962. 29

[48] H.J. Bunge, H.R. Wenk, J. Pannetier, Neutron Diffraction Texture Analysis, Texture Microstruct. 5 (1982) 153. 29

[49] R.A. Schwarzer, Crystal Textur Analysis by Means of Electron Diffraction, Advances and Applications of Quantitative Texture Analysis, DGM Informationsgesellschaft, (1989) 51. 29

[50] S.M. Jogun, C.F. Zukowski, Rheology and Microstructure of Dense Suspensions of Plate-Shaped Colloidal Particles, J. Rheol. 43 (1999) 847. 29

[51] H. Klein, E. Dahlem-Klein \& N.J. Park, Program System, ODF-Analysis, Hrsg.: H.J. Bunge, Cuvillier Verlag Göttingen 1993. 30, 47, 49, 50, 53, 138

[52] L.G. Schulz, A Direct Method of Determining Preferred Orientation of a Flat Reflection Sample Using a Geiger Counter X-Ray Goniometer, J. Appl. Cryst. 20 (1949) 1030. 30, 91

[53] B.F. Decker, E.T. Asp, D. Harker, Preferred Orientation Determination Using a Geiger Counter X-Ray Goniometer, J. Appl. Phys. 19 (1948) 388. 30

[54] L. Wcislak Texture Analysis with a Position Sensitive DetectorHrsg.: H.J. Bunge, Cuvillier Verlag Göttingen 1996. 30, 138 
[55] H.J. Bunge, H. Klein, Determination of Quantitative, High-Resolution Pole Figures with the Area Detector, Z. Metallkd. 87 (1996) 465. 36, 53, 138, 139

[56] A.P. Hammersley,FIT2D http://www.esrf.fr/computing/scientific/fit2d (1997). 38, 70

[57] W.H. Press, S.A. Teukolsky, W.T. Vetterling, B.P. Flannery, Numerical Recipes in C, Cambridge University Press, (1992) 671. 39

[58] W.H. Press, S.A. Teukolsky, W.T. Vetterling, B.P. Flannery, Numerical Recipes in C, Cambridge University Press, (1992) 681. 39

[59] B.F. Decker, Absorption Corrections in X-Ray Examinations of Preferred Orientation in Flat Sheet Specimens, Int. Tables f. X-Ray Cryst. Volume II (1985) 301. 41, 42, 138

[60] S. Brennan and P.L. Cowan, A suite of programs for calculating x-ray absorption, reflection and diffraction performance for a variety of materials at arbitrary wavelengths Rev. Sci. Instrum. 63 (1992) 850 . 42

[61] W. Skrotzki, B. Klöden, R. Tamm, C.-G.Oertel,U. Garbe und E. Rybacki, Torsion Texture Measurement with High-Energy Synchrotron Radiation on NiAl, Textures a. Microstructures 35 (2003) 253. 45, 138

[62] H.J. Bunge, Texture Analysis in Materials Science, Cuvillier Verlag Göttingen 1993. 46, 47, 49, 52, 53, 138

[63] W. Kleber, H.-J. Bautsch, J. Bohm,Einführung in die Kristallographie, Verlag Technik GmbH Berlin 1990. 47, 55, 58, 139

[64] W. Schaumann, Der neue BLV Steine- und Mineralienführer, München: BLV Verlagsgesellschaft 1994. 55, 56, 139

[65] Matthies, Mineralogie, Springer Verlag 1993. 59, 139

[66] A.B.D. Brown, C. Ferrero, T. Narayanan, A.R. Rennie, Phase Seperation and Structure in a Concentrated Colliodal Dispersion of Uniform Plates, Eur. Phys. J. B 11 (1999) 481. 69, 73, 74, 139

[67] M. Günther, Muskovitabbildung, http://www.mineralogischesammlungen.de/muskovit.html (2004). 74, 139

[68] E. Anczurowski, R.G. Cox, S.G. Mason, The Kinetics of Flowing Dispersions, IV. Transient Orientations of Cylinders, J. Colloid Interface Sci. 23 (1967) 547. 77, 126

[69] A. Böcker, H. Klein, H.J. Bunge, Development of Cross-Rolling Textures in AlMn1, Texture Microstruct. 12 (1990) 155. 81, 83

[70] Mar Research (1998). Image Plate Detecting System MAR-345. Technical report. 114 
[71] T. Tsuji, R. Narutomi, T. Yokomine, S Ebara, A. Shimizu, Unsteady Three-Dimensional Simulation of Interactions between Flow and two Particles, J. Multiphase Flow 29 (2003) 1431. 126

[72] A.N. Prokunin, On a Paradox in the Motion of a Rigid Particle along a Wall in a Fluid, Fluid Dyn. 38 (2003) 443. 126

[73] P. Pakdel, S. Kim, Mobility and Stresslet Function of Particles with Rough Surfaces in Viscous Fluids: A Numerical Study, J. Rheol. 35 (1991) 797. 126

[74] J.P. Straley, Ordered Phases of a Liquid of Biaxial Particles, Phys. Rev. A, 10 (1974) 1881. 127

[75] ICDD JCPDS-International Centre for Diffraction Data. PCPDFWIN v. 1.30 (1997). 141, 147, 148 


\section{Abbildungsverzeichnis}

1.1 Scherströmung zwischen einer festen Wand und einer bewegten Platte . . . . . . . . . . . . . . . . 10

1.2 Geschwindigkeitsverteilung einer Scherströmung für eine Flüssigkeit mit schwachen Adhäsionskräften [25] . . . . . . . . . 10

1.3 Geschwindigkeitsverlauf $v=v(r)$ bei laminarer Rohrströmung [24] 11

1.4 Anlaufströmung [24] . . . . . . . . . . . . . . . . . . 11

1.5 Mechanische Ähnlichkeit von Strömungen [27] . . . . . . . . . . . 12

1.6 Geschwindigkeitsverteilung der turbulenten Strömung in einem Rohr mit dem Durchmesser $D[24] \ldots . . . . . . . .13$

1.7 Volumenelement in einer inhomogenen Strömung [23] . . . . . . . 14

1.8 Geschwindigkeitsprofil um eine Kugel, die von einer viskosen Flüssigkeit umströmt wird $[23] \ldots \ldots \ldots \ldots$

1.9 Rotationsellipsoid im kartesischen Koordinatensystem . . . . . . 15

1.10 Darstellung der Flugbahnen für ein Stäbchen in einer Scherströmung $\left(r_{e}>1\right)$ für unterschiedliche Werte der Orbitkonstante

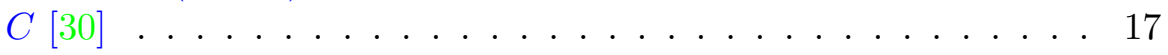

1.11 Stereographische Projektion der Bewegung eines Partikels in einer Scherströmung mit $x$ als Strömungsrichtung und y als Gradientenrichtung für die drei Hauptebenen [7] . . . . . . . . . . 18

1.12 Simulierte Flugbahn eines Partikels als Funktion der Reynolds$z a h l[31] \ldots \ldots \ldots \ldots \ldots$

1.13 Simulation der Fließbewegung von 400 Teilchen in einer periodischen Zellen [32] . . . . . . . . . . . . . . . . . . . . . 20

1.14 Grad der Partikelbewegung für unterschiedliche Partikelgrößen bei $50 \mathrm{Vol} \%$ Feststoffkonzentration [12] . . . . . . . . . . . . 21

1.15 Geschwindigkeitsverteilung der Rohrströmung für verschiedene Feststoffkonzentrationen $[13] \ldots \ldots$. . . . . . . . . 22

1.16 Integrale Orientierungsverteilung $P\left(\phi_{i}\right)$ als Funktion der Winkel $\phi_{i}$ (siehe Abb. 1.11) für a) Stäbchen und b) Plättchen in stark verdünnten Systemen [39] . . . . . . . . . . . . . . . . 23

1.17 Integrale Orientierungsverteilung $P \theta_{i}$ als Funktion der Winkel $\theta_{i}$ (siehe Abb. 1.11) für a) Stäbchen und b) Plättchen in stark verdünnten Systemen [39] . . . . . . . . . . . . . . . . 23

1.18 Projektion der Enden der Polarachsen von Stäbchen mit dem Radienverhältnis $r_{e}=20.8$ nach Anczurowski et al. [39] . . . . . 24 
1.19 Projektion der Enden der Polarachsen von Plättchen mit dem Radienverhältnis $r_{e}=0,26$ nach Anczurowski et al. [39] . . . . . 24

1.20 Unterschiedliche Probenorientierung bei Orientierungsmessungen an strömenden Kaolinit/Wasser Gemischen von Barè et al. [43] . 26

1.21 Schematische Darstellung der Orientierung von Kaolinitpartikeln in einer Strömung nach Barè et al. [43] . . . . . . . . . . . . . . . 26

1.22 a) Orientierung bei geringer Scherrate b) Orientierung bei hoher Scherrate nach Brown et al. [44] . . . . . . . . . . . . . 27

1.23 Intensität für den Basisebenereflex für unterschiedliche Scherraten $0.22 s^{-1}(\bigcirc), 1.0 s^{-1}(\bullet), 1.1 s^{-1}(\mathbf{\square}), 3.6 s^{-1}(\bullet), 8.4 s^{-1}(\mathbf{\Lambda})$, $23 s^{-1}(\mathbf{v})$ nach Brown et al. [44] . . . . . . . . . . . . 28

2.1 Texturgoniometer mit Eulerwiege [51] . . . . . . . . . . . 30

2.2 Texturmessung nach a) dem Rückstrahlverfahren und b) dem Durchstrahlverfahren [54] . . . . . . . . . . . . . . 30

2.3 Messbare Bereiche in der Polfigur nach a) dem Rückstrahlverfahren und b) dem Durchstrahlverfahren . . . . . . . . . . . 31

2.4 Polfigurmessraster a) Winkeltreu und b) Flächentreu . . . . . . . 31

2.5 Schematische Darstellung des Texturmessplatzes BW5 am HASYLAB [19] . . . . . . . . . . . . . . . . . . 32

2.6 Spektrum des Wiggler des BW5 in Abhängigkeit von Photonenfluss (mit u. ohne Kupferfenster) [19] . . . . . . . . . . . . . 33

2.7 Plexiglasquader . . . . . . . . . . . . . . . . . . . 34

2.8 Beugungsbilder von Muskovit/Glyzerin Gemischen a) 10Vol\% b) 20 Vol\% Feststoffkonzentration . . . . . . . . . . . . 34

2.9 Aufteilung eines Beugungsbildes zur Polfigurauswertung . . . . . 35

2.10 Schematische Darstellung der Beugungsvektors [55] . . . . . . . 36

2.11 Schematische Darstellung der Polfigurpositionen $\{\alpha, \beta\}$ in Abhängigkeit von $\omega$ und $\gamma[21] \ldots \ldots \ldots . \ldots . \ldots . \ldots . \ldots 37$

2.12 Die Polfigursoftware . . . . . . . . . . . . . . . . . 39

2.13 Schematische Darstellung des Strahlwegs [59] . . . . . . . . . 42

2.14 Korrekturfaktor der Absorption für unterschiedliche Absorptionskoeffizienten . . . . . . . . . . . . . . . 4 43

2.15 Korrigierte amorphe Intensitäten . . . . . . . . . . . . . 44

$2.162 \theta$-Position für den Wert der $f(2 \theta)$ aus der Untergrundanpassung 44

2.17 Polfigurausschnitt des Messrasters und des äquidistanten Rasters

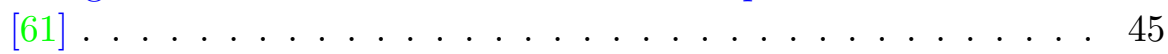

2.18 Probenfestes Koordinatensystem $K_{A}$ und kristallfestes Koordinatensystem $K_{B}[62] \ldots \ldots \ldots \ldots \ldots$

2.19 Definition der Eulerschen Winkel $\left\{\varphi_{1}, \phi, \varphi_{2}\right\}[62] \ldots . . . . .47$

2.20 Eulerraum: a) einzelne Orientierung b) Orientierung aller Kristallite in der Probe c) Orientierungsverteilung [62] . . . . . . . 47

2.21 Anzahl der linear unabhängigen Polfiguren der verschiedenen Symmetrien in Abhängigkeit vom Reihenentwicklungsgrad $l$. . . 51

2.22 Annäherung der unstetigen Funktion $f(g)$ durch ihre Dichtever-

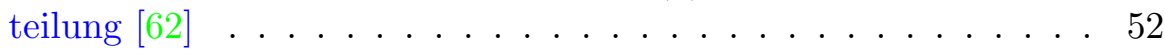


2.23 Darstellung des mittleren Betrags der Koeffizienten $\overline{C_{l}}$ und des Fehlers $\Delta C_{l}[55] \ldots \ldots \ldots \ldots \ldots \ldots$

3.1 Kristallstruktur von Muskovit [63] . . . . . . . . . . . 55

3.2 Natürliches Muskovitmineral [64] . . . . . . . . . . . . . . 56

3.3 Durchlichtmikroskopische Aufnahme des Muskovitpulvers . . . . 56

3.4 Korngrößenverteilung der Muskovitpulverfraktion $<63 \mu \mathrm{m}$. . . . 57

3.5 Korngrößenverteilung der Muskovitpulverfraktion $63-125 \mu \mathrm{m}$. . 57

3.6 Kristallstruktur von $\mathrm{Al}_{2} \mathrm{O}_{3}[63] \ldots \ldots \ldots \ldots$

3.7 Kristallhabitus von Korund [65] . . . . . . . . . . . . . . . . . 59

3.8 REM-Aufnahmen für die Probe A . . . . . . . . . . . . . 60

3.9 REM-Aufnahmen für die Probe B . . . . . . . . . . . . . . 60

3.10 REM-Aufnahmen für die Probe $\mathrm{C} \ldots \ldots$. . . . . . . . 61

3.11 REM-Aufnahme der Probe HVAFG . . . . . . . . . . . 61

3.12 REM-Aufnahme der Probe CTC20 . . . . . . . . . . . . . . 62

3.13 REM-Aufnahme der Probe Gilox . . . . . . . . . . . . . . . . 62

4.1 Scherküvette mit rotierenden Zylindern [44] . . . . . . . . . . 63

4.2 Schematische Darstellung einer Rotationsküvette im Strahlen-

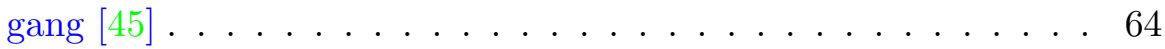

4.3 Die Rotationsküvette . . . . . . . . . . . . . . . . . . . . 64

4.4 Die Rotationsküvette in schematischer Darstellung . . . . . . . . 65

4.5 Spezielle Dichtung der Rotationsküvette . . . . . . . . . . . 65

4.6 Schematische Darstellung der Orientierungsbestimmung von ZweiPhasen-Gemischen mittels Spritzenpumpe [43] . . . . . . . 66

4.7 Die Durchflussküvette . . . . . . . . . . . . . . . . . 66

4.8 Schematische Darstellung der Durchflussküvette . . . . . . . . . 67

5.1 Beugungsbild von Muskovit (Korngröße $<63 \mu \mathrm{m}$ ) in Glyzerin . . 69

5.2 Beugungsdiagramme für Muskovit . . . . . . . . . . . . 70

5.3 Gemessener (dunkelgrau) und nicht gemessener Bereich (hellgrau) in der Polfigur . . . . . . . . . . . . . . . 71

5.4 Muskovitpolfiguren $(<63 \mu m)$ gemessen bei a) $0 \mathrm{U} / \mathrm{min}$ b) eine Drehung c) $1 \mathrm{U} / \mathrm{min}$ d) $4 \mathrm{U} / \mathrm{min} \ldots . . .72$

5.5 Agglomerat hexagonaler Nickel(II)Hydroxid Partikel nach Brown et al. [66] . . . . . . . . . . . . . . . . . . 74

5.6 Pseudohexagonales Muskovitpartikel [67] . . . . . . . . . . . . 74

5.7 Schematische Darstellung der Orientierung eines Muskovitteilchens in einer Polfigur für die Rotationsküvette . . . . . . . . . 75

5.8 Muskovitpolfiguren (Korngröße 63-125 $\mu \mathrm{m}$ ) gemessen bei a) $0 \mathrm{U} / \min$ b) $0.1 \mathrm{U} / \min$ c) $0.3 \mathrm{U} / \min$ d) $1 \mathrm{U} / \min \ldots . . .76$

5.9 Darstellung des Orientierungswinkels $\gamma$ in Abhängigkeit von der Rotationsgeschwindigkeit ("'guide to the eye"') . . . . . . . 77

5.10 Muskovitpolfiguren (Korngröße $<63 \mu \mathrm{m}$ ) gemessen bei unterschiedlichen Fliessgeschwindigkeiten a) $0,2 \mathrm{ml} / \mathrm{s}$ b) $0,5 \mathrm{ml} / \mathrm{s}$ c) $1 m l \ldots \ldots \ldots \ldots \ldots \ldots \ldots \ldots$ 
5.11 Schematische Darstellung der Orientierung eines Muskovitteilchen in einer Polfigur im Strömungsfeld der Durchflussküvette . . 79

5.12 Beugungsbild von $\mathrm{Al}_{2} \mathrm{O}_{3}$-Partikeln in Glyzerin in der Rotationsküvette . . . . . . . . . . . . . . 80

5.13 Beugungsdiagramme für $\mathrm{Al}_{2} \mathrm{O}_{3} \ldots \ldots \ldots \ldots$. . . . . . . 81

$5.14\left|C_{l}^{m n}\right|$ für $l=6$ und $m=1$ für die Proben A,B,C in der Rotationsküvette . . . . . . . . . . . . . . 83

5.15 C-Koeffizienten $l=6$ für die Proben CTC20, Gilox und HVAFG in der Rotationsküvette . . . . . . . . . . . . . . . 83

5.16 C-Koeffizienten $l=6$ für die Proben CTC20, Gilox und HVAFG in der Durchflussküvette . . . . . . . . . . . . . . . . 84

5.17 Darstellung der kleinsten asymmetrischen Einheit einer rückgerechneten Polfigur mit orthorhombischer Probensymmetrie . . . 85

5.18 Ergebnisse der ODF-Rechnung für die Probe Gilox (10Vol\%) in der Rotationsküvette bei $1 \mathrm{ml} / \mathrm{s}$ Fluss . . . . . . . . . . . . 86

5.19 Polfigurschnitt bei $\beta=0^{\circ}$ für verschiedene $L_{\max }$ von HVAFG in der Durchflussküvette . . . . . . . . . . . . . . . 86

5.20 Polfigurschnitt bei $\beta=0^{\circ}$ für verschiedene $L_{\max }$ von CTC20 in der Durchflussküvette . . . . . . . . . . . . . . . 87

5.21 Polfigurschnitt bei $\beta=0^{\circ}$ für verschiedene $L_{\max }$ von Gilox in der Durchflussküvette . . . . . . . . . . . . . . 87

5.22 Gemessene und rückgerechnete Polfiguren der Probe B (36Vol\%) (Rotationsküvette $0,25 \mathrm{U} / \mathrm{min} \ldots \ldots$. . . . . . . . 88

5.23 Gemessene und rückgerechnete Polfiguren von Gilox (13Vol\%) der Rotationsküvette bei 1U/min . . . . . . . . . . . . . . 89

5.24 Gemessene und rückgerechnete Polfiguren von Gilox (10Vol\%) der Durchflussküvette bei $1 \mathrm{ml} / \mathrm{s}$. . . . . . . . . . . . . . . 90

5.25 Schematische Darstellung des nicht messbaren Bereichs nach dem Durchstrahlverfahren . . . . . . . . . . . . . . . . . 91

5.26 Rückgerechnete Polfiguren der Proben H1 aus konventionell röntgenographisch ermittelten Daten nach dem Reflexionsverfahren . 91

5.27 Rückgerechnete Polfiguren der Proben H1 aus 6 gemessen Polfiguren in der Rotationsküvette . . . . . . . . . . . . . . . . . . 92

5.28 Rückgerechnete Polfiguren der Proben H1 aus 4 gemessen Polfiguren in der Rotationsküvette . . . . . . . . . . . . . . . . . . . 93

5.29 Horizontaler (001)-Polfigurschnitt in Querrichtung der Probe H1 in der Rotationsküvette . . . . . . . . . . . . . . . . . 93

5.30 (001)-Polfiguren der Proben A,B,C bei $1 \mathrm{U} / \mathrm{min}$. . . . . . . . . . 96

5.31 (001)-Polfigurschnitt in Scherrichtung der Proben A,B,C bei 0,25 $\mathrm{U} / \mathrm{min} \ldots \ldots \ldots \ldots \ldots \ldots$

5.32 (001)-Polfigurschnitt senkrecht zur Scherrichtung der Proben A,B,C bei $0,25 \mathrm{U} / \mathrm{min} \ldots \ldots \ldots \ldots \ldots \ldots$

5.33 (110)-Polfiguren der Proben A,B,C bei 1U/min . . . . . . . . . 98

5.34 (001)-Polfigurschnitt in Scherrichtung der Proben A für unterschiedliche Strömungsgeschwindigkeiten . . . . . . . . . . . 99

5.35 (110)-Polfigurschnitt bei $\alpha=90^{\circ}$ der Proben A für unterschiedliche Strömungsgeschwindigkeiten . . . . . . . . . . . . . 100 
5.36 (001)-Polfigurschnitt senkrecht zu SR der Proben B unterschiedlichen Feststoffkonzentrationen . . . . . . . . . . . . . . 101

5.37 schematische Darstellung der Braggblende im Strahlengang . . . 104

5.38 (001)-Polfiguren der Proben HVAFG, CTC20 und Gilox bei 1U/min 105

5.39 (001)-Polfigurschnitte senkrecht zu SR der Proben HVAFG, CTC20

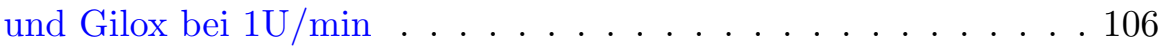

5.40 (001)-Polfigurschnitte in Richtung SR der Proben HVAFG, CTC20 und Gilox bei $1 \mathrm{U} / \mathrm{min} \ldots \ldots \ldots$. . . . . . . . . 106

5.41 (110)-Polfiguren der Proben HVAFG, CTC20 und Gilox bei 1U/min 107

5.42 (001)-Polfigurschnitte senkrecht zu SR der Proben CTC20 mit $13 \mathrm{Vol} \% \ldots \ldots \ldots \ldots \ldots$

5.43 (110)-Polfigurschnitte mit $\alpha=90^{\circ}$ der Proben CTC20 bei unterschiedlichen Strömungsgeschwindigkeiten . . . . . . . . . . 109

5.44 001)-Polfigurschnitt senkrecht zu SR von CTC20 bei unterschiedlichen Feststoffkonzentrationen . . . . . . . . . . . . . . 110

5.45 001)-Polfigurschnitt senkrecht zu SR von HVAFG bei unterschiedlichen Feststoffkonzentrationen . . . . . . . . . . . . . . . . 110

5.46 Intensität der Vorzugsorientierung bei $\{0,0\}$ von HVAFG und CTC20 für unterschiedliche Feststoffkonzentrationen . . . . . . . 111

5.47 (001)-Polfiguren der Proben HVAFG, CTC20 und Gilox bei 1ml/s114

5.48 (001)-Polfigurschnitte in Richtung FR der Proben HVAFG, CTC20 und Gilox bei 1ml/s . . . . . . . . . . . . . . . . 115

5.49 (001)-Polfigurschnitte senkrecht zu FR der Proben HVAFG, CTC20

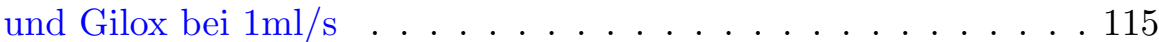

5.50 (001)-Polfiguren der Proben HVAFG, CTC20 und Gilox bei 1ml/s116

5.51 (001)-Polfigurschnitte senkrecht zu FR der Proben Gilox bei unterschiedlichen Strömungsgeschwindigkeiten . . . . . . . . . 117

5.52 (110)-Polfigurschnitte mit $\alpha=90^{\circ}$ der Proben Gilox bei unterschiedlichen Strömungsgeschwindigkeiten . . . . . . . . . 118

5.53 (001)-Polfigurschnitte senkrecht zu FR der Proben Gilox bei unterschiedlichen Strömungsgeschwindigkeiten . . . . . . . . . . 119

5.54 Intensität der Vorzugsorientierung bei $\{0,0\}$ von Gilox für unterschiedliche Feststoffkonzentrationen . . . . . . . . . . . . 120

5.55 Intensität der Vorzugsorientierung bei $\{0,0\}$ von CTC20 für unterschiedliche Feststoffkonzentrationen . . . . . . . . . . . 121

A.1 Auszug aus Pulverdatenbank JCPDS für Muskovit [75] . . . . . . 147

A.2 Auszug aus Pulverdatenbank JCPDS für $\mathrm{Al}_{2} \mathrm{O}_{3}[75] \ldots \ldots$. . . . 148

B.1 gemessene Polfiguren Probe A 36Vol\% 0,25 U/min . . . . . . . . 150

B.2 Rückgerechnete Polfiguren, C-Koeffizienten und Fehler der Probe A $36 \mathrm{Vol} \%, 0,25 \mathrm{U} / \mathrm{min} \ldots \ldots \ldots \ldots . \ldots \ldots 151$

B.3 gemessene Polfiguren Probe A 36Vol\% 0,5 U/min . . . . . . . . . 152

B.4 Rückgerechnete Polfiguren, C-Koeffizienten und Fehler der Probe A $36 \mathrm{Vol} \% 0,5 \mathrm{U} / \mathrm{min} \ldots \ldots \ldots \ldots . \ldots \ldots 3$

B.5 gemessene Polfiguren Probe A 36Vol\% 1 U/min . . . . . . . . . 154 
B.6 Rückgerechnete Polfiguren, C-Koeffizienten und Fehler der Probe A $36 \mathrm{Vol} \% 1 \mathrm{U} / \mathrm{min} \ldots \ldots \ldots \ldots . \ldots \ldots \ldots$

B.7 gemessene Polfiguren Probe B 36Vol\% 0,25 U/min . . . . . . 156

B.8 Rückgerechnete Polfiguren, C-Koeffizienten und Fehler der Probe

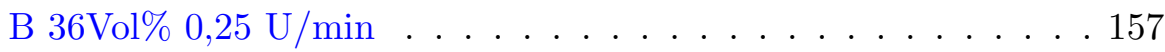

B.9 gemessene Polfiguren Probe B 36Vol\% 0,5 U/min . . . . . . . . 158

B.10 Rückgerechnete Polfiguren, C-Koeffizienten und Fehler der Probe

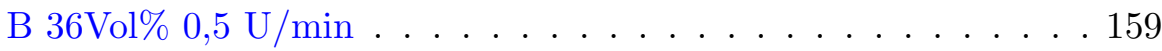

B.11 gemessene Polfiguren Probe B 36Vol\% 1 U/min . . . . . . . . . 160

B.12 Rückgerechnete Polfiguren, C-Koeffizienten und Fehler der Probe B $36 \mathrm{Vol} \% 1 \mathrm{U} / \mathrm{min} \ldots \ldots \ldots 16 \ldots$

B.13 gemessene Polfiguren Probe B 24Vol\% 0,25 U/min . . . . . . . 162

B.14 Rückgerechnete Polfiguren, C-Koeffizienten und Fehler der Probe B $24 \mathrm{Vol} \% 0,25 \mathrm{U} / \mathrm{min} \ldots \ldots \ldots 3$

B.15 gemessene Polfiguren Probe B 24Vol\% 0,5 U/min . . . . . . . . 164

B.16 Rückgerechnete Polfiguren, C-Koeffizienten und Fehler der Probe B $24 \mathrm{Vol} \% 0,5 \mathrm{U} / \mathrm{min} \ldots \ldots \ldots \ldots$

B.17 gemessene Polfiguren Probe B 24Vol\% 1 U/min . . . . . . . . . 166

B.18 Rückgerechnete Polfiguren, C-Koeffizienten und Fehler der Probe B $24 \mathrm{Vol} \% 1 \mathrm{U} / \mathrm{min} \ldots \ldots \ldots$. . . . . . . . . . . 167

B.19 gemessene Polfiguren Probe C 28Vol\% 0,25 U/min . . . . . . . 168

B.20 Rückgerechnete Polfiguren, C-Koeffizienten und Fehler der Probe $\mathrm{C} 28 \mathrm{Vol} \% 0,25 \mathrm{U} / \mathrm{min} \ldots \ldots \ldots$

B.21 gemessene Polfiguren Probe C 28Vol\% 0,5 U/min . . . . . . . 170

B.22 Rückgerechnete Polfiguren, C-Koeffizienten und Fehler der Probe $\mathrm{C} 28 \mathrm{Vol} \% 0,5 \mathrm{U} / \mathrm{min} \ldots \ldots \ldots \ldots . \ldots \ldots 171$

B.23 gemessene Polfiguren Probe C 28Vol\% 1 U/min . . . . . . . . . 172

B.24 Rückgerechnete Polfiguren, C-Koeffizienten und Fehler der Probe

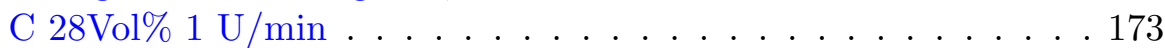

C.1 Gemessene Polfiguren HVAFG 26Vol\% 0,5 U/min . . . . . . . . 176

C.2 Rückgerechnete Polfiguren, C-Koeffizienten und Fehler, HVAFG

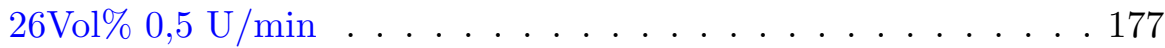

C.3 Gemessene Polfiguren HVAFG 26Vol\% 1 U/min . . . . . . . . . 178

C.4 Rückgerechnete Polfiguren, C-Koeffizienten und Fehler, HVAFG

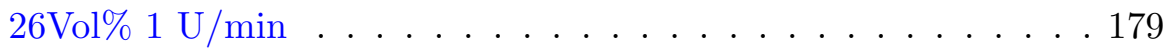

C.5 Gemessene Polfiguren HVAFG 26Vol\% 2 U/min . . . . . . . . . . 180

C.6 Rückgerechnete Polfiguren, C-Koeffizienten und Fehler, HVAFG $26 \mathrm{Vol} \% 2 \mathrm{U} / \mathrm{min} \ldots \ldots \ldots . \ldots . \ldots . \ldots 181$

C.7 Gemessene Polfiguren HVAFG 13Vol\% 0,5 U/min . . . . . . . . 182

C.8 Rückgerechnete Polfiguren, C-Koeffizienten und Fehler, HVAFG

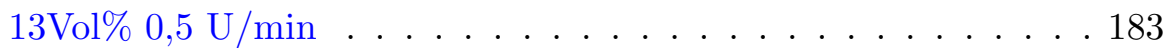

C.9 Gemessene Polfiguren HVAFG $13 \mathrm{Vol} \% 1 \mathrm{U} / \mathrm{min}$. . . . . . . . . . 184

C.10 Rückgerechnete Polfiguren, C-Koeffizienten und Fehler, HVAFG $13 \mathrm{Vol} \% 1 \mathrm{U} / \mathrm{min} \ldots \ldots \ldots . \ldots \ldots$

C.11 Gemessene Polfiguren HVAFG 9Vol\% 0,5 U/min . . . . . . . 186 
C.12 Rückgerechnete Polfiguren, C-Koeffizienten und Fehler, HVAFG $9 \mathrm{Vol} \%$ 0,5 U/min . . . . . . . . . . . . . . . . . 187

C.13 Gemessene Polfiguren HVAFG 9Vol\% 1 U/min . . . . . . . . . 188

C.14 Rückgerechnete Polfiguren, C-Koeffizienten und Fehler, HVAFG

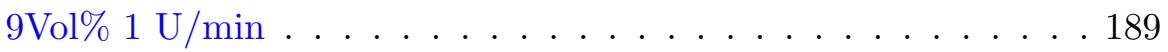

C.15 Gemessene Polfiguren HVAFG 9Vol\% 2 U/min . . . . . . . . . 190

C.16 Rückgerechnete Polfiguren, C-Koeffizienten und Fehler, HVAFG

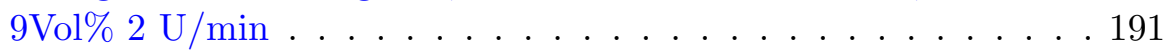

C.17 Gemessene Polfiguren CTC20 26Vol\% 0,5 U/min . . . . . . . . . 192

C.18 Rückgerechnete Polfiguren, C-Koeffizienten und Fehler, CTC20

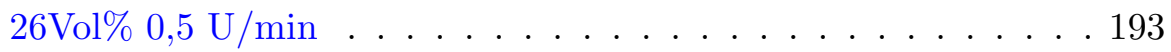

C.19 Gemessene Polfiguren CTC20 26Vol\% 1 U/min . . . . . . . . . . 194

C.20 Rückgerechnete Polfiguren, C-Koeffizienten und Fehler, CTC20 $26 \mathrm{Vol} \% 1 \mathrm{U} / \mathrm{min} \ldots \ldots \ldots \ldots . \ldots \ldots$

C.21 Gemessene Polfiguren CTC20 26Vol\% 2 U/min . . . . . . . . . . 196

C.22 Rückgerechnete Polfiguren, C-Koeffizienten und Fehler, CTC20 $26 \mathrm{Vol} \% 2 \mathrm{U} / \mathrm{min} \ldots \ldots \ldots \ldots . \ldots \ldots$

C.23 Gemessene Polfiguren CTC20 13Vol\% 0,5 U/min . . . . . . . . . 198

C.24 Rückgerechnete Polfiguren, C-Koeffizienten und Fehler, CTC20

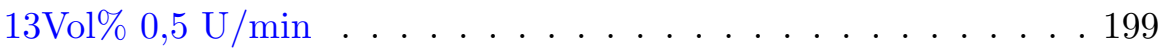

C.25 Gemessene Polfiguren CTC20 13Vol\% 1 U/min . . . . . . . . . 200

C.26 Rückgerechnete Polfiguren, C-Koeffizienten und Fehler, CTC20

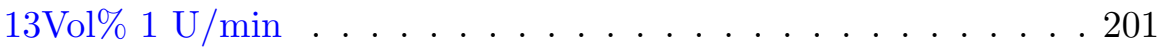

C.27 Gemessene Polfiguren CTC20 13Vol\% 2 U/min . . . . . . . . . . 202

C.28 Rückgerechnete Polfiguren, C-Koeffizienten und Fehler, CTC20 $13 \mathrm{Vol} \% 2 \mathrm{U} / \mathrm{min} \ldots . . \ldots . \ldots . \ldots . . \ldots 203$

C.29 Gemessene Polfiguren CTC20 9Vol\% 0,5 U/min . . . . . . . . . . 204

C.30 Rückgerechnete Polfiguren, C-Koeffizienten und Fehler, CTC20

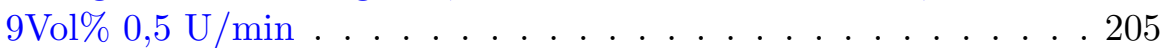

C.31 Gemessene Polfiguren CTC20 9Vol\% 1 U/min . . . . . . . . . . 206

C.32 Rückgerechnete Polfiguren, C-Koeffizienten und Fehler, CTC20 9Vol\% $1 \mathrm{U} / \mathrm{min} \ldots \ldots \ldots . \ldots . \ldots . \ldots 207$

C.33 Gemessene Polfiguren CTC20 9Vol\% 2 U/min . . . . . . . . . . . 208

C.34 Rückgerechnete Polfiguren, C-Koeffizienten und Fehler, CTC20

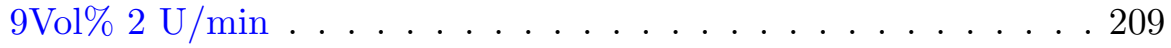

C.35 Gemessene Polfiguren Gilox 13Vol\% 0,5 U/min . . . . . . . . . 210

C.36 Rückgerechnete Polfiguren, C-Koeffizienten und Fehler, Gilox 13Vol\%

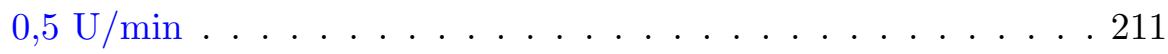

C.37 Gemessene Polfiguren Gilox 13Vol\% 1 U/min . . . . . . . . . . 212

C.38 Rückgerechnete Polfiguren, C-Koeffizienten und Fehler, Gilox 13Vol\% $1 \mathrm{U} / \min \ldots \ldots \ldots \ldots \ldots \ldots . \ldots \ldots \ldots$

C.39 Gemessene Polfiguren Gilox 13Vol\% 2 U/min . . . . . . . . . . . 214

C.40 Rückgerechnete Polfiguren, C-Koeffizienten und Fehler, Gilox 13Vol\%

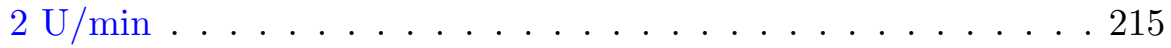

D.1 Gemessene Polfiguren HVAFG $10 \mathrm{Vol} \%$ 0,2 ml/s . . . . . . . . . 218 
D.2 Rückgerechnete Polfiguren, C-Koeffizienten und Fehler, HVAFG

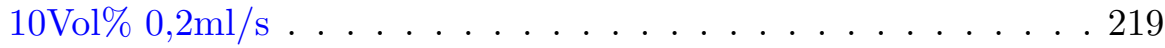

D.3 Gemessene Polfiguren HVAFG $10 \mathrm{Vol} \%$ 0,5 ml/s . . . . . . . . . 220

D.4 Rückgerechnete Polfiguren, C-Koeffizienten und Fehler, HVAFG

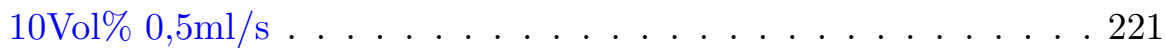

D.5 Gemessene Polfiguren HVAFG $10 \mathrm{Vol} \% 1 \mathrm{ml} / \mathrm{s}$. . . . . . . . . . . 222

D.6 Rückgerechnete Polfiguren, C-Koeffizienten und Fehler, HVAFG $10 \mathrm{Vol} \% 1 \mathrm{ml} / \mathrm{s} \ldots \ldots \ldots \ldots . \ldots . \ldots . \ldots . \ldots . \ldots 223$

D.7 Gemessene Polfiguren HVAFG $10 \mathrm{Vol} \% 2 \mathrm{ml} / \mathrm{s}$. . . . . . . . . . . 224

D.8 Rückgerechnete Polfiguren, C-Koeffizienten und Fehler, HVAFG

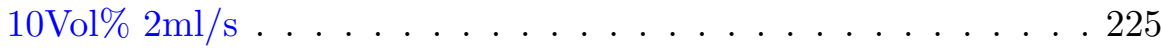

D.9 Gemessene Polfiguren HVAFG 10Vol\% $5 \mathrm{ml} / \mathrm{s} \ldots \ldots \ldots . . . .226$

D.10 Rückgerechnete Polfiguren, C-Koeffizienten und Fehler, HVAFG $10 \mathrm{Vol} \% 5 \mathrm{ml} / \mathrm{s} \ldots \ldots \ldots \ldots . \ldots . \ldots . \ldots 227$

D.11 Gemessene Polfiguren HVAFG 12,5Vol\% 0,2 ml/s . . . . . . . . 228

D.12 Rückgerechnete Polfiguren, C-Koeffizienten und Fehler, HVAFG $12,5 \mathrm{Vol} \% 0,2 \mathrm{ml} / \mathrm{s} \ldots \ldots \ldots \ldots \ldots$

D.13 Gemessene Polfiguren HVAFG 12,5Vol\% 0,5 ml/s . . . . . . . . 230

D.14 Rückgerechnete Polfiguren, C-Koeffizienten und Fehler, HVAFG $12,5 \mathrm{Vol} \% 0,5 \mathrm{ml} / \mathrm{s} \ldots \ldots \ldots \ldots \ldots \ldots \ldots$

D.15 Gemessene Polfiguren HVAFG $12,5 \mathrm{Vol} \% 1 \mathrm{ml} / \mathrm{s}$. . . . . . . . . . 232

D.16 Rückgerechnete Polfiguren, C-Koeffizienten und Fehler, HVAFG $12,5 \mathrm{Vol} \% 1 \mathrm{ml} / \mathrm{s} \ldots \ldots \ldots \ldots . \ldots \ldots . \ldots \ldots$

D.17 Gemessene Polfiguren HVAFG $12,5 \mathrm{Vol} \% 2 \mathrm{ml} / \mathrm{s}$. . . . . . . . . 234

D.18 Rückgerechnete Polfiguren, C-Koeffizienten und Fehler, HVAFG

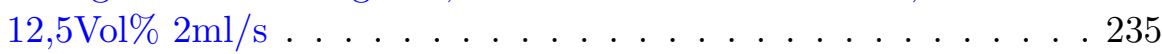

D.19 Gemessene Polfiguren HVAFG $12,5 \mathrm{Vol} \% 5 \mathrm{ml} / \mathrm{s}$. . . . . . . . . 236

D.20 Rückgerechnete Polfiguren, C-Koeffizienten und Fehler, HVAFG $12,5 \mathrm{Vol} \% 5 \mathrm{ml} / \mathrm{s} \ldots \ldots \ldots \ldots \ldots \ldots . \ldots \ldots \ldots$

D.21 Gemessene Polfiguren CTC20 7,5Vol\% 0,2 ml/s . . . . . . . . 238

D.22 Rückgerechnete Polfiguren, C-Koeffizienten und Fehler, CTC20 $7,5 \mathrm{Vol} \% 0,2 \mathrm{ml} / \mathrm{s} \ldots \ldots \ldots \ldots \ldots \ldots$

D.23 Gemessene Polfiguren CTC20 7,5Vol\% 0,5 ml/s . . . . . . . . . 240

D.24 Rückgerechnete Polfiguren, C-Koeffizienten und Fehler, CTC20 $7,5 \mathrm{Vol} \% 0,5 \mathrm{ml} / \mathrm{s} \ldots \ldots \ldots \ldots \ldots \ldots \ldots$

D.25 Gemessene Polfiguren CTC20 7,5Vol\% $1 \mathrm{ml} / \mathrm{s}$. . . . . . . . . . 242

D.26 Rückgerechnete Polfiguren, C-Koeffizienten und Fehler, CTC20 $7,5 \mathrm{Vol} \% 1 \mathrm{ml} / \mathrm{s} \ldots \ldots \ldots \ldots \ldots . \ldots \ldots . \ldots \ldots$

D.27 Gemessene Polfiguren CTC20 7,5Vol\% $2 \mathrm{ml} / \mathrm{s}$. . . . . . . . . . . 244

D.28 Rückgerechnete Polfiguren, C-Koeffizienten und Fehler, CTC20

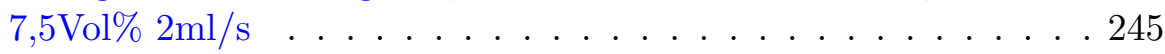

D.29 Gemessene Polfiguren CTC20 7,5Vol\% $5 \mathrm{ml} / \mathrm{s}$. . . . . . . . . . . 246

D.30 Rückgerechnete Polfiguren, C-Koeffizienten und Fehler, CTC20 $7,5 \mathrm{Vol} \% 5 \mathrm{ml} / \mathrm{s} \ldots \ldots \ldots \ldots \ldots \ldots \ldots . \ldots \ldots \ldots$

D.31 Gemessene Polfiguren CTC20 10Vol\% 0,2 ml/s . . . . . . . . . 248

D.32 Rückgerechnete Polfiguren, C-Koeffizienten und Fehler, CTC20

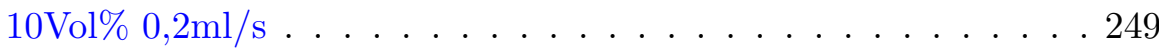


D.33 Gemessene Polfiguren CTC20 10Vol\% 0,5 ml/s . . . . . . . . . 250

D.34 Rückgerechnete Polfiguren, C-Koeffizienten und Fehler, CTC20

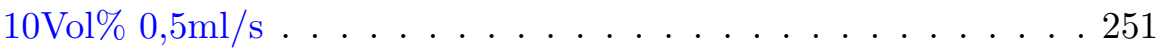

D.35 Gemessene Polfiguren CTC20 10Vol\% $1 \mathrm{ml} / \mathrm{s} \ldots . . . . . . .252$

D.36 Rückgerechnete Polfiguren, C-Koeffizienten und Fehler, CTC20 $10 \mathrm{Vol} \% 1 \mathrm{ml} / \mathrm{s} \ldots \ldots \ldots \ldots \ldots . \ldots \ldots . \ldots \ldots$

D.37 Gemessene Polfiguren CTC20 10Vol\% $2 \mathrm{ml} / \mathrm{s} \ldots \ldots . . . . . .254$

D.38 Rückgerechnete Polfiguren, C-Koeffizienten und Fehler, CTC20

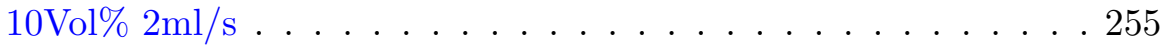

D.39 Gemessene Polfiguren CTC20 10Vol\% $5 \mathrm{ml} / \mathrm{s} \ldots \ldots . . . . .256$

D.40 Rückgerechnete Polfiguren, C-Koeffizienten und Fehler, CTC20 $10 \mathrm{Vol} \% 5 \mathrm{ml} / \mathrm{s} \ldots \ldots \ldots \ldots \ldots \ldots . \ldots \ldots \ldots$

D.41 Gemessene Polfiguren CTC20 12,5Vol\% 0,2 ml/s . . . . . . . 258

D.42 Rückgerechnete Polfiguren, C-Koeffizienten und Fehler, CTC20 $12,5 \mathrm{Vol} \% 0,2 \mathrm{ml} / \mathrm{s} \ldots \ldots \ldots \ldots \ldots$

D.43 Gemessene Polfiguren CTC20 12,5Vol\% 0,5 ml/s . . . . . . . . 260

D.44 Rückgerechnete Polfiguren, C-Koeffizienten und Fehler, CTC20 $12,5 \mathrm{Vol} \% 0,5 \mathrm{ml} / \mathrm{s} \ldots \ldots \ldots \ldots \ldots \ldots$

D.45 Gemessene Polfiguren CTC20 12,5Vol\% $1 \mathrm{ml} / \mathrm{s}$. . . . . . . . . . 262

D.46 Rückgerechnete Polfiguren, C-Koeffizienten und Fehler, CTC20 $12,5 \mathrm{Vol} \% 1 \mathrm{ml} / \mathrm{s} \ldots \ldots \ldots \ldots . \ldots \ldots . \ldots \ldots$

D.47 Gemessene Polfiguren CTC20 12,5Vol\% $2 \mathrm{ml} / \mathrm{s}$. . . . . . . . . . 264

D.48 Rückgerechnete Polfiguren, C-Koeffizienten und Fehler, CTC20 $12,5 \mathrm{Vol} \% 2 \mathrm{ml} / \mathrm{s} \ldots \ldots \ldots \ldots \ldots$

D.49 Gemessene Polfiguren CTC20 12,5Vol\% $5 \mathrm{ml} / \mathrm{s} \ldots . . . . .266$

D.50 Rückgerechnete Polfiguren, C-Koeffizienten und Fehler, CTC20 $12,5 \mathrm{Vol} \% 5 \mathrm{ml} / \mathrm{s} \ldots \ldots \ldots \ldots \ldots \ldots \ldots \ldots \ldots$

D.51 Gemessene Polfiguren Gilox 7,5Vol\% 0,2 ml/s . . . . . . . . 268

D.52 Rückgerechnete Polfiguren, C-Koeffizienten und Fehler, Gilox 7,5Vol\% $0,2 \mathrm{ml} / \mathrm{s} \ldots \ldots \ldots \ldots \ldots \ldots \ldots \ldots$

D.53 Gemessene Polfiguren Gilox 7,5Vol\% 0,5 ml/s . . . . . . . . 270

D.54 Rückgerechnete Polfiguren, C-Koeffizienten und Fehler, Gilox 7,5Vol\%

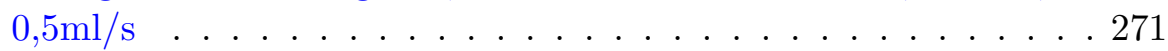

D.55 Gemessene Polfiguren Gilox 7,5Vol\% $1 \mathrm{ml} / \mathrm{s} \ldots \ldots . . . . . .272$

D.56 Rückgerechnete Polfiguren, C-Koeffizienten und Fehler, Gilox 7,5Vol\% $1 \mathrm{ml} / \mathrm{s} \ldots \ldots \ldots \ldots \ldots \ldots \ldots \ldots$

D.57 Gemessene Polfiguren Gilox 7,5Vol\% $2 \mathrm{ml} / \mathrm{s} \ldots \ldots . . . . .274$

D.58 Rückgerechnete Polfiguren, C-Koeffizienten und Fehler, Gilox 7,5Vol\%

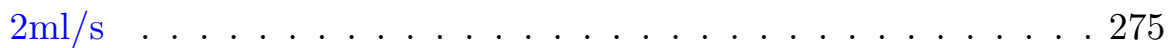

D.59 Gemessene Polfiguren Gilox 7,5Vol\% $5 \mathrm{ml} / \mathrm{s} \ldots \ldots . . .276$

D.60 Rückgerechnete Polfiguren, C-Koeffizienten und Fehler, Gilox 7.5Vol\% $5 \mathrm{ml} / \mathrm{s} \ldots \ldots \ldots \ldots \ldots \ldots \ldots \ldots \ldots$

D.61 Gemessene Polfiguren Gilox 10Vol\% 0,2 ml/s . . . . . . . . . 278

D.62 Rückgerechnete Polfiguren, C-Koeffizienten und Fehler, Gilox 10Vol\%

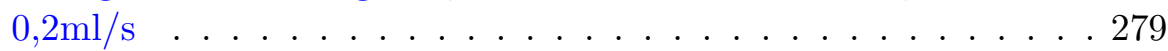

D.63 Gemessene Polfiguren Gilox 10Vol\% 0,5 ml/s . . . . . . . . . 280 
D.64 Rückgerechnete Polfiguren, C-Koeffizienten und Fehler, Gilox 10Vol\%

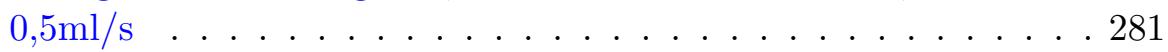

D.65 Gemessene Polfiguren Gilox 10Vol\% $1 \mathrm{ml} / \mathrm{s}$. . . . . . . . . . 282

D.66 Rückgerechnete Polfiguren, C-Koeffizienten und Fehler, Gilox 10Vol\% $1 \mathrm{ml} / \mathrm{s} \ldots \ldots \ldots \ldots \ldots \ldots . \ldots \ldots 28 \ldots \ldots \ldots$

D.67 Gemessene Polfiguren Gilox 10Vol\% $2 \mathrm{ml} / \mathrm{s}$. . . . . . . . . . . 284

D.68 Rückgerechnete Polfiguren, C-Koeffizienten und Fehler, Gilox 10Vol\% $2 \mathrm{ml} / \mathrm{s} \ldots \ldots \ldots \ldots \ldots \ldots \ldots \ldots$

D.69 Gemessene Polfiguren Gilox 10Vol\% $5 \mathrm{ml} / \mathrm{s}$. . . . . . . . . . 286

D.70 Rückgerechnete Polfiguren, C-Koeffizienten und Fehler, Gilox 10Vol\% $5 \mathrm{ml} / \mathrm{s} \ldots \ldots \ldots \ldots \ldots \ldots . \ldots \ldots 287 \ldots \ldots \ldots$

D.71 Gemessene Polfiguren Gilox 12,5Vol\% 0,2 ml/s . . . . . . . . . 288

D.72 Rückgerechnete Polfiguren, C-Koeffizienten und Fehler, Gilox 12,5Vol\% $0,2 \mathrm{ml} / \mathrm{s} \ldots \ldots \ldots \ldots \ldots \ldots \ldots$

D.73 Gemessene Polfiguren Gilox 12,5Vol\% 0,5 ml/s . . . . . . . . 290

D.74 Rückgerechnete Polfiguren, C-Koeffizienten und Fehler, Gilox 12,5Vol\% $0,5 \mathrm{ml} / \mathrm{s} \ldots \ldots \ldots \ldots \ldots \ldots \ldots$

D.75 Gemessene Polfiguren Gilox 12,5Vol\% $1 \mathrm{ml} / \mathrm{s}$. . . . . . . . . 292

D.76 Rückgerechnete Polfiguren, C-Koeffizienten und Fehler, Gilox 12,5Vol\% $1 \mathrm{ml} / \mathrm{s} \ldots \ldots \ldots \ldots \ldots . \ldots \ldots . \ldots \ldots$

D.77 Gemessene Polfiguren Gilox 12,5Vol\% $2 \mathrm{ml} / \mathrm{s}$. . . . . . . . . . 294

D.78 Rückgerechnete Polfiguren, C-Koeffizienten und Fehler, Gilox 12,5Vol\% $2 \mathrm{ml} / \mathrm{s} \ldots \ldots \ldots \ldots \ldots \ldots \ldots \ldots$

D.79 Gemessene Polfiguren Gilox 12,5Vol\% $5 \mathrm{ml} / \mathrm{s}$. . . . . . . . . 296

D.80 Rückgerechnete Polfiguren, C-Koeffizienten und Fehler, Gilox 12,5Vol\% $5 \mathrm{ml} / \mathrm{s} \ldots \ldots \ldots \ldots \ldots \ldots \ldots$ 


\section{Anhang A}

\section{Diffraktionsdaten}

\section{A.1 Pulverdaten für Muskovit}

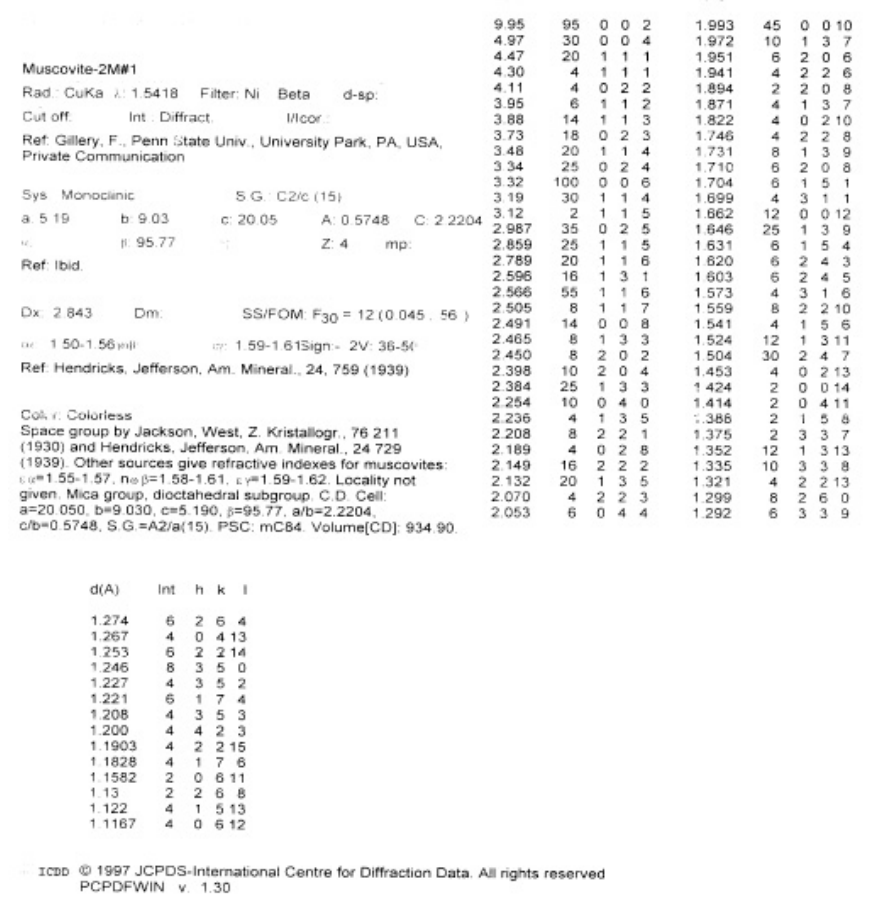

Abbildung A.1: Auszug aus Pulverdatenbank JCPDS für Muskovit [75] 


\section{A.2 Pulverdaten für Aluminiumoxid}

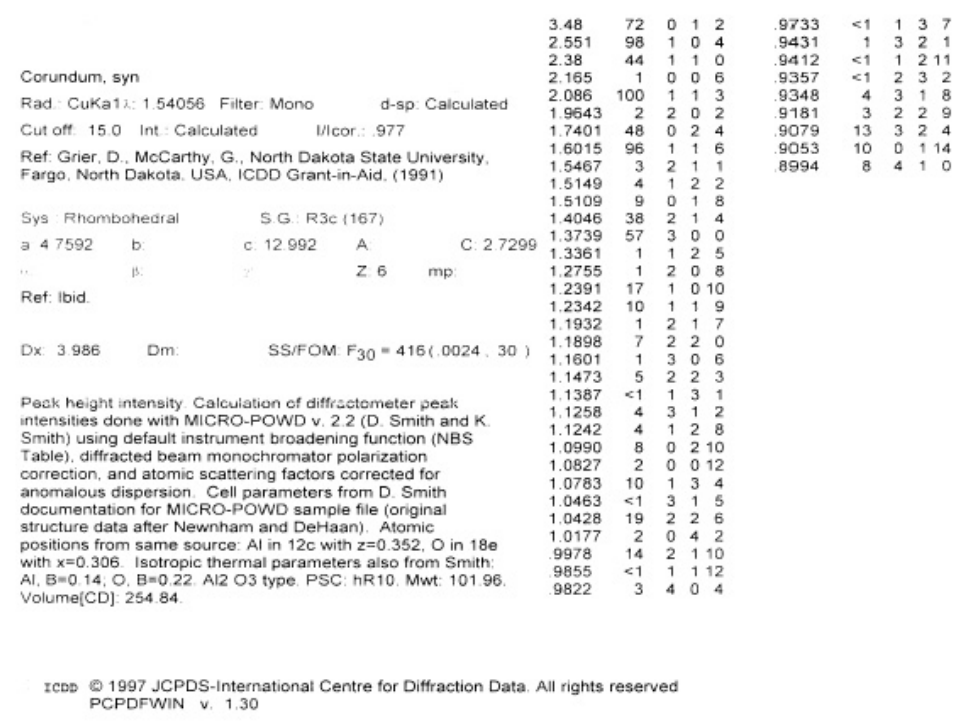

Abbildung A.2: Auszug aus Pulverdatenbank JCPDS für $\mathrm{Al}_{2} \mathrm{O}_{3}[75]$ 
Anhang B

Rotationsküvette (A, B, C) 


\section{B.1 Probe A}
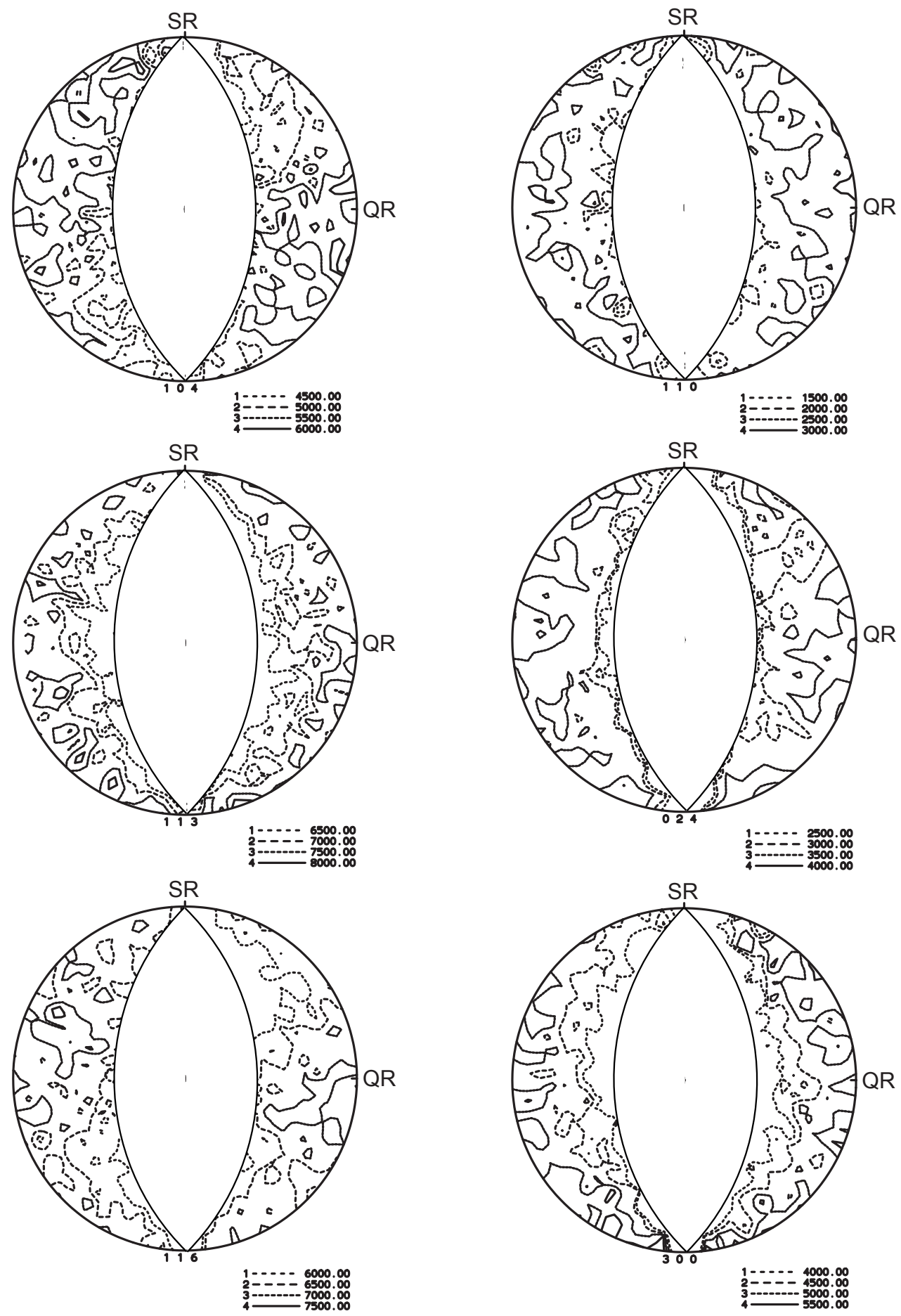

Abbildung B.1: gemessene Polfiguren Probe A 36Vol\% 0,25 U/min 

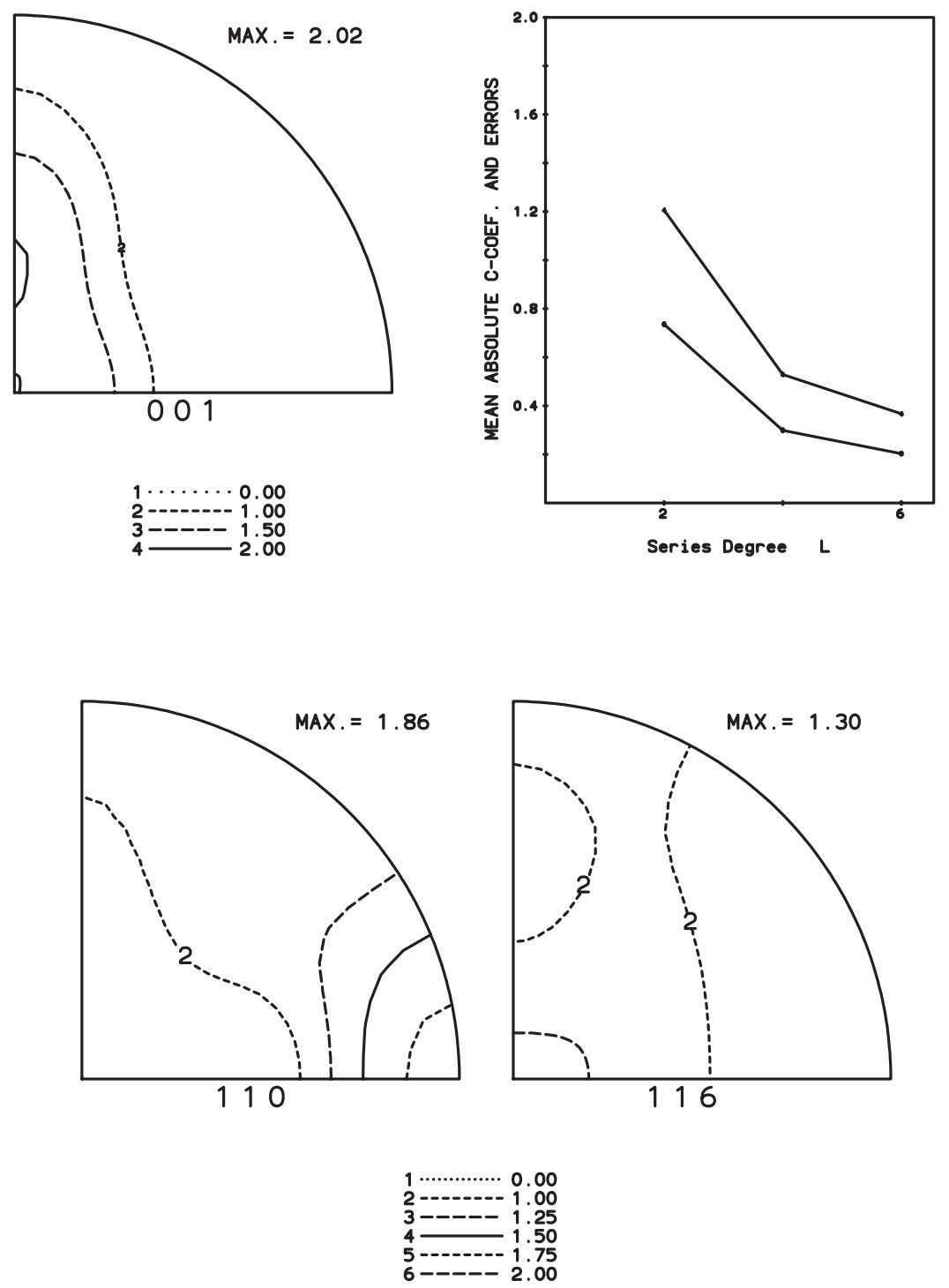

Abbildung B.2: Rückgerechnete Polfiguren, C-Koeffizienten und Fehler der Probe A $36 \mathrm{Vol} \%, 0,25 \mathrm{U} / \mathrm{min}$ 

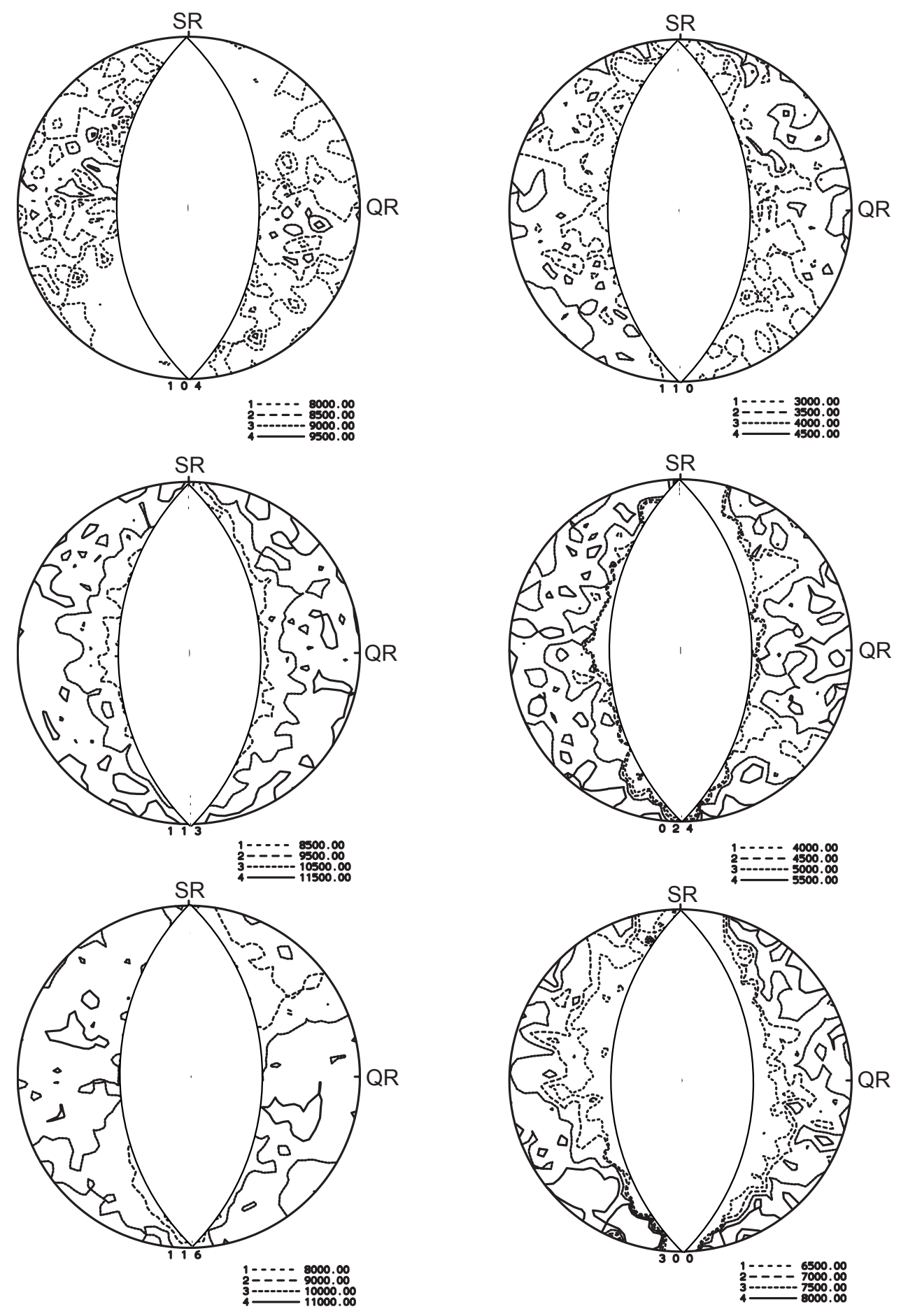

Abbildung B.3: gemessene Polfiguren Probe A 36Vol\% 0,5 U/min 


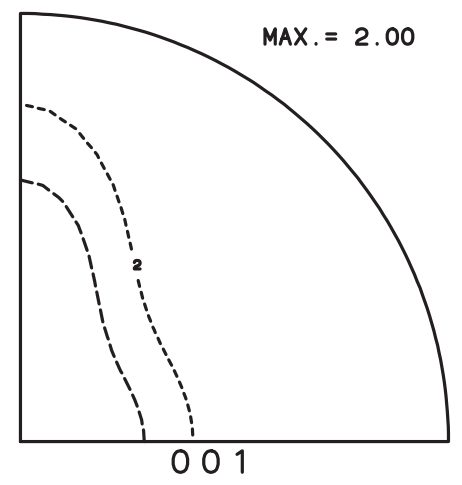

$1 \cdots \cdots \times 0.00$

0.00
1.00
1.50
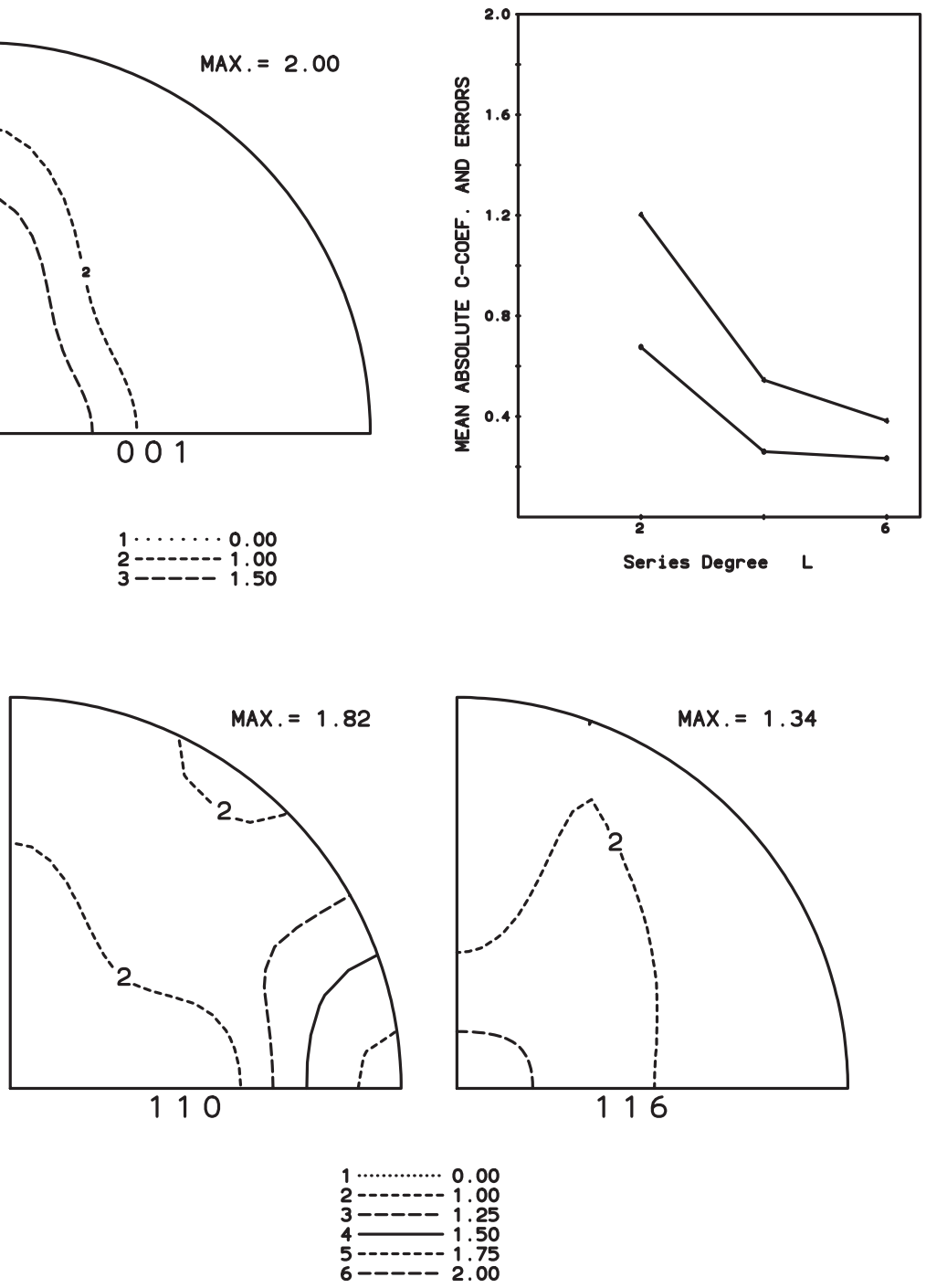

Abbildung B.4: Rückgerechnete Polfiguren, C-Koeffizienten und Fehler der Probe A $36 \mathrm{Vol} \% 0,5 \mathrm{U} / \mathrm{min}$ 

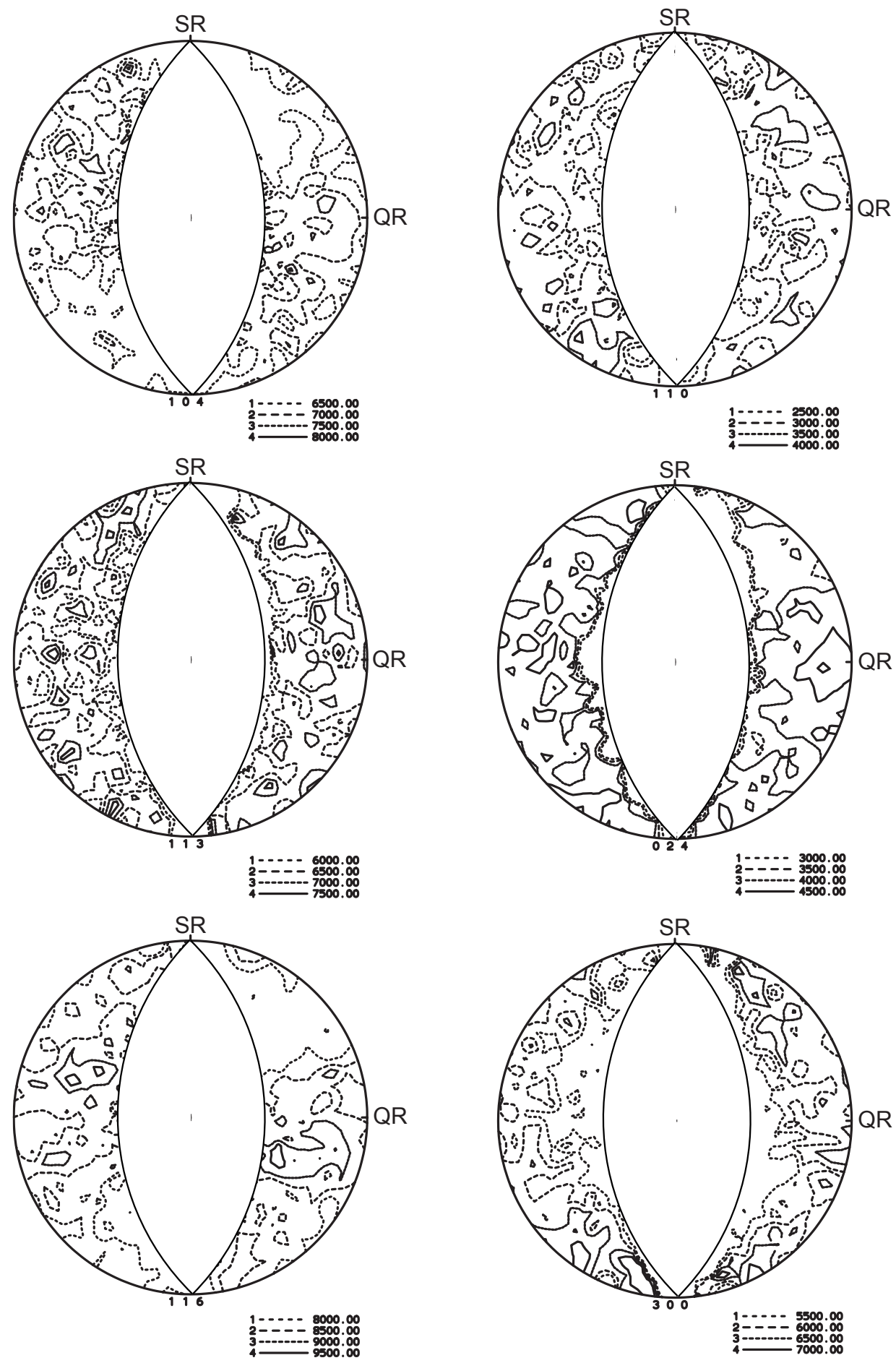

Abbildung B.5: gemessene Polfiguren Probe A 36Vol\% 1 U/min 

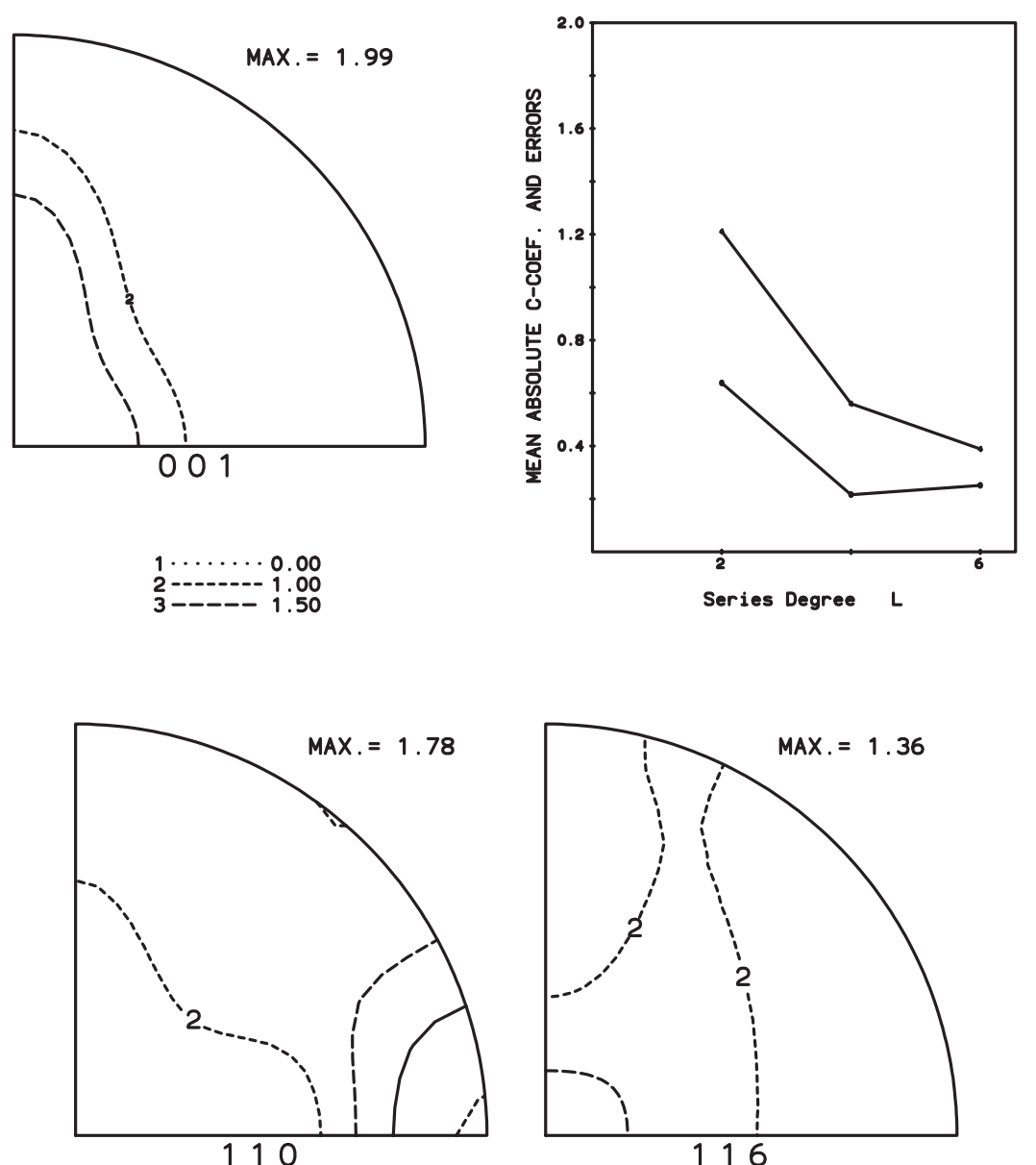

$$
\begin{aligned}
& 1 \ldots \ldots \ldots \ldots \ldots .0 \\
& 2-00 \\
& 3-\cdots-1.00 \\
& 4--1.25 \\
& 5-\cdots-1.50 \\
& 6---1.00
\end{aligned}
$$

Abbildung B.6: Rückgerechnete Polfiguren, C-Koeffizienten und Fehler der Probe A $36 \mathrm{Vol} \% 1 \mathrm{U} / \mathrm{min}$ 


\section{B.2 Probe B}
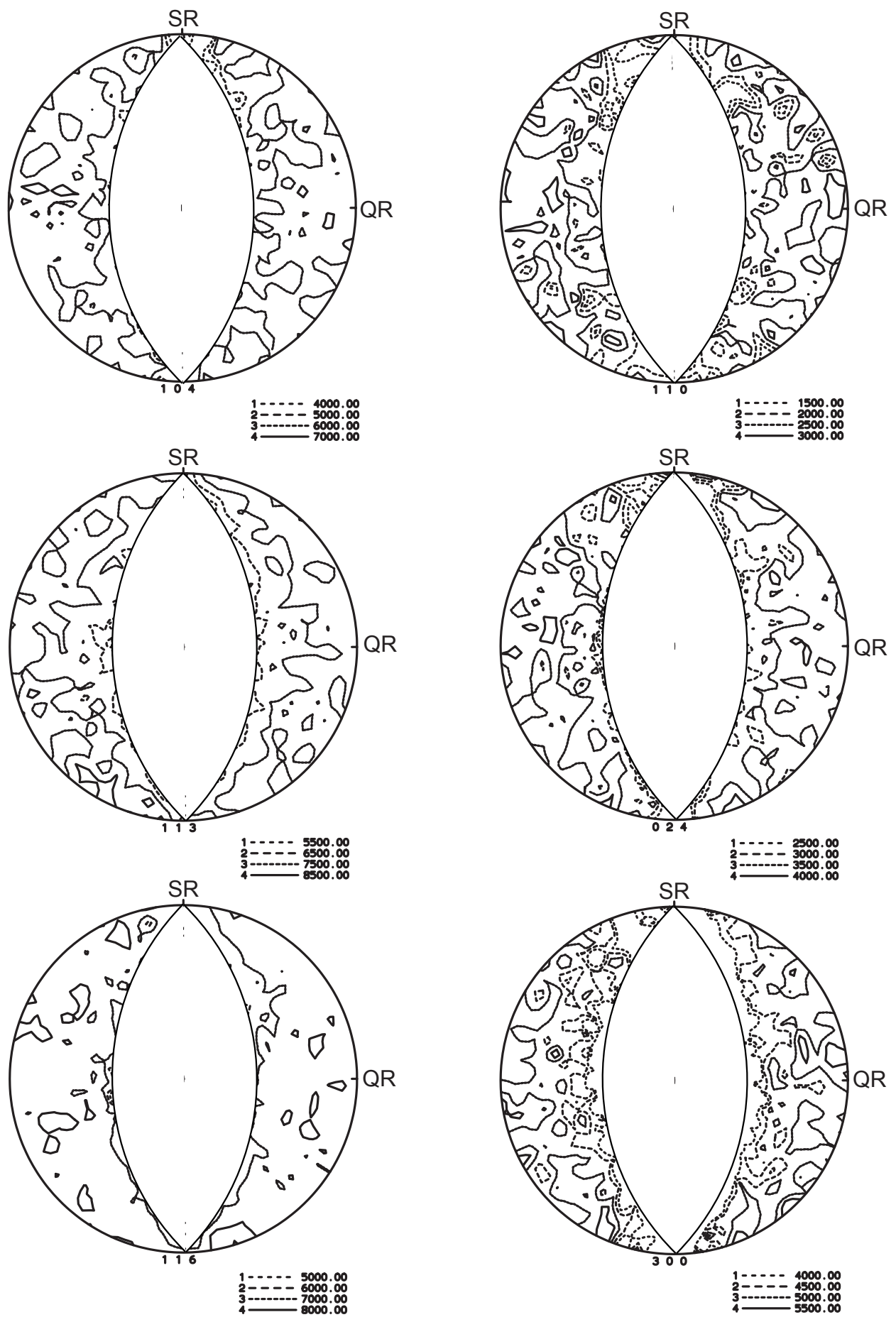

Abbildung B.7: gemessene Polfiguren Probe B 36Vol\% 0,25 U/min 

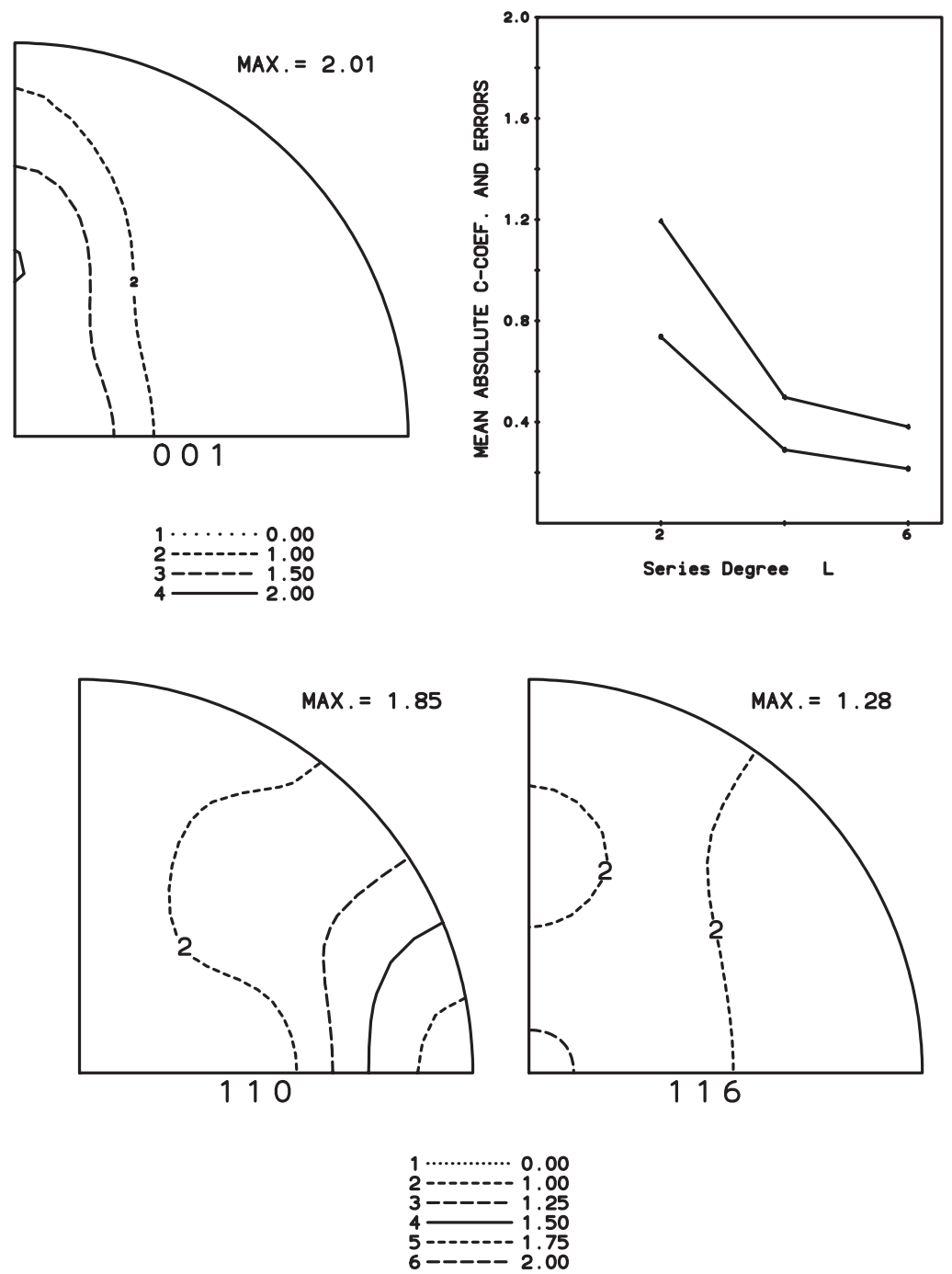

Abbildung B.8: Rückgerechnete Polfiguren, C-Koeffizienten und Fehler der Probe B $36 \mathrm{Vol} \%$ 0,25 U/min 

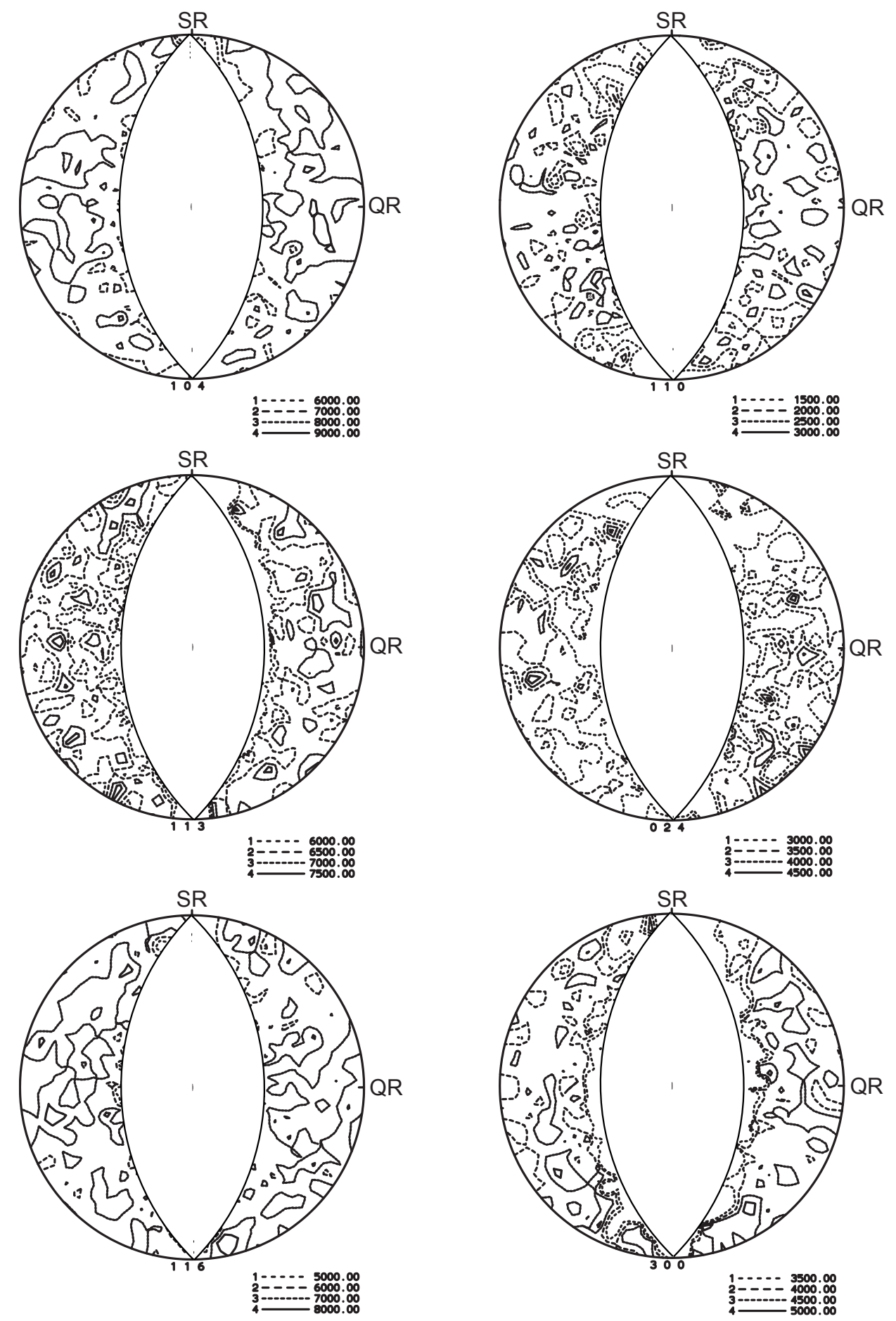

Abbildung B.9: gemessene Polfiguren Probe B 36Vol\% 0,5 U/min 

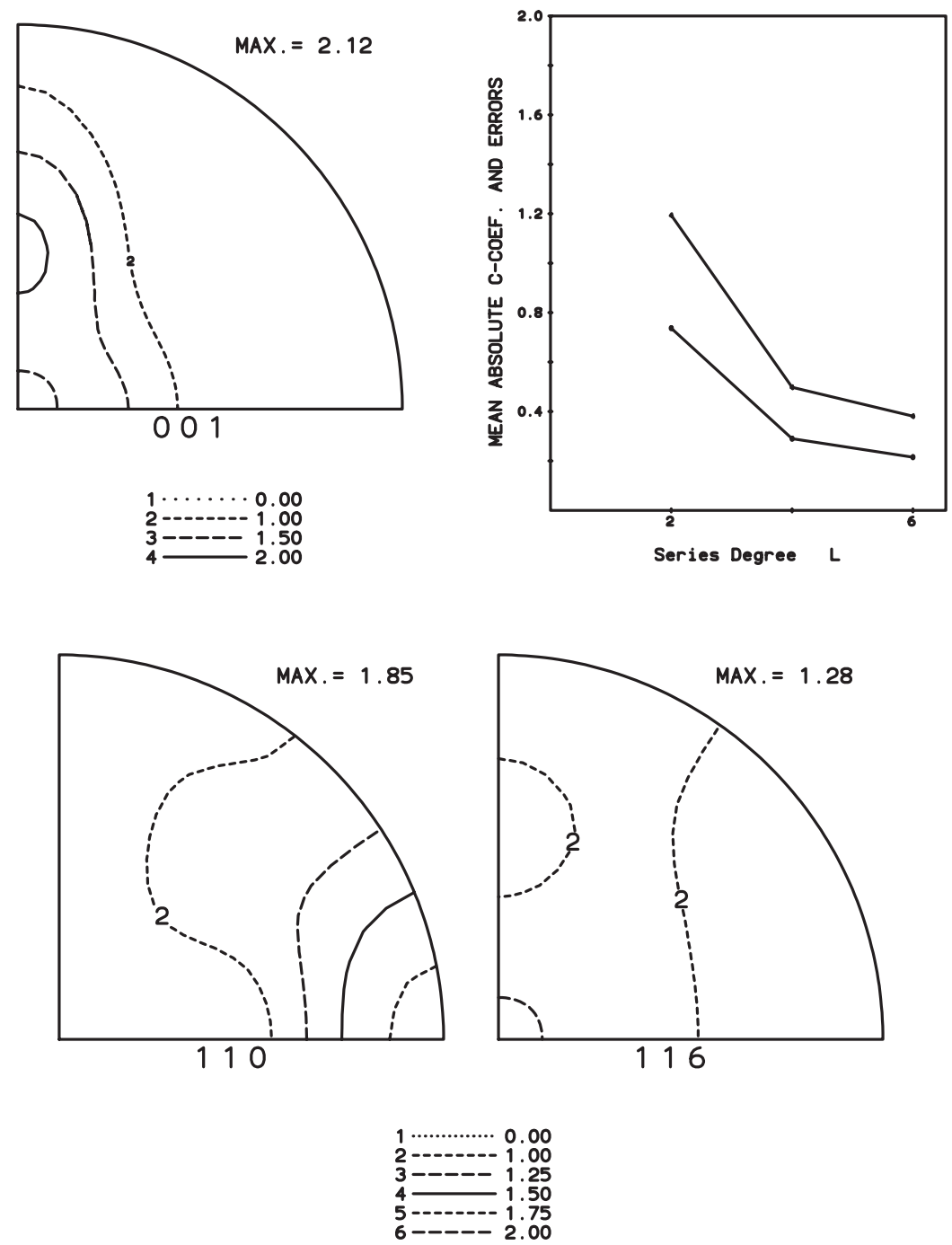

Abbildung B.10: Rückgerechnete Polfiguren, C-Koeffizienten und Fehler der Probe B 36Vol\% 0,5 U/min 

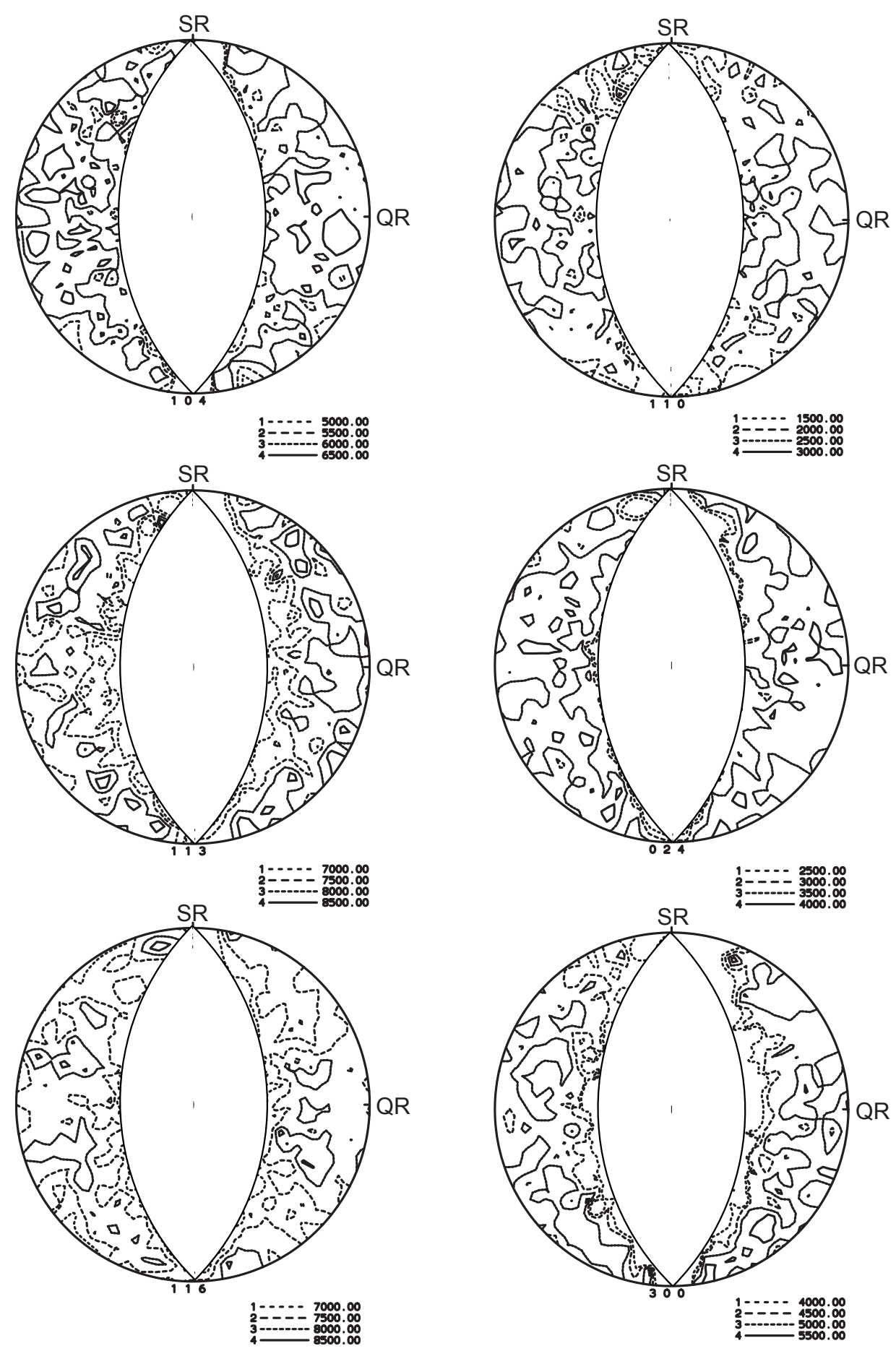

Abbildung B.11: gemessene Polfiguren Probe B 36Vol\% 1 U/min 


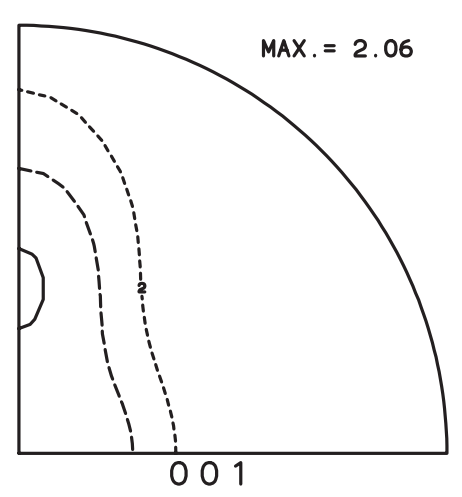

$1 \ldots . . .000$

1
$3----1.50$
$4-\cdots-1.00$
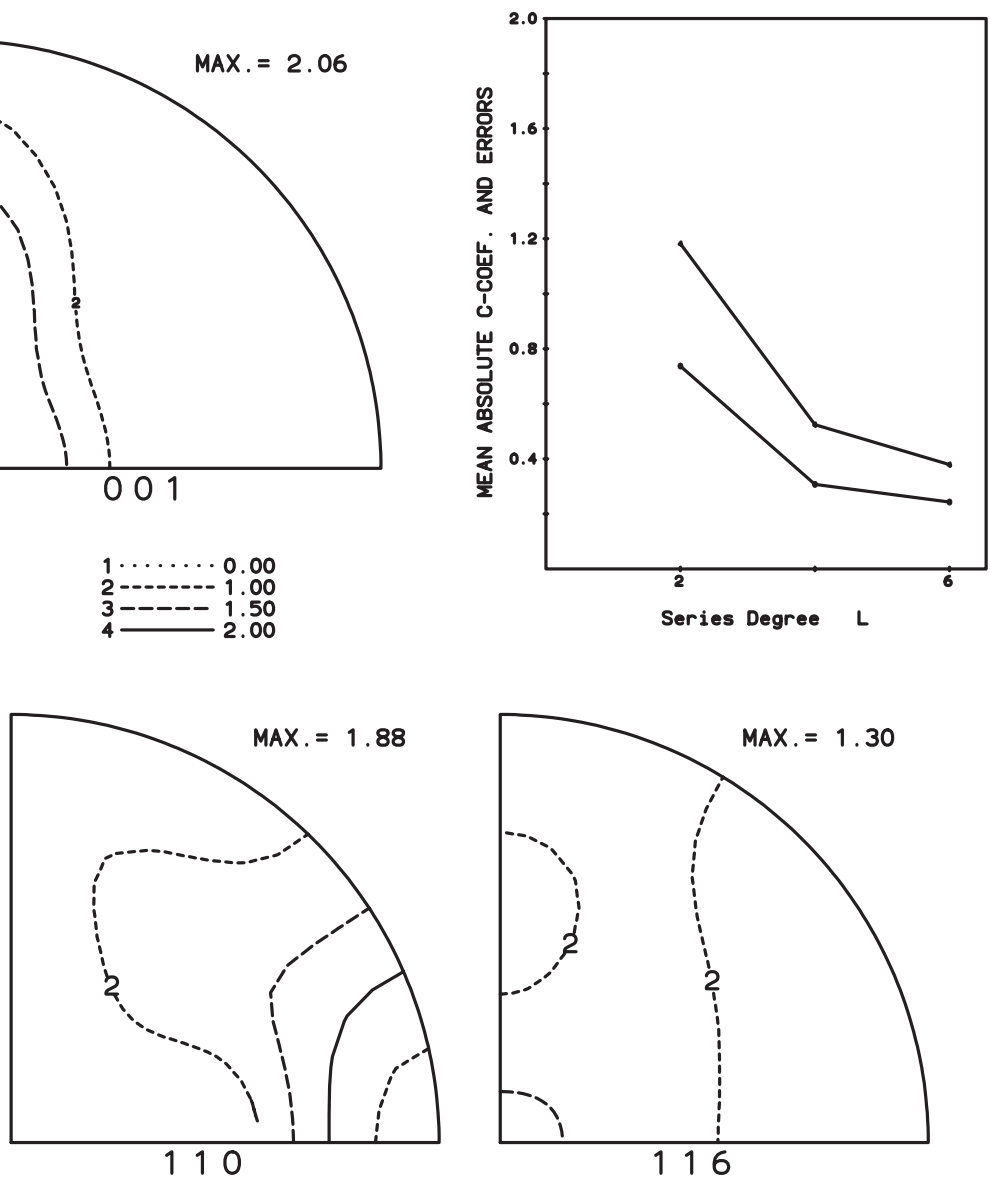

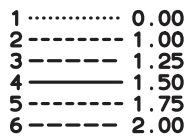

Abbildung B.12: Rückgerechnete Polfiguren, C-Koeffizienten und Fehler der Probe B 36Vol\% $1 \mathrm{U} / \mathrm{min}$ 

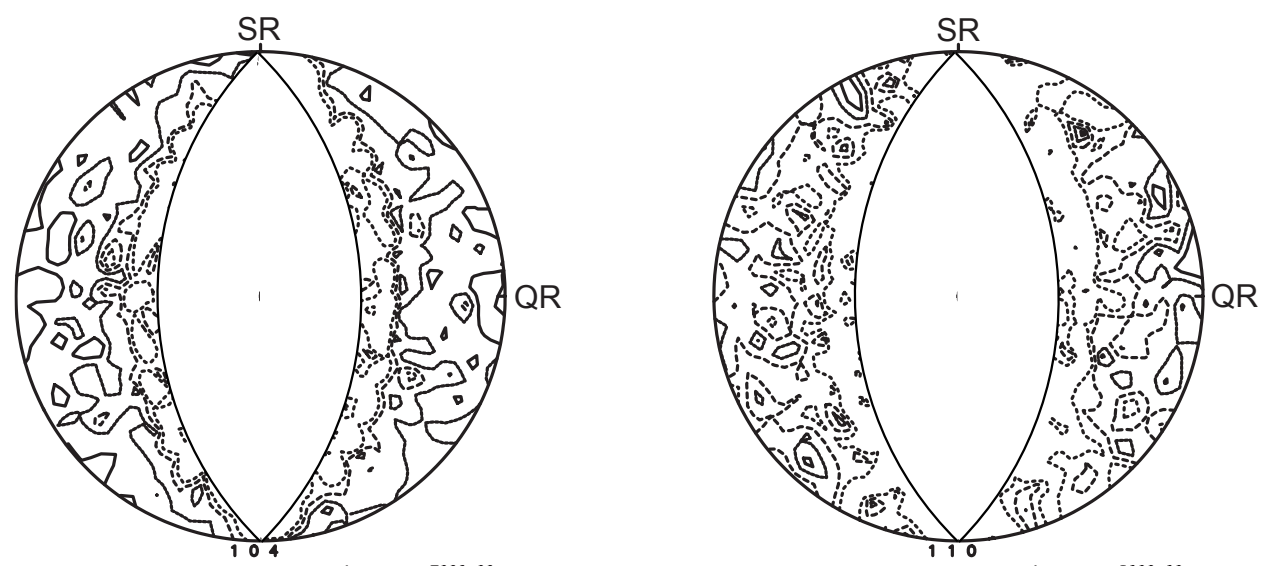

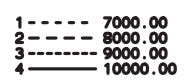
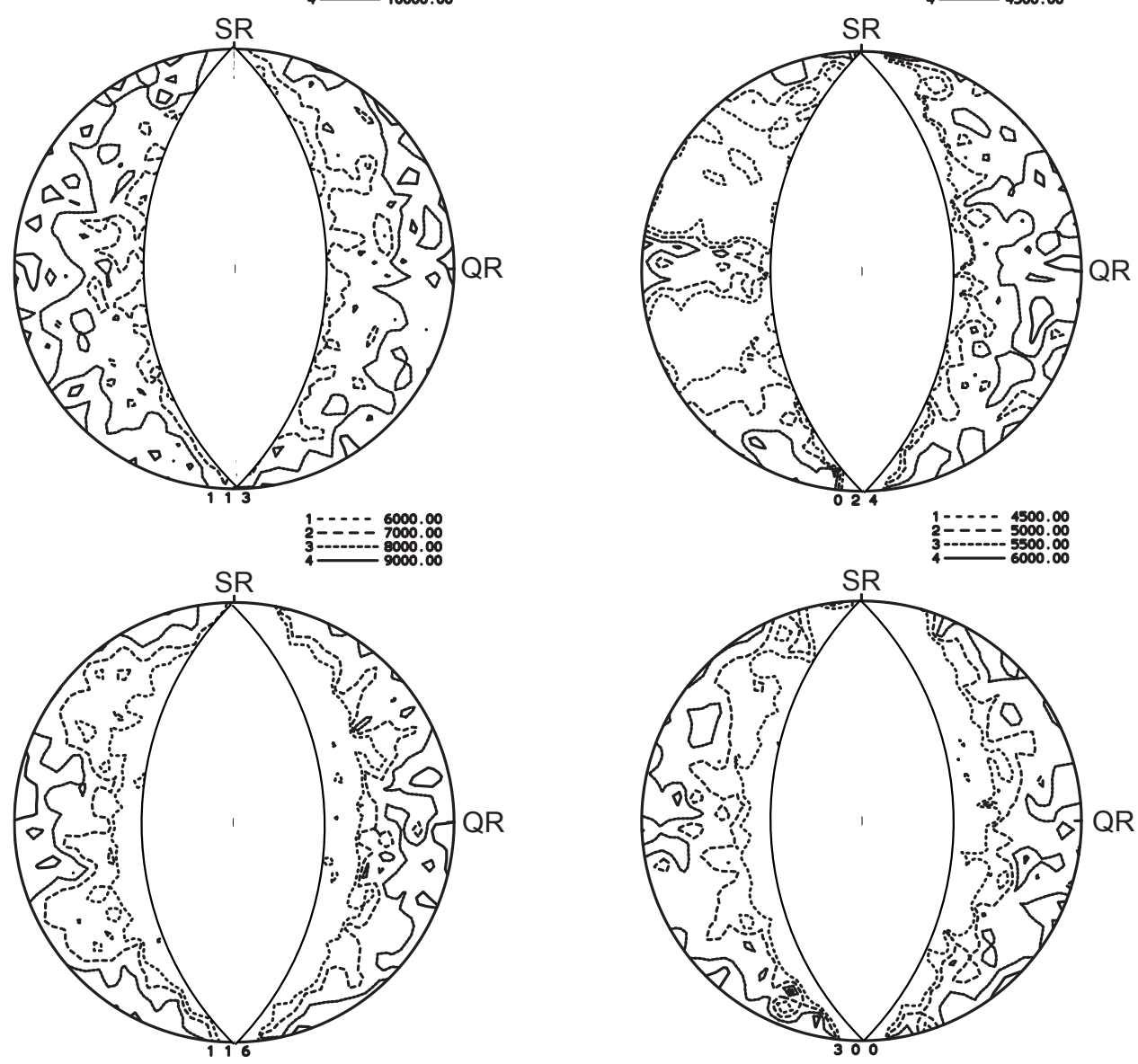

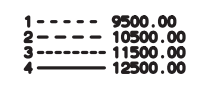

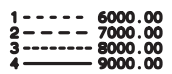

Abbildung B.13: gemessene Polfiguren Probe B 24Vol\% 0,25 U/min 

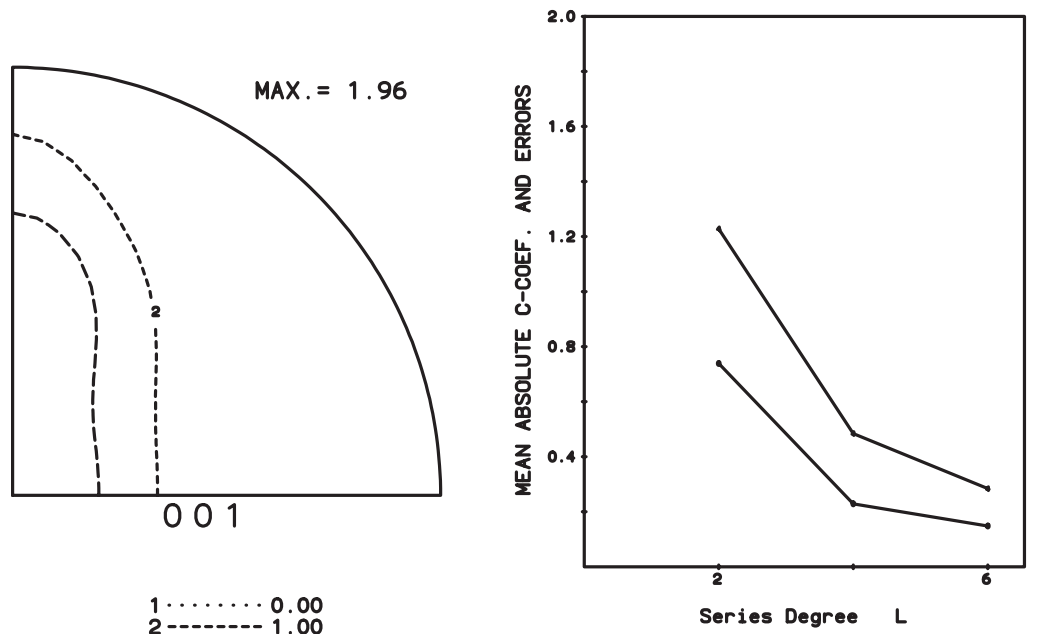

$1 \cdots \cdots \cdots 0.00$
$2 \cdots-1.00$
$3----1.50$
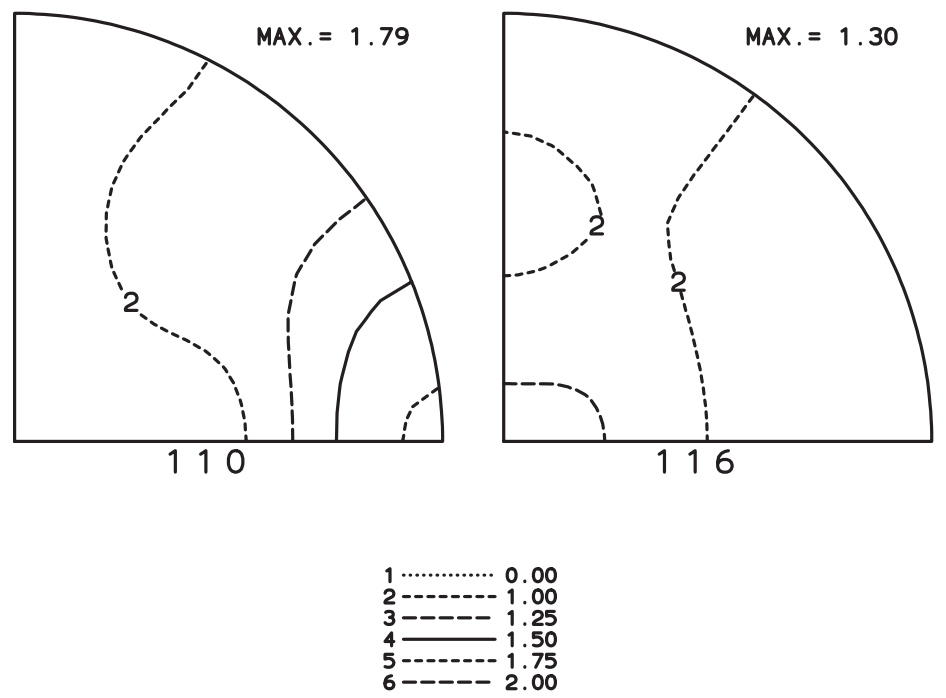

Abbildung B.14: Rückgerechnete Polfiguren, C-Koeffizienten und Fehler der Probe B 24Vol\% 0,25 U/min 

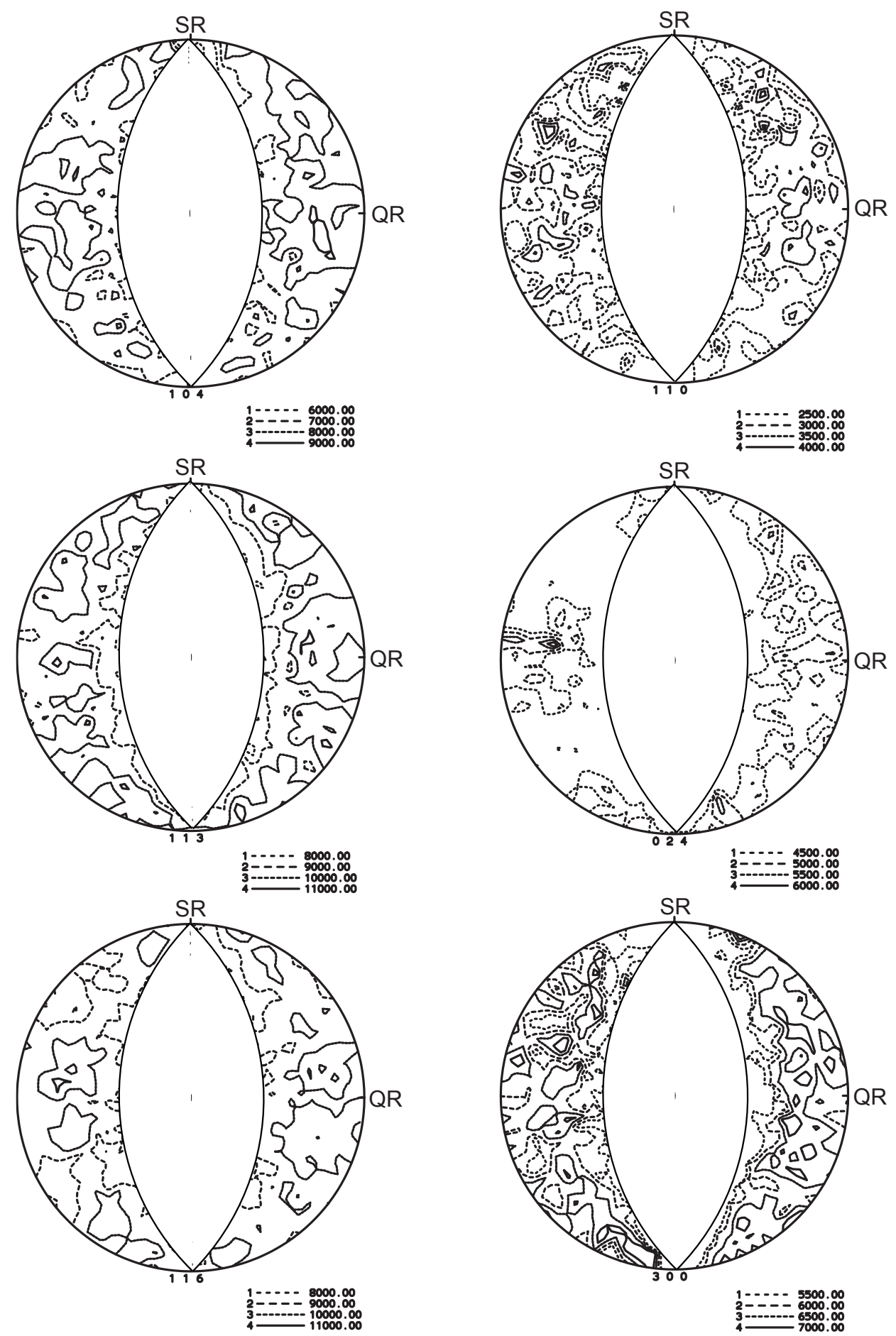

Abbildung B.15: gemessene Polfiguren Probe B 24Vol\% 0,5 U/min 

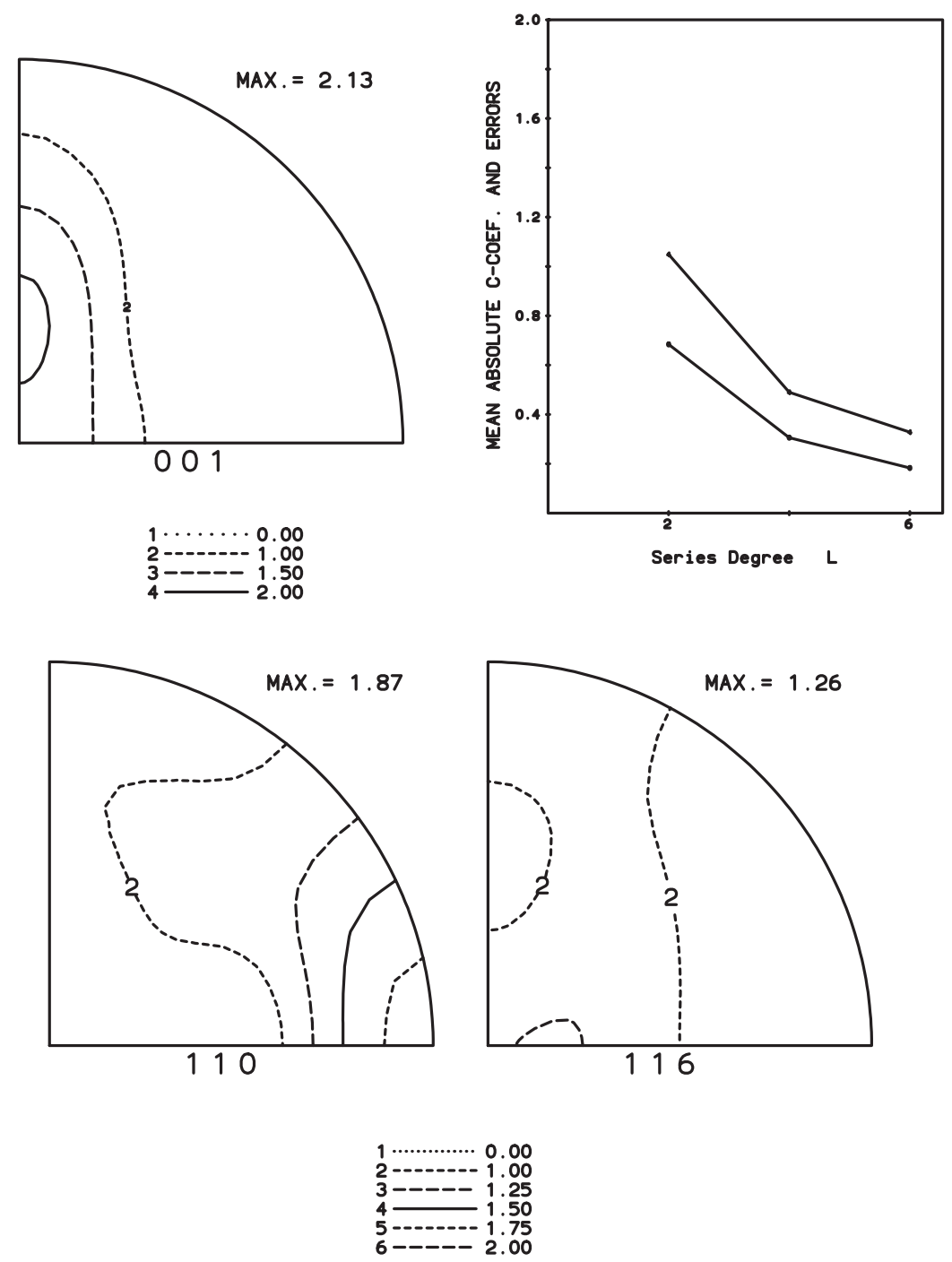

Abbildung B.16: Rückgerechnete Polfiguren, C-Koeffizienten und Fehler der Probe B 24Vol\% 0,5 U/min 

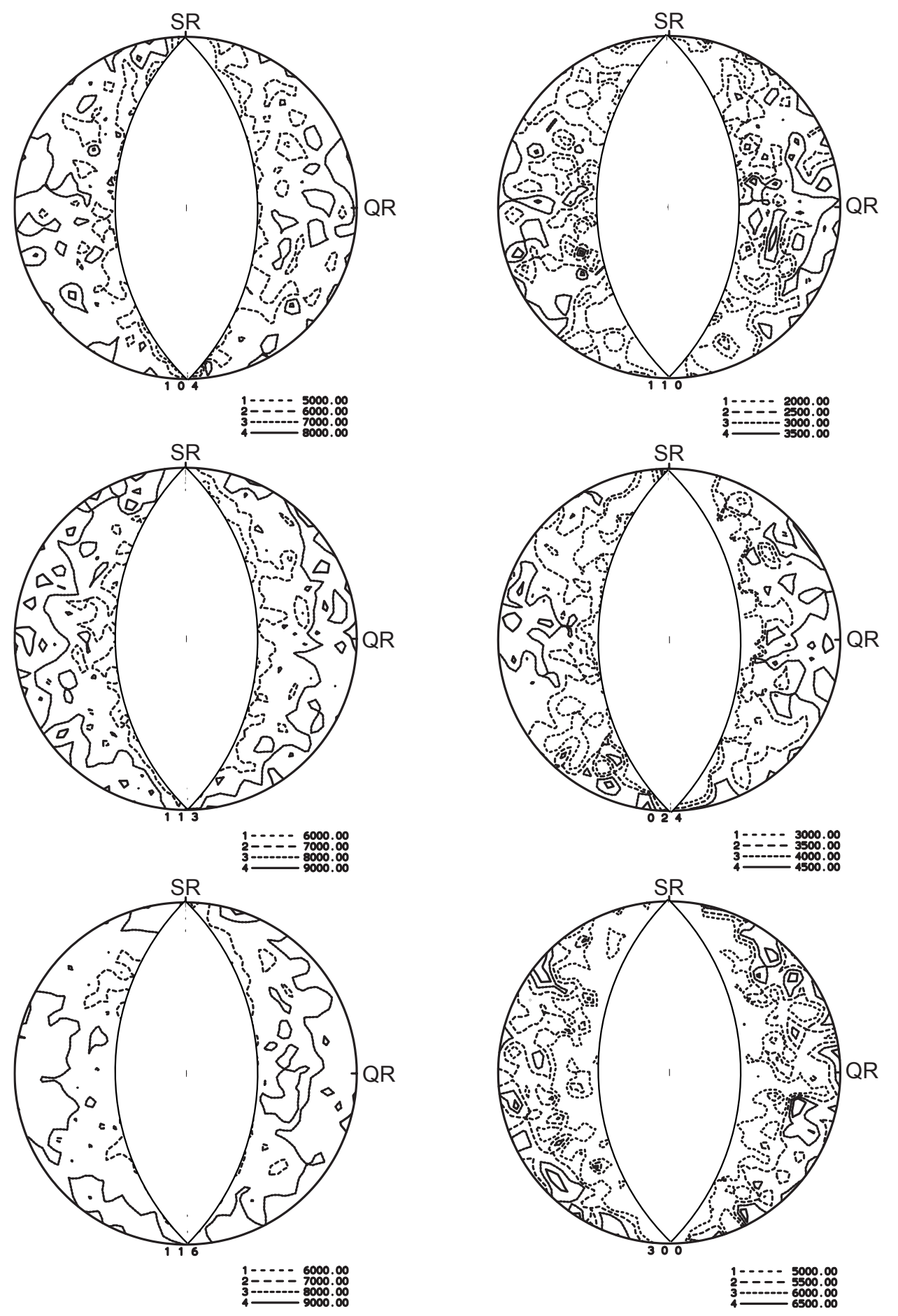

Abbildung B.17: gemessene Polfiguren Probe B 24Vol\% 1 U/min 

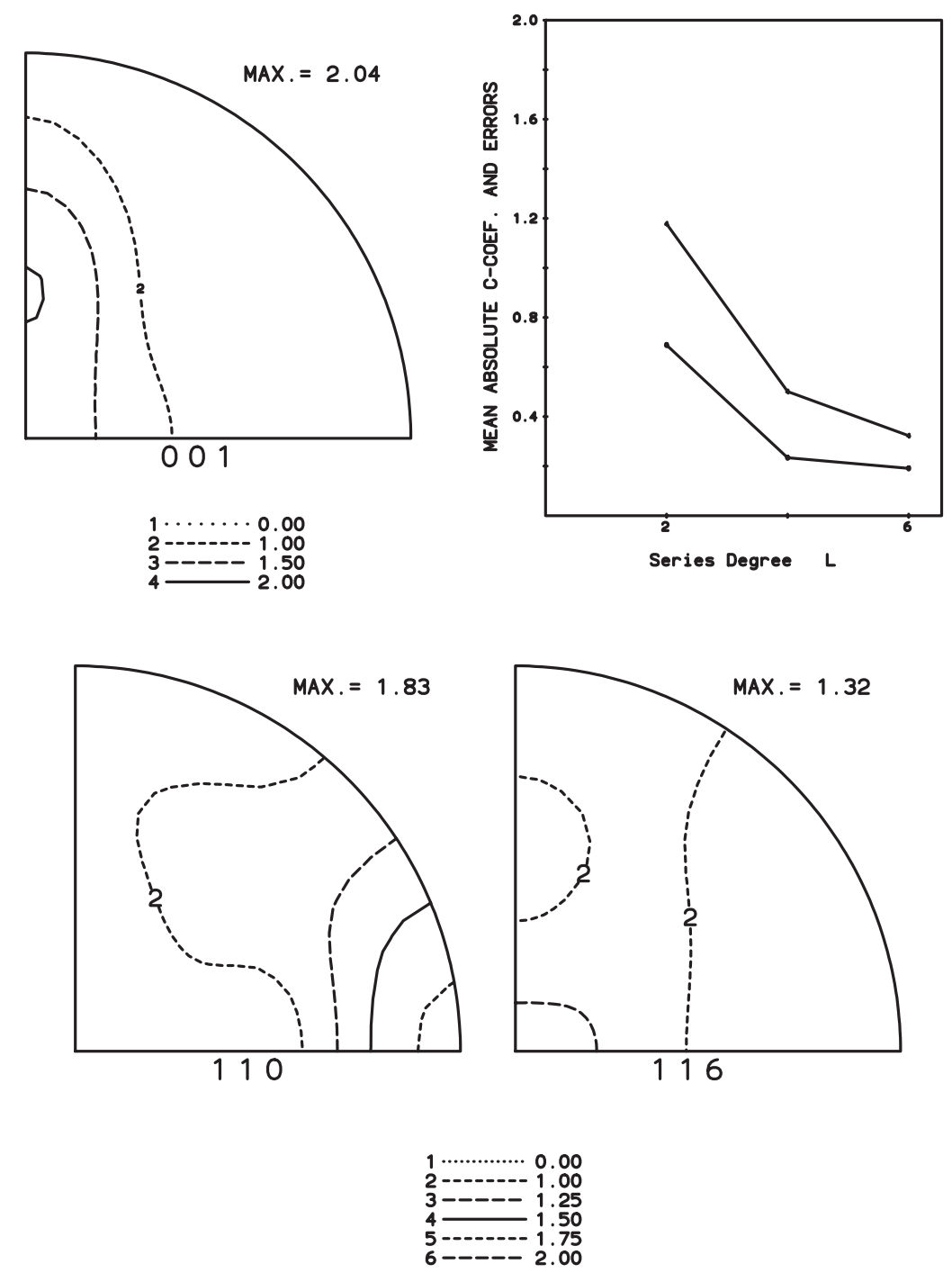

Abbildung B.18: Rückgerechnete Polfiguren, C-Koeffizienten und Fehler der Probe B 24Vol\% $1 \mathrm{U} / \mathrm{min}$ 


\section{B.3 Probe C}
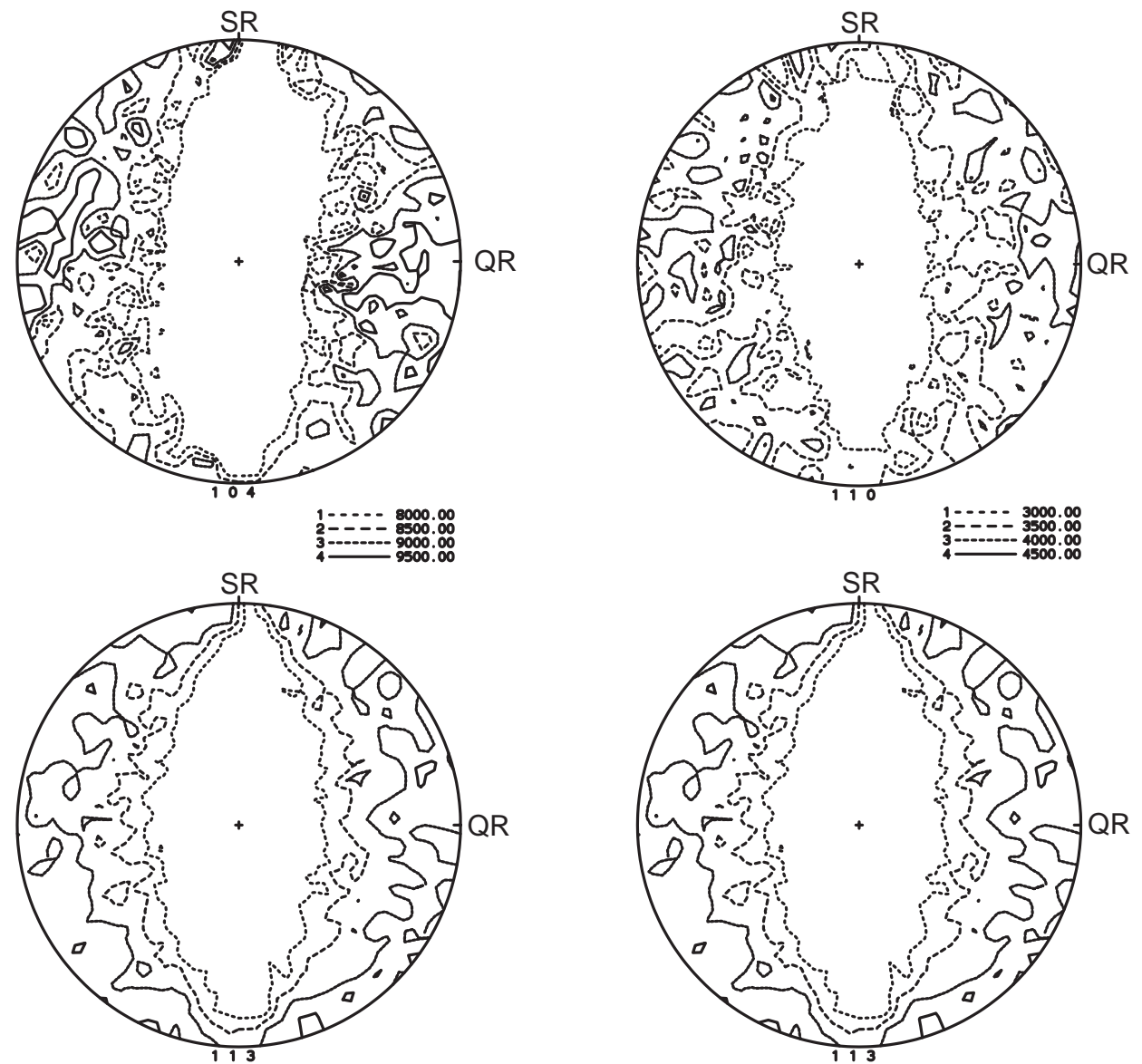

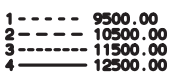
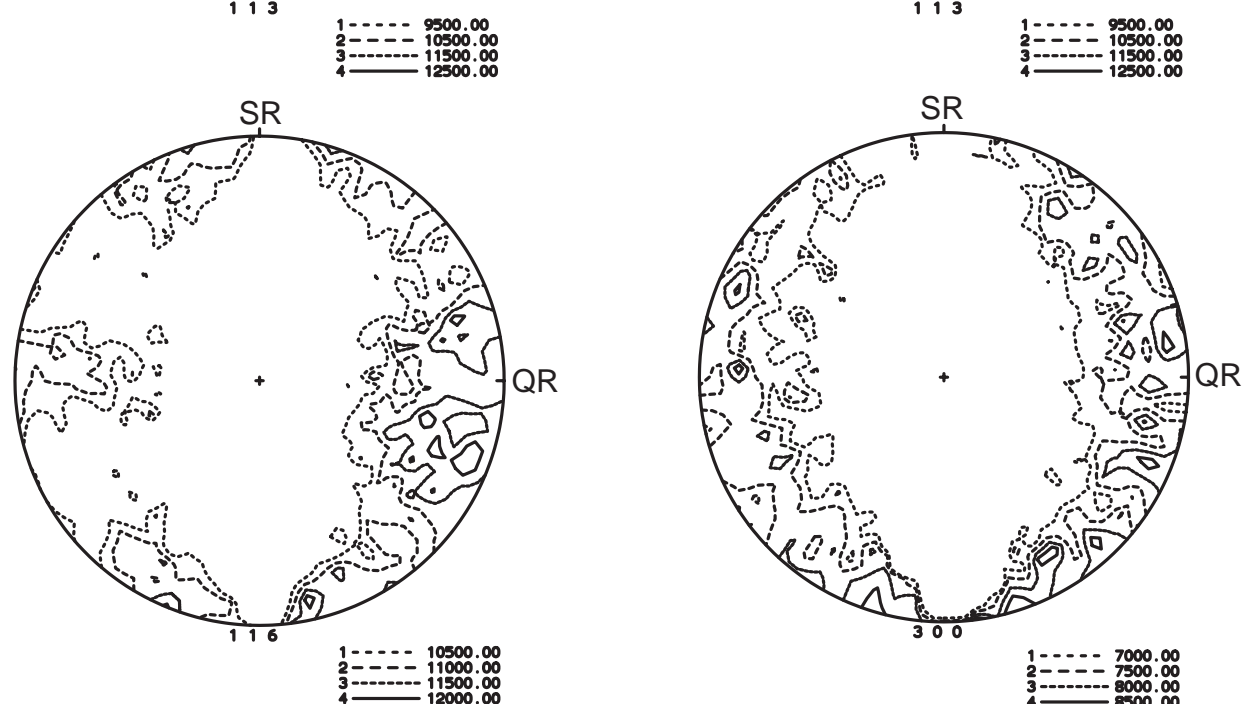

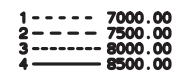

Abbildung B.19: gemessene Polfiguren Probe C 28Vol\% 0,25 U/min 


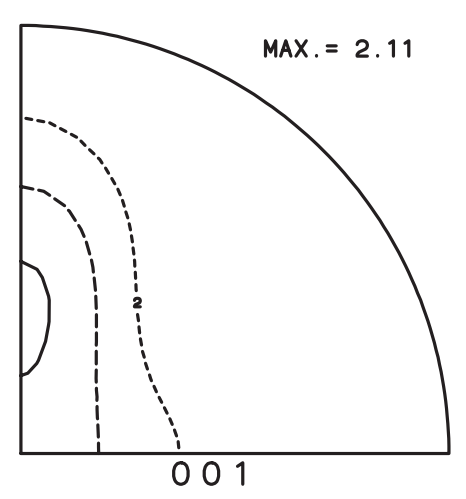

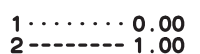

$\begin{array}{r}3----1.50 \\ \hline\end{array}$
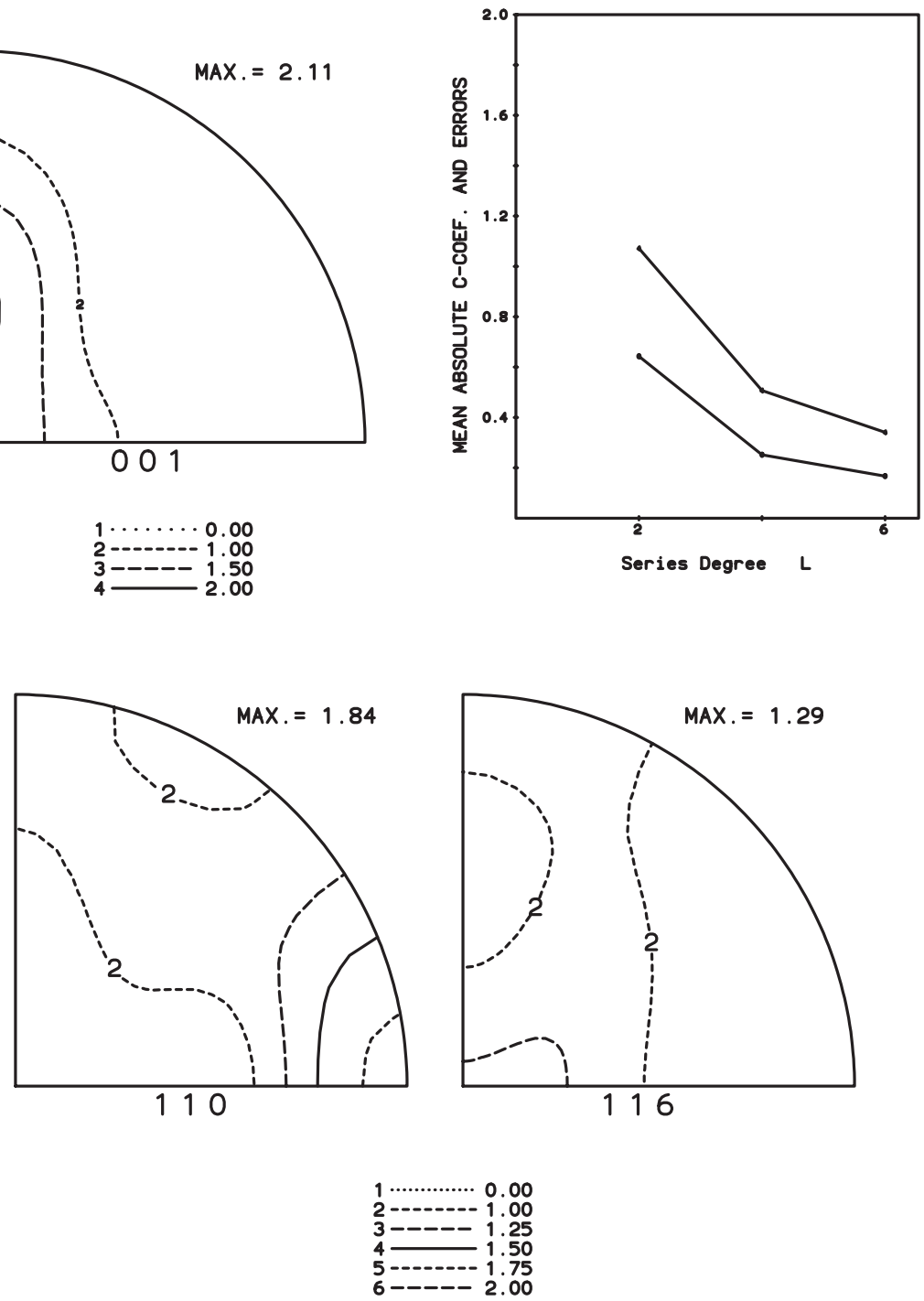

Abbildung B.20: Rückgerechnete Polfiguren, C-Koeffizienten und Fehler der Probe C 28Vol\% 0,25 U/min 

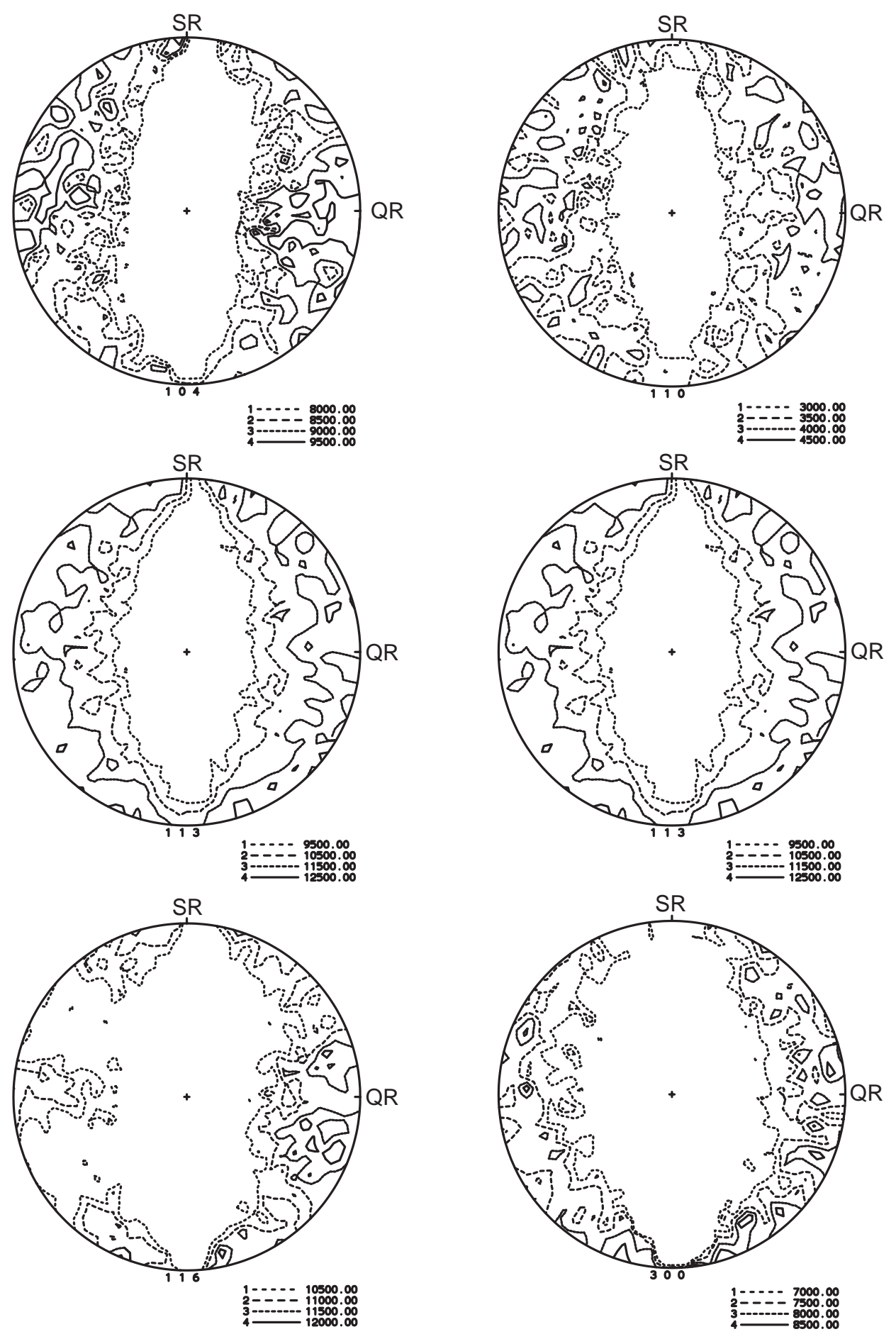

Abbildung B.21: gemessene Polfiguren Probe C 28Vol\% 0,5 U/min 

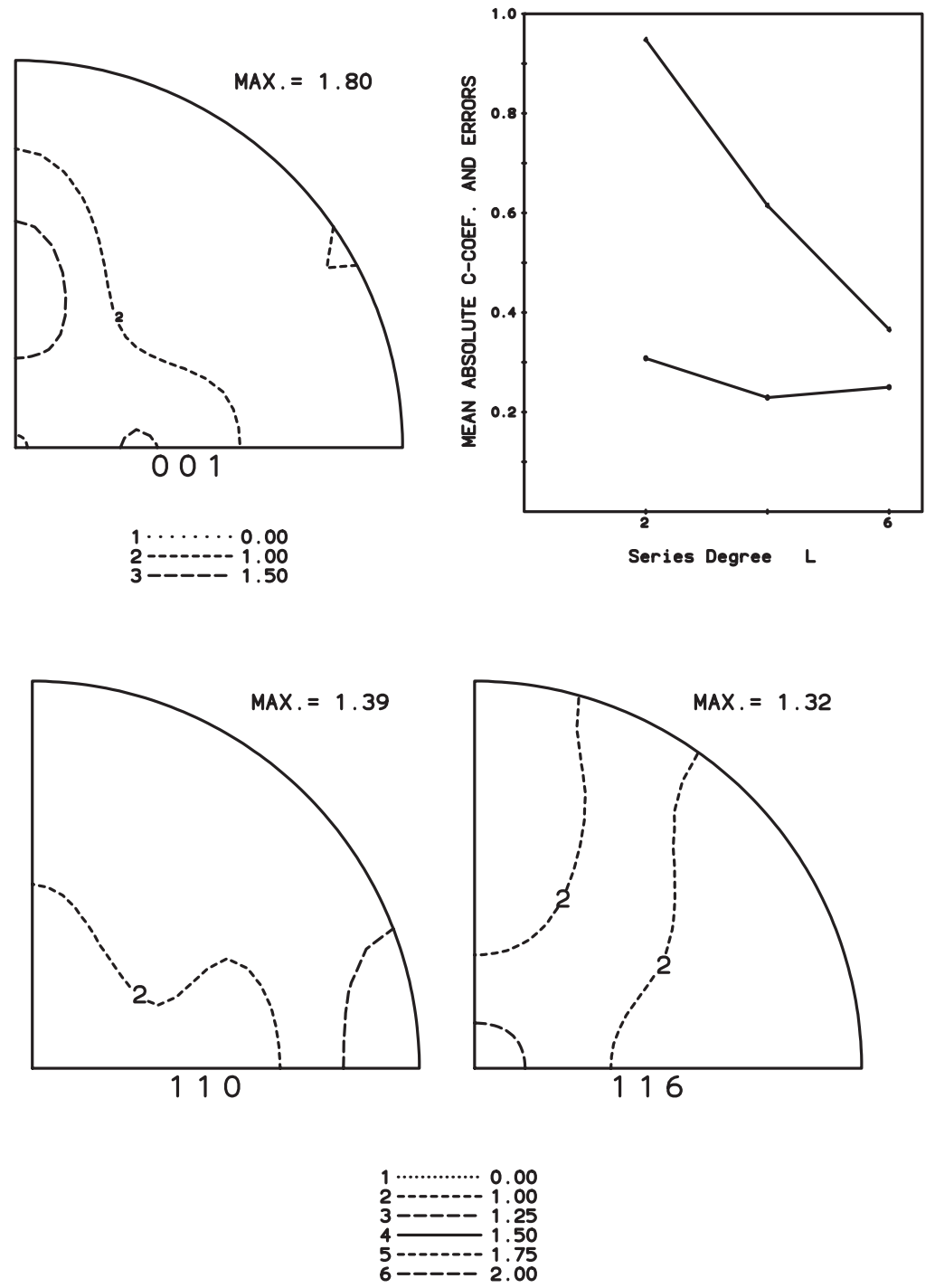

Abbildung B.22: Rückgerechnete Polfiguren, C-Koeffizienten und Fehler der Probe C 28Vol\% 0,5 U/min 

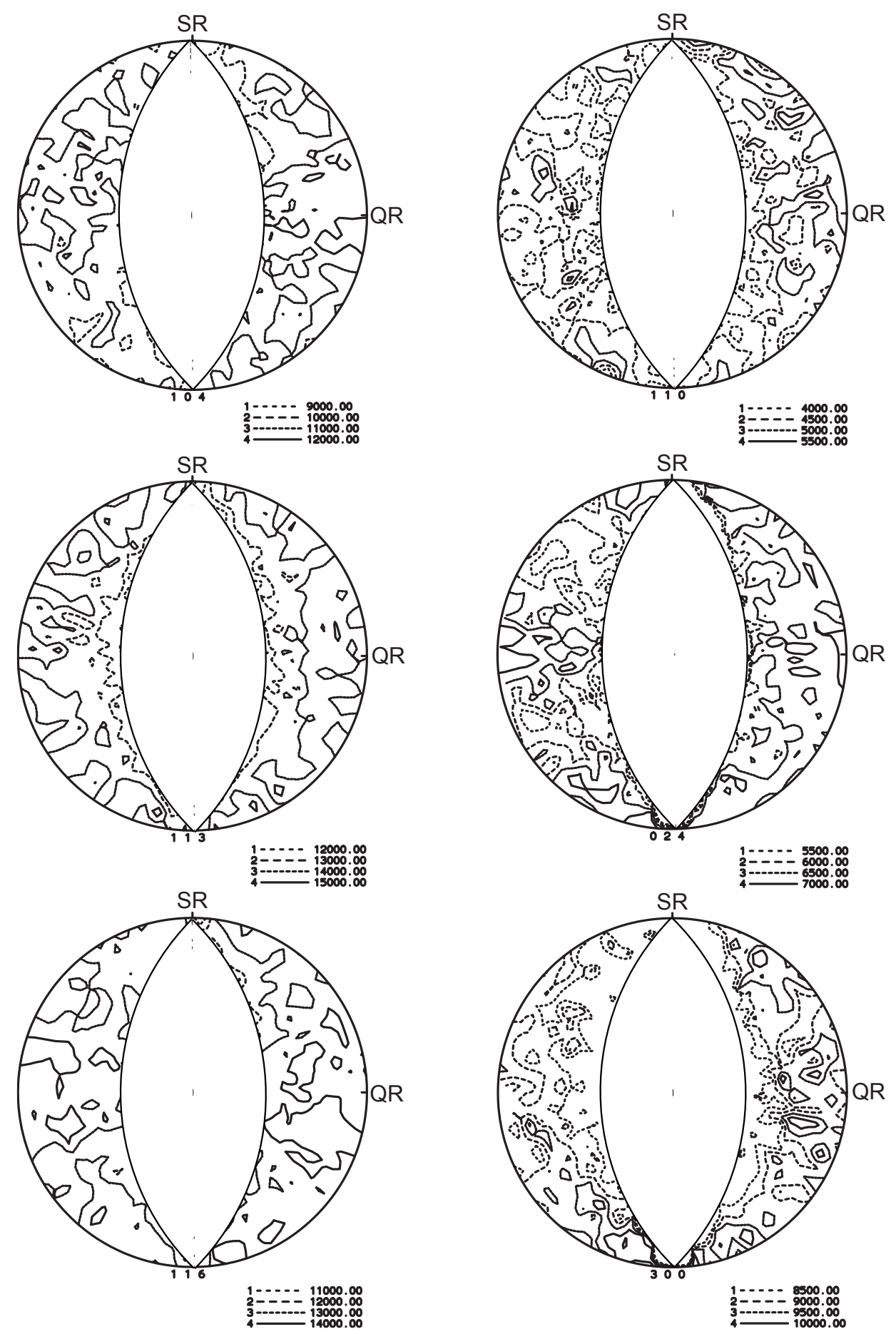

Abbildung B.23: gemessene Polfiguren Probe C 28Vol\% 1 U/min 

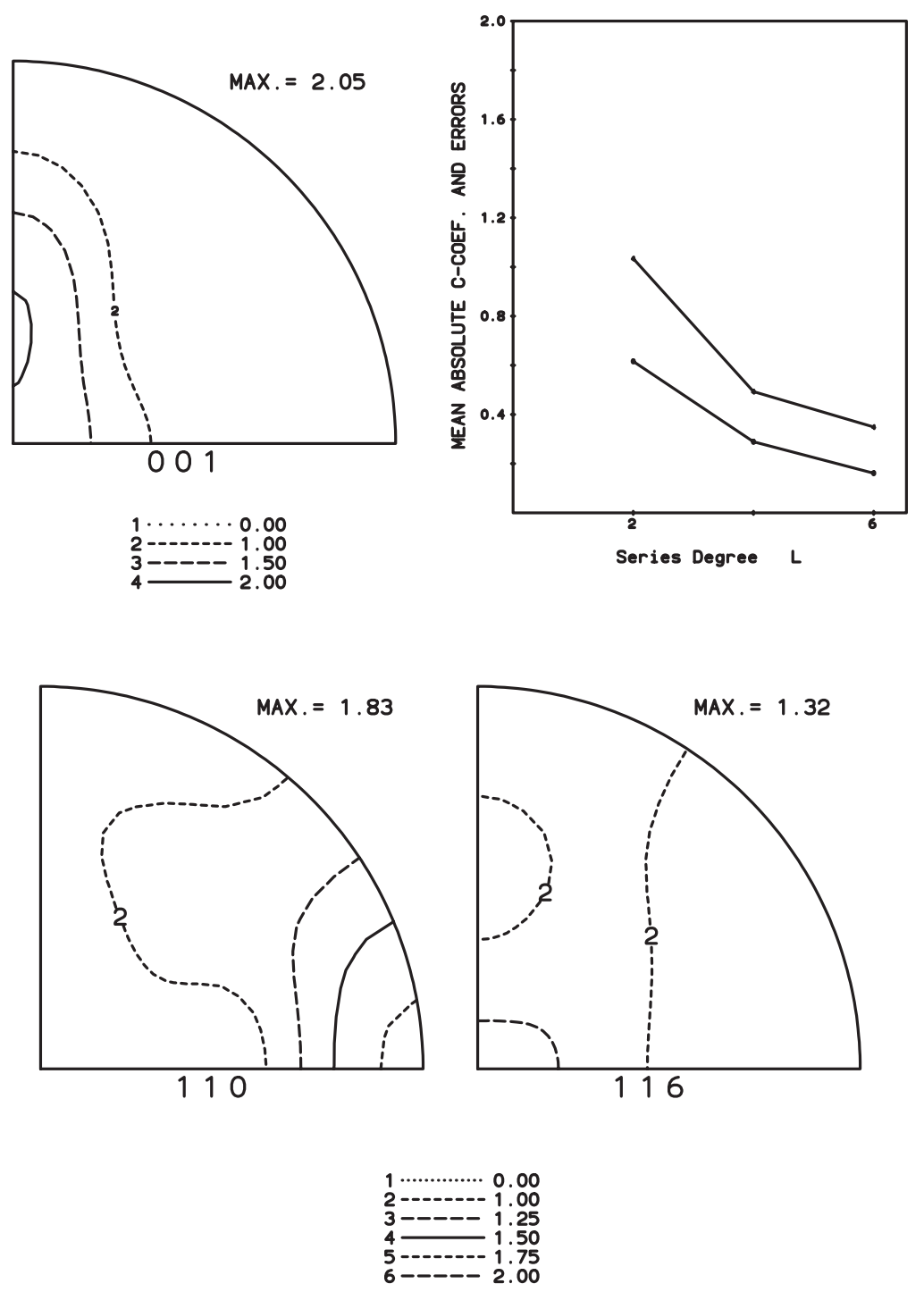

Abbildung B.24: Rückgerechnete Polfiguren, C-Koeffizienten und Fehler der Probe C $28 \mathrm{Vol} \% 1 \mathrm{U} / \mathrm{min}$ 
Anhang C

Rotationsküvette (HVAFG, CTC20, Gilox) 


\section{C.1 Probe HVAFG 26Vol\%}
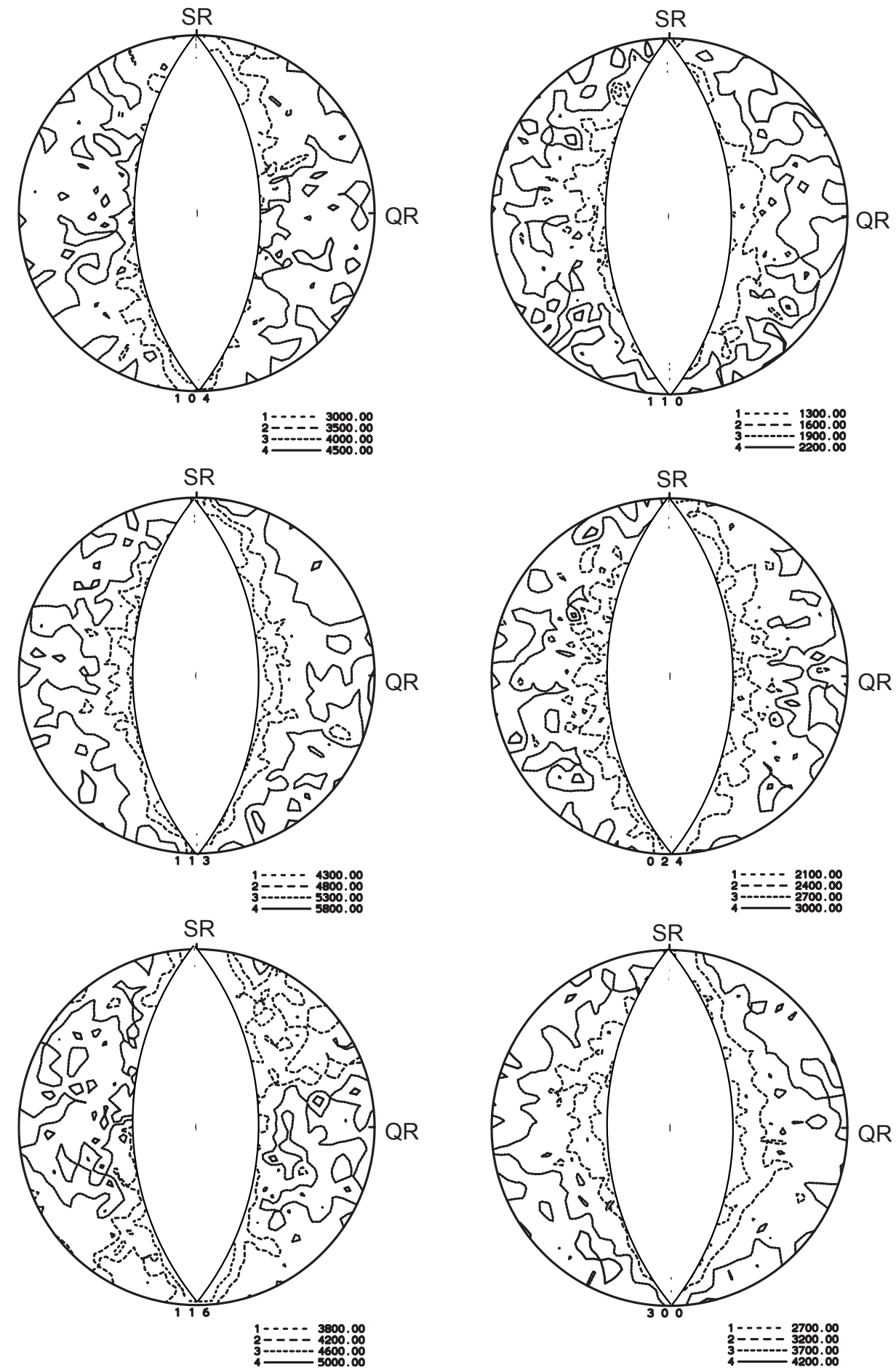

Abbildung C.1: Gemessene Polfiguren HVAFG 26Vol\% 0,5 U/min 

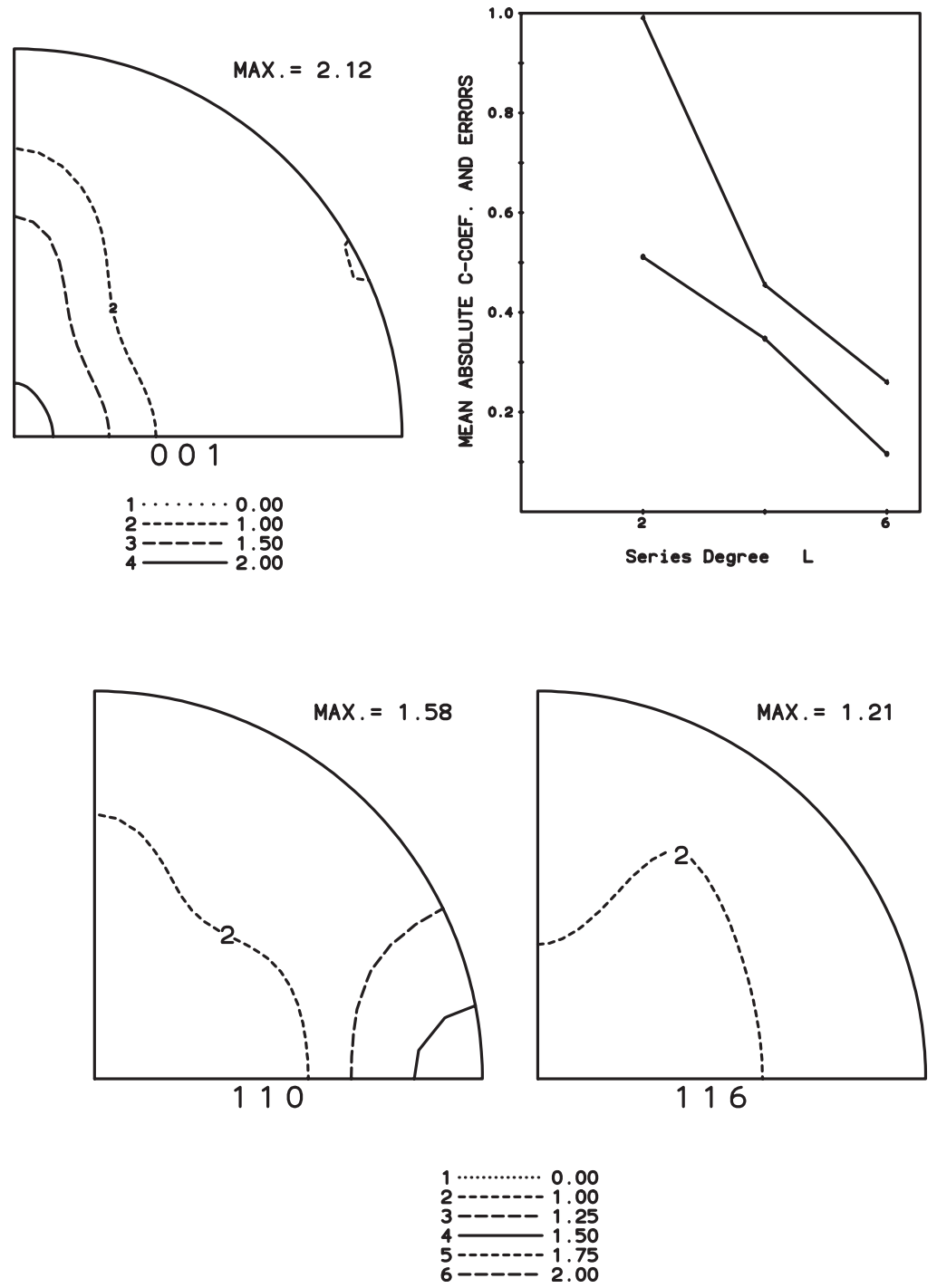

Abbildung C.2: Rückgerechnete Polfiguren, C-Koeffizienten und Fehler, HVAFG 26Vol\% 0,5 U/min 

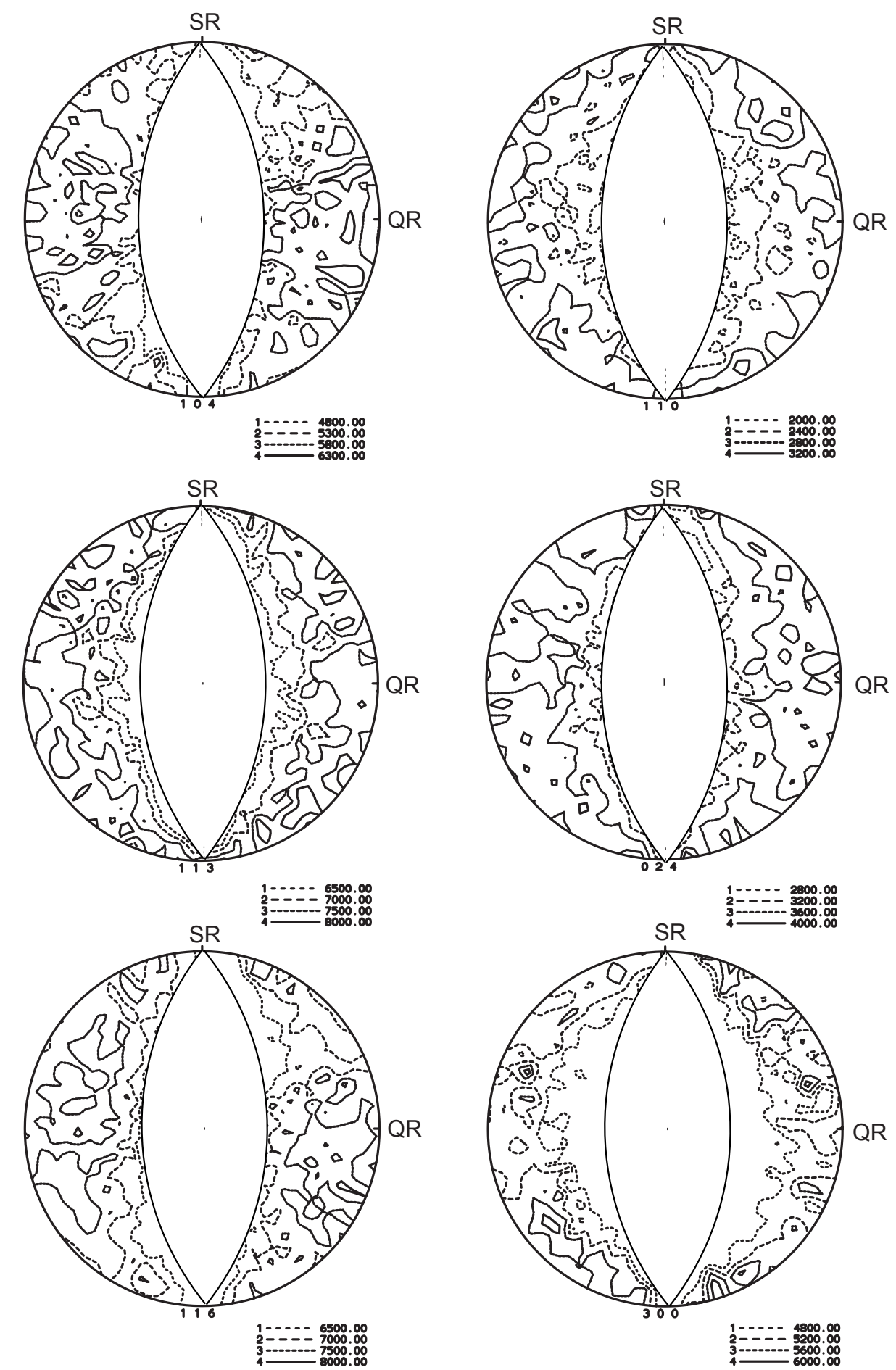

Abbildung C.3: Gemessene Polfiguren HVAFG 26Vol\% 1 U/min 

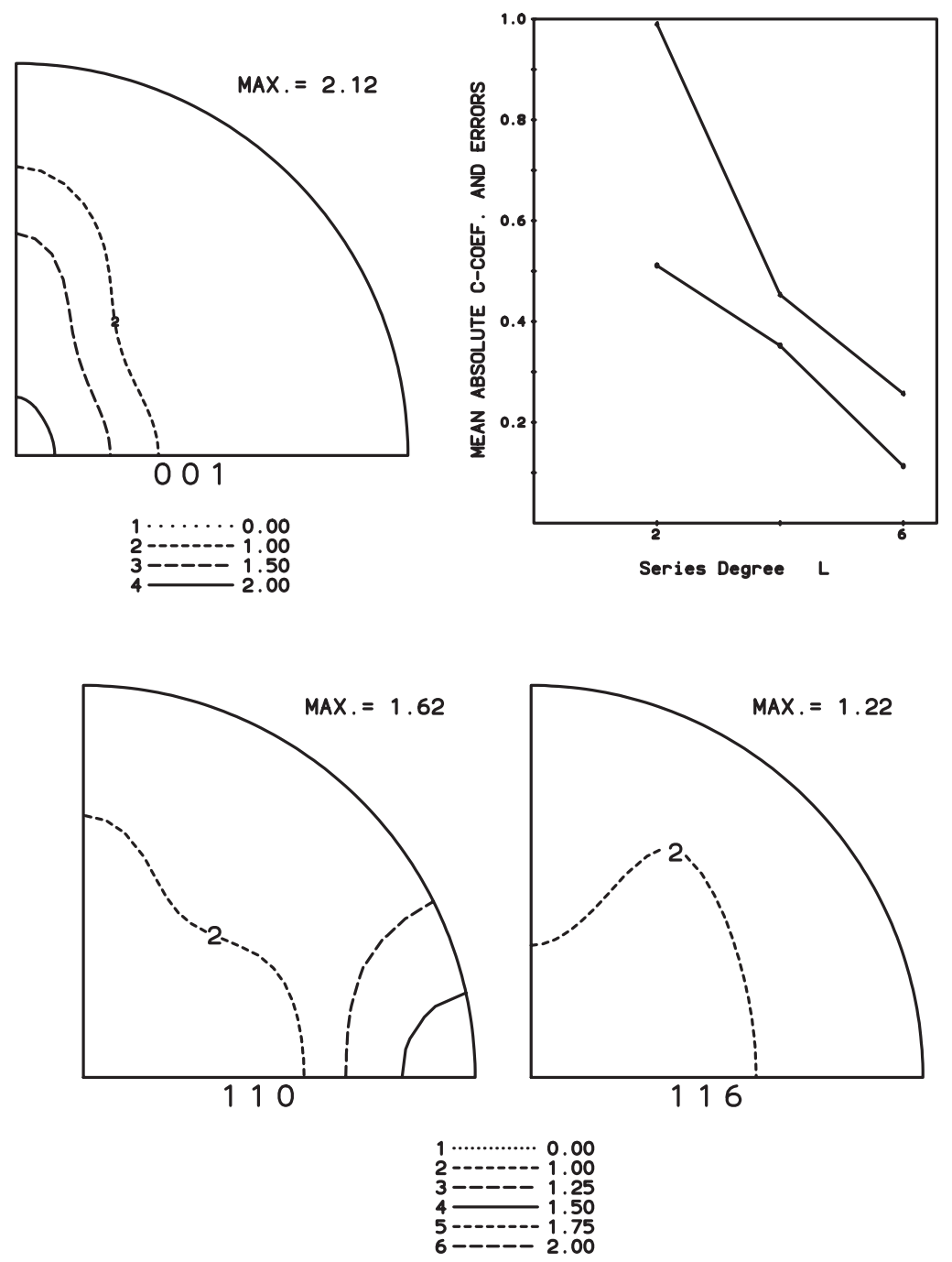

Abbildung C.4: Rückgerechnete Polfiguren, C-Koeffizienten und Fehler, HVAFG 26Vol\% $1 \mathrm{U} / \mathrm{min}$ 

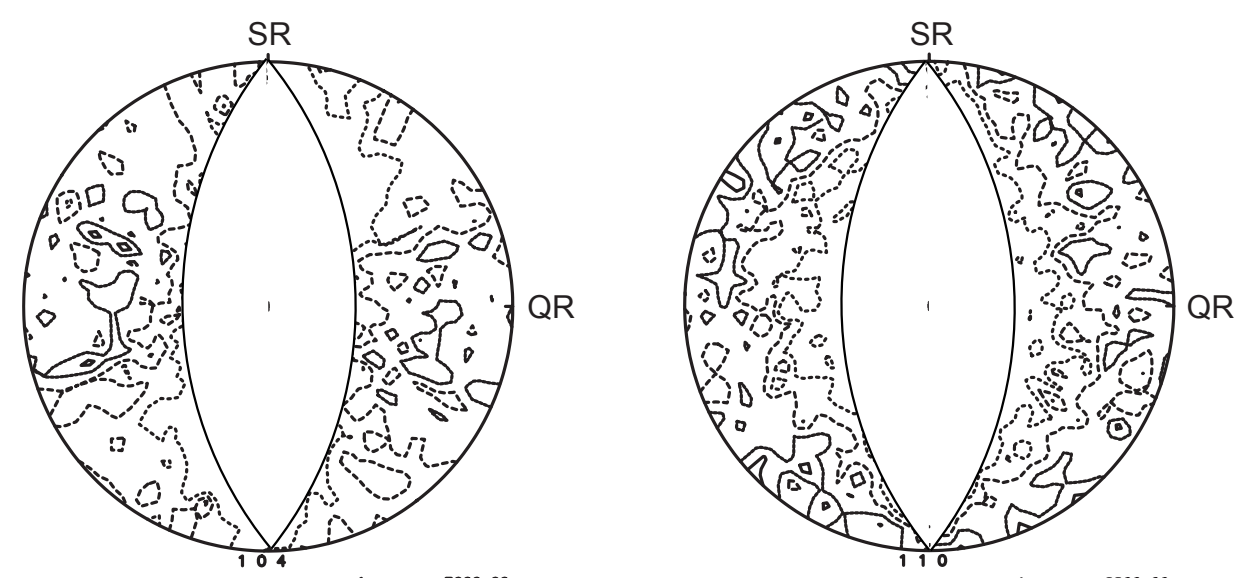

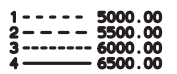
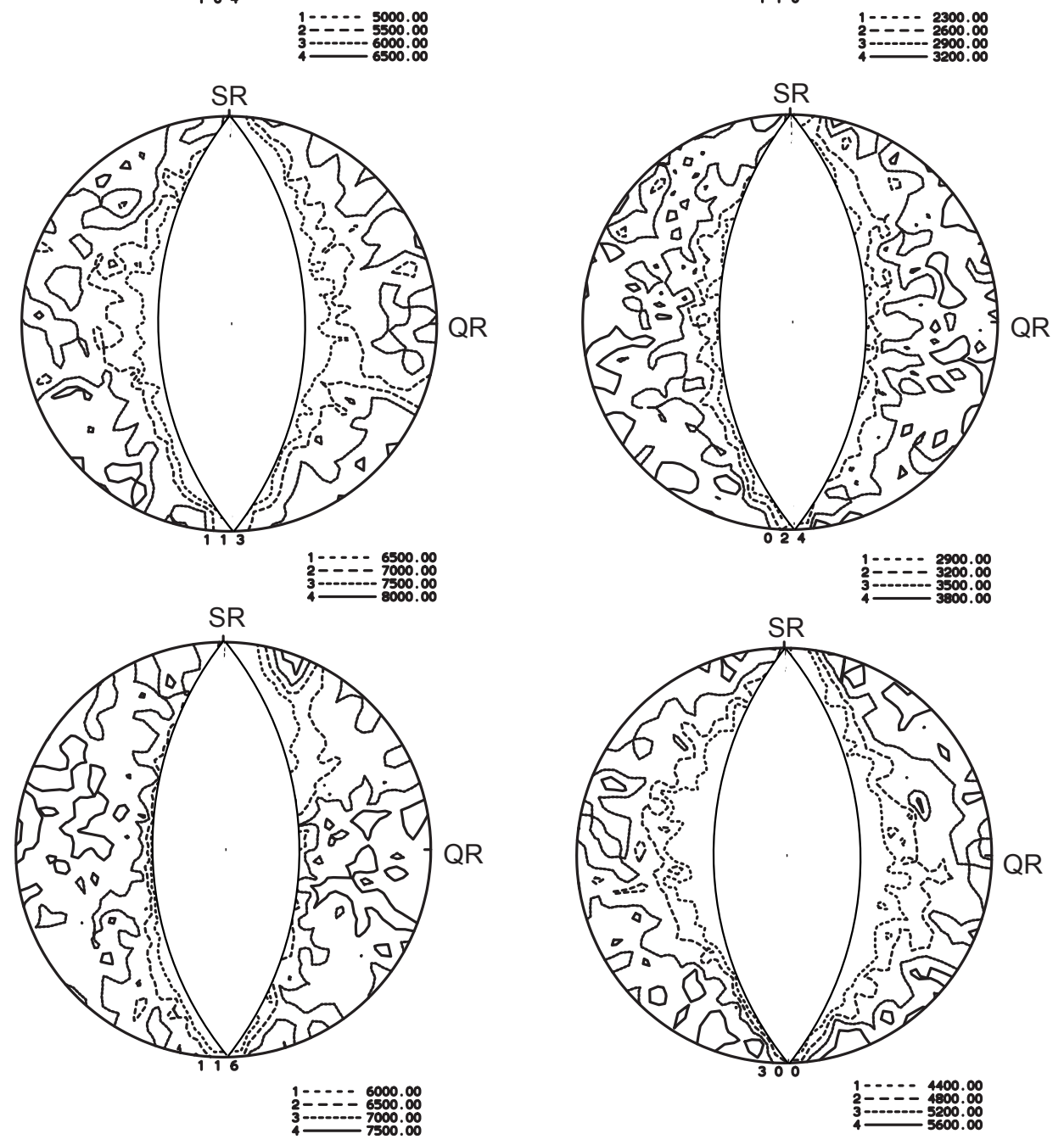

Abbildung C.5: Gemessene Polfiguren HVAFG 26Vol\% 2 U/min 

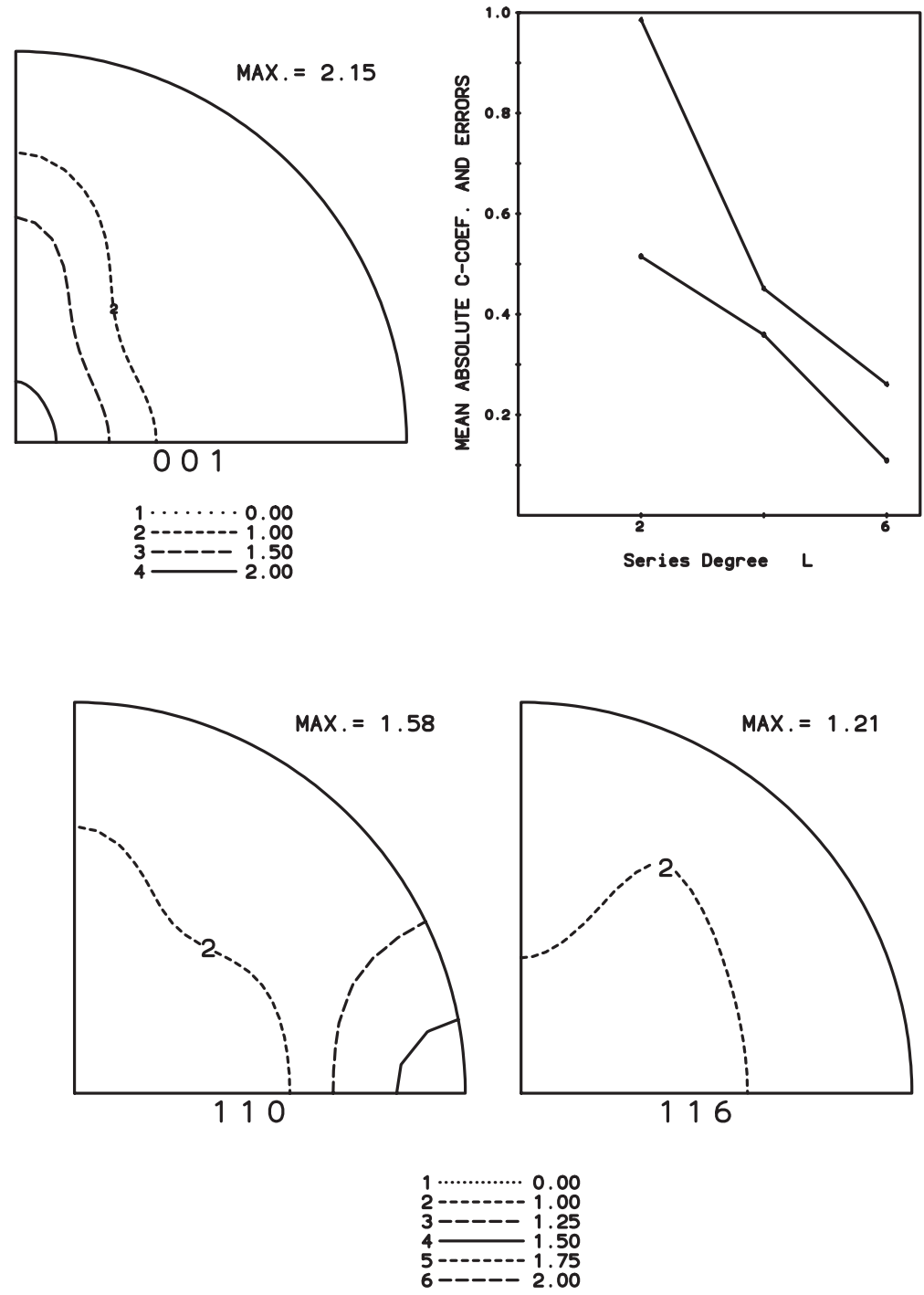

Abbildung C.6: Rückgerechnete Polfiguren, C-Koeffizienten und Fehler, HVAFG 26Vol\% $2 \mathrm{U} / \mathrm{min}$ 


\section{C.2 Probe HVAFG 13 Vol\%}
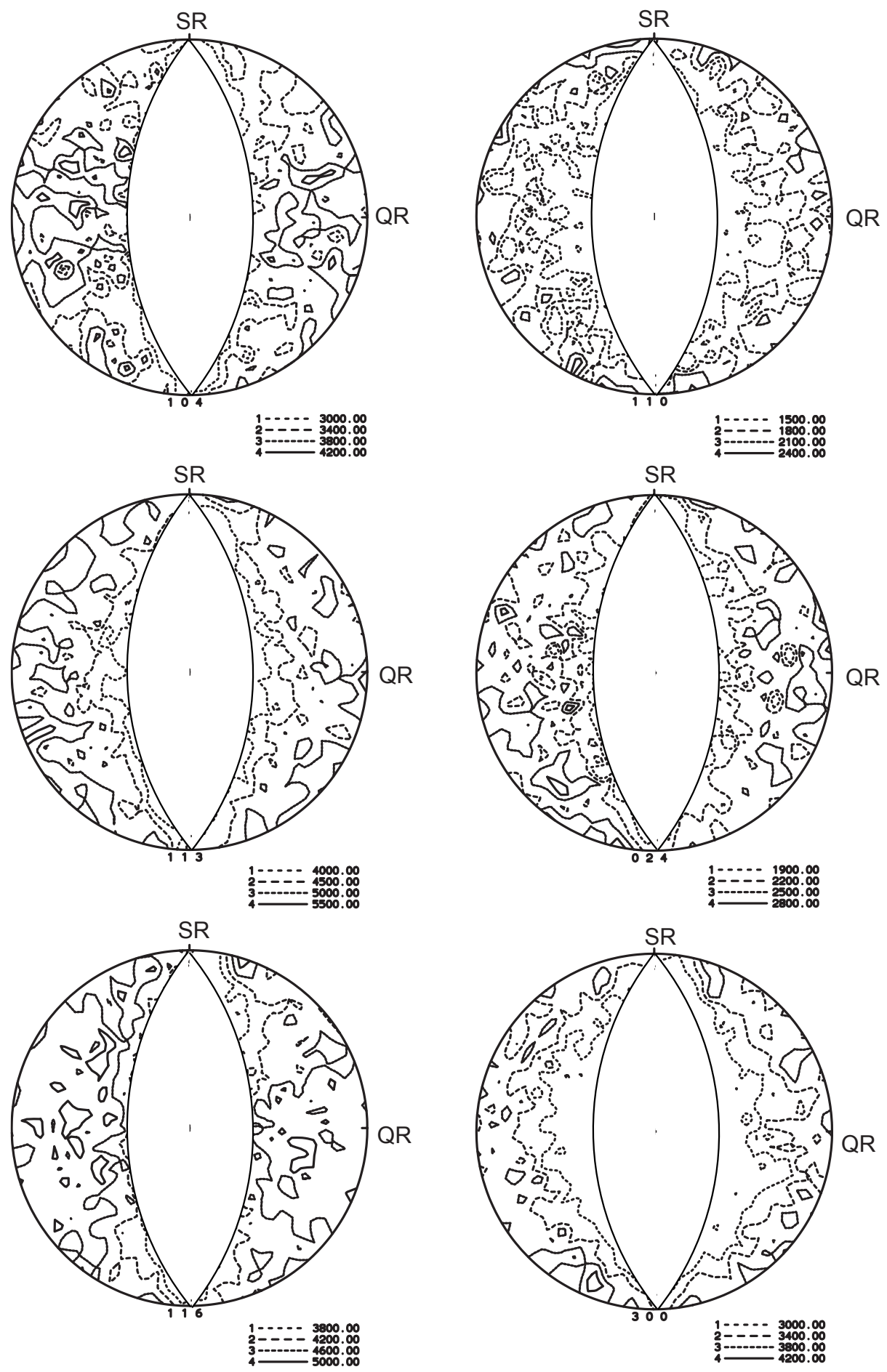

Abbildung C.7: Gemessene Polfiguren HVAFG 13Vol\% 0,5 U/min 

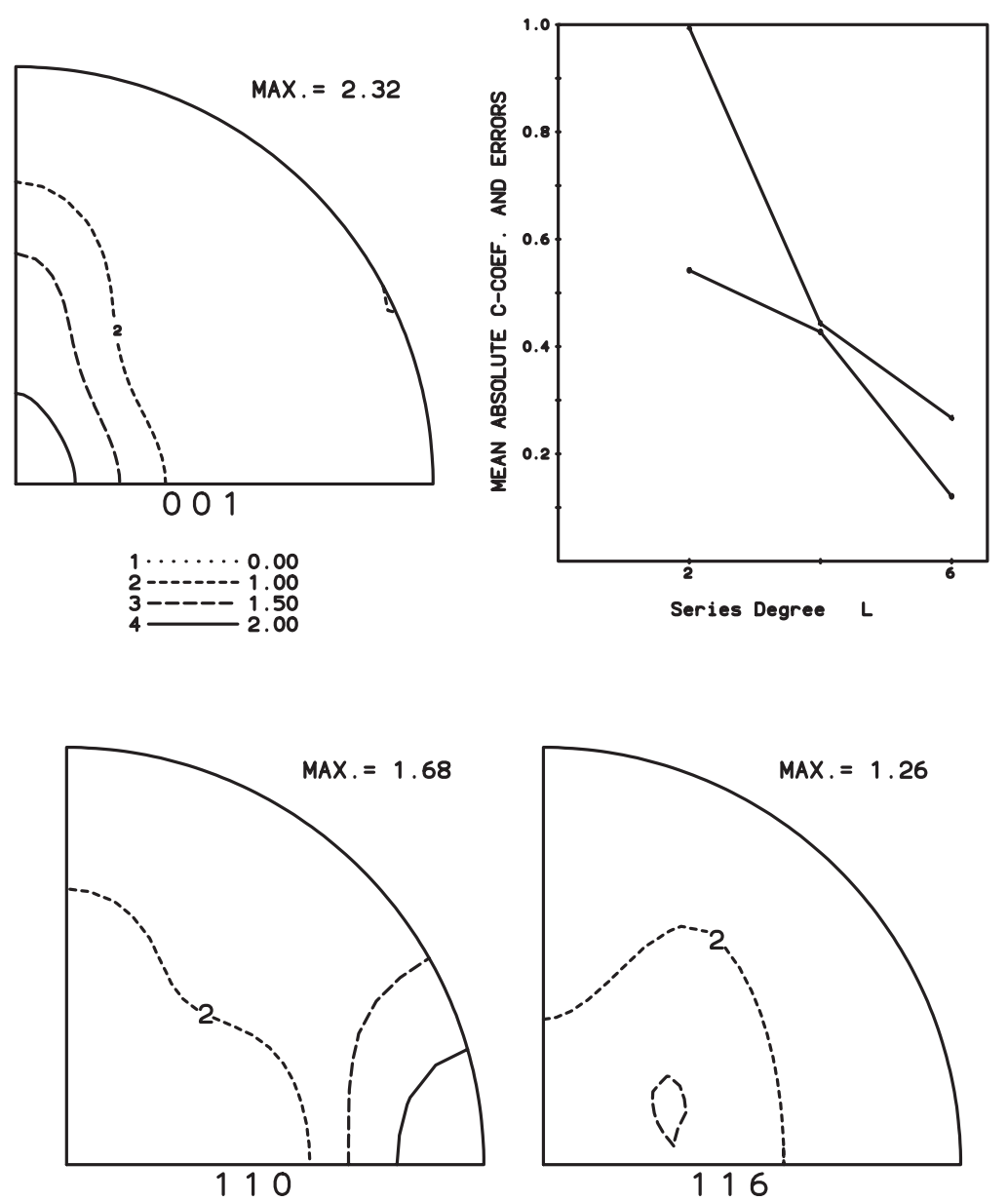

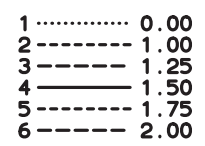

Abbildung C.8: Rückgerechnete Polfiguren, C-Koeffizienten und Fehler, HVAFG $13 \mathrm{Vol} \%$ 0,5 U/min 

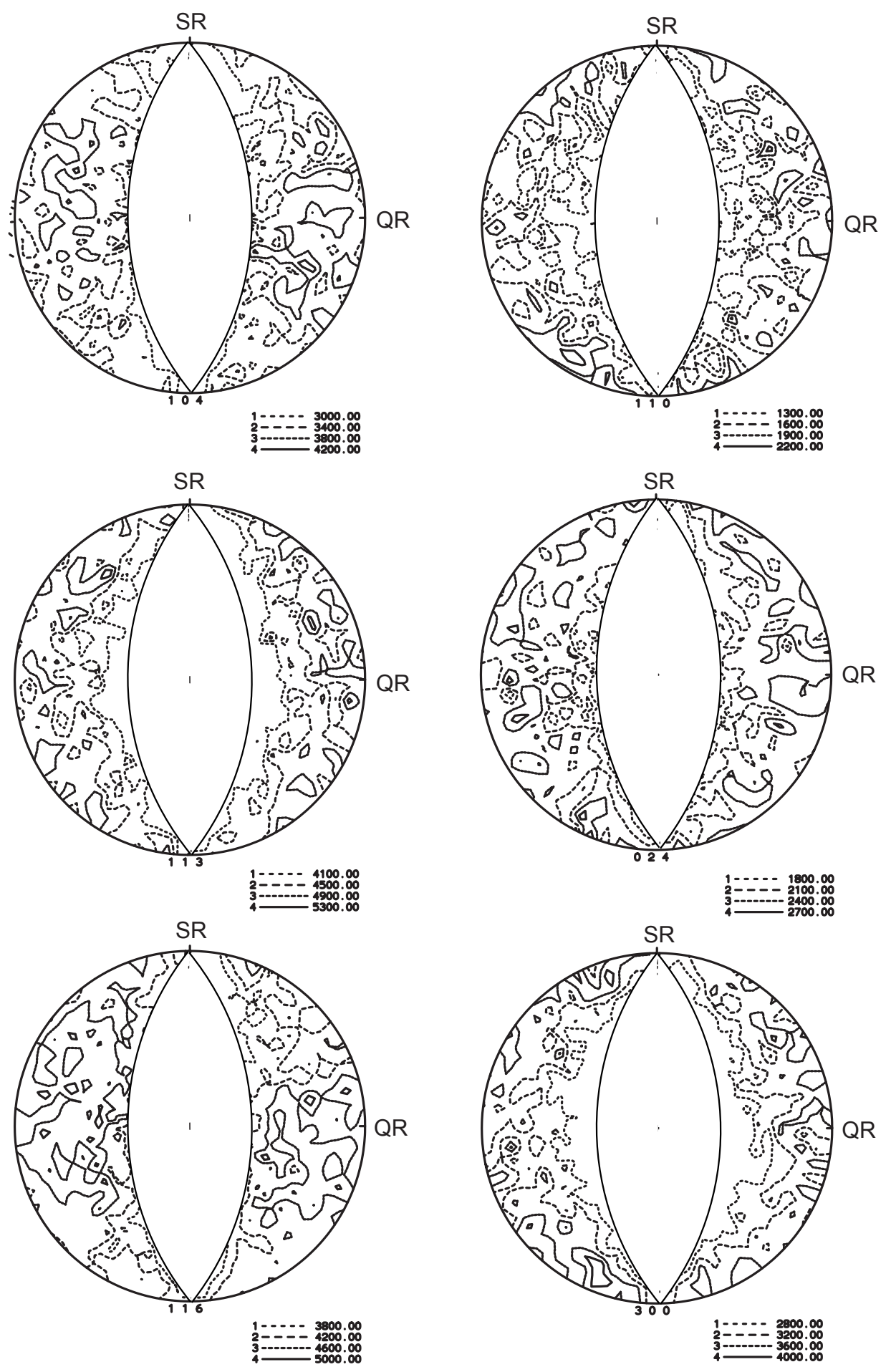

Abbildung C.9: Gemessene Polfiguren HVAFG 13Vol\% 1 U/min 

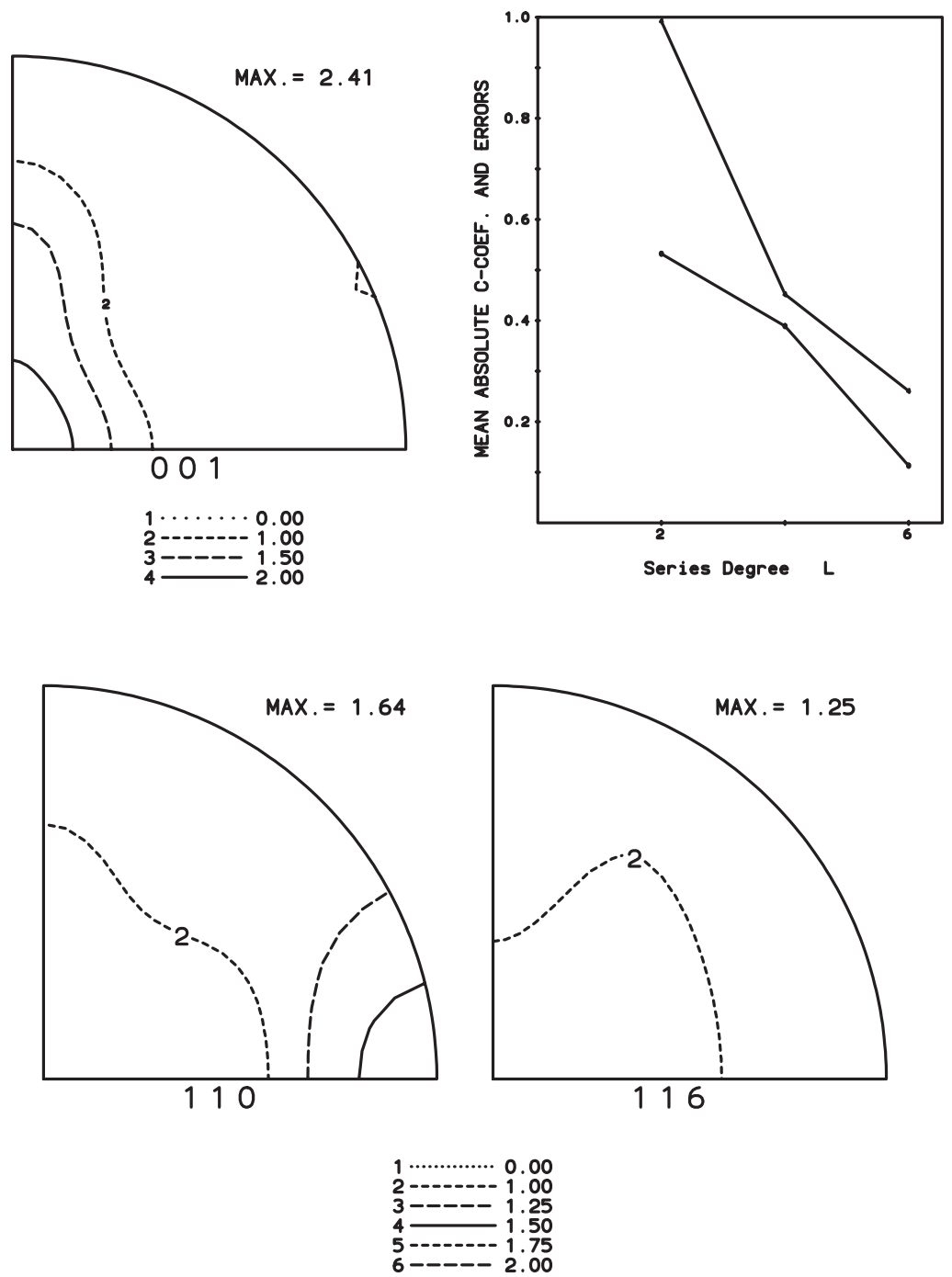

Abbildung C.10: Rückgerechnete Polfiguren, C-Koeffizienten und Fehler, HVAFG $13 \mathrm{Vol} \% 1 \mathrm{U} / \mathrm{min}$ 


\section{C.3 Probe HVAFG 9Vol\%}
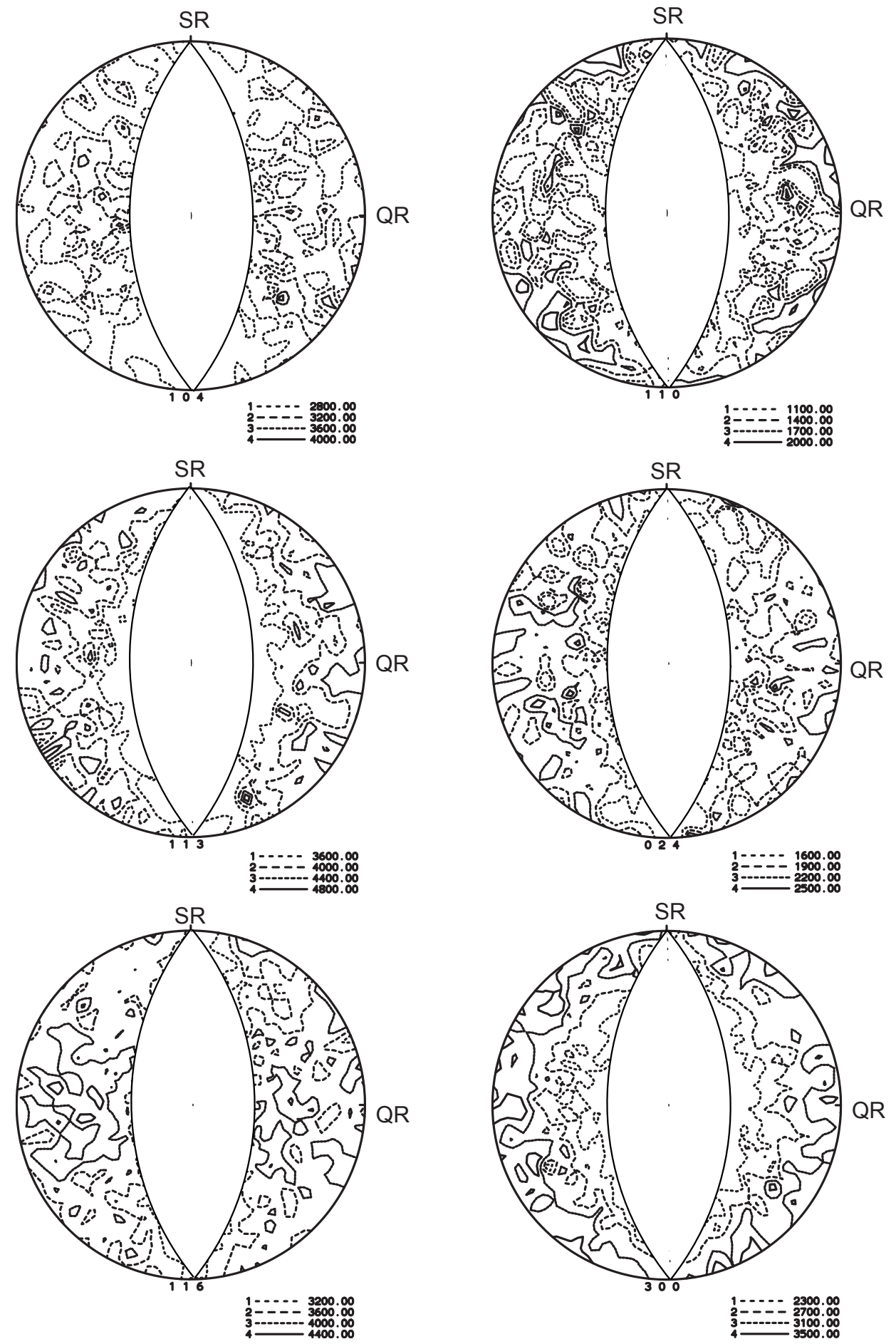

Abbildung C.11: Gemessene Polfiguren HVAFG 9Vol\% 0,5 U/min 

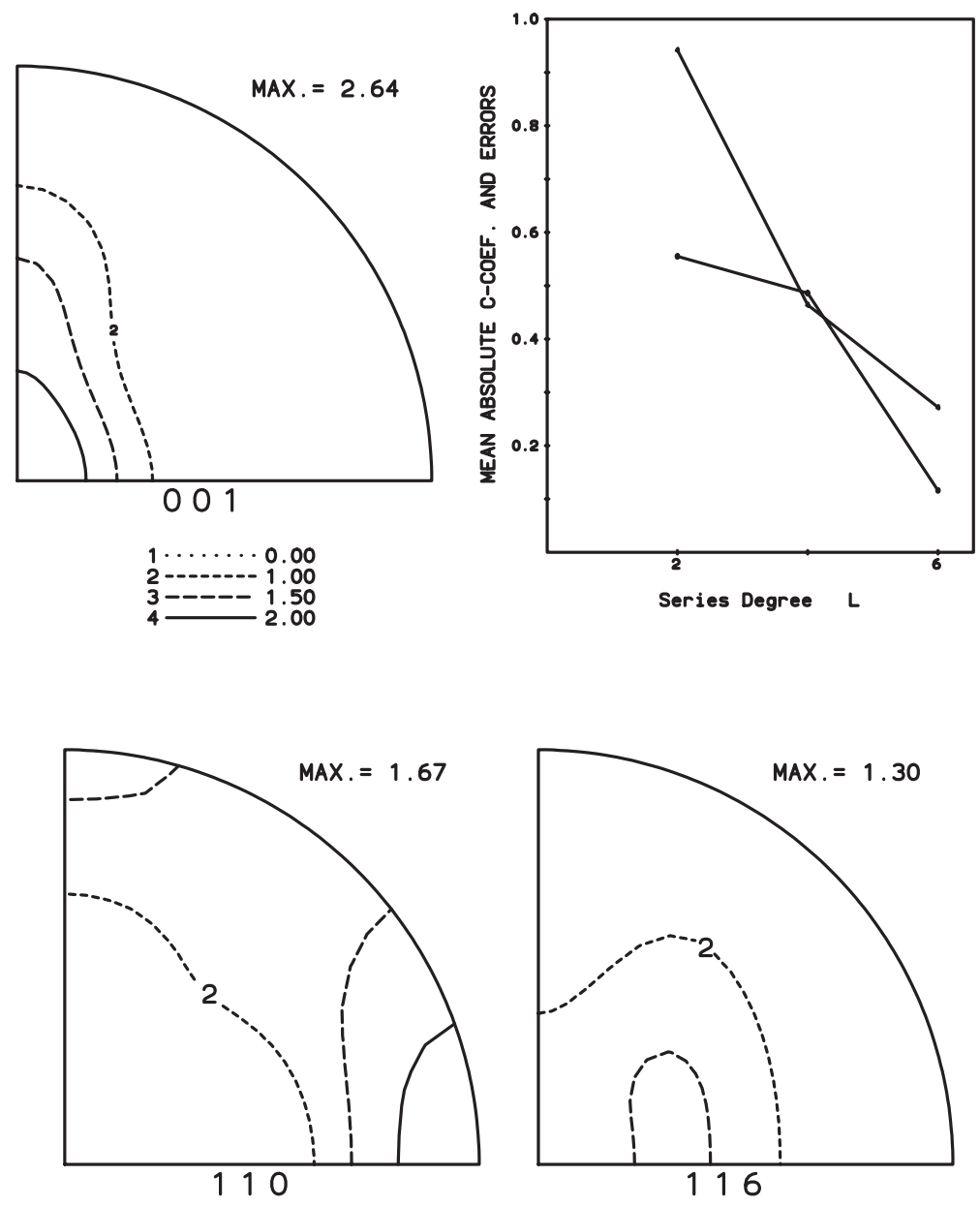

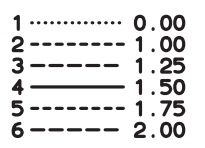

Abbildung C.12: Rückgerechnete Polfiguren, C-Koeffizienten und Fehler, HVAFG 9Vol\% 0,5 U/min 

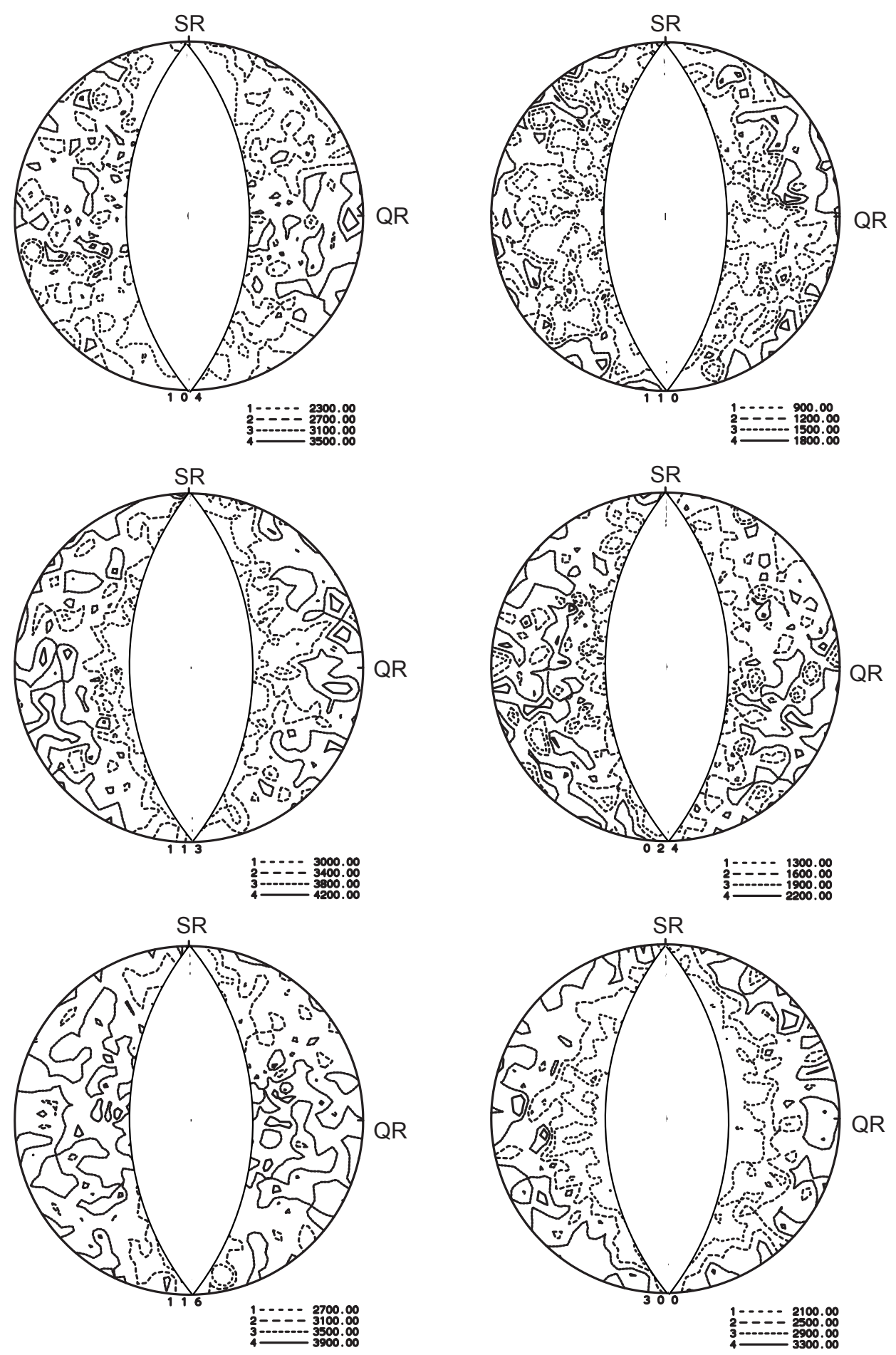

Abbildung C.13: Gemessene Polfiguren HVAFG 9Vol\% 1 U/min 

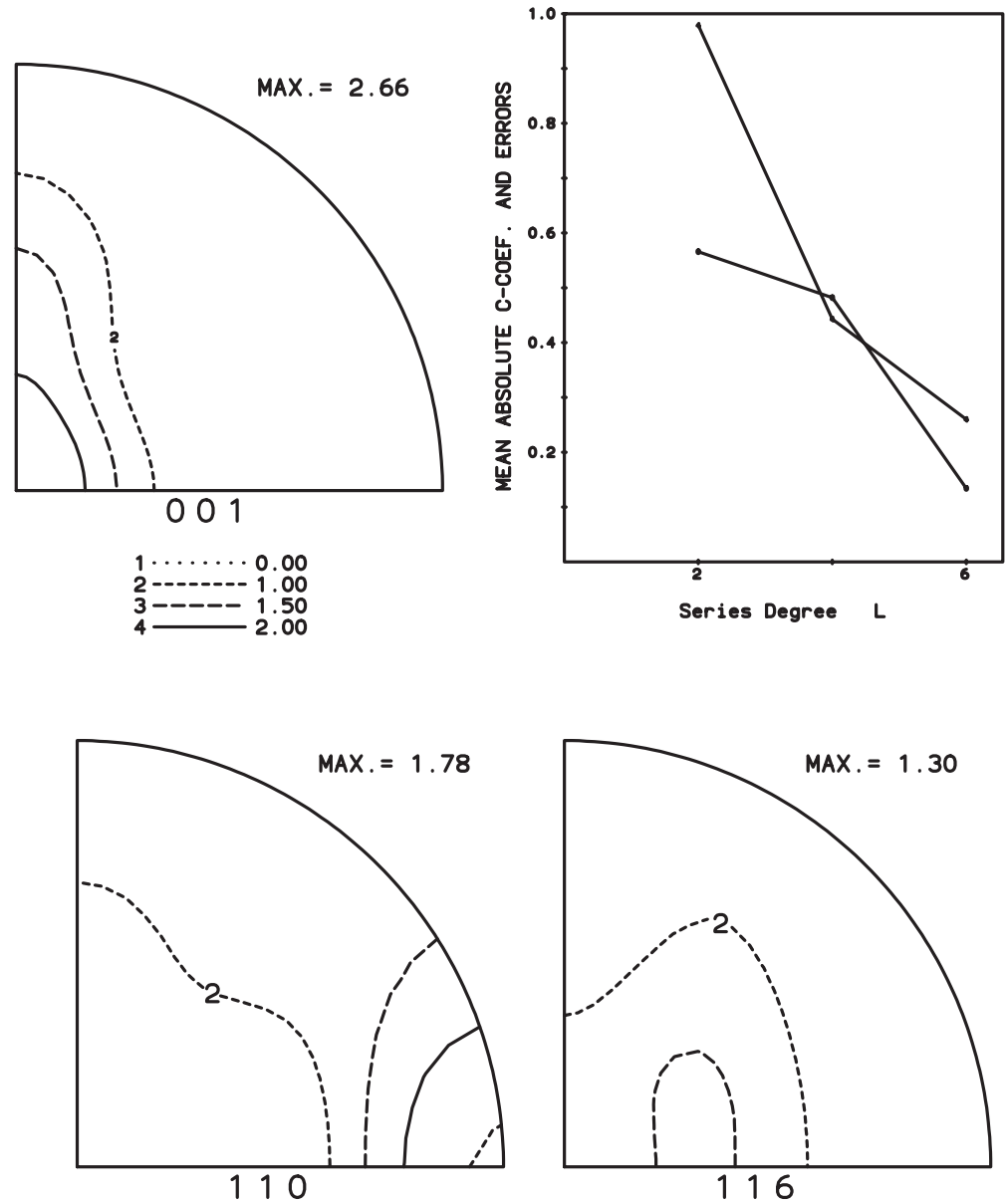

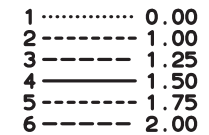

Abbildung C.14: Rückgerechnete Polfiguren, C-Koeffizienten und Fehler, HVAFG 9Vol\% $1 \mathrm{U} / \mathrm{min}$ 

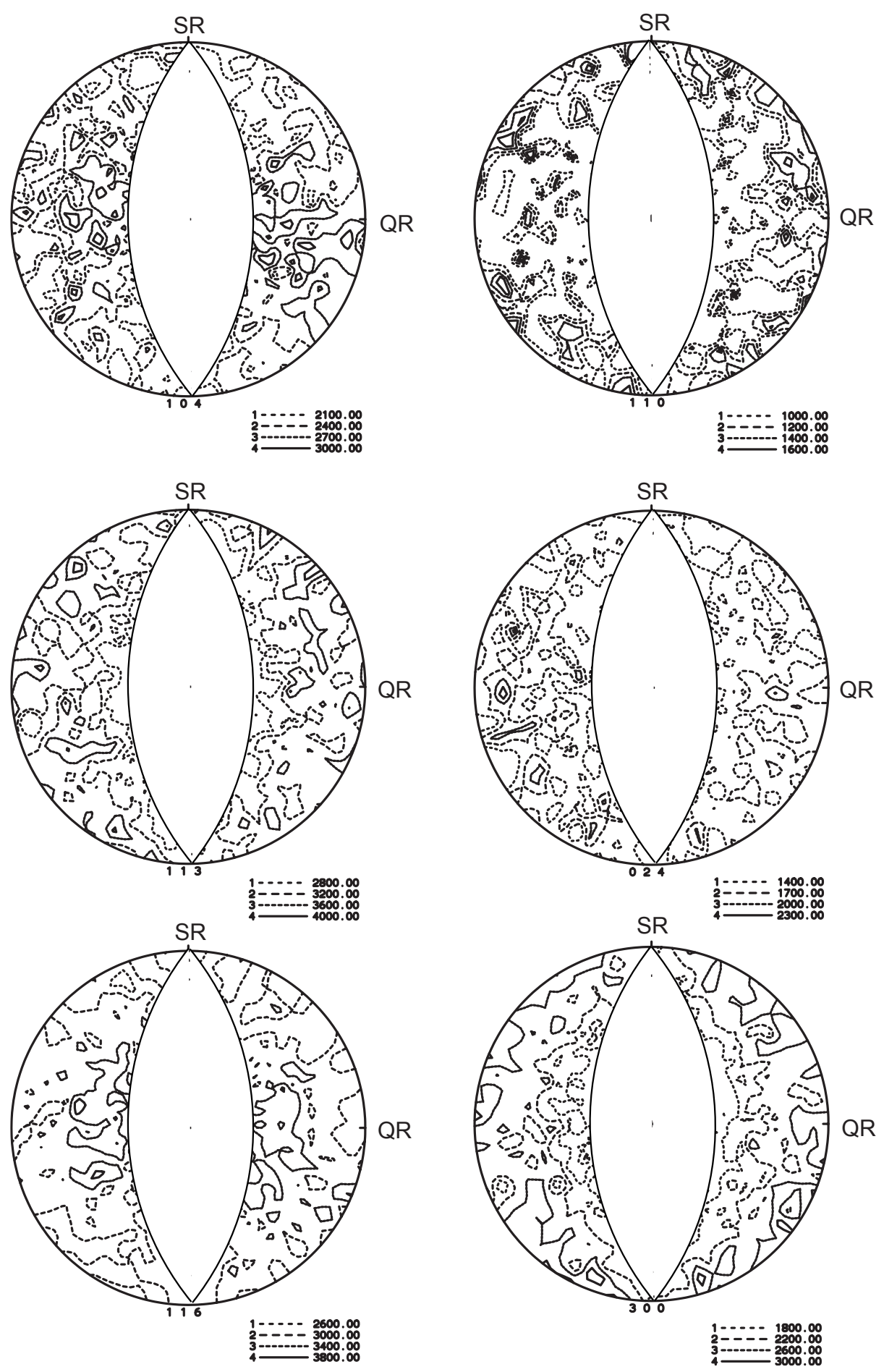

Abbildung C.15: Gemessene Polfiguren HVAFG 9Vol\% 2 U/min 

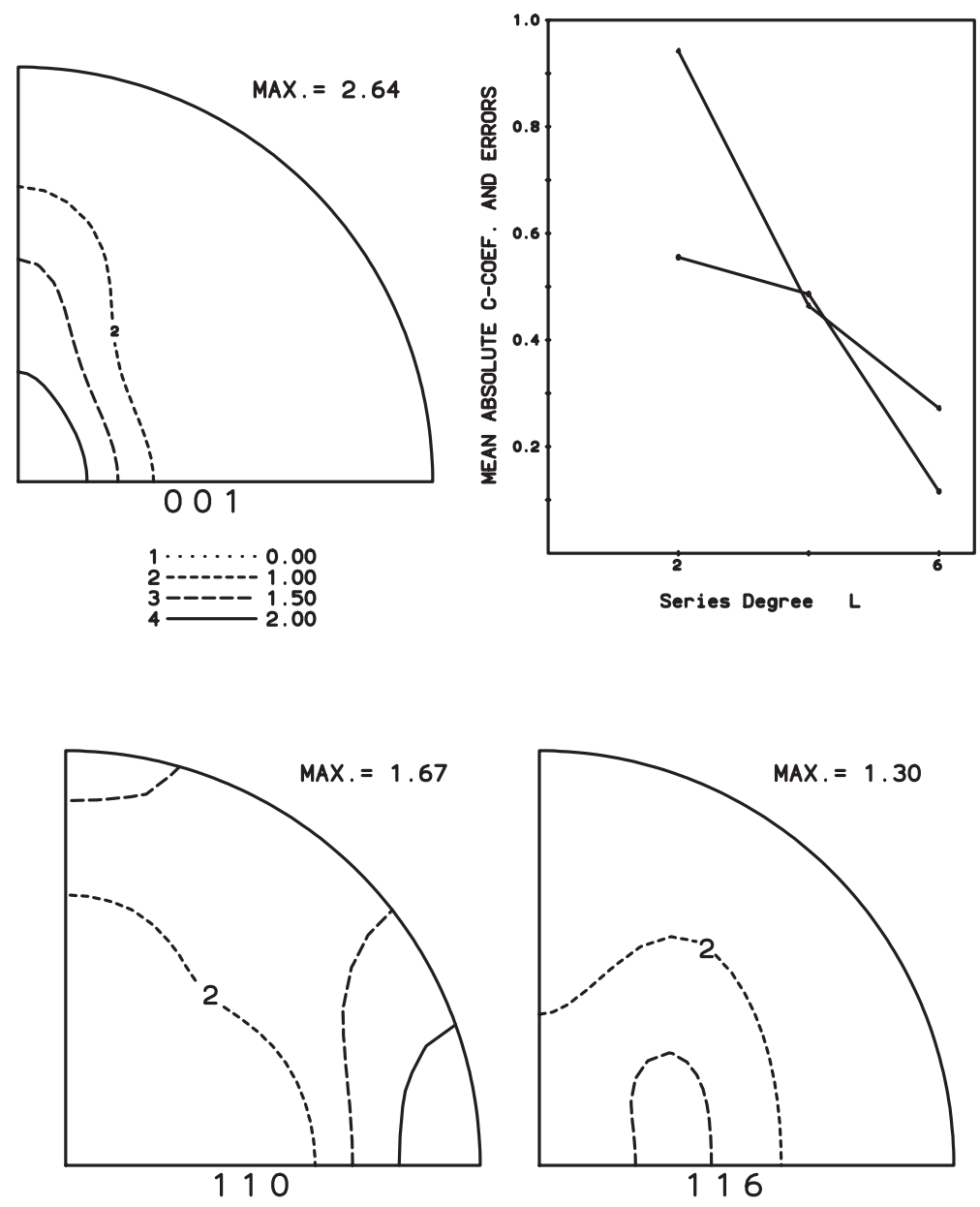

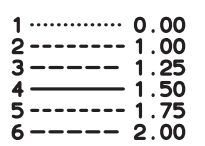

Abbildung C.16: Rückgerechnete Polfiguren, C-Koeffizienten und Fehler, HVAFG 9Vol\% $2 \mathrm{U} / \mathrm{min}$ 


\section{C.4 Probe CTC20 26Vol\%}
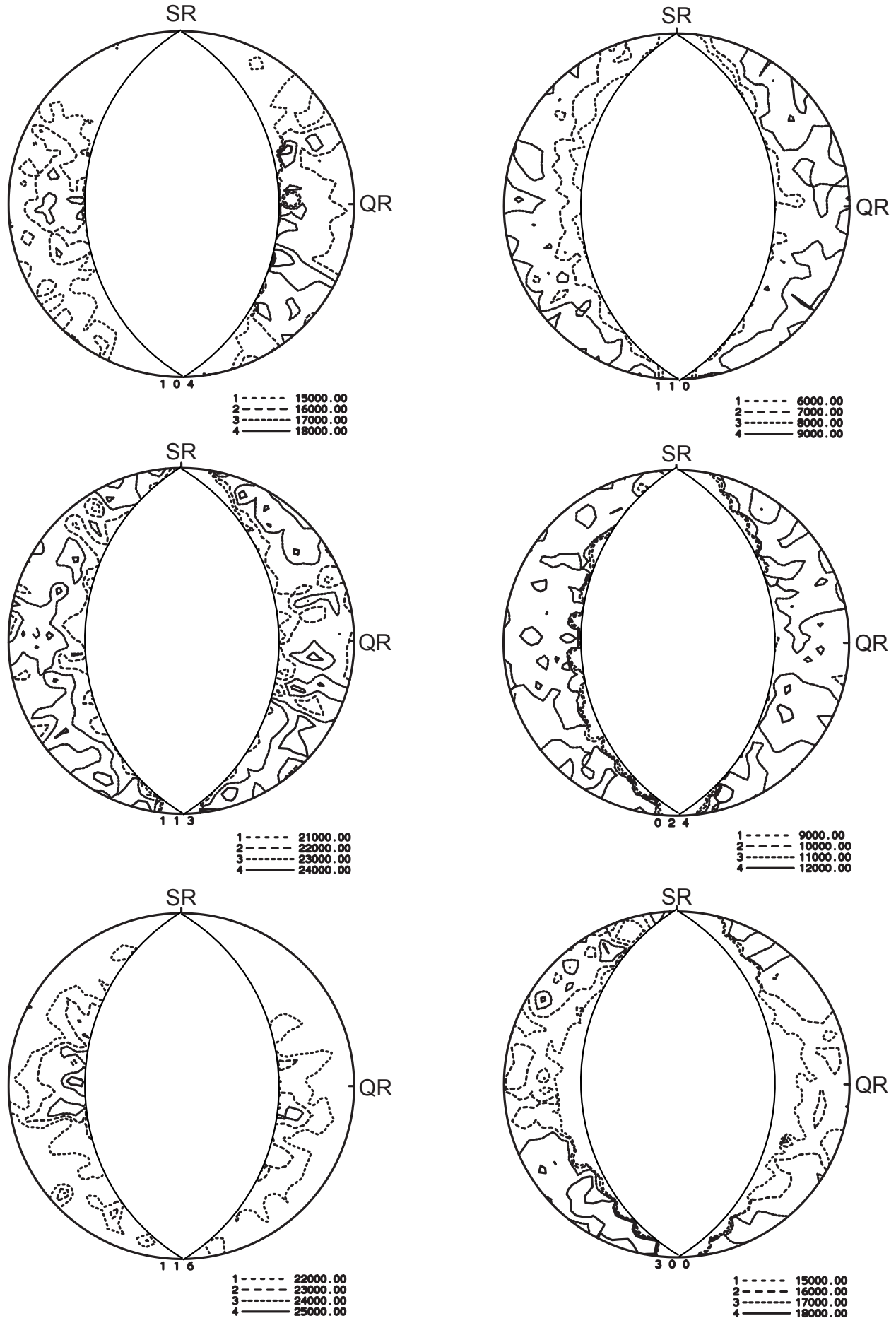

Abbildung C.17: Gemessene Polfiguren CTC20 26Vol\% 0,5 U/min 

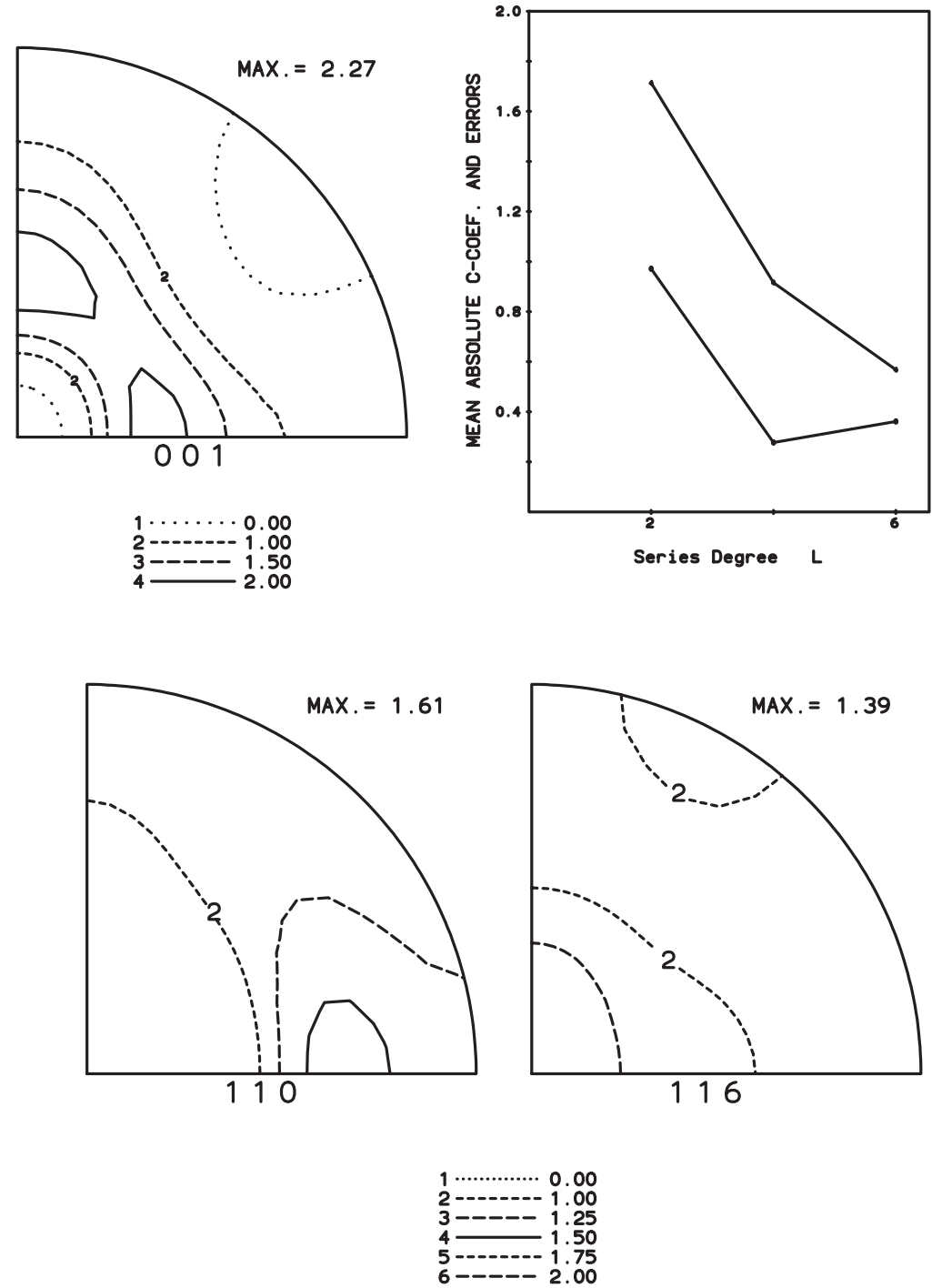

Abbildung C.18: Rückgerechnete Polfiguren, C-Koeffizienten und Fehler, CTC20 26Vol\% 0,5 U/min 

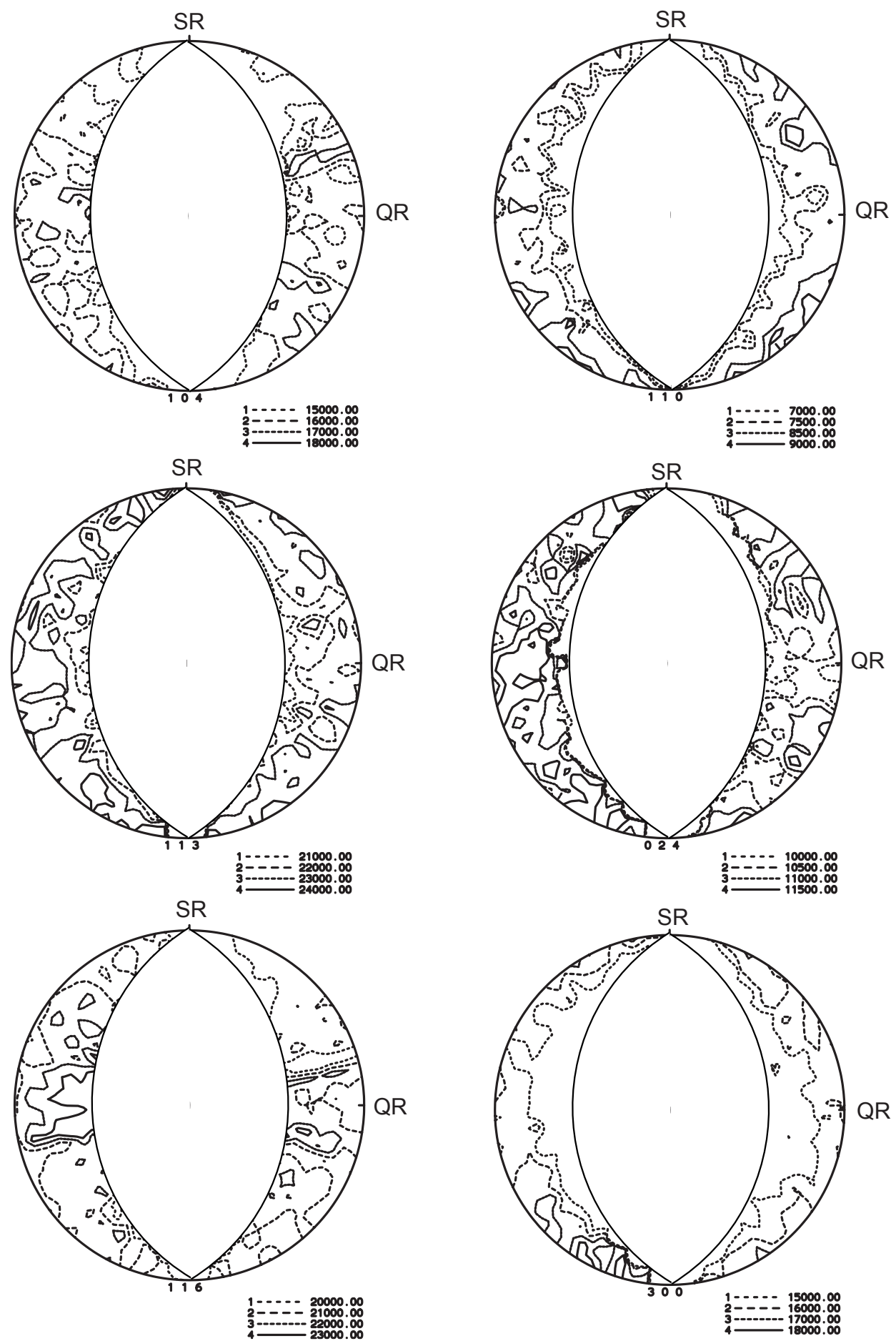

Abbildung C.19: Gemessene Polfiguren CTC20 26Vol\% 1 U/min 

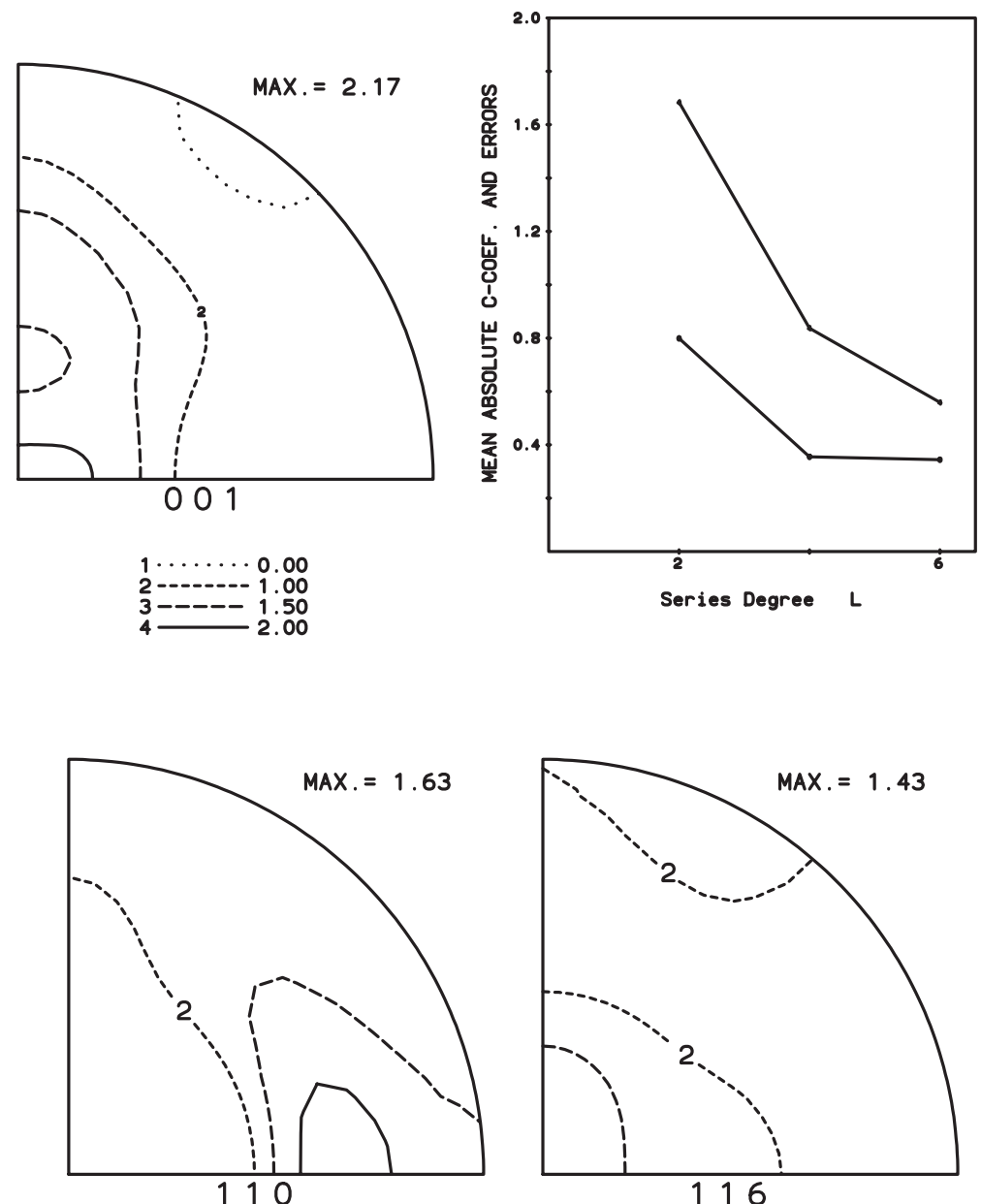

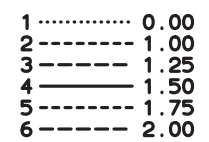

Abbildung C.20: Rückgerechnete Polfiguren, C-Koeffizienten und Fehler, CTC20 26Vol\% $1 \mathrm{U} / \mathrm{min}$ 

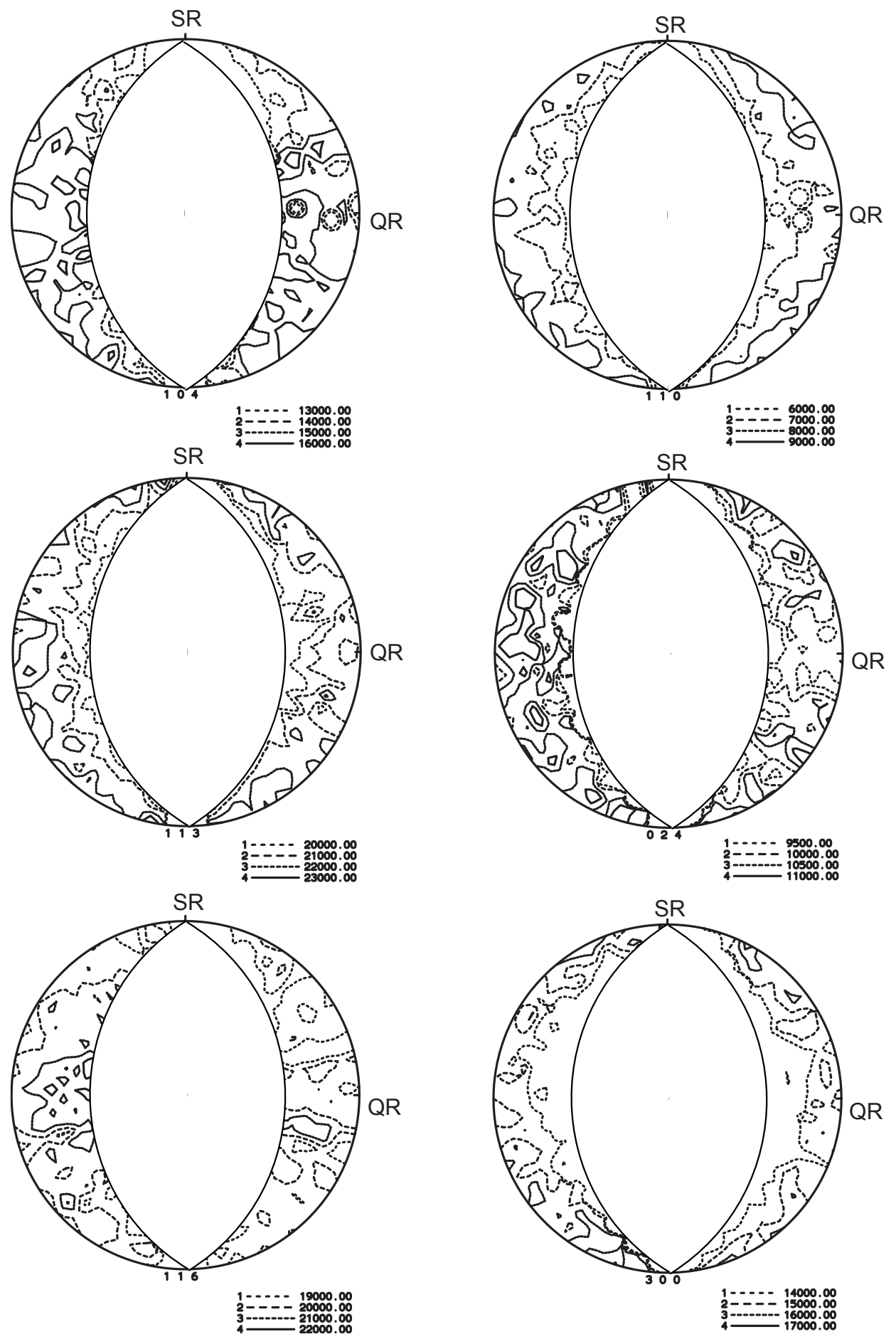

Abbildung C.21: Gemessene Polfiguren CTC20 26Vol\% 2 U/min 

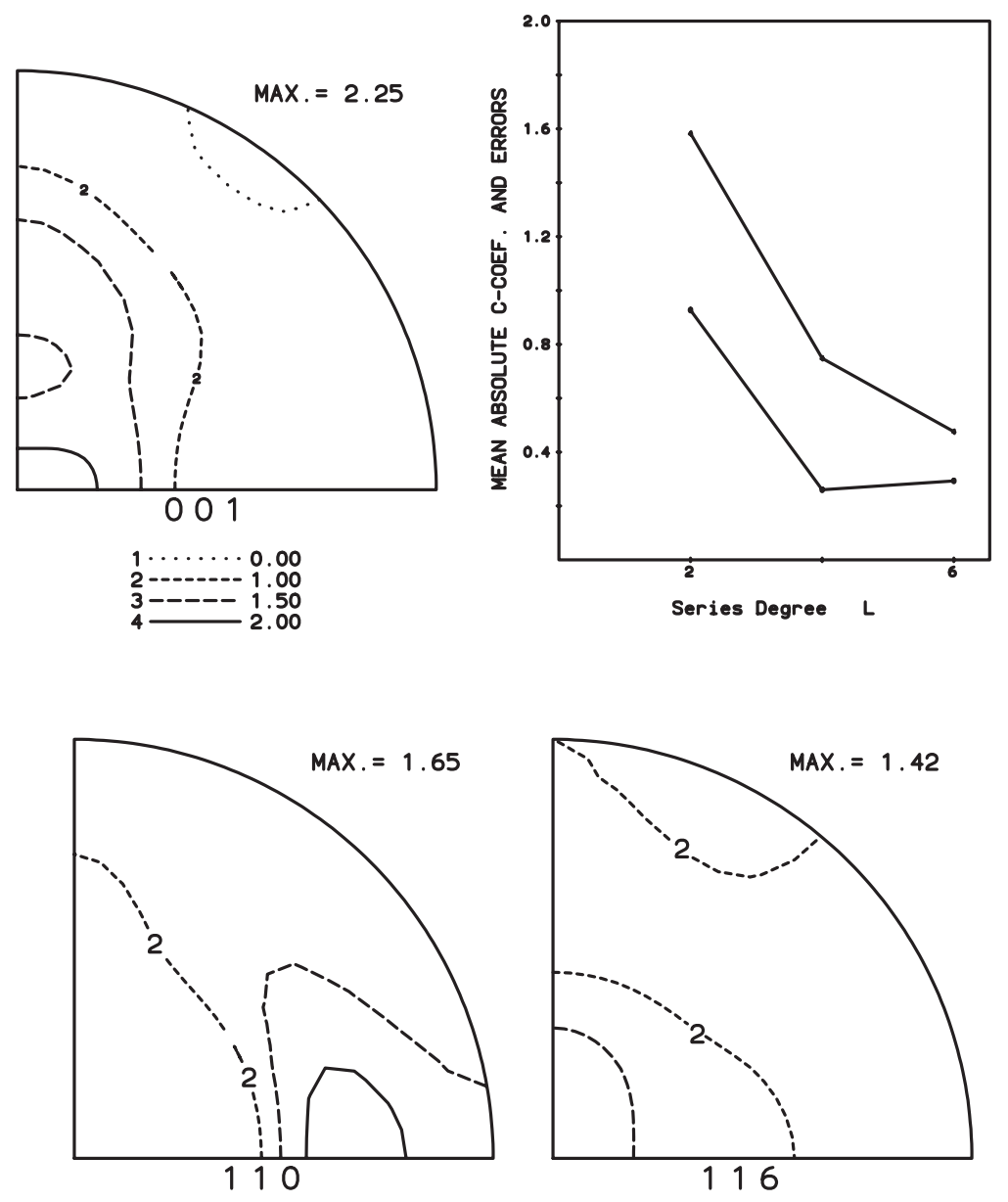

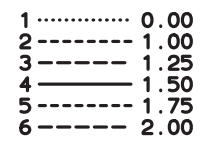

Abbildung C.22: Rückgerechnete Polfiguren, C-Koeffizienten und Fehler, CTC20 26Vol\% $2 \mathrm{U} / \mathrm{min}$ 


\section{C.5 Probe CTC20 13Vol\%}
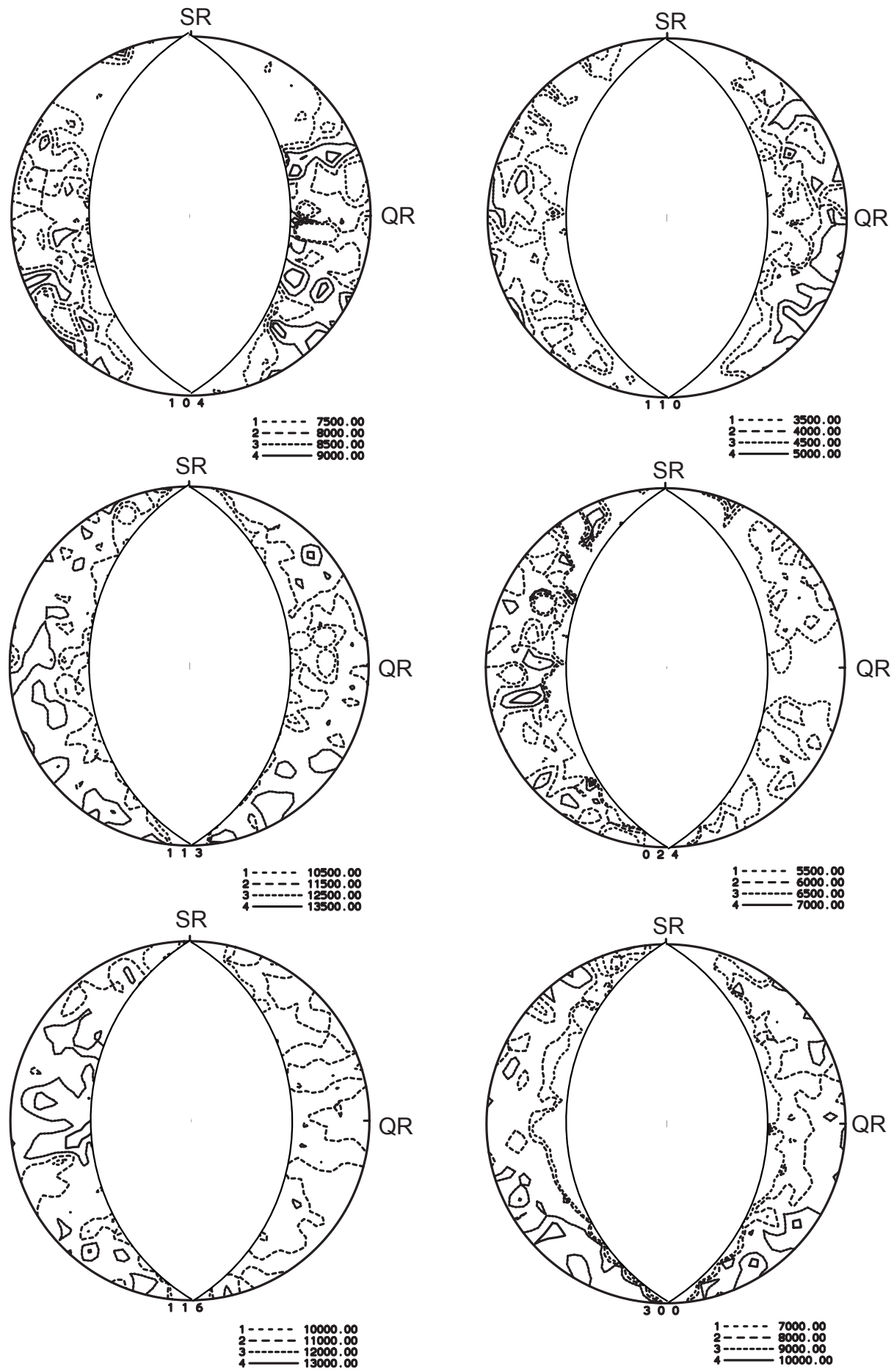

Abbildung C.23: Gemessene Polfiguren CTC20 13Vol\% 0,5 U/min 

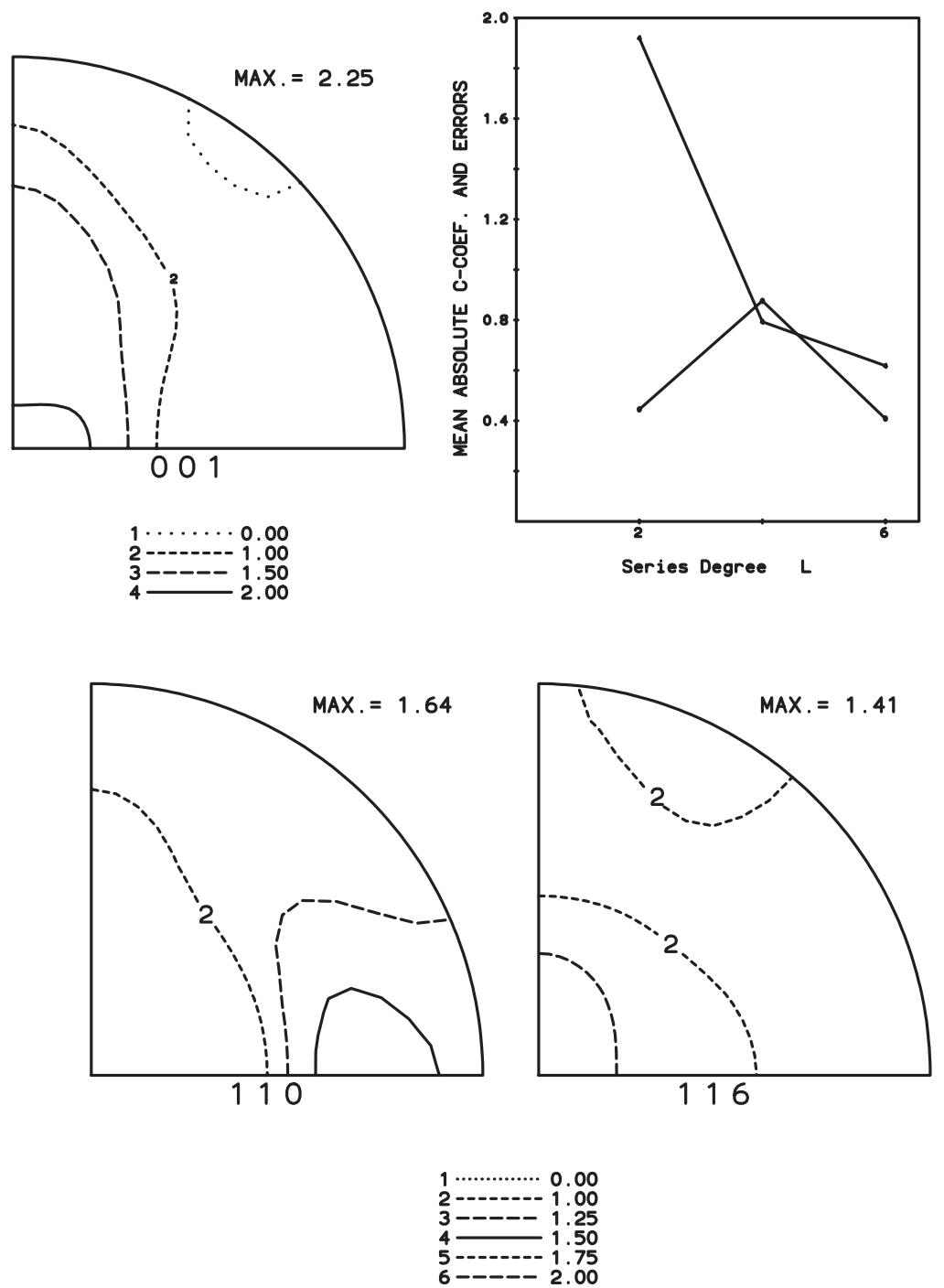

Abbildung C.24: Rückgerechnete Polfiguren, C-Koeffizienten und Fehler, CTC20 13Vol\% 0,5 U/min 

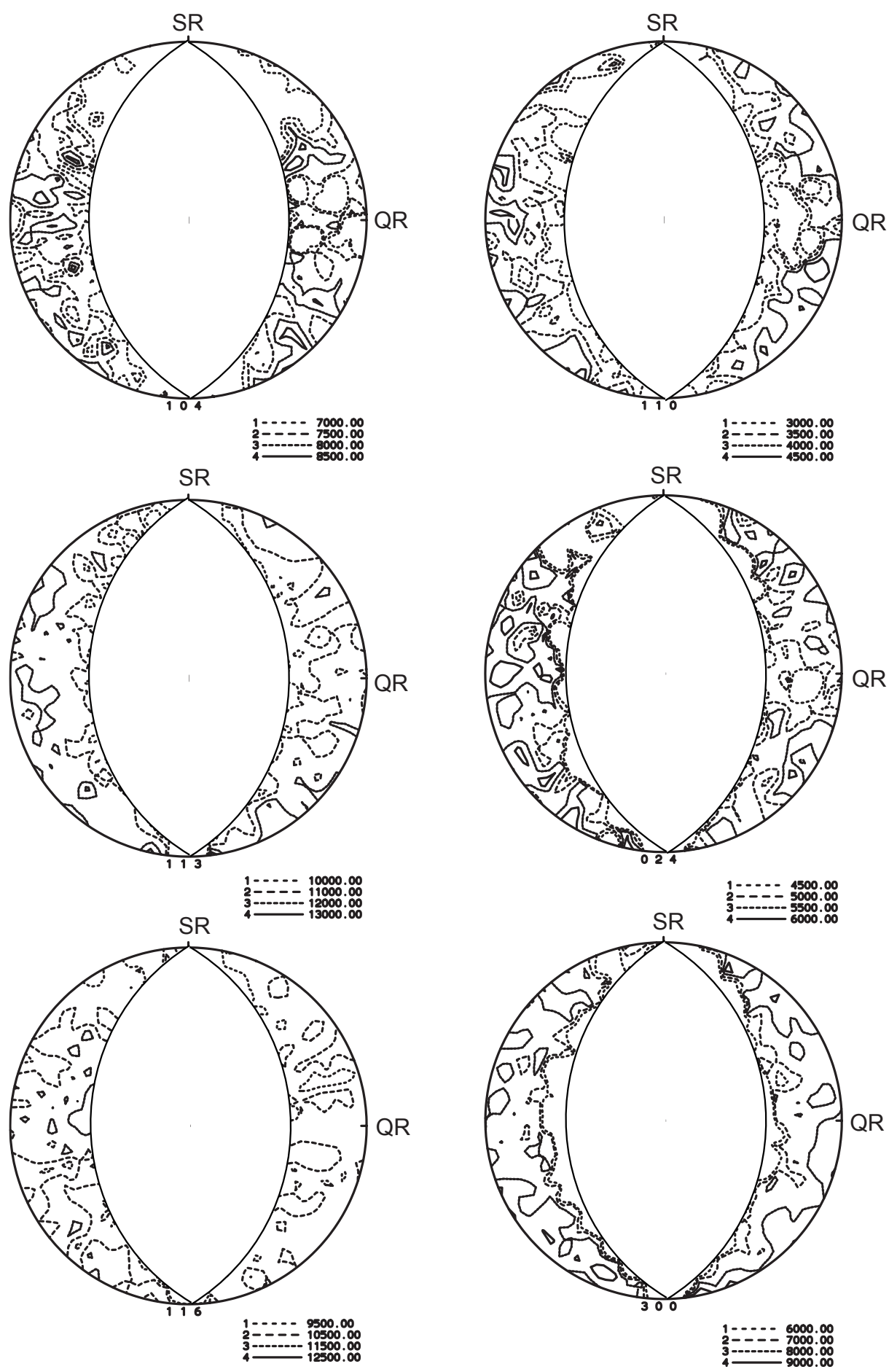

Abbildung C.25: Gemessene Polfiguren CTC20 13Vol\% 1 U/min 

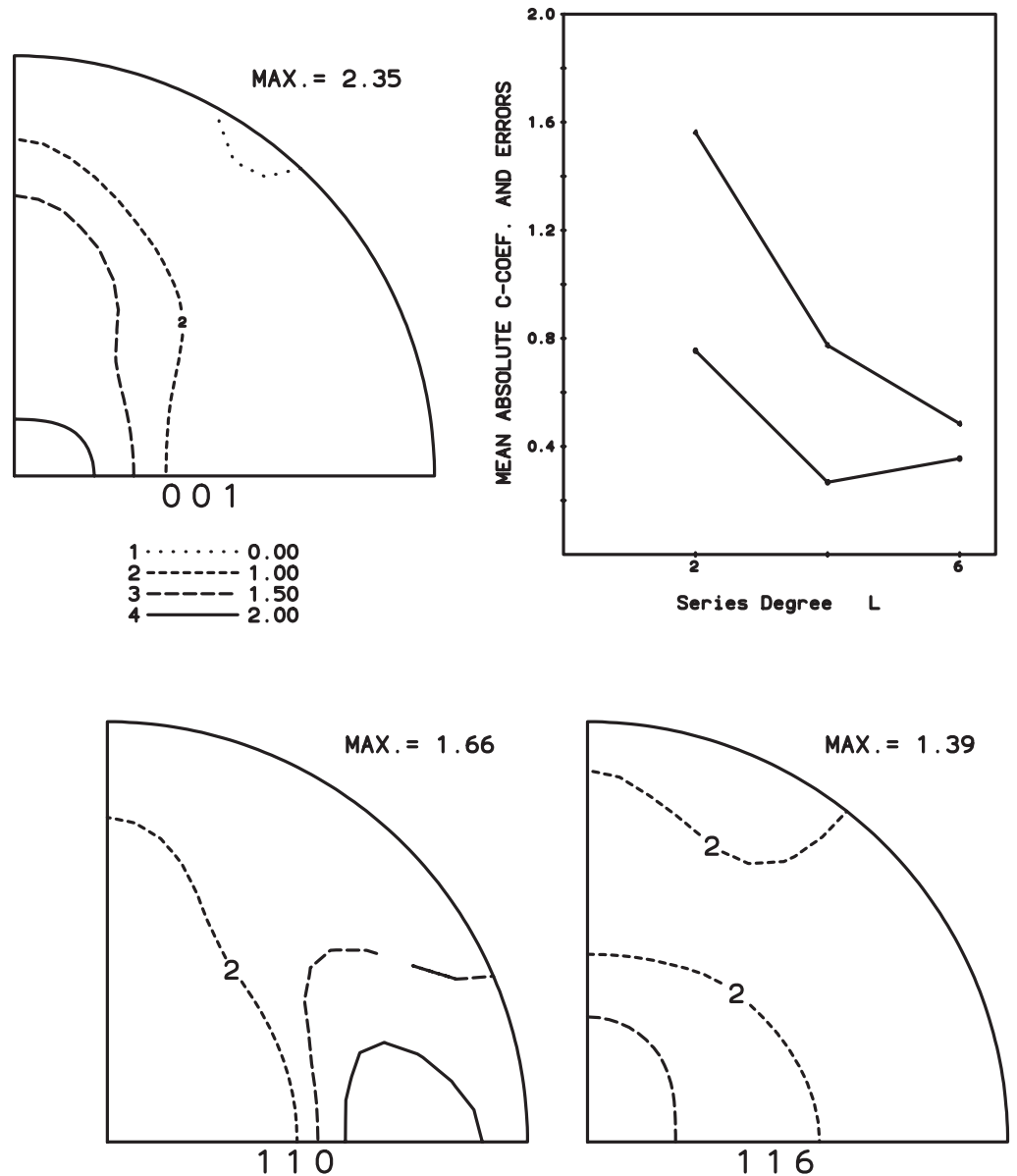

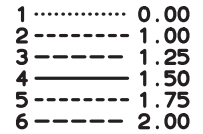

Abbildung C.26: Rückgerechnete Polfiguren, C-Koeffizienten und Fehler, CTC20 13Vol\% $1 \mathrm{U} / \mathrm{min}$ 

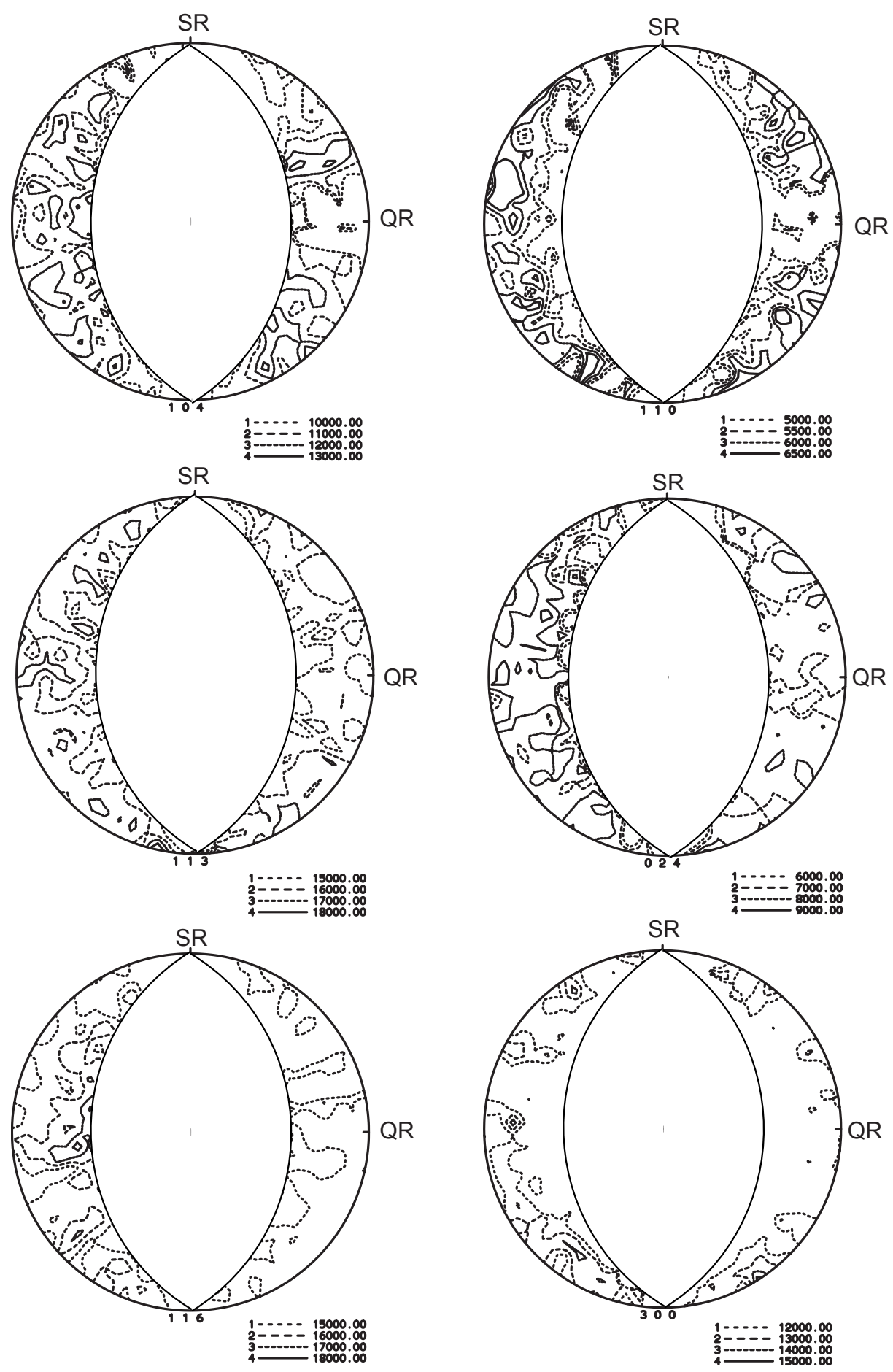

Abbildung C.27: Gemessene Polfiguren CTC20 13Vol\% 2 U/min 

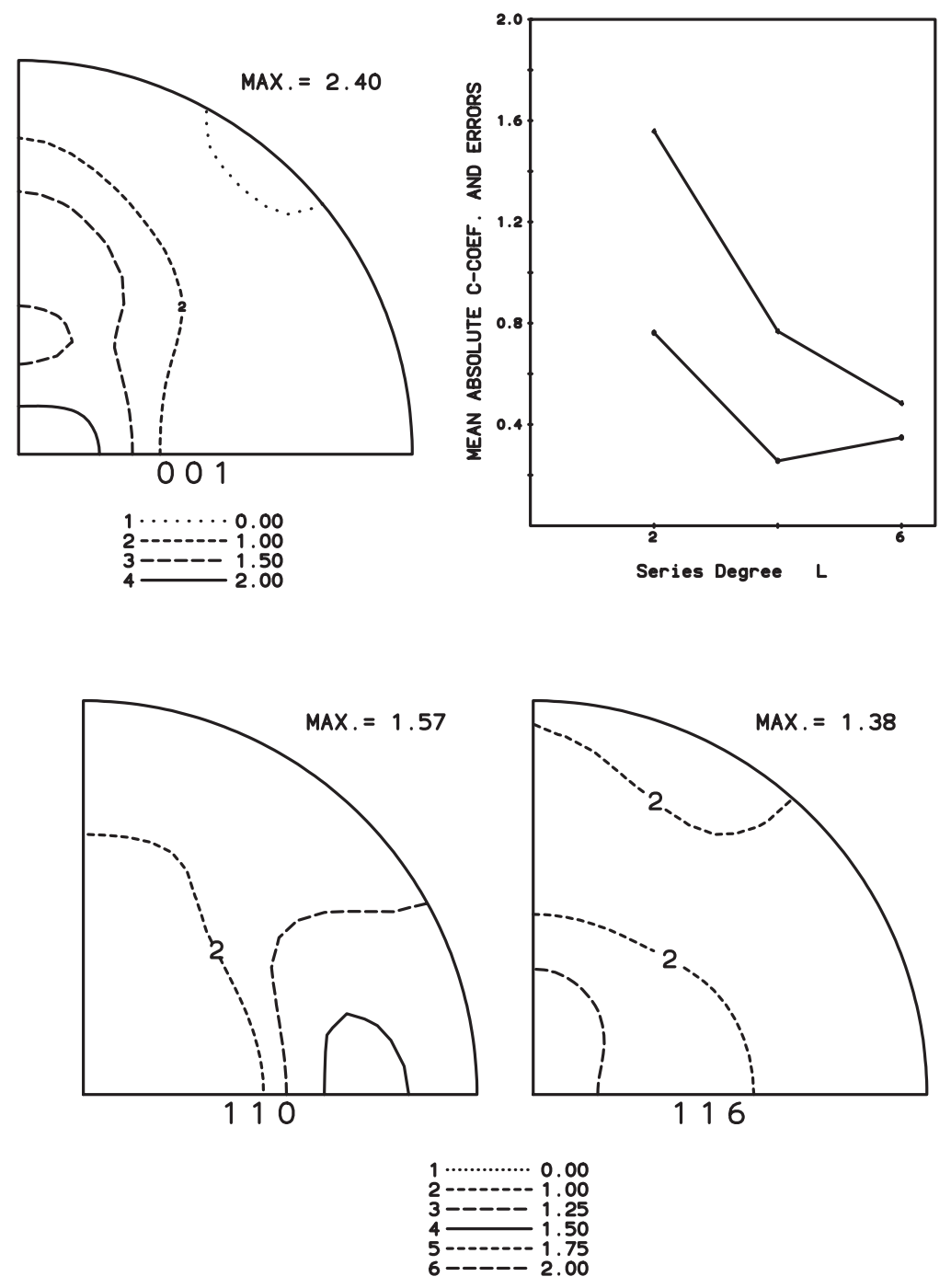

Abbildung C.28: Rückgerechnete Polfiguren, C-Koeffizienten und Fehler, CTC20 13Vol\% $2 \mathrm{U} / \mathrm{min}$ 


\section{C.6 Probe CTC20 9Vol\%}
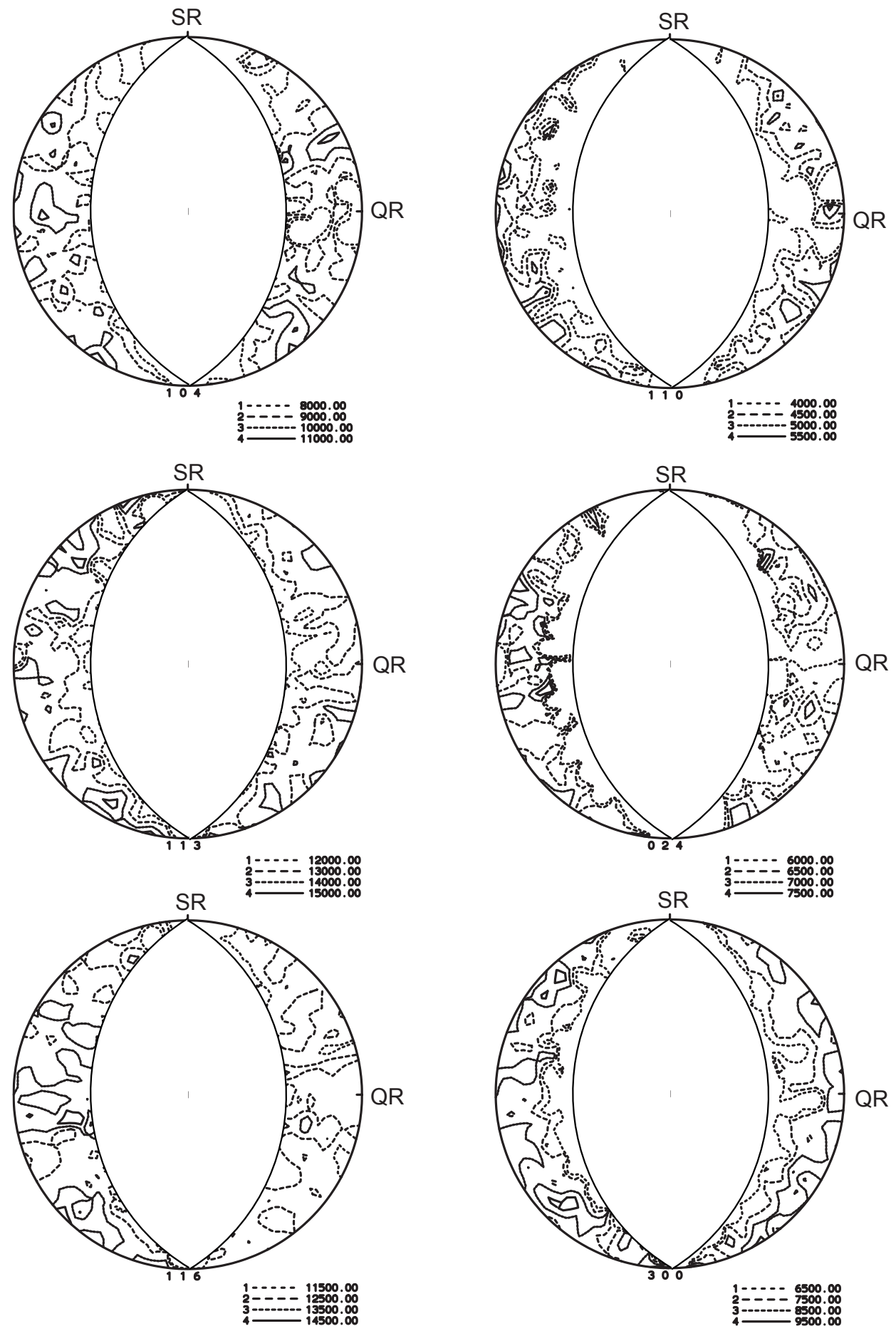

Abbildung C.29: Gemessene Polfiguren CTC20 9Vol\% 0,5 U/min 

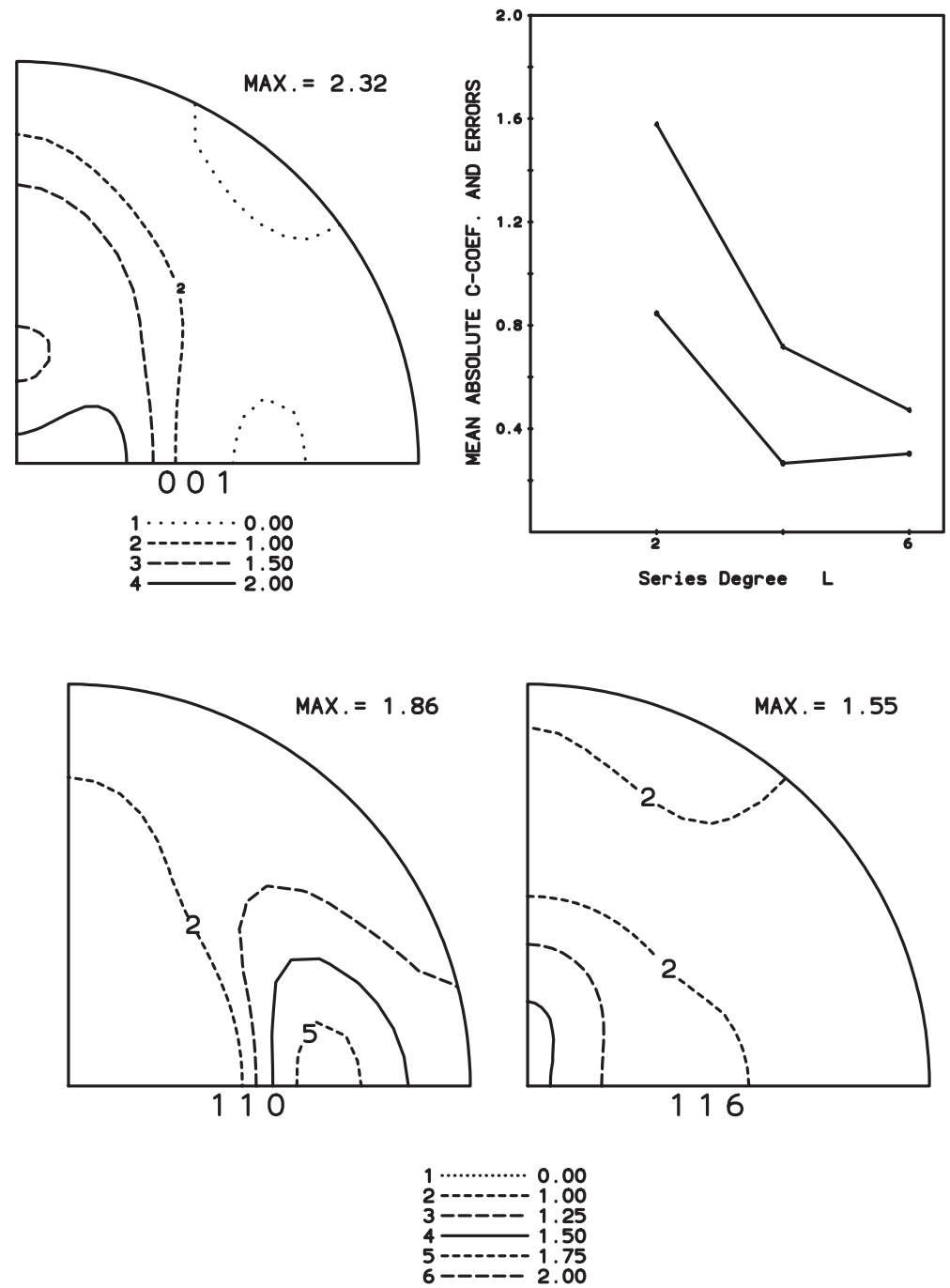

Abbildung C.30: Rückgerechnete Polfiguren, C-Koeffizienten und Fehler, CTC20 9Vol\% 0,5 U/min 

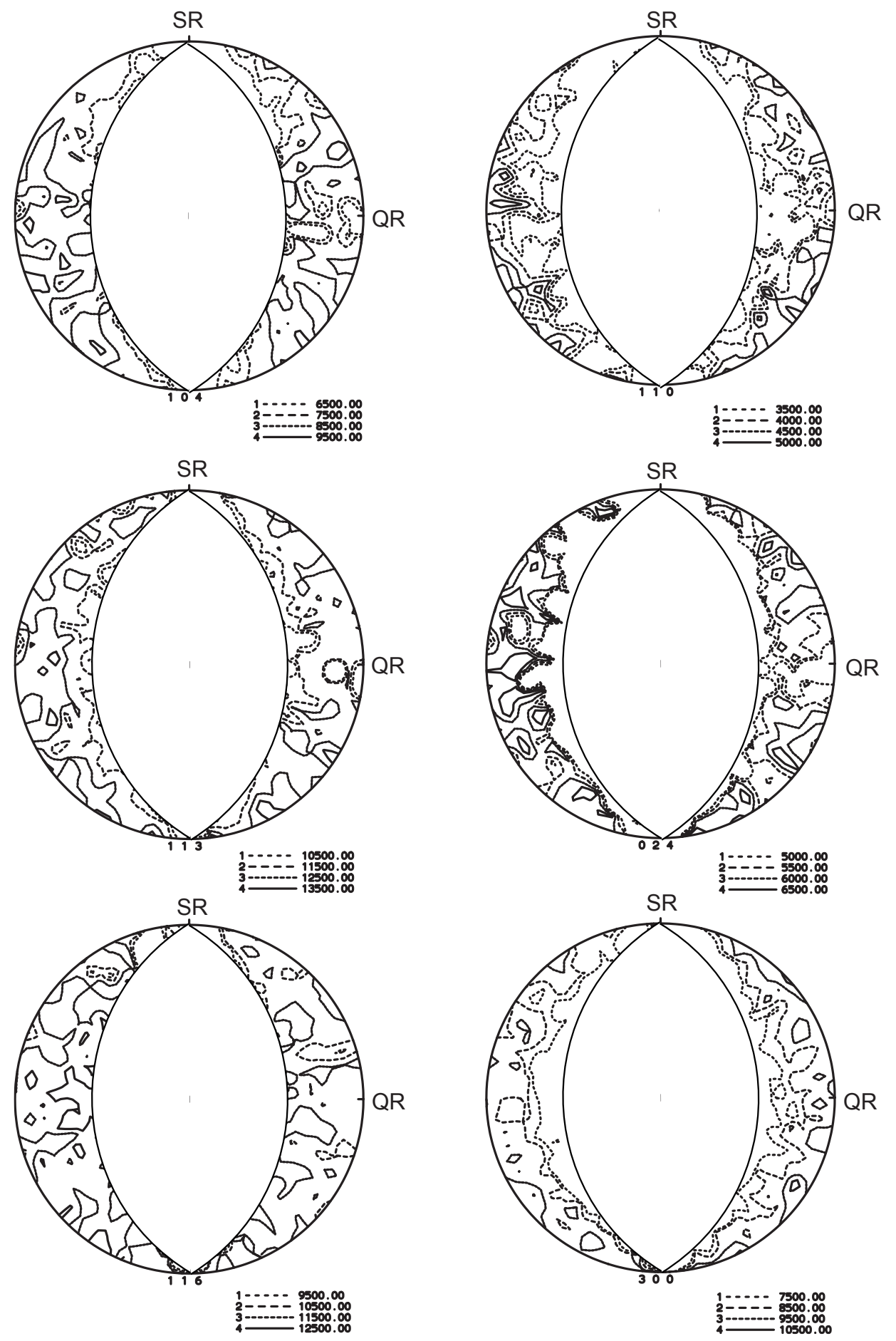

Abbildung C.31: Gemessene Polfiguren CTC20 9Vol\% 1 U/min 

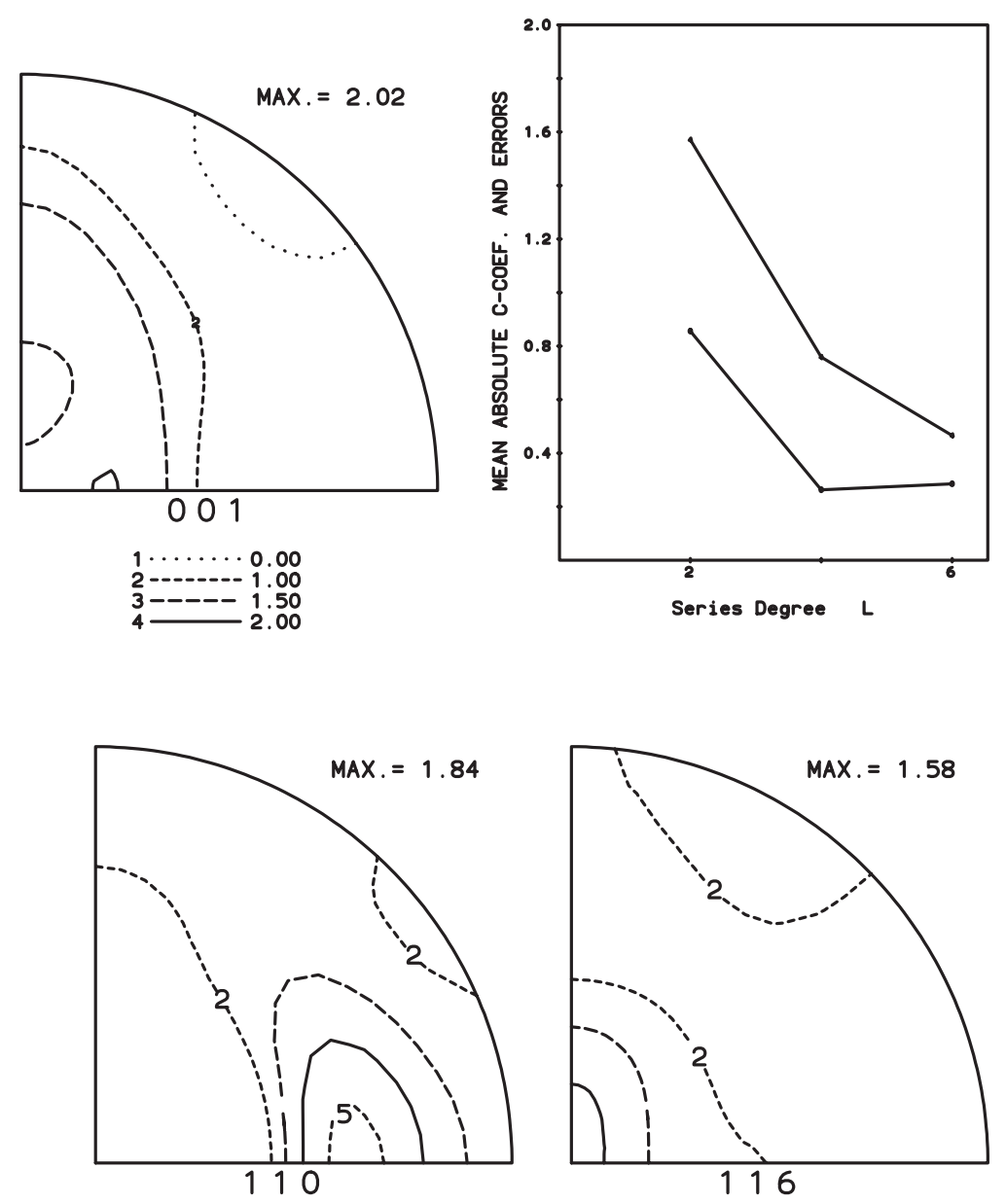

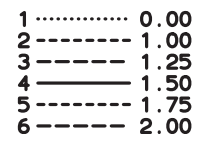

Abbildung C.32: Rückgerechnete Polfiguren, C-Koeffizienten und Fehler, CTC20 9Vol\% $1 \mathrm{U} / \mathrm{min}$ 

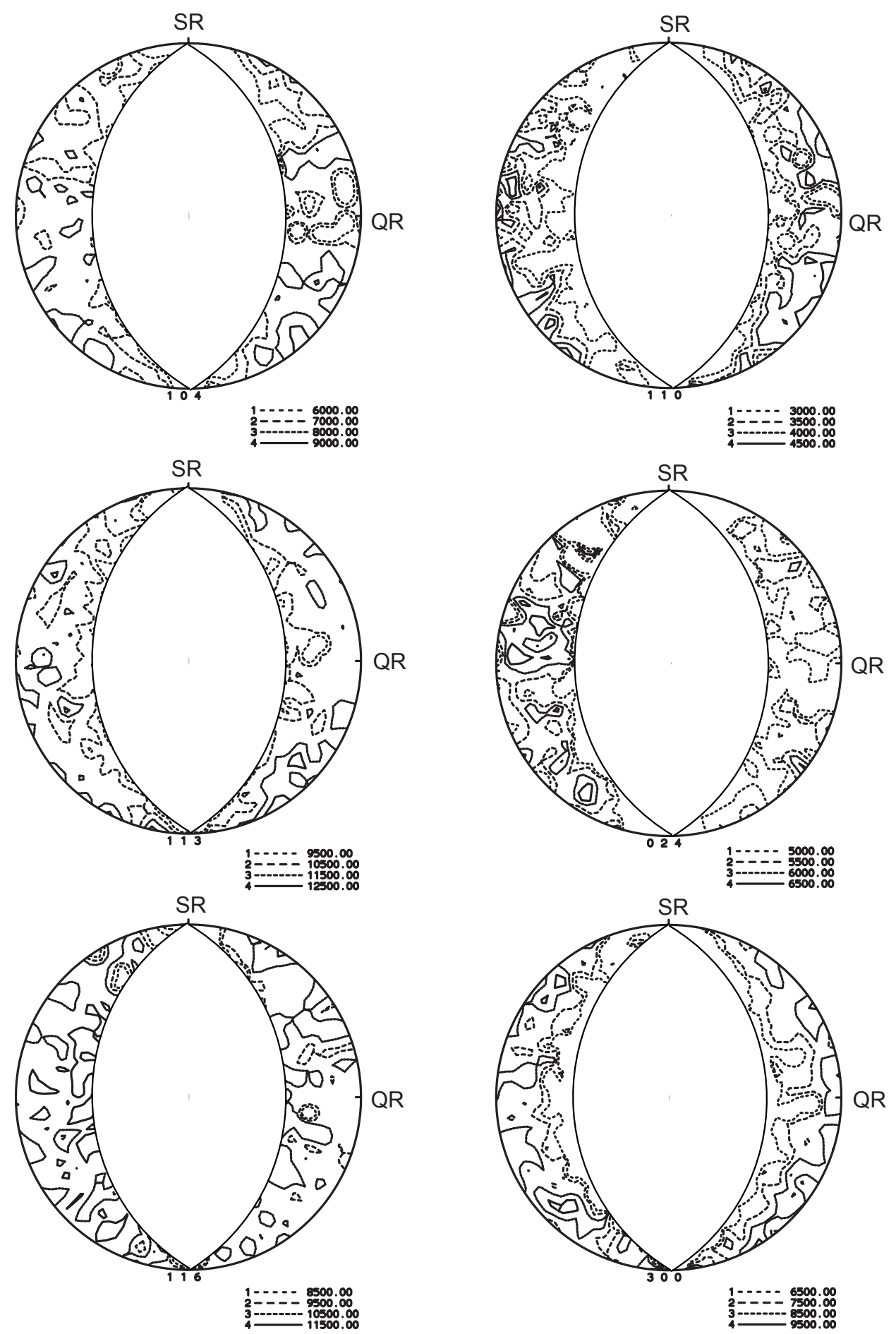

Abbildung C.33: Gemessene Polfiguren CTC20 9Vol\% 2 U/min 

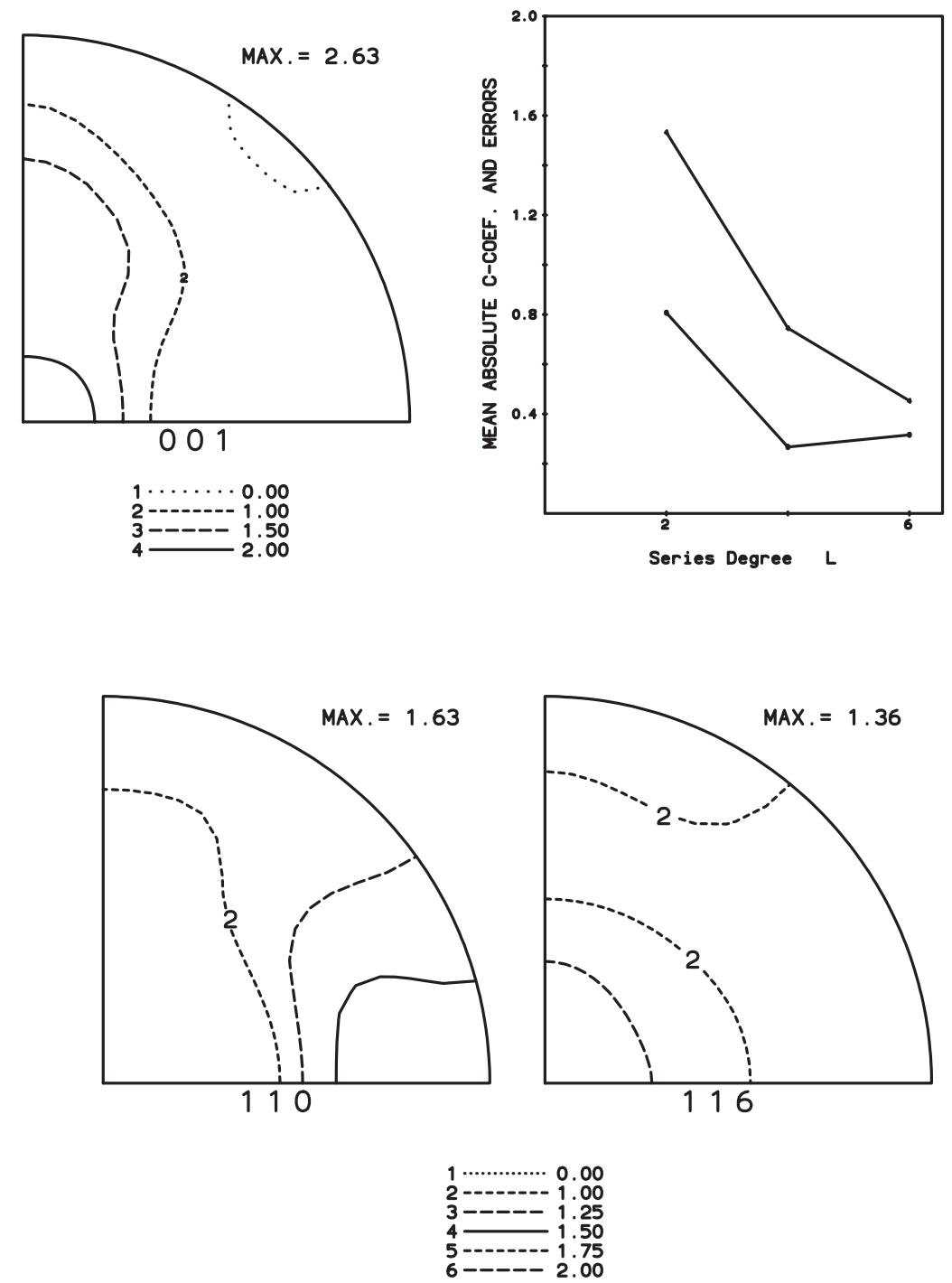

Abbildung C.34: Rückgerechnete Polfiguren, C-Koeffizienten und Fehler, CTC20 9Vol\% $2 \mathrm{U} / \mathrm{min}$ 


\section{C.7 Probe Gilox 13Vol\%}
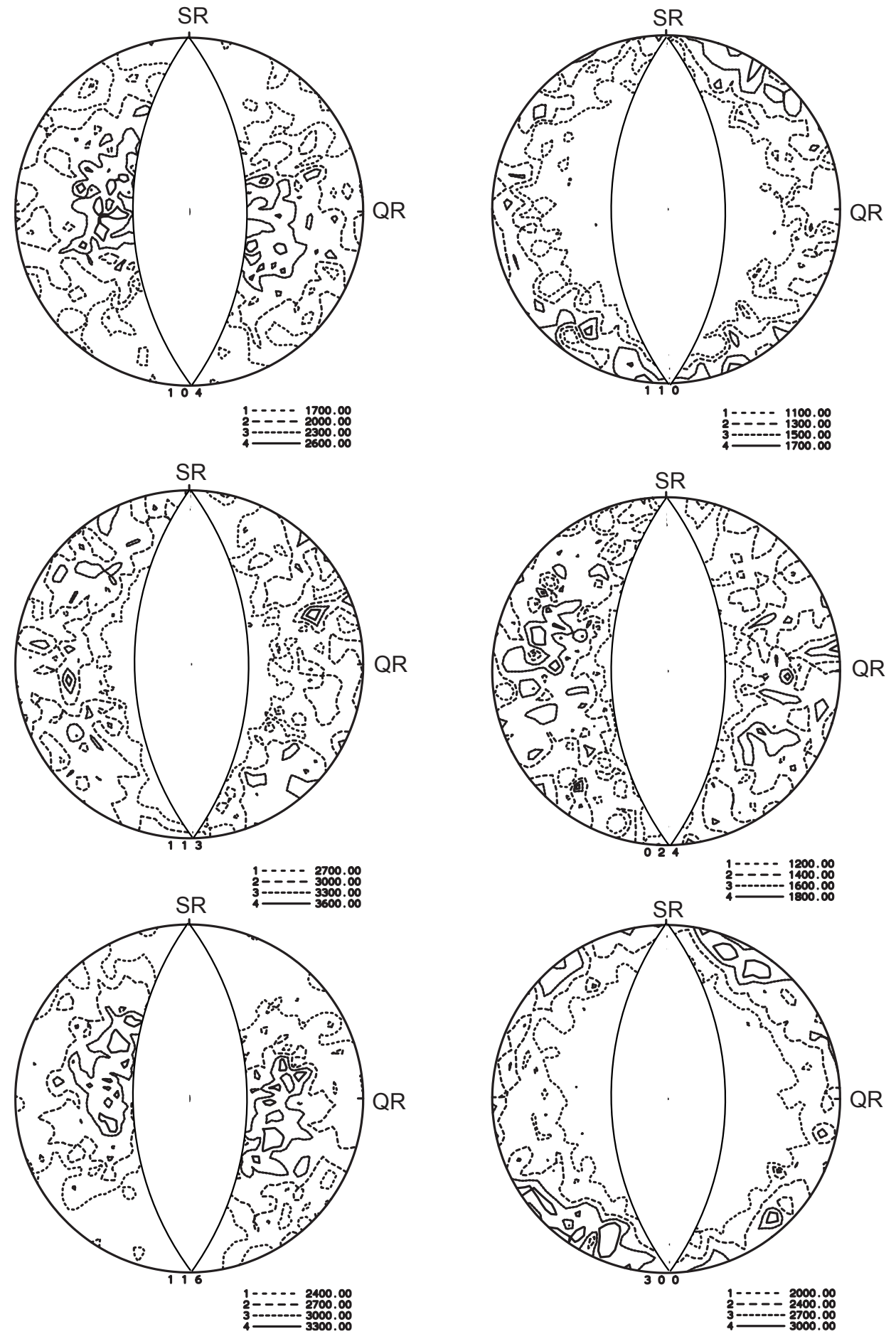

Abbildung C.35: Gemessene Polfiguren Gilox 13Vol\% 0,5 U/min 

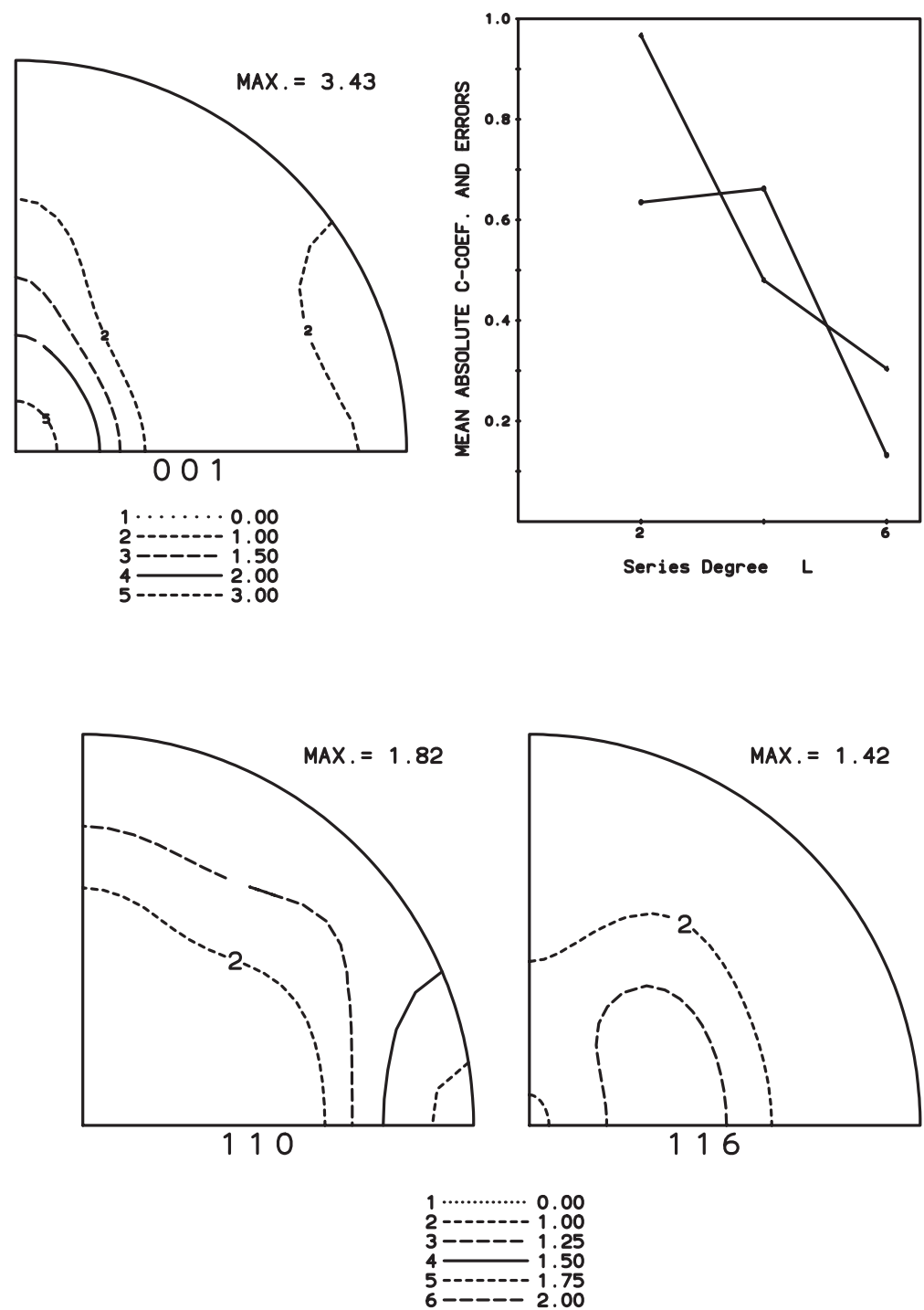

Abbildung C.36: Rückgerechnete Polfiguren, C-Koeffizienten und Fehler, Gilox $13 \mathrm{Vol} \% 0,5 \mathrm{U} / \mathrm{min}$ 

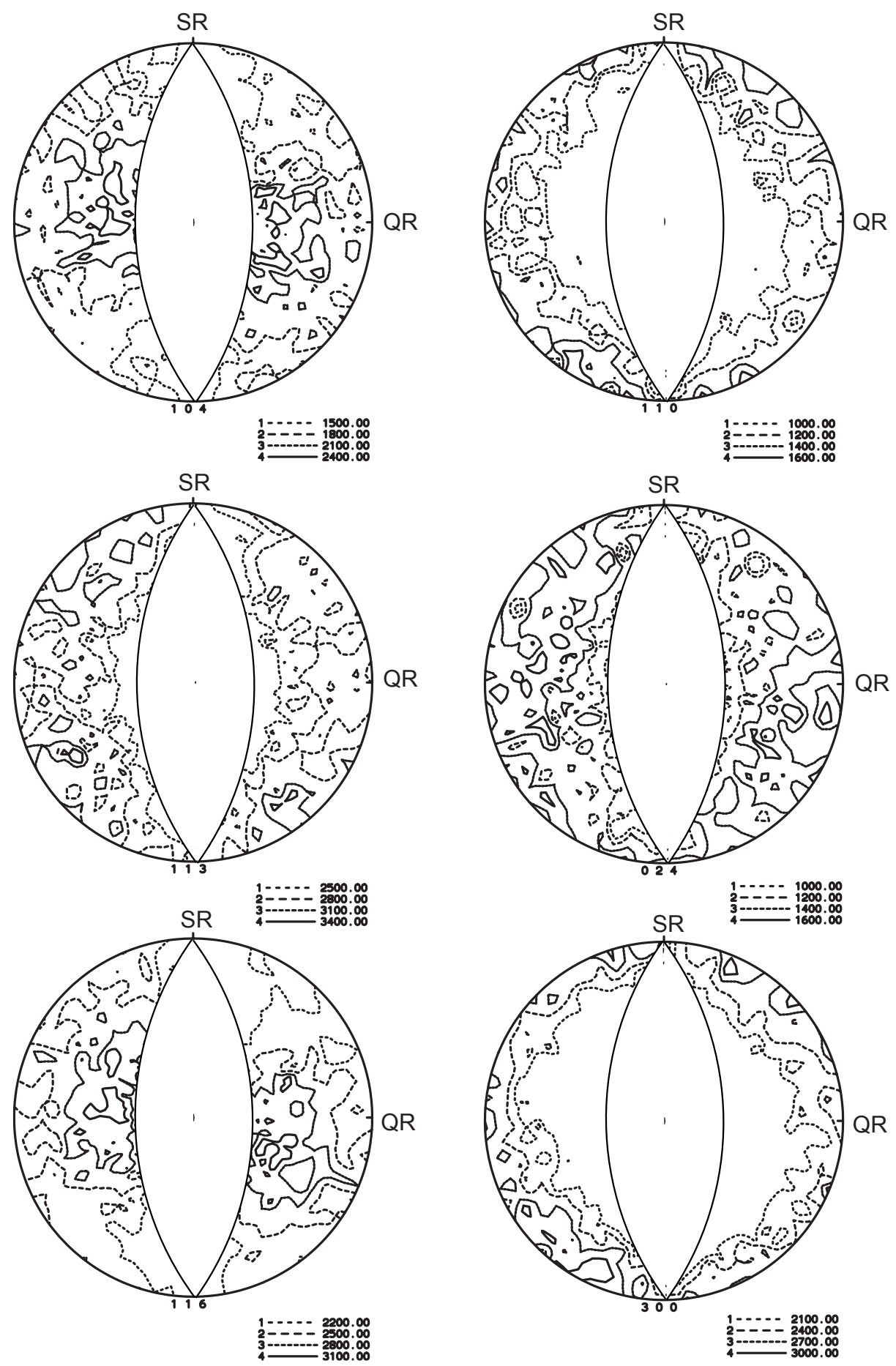

Abbildung C.37: Gemessene Polfiguren Gilox 13Vol\% 1 U/min 

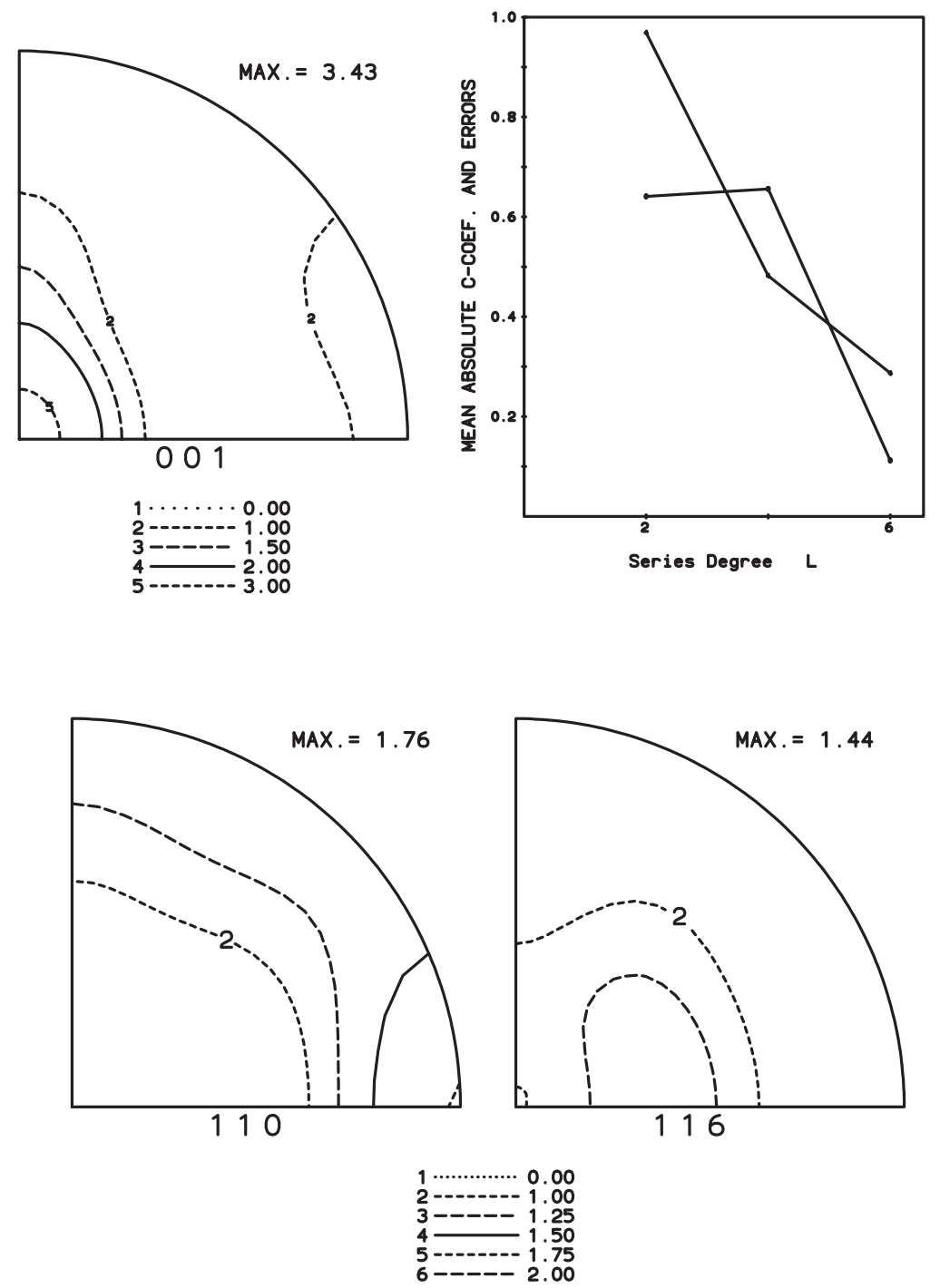

Abbildung C.38: Rückgerechnete Polfiguren, C-Koeffizienten und Fehler, Gilox $13 \mathrm{Vol} \% 1 \mathrm{U} / \mathrm{min}$ 

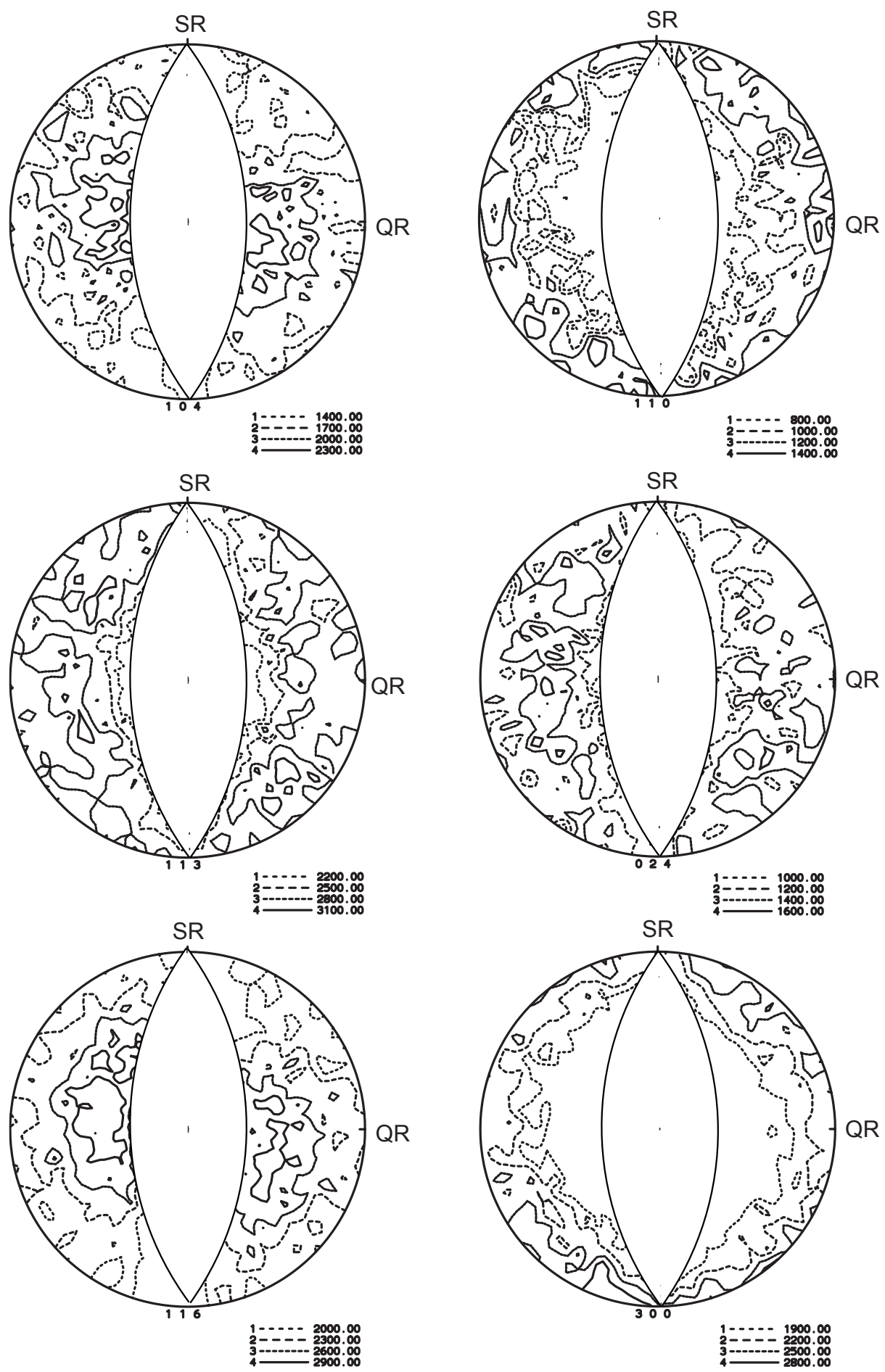

Abbildung C.39: Gemessene Polfiguren Gilox 13Vol\% 2 U/min 

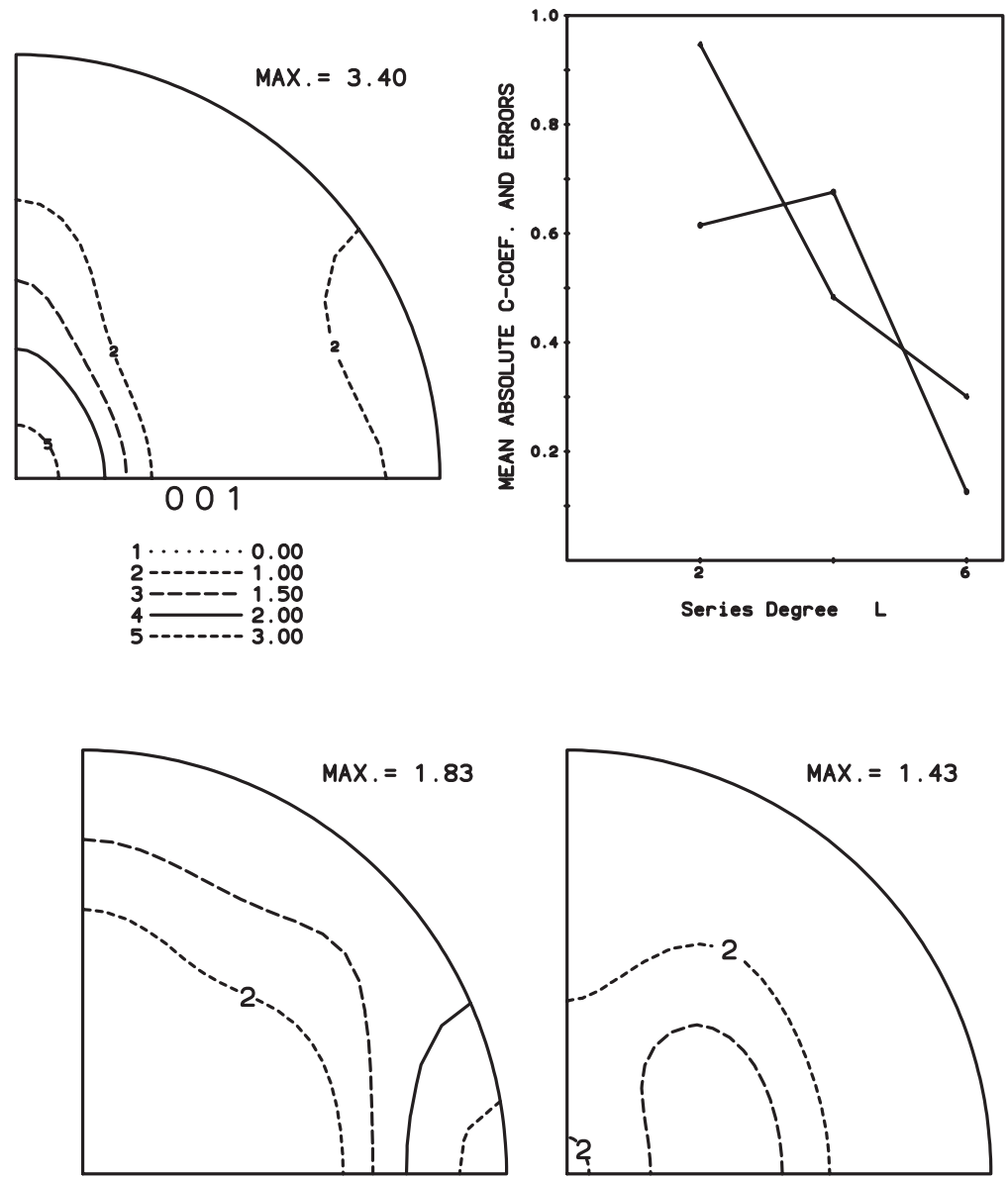

110
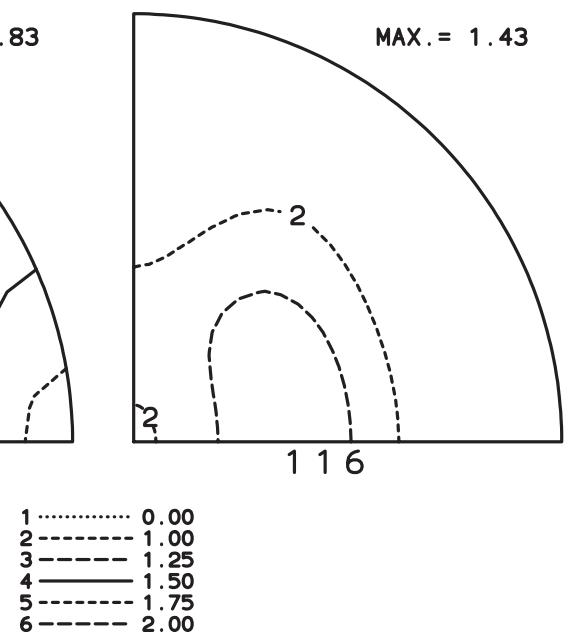

Abbildung C.40: Rückgerechnete Polfiguren, C-Koeffizienten und Fehler, Gilox $13 \mathrm{Vol} \% 2 \mathrm{U} / \mathrm{min}$ 
Anhang D

Durchflussküvette (HVAFG, CTC20, GILOX) 


\section{D.1 Probe HVAFG 10Vol\%}
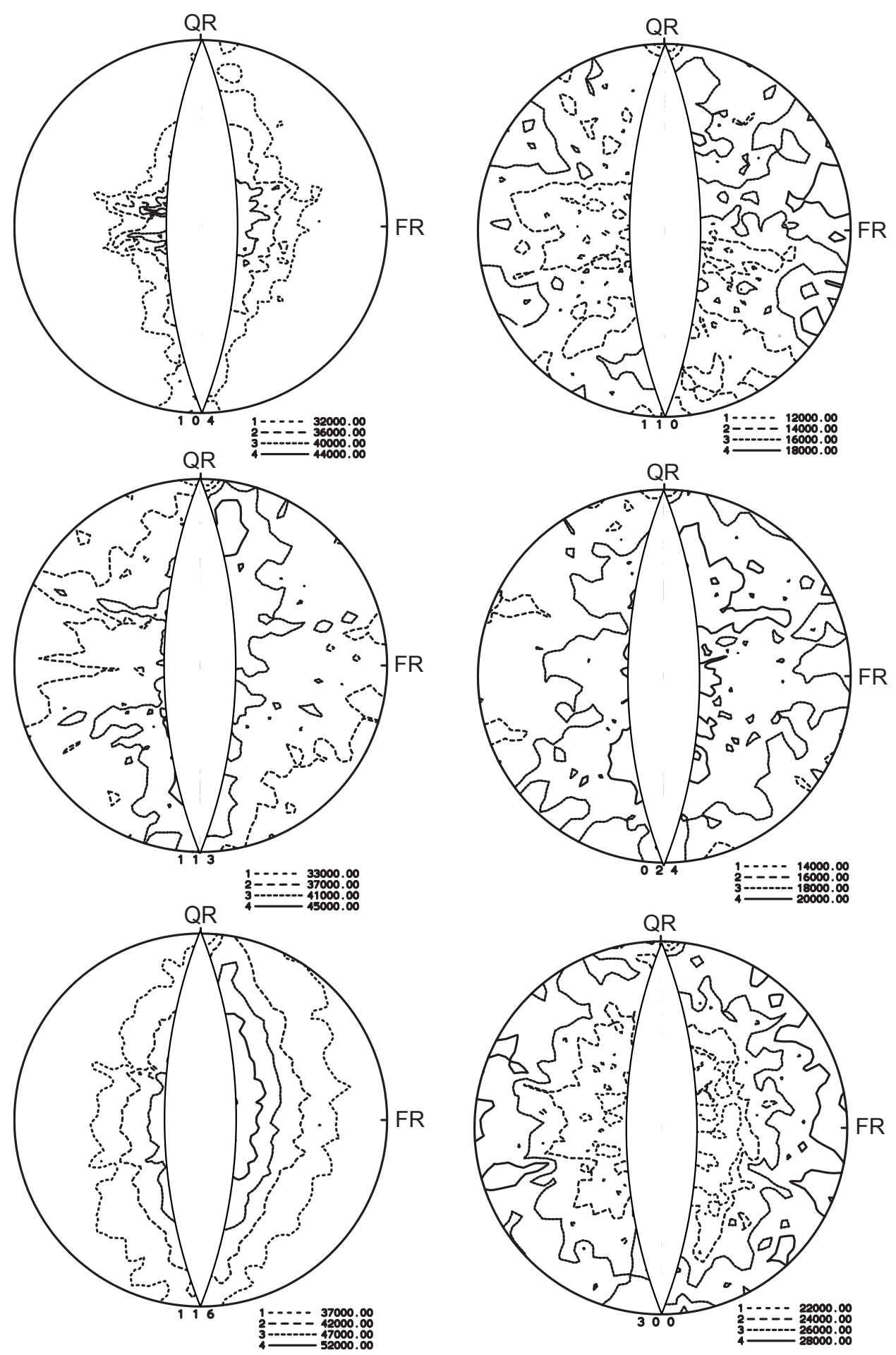

Abbildung D.1: Gemessene Polfiguren HVAFG 10Vol\% 0,2 ml/s 

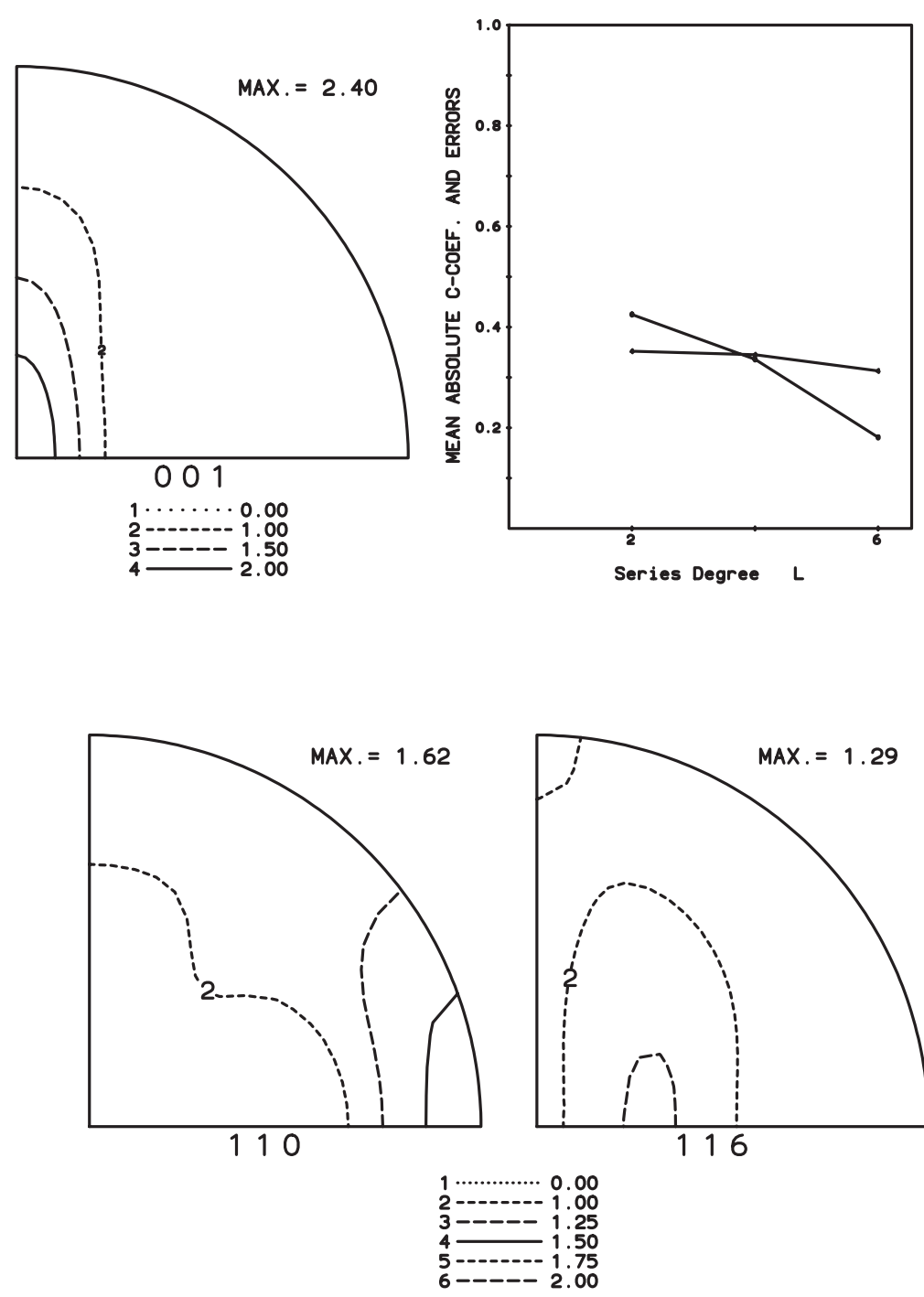

Abbildung D.2: Rückgerechnete Polfiguren, C-Koeffizienten und Fehler, HVAFG $10 \mathrm{Vol} \% 0,2 \mathrm{ml} / \mathrm{s}$ 

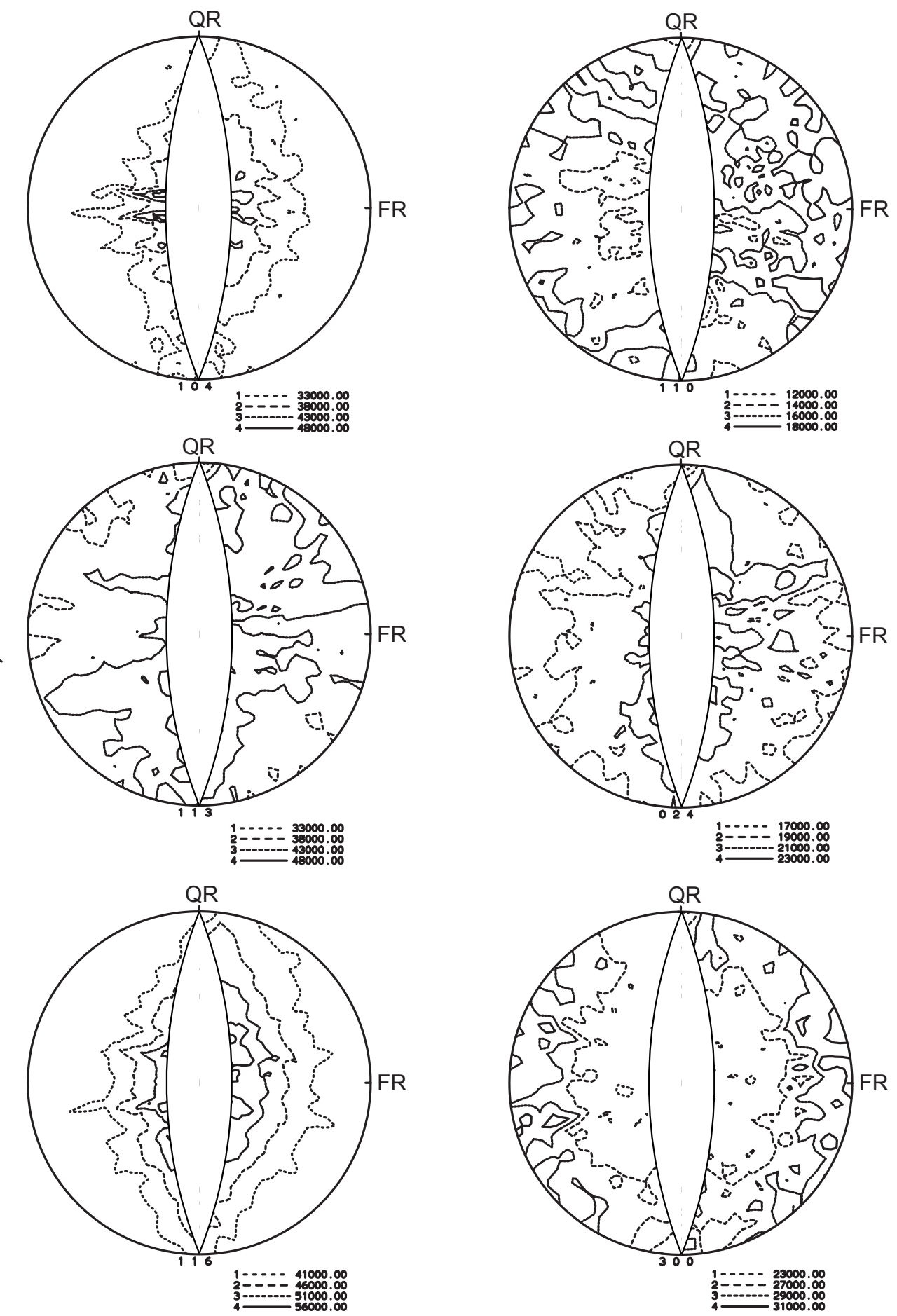

Abbildung D.3: Gemessene Polfiguren HVAFG 10Vol\% 0,5 ml/s 

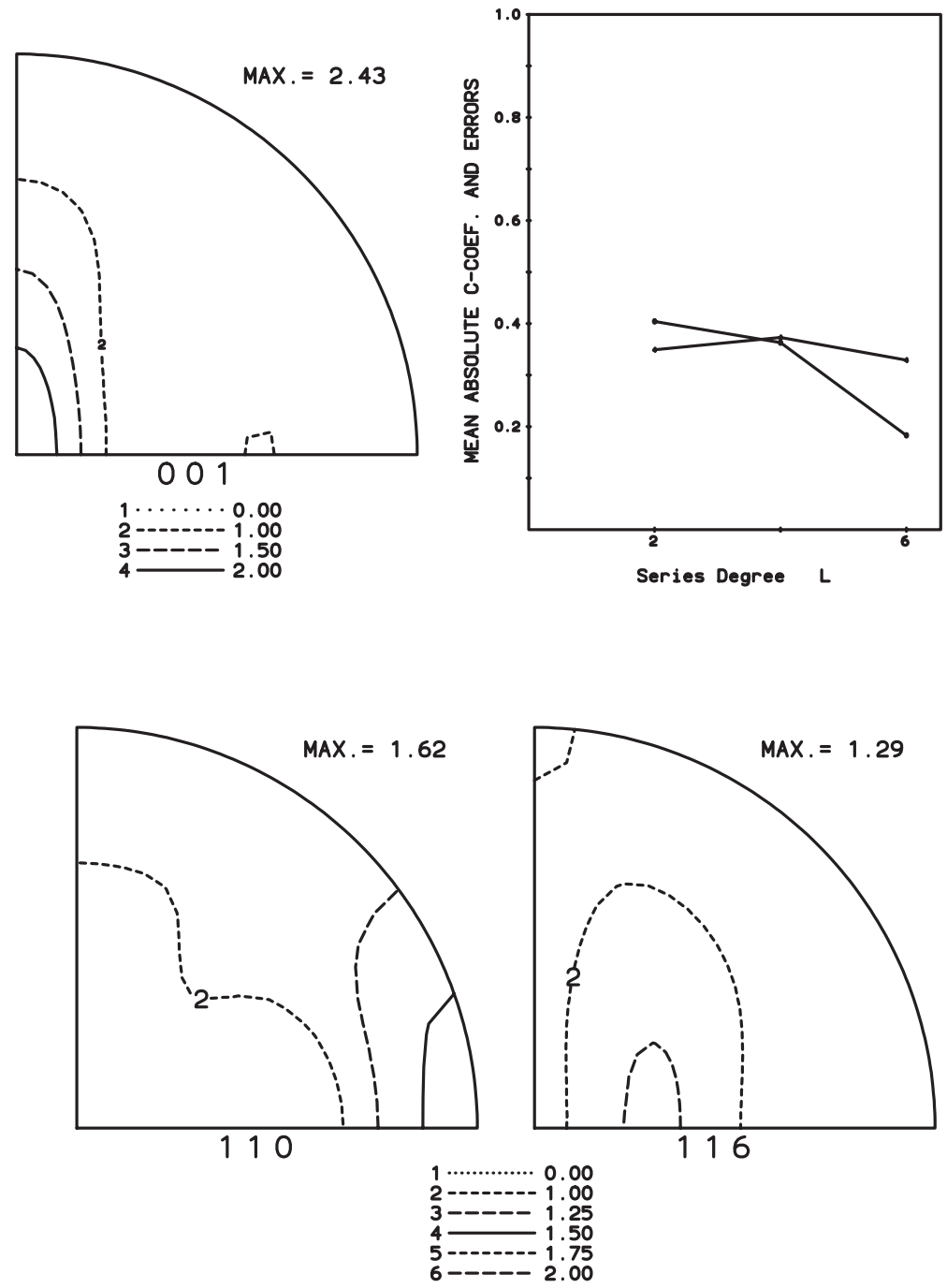

Abbildung D.4: Rückgerechnete Polfiguren, C-Koeffizienten und Fehler, HVAFG $10 \mathrm{Vol} \%$ 0,5ml/s 

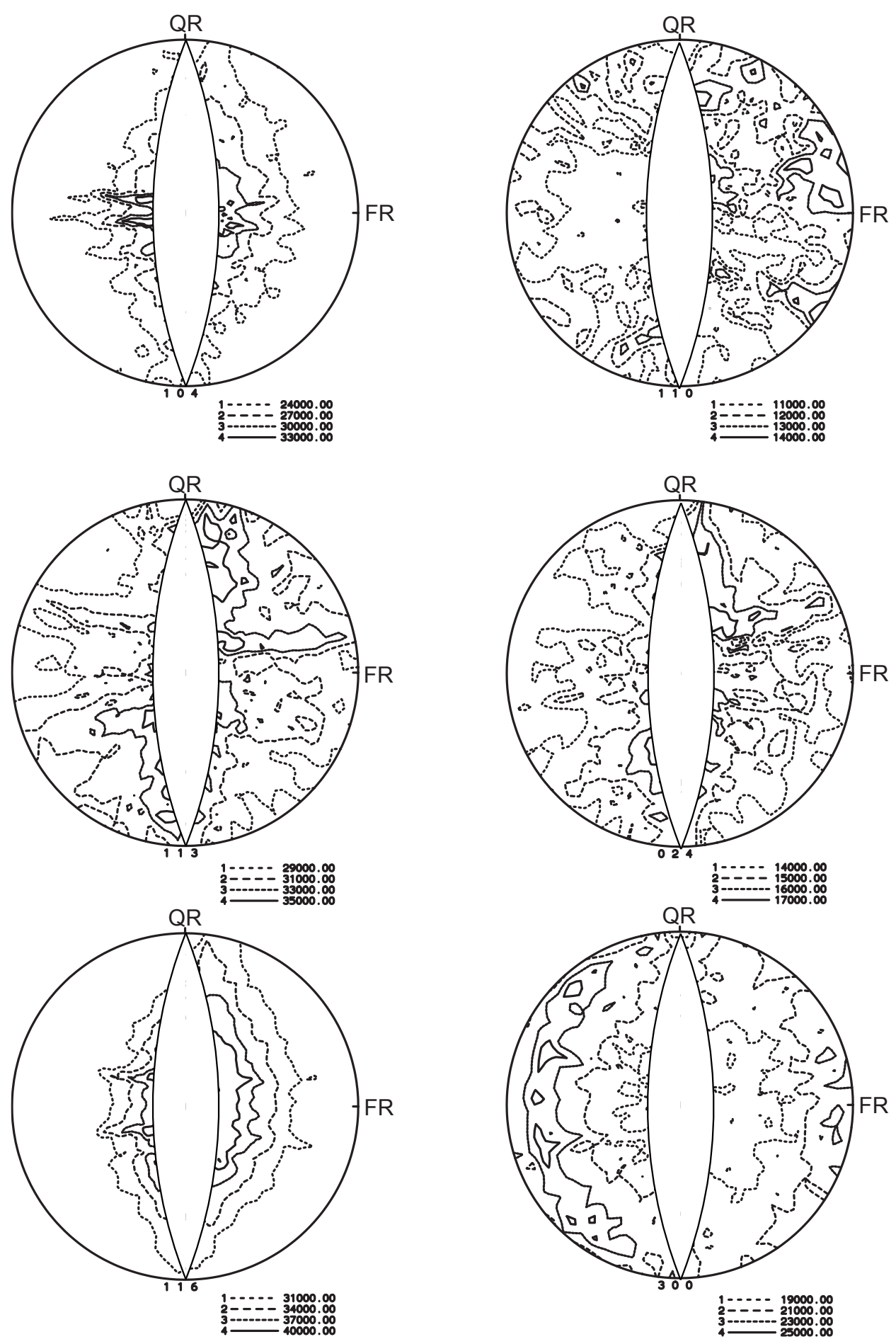

Abbildung D.5: Gemessene Polfiguren HVAFG 10Vol\% $1 \mathrm{ml} / \mathrm{s}$ 

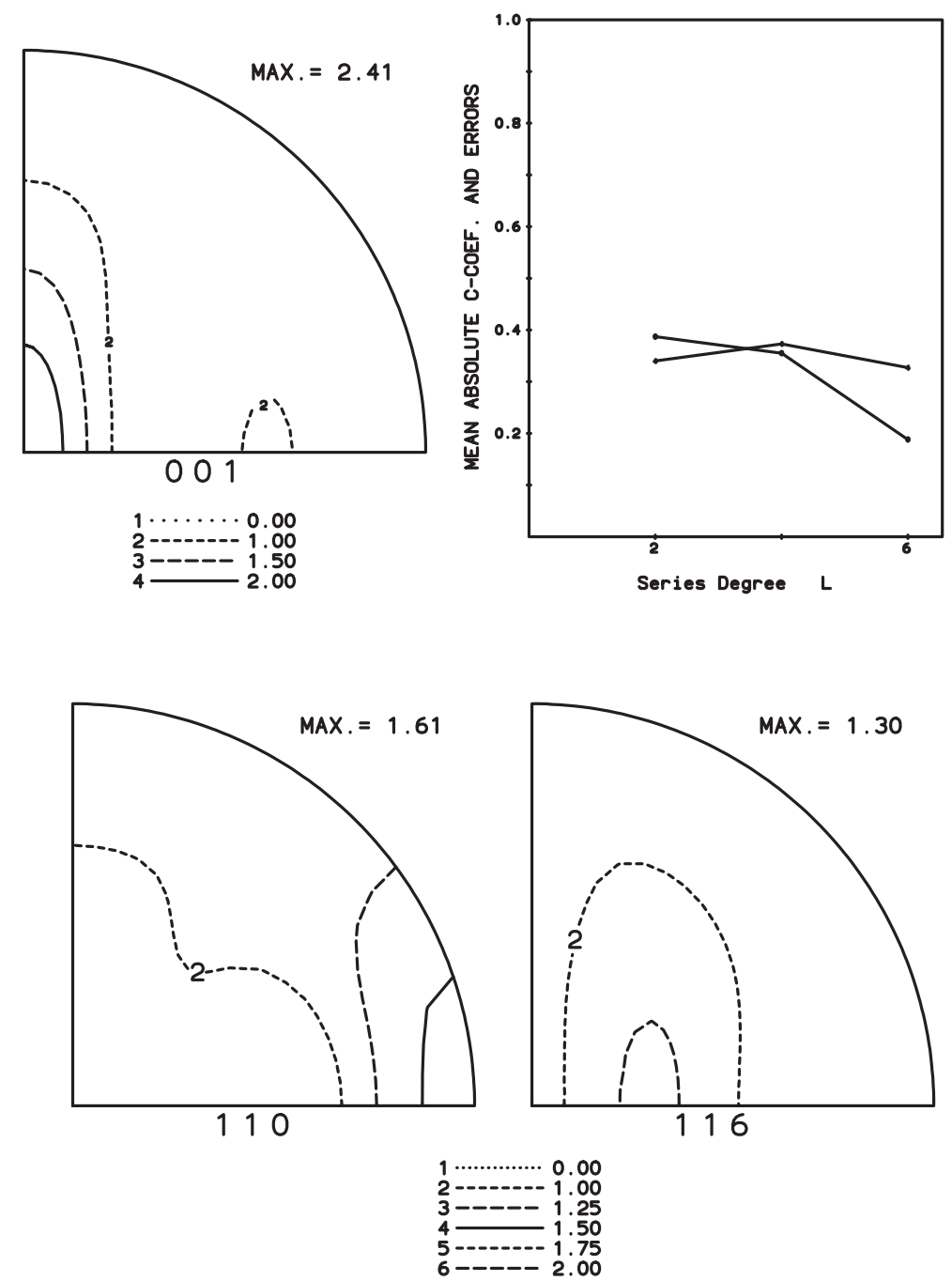

Abbildung D.6: Rückgerechnete Polfiguren, C-Koeffizienten und Fehler, HVAFG $10 \mathrm{Vol} \% 1 \mathrm{ml} / \mathrm{s}$ 

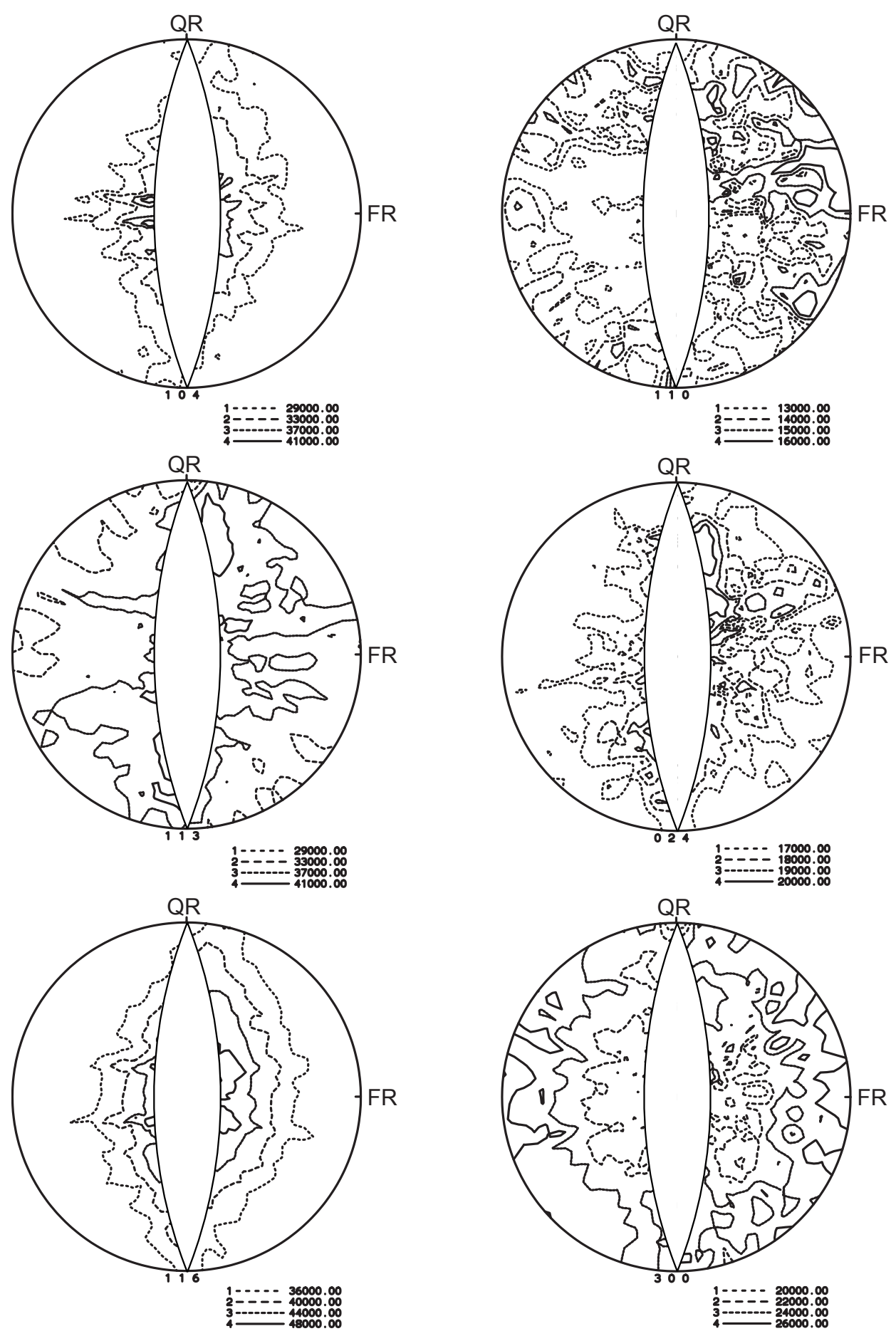

Abbildung D.7: Gemessene Polfiguren HVAFG 10Vol\% $2 \mathrm{ml} / \mathrm{s}$ 

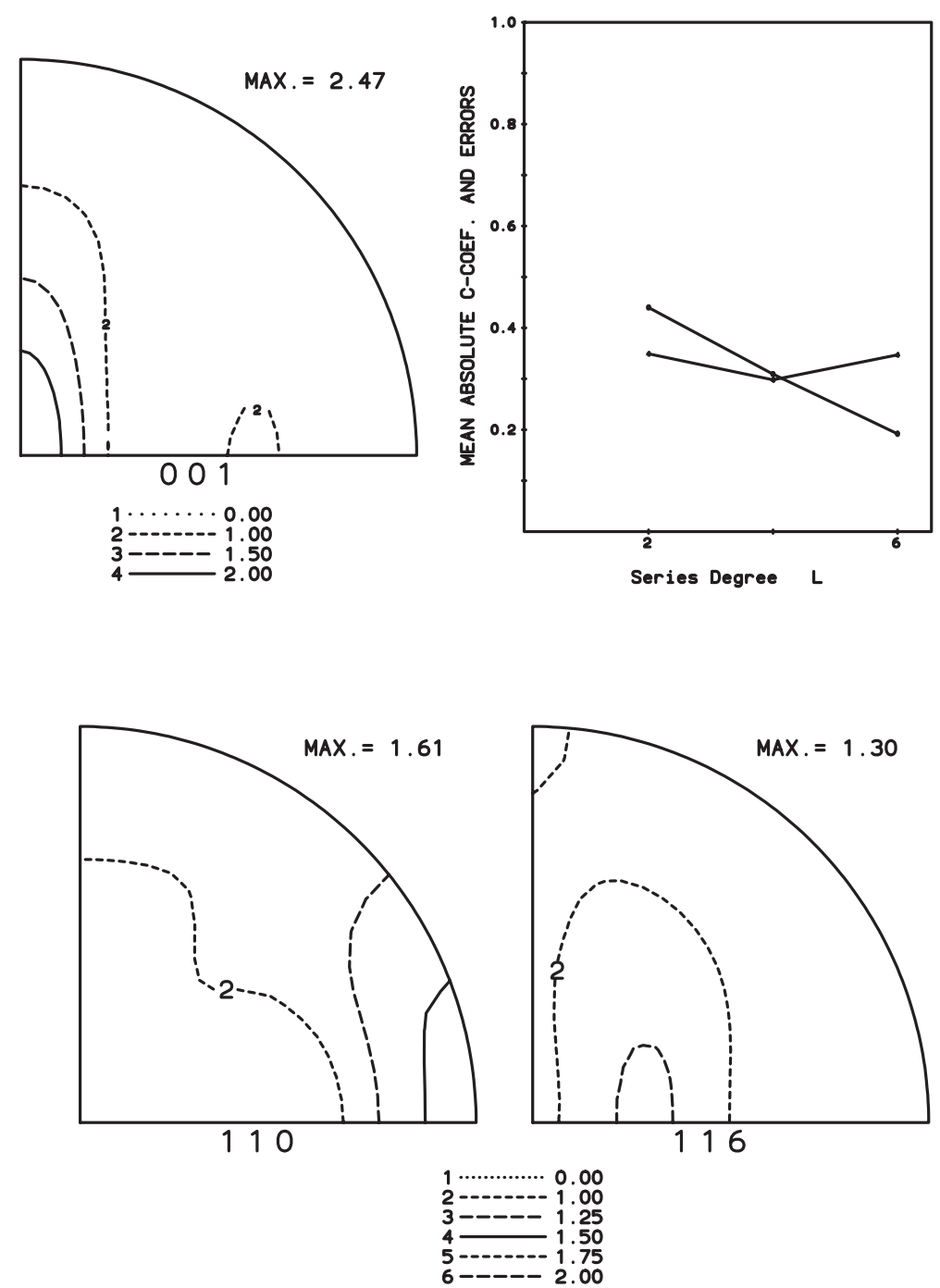

Abbildung D.8: Rückgerechnete Polfiguren, C-Koeffizienten und Fehler, HVAFG $10 \mathrm{Vol} \% 2 \mathrm{ml} / \mathrm{s}$ 

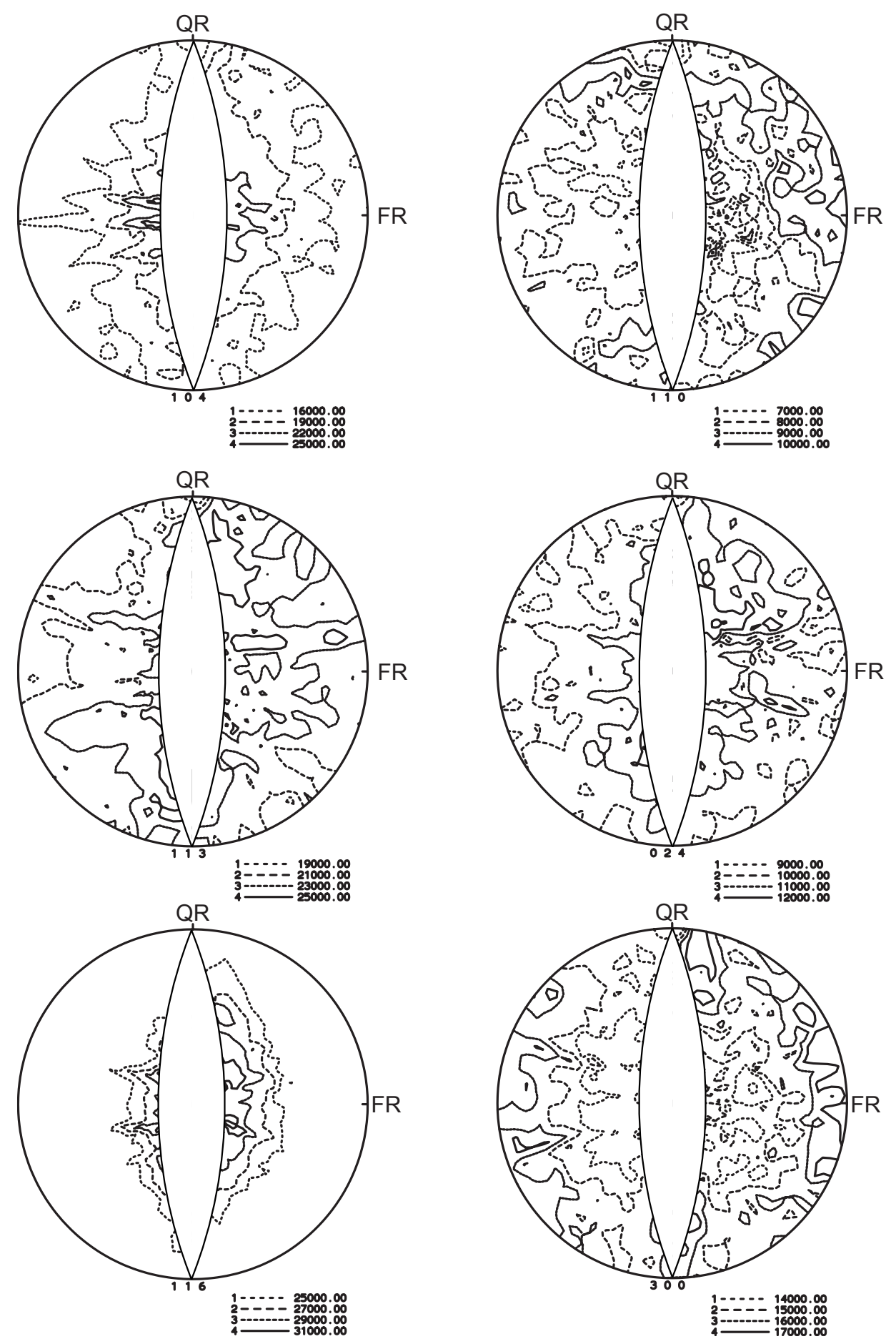

Abbildung D.9: Gemessene Polfiguren HVAFG 10Vol\% $5 \mathrm{ml} / \mathrm{s}$ 

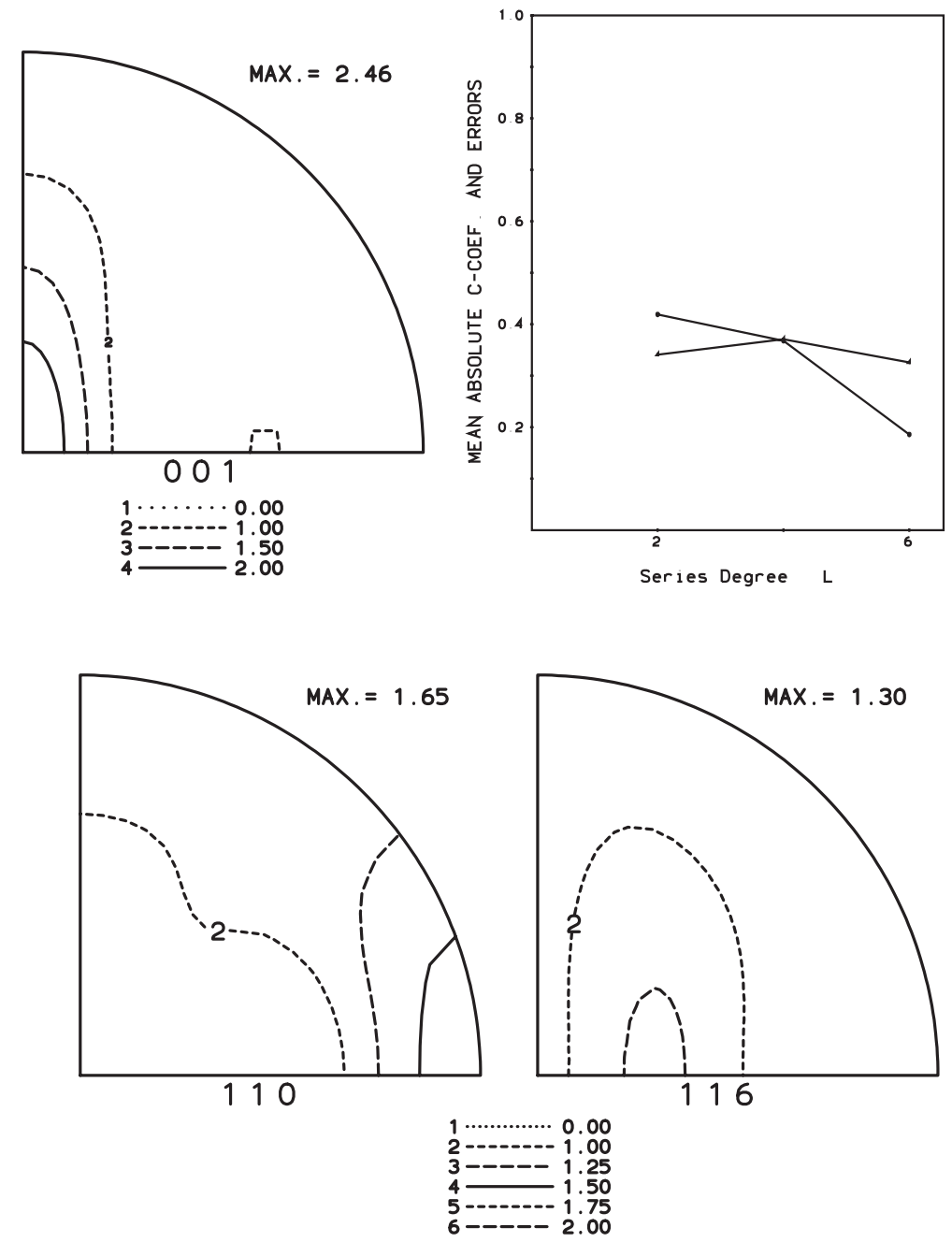

Abbildung D.10: Rückgerechnete Polfiguren, C-Koeffizienten und Fehler, HVAFG $10 \mathrm{Vol} \% 5 \mathrm{ml} / \mathrm{s}$ 


\section{D.2 HVAFG $12,5 \mathrm{Vol} \%$}
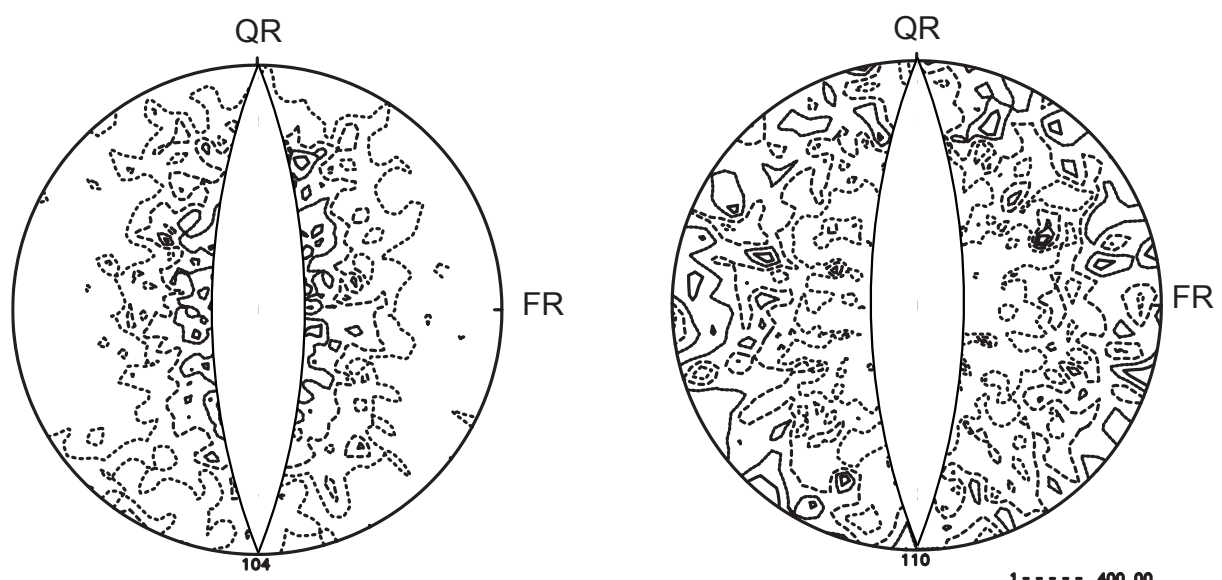

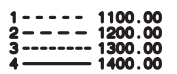

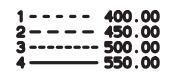
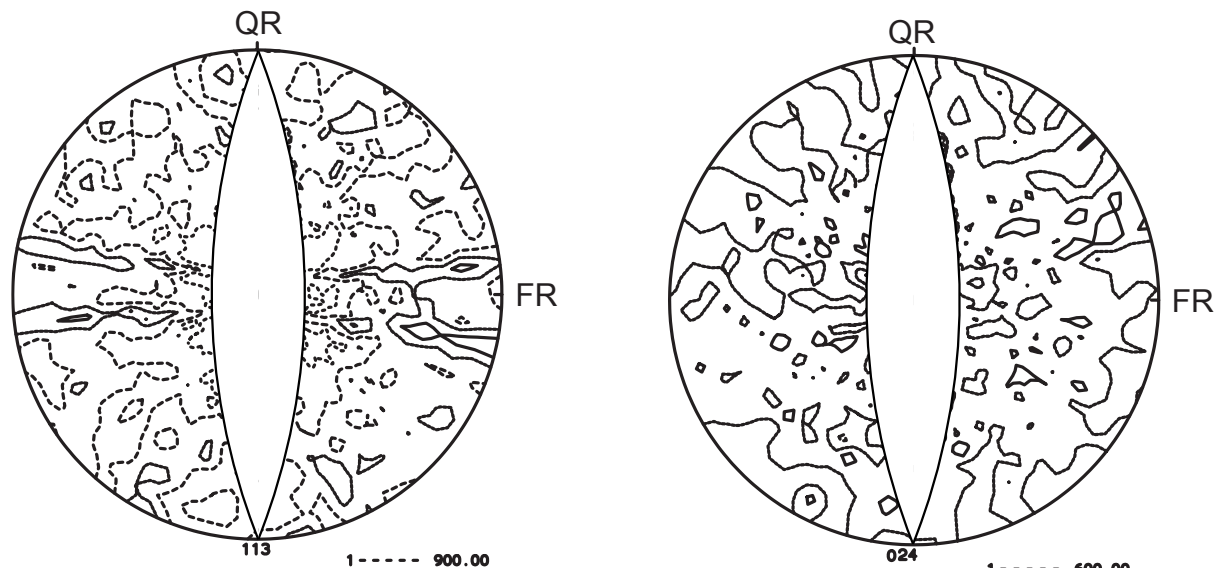

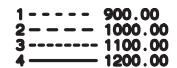

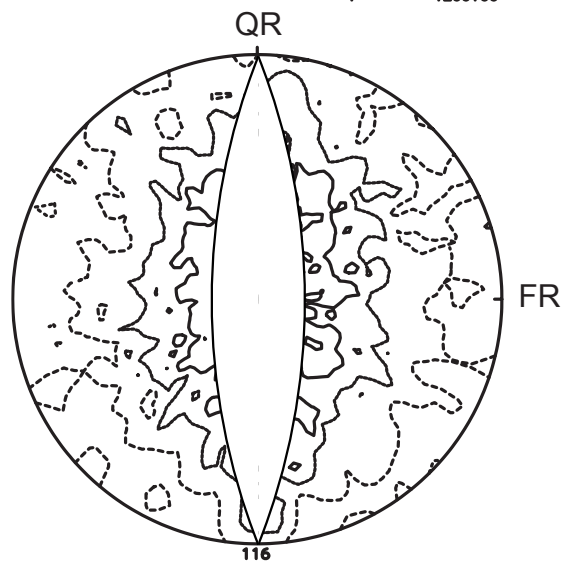

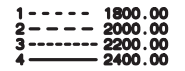

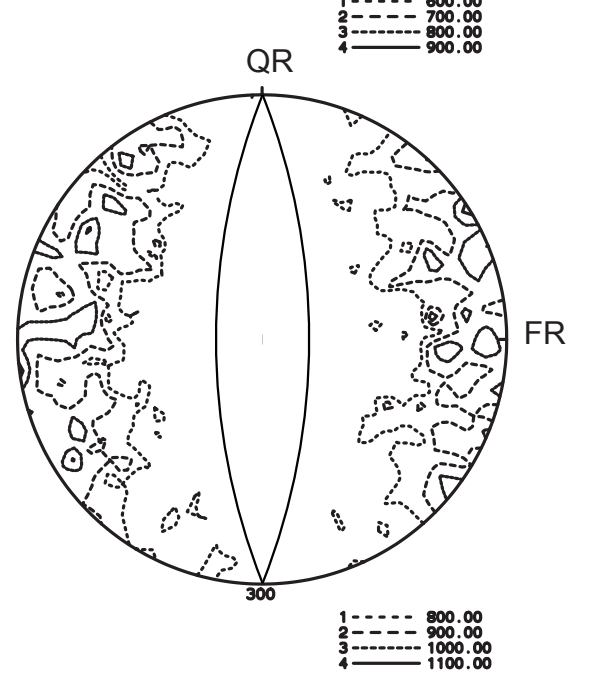

Abbildung D.11: Gemessene Polfiguren HVAFG 12,5Vol\% 0,2 ml/s 

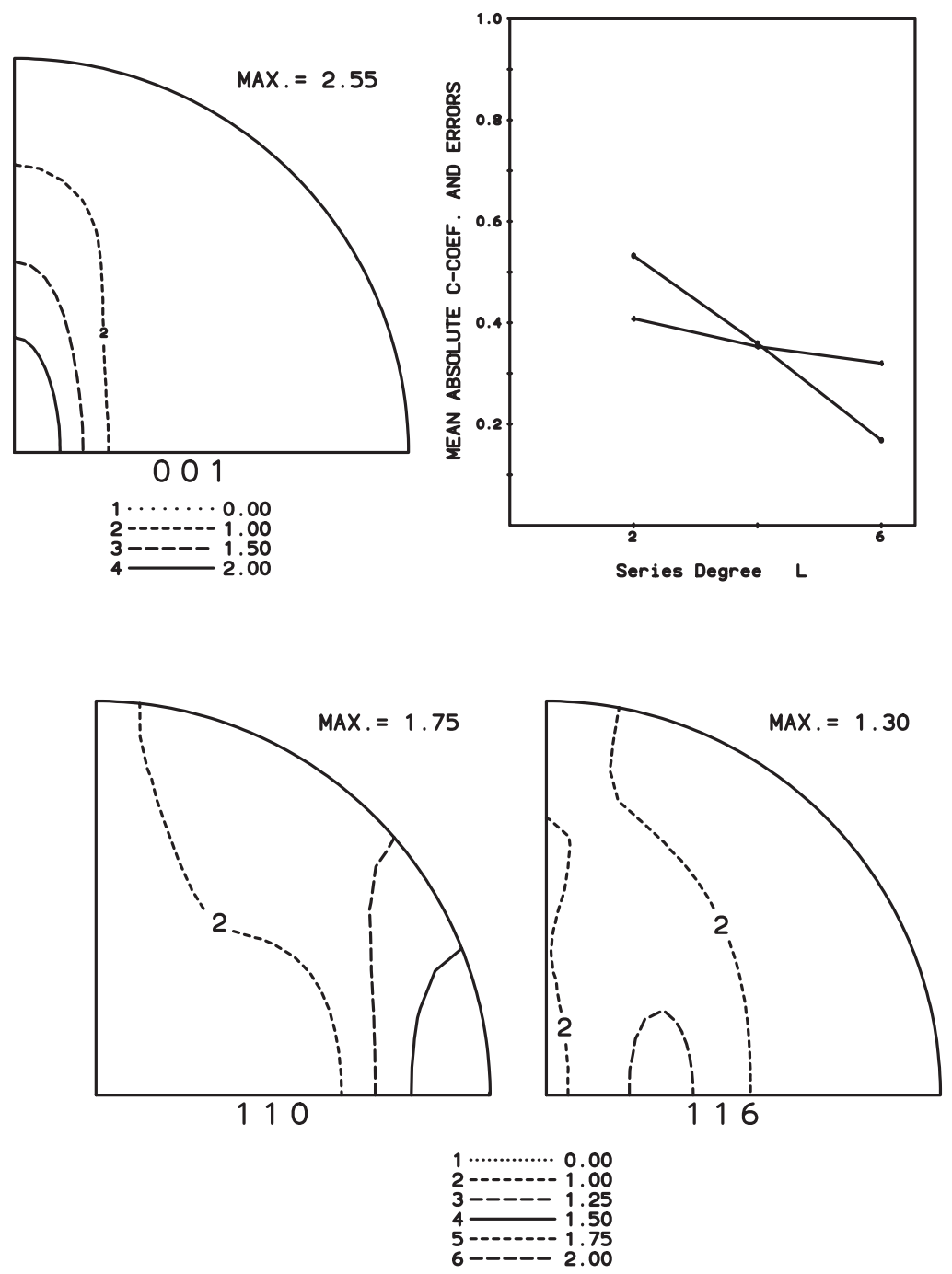

Abbildung D.12: Rückgerechnete Polfiguren, C-Koeffizienten und Fehler, HVAFG $12,5 \mathrm{Vol} \%$ 0,2ml/s 

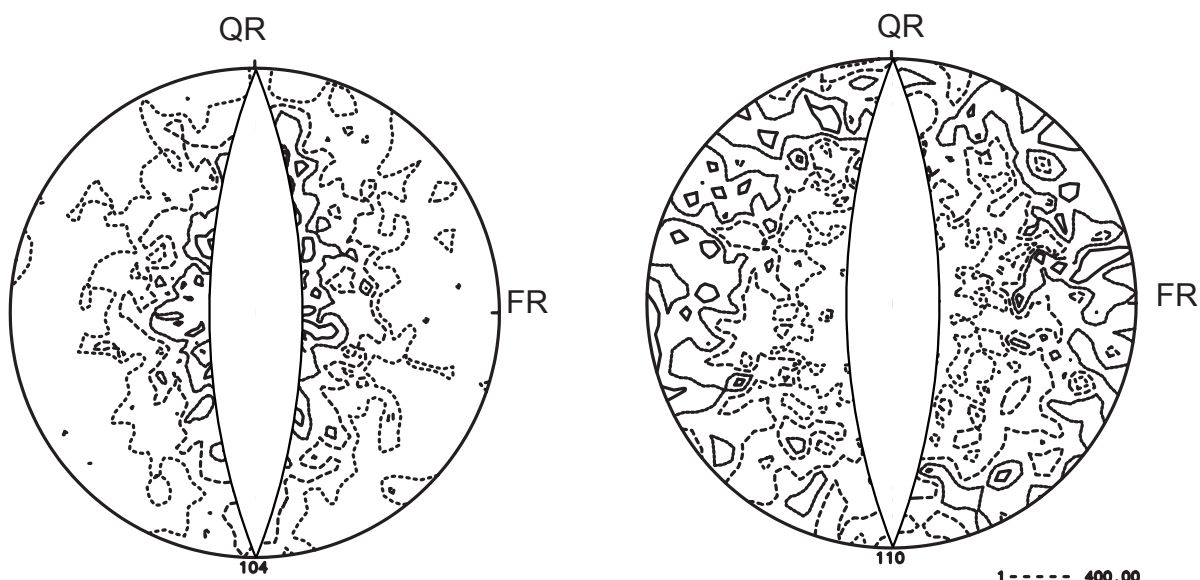

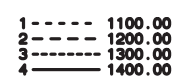
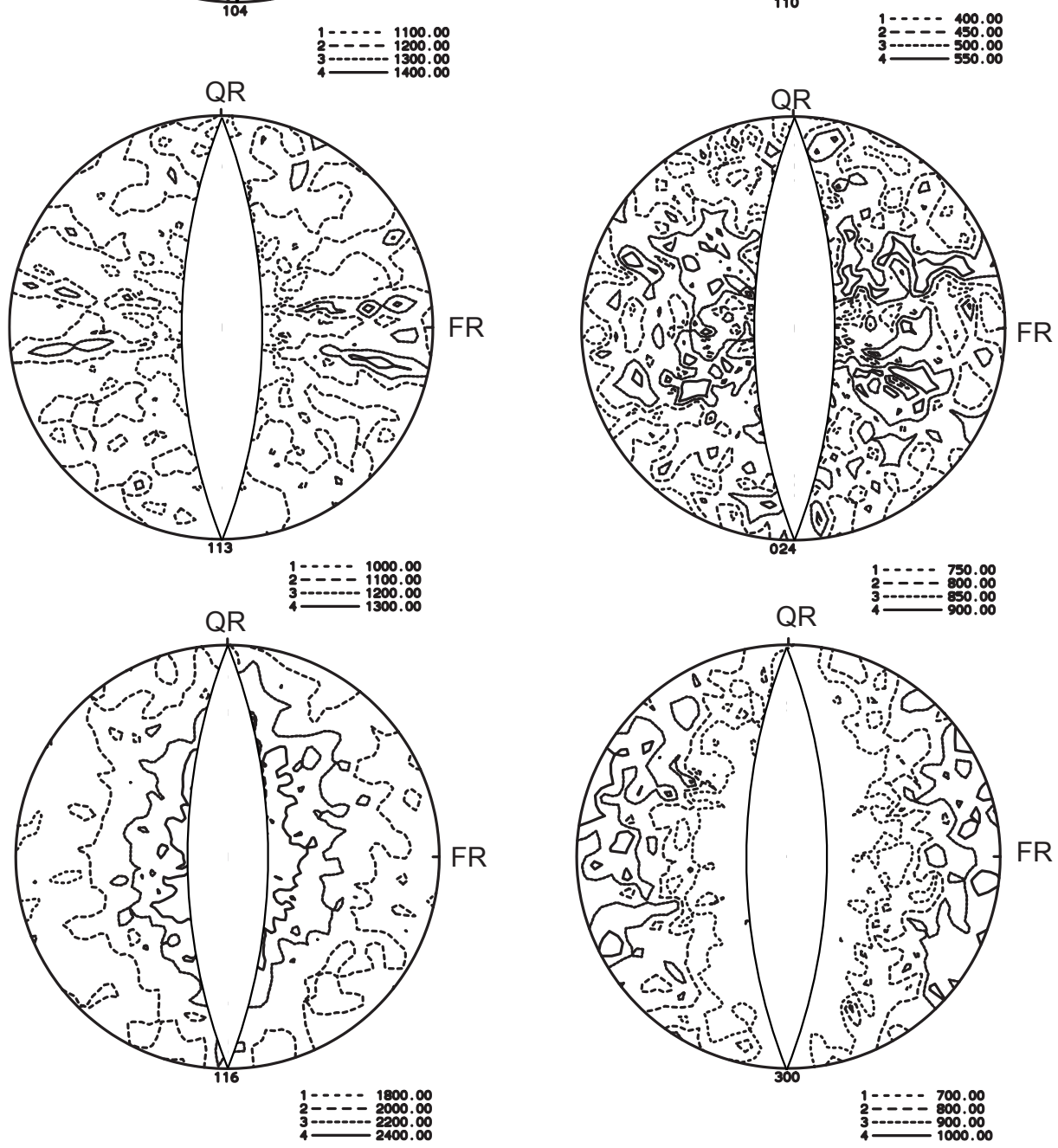

Abbildung D.13: Gemessene Polfiguren HVAFG 12,5Vol\% 0,5 ml/s 

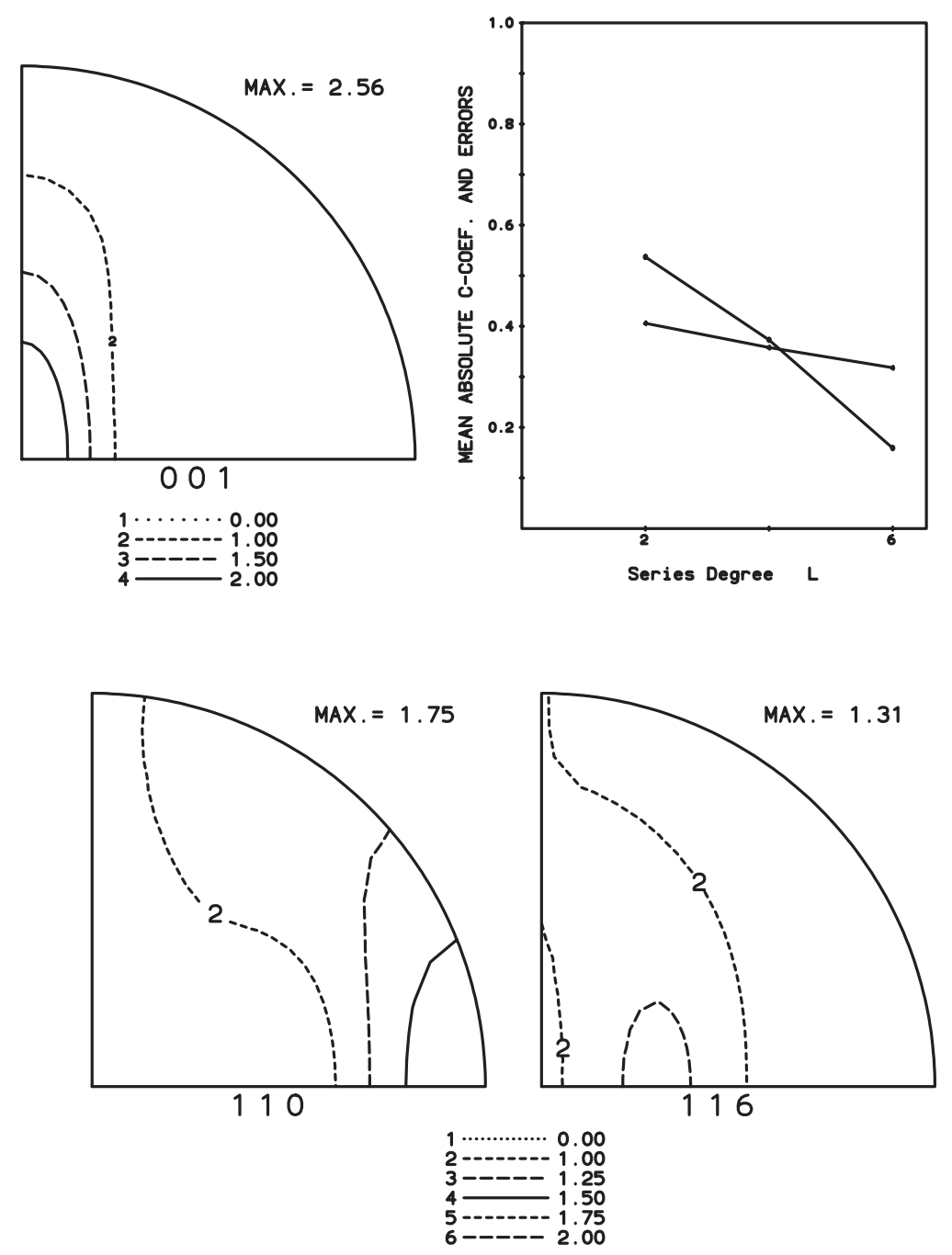

Abbildung D.14: Rückgerechnete Polfiguren, C-Koeffizienten und Fehler, HVAFG $12,5 \mathrm{Vol} \%$ 0,5ml/s 

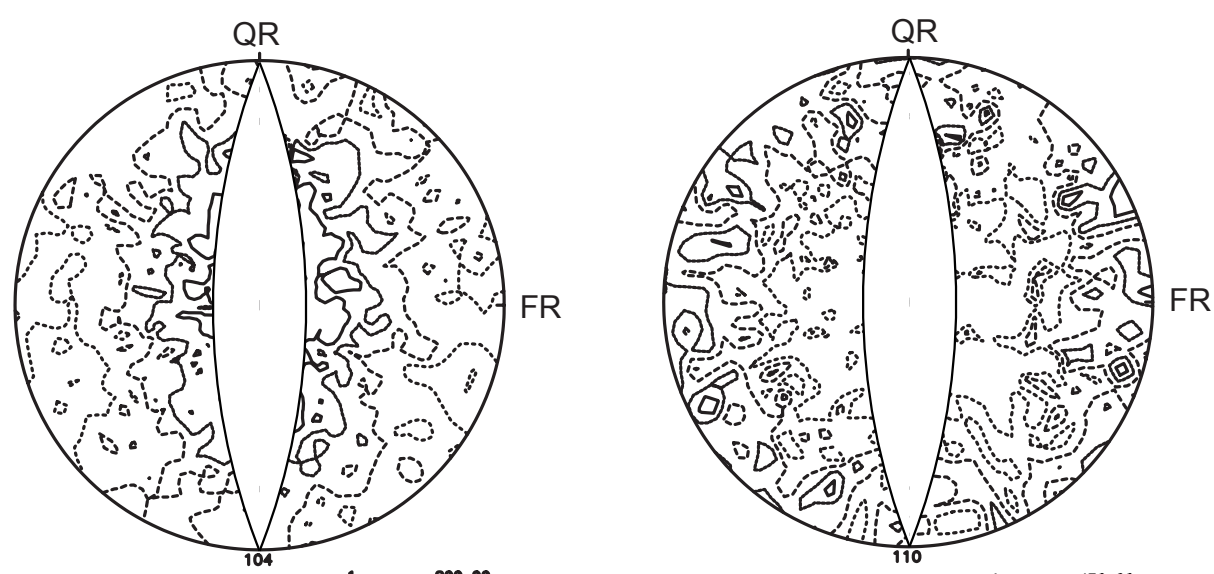

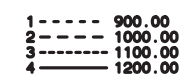
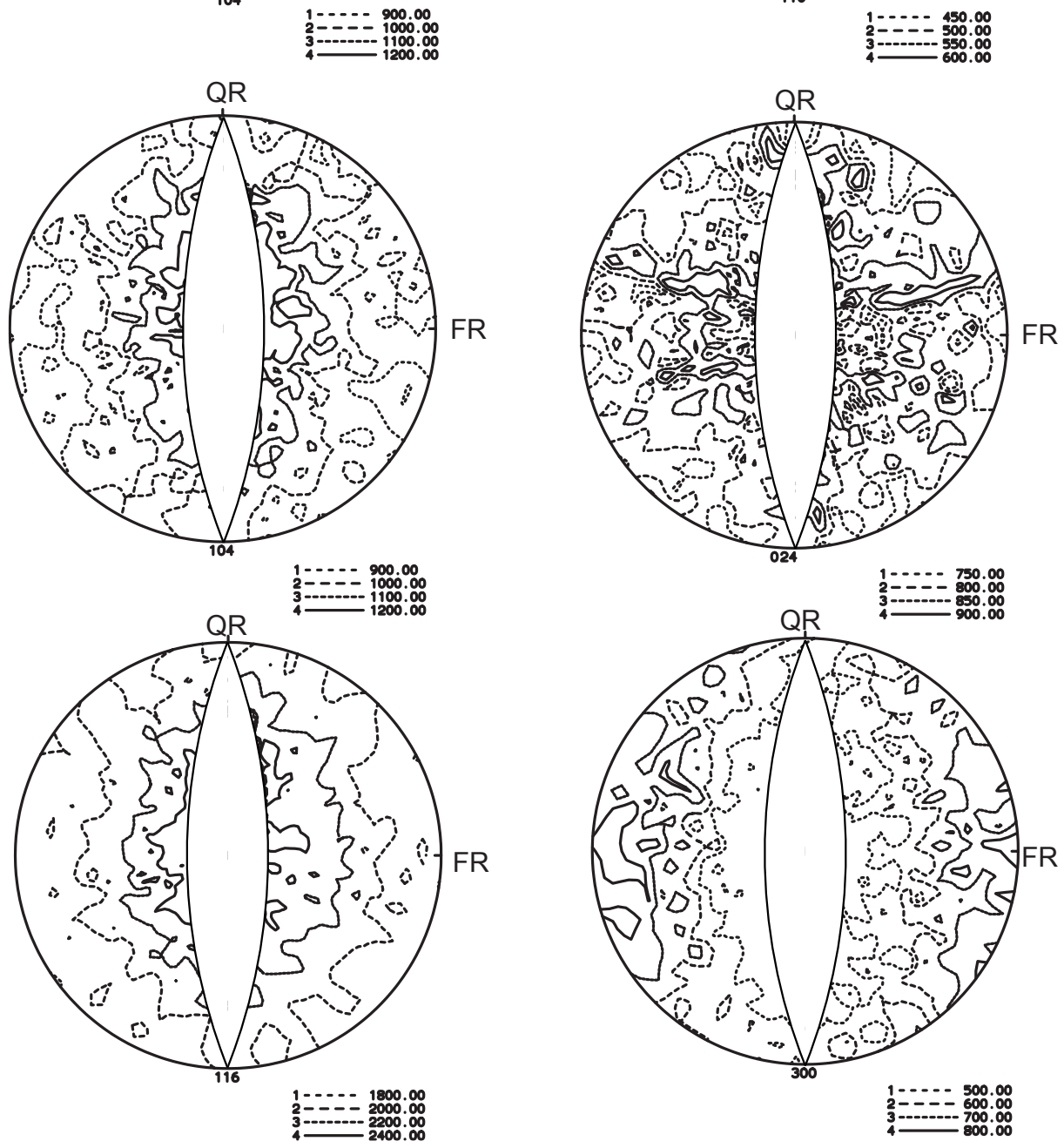

Abbildung D.15: Gemessene Polfiguren HVAFG 12,5Vol\% $1 \mathrm{ml} / \mathrm{s}$ 

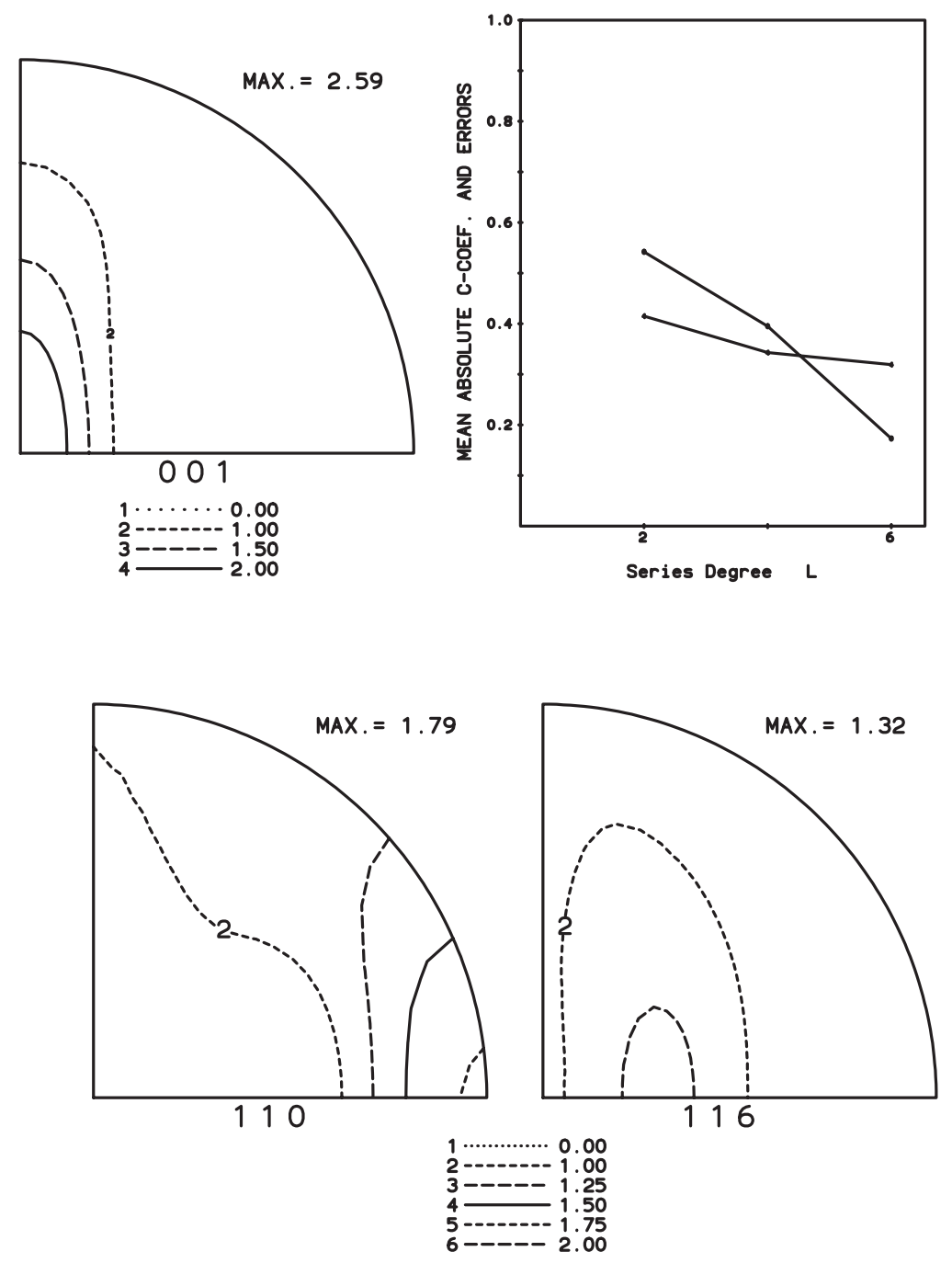

Abbildung D.16: Rückgerechnete Polfiguren, C-Koeffizienten und Fehler, HVAFG $12,5 \mathrm{Vol} \% 1 \mathrm{ml} / \mathrm{s}$ 

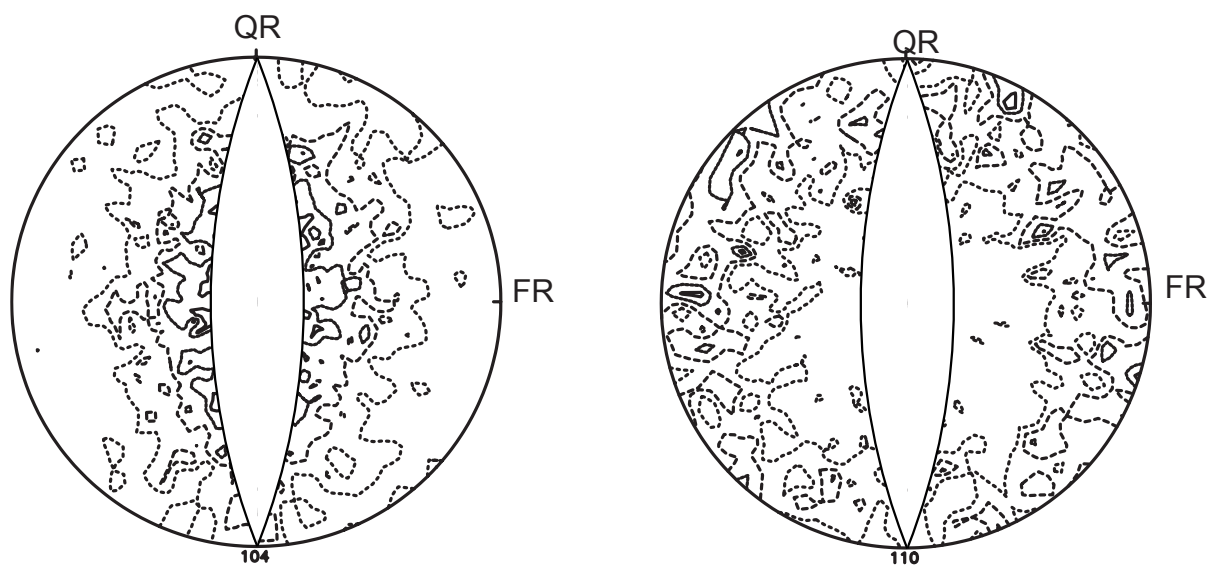

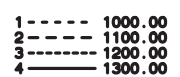

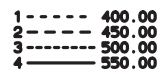
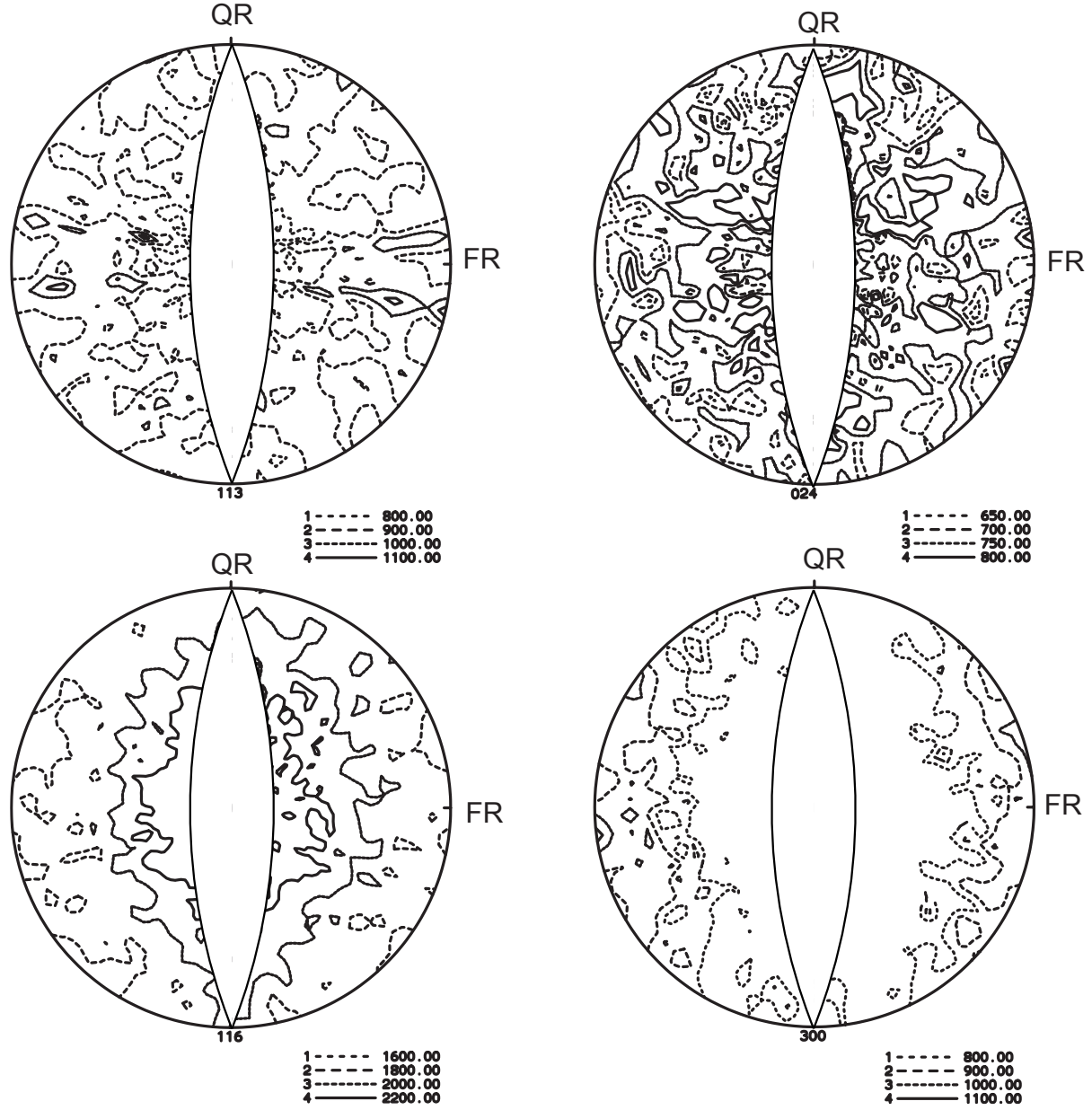

Abbildung D.17: Gemessene Polfiguren HVAFG 12,5Vol\% 2 ml/s 

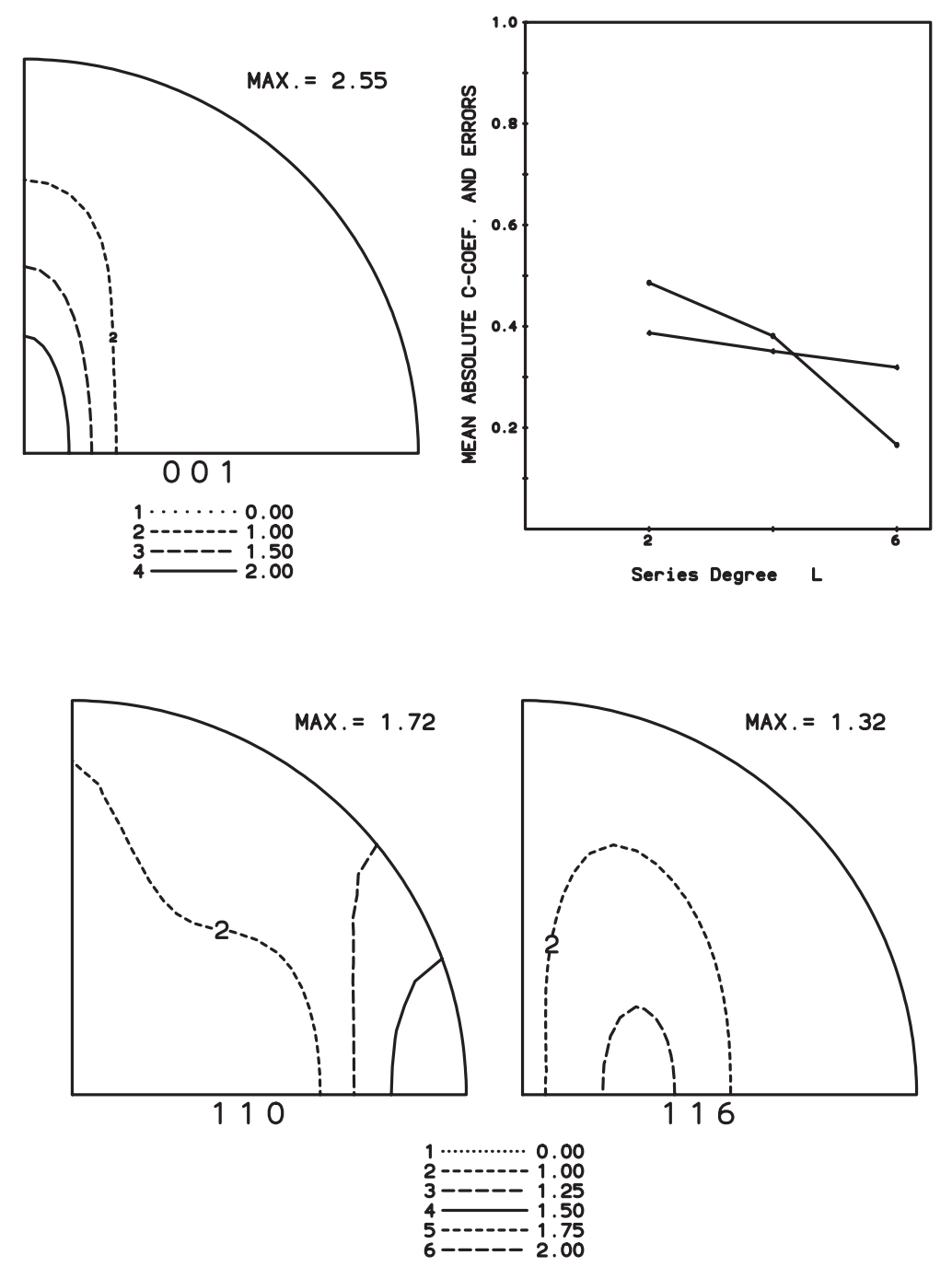

Abbildung D.18: Rückgerechnete Polfiguren, C-Koeffizienten und Fehler, HVAFG $12,5 \mathrm{Vol} \% 2 \mathrm{ml} / \mathrm{s}$ 

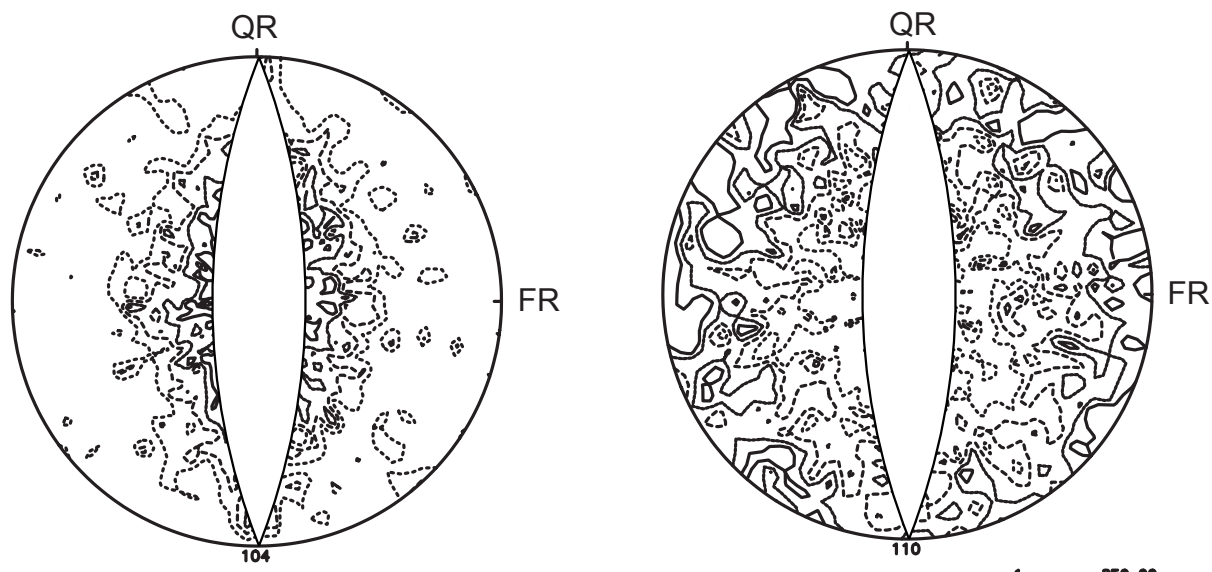

1
2
3
3
4
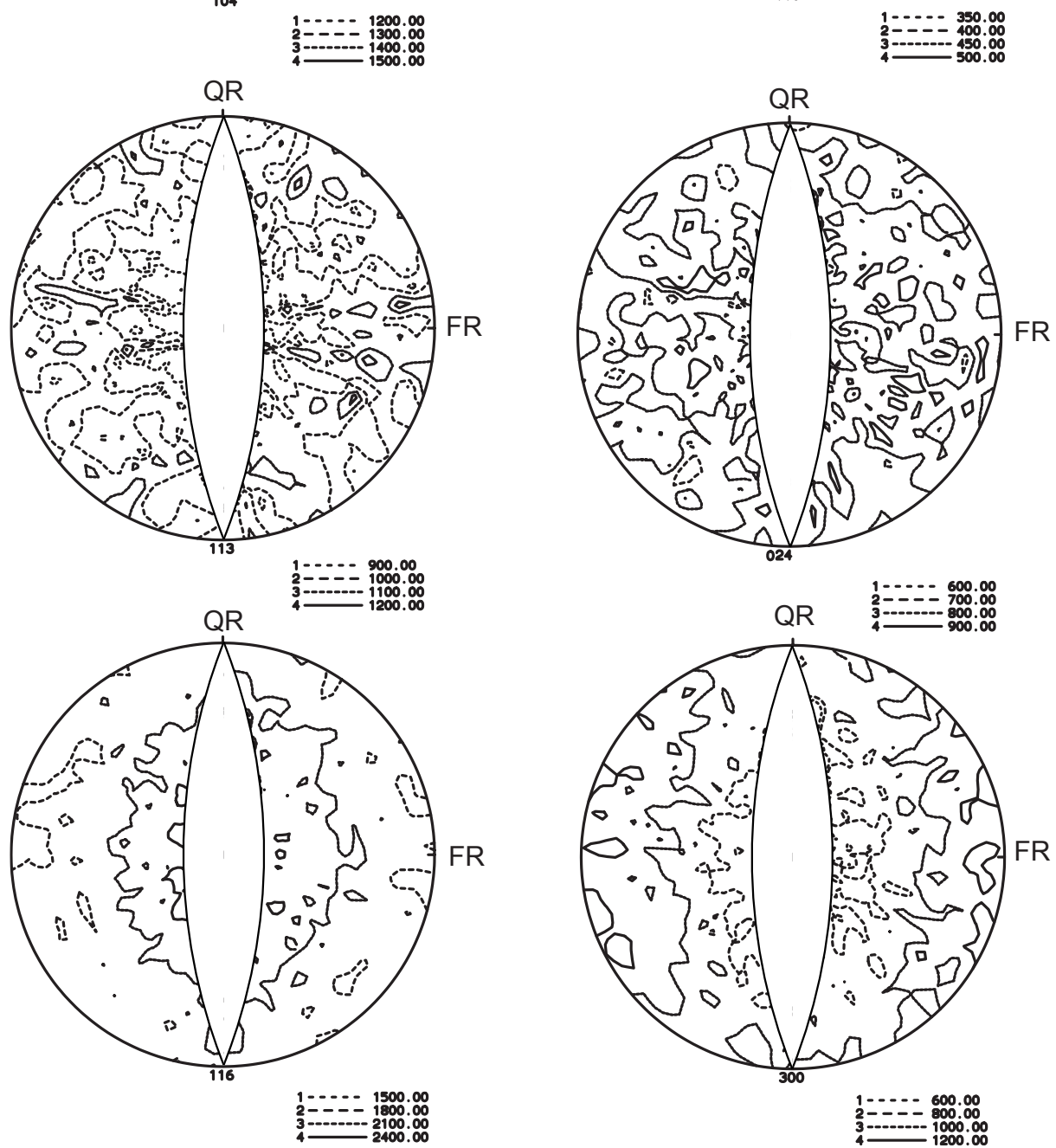

Abbildung D.19: Gemessene Polfiguren HVAFG 12,5Vol\% 5 ml/s 

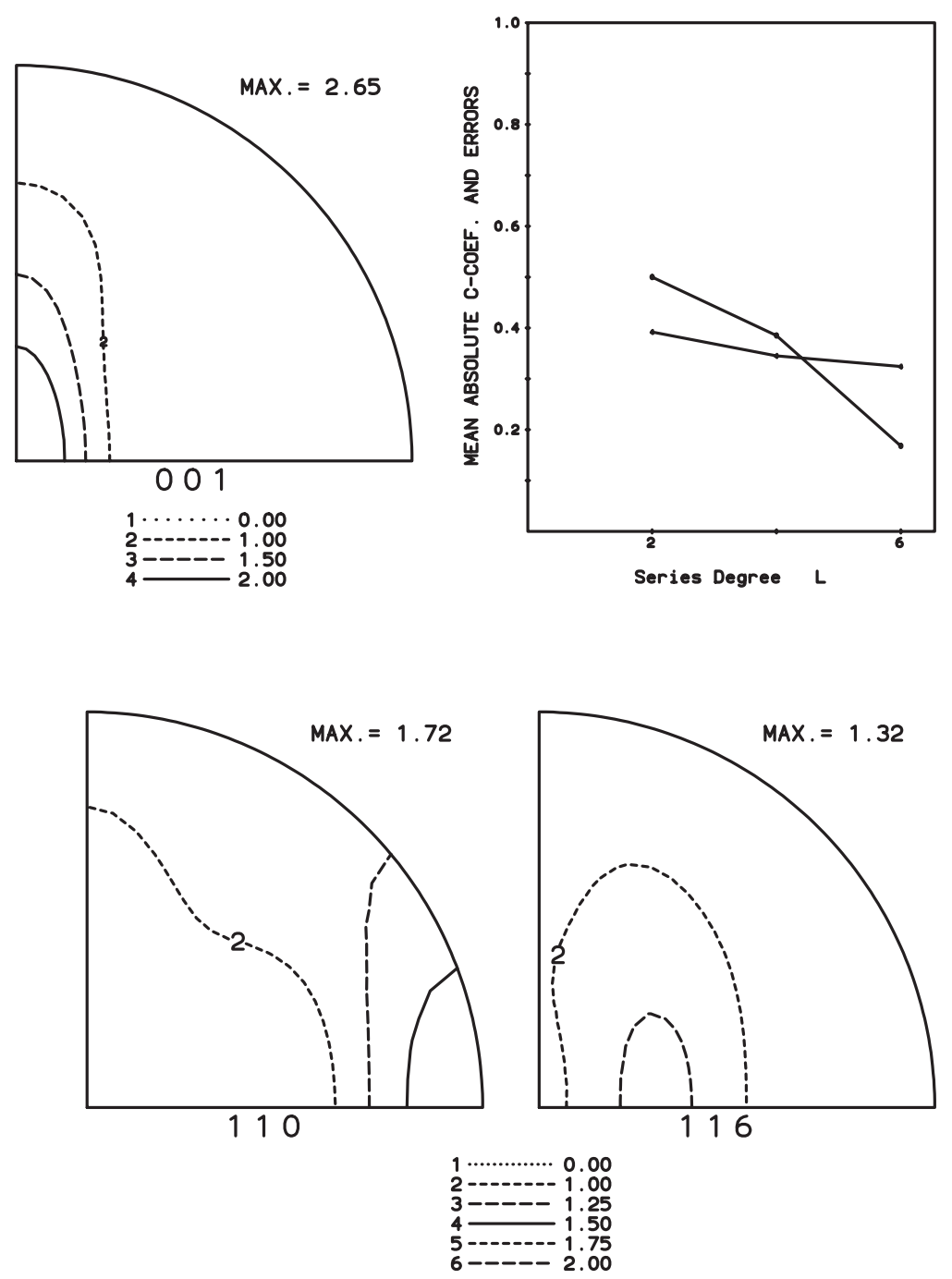

Abbildung D.20: Rückgerechnete Polfiguren, C-Koeffizienten und Fehler, HVAFG $12,5 \mathrm{Vol} \% 5 \mathrm{ml} / \mathrm{s}$ 


\section{D.3 Probe CTC20 7,5Vol\%}
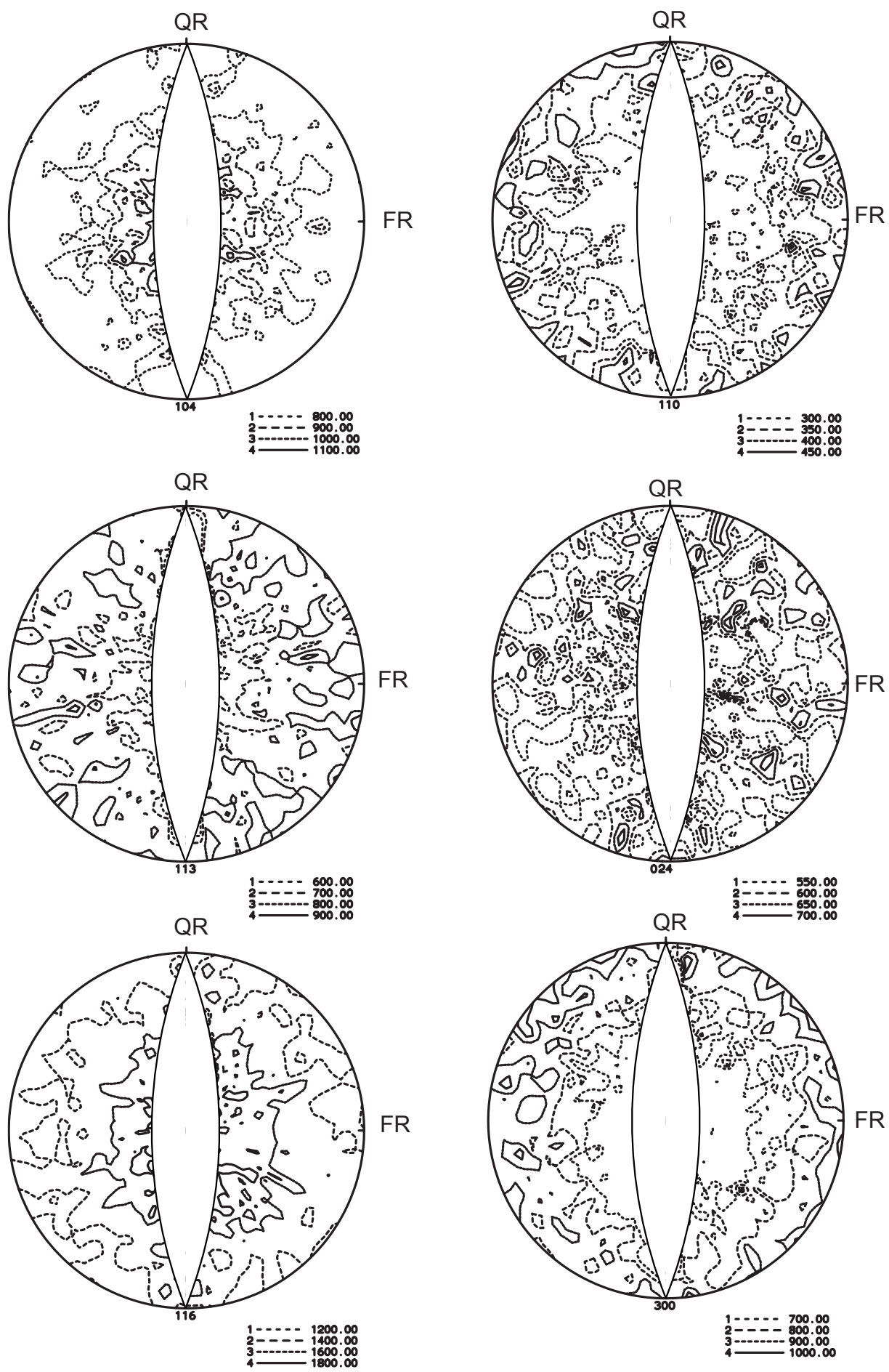

Abbildung D.21: Gemessene Polfiguren CTC20 7,5Vol\% 0,2 ml/s 

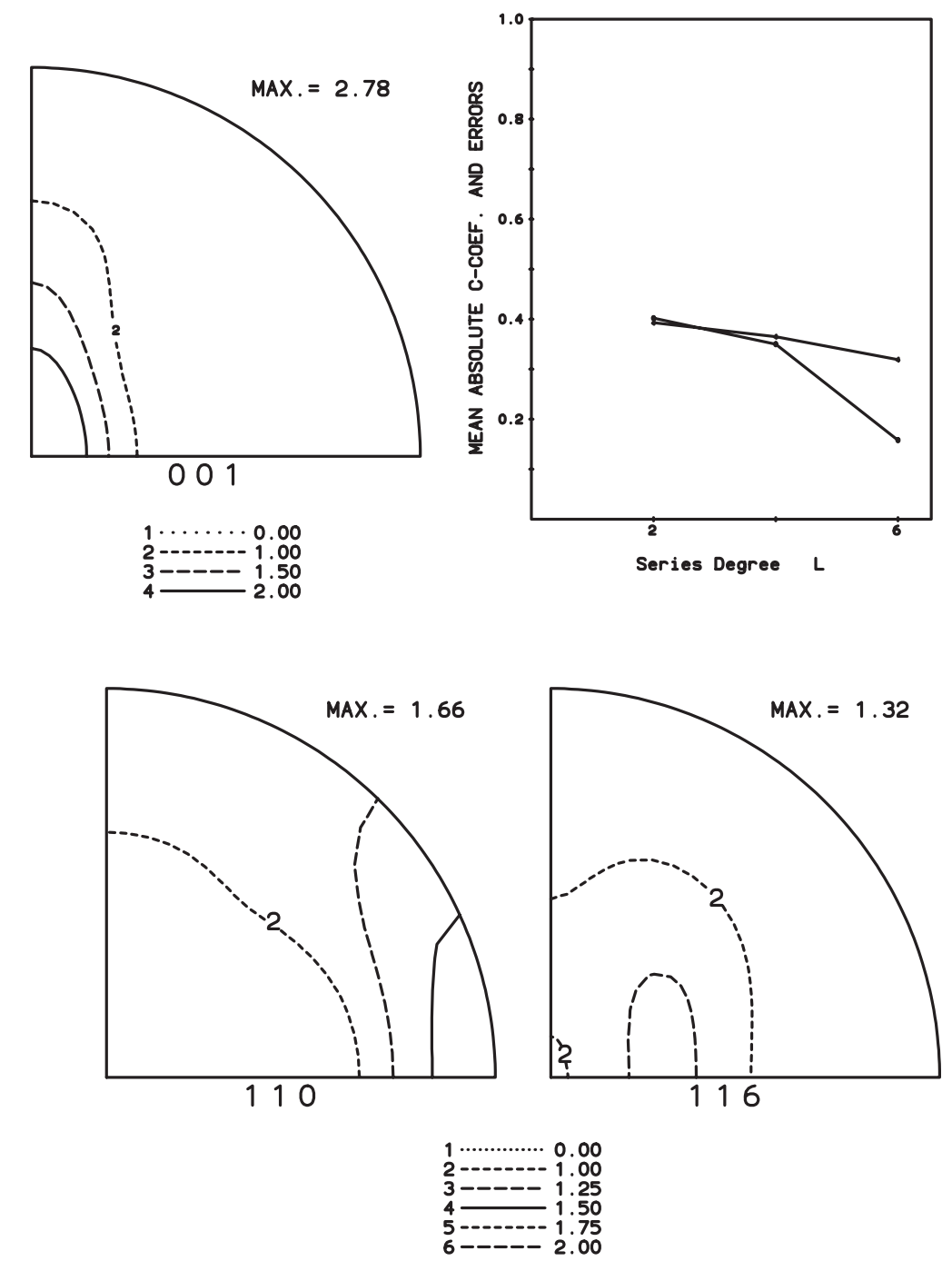

Abbildung D.22: Rückgerechnete Polfiguren, C-Koeffizienten und Fehler, CTC20 7,5Vol\% 0,2ml/s 

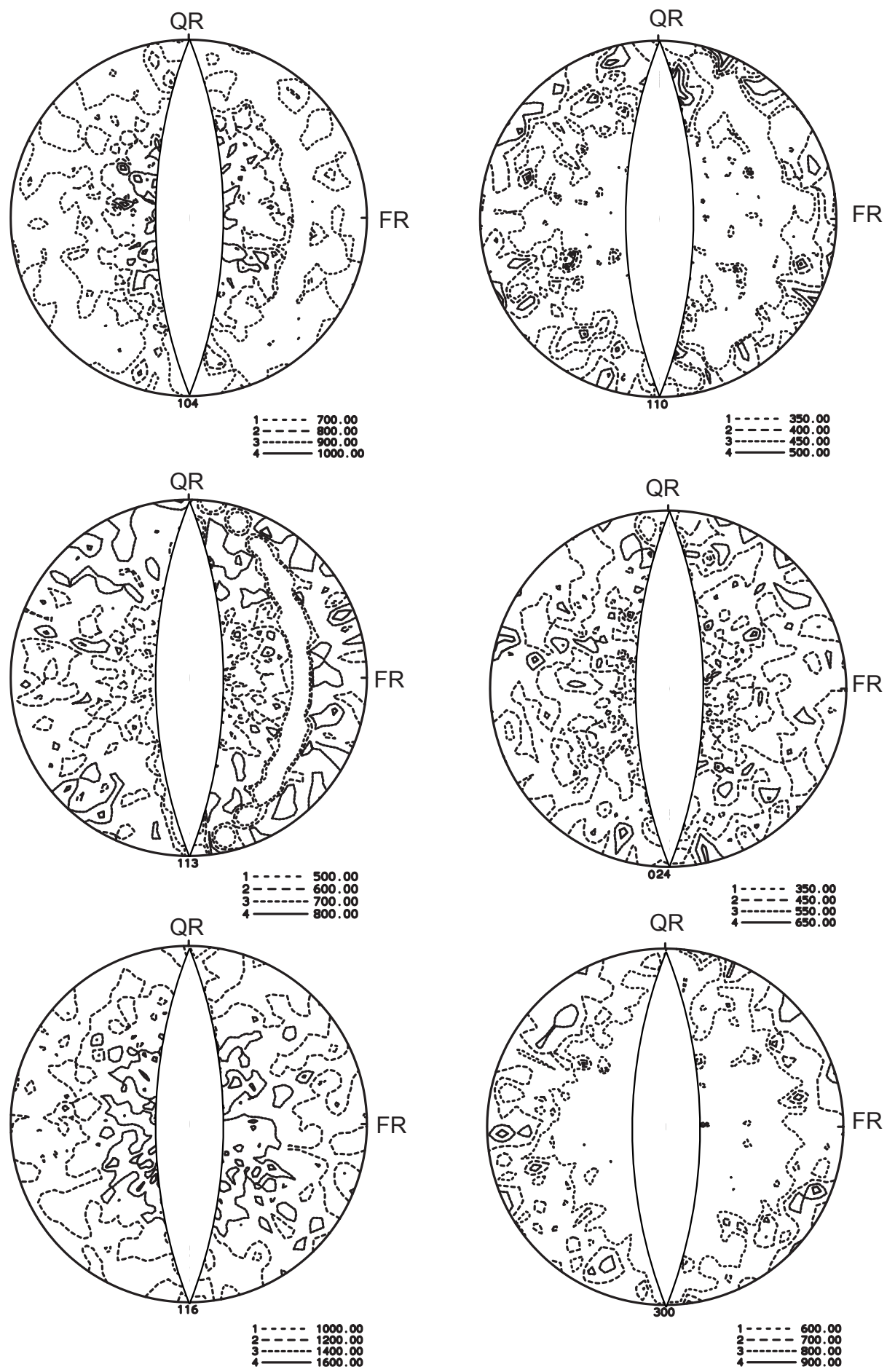

Abbildung D.23: Gemessene Polfiguren CTC20 7,5Vol\% 0,5 ml/s 

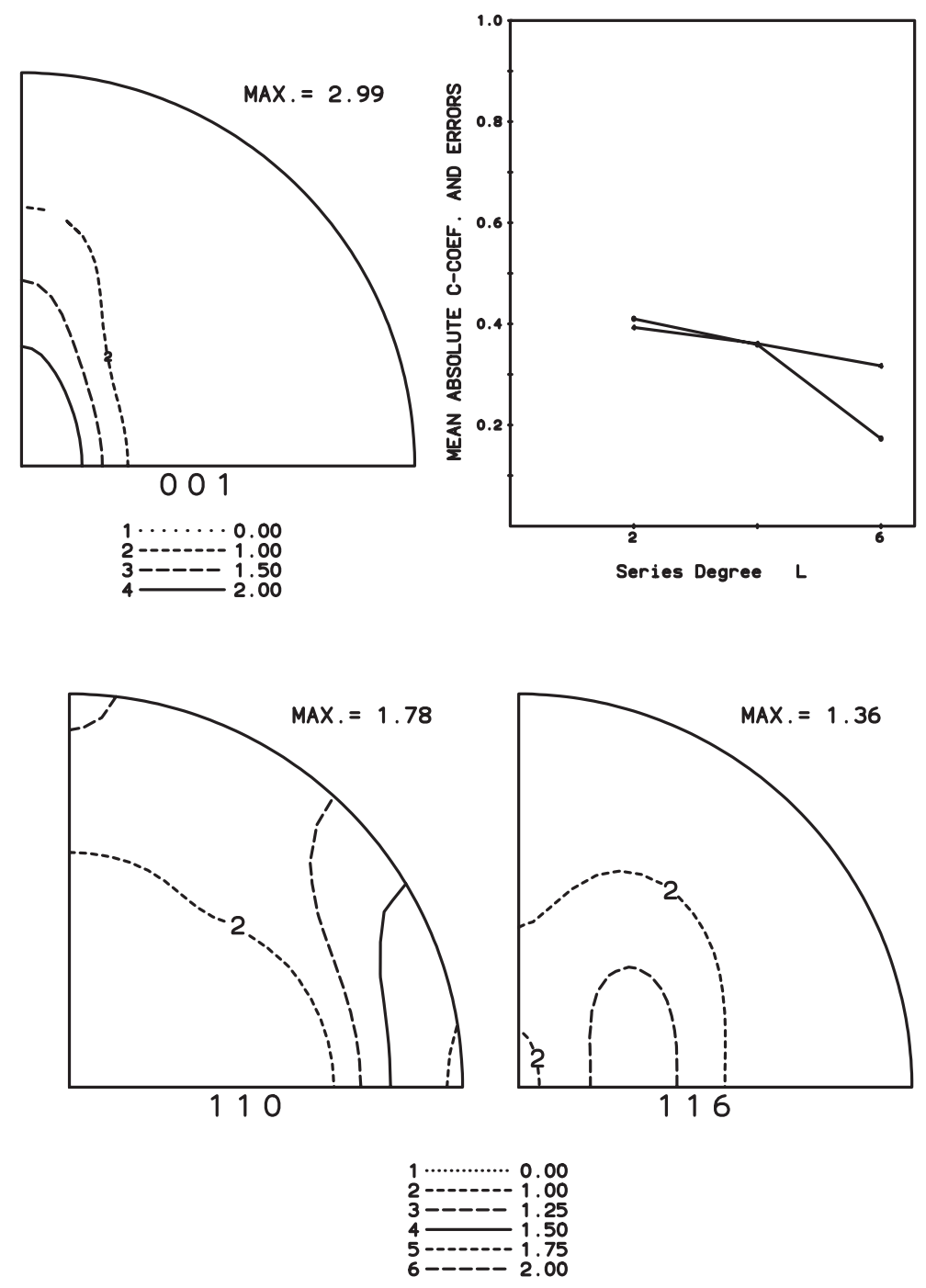

Abbildung D.24: Rückgerechnete Polfiguren, C-Koeffizienten und Fehler, CTC20 7,5 Vol\% 0,5ml/s 

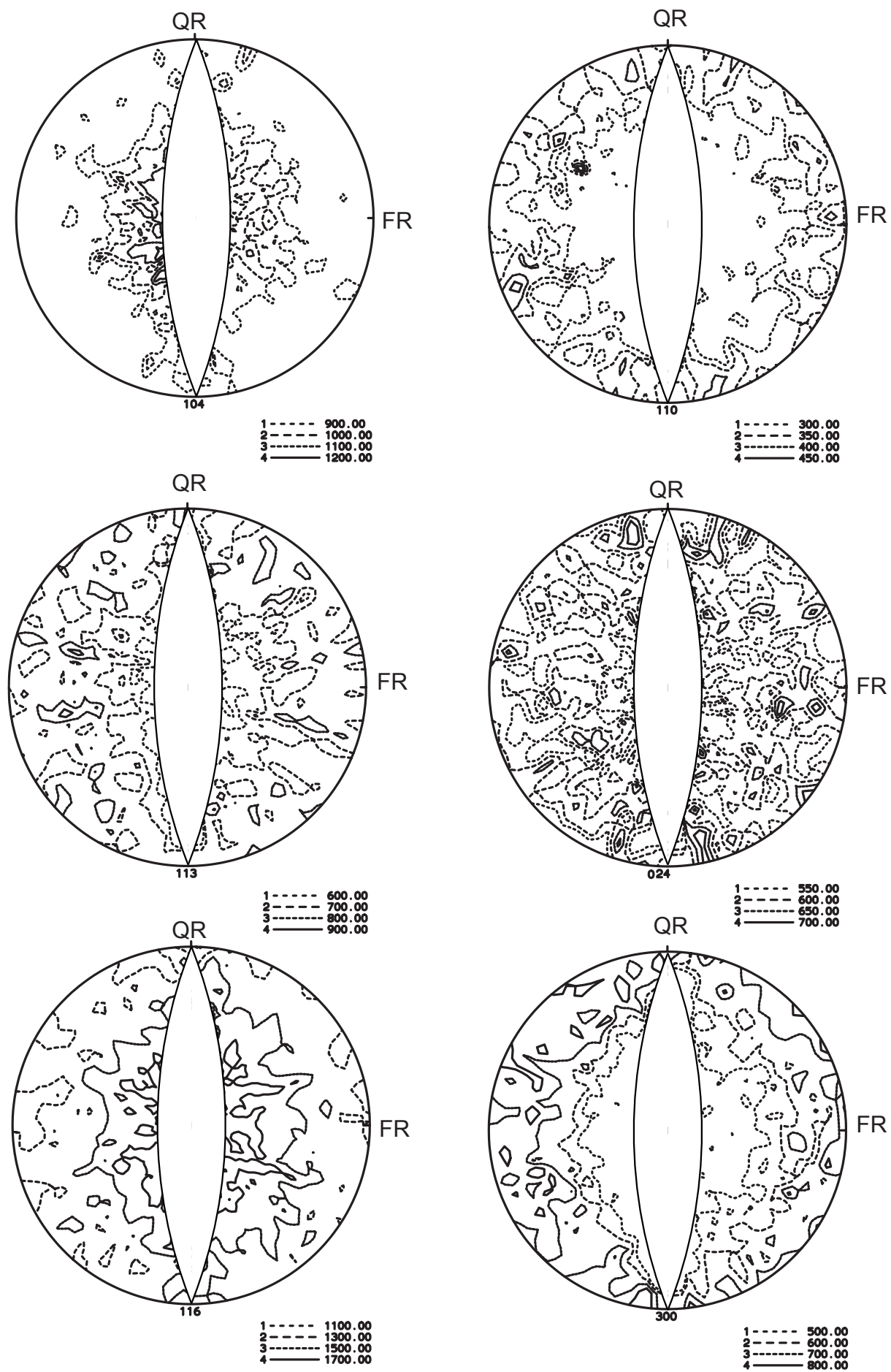

Abbildung D.25: Gemessene Polfiguren CTC20 7,5Vol\% $1 \mathrm{ml} / \mathrm{s}$ 

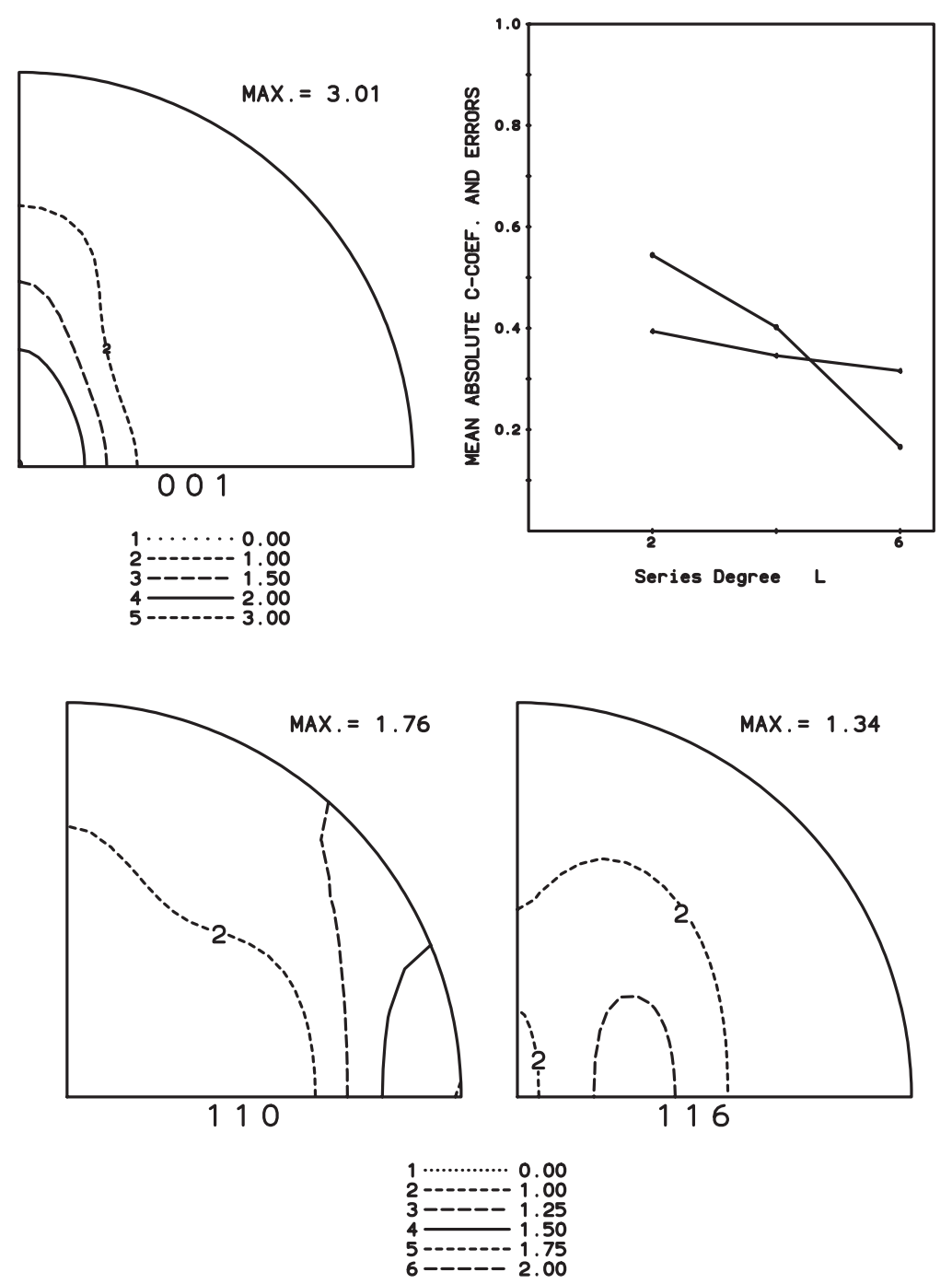

Abbildung D.26: Rückgerechnete Polfiguren, C-Koeffizienten und Fehler, CTC20 7,5Vol\% 1ml/s 

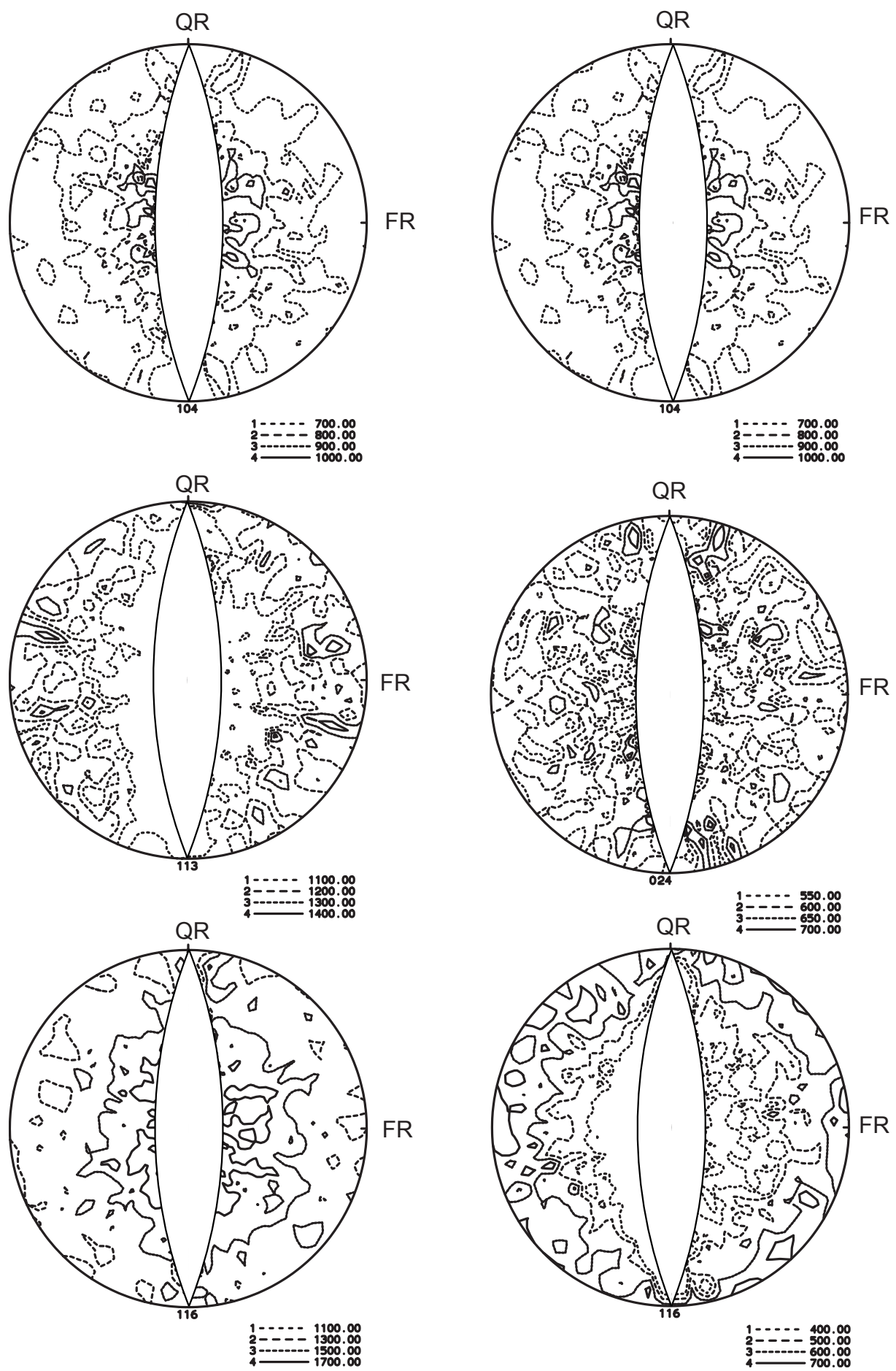

Abbildung D.27: Gemessene Polfiguren CTC20 7,5Vol\% $2 \mathrm{ml} / \mathrm{s}$ 

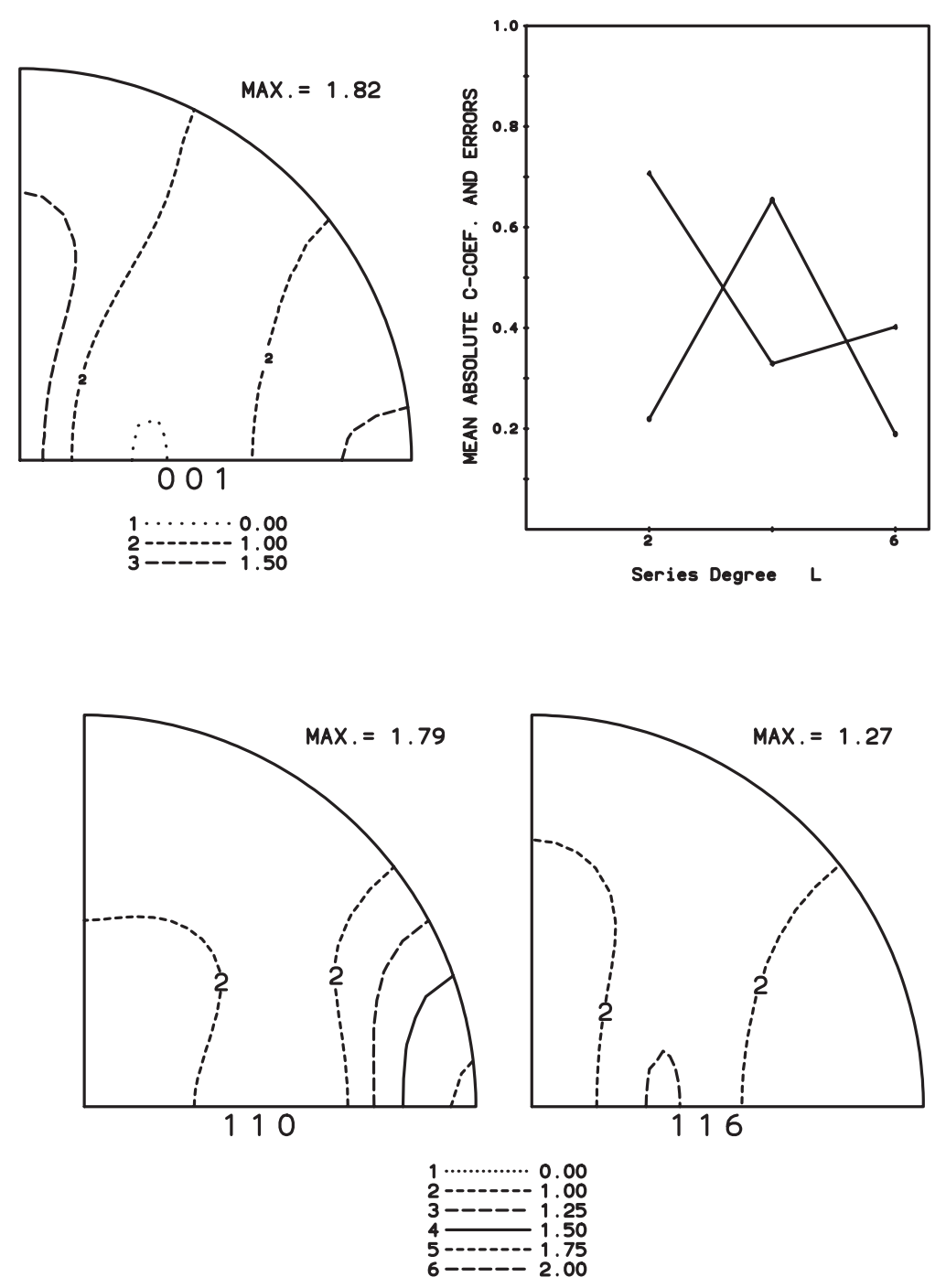

Abbildung D.28: Rückgerechnete Polfiguren, C-Koeffizienten und Fehler, CTC20 7,5Vol\% 2ml/s 

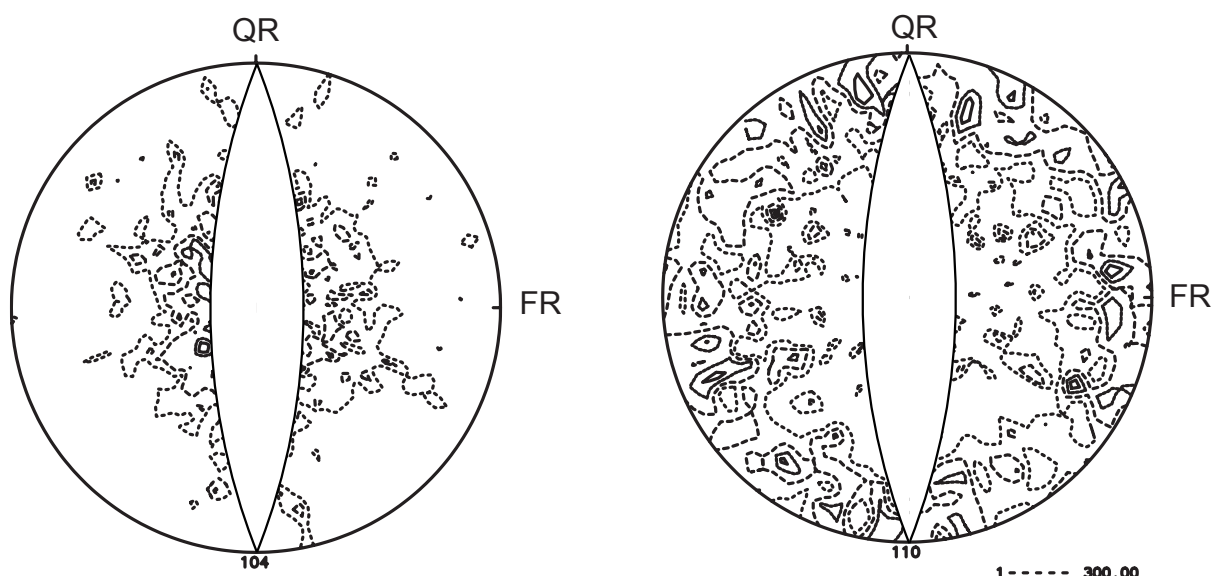

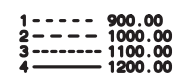
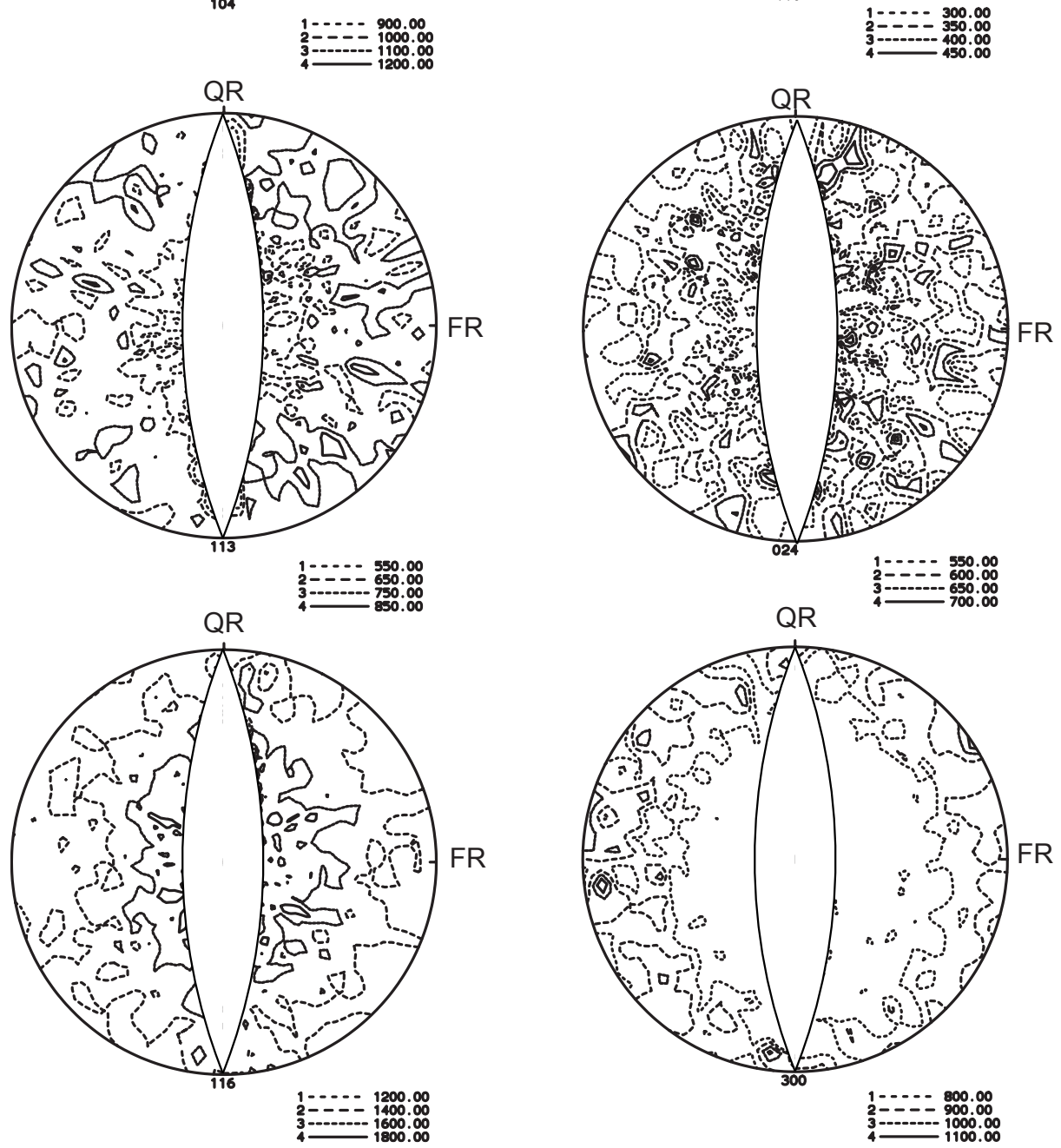

Abbildung D.29: Gemessene Polfiguren CTC20 7,5Vol\% 5 ml/s 

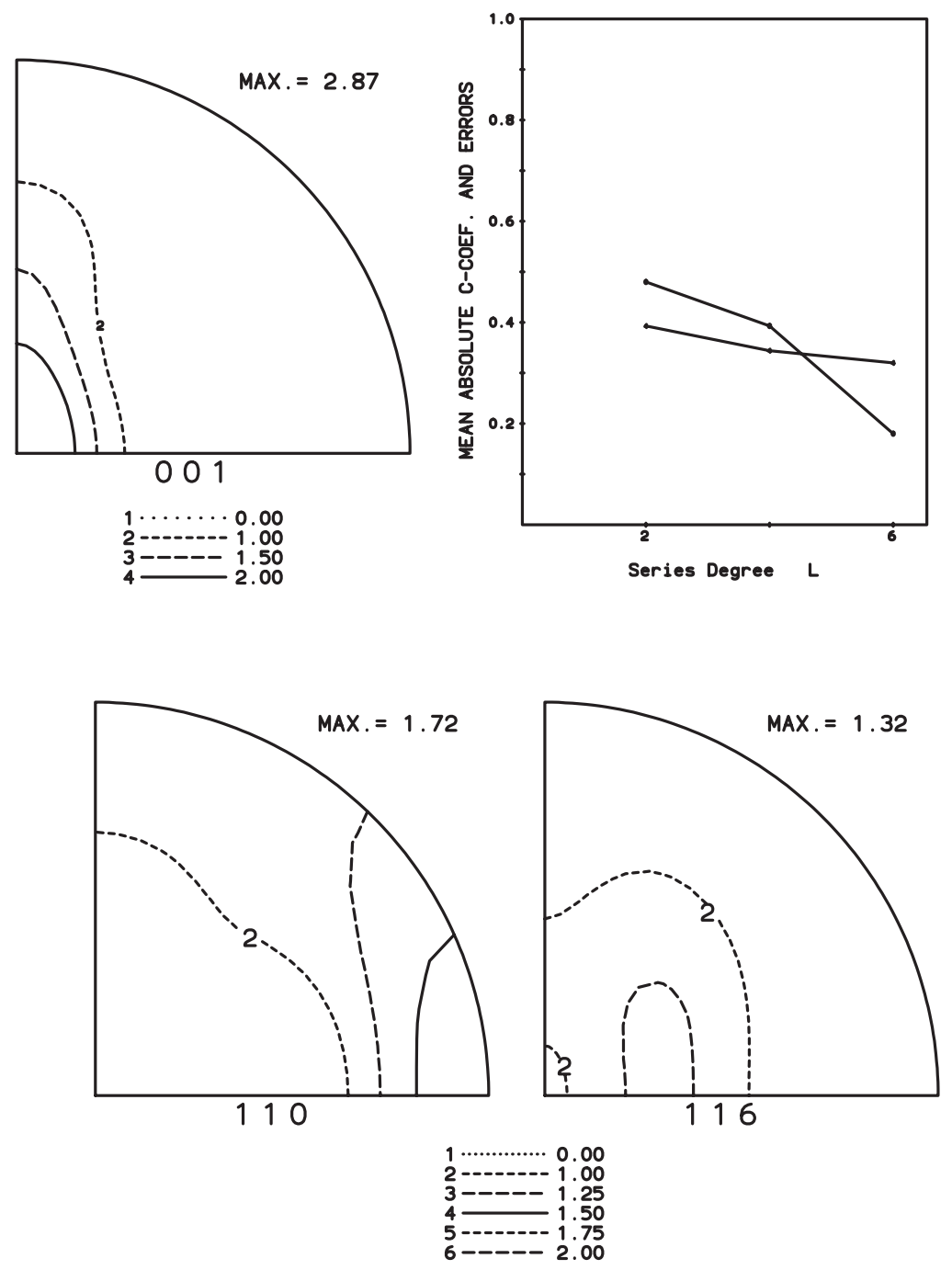

Abbildung D.30: Rückgerechnete Polfiguren, C-Koeffizienten und Fehler, CTC20 7,5Vol\% 5ml/s 


\section{D.4 Probe CTC20 10Vol\%}
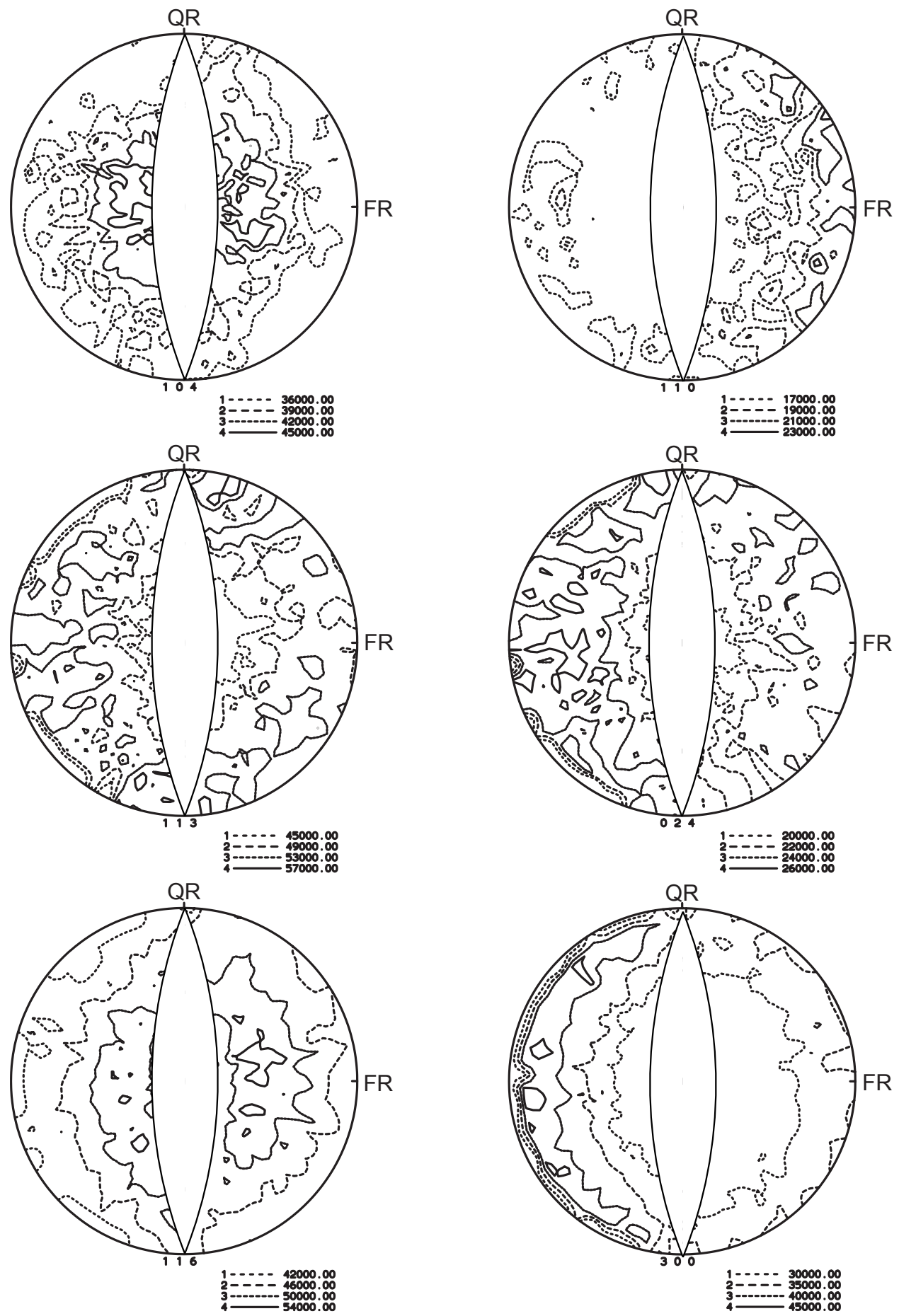

Abbildung D.31: Gemessene Polfiguren CTC20 10Vol\% 0,2 ml/s 

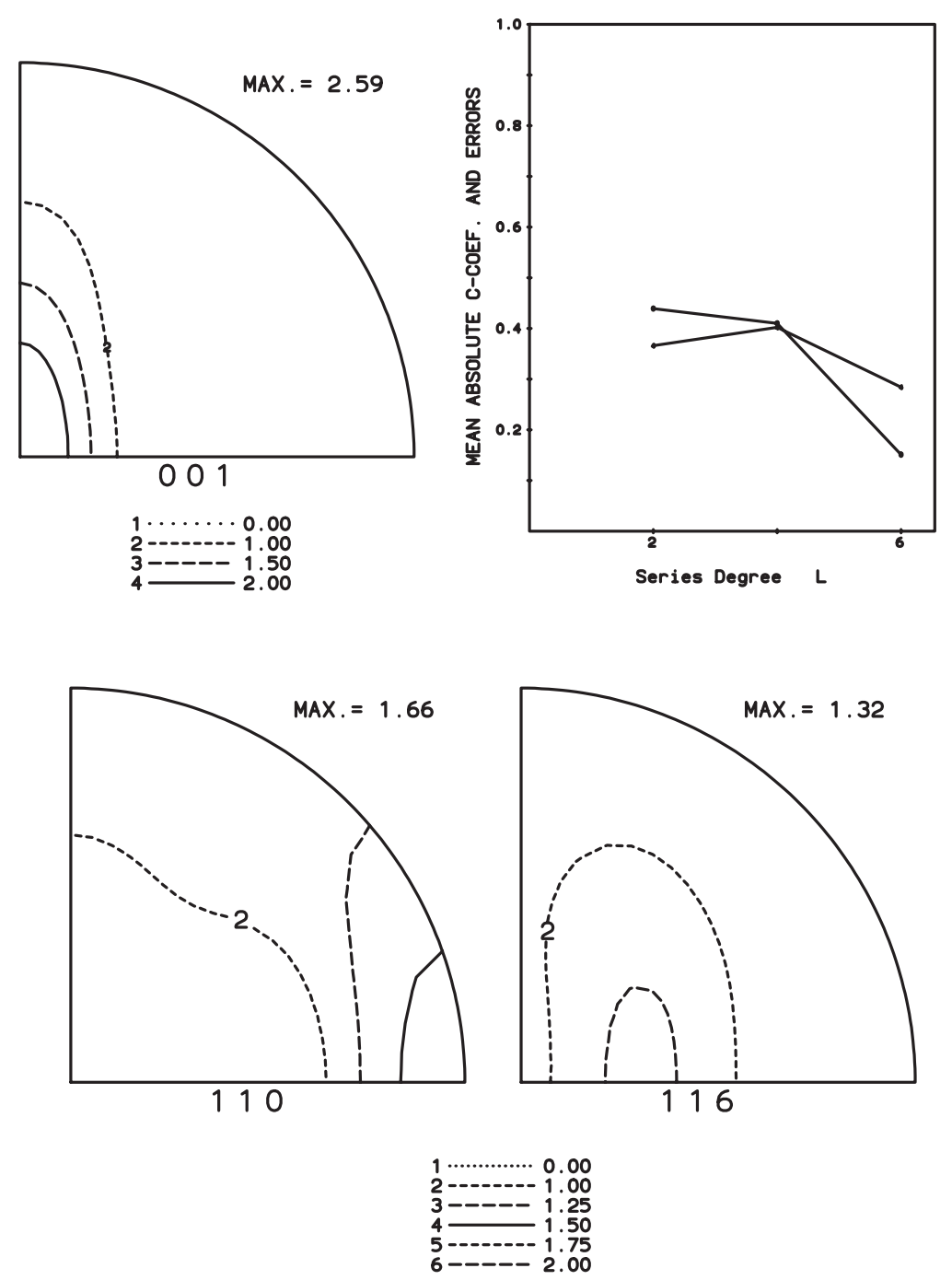

Abbildung D.32: Rückgerechnete Polfiguren, C-Koeffizienten und Fehler, CTC20 10Vol\% 0,2ml/s 

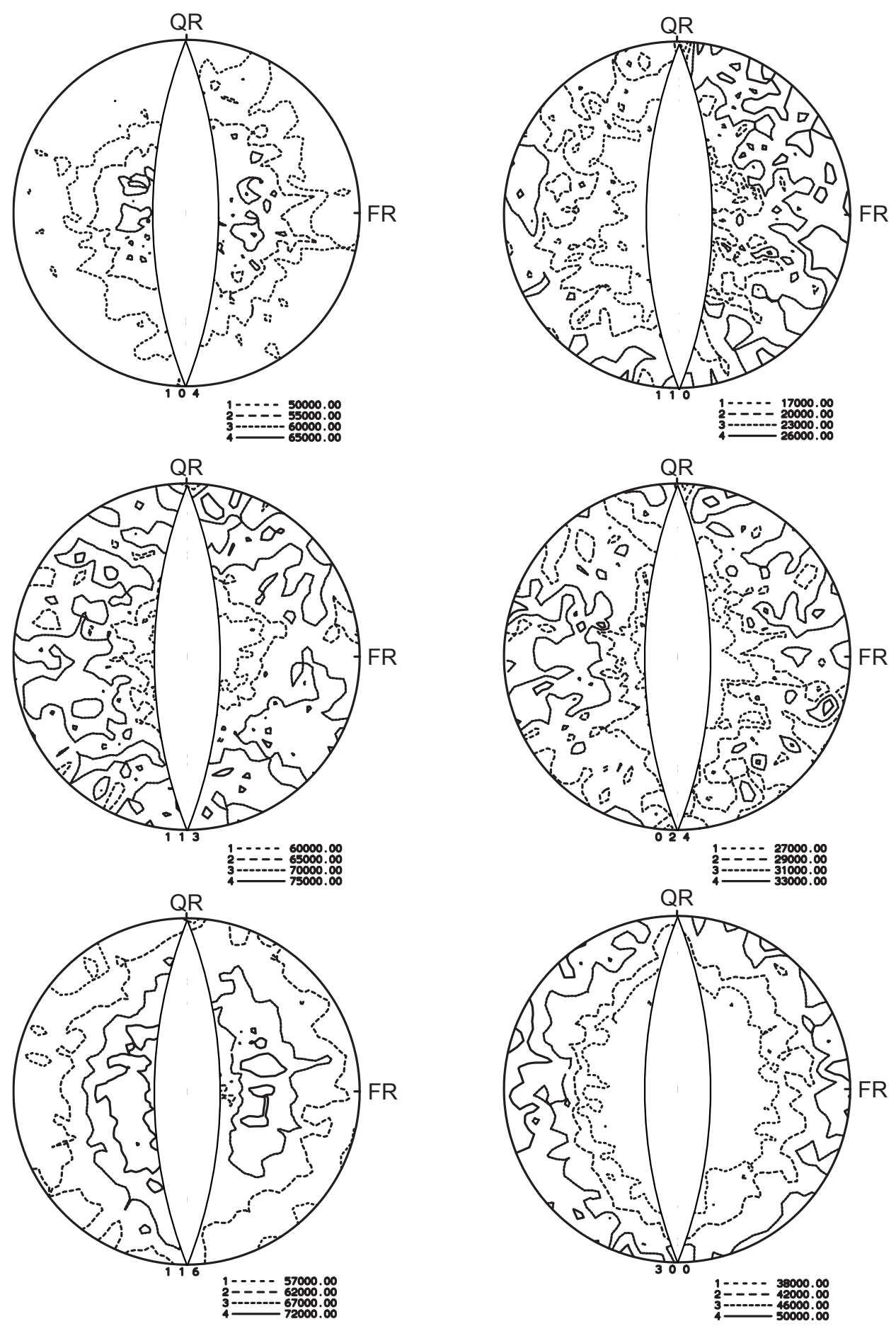

Abbildung D.33: Gemessene Polfiguren CTC20 10Vol\% 0,5 ml/s 

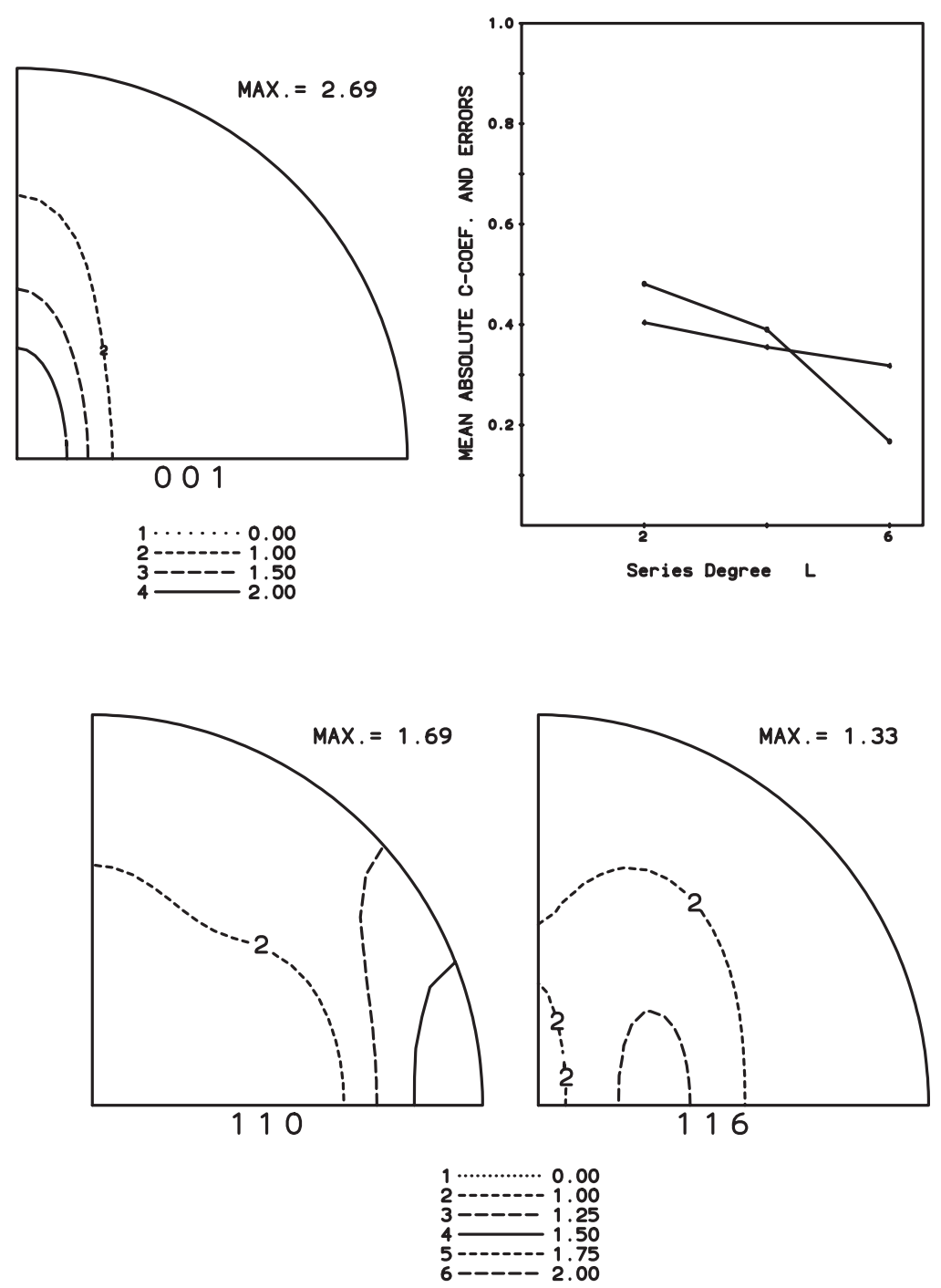

Abbildung D.34: Rückgerechnete Polfiguren, C-Koeffizienten und Fehler, CTC20 10Vol\% 0,5ml/s 

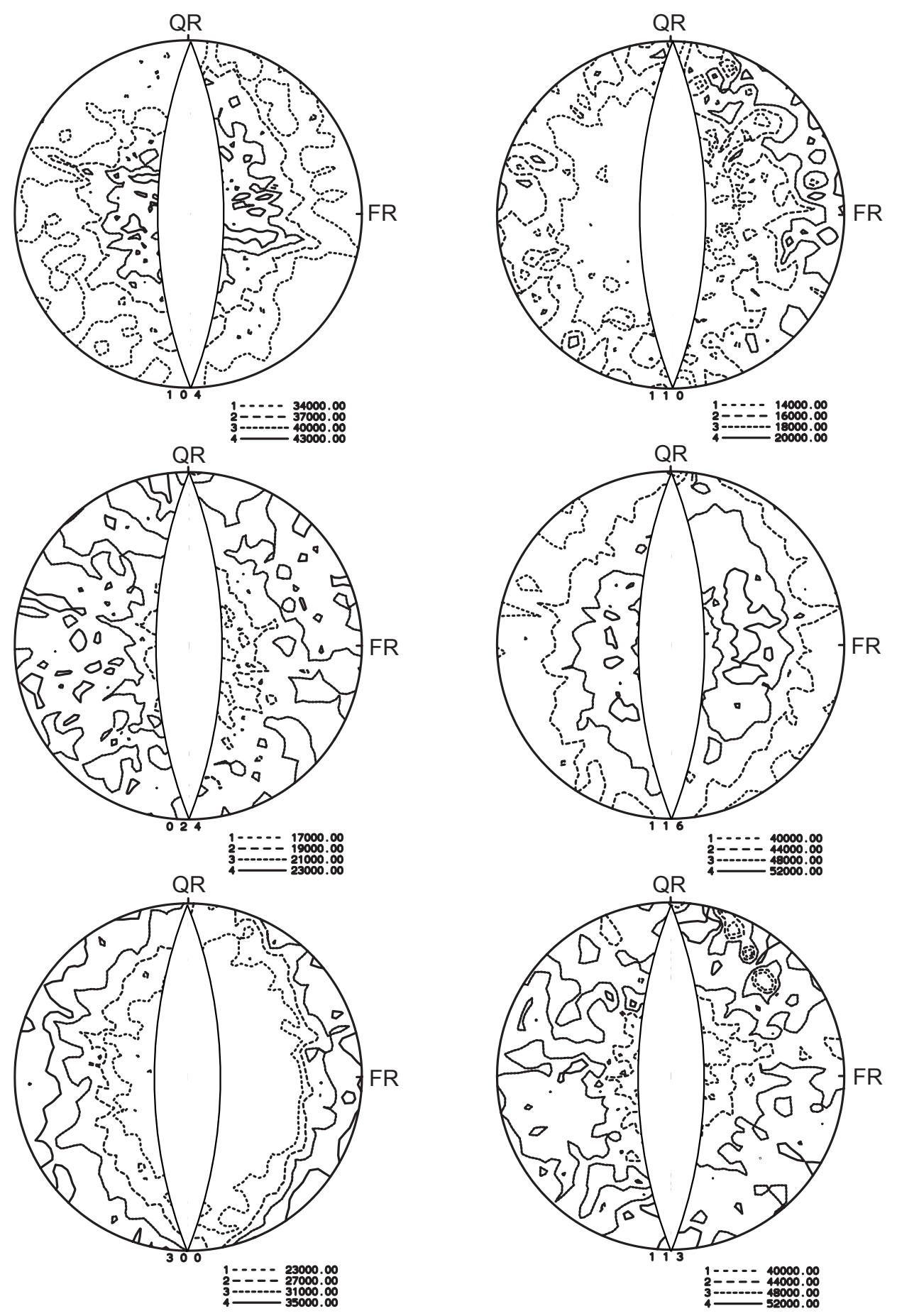

Abbildung D.35: Gemessene Polfiguren CTC20 10Vol\% $1 \mathrm{ml} / \mathrm{s}$ 

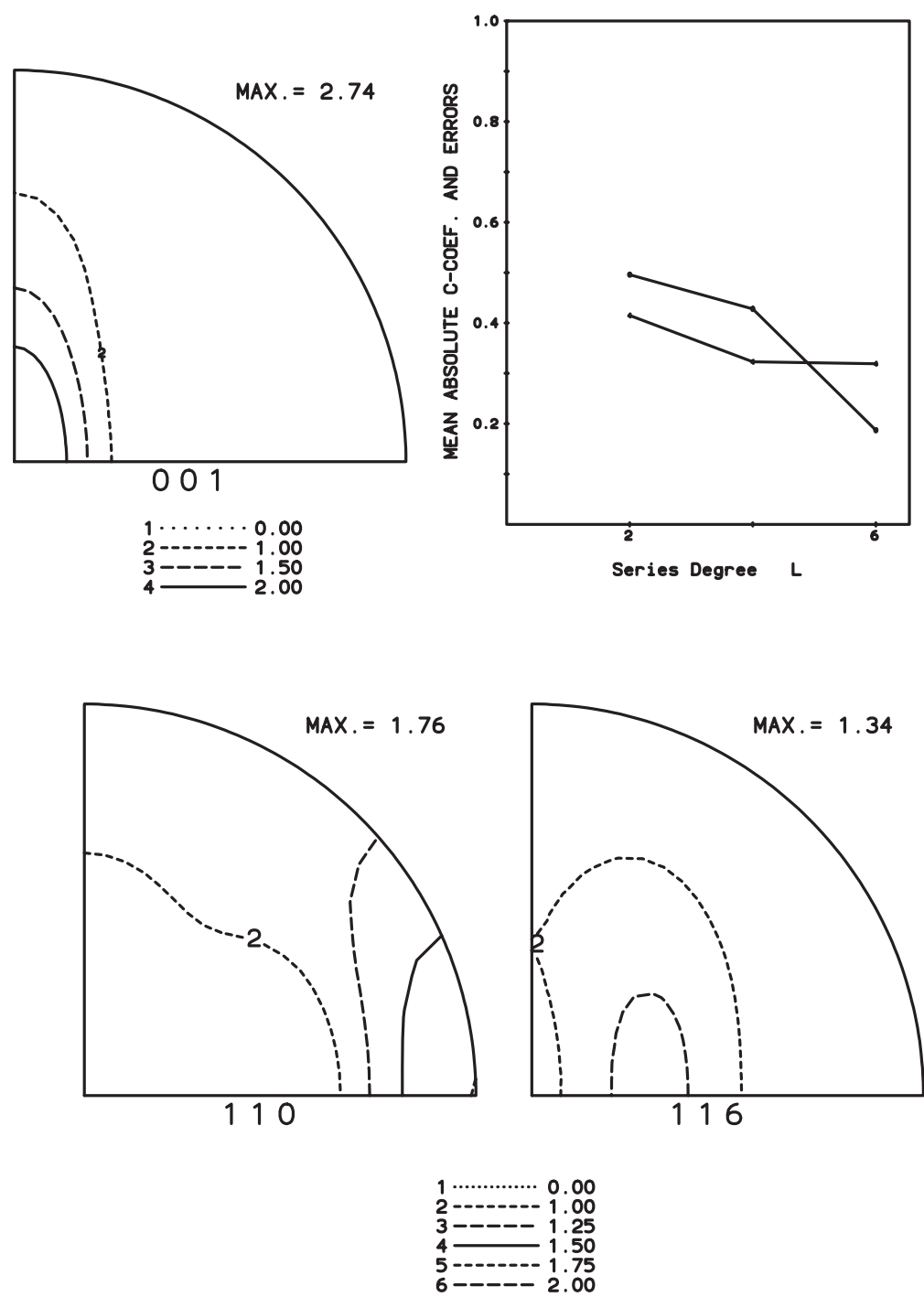

Abbildung D.36: Rückgerechnete Polfiguren, C-Koeffizienten und Fehler, CTC20 10Vol\% 1ml/s 

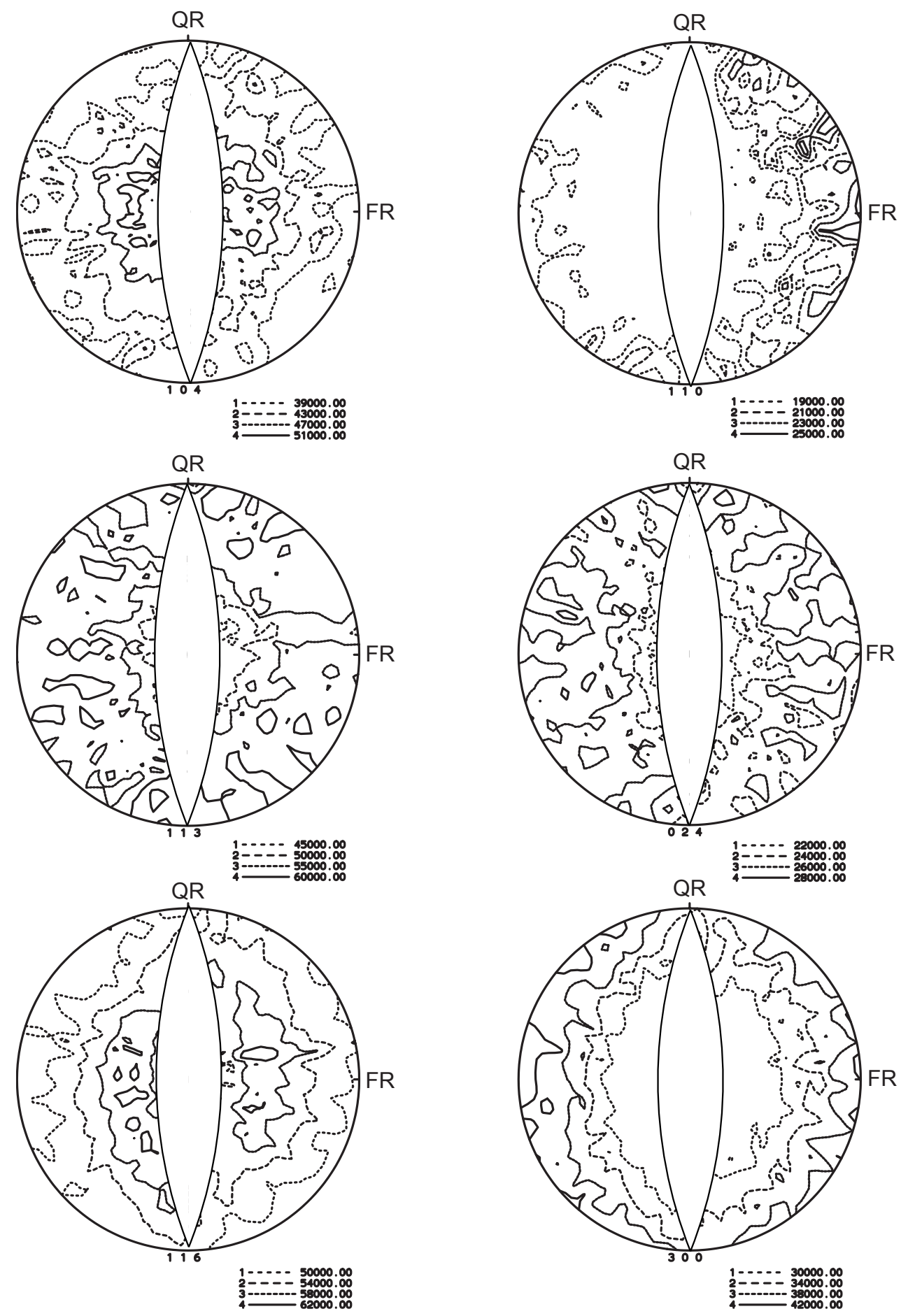

Abbildung D.37: Gemessene Polfiguren CTC20 10Vol\% 2 ml/s 

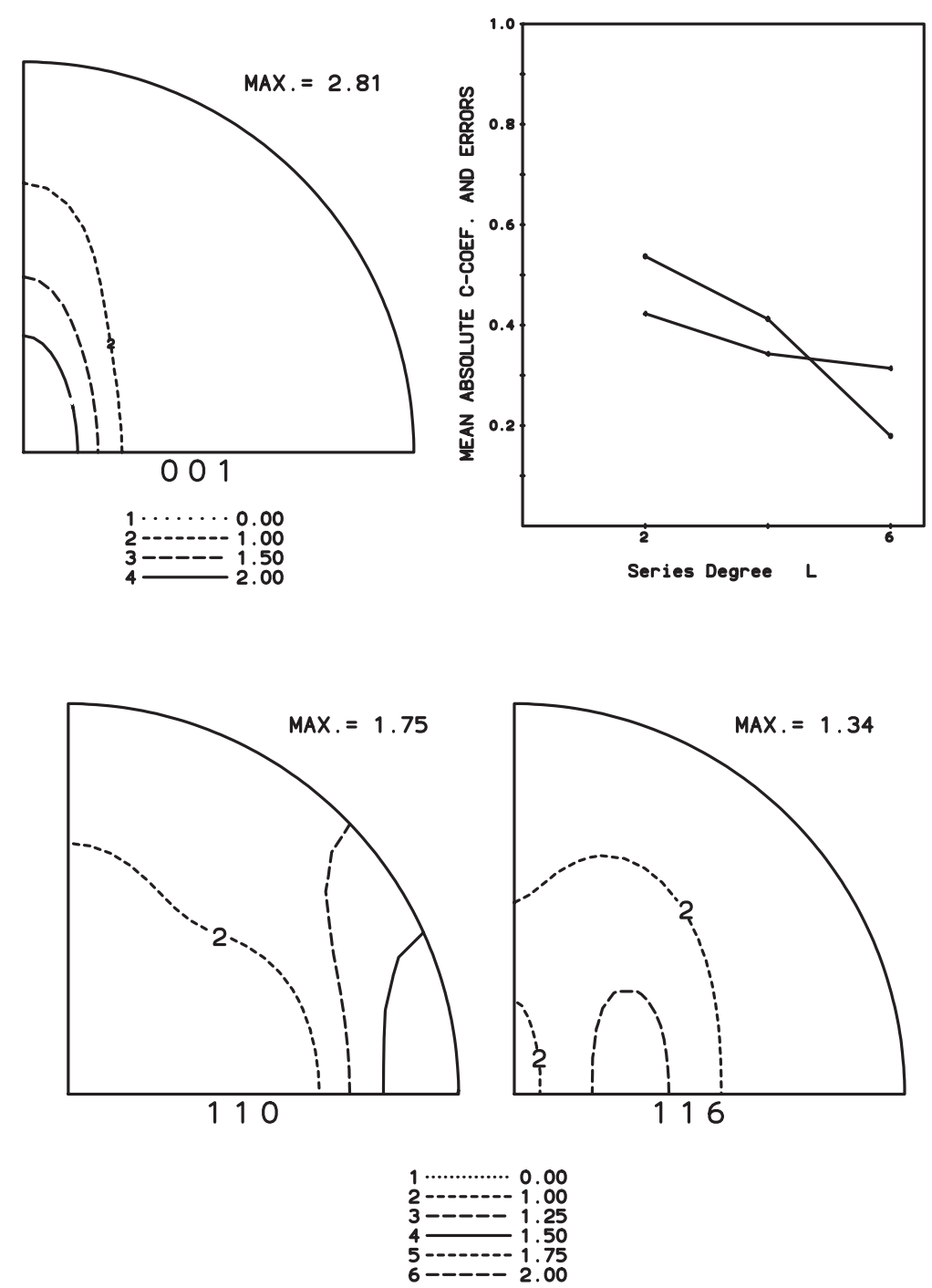

Abbildung D.38: Rückgerechnete Polfiguren, C-Koeffizienten und Fehler, CTC20 10Vol\% 2ml/s 

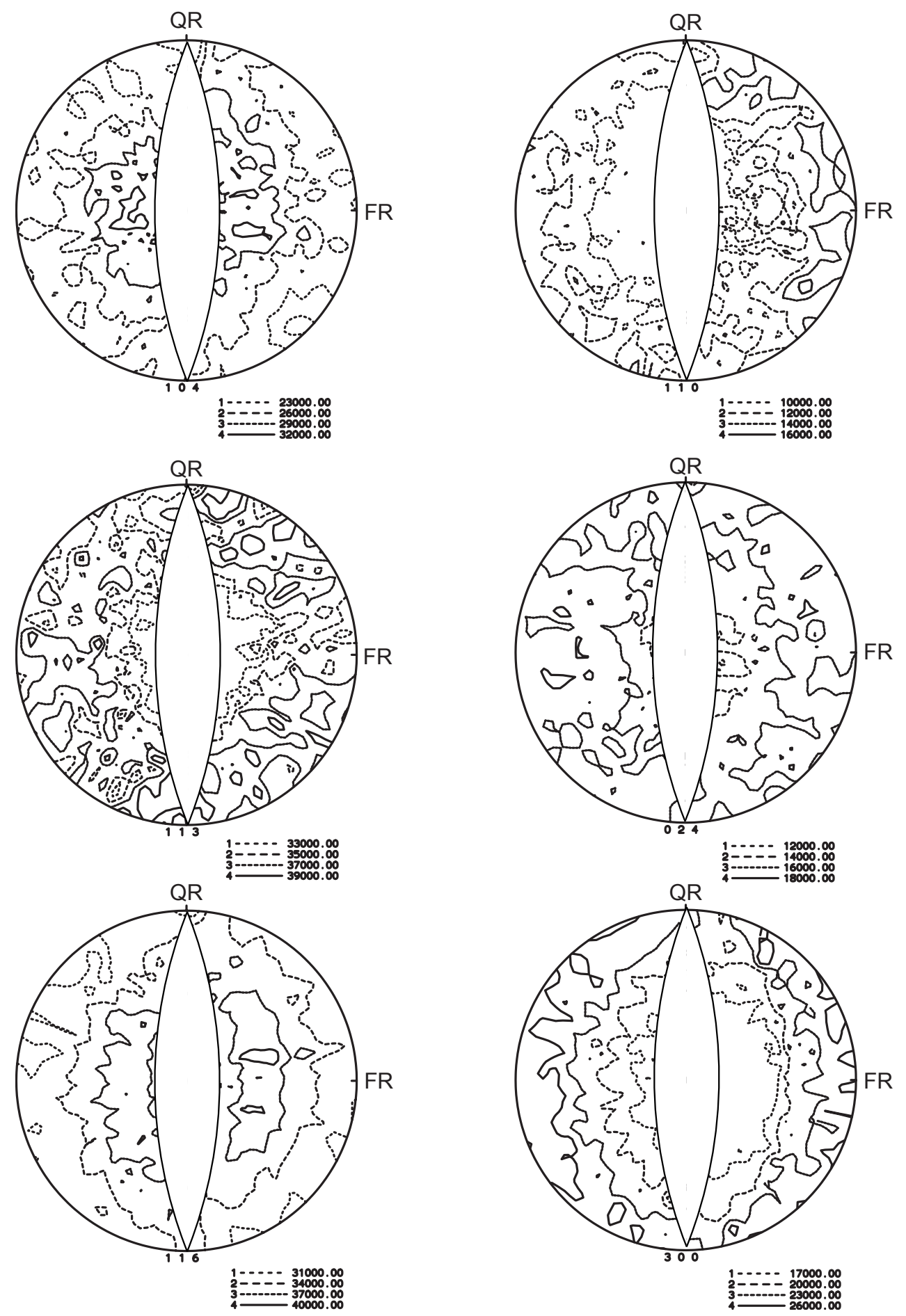

Abbildung D.39: Gemessene Polfiguren CTC20 10Vol\% 5 ml/s 

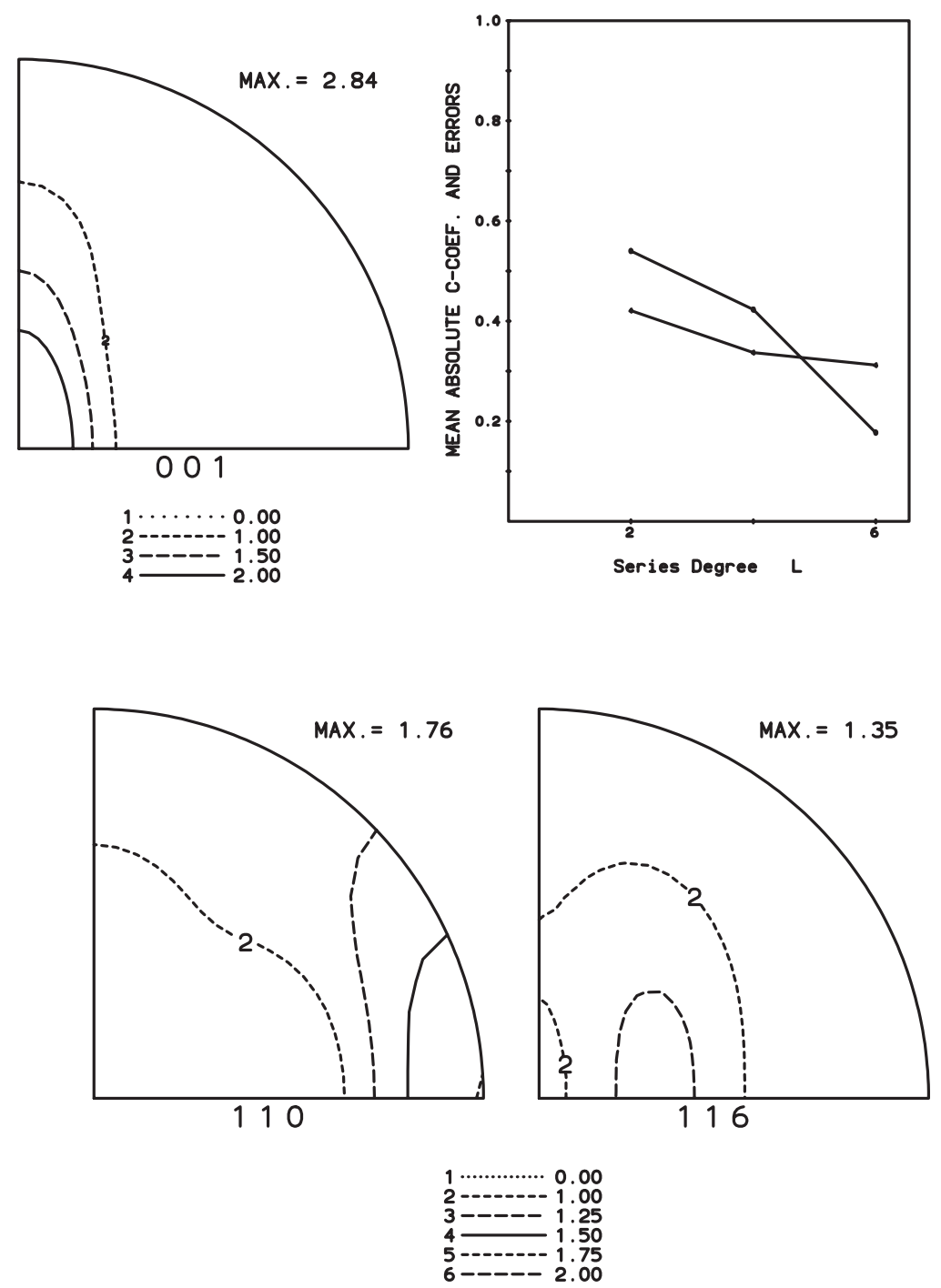

Abbildung D.40: Rückgerechnete Polfiguren, C-Koeffizienten und Fehler, CTC20 10Vol\% $5 \mathrm{ml} / \mathrm{s}$ 


\section{D.5 Probe CTC20 12,5 Vol\%}
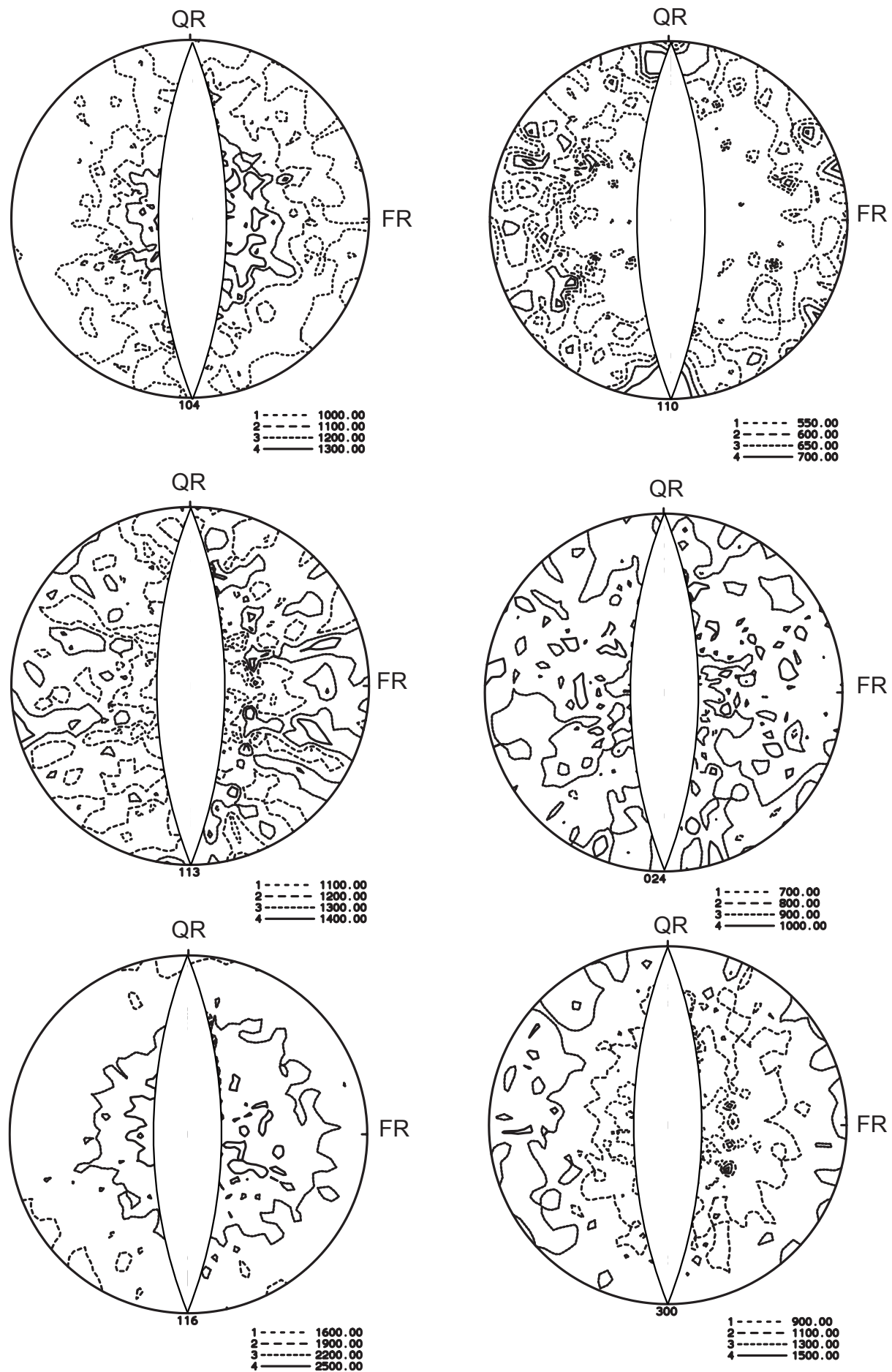

Abbildung D.41: Gemessene Polfiguren CTC20 12,5Vol\% 0,2 ml/s 

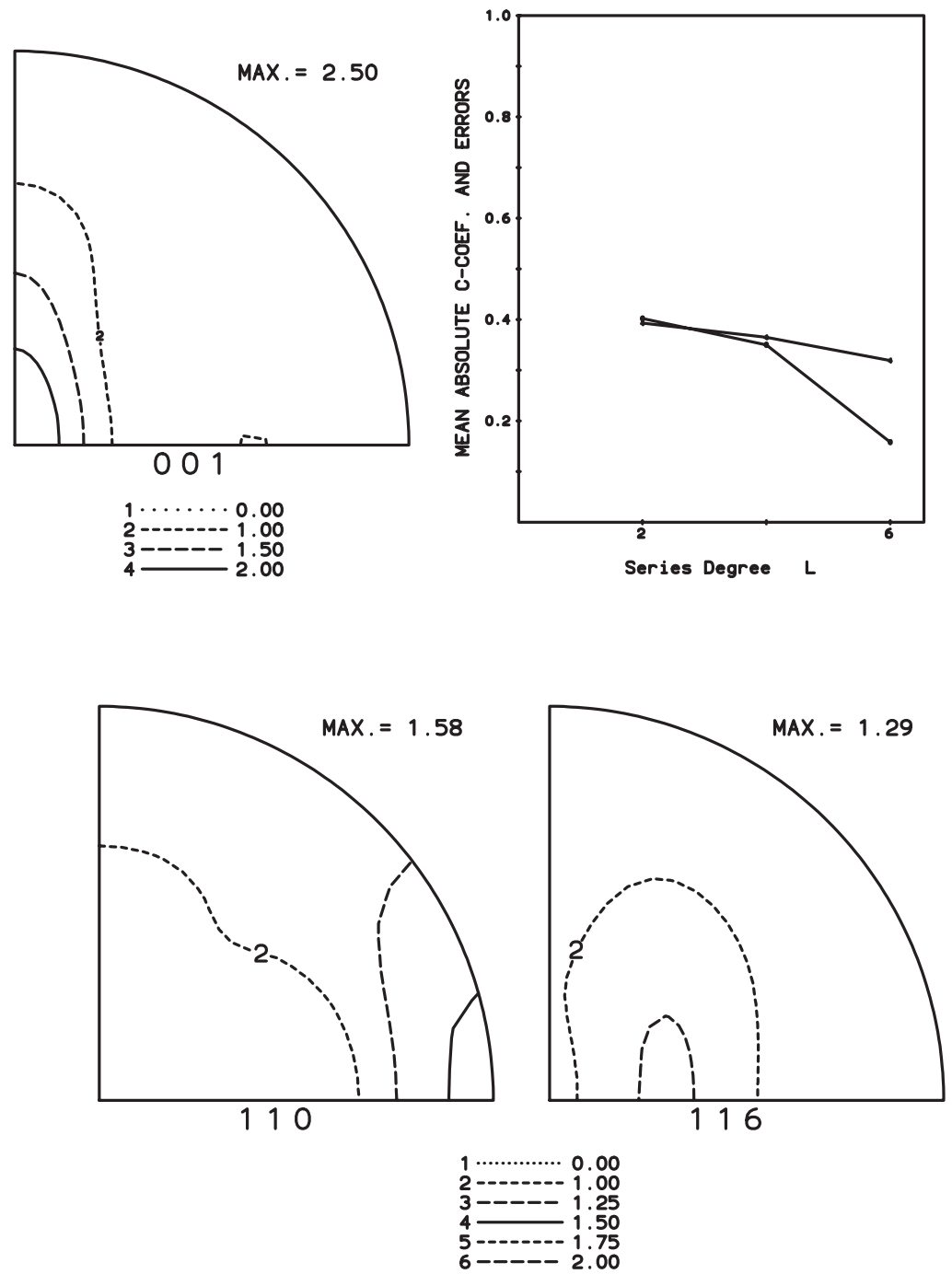

Abbildung D.42: Rückgerechnete Polfiguren, C-Koeffizienten und Fehler, CTC20 12,5Vol\% 0,2ml/s 

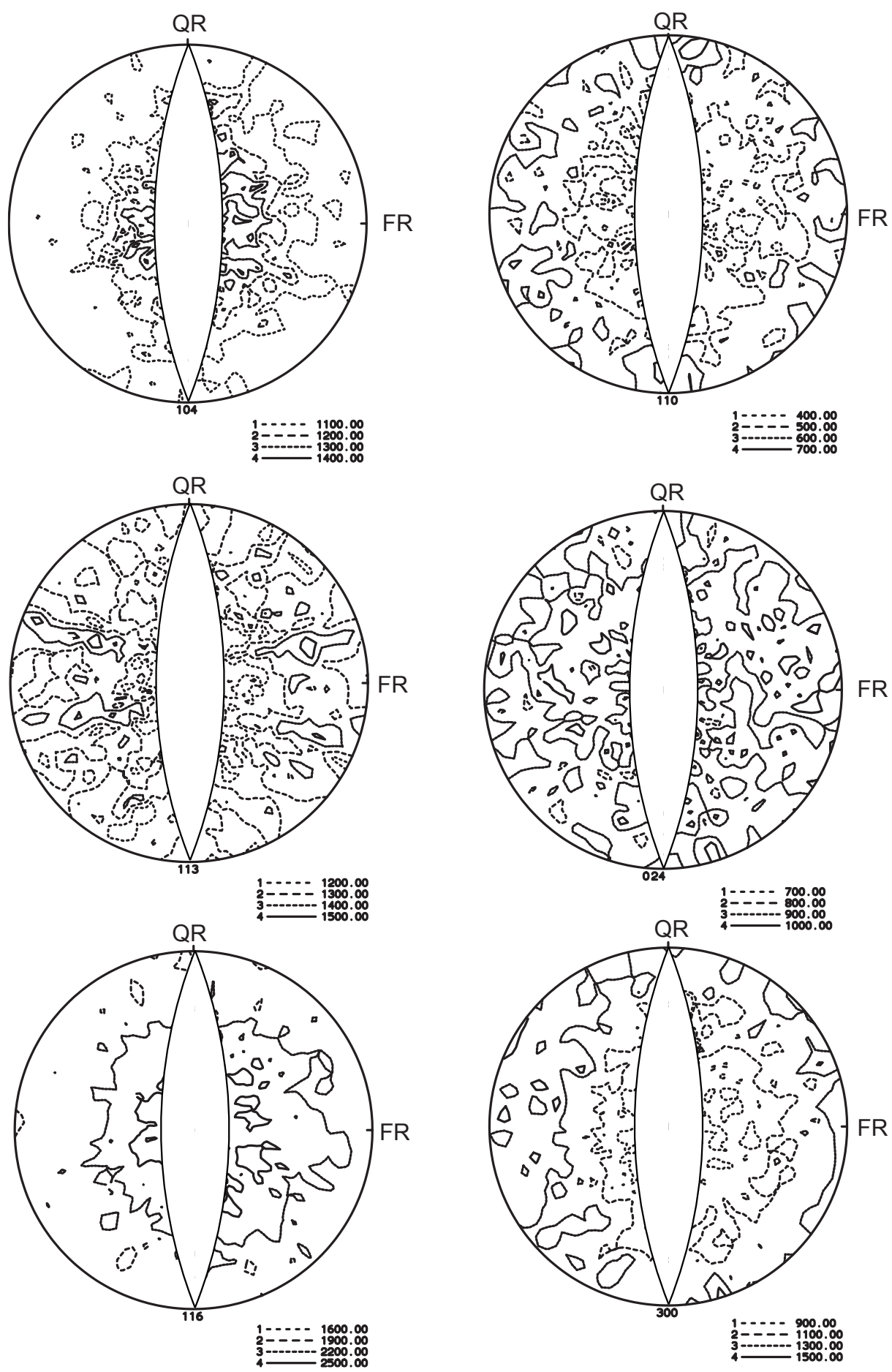

Abbildung D.43: Gemessene Polfiguren CTC20 12,5Vol\% 0,5 ml/s 

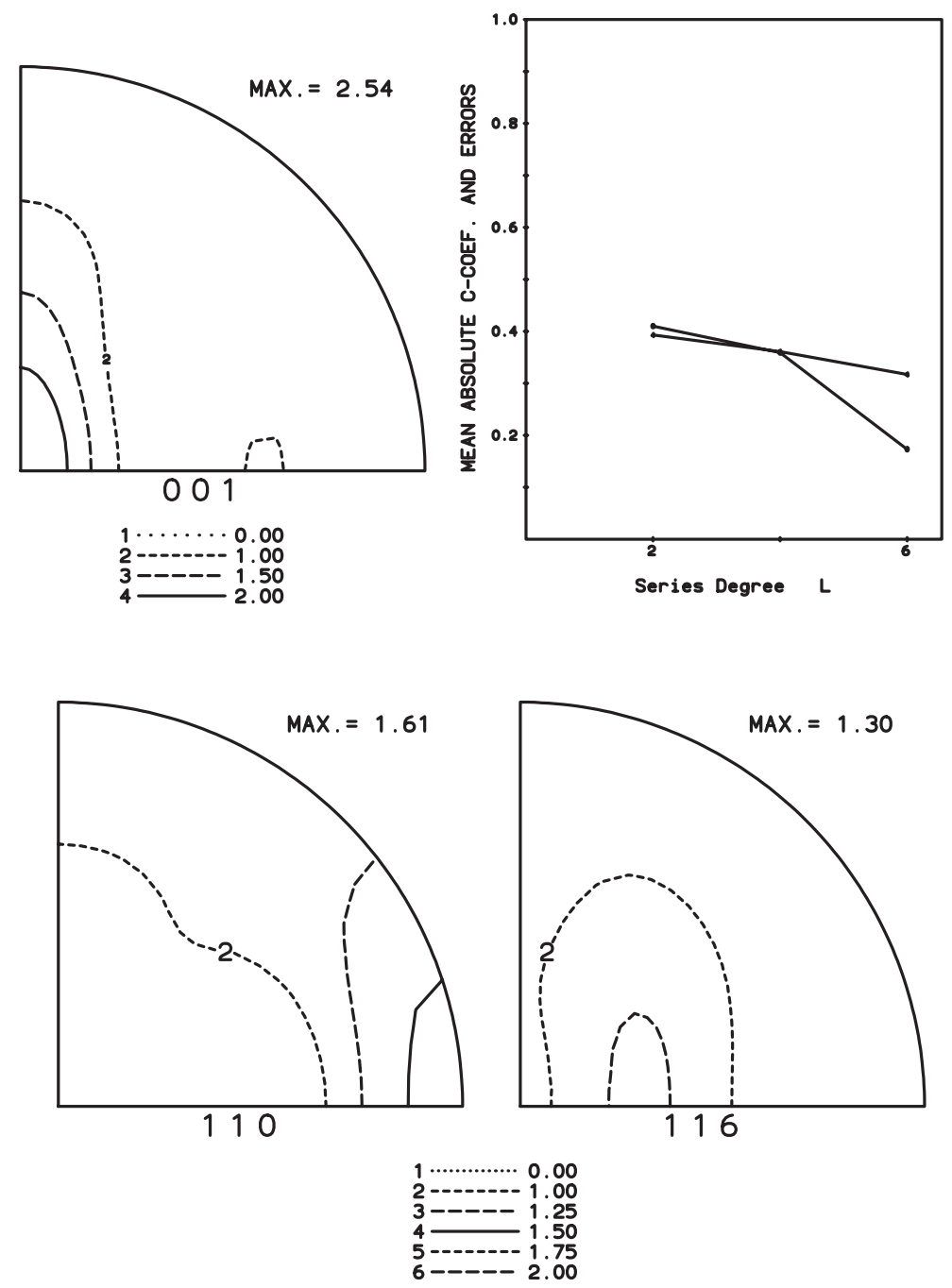

Abbildung D.44: Rückgerechnete Polfiguren, C-Koeffizienten und Fehler, CTC20 12,5Vol\% 0,5ml/s 

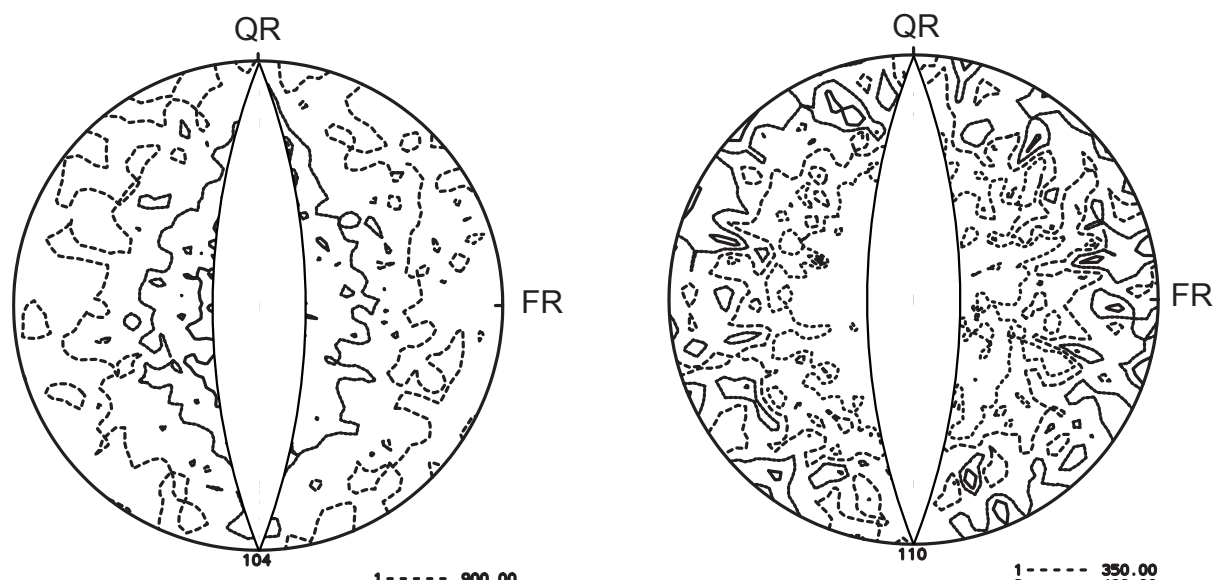

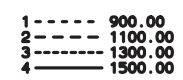
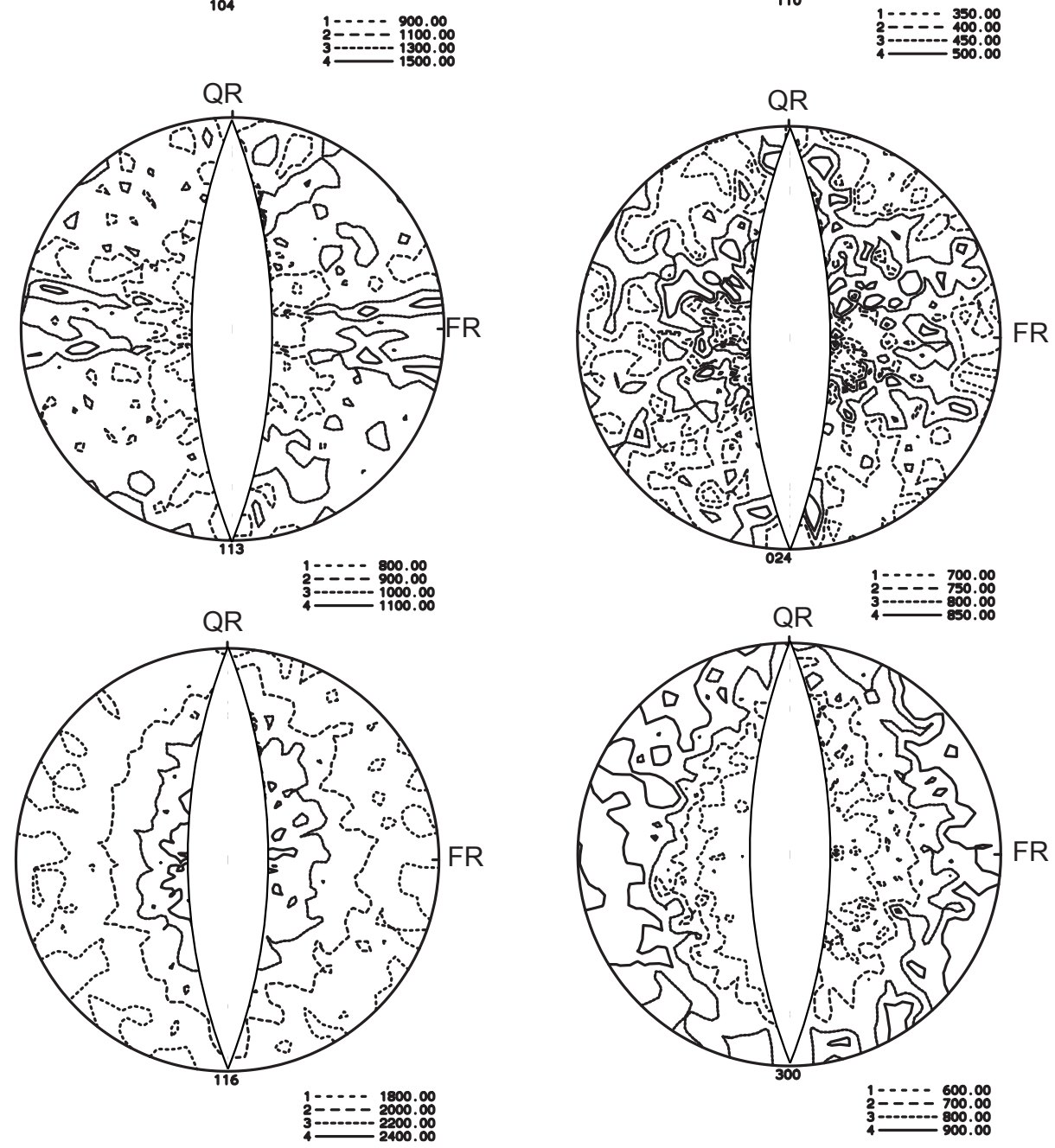

Abbildung D.45: Gemessene Polfiguren CTC20 12,5Vol\% $1 \mathrm{ml} / \mathrm{s}$ 

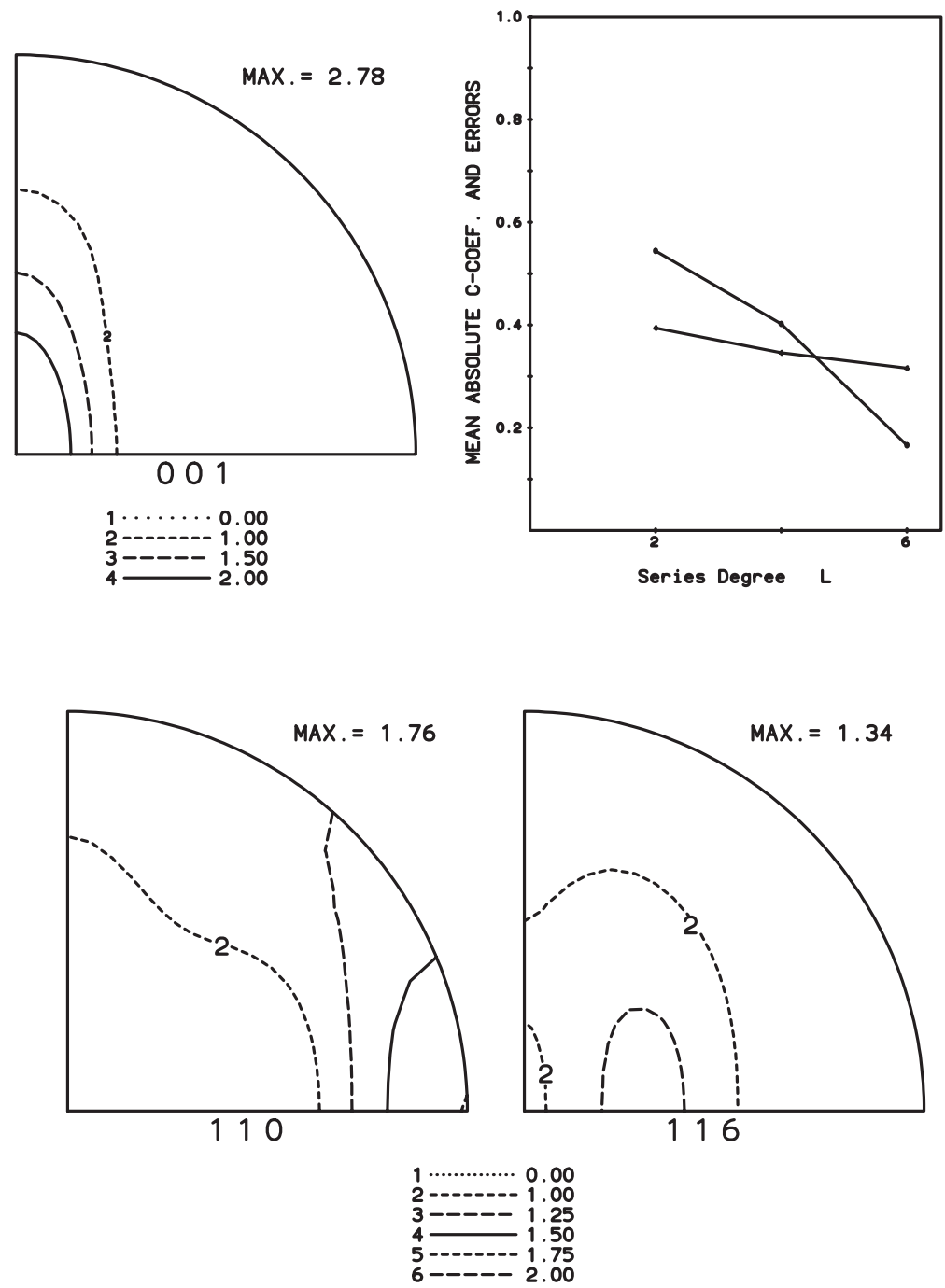

Abbildung D.46: Rückgerechnete Polfiguren, C-Koeffizienten und Fehler, CTC20 12,5Vol\% $1 \mathrm{ml} / \mathrm{s}$ 

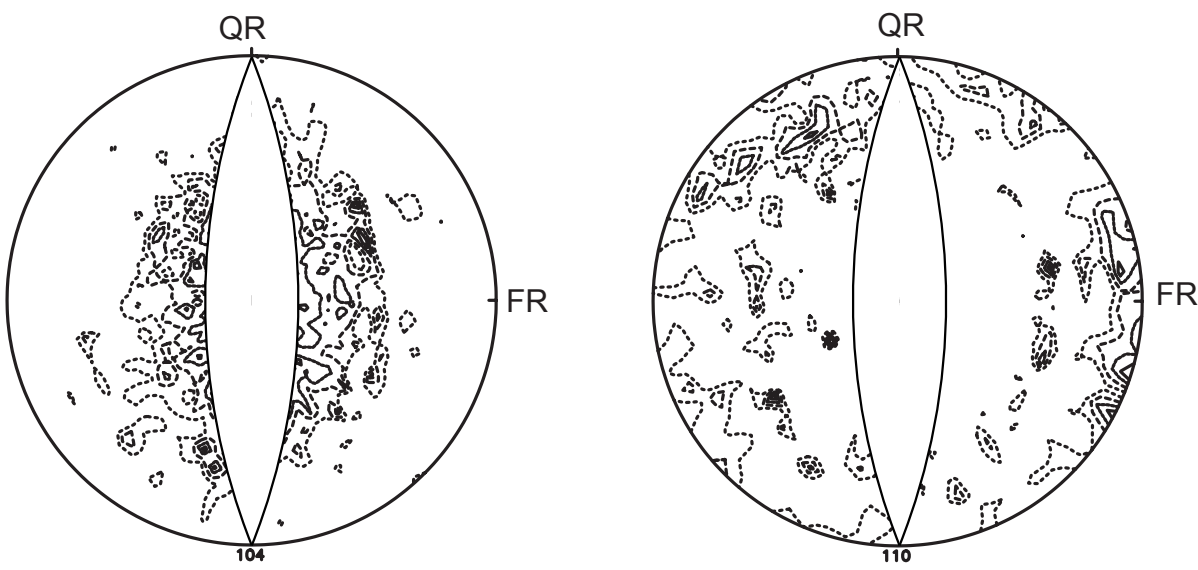

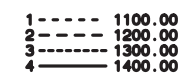
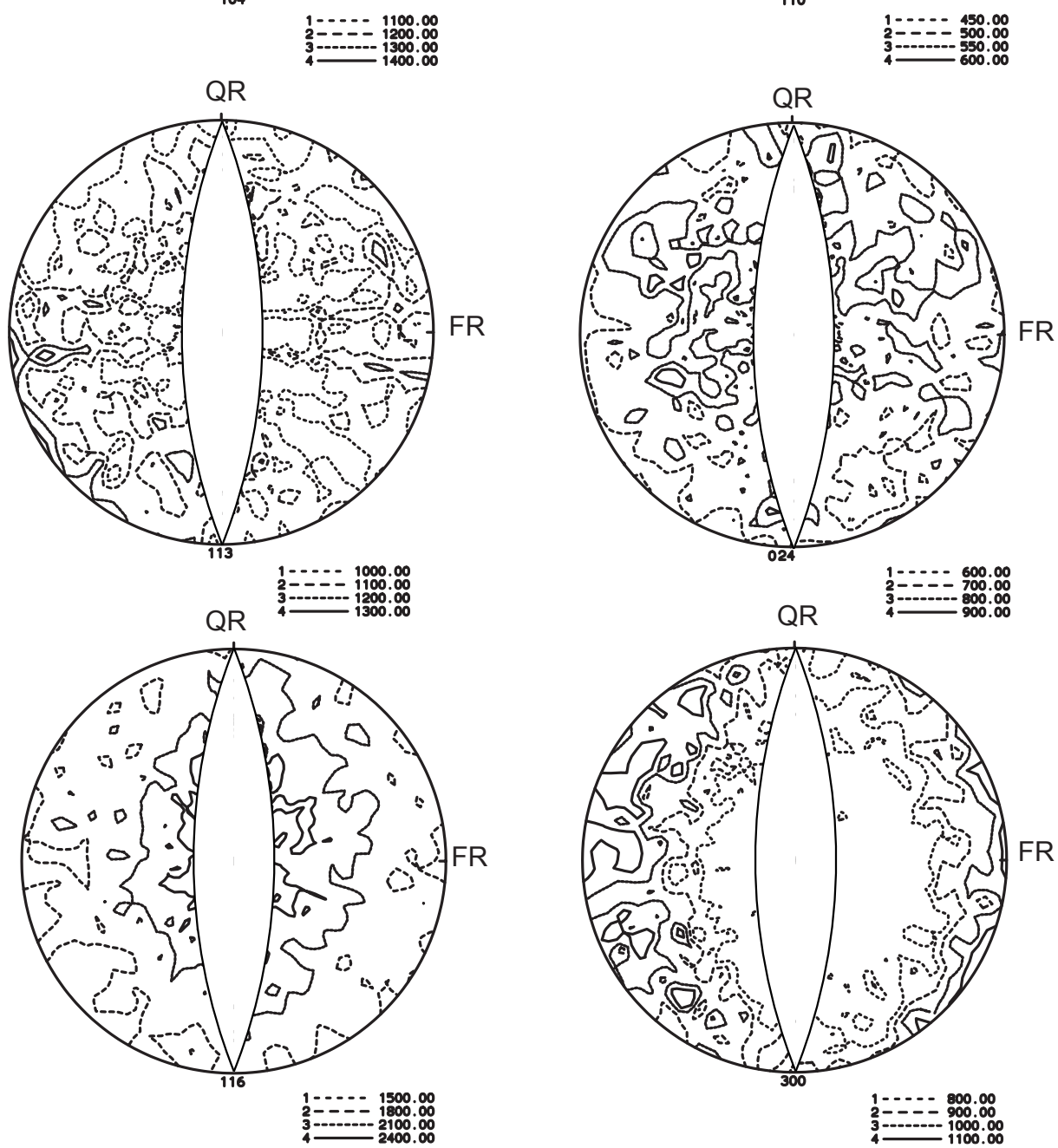

Abbildung D.47: Gemessene Polfiguren CTC20 12,5Vol\% $2 \mathrm{ml} / \mathrm{s}$ 

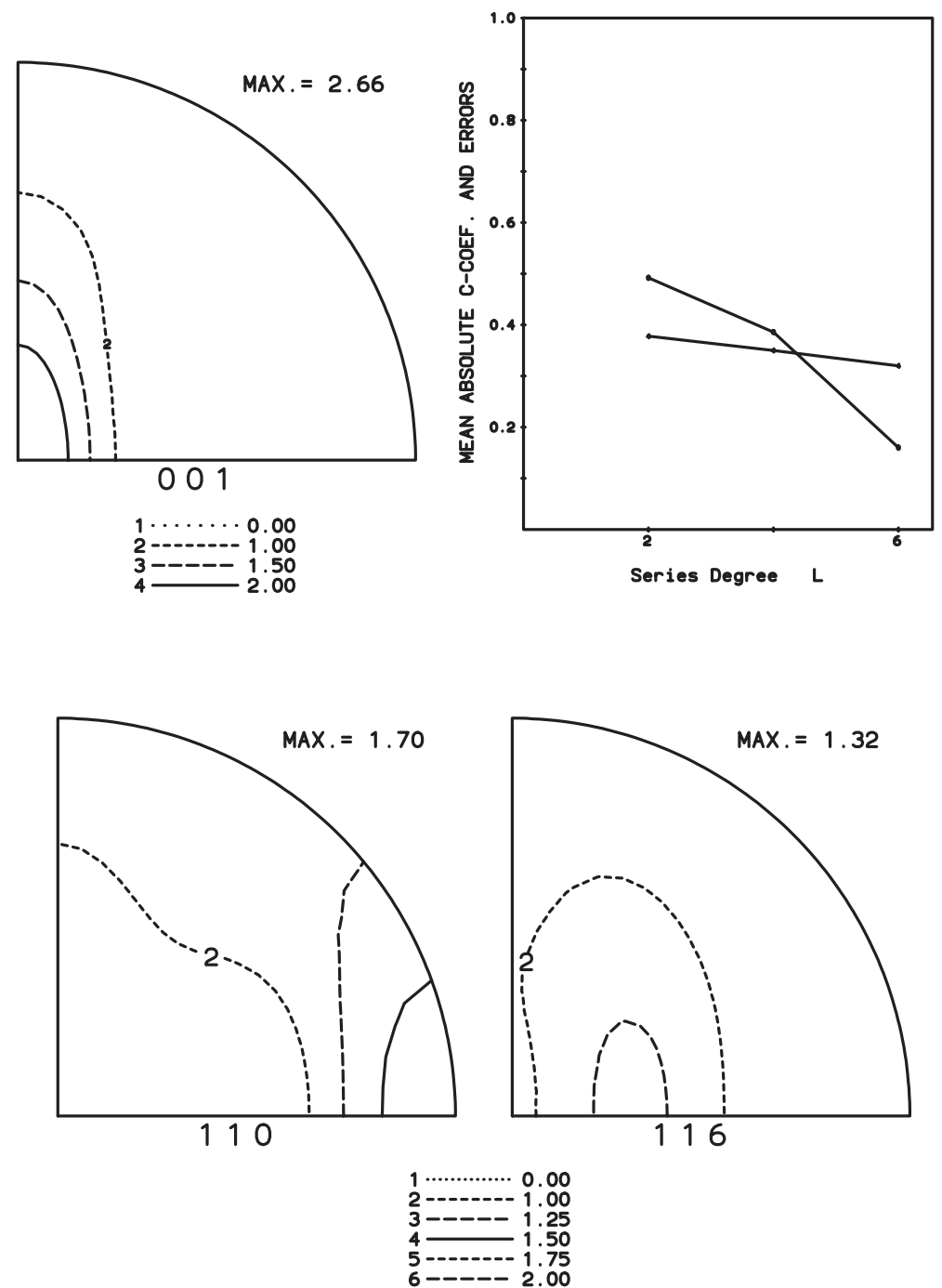

Abbildung D.48: Rückgerechnete Polfiguren, C-Koeffizienten und Fehler, CTC20 12,5Vol\% 2ml/s 

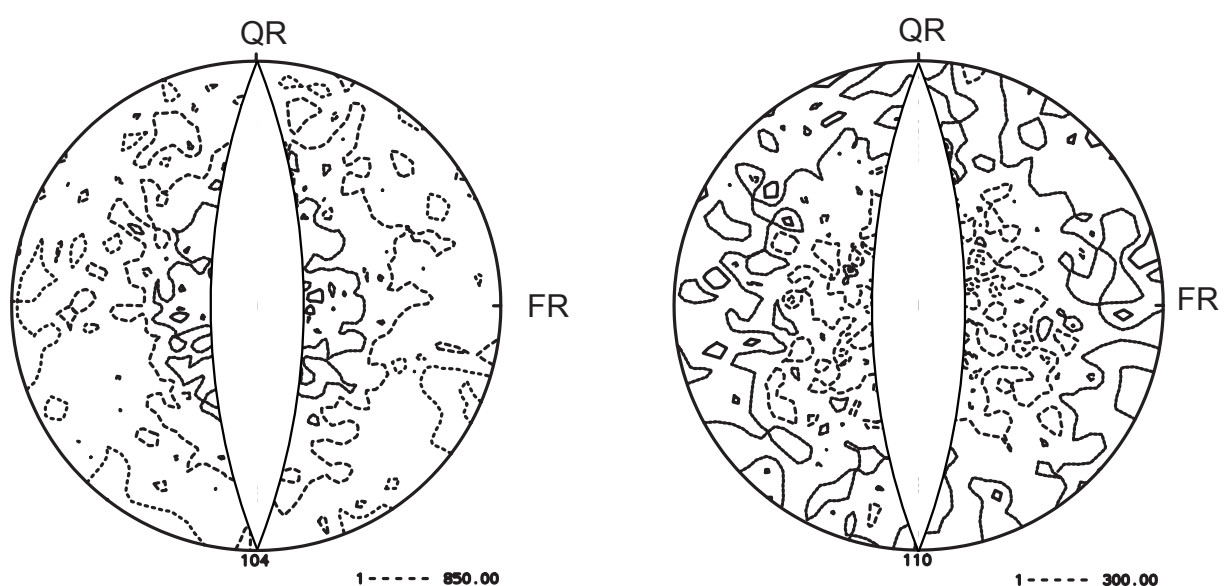

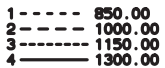
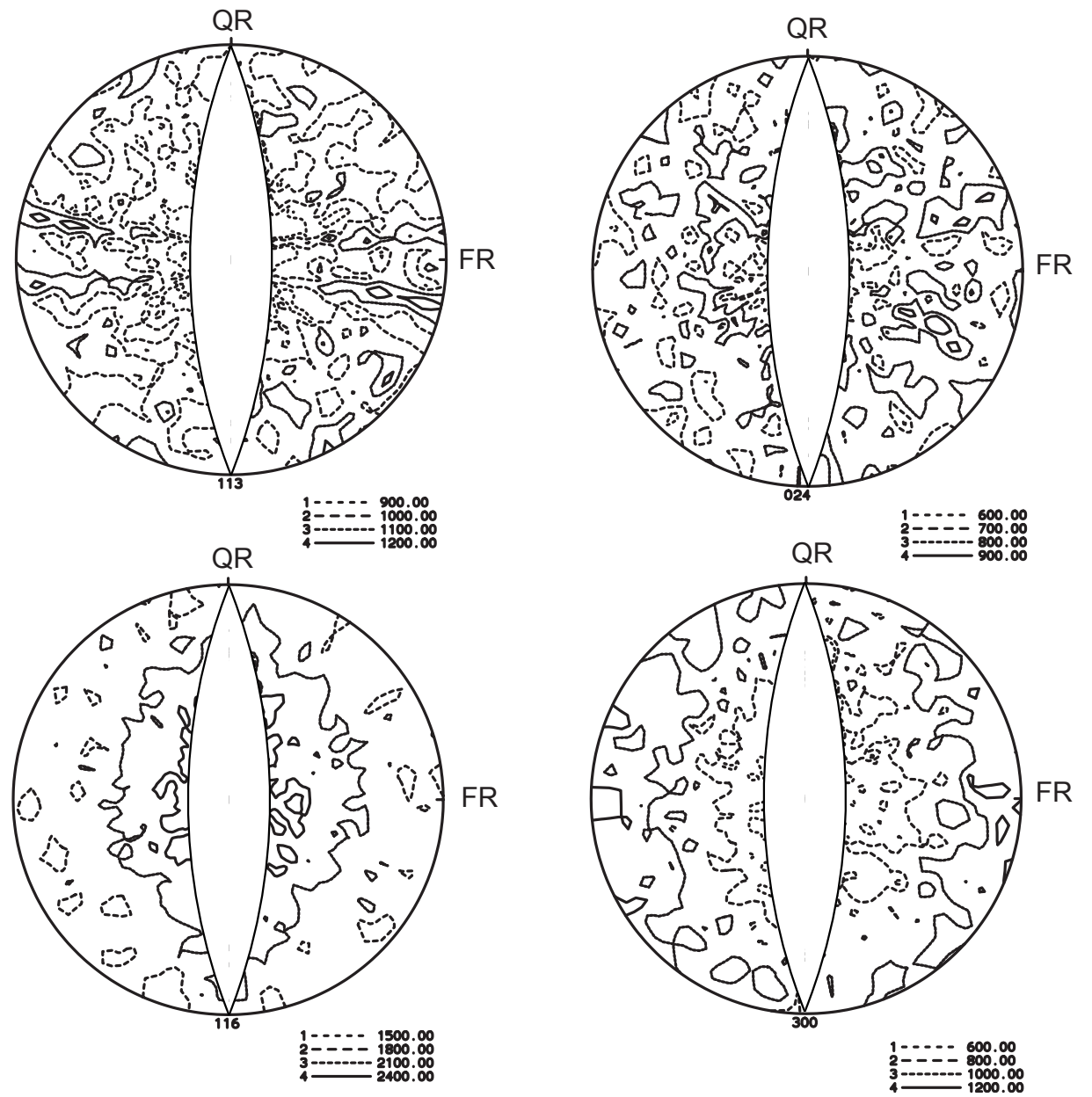

Abbildung D.49: Gemessene Polfiguren CTC20 12,5Vol\% $5 \mathrm{ml} / \mathrm{s}$ 

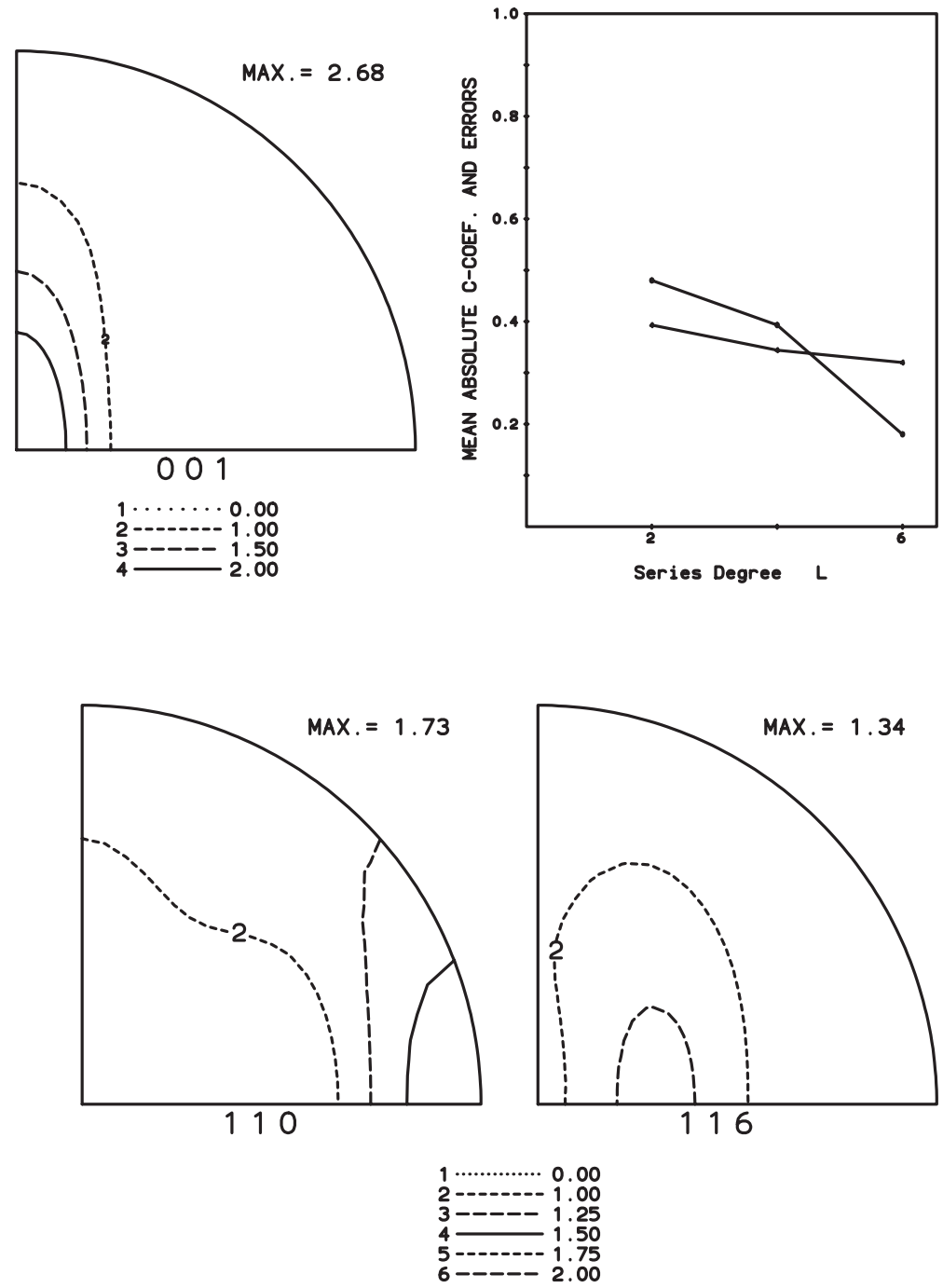

Abbildung D.50: Rückgerechnete Polfiguren, C-Koeffizienten und Fehler, CTC20 12,5Vol\% 5ml/s 


\section{D.6 Probe Gilox 7,5 Vol\%}
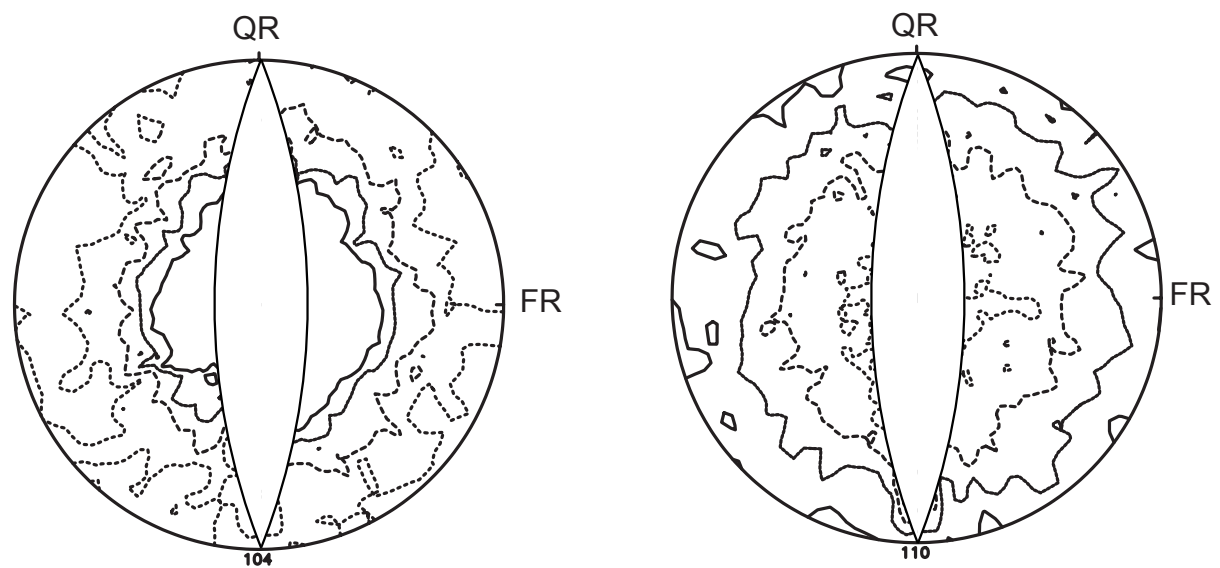

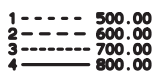
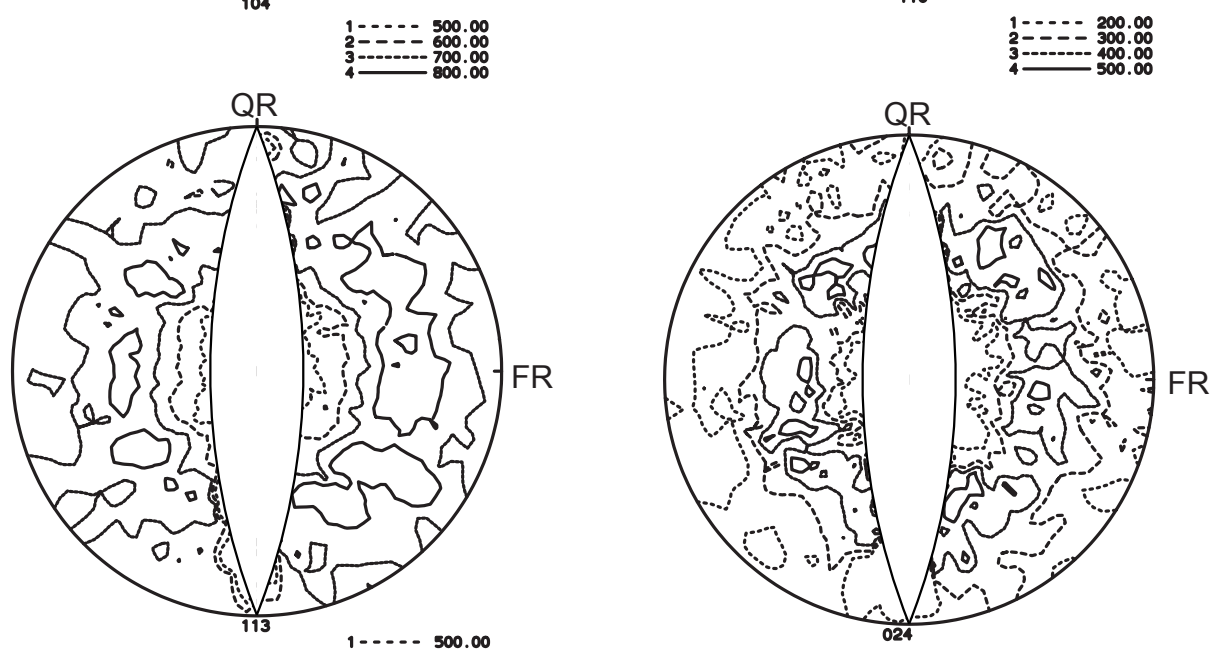

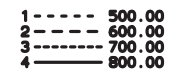

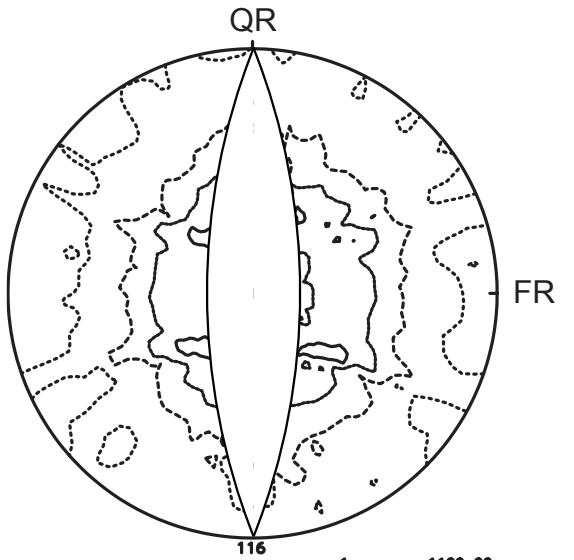

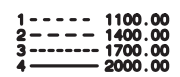

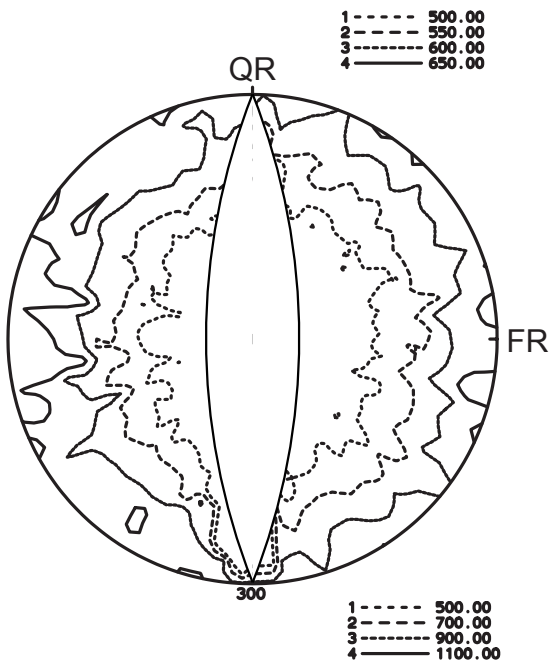

Abbildung D.51: Gemessene Polfiguren Gilox 7,5Vol\% 0,2 ml/s 

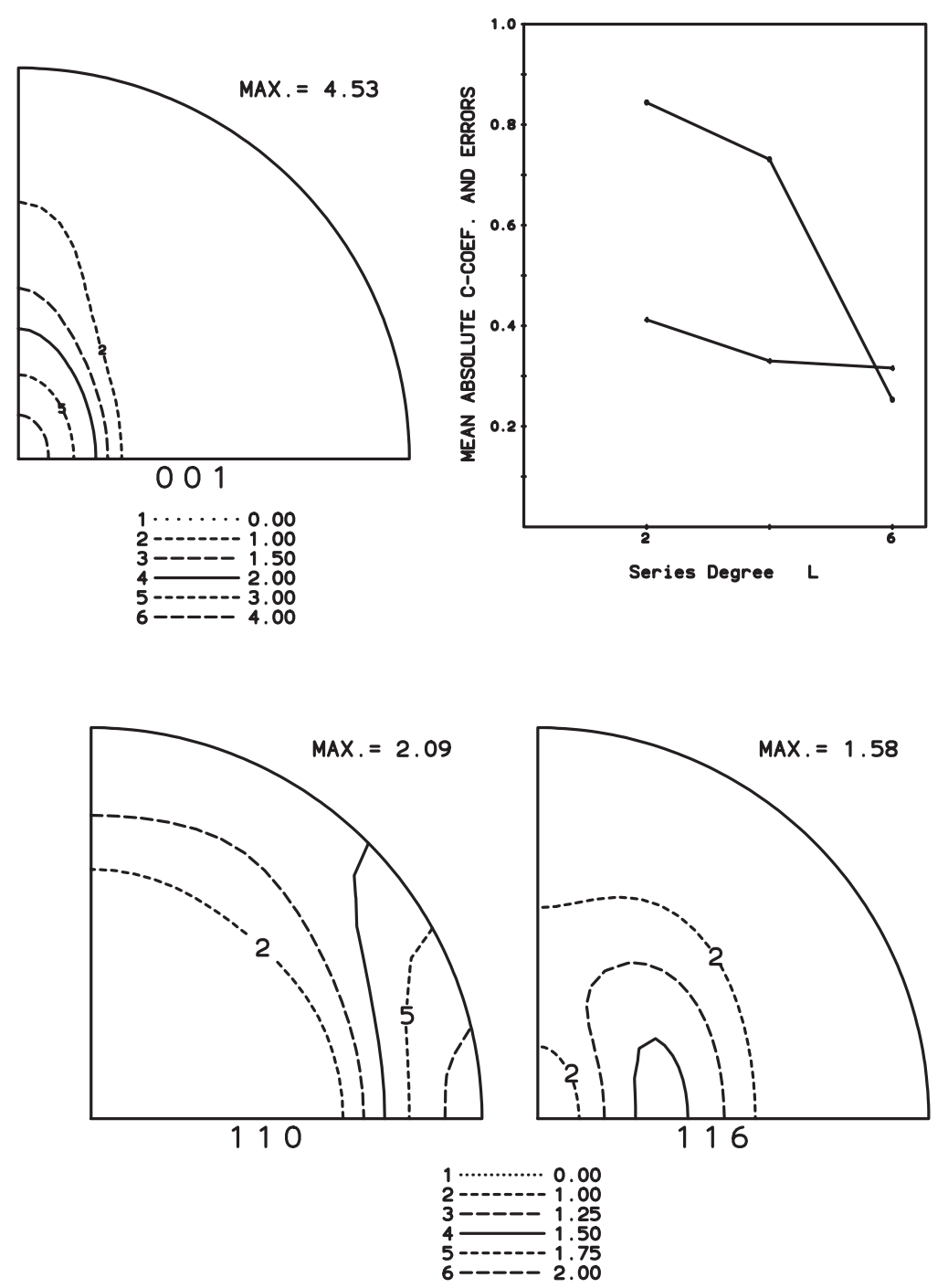

Abbildung D.52: Rückgerechnete Polfiguren, C-Koeffizienten und Fehler, Gilox $7,5 \mathrm{Vol} \% 0,2 \mathrm{ml} / \mathrm{s}$ 

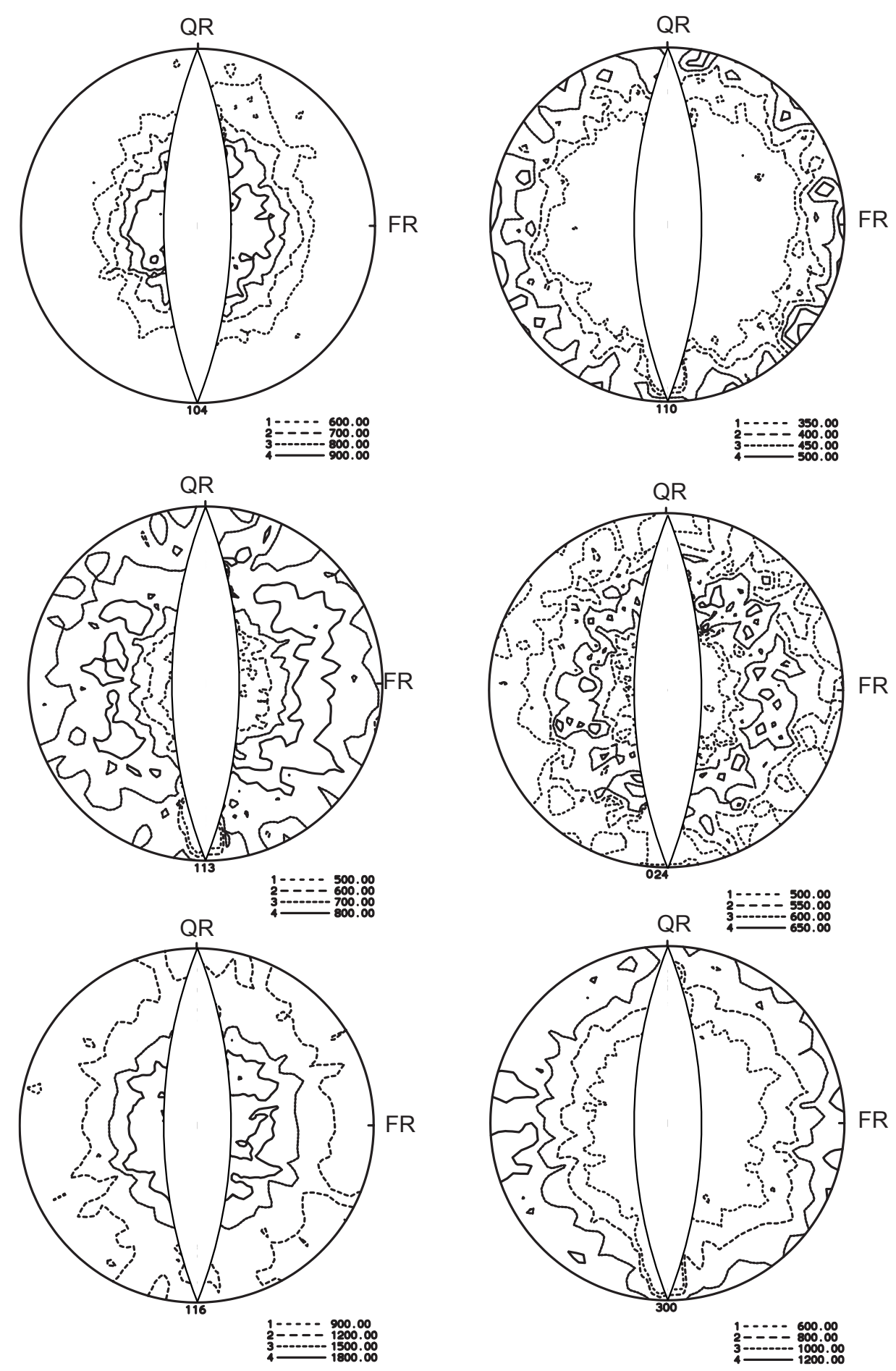

Abbildung D.53: Gemessene Polfiguren Gilox 7,5Vol\% 0,5 ml/s 

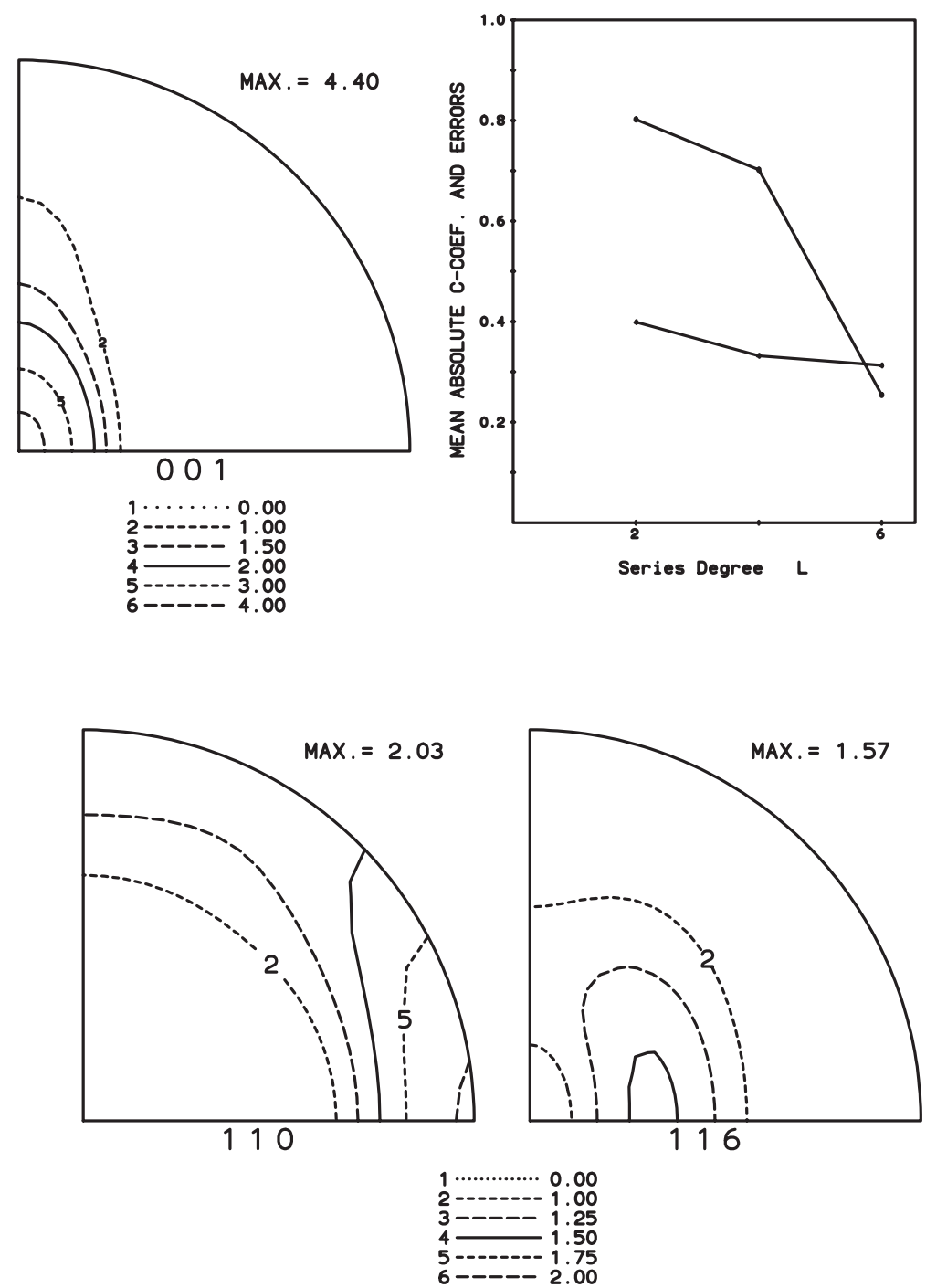

Abbildung D.54: Rückgerechnete Polfiguren, C-Koeffizienten und Fehler, Gilox $7,5 \mathrm{Vol} \% 0,5 \mathrm{ml} / \mathrm{s}$ 

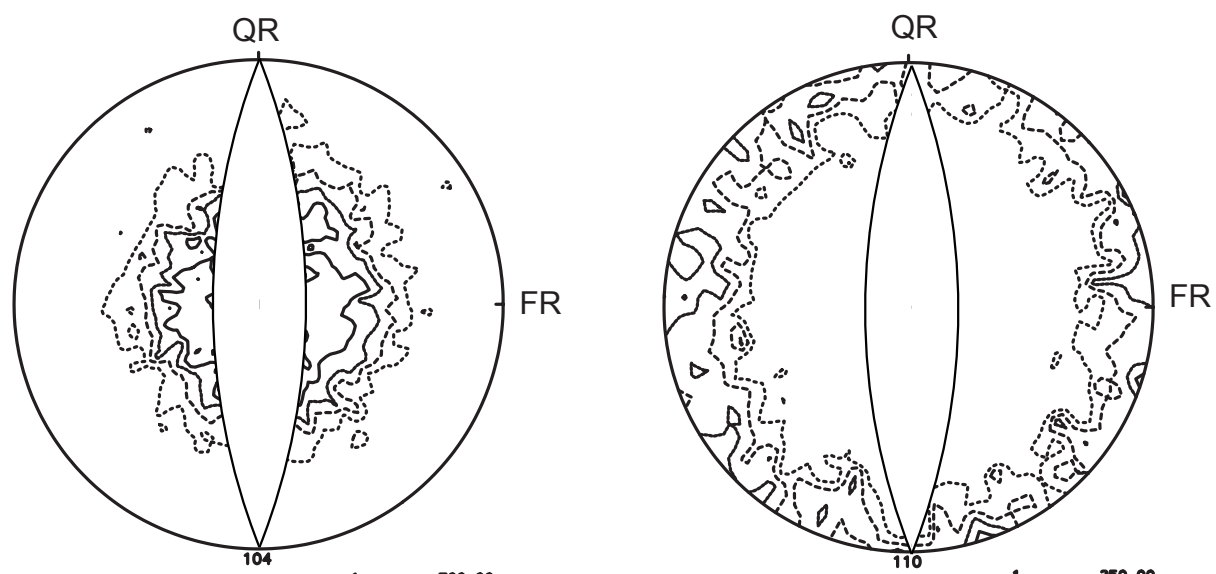

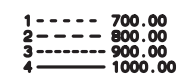
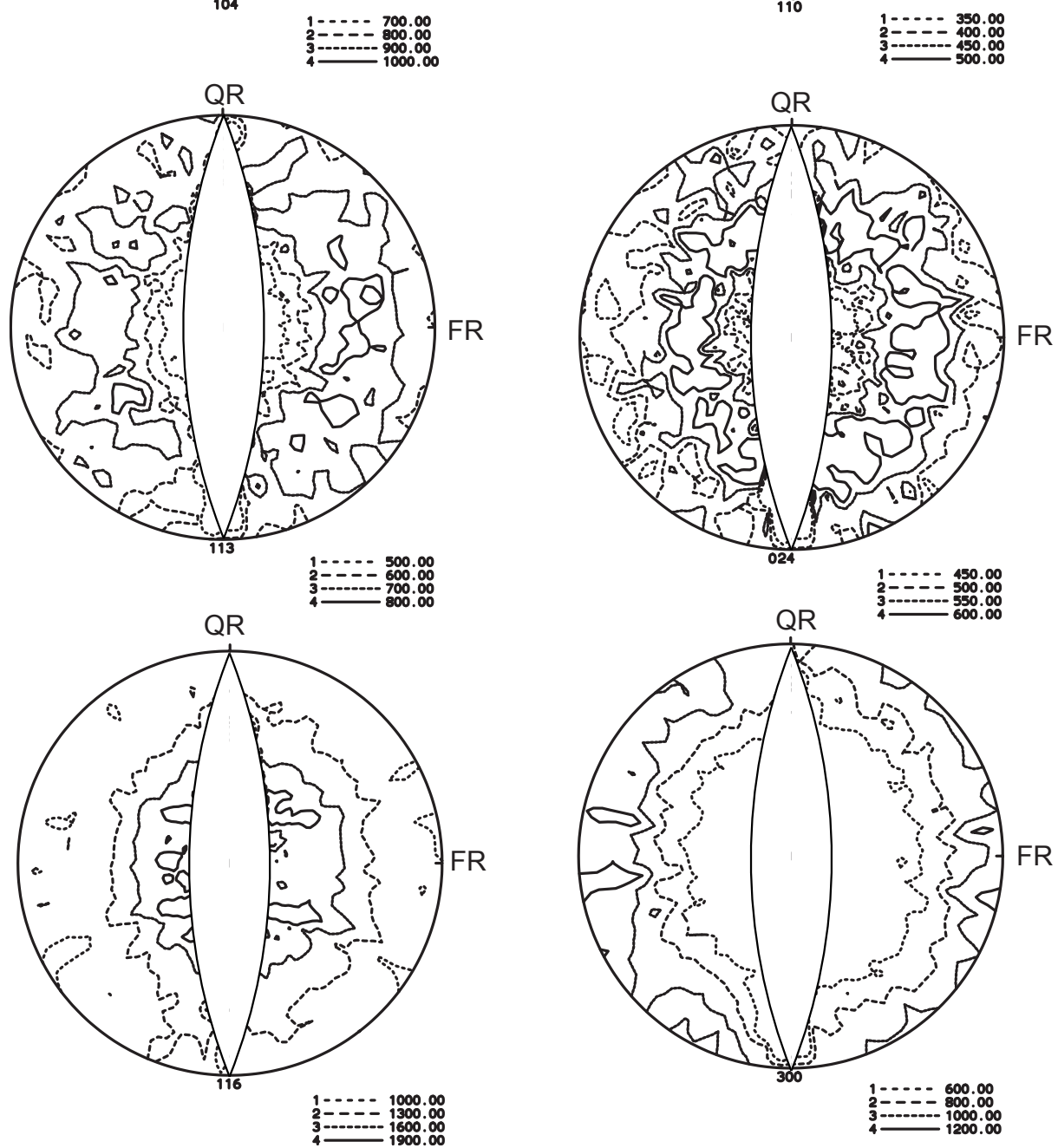

Abbildung D.55: Gemessene Polfiguren Gilox 7,5Vol\% $1 \mathrm{ml} / \mathrm{s}$ 

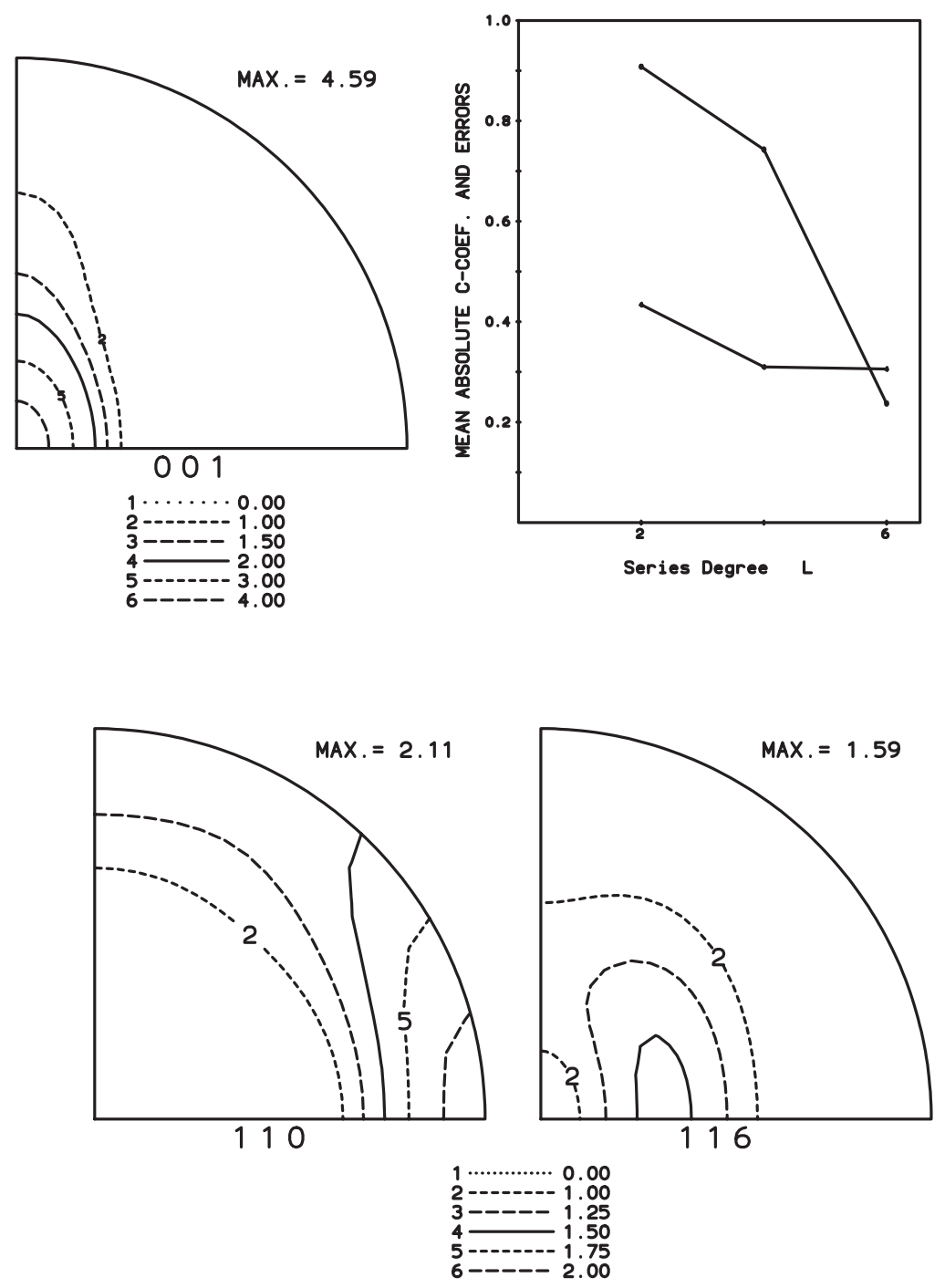

Abbildung D.56: Rückgerechnete Polfiguren, C-Koeffizienten und Fehler, Gilox $7,5 \mathrm{Vol} \% 1 \mathrm{ml} / \mathrm{s}$ 

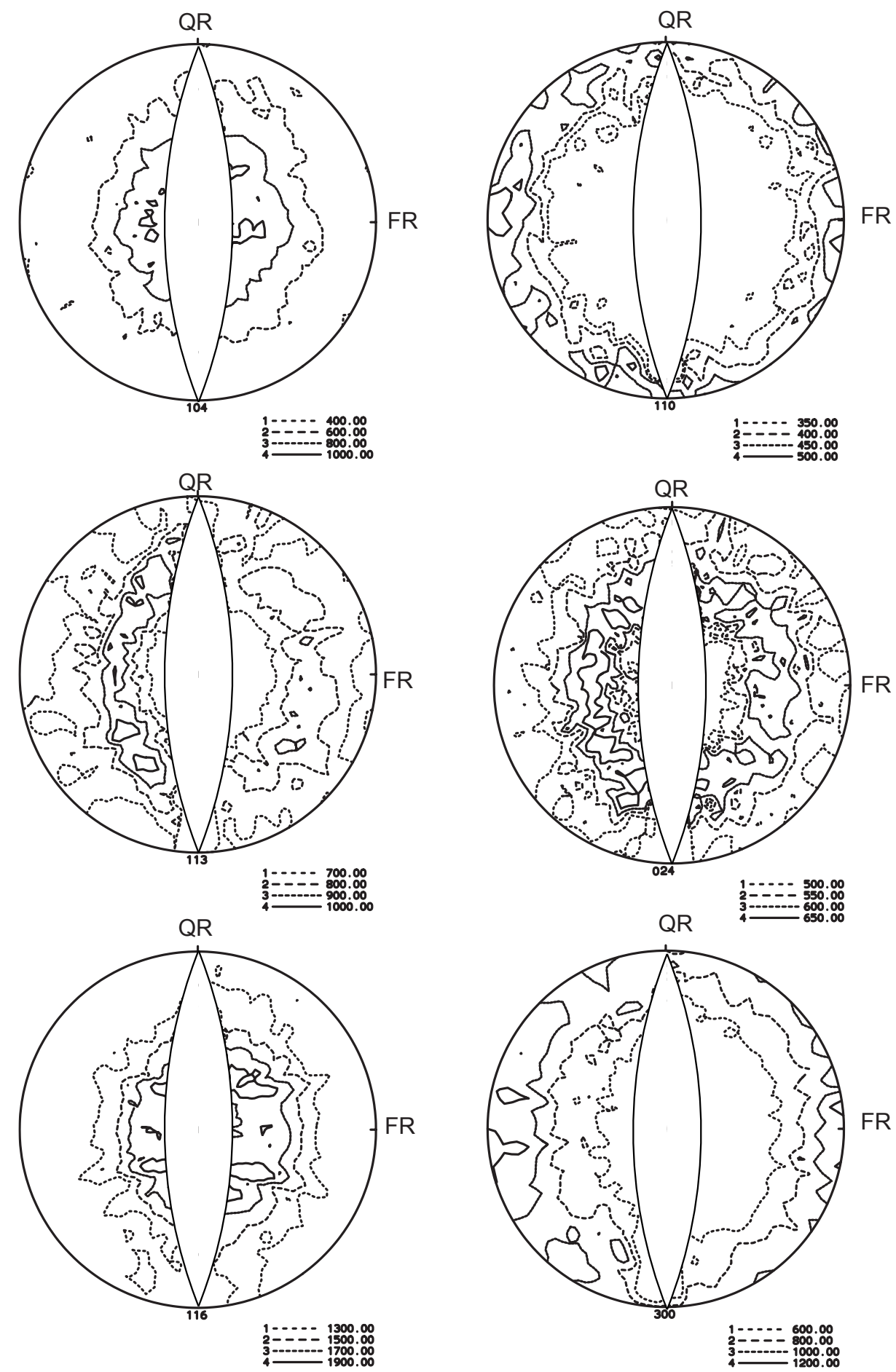

Abbildung D.57: Gemessene Polfiguren Gilox 7,5Vol\% $2 \mathrm{ml} / \mathrm{s}$ 

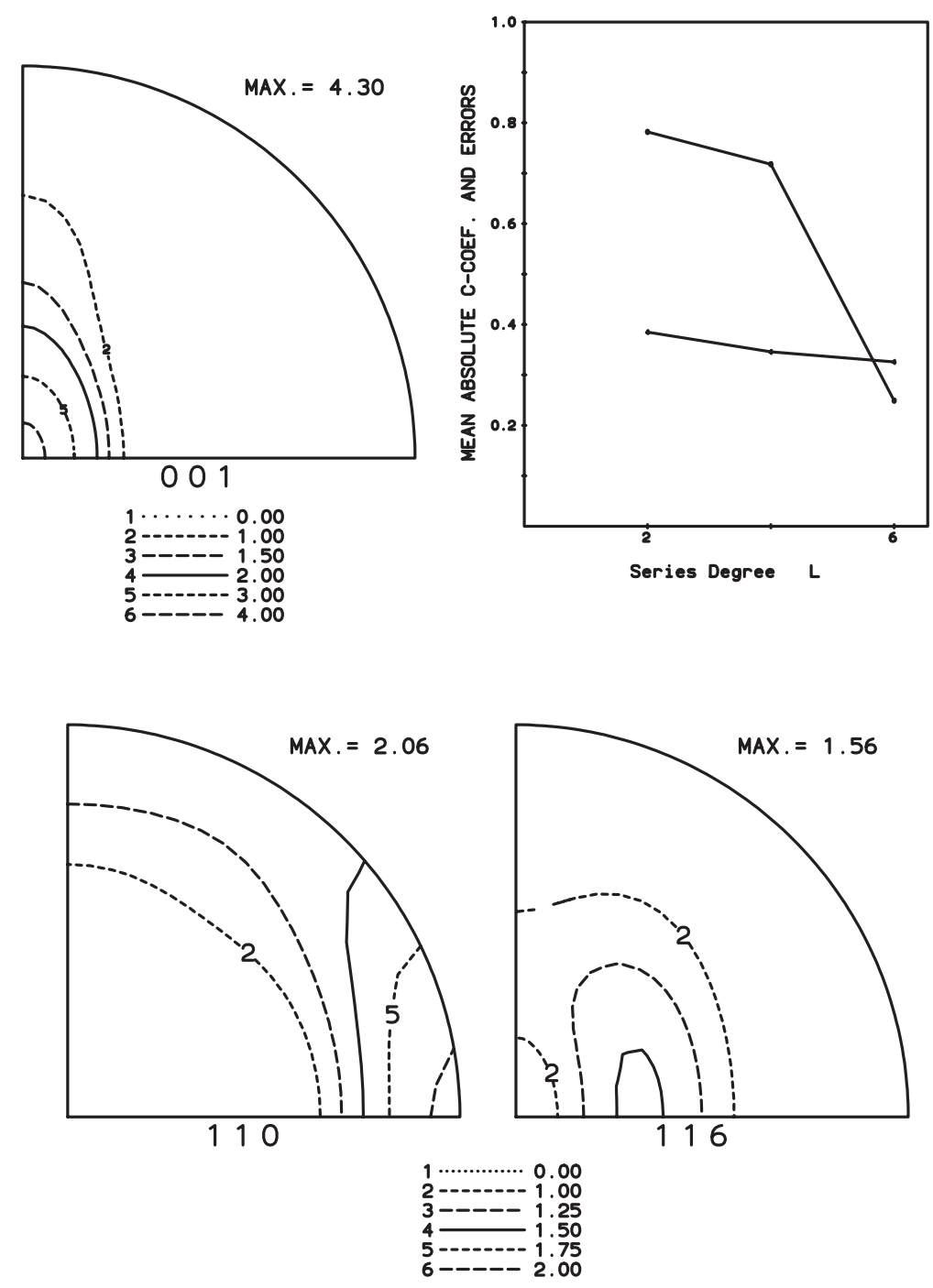

Abbildung D.58: Rückgerechnete Polfiguren, C-Koeffizienten und Fehler, Gilox $7,5 \mathrm{Vol} \% 2 \mathrm{ml} / \mathrm{s}$ 

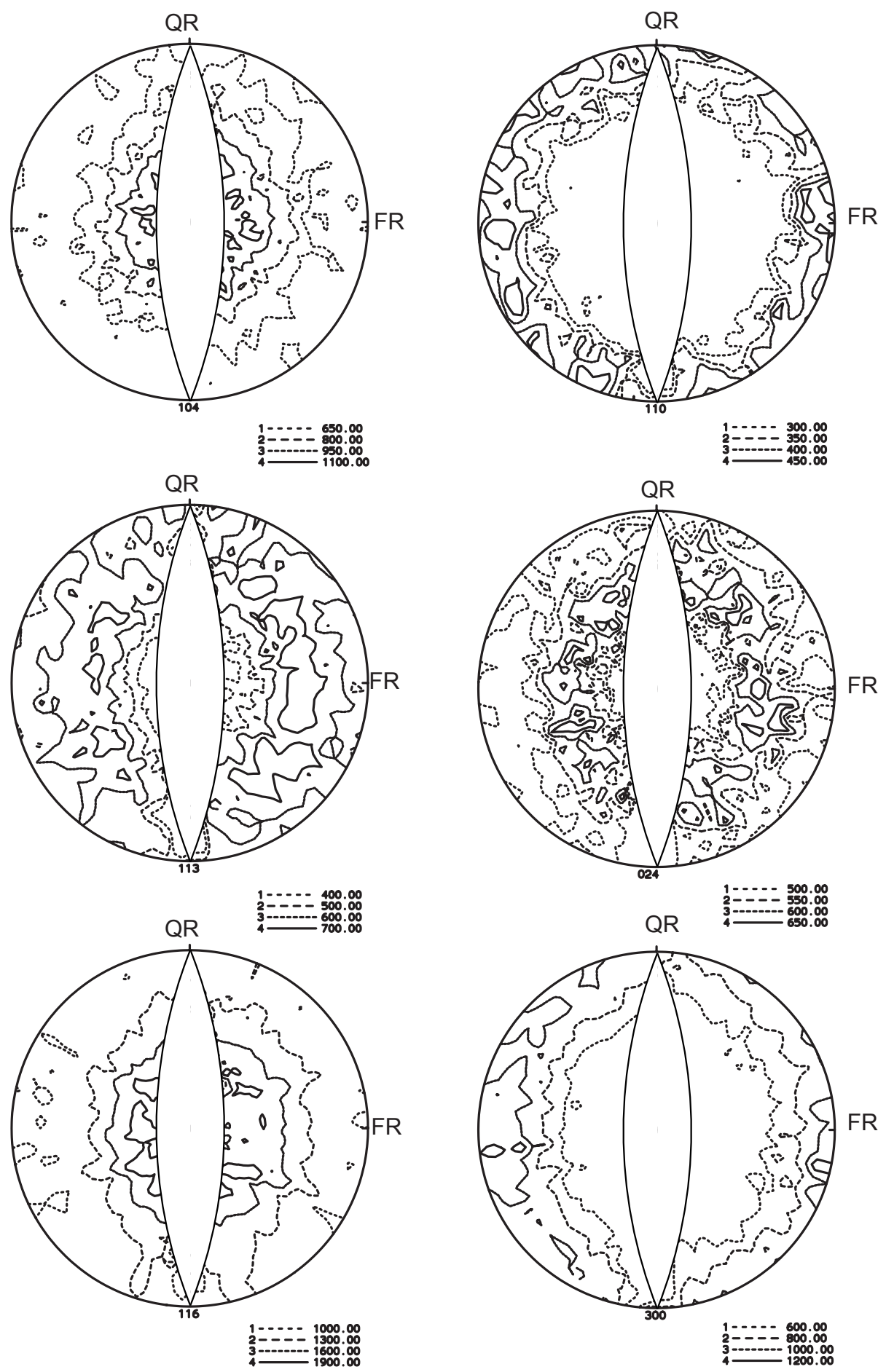

Abbildung D.59: Gemessene Polfiguren Gilox 7,5Vol\% $5 \mathrm{ml} / \mathrm{s}$ 

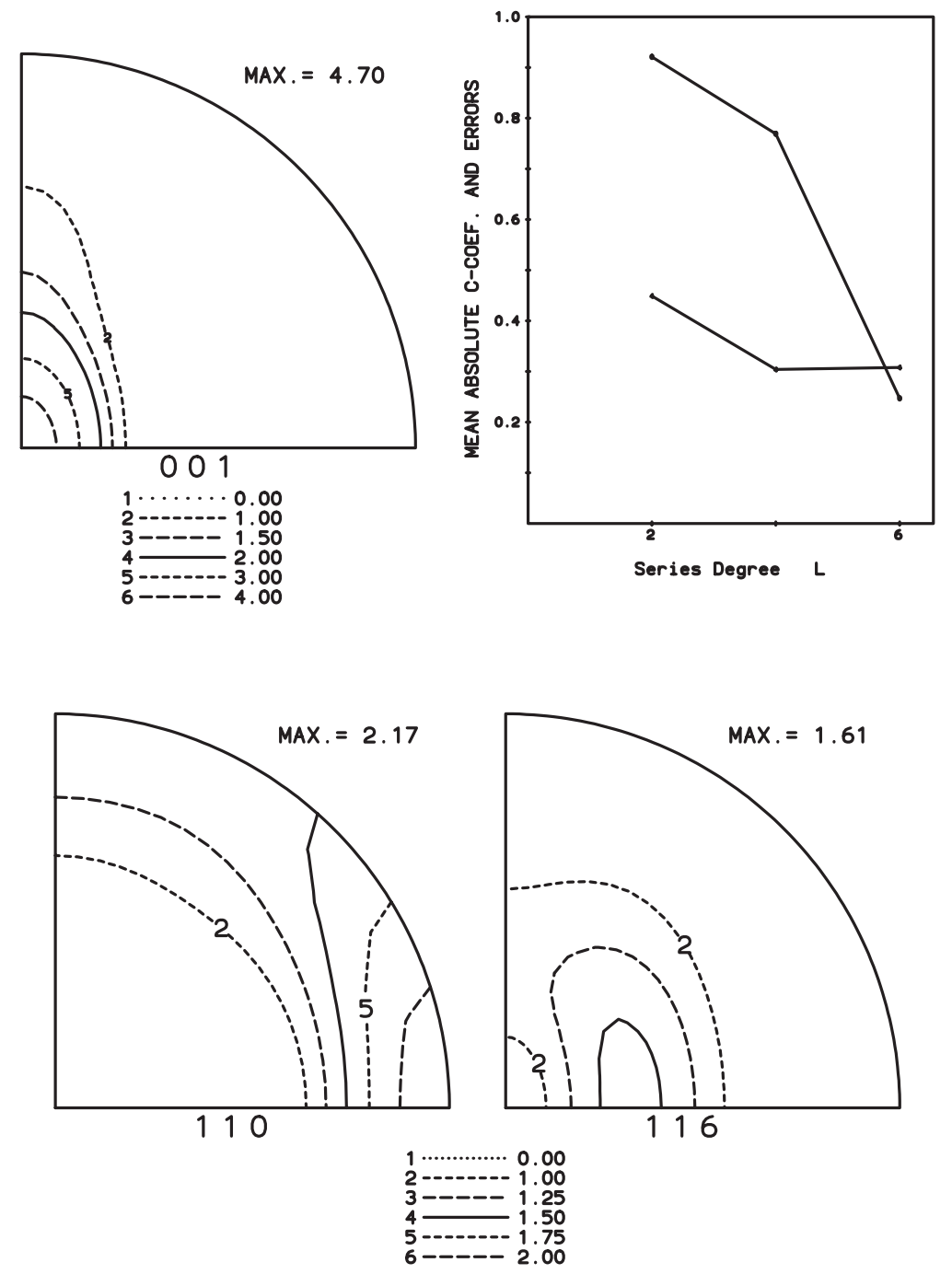

Abbildung D.60: Rückgerechnete Polfiguren, C-Koeffizienten und Fehler, Gilox $7.5 \mathrm{Vol} \% 5 \mathrm{ml} / \mathrm{s}$ 


\section{D.7 Probe Gilox 10Vol\%}
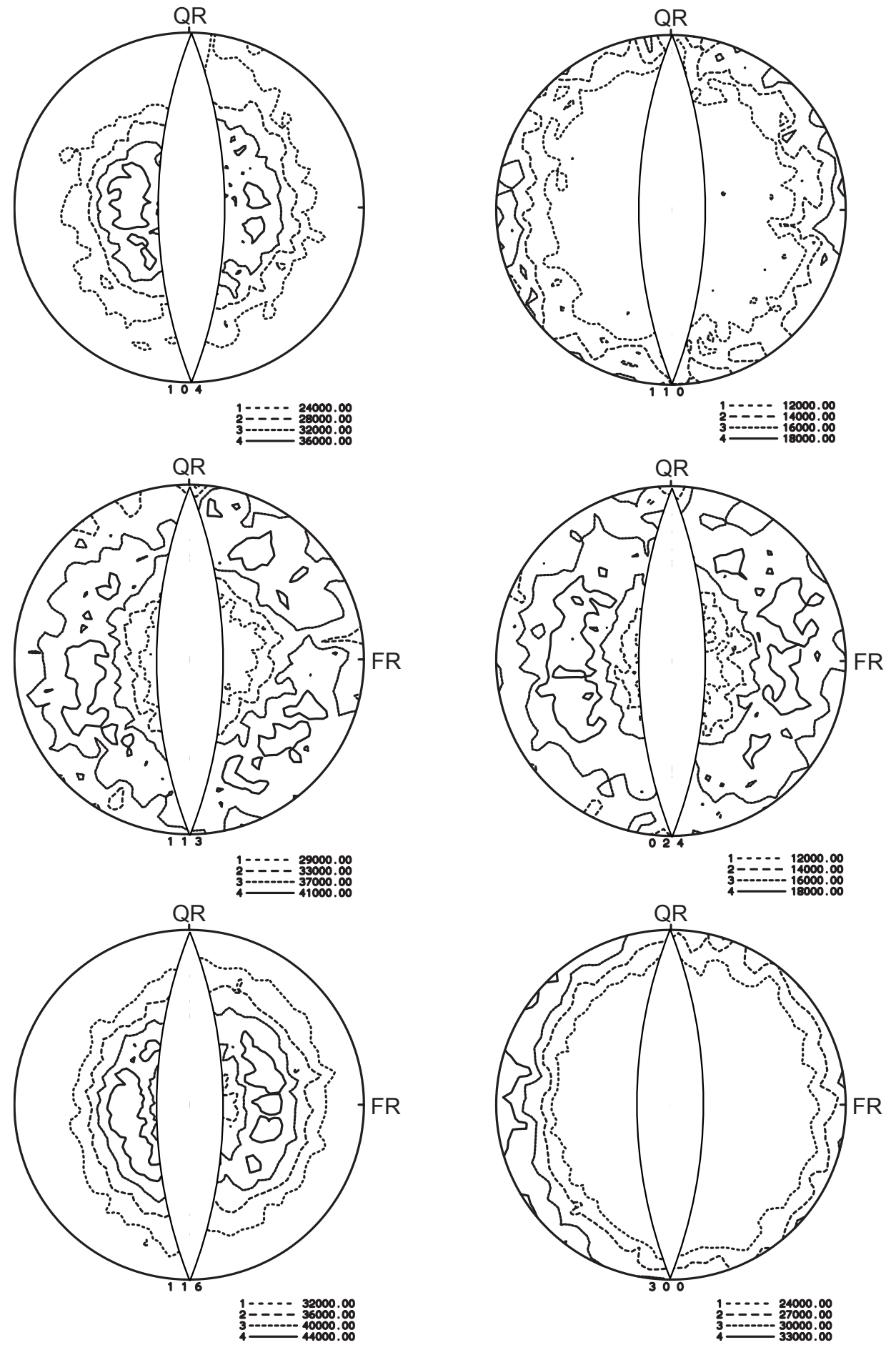

Abbildung D.61: Gemessene Polfiguren Gilox 10Vol\% 0,2 ml/s 

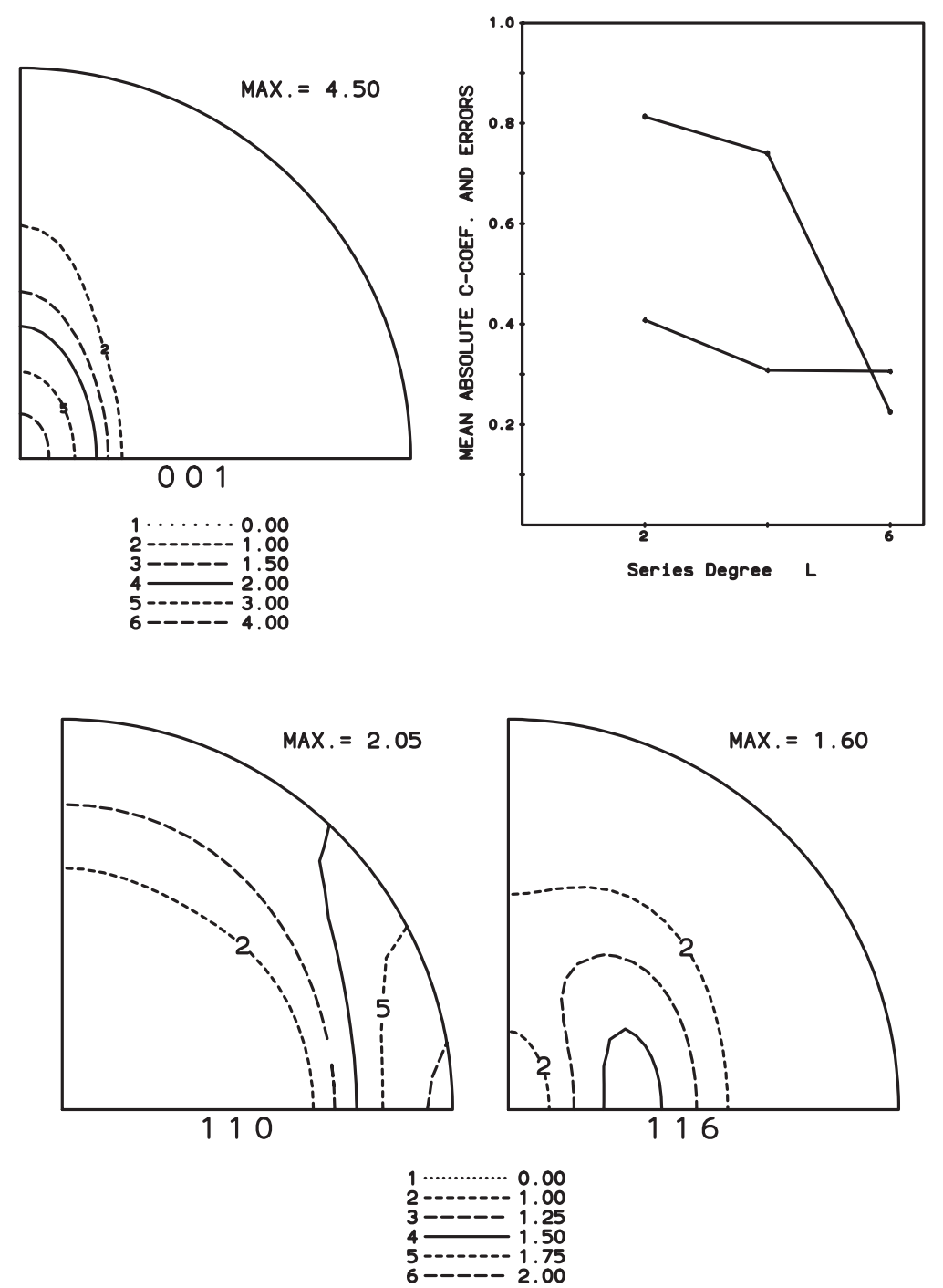

Abbildung D.62: Rückgerechnete Polfiguren, C-Koeffizienten und Fehler, Gilox $10 \mathrm{Vol} \% 0,2 \mathrm{ml} / \mathrm{s}$ 

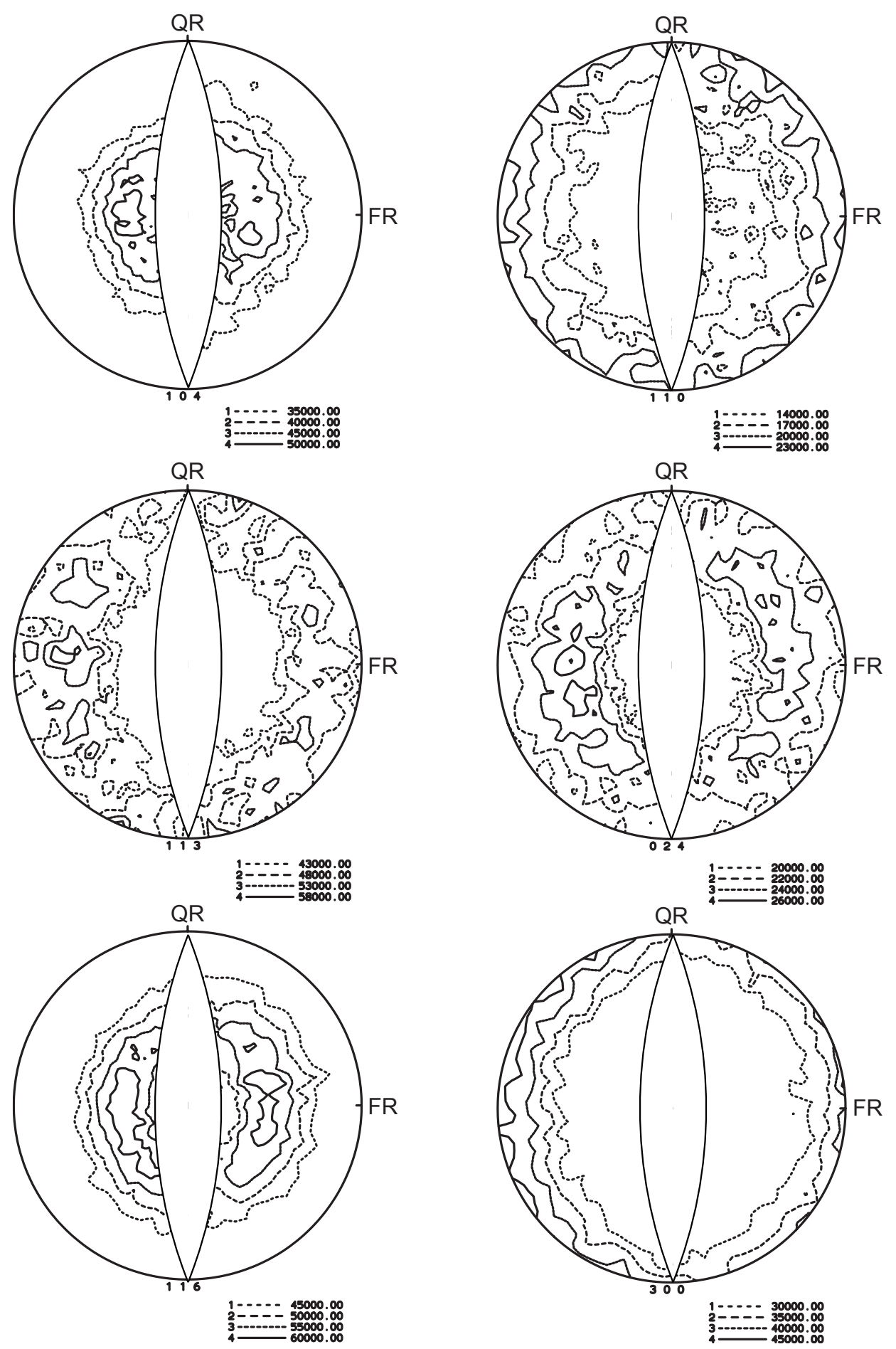

Abbildung D.63: Gemessene Polfiguren Gilox 10Vol\% 0,5 ml/s 

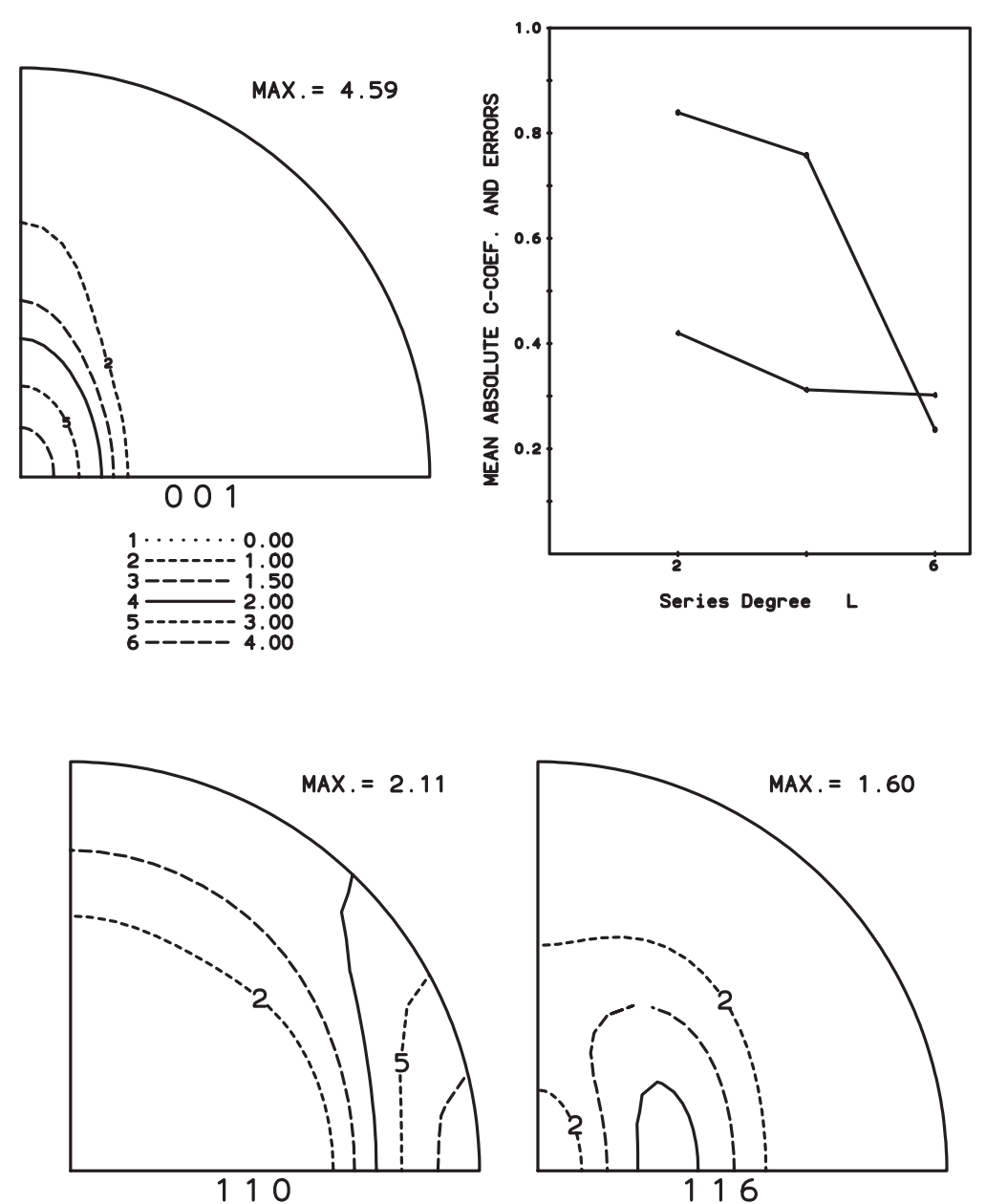

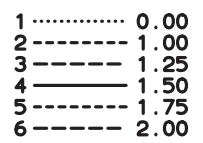

Abbildung D.64: Rückgerechnete Polfiguren, C-Koeffizienten und Fehler, Gilox $10 \mathrm{Vol} \% 0,5 \mathrm{ml} / \mathrm{s}$ 

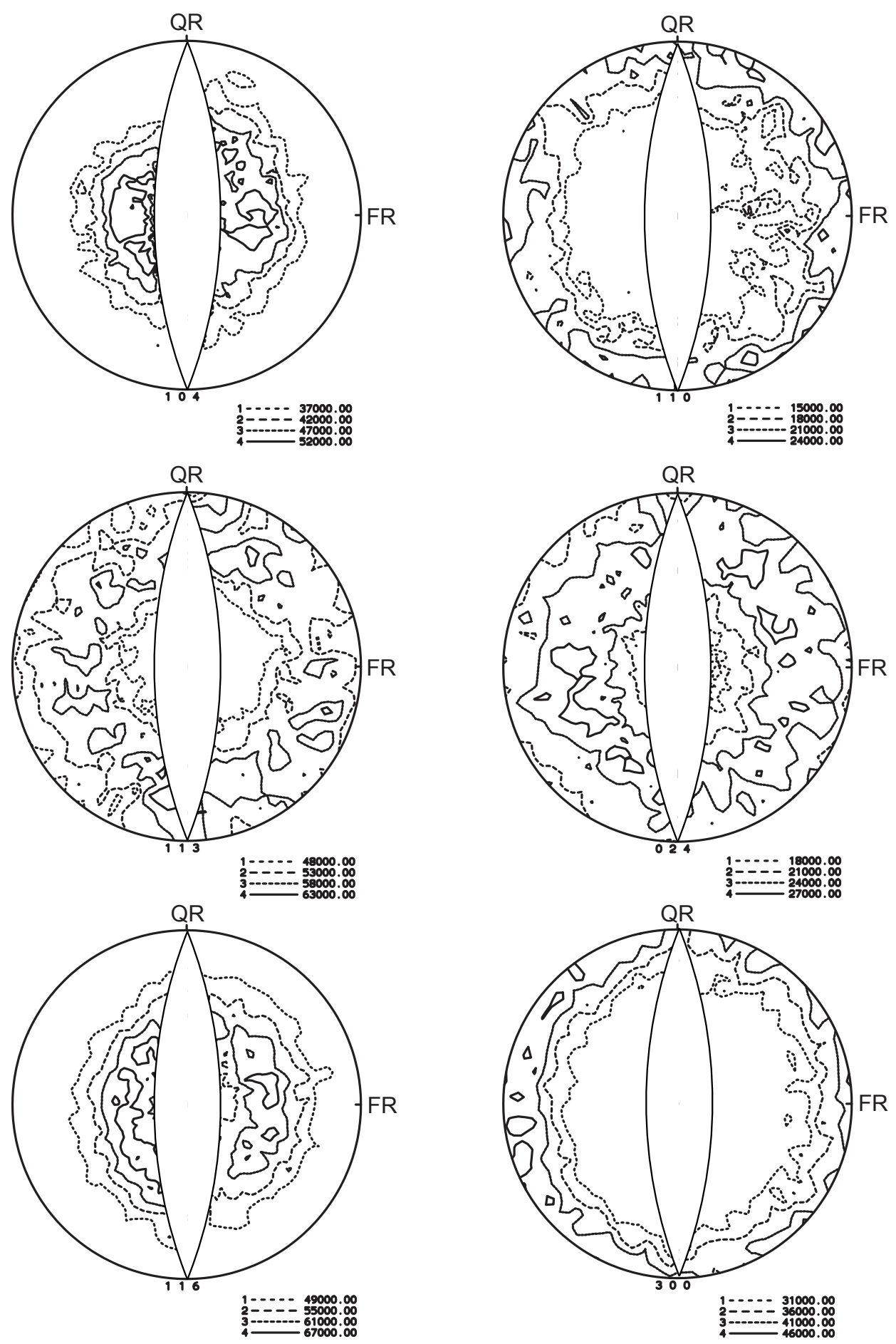

Abbildung D.65: Gemessene Polfiguren Gilox 10Vol\% $1 \mathrm{ml} / \mathrm{s}$ 

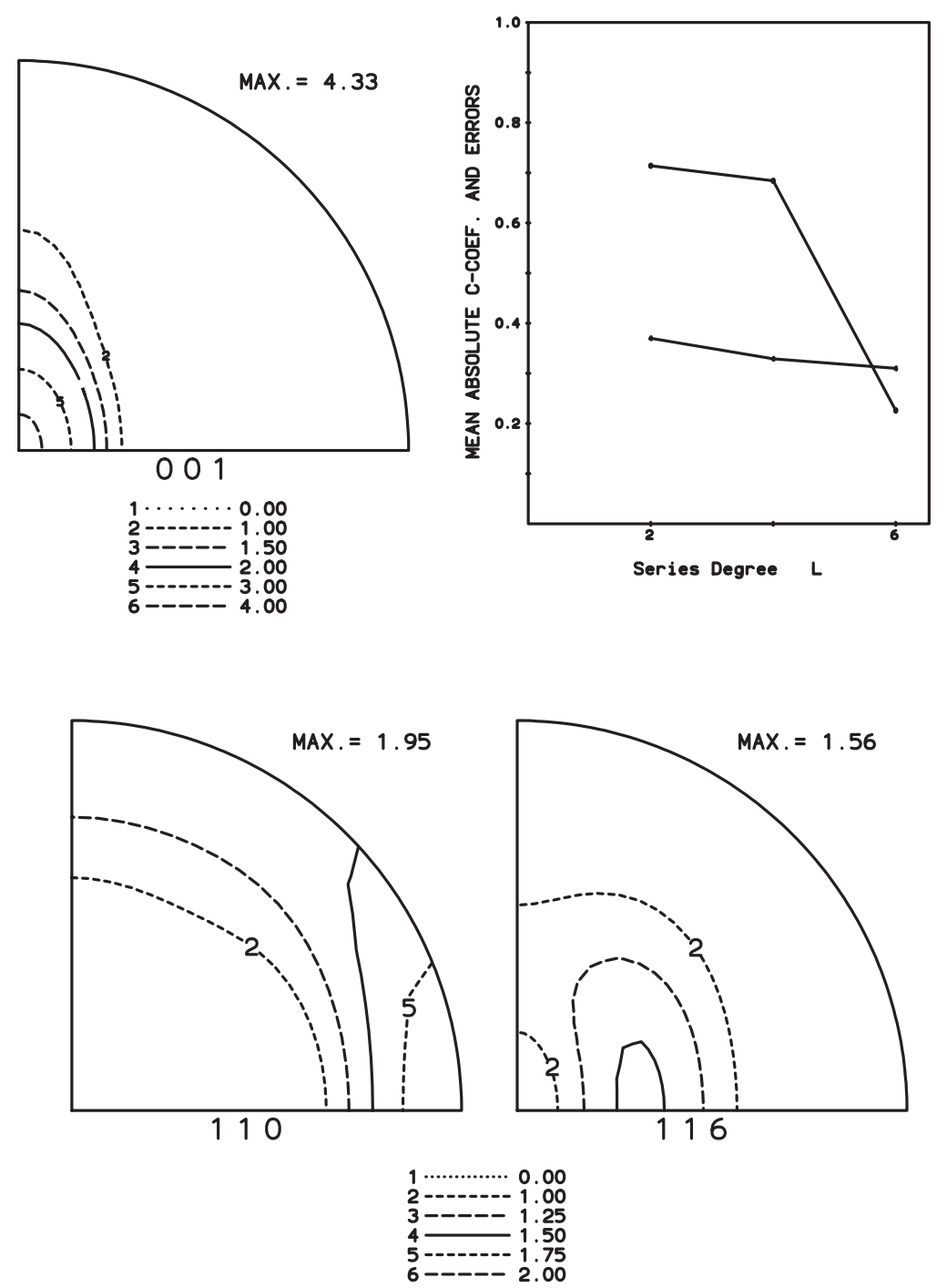

Abbildung D.66: Rückgerechnete Polfiguren, C-Koeffizienten und Fehler, Gilox $10 \mathrm{Vol} \% 1 \mathrm{ml} / \mathrm{s}$ 

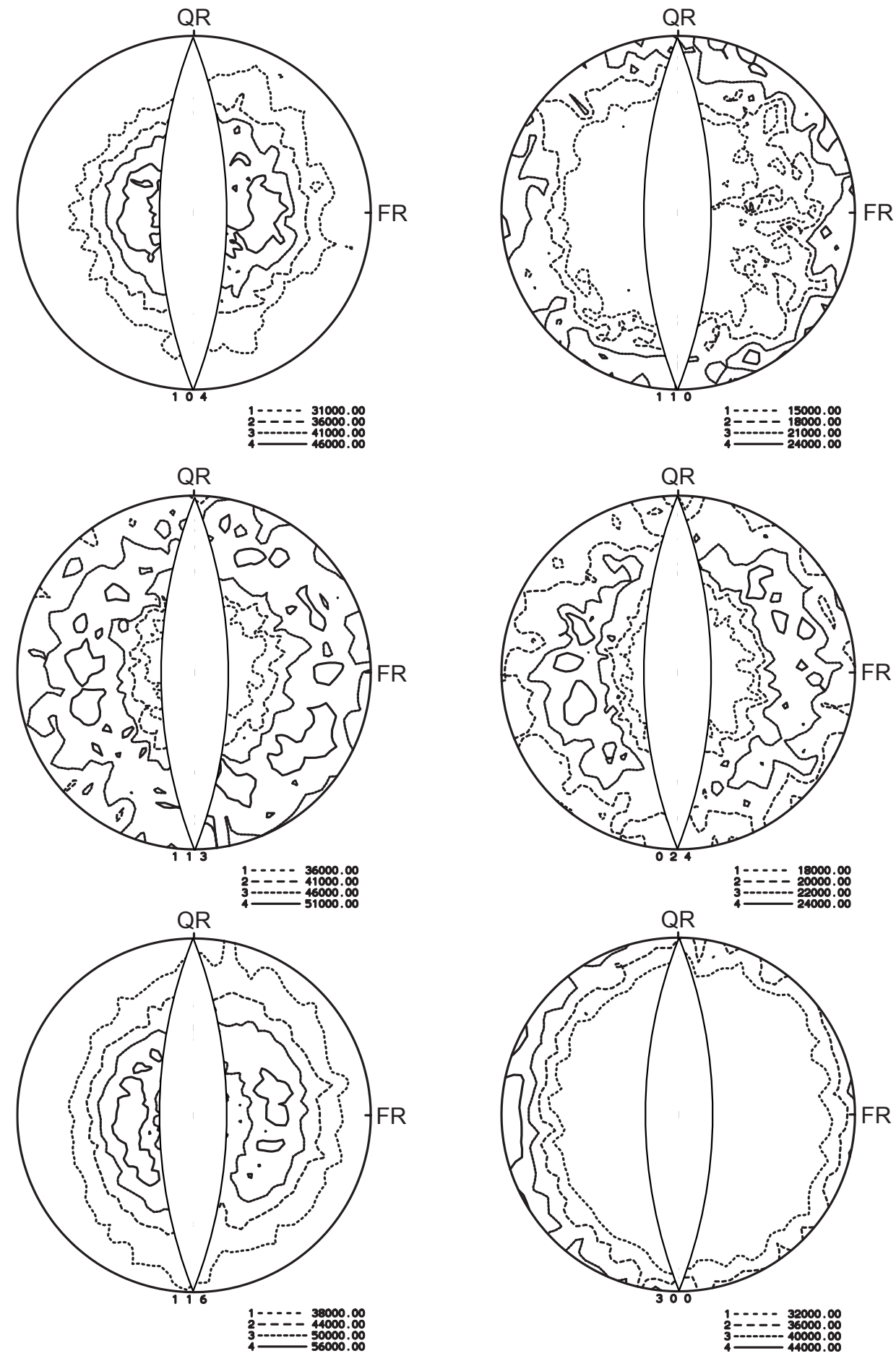

Abbildung D.67: Gemessene Polfiguren Gilox 10Vol\% $2 \mathrm{ml} / \mathrm{s}$ 

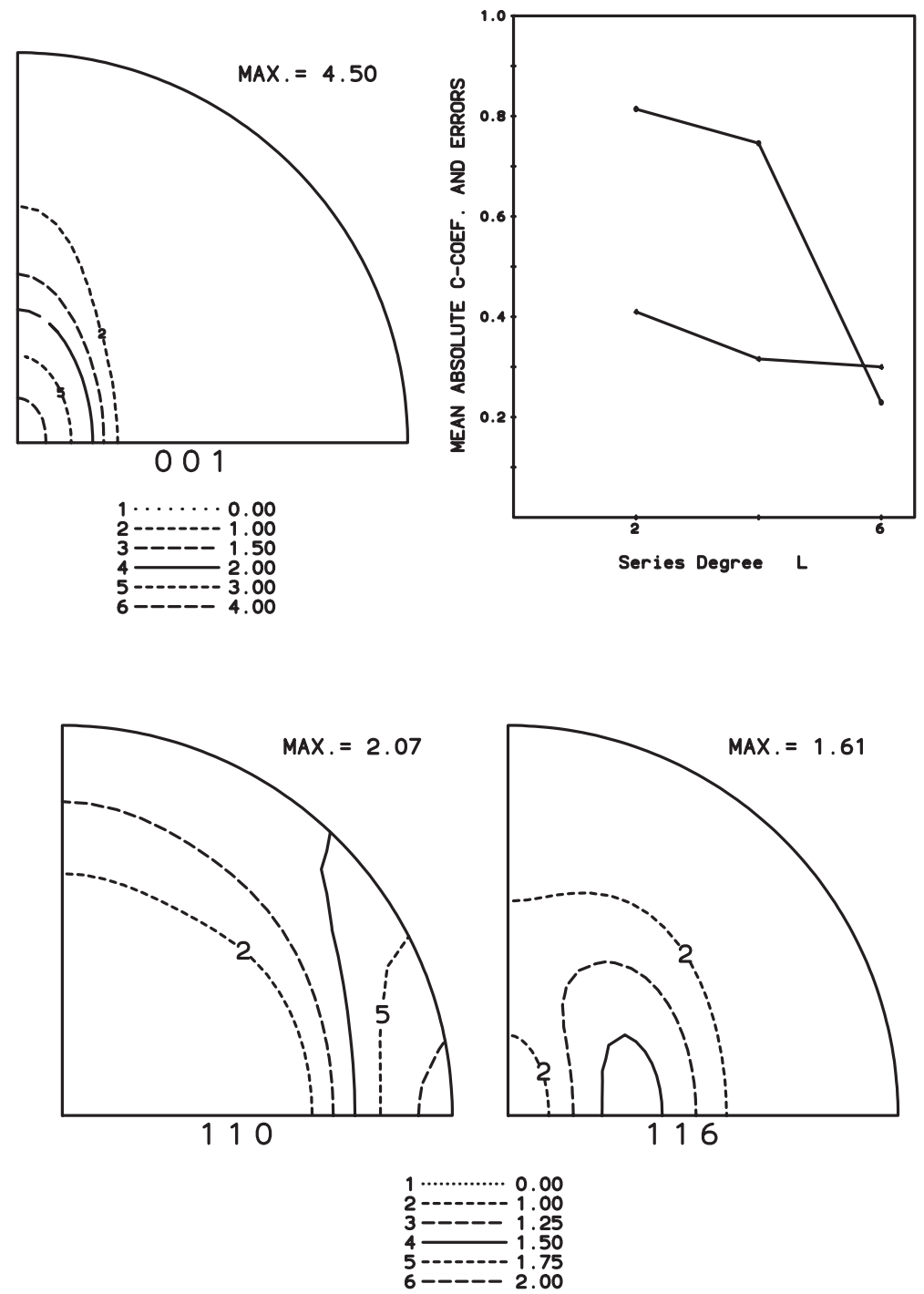

Abbildung D.68: Rückgerechnete Polfiguren, C-Koeffizienten und Fehler, Gilox $10 \mathrm{Vol} \% 2 \mathrm{ml} / \mathrm{s}$ 

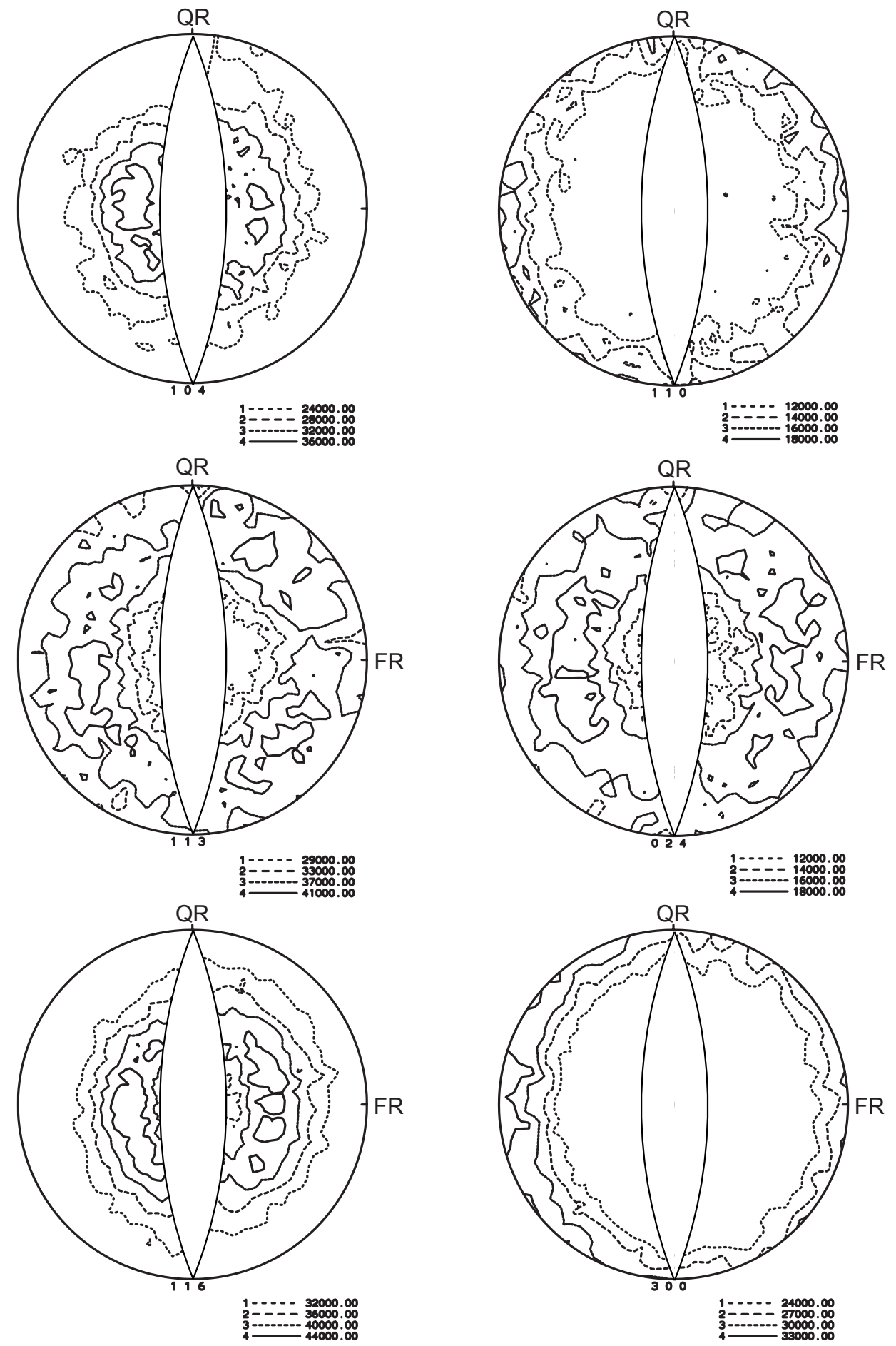

Abbildung D.69: Gemessene Polfiguren Gilox 10Vol\% 5 ml/s 

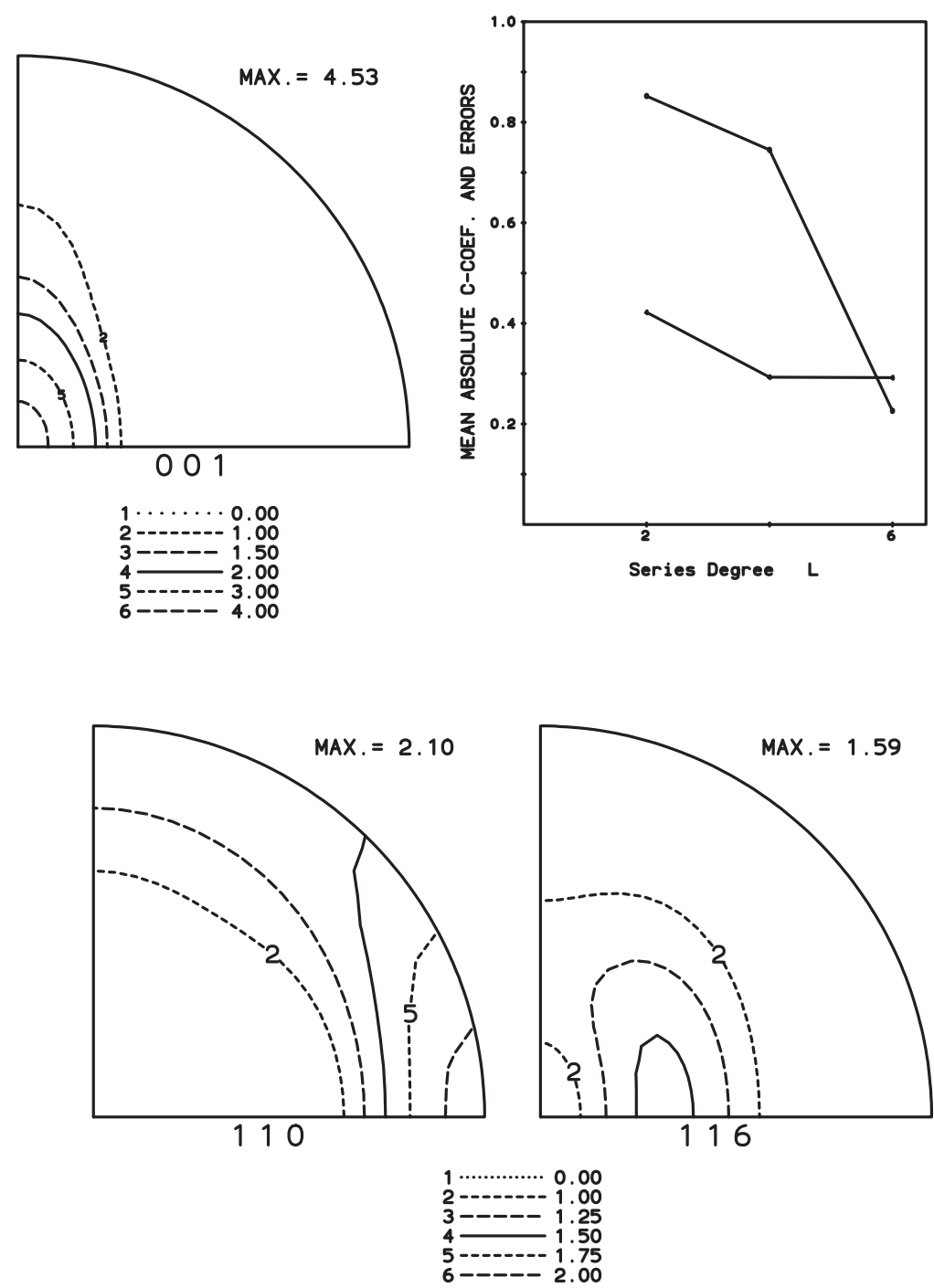

Abbildung D.70: Rückgerechnete Polfiguren, C-Koeffizienten und Fehler, Gilox $10 \mathrm{Vol} \% 5 \mathrm{ml} / \mathrm{s}$ 


\section{D.8 Probe Gilox $12,5 \mathrm{Vol} \%$}
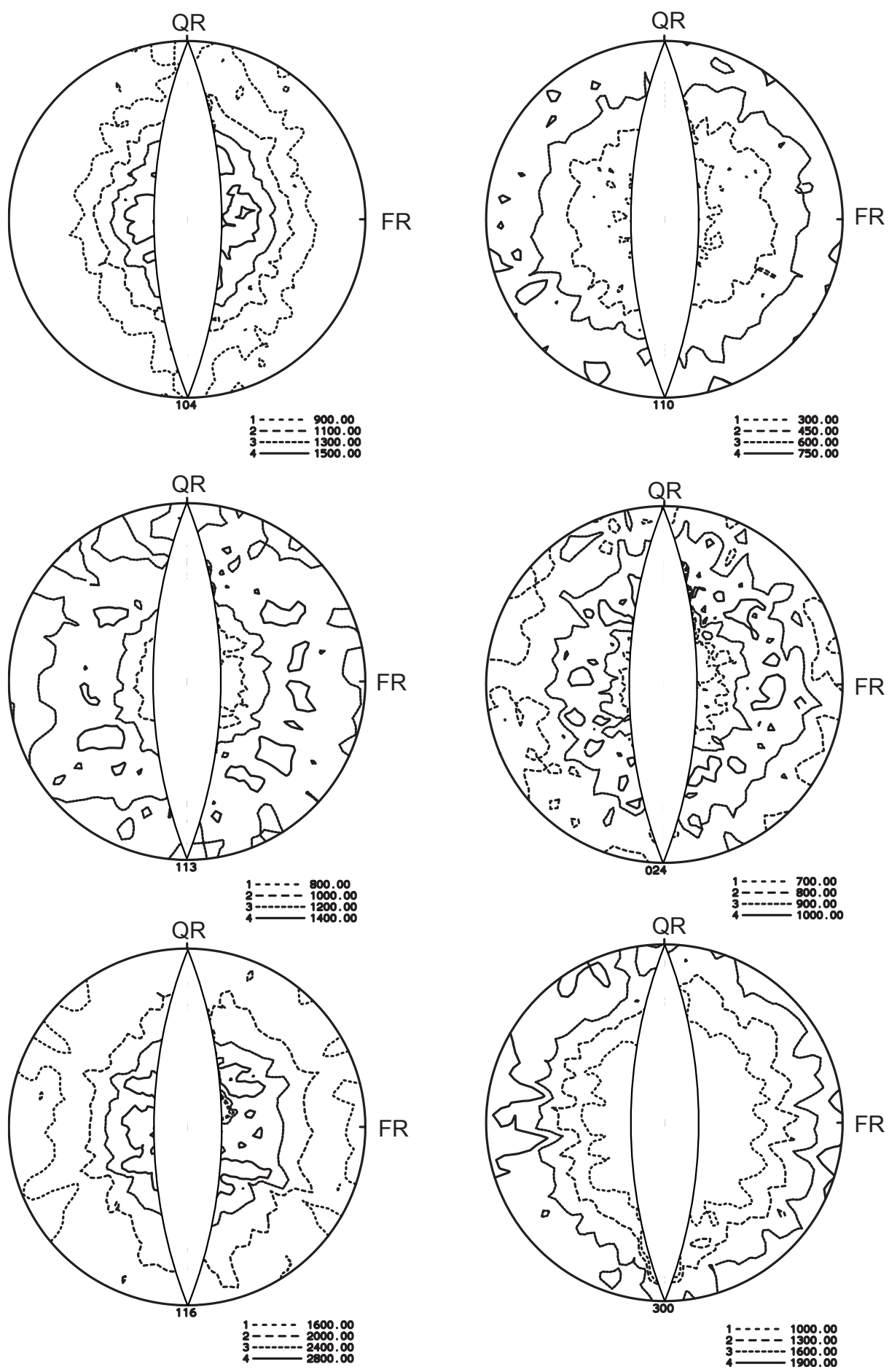

Abbildung D.71: Gemessene Polfiguren Gilox 12,5Vol\% 0,2 ml/s 

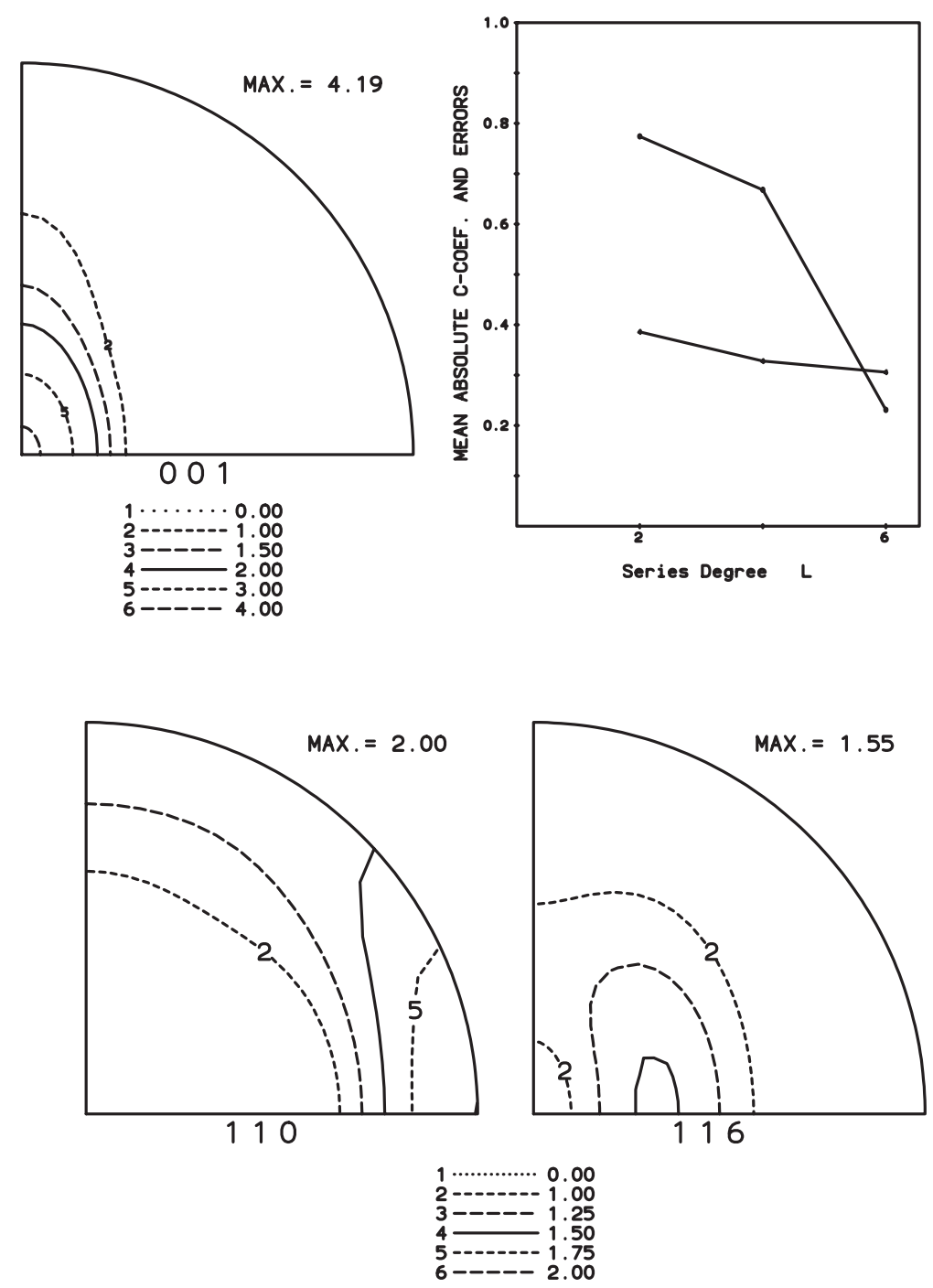

Abbildung D.72: Rückgerechnete Polfiguren, C-Koeffizienten und Fehler, Gilox $12,5 \mathrm{Vol} \% 0,2 \mathrm{ml} / \mathrm{s}$ 

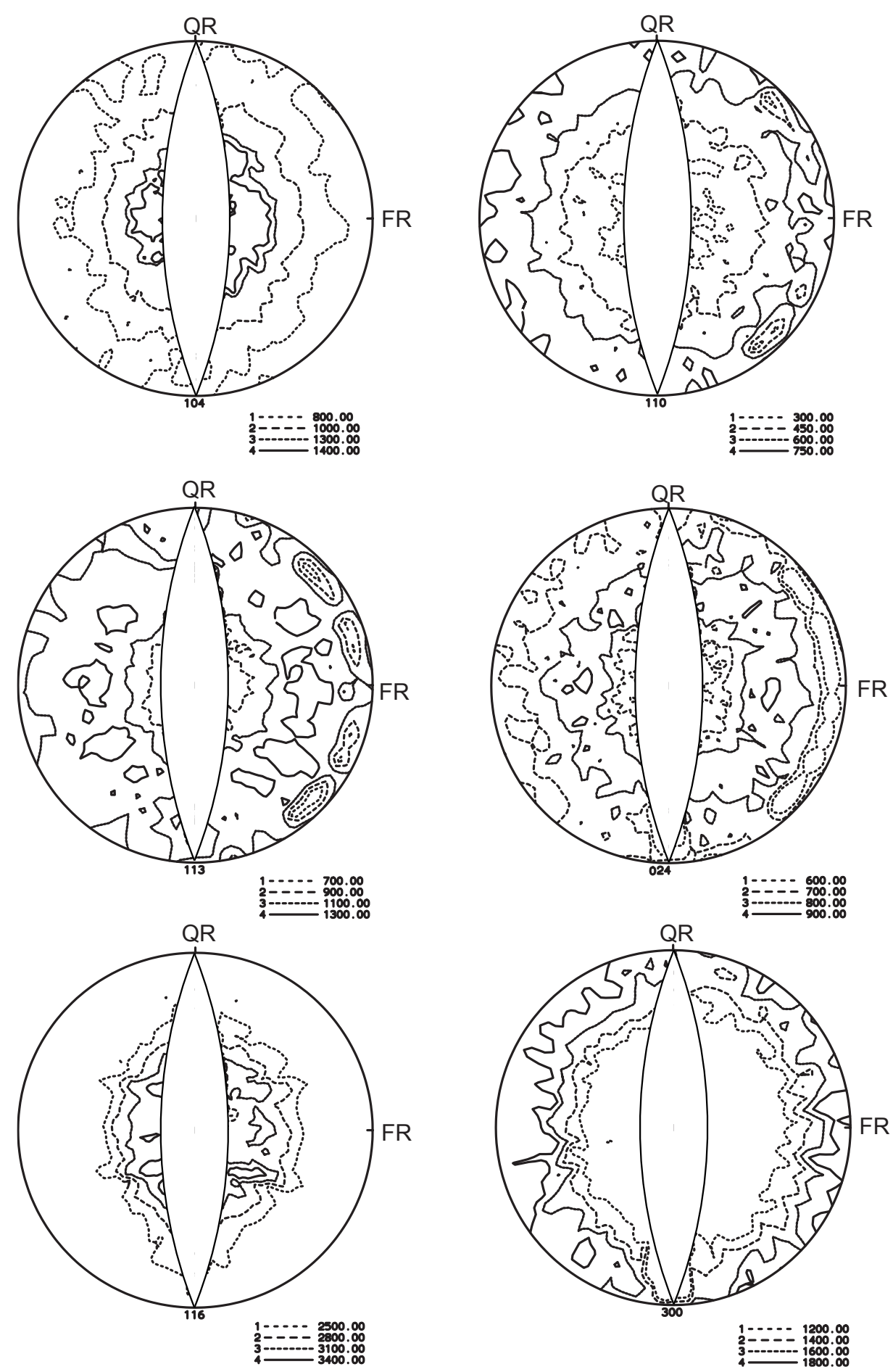

Abbildung D.73: Gemessene Polfiguren Gilox 12,5Vol\% 0,5 ml/s 

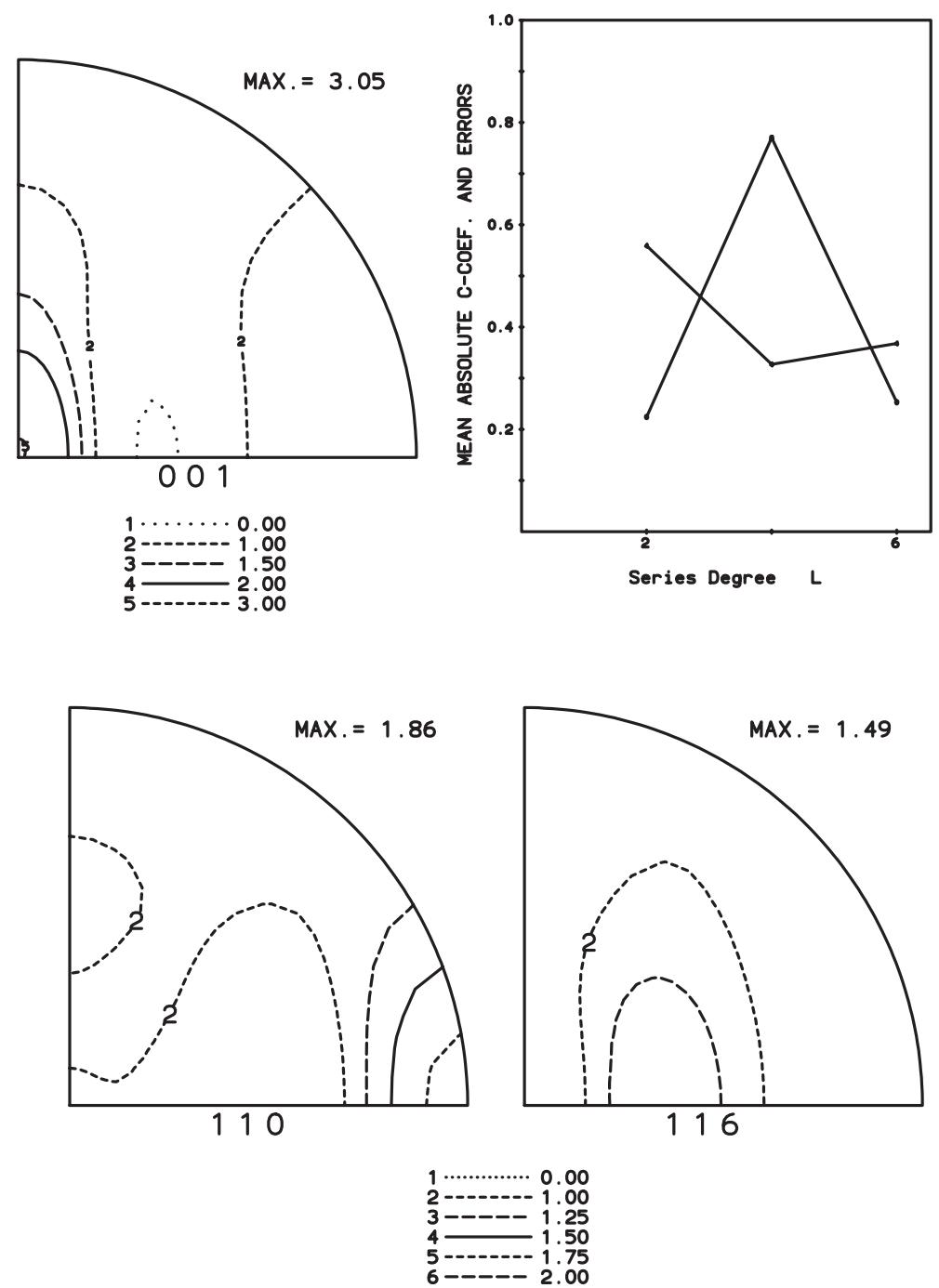

Abbildung D.74: Rückgerechnete Polfiguren, C-Koeffizienten und Fehler, Gilox $12,5 \mathrm{Vol} \% 0,5 \mathrm{ml} / \mathrm{s}$ 

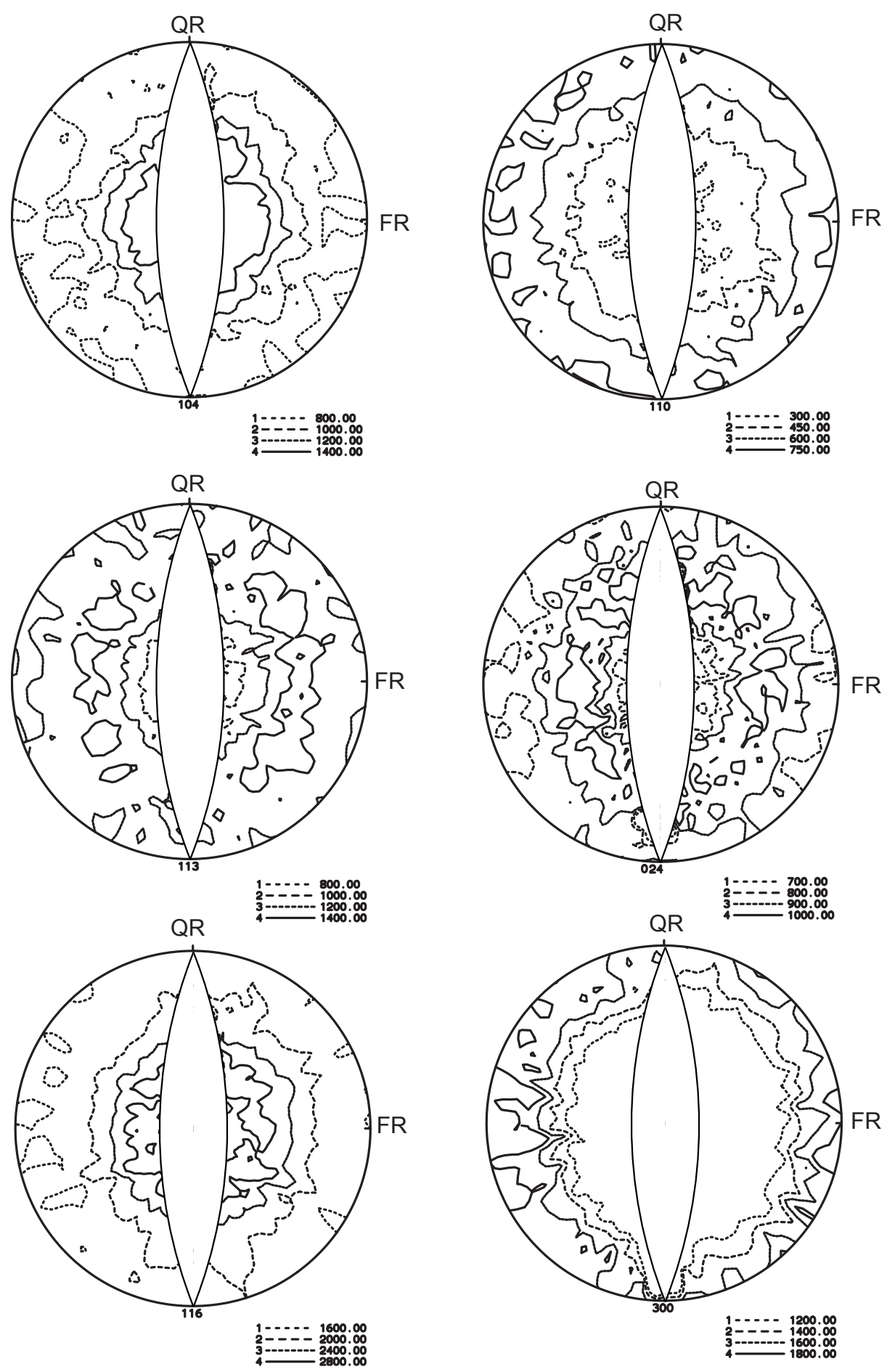

Abbildung D.75: Gemessene Polfiguren Gilox 12,5Vol\% $1 \mathrm{ml} / \mathrm{s}$ 

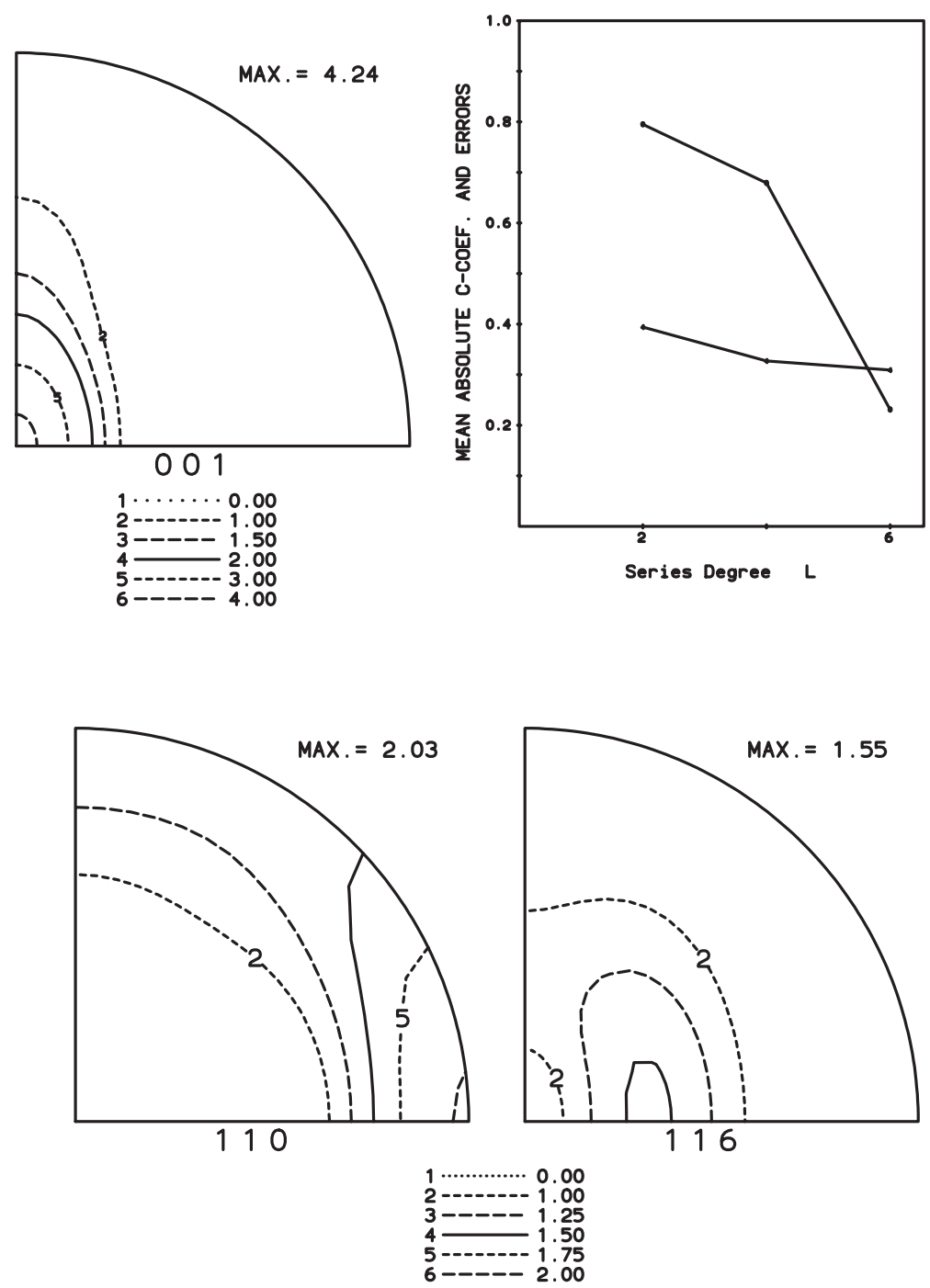

Abbildung D.76: Rückgerechnete Polfiguren, C-Koeffizienten und Fehler, Gilox $12,5 \mathrm{Vol} \% 1 \mathrm{ml} / \mathrm{s}$ 

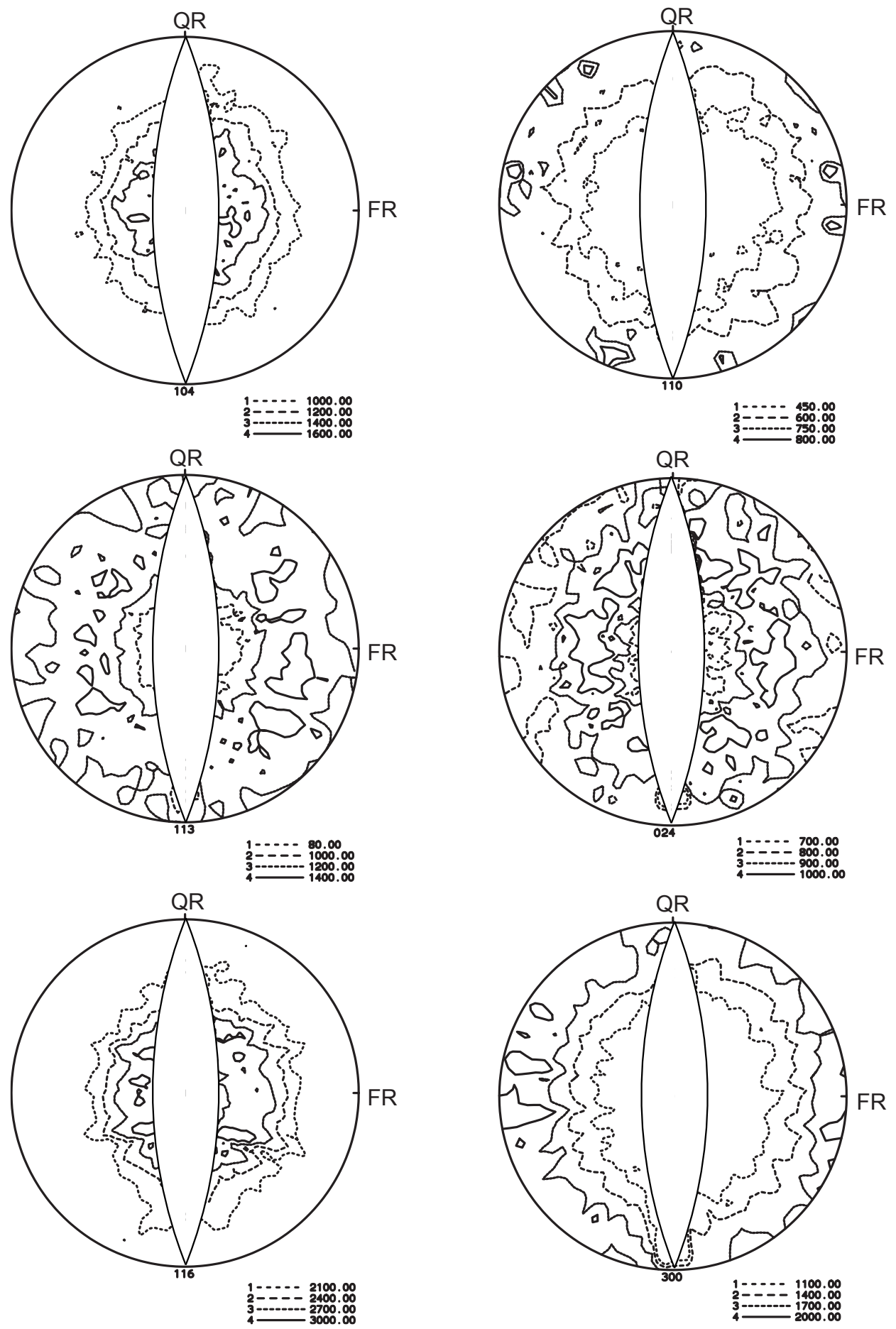

Abbildung D.77: Gemessene Polfiguren Gilox 12,5Vol\% $2 \mathrm{ml} / \mathrm{s}$ 

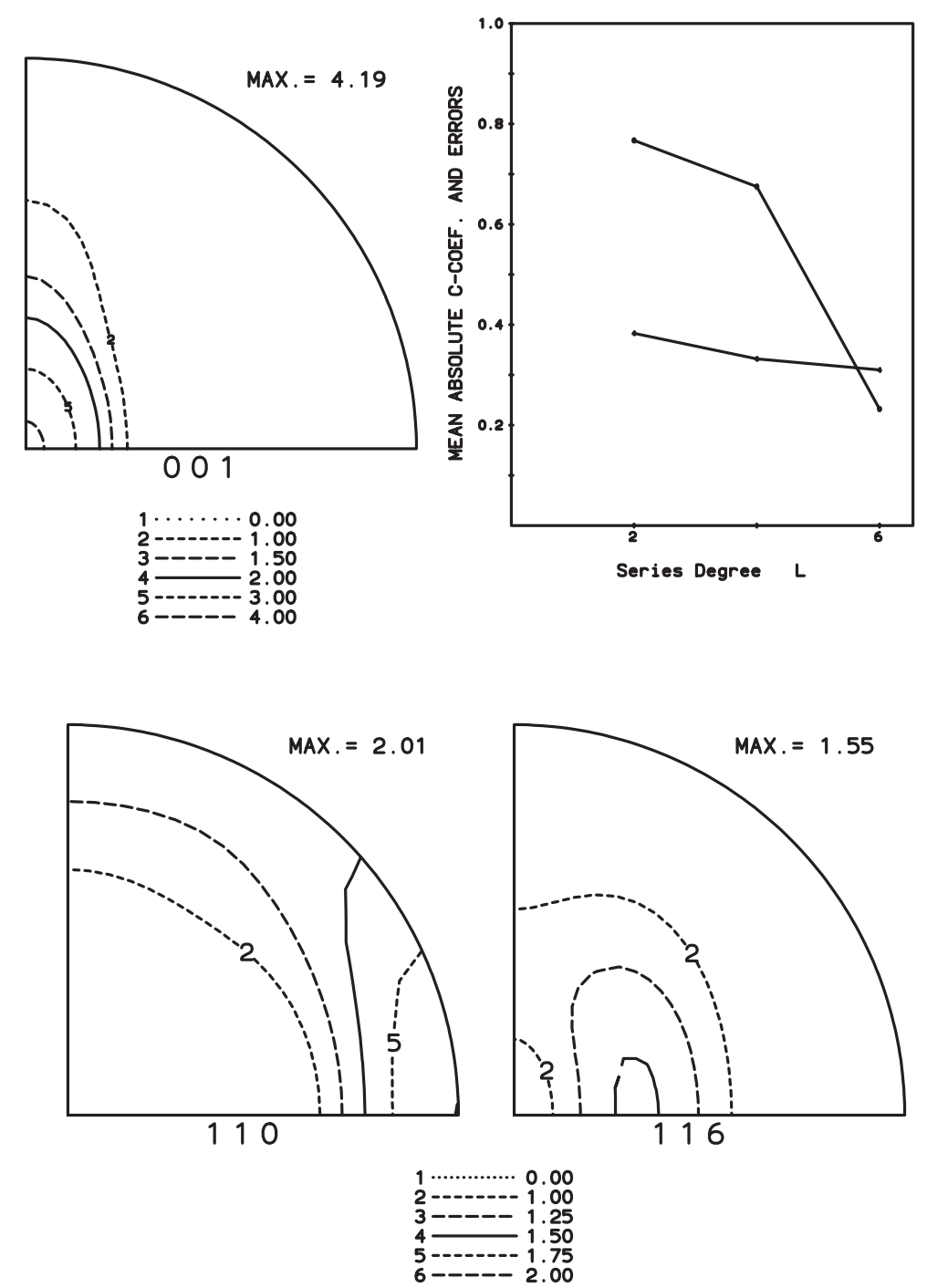

Abbildung D.78: Rückgerechnete Polfiguren, C-Koeffizienten und Fehler, Gilox $12,5 \mathrm{Vol} \% 2 \mathrm{ml} / \mathrm{s}$ 

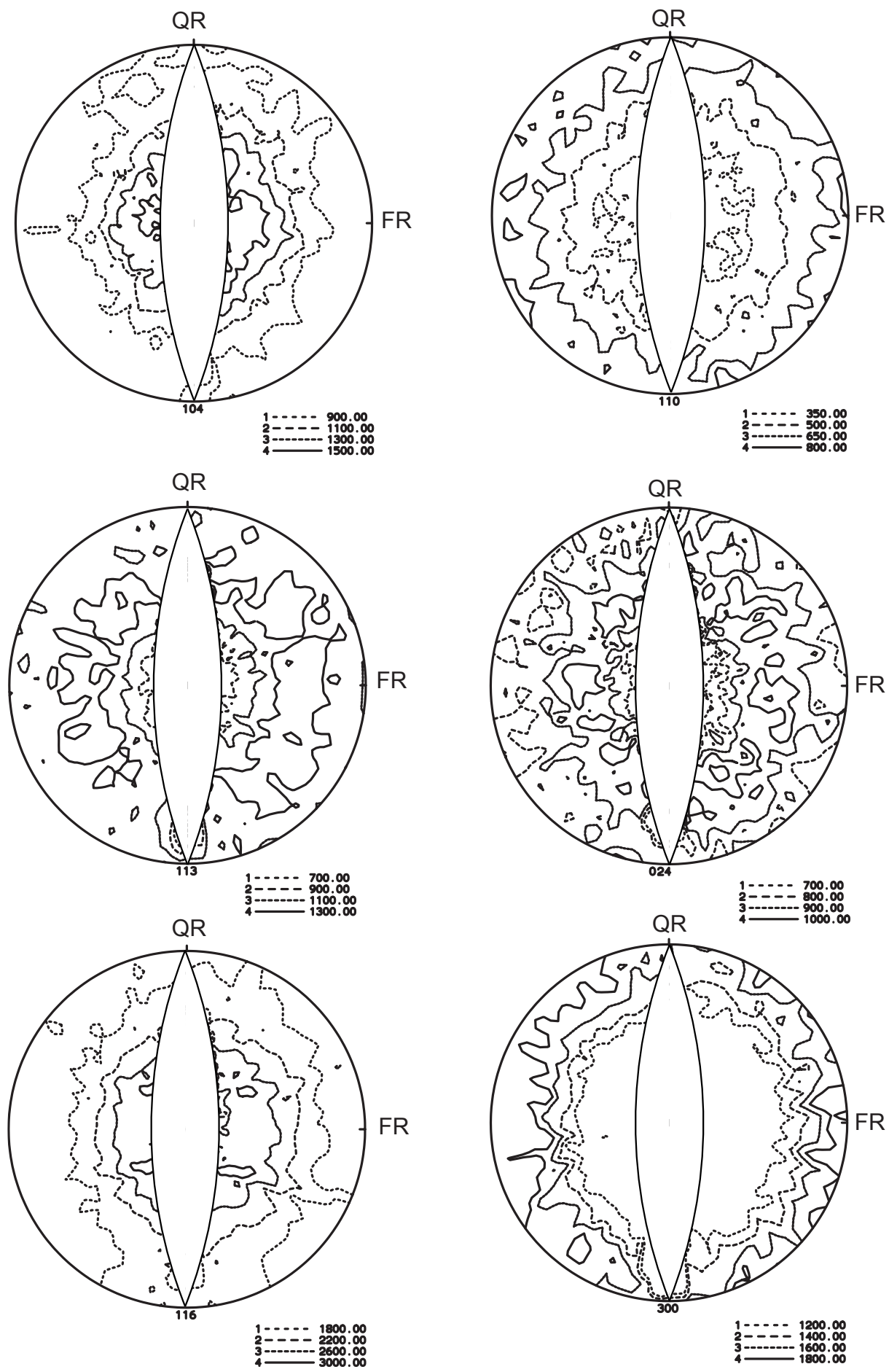

Abbildung D.79: Gemessene Polfiguren Gilox 12,5Vol\% $5 \mathrm{ml} / \mathrm{s}$ 

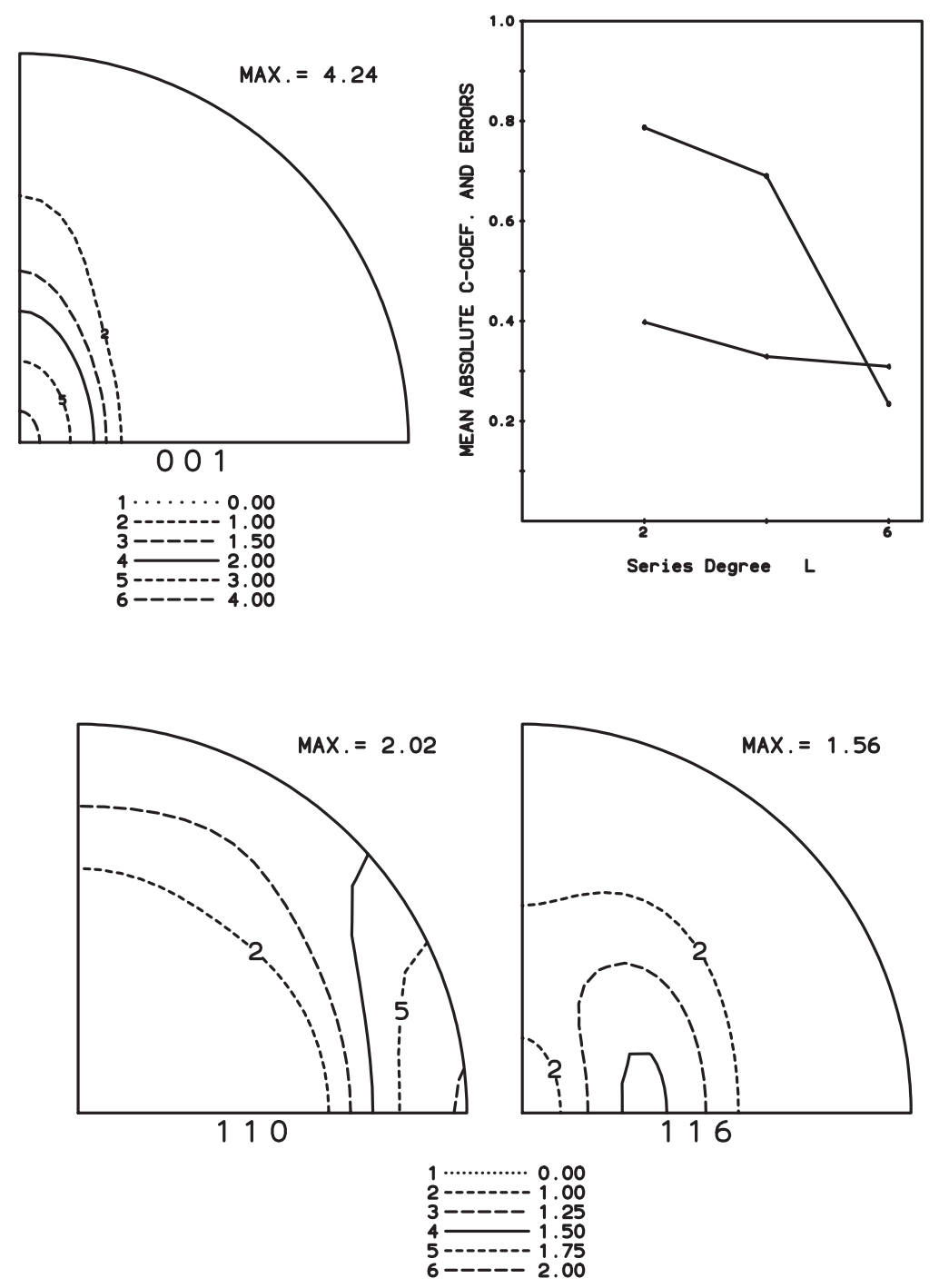

Abbildung D.80: Rückgerechnete Polfiguren, C-Koeffizienten und Fehler, Gilox $12,5 \mathrm{Vol} \% 5 \mathrm{ml} / \mathrm{s}$ 


\section{Lebenslauf}

Name:

Geburtstag:

Geburtsort:

Staatsangehörigkeit:

\section{Schulbildung:}

$08.81-06.88$

\section{Hochschulbildung:}

$04.90-09.95$

$10.95-06.00$

Thema der Diplomarbeit:

$03.01-11.04$

Thema der Promotion:

$12.04-03.05$
Ulf Garbe

20.03.1969

Höxter

deutsch

Gymnasium Liebigstrasse Holzminden;

Abschluss: Abitur

Georg-August-Universität Göttingen

Studienrichtung: Chemie

Georg-August-Universität Göttingen

Studienrichtung: Mineralogie

Abschluss: Diplom-Mineraloge

Ortsaufgelöste röntgenographische

Texturanalyse

Doktorat am Institut für Physik und Physikalische Technologien der Technischen Universität Clausthal

Texturentwicklung in Zwei-Phasen Strömungen

Doktorat am Geowissenschaftlichen Zentrum

der Universität Göttingen Abt. Kristallographie

Abschluss der Promotion 